
no. 3 

Marine Biological Laboritory

IIE A R Y

OCT $1-191 \%$

WOODS HOLE, MASS. 

THE AMERICAN MIDLAND NATURALIST Monograph No. 3 


\section{THE AMERICAN MIDLAND NATURALIST \\ Monograph Series}

\section{EDITORIAL STAFF}

THEODOR JUST

Editor, University of Notre Dame

Botany

Edward A. Chapin

Entomology

U. S. National Museum, Washington, D. C.

KENNETH W. COOPER

Princeton University, Princeton, N. J.

Carroll Lane Fenton

Rutgers University, New Brunswick, N. J.

Invertebrate Paleontology

John Hobart Hoskins

Paleobotany

University of Cincinnati, Cincinnati, Ohio

Remington Kellogg

Mammalogy

U. S. National Museum, Washington, D. C.

Jean Mrron Linsdale.

Ornithology

Hastings Reservation, Monterey, California

George Willard Martin Mycology

State University of Iowa, lowa City, lowa

Karl Patterson Schmidt.

Ichthyology and Herpetology

Chicago Natural History Museum, Chicago, Illinois

Harley Jones Van Cleave

Invertebrate Zoology

University of Illinois, Urbana, Illinois 


\title{
THE AMERICAN MIDLAND NATURALIST
}

Monograph No. 3

Edited by Theodor Just

Published by the University of Notre Dame,

Notre Dame, Ind.

\section{The Mosquitoes of the Southern United States East of Oklahoma and Texas}

\author{
By STANLEY J. CARPENTER \\ (Entomologist, National Biscuit Co., New York, N. Y.) \\ formerly \\ (Lt. Col., Sanitary Corps, A. U. S.) \\ and \\ WOODROW W. MIDDLEKAUFF \\ (Assistant Professor of Economic Entomology \\ University of California, Berkeley) \\ formerly \\ (Major, Sanitary Corps, A. U. S.) \\ and \\ ROY W. CHAMBERLAIN \\ (lst Lt., Sanitary Corps, A. U. S.)
}


Copyright, 1946

by

The American Midland Naturalist

University of Notre Dame,

Notre Dame, Ind. 


\section{CONTENTS}

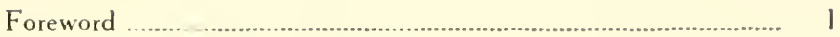

Bionomics and Relation to Disease ............................................ 2

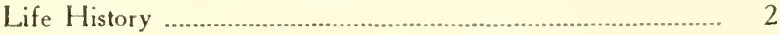

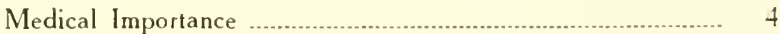

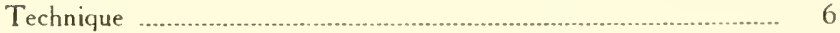

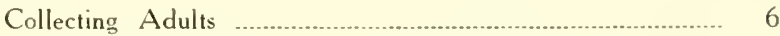

Transporting, Mounting and Storing Adult Specimens ......... 13

Preparing Male Terminalia for Study ............................. 15

Preparing Cibarial Armature for Study …....................... 16

Dissection for Malaria Parasites ........................................ 16

Collecting Mosquito Eggs .................................................... 18

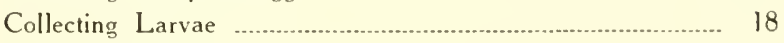

Rearing, Storing and Mounting Larvae .......................... 20

Mosquito Identification ........................................................ 23

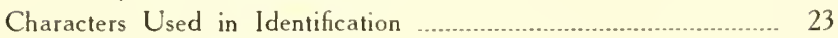

Adult Characters ........................................................... 23

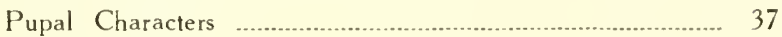

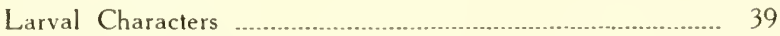

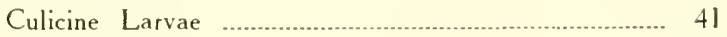

Anopheline Larvae ................................................. 44

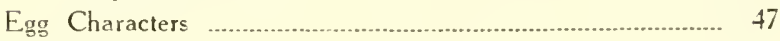

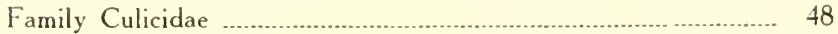

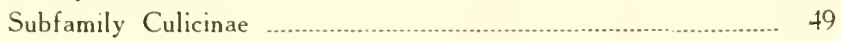

Tribe Anophelini ................................................................ 50

Genus Anopheles Meigen ................................................. 50

Anopheles atropos Dyar and Knab.............................. 52

Anopheles barberi Coquillett ................................. 55

Anopheles crucians bradleyi King................................. 58

Anopheles crucians crucians Wiedemann .................... 60

Anopheles crucians georgianus King............................. 64

Anopheles pseudopunctipennis pseudopunctipennis Theo-

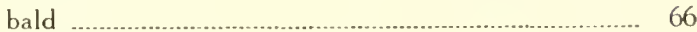

Anophel $r$ punctipennis (Say) ......................................... 69

Anopheles quadrimaculatus Say ................................... 73

Anopheles walkeri Theobald ….................................. 76

Anopheles albimanus Wiedemann ........................... 80

Tribe Megarhinini ....................................................................... 83

Genus Megarhinus Robineau-Desvoidy .............................. 83

Megarhinus rutilus Coquillett …................................. 84

Megarhinus septentrionalis Dyar and Knab ................... 84

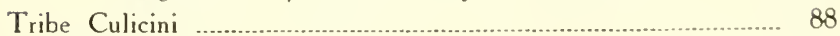

Genus Wyeomyia Theobald _................................... 89

Wyeomyia mitchellii (Theobald) ….......................... 90 
Wyeomyia smithii (Coquillett) …................................ 93

Wyeomyia vanduzeei Dyar and Knab .......................... 96

Genus Uranotaenia Lynch-Arribalzaga ................................. 100

Uranolaenia lowii Theobald _.._. 101

Uranotaenia sapphirina (Osten Sacken) ........................ 104

Genus Culiseta Felt _............................................................ 107

Culiseta inornata (Williston) ....................................... 108

Culiseta me! anura (Coquillett) .............................................. 112

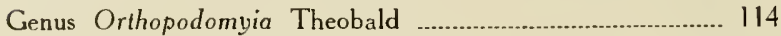

Orthopodomyia alba Baker ............................................... 114

Orthopodomyia signifera (Coquillett) .......................... 116

Genus Mansonia Blanchard ................................................... 120

Mansonia indubitans Dyar and Shannon ....................... 121

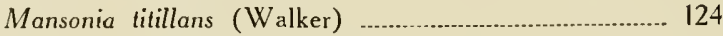

Mansonia perturbans (Walker) .................................. 127

Genus Psorophora Robineau-Desvoidy ............................... 130

Psorophora ciliata (Fabricius) _............................................. 133

Psorophora howardii Coquillett _................................ 136

Psorophora cyanescens (Coquillett) …...................... 139

Psorophora ferox (Humboldt) .................................. 142

Psorophora horrida (Dyar and Knab) ...................... 145

Psorophora longipalpis Roth ........................................ 147

Psorophora varipes (Coquillett) .............................. 150

Psorophora confinnis (Lynch-Arribalzaga) ................... 153

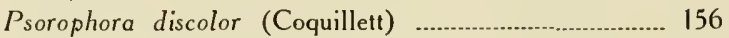

Psorophora pygmaea (Theobald) ............................. 160

Psorophora signipennis (Coquillett) .............................. 162

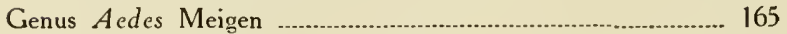

Aedes atlanticus Dyar and Knab ............................ 170

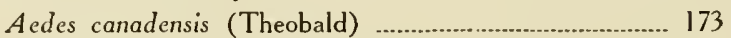

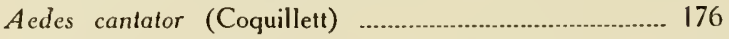

Aedes dorsalis (Meigen) ............................................... 179

Aedos dupreei (Coquillett) ................................. 182

Aedes fulvus pallens Ross ............................................ 185

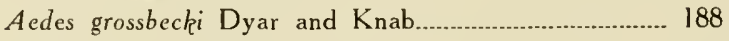

Aedes infirmatus Dyar and Knab ........................................

Aedes mathesoni Middlekauff ...................................... 194

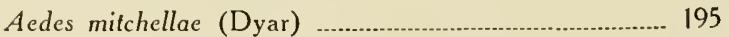

Aedes nigromaculis (Ludlow) ............................................ 197

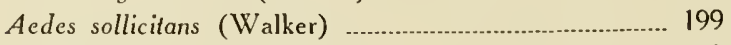

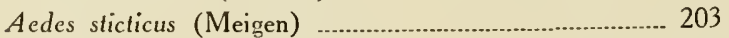

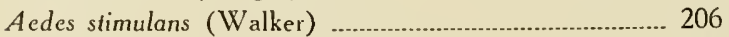

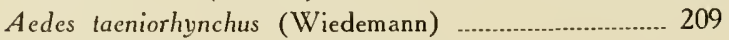

Aedes thibaulti Dyar and Knab ................................... 211

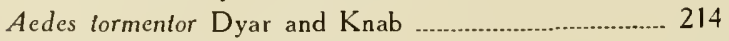

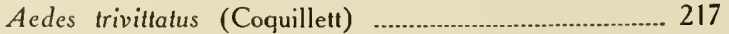

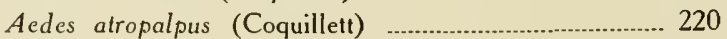

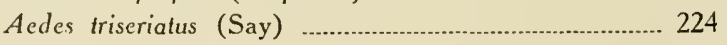


Aedes aegypti (Linnaeus)

Aedes vexans (Meigen)

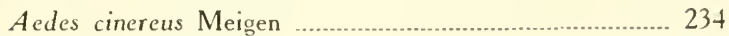

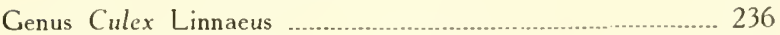

Culex bahamensis Dyar and Knab …....................... 240

Culex nigripalpus Theobald ......................................... 242

Culex pipiens Linnaeus ................................................ 245

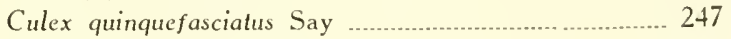

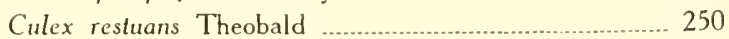

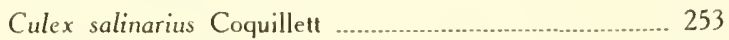

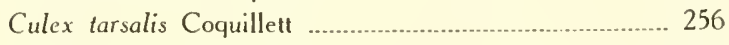

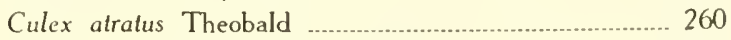

Culex erraticus (Dyar and Knab) _.................................. 262

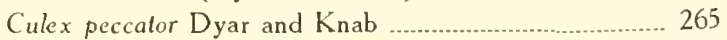

Culex pilosus (Dyar and Knab) ........................... 268

Culex apicalis Adams ........................................................... 271

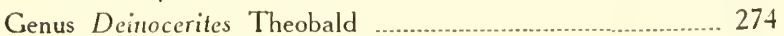

Deinocerites cancer Theobald ….................................. 275

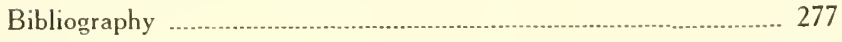

Index 


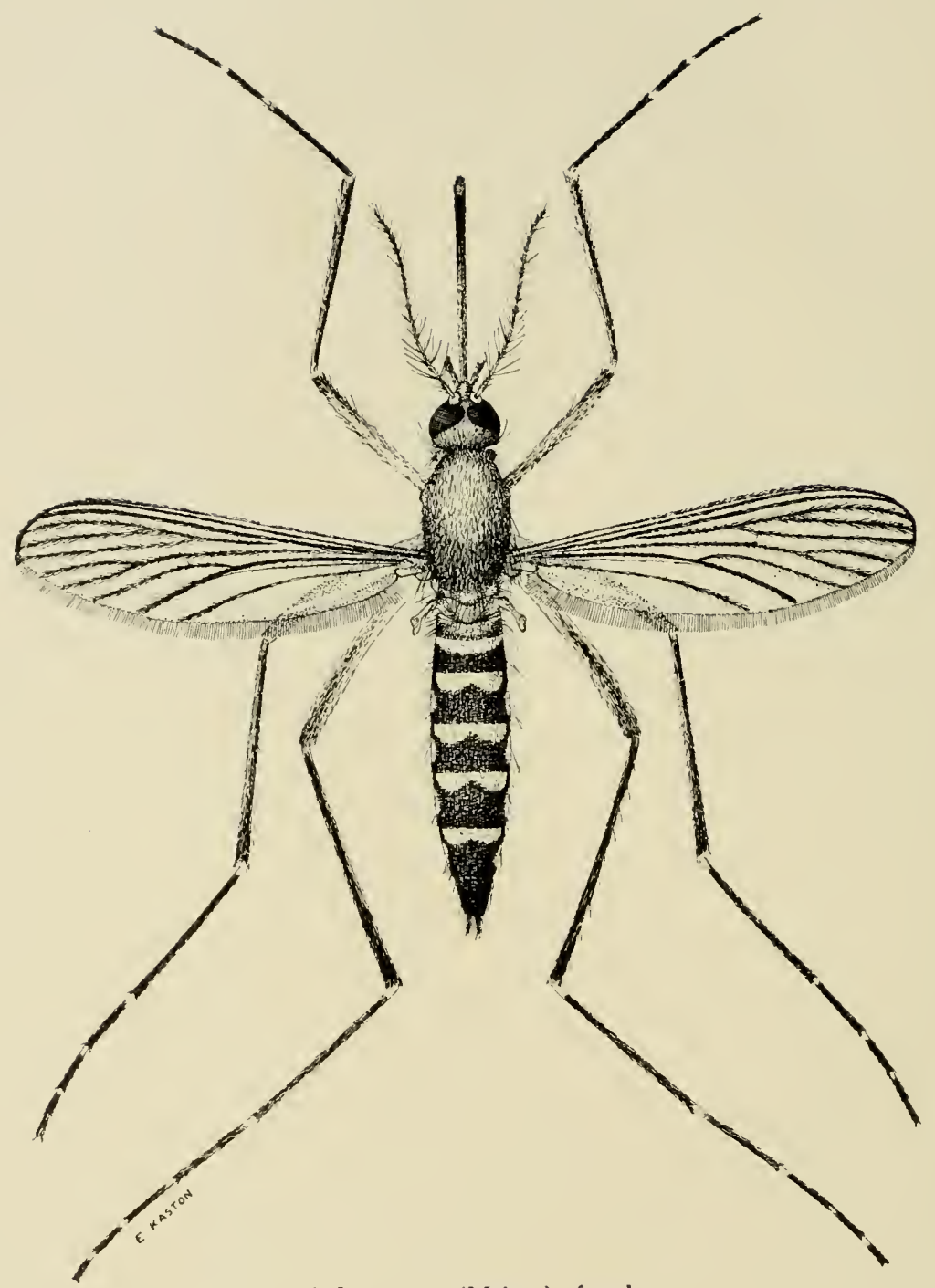

Aedes vexans (Meigen), female. 


\section{The Mosquitoes of the Southern United States, East of Oklahoma and Texas}

\section{Foreword}

In this monograph the authors have attempted to give an up-to-date account of the mosquitoes occurring in the southern United States east of Oklahoma and Texas, and it is hoped that the publication will be a useful reference for those interested in the mosquitoes of this region.

The keys and descriptions are based on the known mosquito fauna of Alabama, Arkansas, Florida, Georgia, Kentucky, Louisiana, Mississippi, Missouri, North Carolina, South Carolina, Tennessee, and Virginia. With rare exceptions, the work is also applicable to the species found in bordering areas, particularly the eastern portions of Texas, Oklahoma, and Kansas, and the southern portions of Illinois, Indiana, Ohio, West Virginia, and Maryland.

The mosquitoes of the southern United States differ greatly in their habits and importance, thus making identification a necessary prerequisite for a successful control program. The lack of adequate keys, descriptions of species and illustrations has often contributed to the difficulty of making accurate identifications. We have attempted to fill this need by including necessary keys, specific descriptions, and illustrations showing diagnostic features of adult females, male terminalia, and larvae.

Distribution records given for each species have been compiled largely from published state and regional lists. We have attempted, insofar as possible, to cite the more recent and readily available references to state collection records rather than earlier references, many of which are no longer available. References to seasonal distribution are based principally upon Army mosquito collection records of the Fourth Service Command Medical Laboratory.

The systematic arrangement of genera and subgenera follows rather closely that of Edwards (1932). Since the species are well catalogued in available works, including Dyar (1928), Edwards (1932), and Matheson (1944), few citations other than the original ones are given under the species.

Techniques for collecting and studying mosquitoes and discussions on bionomics and medical importance are included to make the monograph a practical handbook for entomologists and public health workers.

For other comprehensive works which include the mosquitoes found in the southern United States the reader is referred to Howard, Dyar and Knab (1912-17); Dyar (1922); Dyar (1928); King, Bradley and McNeel (1944); and Matheson (1944). 
The authors are indebted to Drs. E. A. Chapin and Alan Stone of the U. S. National Museum for helpful suggestions, for assistance in locating critical specimens and for reviewing the manuscript. We wish to express our gratitude to Brigadier General James Stevens Simmons, M. C., Chief, Division of Preventive Medicine, Office of The Surgeon General, U. S. Army; Colonel W. A. Hardenbergh, SnC., Chief, Sanitary Engineering Section of that office; and Colonel Loren D. Moore, M. C., Commanding Officer, 4th Sv.C. Med. Lab., for encouragement and aid. It is also desired to express appreciation to Mrs. Elizabeth Kaston for the illustrations and to the following members of the Entomology Department of the 4th Sv. C. Med. Lab. for valuable assistance: Capt. James P. Toffaleti, SnC., Capt. Harry H. Hoogstraal, SnC., Capt. John A. Carollo, SnC., 2nd Lt. John F. Wanamaker, SnC., Sgts. Daniel Sudia and T. E. Duffey, Leonora Peeples, Jeanne Spence, Frances E. Smith, Winona Gilstrap, Rosemary Baker, and Evelynne Cox.

\section{Bionomics and Relation to Disease}

\section{LifE History}

Mosquitoes undergo a complete metamorphosis, thus passing through four distinct stages during their life cycle; namely, egg, larva, pupa, and adult or imago.

Egg.-The choice of the larval habitat is made by the female at the time of oviposition. The egg-laying habits of mosquitoes as well as the type of eggs deposited are quite variable but are reasonably similar within a genus. The eggs are deposited singly or in boat-shaped rafts. The females of the genera Culex, Mansonia, Culiseta, and Uranotaenia generally place their rafts of eggs upon the surface of quiet waters, however, the egg raft of Culex apicalis is deposited above the water level on the banks of pools where they remain unhatched until a rainfall causes them to be launched upon the surface of the adjacent water. Anopheles females usually lay their eggs on the surface of still waters and as a result of surface tension they are frequently drawn together to form star-shaped clusters. Psorophora and many Aedes deposit their eggs singly in protected places in moist or dry depressions in the ground, where they remain dormant for a considerable period of time before rains, fluctuating tides, floods or melting snows submerge them and permit hatching. In the absence of water many of these eggs are capable of remaining viable for months or even several years.

Larva.-After the mosquito egg has been in contact with water for a sufficient length of time, the larva cuts its way out by means of the egg breaker on the head and escapes into the water. During growth the larva sheds its skin four times, the stages between moults being called instars.

The food of mosquito larvae consists chiefly of minute plant and animal life and fragments of organic and inorganic debris which are swept into the mouth by the sweeping action of the mouth brushes. Anopheles larvae feed on the surface, while most Culicine larvae feed below the surface of the water. Anopheles larvae rotate the head $180^{\circ}$ and with the mouth brushes upward, sweep the surface film for food. Megarhinus larvae and several species of Psorophora are predacious and feed primarily upon other species of mosquito larvae. 
The duration of the larval stage depends upon inherent characteristics of the species concerned and upon several external factors, such as water, temperature, and the availability of a suitable food supply. Under optimum conditions many species of mosquitoes pass through the larval stage in about a week to ten days, while others may stretch this period into several months. Some of the temporary pool breeders pass through this stage in 4 to 5 days. Mosquito larvae are found in nearly all types of permanent and temporary waters, both fresh and brackish. Specific reference to the typical larval habitats will be found under the discussions of the individual species.

All mosquito larvae, except those in the genus Mansonia, must come to the surface of the water at periodic intervals to renew their supply of oxygen. The Mansonia larvae as well as pupae attach themselves to the underwater portions of certain aquatic plants and secure their oxygen from the plant tissues.

Pupa.-With the fourth moult the pupa or "tumbler" stage appears. Unlike the larvae, the pupae are lighter than water and do not feed. They are very sensitive to disturbances of the water and will go downward very rapidly in a jerking, trembling motion when disturbed. A pair of large breathing tubes (trumpets) on the cephalothorax enables the pupa to break the surface film and obtain atmospheric oxygen. The pupal stage lasts but a few days (usually 2 or 3 ) then the pupa extends its abdomen parallel to the surface film in preparation for the emergence of the adult.

Adult.-When the adult is ready to emerge it swallows some of the air enclosed within the pupal skin and by increased muscular action and internal pressure causes a split to occur on the dorsum of the cephalothorax, permitting the adult to emerge. The newly emerged adult uses the cast skin or adjacent vegetation as a float and remains quiescent for a short period of time sufficient to dry and harden its body before flying away.

The species of Anophelines and Culicines vary considerably in their habits, and the habits of the larvae and adults are particularly diversified. Only a brief discussion of the more obvious characteristics is included here.

The food of a female mosquito includes not only the nectar of flowers and the juices of fruits, but also, in all but a few of our species, animal blood. The maxillae and mandibles of male mosquitoes are insufficiently developed for blood sucking purposes, consequently the male is an obligatory vegetarian.

The majority of the Anophelines and many of the Culicines feed by preference during the twilight hours of morning and evening, although some will attack in direct sunlight as well. The females continue to feed throughout their life, which in non-nibernating species may, in exceptional cases, be as long as several months.

It appears that warm-biooded animals and birds are the most common sources of blood, however, even cold-blooded vertebrates are attacked by some species. Some mosquitoes are opportunists and will attack indiscriminately any warm-blooded animal encountered (zoophilism). On the other hand, certain species, among which are numbered some of the Anopheles, show a marked predilection for the blood of man (androphilism). Domes- 
ticated species such as Aedes aegypti feed eagerly on human blood and definitely prefer it. Semi-domesticated and wild species vary in their feeding preferences.

The length of life of adult mosquitoes under natural conditions is diffcult to determine and great variation occurs. Appearance of males in the spring is usually taken as indicating the emergence of the first brood.

The mating habits vary considerably among the different species. With many species of Anophelines and Culicines the males swarm together in large numbers in the evening at which time the females invade the swarm, each emerging united with a male in the act of copulation. Some mosquitoes will mate in very small containers while in captivity. Males and females of a few species have been observed in copulation while resting on objects near their breeding places.

The flight range varies considerably with different species of mosquitoes. The salt marsh mosquitoes are notorious wanderers, a flight of 50 miles or more is not considered too unusual. Aedes vexans is a strong flier and has been taken 10 to 15 miles from points where stained specimens were released. Most Anophelines are considered to be weak fliers and seldom disperse more than a mile from their breeding areas, however, many exceptions to this broad statement have been recorded as a result of the recapture of marked specimens.

Males are seldom found far from their breeding grounds, although upon occasions they are reported to accompany the migrating females. Flight may be with or against winds of moderate velocity. Few mosquitoes are on the wing when the velocity of the wind exceeds 10 miles per hour.

Mosquitoes may, on occasions, be dispersed by artificial means, including automobiles, trains, ships, and airplanes; and the danger of introducing disease-bearing species is now more critical than it ever has been in the past.

Many of our southern species retire to sheltered locations during the daytime to rest and digest their blood meals. Such places as hollow trees, culverts, caves, stables, etc., are especially attractive and are frequently used as collecting stations in mosquito control operations to determine the species present and their relative abundance.

A great many of our species of Anopheles and Culex hibernate as adult females in protected, moist, semi-dark shelters and come out in the spring in search of food and suitable places in which to deposit their eggs. Males do not overwinter but die with the advent of cold weather.

\section{Medical Importance}

Mosquitoes are known to transmit several important diseases of man and animals. These diseases are briefly described as follows:

Human Malaria.-This is an acute and chronic febrile disease caused by the invasion of the red blood corpuscles by protozoa belonging to the genus Plasmodium and transmitted by Anopheles mosquitoes. Four species of parasites are pathogenic to man; namely $P$. vivax, the causative agent of benign 
or tertian malaria; P. falciparum, which causes malignant tertian, estivoautumnal or subtertian malaria; $P$. malariae, which causes quartan malaria; and $P$. ovale, the cause of ovale malaria.

The life cycle of the parasite consists of a sexual (sporogony) phase with multiplication in Anopheles mosquitoes and an asexual (schizogony) phase with multiplication in man.

The primary vectors of human malaria in the United States are Anopheles quadrimaculatus in the eastern and southern states, and A. maculipennis freeborni in the western states.

Bird Malaria.-This disease is caused by an infection with one or more species of blood-inhabiting protozoa belonging to the genus Plasmodium and is transmitted by various species of mosquitoes. Since the behavior of the parasites causing bird malaria resembles closely that of the plasmodia of human malaria, experimental work with these forms have helped to solve many of the problems of human malaria. Mosquitoes which have been shown to be susceptible to infection with bird malaria parasites are found in the following genera: Aedes, Anopheles, Culex and Culiseta. Culex pipiens and C. quinquefasciatus are generally considered to be important vectors.

Yellow fever.-This virus disease often reaches epidemic proportions producing high mortality among non-immune populations. It occurs in the urban or epidemic form in the presence of the Aedes aegypti mosquito and in the jungle form as an infection of certain animals of tropical forests, transmitted from animal to animal, and occasionally to man, by various species of jungle mosquitoes. The two forms of the disease are quite similar in all respects except for variations in the epidemiology.

The disease is known to occur in certain portions of Central and South America, the Caribbean region and west and central Africa. It has not occurred in the United States during recent years.

Wild primates, marsupials and rodents have been shown to be susceptible carriers in whose blood the virus circulates for the initial few days of the disease and thus serve as a source of infection to susceptible mosquitoes.

An impressive list of Culicine mosquitoes belonging to the genera Acdes, Haemagogus, Eretmapodites, Culex, Mansonia, Psorophora, Wyeomyia, and Limatus, have either been found naturally infected or have been shown experimentally to be capable of harboring and transmitting the virus.

Dengue.-Dengue or "breakbone" fever is an acute infectious disease of man caused by a filterable virus and transmitted principally by Aedes aegypti and Aedes albopictus. It is endemic throughout the tropical and subtropical regions of the world and while it may occur sporadically, it usually appears in epidemic form.

Filariasis.-Filariasis is a disease of man caused by the presence of a parasitic nematode worm, usually $W$ uchereria bancrofti, the immature forms (microfilaria) of which are transmitted by the bite of certain species of mosquitoes. The dramatic clinical manifestation of enlarged extremities has given this disease the common name of elephantiasis. Wuchereria bancrofti, the 
causative agent of Bancroft's filariasis, is the species most frequently encountered in this disease, but in the Asiatic-Pacific zone Wuchereria malayi also occurs. The disease is endemic throughout the moist, warm regions of the world from about $36^{\circ} \mathrm{N}$. to $25^{\circ} \mathrm{S}$. latitude. In the Americas it is sporadic from about Central Mexico to Argentina, being confined on the mainland to coastal areas and river valleys. The West Indies and adjacent islands are endemic centers. More than 50 species of mosquitoes are reported to be natural or experimental vectors of filariasis. The following genera are represented: Culex, Aedes, Marnsonia, and Anopheles.

Heartworm of Dogs.-Mosquitoes belonging to the genera Culex, Anopheles, and Aedes, are known to transmit Dirofilaria immitis, a parasitic nematode found in dogs, cats and various wild carnivores. The worms invade the heart (usually the right ventricle) and the pulmonary artery of the host where they often form tangled knots and may cause the death of the host.

Mosquito-Borne Encephalitides.-These consist of several immunologically distinct epidemic virus diseases which have an affinity for the central nervous system and may be disseminated by the bites of arthropods, including various mosquitoes. The diseases are closely related both epidemilogically and clinically. The principal types are classified as follows: Equine encephalomyelitis (Eastern strain, Western strain and Venezuelan strain), St. Louis encephalitis, and Japanese B encephalitis.

Culex tarsalis, C. pipiens, Culiseta inornata and Anopheles maculipennis freeborni have been found naturally infected with the virus of western equine encephalomyelitis and the disease has been transmitted in the laboratory by mosquitoes belonging to the genera Aedes, Culex and Culiseta. Culex tarsalis and $C$. pipiens have been found naturally infected with the virus of St. Louis encephalitis and several species belonging to the genera Aedes, Culex, and Culiseta will transmit the virus experimentally. Culex tritaeniorhynchus is believed to be the principal vector of Japanese B encephalitis.

Mosquito Vectors of Dermatobia.-Eggs of the tropical warble fly (Dermatobia hominis) are carried by mosquitoes belonging to the genera Aedes, Culex, Trichoprosopon, Haemagogus, Wyeomyia, Mansonia, Anopheles, and Psorophora. Psorophora lutzii and P. ferox are considered to be important carrier hosts in Central America and northern South America.

Other diseases which may be transmitted by mosquitoes are leishmaniasis, tularemia, fowl-pox, and Rift Valley fever.

\section{Technique}

\section{Collecting Adults}

Due to the varied habits of different species of mosquitoes, many types of adult collections made in different situations may be required to obtain a satisfactory picture of the mosquito population in an area. Many kinds of mosquitoes are attracted to relativelv dark shelters with little air movement and a high atmospheric humiditv for their davtime resting. Some species remain outdoors, apparently avoiding dwellings, and are commonly referred 
to as wild or sylvan species; while those which readily enter buildings to feed are usually referred to as domestic or house-frequenting species. Most species of Anopheles and Culex found in the southern United States prefer to rest by day in such places as buildings, culverts, hollow trees and caves. Their relative abundance can usually be determined by making periodical collections in these shelters. Several techniques for collecting adult mosquitocs are discussed here.

Natural Shelters.- In making collections of mosquitoes in diurnal resting places for the purpose of determining densities, numerous favorable shelters should be examined. After an extensive preliminary survey has been made, stations should be selected from which regular weekly collections are to be made throughout the active mosquito breeding season.

When collecting mosquitoes inside houses, one should search carefully, particularly in dark, protected places. Large numbers of mosquitoes are sometimes found on window screens after sundown, where they may be attempting to leave the building in which they found refuge during the day. On the other hand, they may be collected during the night or early morning on the outer surface of window and door screens where they may be trying to enter. Window traps are used in some areas to sample species attempting to enter a building. Mosquitoes are frequently found concentrated in large numbers in stables where domestic animals are housed.

The coloration of some species enable them to blend with their surroundings, making it very difficult for them to be seen. A flashlight properly focused is usually necessary for spotting specimens resting in dark places. After one has collected all visible specimens, it is frequently advisable to prod in corners, trash heaps, and dark places, collecting the disturbed mosquitoes in flight with a midge net.

Artificial Shelters.- In army camps where practically all the buildings are well-screened, it is often difficult to find well-located stations in which to make collections. Nail kegs and small boxes are claimed by some workers to be satisfactory for this purpose when placed near extensive breeding areas $(68,175)$. A small privy-type house, however, has been proven by malariacontrol workers in the southern United States to be more satisfactory (Fig. 1). The privy-type house may be constructed of weather-beaten lumber and should be approximately 4 feet square and 7 feet high. The front is lefi open to a height of about 30 inches from the ground.

Mosquito Traps.-Several types of traps have been devised for use in connection with mosquito surveys and mosquito control. It cannot be said that any of the traps now in use are of much value as an anti-mosquito measure, but some of them do provide valuable information as to the species present in a given locality and their abundance. The more common types of traps used for sampling and evaluating mosquito densities are the New Jersey Mosquito Trap (Fig. 2) and stable traps.

The New Jersey trap should be fastened to a post by means of bolts, or 
hung from the limb of a tree with the light approximately $51 / 2$ to 6 feet from the ground. Care must be exercised in selecting trap locations. Traps should not be located near buildings where animals are housed, in the vicinity of lights which may serve as counter attractants, in locations open to prevailing strong winds, near industrial plants giving off a large amount of smoke or gas which might possibly serve as a repellant, or where any other existing condition would tend to cause abnormal mosquito catches.

The New Jersey trap should be placed on a regular schedule of operation of 3 to 7 nights each week throughout the mosquito-breeding season. It is usually operated from 7:00 P. M. to 7:00 A. M. in this region. A 25- or 40-watt frosted bulb is generally used, and an automatic time switch may be provided to start and stop the trap, or it may be turned on and off by hand. The mosquitoes from each collection should be promptly separated from other insect specimens to prevent molding and placed in properly labelled pill boxes for subsequent identification.

Light traps have been widely employed by mosquito-abatement districts in the United States for making comparative studies in connection with the

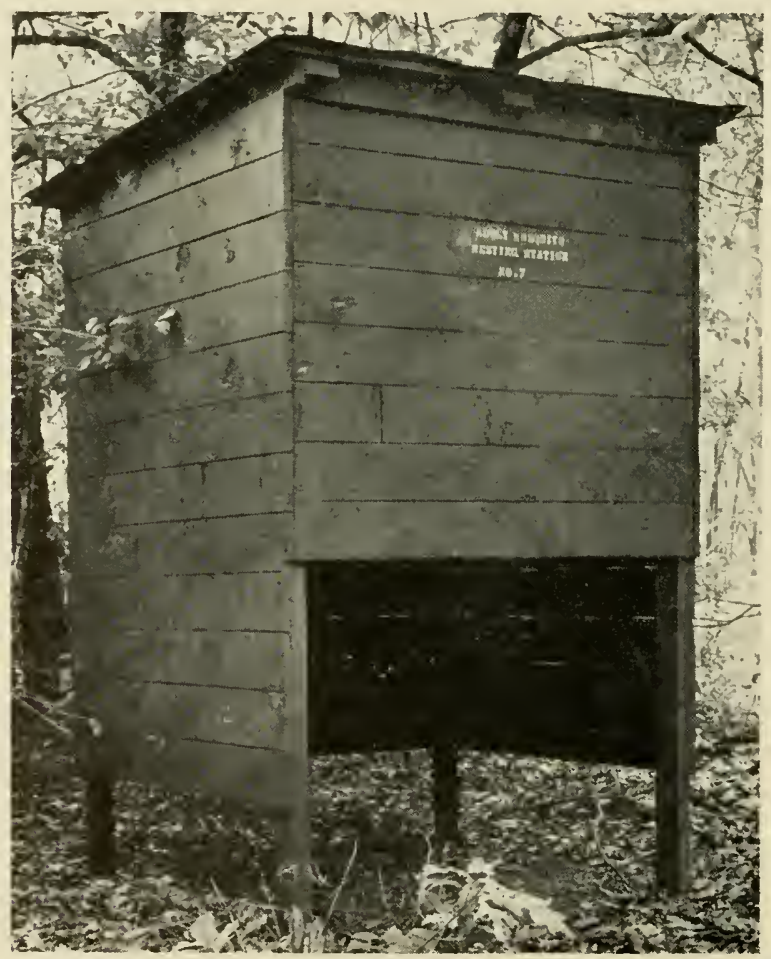

Fig. 1. Artificial shelter for measuring mosquito densities (U. S. Army Signal Corps photograph). 
control of salt-marsh mosquitoes, particularly Aedes sollicitans and A. taeniorhynchus. They often yield adult specimens, particularly males, of species seldom taken by other collecting methods. There has been a considerable difference of opinion among workers in the United States as to the value of

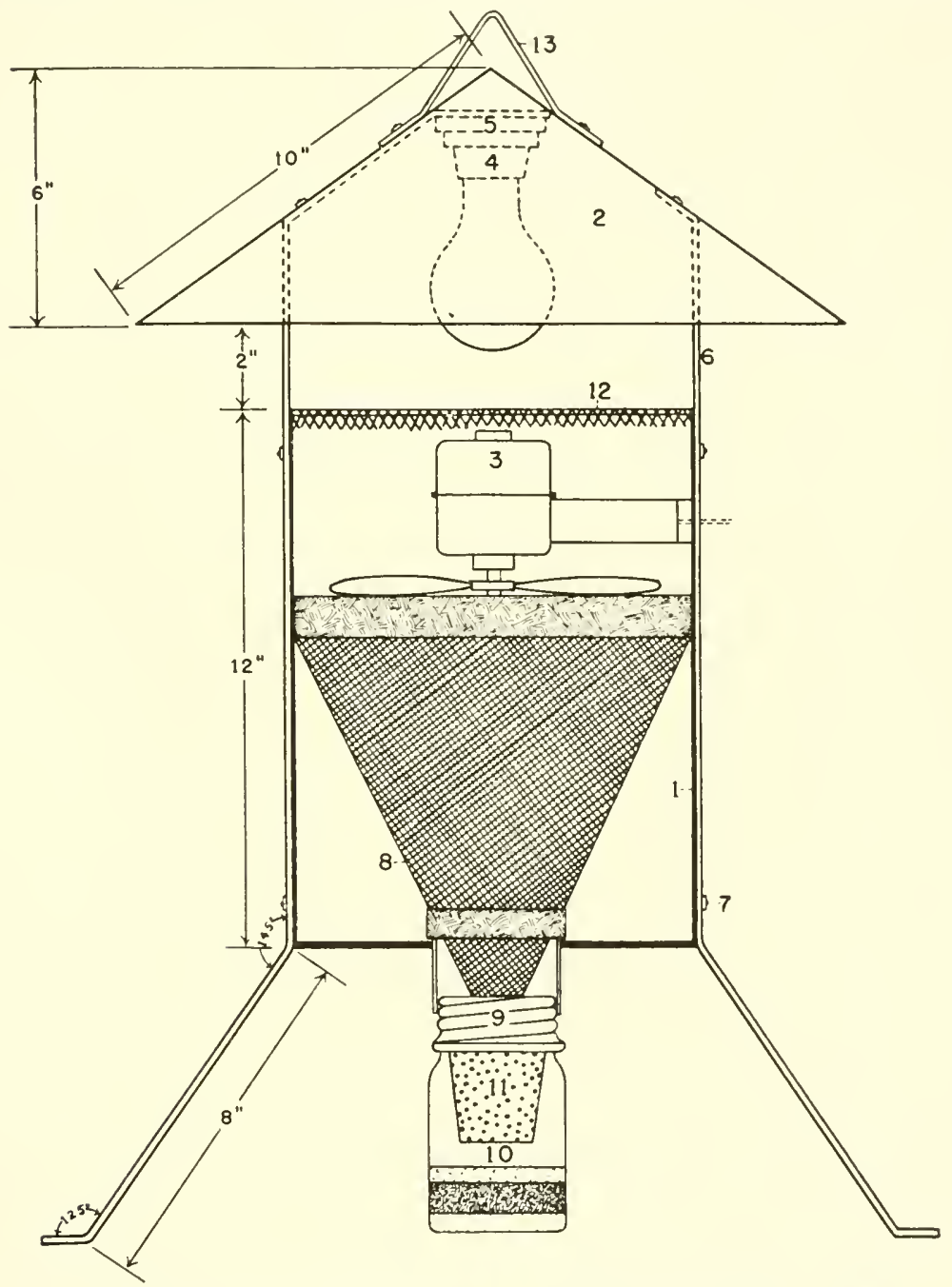

Fig. 2. The New Jersey Mosquito Trap (Modified from Mulhern, 1942): (1) galvanized iron tube (22 ga.), cut away to show interior; (2) roof of galvanized iron: (3) motor and 8 inch fan; (4) porcelain receptacle for light bulb; (5) sponge rubber to absorb vibration; (6) three supporting ribs of band iron $\left(1 / 8^{\prime \prime} \times 1^{\prime \prime}\right)$; (7) screws and nuts for assembling trap; (8) screen funnel of 16 mesh bronze wire; (9) Mason jar cap; (10) jar with cyanide for killing specimens; (11) perforated paper cup: (12) galvanized screen, $3 / 8$ " mesh; (13) loop for carrying or hanging trap. 
the New Jersey light trap for gauging Anopheline populations, however, since many of the factors relating to light reactions of mosquitoes and other stimuli which may attract or repel them are still unknown. It is known that Anopheles crucians, $A$. walkeri and $A$. atropos are readily attracted to these traps, but $A$. quadrimaculatus is known to be less readily attracted. Due to this fact, light traps have not been used on a large scale in connection with malaria control in the southern states.

Stable traps are used for determining densities of Anopheline mosquitoes in many regions of the world. Two types, the Caribbean or Magoon trap (111, 136) and the Egyptian trap (13) are in general use. Each trap consists of a small portable building for housing an animal and is equipped with an ingress baffle along each side wall (Fig. 3). In both the Caribbean

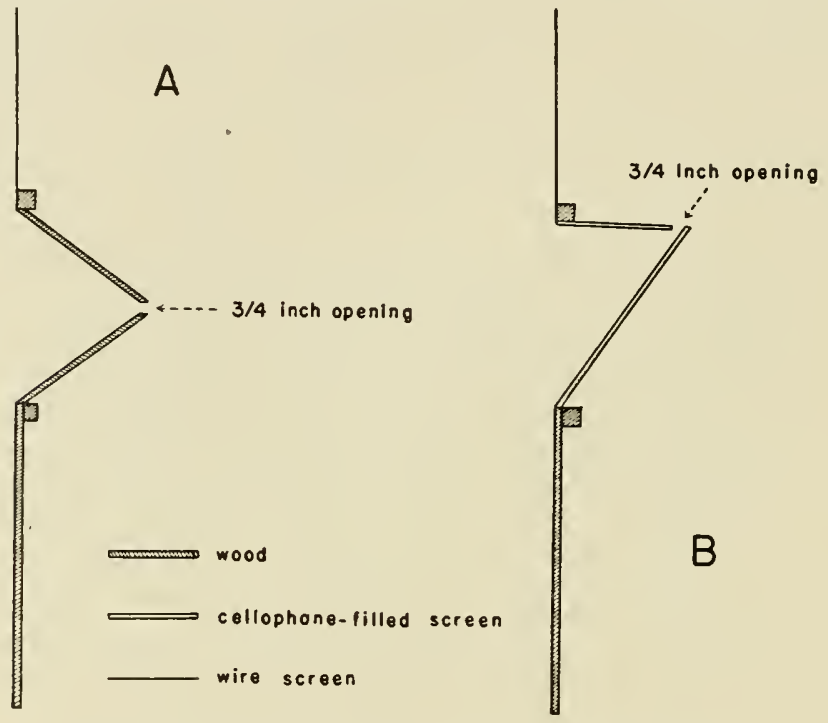

Fig. 3. Side walls of stable traps, showing ingress baffles.

A, Magoon or Caribbean trap. B, Egyptian trap.

and Egyptian traps, the ingress baffle is so constructed as to permit mosquitoes to enter the trap and to discourage their exit. A suitable animal, usually a calf, small horse or goat, is kept overnight in the trap and the trapped mosquitoes are caught by collectors the following morning.

Biting Collections.-Records of the number of mosquitoes attempting to feed on a favorable host give direct information as to the species causing annoyance and their relative abundance. Both nocturnal and diurnal biting collections are important phases of a mosquito survey. Frequent collections should be made of hungry mosquitoes attracted to the collector or some other host provided for this purpose. Horses, mules, cows, and other docile animals are frequently used as attractants. Biting catches can be made by 
two persons, one collecting from the other, or by one person collecting alone. In the latter case, it is best for the collector to be seated with his sleeves rolled above the elbows and his trouscrs rolled above the knees. The collector should sit quietly and capture the specimens with an aspirator or a chloroform tube as they alight or begin to feed. A flashlight may be used, but the amount of light should be reduced by removing the lens or covering it with a semitransparent paper. The collecting at any one place should be done for a set period, usually 20 or 30 minutes. In a malarious area, this type of human exposure is to be discouraged.

Many woodland species, particularly Aedes and Psorophora, may be captured during the daytime in thickets, woodlands, and on occasions in open areas, while attempting to bite. The same method given for making nightbiting collections is used except that the flashlight is not needed.

Sweeping Vegetation.-Certain species of mosquitoes, particularly Aedes and Psorophora, in this region are seldom encountered in resting stations, but shelter during the day amongst the vegetation i: moist, shady places, and especially near the water in which they breed. Mosquitozs can be captured in situations of this kind by sweeping with a net or by disturbing them and catching the specimens in the net while they are in flight. It is frequently advisable to sweep the vegetation around the margins of a breeding place in order to obtain adult specimens which may have recently emerged. Male specimens of species seldom obtained by other collecting methods are occasionally taken by this latter procedure.

Equipment.-A chloroform killing tube (Fig. 4, B) can be made from a large cork-stoppered shell vial or test tube about 1 inch in diameter and 6 or 8 inches long. The tube can be prepared as follows: Cut a scrap of old auto innertube into enough small cubes to occupy the bottom linear inch of space in the tube (rubber bands may be substituted if desired). Pour chloroform into the tube until level with the top of the layer of rubber. After the chloroform has been absorbed by the rubber, a plug of tissue paper should be packed tightly into the tube and covered with one or two close-fitting circular sections of blotter or cardboard. To recharge the tube, remove the blotter or cardboard discs and tissue paper and add chloroform to the rubber as before. Cyanide may be used in the killing tube instead of rubber and chloroform, tut should be handled with caution since it is extremely poisonous.

An aspirator (Fig. 4, D) is a convenient device for collecting mosquitoes in diurnal shelters or while they are biting or attempting to bite. The aspirator is made from a piece of glass tubing about $1 / 2$ inch in diameter and about 6 inches long, fitted with a piece of rubber tubing about 30 inches long. A chemical test tube with the bottom end cut away makes a satisfactory tube. A cloth screen is held in place in the base of the glass tube by a cylindrically-coiled flexible card. Captured mosquitoes are blown from the aspirator into a small cage or directly into a killing tube.

A folding midge net 8 inches in diameter when open, with a short 

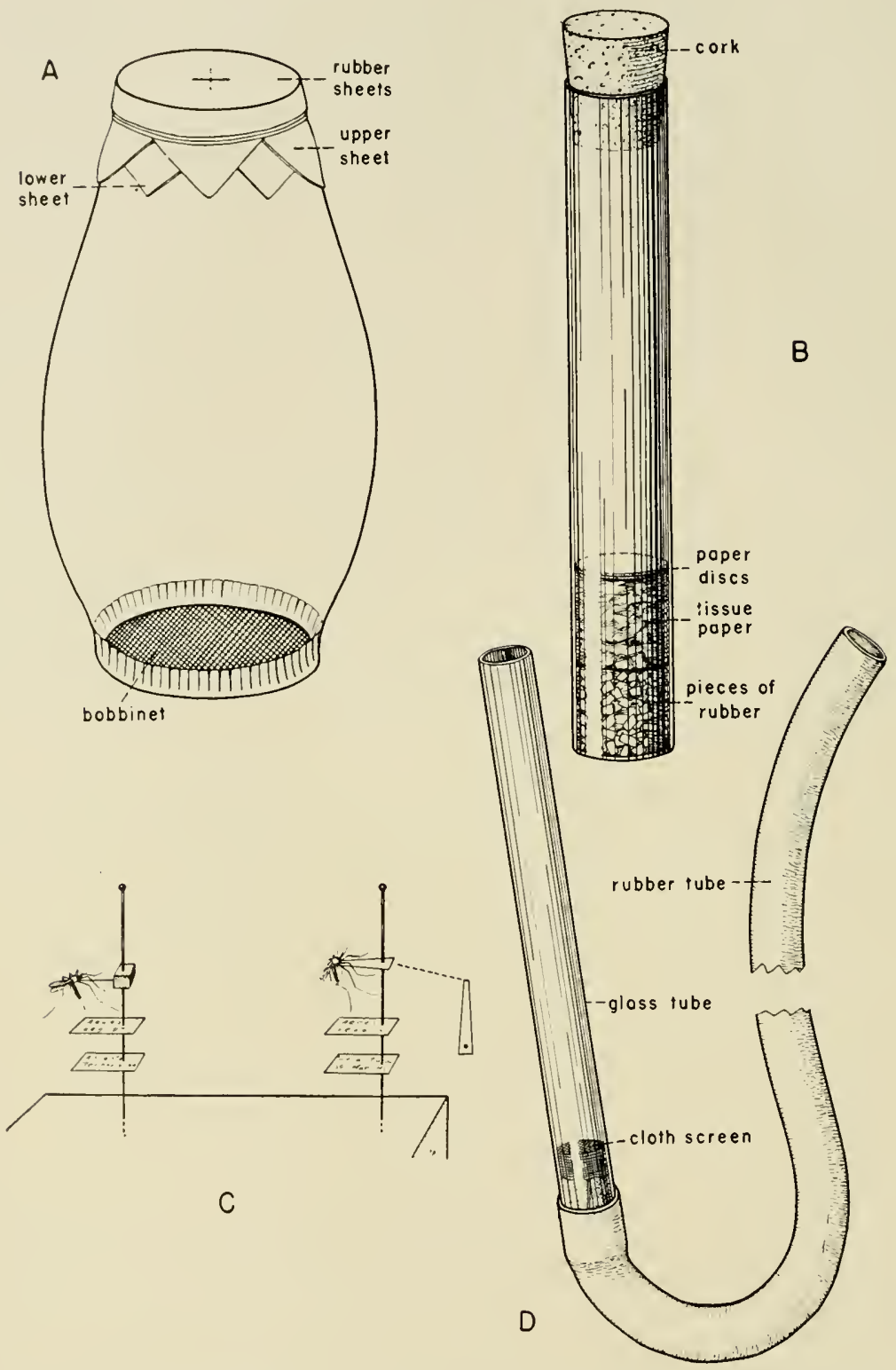

Fig. 4. A, Small lantern chimney cage for holding live adult mosquito specimens. B, Chloroform tube for killing adults. C, Adult specimens mounted with micro-pin and triangular paper point. D. Aspirator for collecting adults. 
handle (approximately 12 to 18 inches) and with an 18-inch bag of good quality netting, is convenient for collecting mosquitoes in the field. It is particularly useful for catching mosquitoes on the wing. Larger nets may be used for catching mosquitoes under special concitions, such as when they are driven out of hollow trees or culverts by the aid of smoke or burning pyrethrum.

A cage made of a small lantern chimney, the bottom of which is closed with bobbinet and the top with a double layer of thin sheet rubber, each layer slit in opposite directions is useful for holding adults while collecting in a resting shelter (Fig. 4, A). The specimens may be emptied from the aspirator linto the cage as often as necessary. They can be stupefied by placing the bottom of the cage firmly against the leg above the knee, thus closing the bottom, and inserting the open end of a chloroform tube through the slit openings in the rubber covers. After they are stupefied, they should be removed and placed in the chloroform tube until dead before finally packing them in pill boxes for study.

\section{Transporting, Mounting and Storing Adult Specimens}

Transporting Specimens.-It is often necessary for specimens to be carried or shipped to a laboratory before they can be properly studied. The specimens may be carried either in the living or dead state, according to the use to be made of them. Heavy mortality usually takes place among live specimens in transit unless great care is exercised. The receptacles must not be overcrowded and the atmosphere in the containers must be kept humid. This can easily be accomplished by wrapping the cage with a damp cloth. Mosquitoes will die quickly when cages or boxes containing collections are exposed to direct sunlight. Live specimens can be carried short distances in small cages made of lantern chimneys. Since glass cages break easily, small square cages approximately $4 \times 4 \times 6$ inches, made of mesquito netting on a wooden or wire frame are preferable for shipping specimens. A cage approximately $18 \times 18 \times 18$ inches will accomodate as many as 1,000 living adults during transit if proper precautions are taken. A cage of this type can be placed on the back seat of an automobile and carried on a trip requiring several hours, even during summer weather, if protected from direct sunlight and draped with wet cloths.

Dead mosquito specimens can be transported in circular pill boxes, each containing a layer of glazed cotton pressed down over the bottom to form a smooth layer. This layer of cotton should not be more than one-eighth to one-fourth inch thick and the specimens should not be covered with cotton. If the cotton is pressed firmly into the box, it will hold the specimens sufficiently to prevent them from sliding about without their becoming excessively entangled in the fibers. One should avoid placing too many specimens in a single box. Some workers prefer to pack specimens on cellucotton or crinkled lens paper. Specimens packed in the above manner may be satisfactorily shipped throush the mails if tightly placed in a mailing container and sufficiently insulated against excessive jarring. Pinned specimens may 
be prepared for shipment by setting them firmly by means of pinning forceps in an insect box. It is preferable to spread a thin layer of cotton over the bottom of the box before setting the pins to protect any specimens which may become detached.

Killing and Mounting.-Mosquitoes which have been reared from larvae or pupae should not be killed immediately after emergence but should be kept alive for at least 24 hours to permit the body to harden. They should not be left long in small vials after emergence, as they may become wet or badly rubbed. Better results are obtained if emergence is allowed to occur in a suitable cage.

When recently killed specimens are available for pinning, micro-pins (minuten nadeln) should always be used. The micro-pin is pushed through a small rectangular piece of cork through which a larger insect pin (No. 3 or 4) is passed at right angle. The point of the micro-pin is thrust into the thorax of the mosquito, preferably between the coxae, directed toward but not piercing the scutum (Fig. 4, C).

Dry specimens can be mounted on triangular paper points using cellulose cement ${ }^{1}$ or shellac as an adhesive. An insect pin (No. 3 or 4 ) is passed through the base of the paper point. A small drop of cement is placed on the tip of the poirt and pressed against the left side of the thorax of the specimen with the tip directed toward the scutum (Fig. 4, C).

Storage of Specimens.-Glass-topped cabinet drawers are desirable for storing reference collections. This is probably the most practical method of housing pinned specimens, especially if pinning trays are used for storage of a series of each species. At least two styles of insect drawers, the Cornell University type and the National Museum type, can be obtained from supply houses in this country.

Pinned specimens to te used for teaching purposes, or small personal collcctions, may be stored in Schmitt boxes or other insect boxes of tight construction having bottoms lined with sheet cork or balsa wood. Unpinned specimens can te stored in labeled pill boxes prepared in the same manner as those used for shipping.

Care must be taken to protect stored specimens from insect pests. If collections are neglected for even a few months, they may be completely destroyed. Flake napthalene and paradichlorobenzene are generally used for this purpose. Either of these chemicals or a mixture of the two should be placed in a small perforated container and fastened in one corner of the insect box. In damp climates, extra precautions mav be necessary to deter the growth of destructive molds. Mold growth may be prevented by storage in dry chambers, or by thinly painting the insides of the boxes with creosote

1 Cellulose cement can be prepared by dissolving celluloid in amyl acetate. Amyl acetate is a volatile substance and the stock cement will need to be thinned frequently. Cellulose cement can also be purchased in small tubes. When about one part balsam is added to three parts of the cellulose cement, a more adhesive, less brittle, and slower drying product is obtained. 
or pure carbolic acid. Specimens that are kept in pill boxes should be stored in large cans or jars containing napthalene or paradichlorobenzene.

\section{Preparing Male Terminalia for Study}

For making specific identifications and for studying relationships between the different species of mosquitoes, the examination of male terminalia is invaluable. Any medium used for mounting larvae may also be used for mounting terminalia. It is often not desirable, however, to make a permanent mount but merely to examine the specimen in water or gylcerin, and then store in 70 to 80 per cent alcohol for future reference.

To prepare terminalia for study, carefully clip off the apical fourth of the abdomen with sharp scissors or forceps and place in a small casserole containing a 10 to 20 per cent solution of potassium hydroxide. Heat the solution to near the boiling point, and then transfer the terminalia to a beaker of water and allow to rinse for several minutes; then place it in a drop of water or gylcerin on a slide and examine with a dissecting microscope.

To mount in chloral gum, transfer the specimen with a dissecting needle from water to a drop of the mounting medium on a clean slide. Remove the superfluous abdominal segments from the terminalia proper, orient it with the dorsal side up and extend the dististyles. Since the terminalia of the genus Culex have stout basistyles, either these structures should be dissected loose at their bases and laid flat, or the cover glass should be blocked up with small pieces of glass. In the Anophelines, the anal lobe and the ninth tergite often obscure characters of the phallosome; therefore it may be necessary to dissect these structures away from the rest of the specimen, but they should be left in the same field. Anopheline terminalia should be mounted flat so that the leaflets of the phallosome will straighten out and be easily seen. A specimen may tend to shift out of position when a cover slip is applied, but this can usually be prevented if slight pressure is exerted on the cover slip with a pair of forceps as it settles into place.

Chloral gum is a convenient medium to use for mounting terminalia, but the mount usually lasts for only a few years. Specimens mounted in chloral gum are extremely easy to remount, however, which is a point not to be overlooked if a detailed study of the specimen in different positions is desired. For a more permanent mount, however, Canada balsam, clarite, creosote-balsam, or Euparal are preferred (see methods for mounting mosquito larvae).

If creosote-balsam is used, leave the specimen in 80 per cent and 95 per cent alcohol for 5 minutes each, and in absolute alcohol for 30 seconds; then transfer with a dissecting needle to a clean slide on which has been placed a drop of the creosote-balsam medium. If the standard Canada balsam method is followed, the same procedure as above can be used, except that a 2- or 3-minute clearing step must be introduced between the absolute alcohol and final mounting medium. The authors prefer creosote U. S. P., oil of wintergreen, or clove oil over xylene as clearing agents since they do not cause the specimens to become brittle. 
Some workers stain terminalia in order to make some of the delicate structures show up more clearly. Staining is seldom necessary, so the techniques have been omitted here. Good accounts of staining techniques are given by Edwards (56) and Komp (100).

\section{Preparing Cibarial Armature for Study}

The cibarial ("pharyngeal") armature has been most extensively used in separating subgenera and certain Old World species of Anopheles. Michener (122) discusses its use in separating mosquitoes of the Culex (Melanoconion) group in the southern United States. The methods for mounting male terminalia are also applicable to mounting the cibarial armature. Since these structures are so small, the chloral gum method will no doubt be preferred because of the fewer steps required. Specimens may be prepared for study in the following manner: Heat the head of a female mosquito in a 10 to 20 per cent solution of potassium hydroxide for a short time; then rinse in a small beaker of water for a few minutes. Transfer the specimen to a drop of water on a slide under a dissecting microscope and break away the head capsule with fine needles. The pharynx and cibarium are attached to the hypopharynx, which may be transferred to a drop of chloral gum mounting medium for further dissection. Application of a small cover slip completes the mount.

\section{Dissection for Malaria Parasites}

Specimens containing blood should be kept either in test tubes plugged with damp cotton or in humid lamp chimneys for about 48 hours, thus allowing time for digestion. If a large number of specimens are to be dissected, considerable time may be saved by anaesthetizing several at once and placing them in a moist chamber. Care should be exercised not to prepare more than can be examined in a half hour. If only a few specimens are to be dissected, they may be transferred one at a time to a test tube and killed by striking the distal end of the tube smartly against the palm of the hand. It is not always necessary to remove wings and legs, especially where speed is of primary consideration.

Several techniques of dissecting mosquitoes for malarial oocysts and sporozoites have been described by various workers, including Barber and Rice (10), Blacklock and Wilson (20), Boyd (22), Christophers et al. (41), Puri (139), Simmons and Aitken (173), and Wilcox and Logan (187). The following technique has proven satisfactory for dissecting and preparing the salivary glands and mid-gut for examination. Certain internal organs of a female mosquito are shown in Fig. 5.

Mid-gut.-Orient the body under a dissecting microscope on a slide containing a drop of physiological saline with the abdomen pointing toward the observer and touching the drop of saline. Nick the integument on both sides of the penultimate abdominal segment, being careful not to sever the hind-gut. With a needle held in the left hand, press down or transfix the thorax in the region of the hind legs. Take the second needle in the right 
hand, place it on the partially separated tip of the abdomen, and by gentle, intermittent traction, draw out the alimentary canal with attached Malpighian tubules and ovaries into the droplet of saline. Set aside the remainder of the specimen in physiological saline for salivary gland dissection.

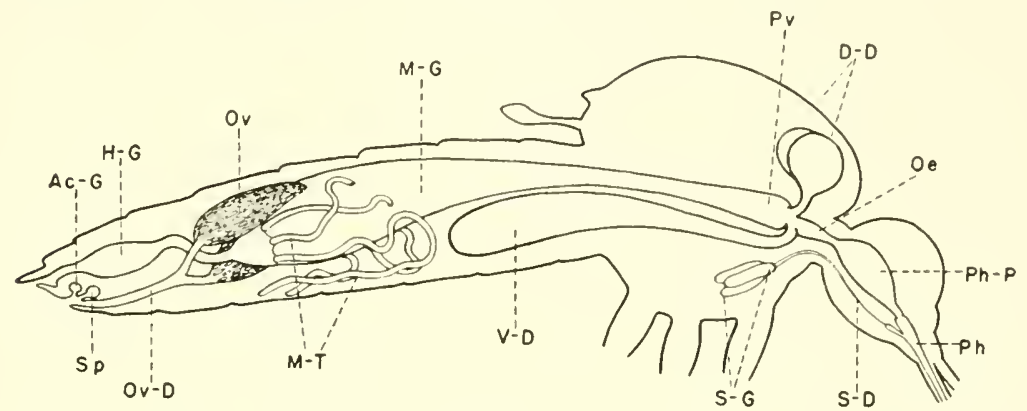

Fig. 5. Iniernal anatomy of a female mosquito (adapted from Marshall, 1938). Ac-G, accessory gland; D-D, dorsal diverticula; H-G, hind-gut; $M-G$, mid-gut; M-T, malpighian tubules; Oe, oesophagus; Ov, ovary; Ov-D, oviduct: Ph, pharynx; Ph-P, pharyngeal pump; Pv, proventriculus; S-D, salivary duct; S-G, salivary glands; $S p$, spermatheca; V-D, ventral diverriculum.

Prepare the mid-gut for examination for oocysts as follows: Carefully sever the digestive tract at the point of origin of the Malpighian tubules and transfer the mid-gut to a fresh drop of saline, discarding the Malipghian tubules, hind-gut, ovaries and other debris; apply cover slip; remove excess saline with filter paper. Transfer the slide to a compound microscope and carefully examine the stomach wall for the presence of oocysts. The younger forms of oocysts are clear, oval or round bodies and contain distinct pigment granules; larger forms are similarly shaped but have distinct cyst walls and contain pigment clumps. The mature oocysts have lost their pigment and are filled with hundreds of slightly curved sporozoites. Before calling a slide negative, the mid-gut should be rolled over by sliding the cover slip so that all surfaces may be observed in profile.

Salivary glands.-Place the remainder of the specimen in a drop of physiological saline tinted with methylene blue and orient it so that the head will point away from the observer. With a needle held in the left hand, press Jown on the thorax across the region of the mid-coxae, causing the neck to bulge slightly; at the same time, with a needle in the right hand, transfix the head capsule, and with gentle traction, pull the head away from the thorax. As the head tears away, a small tag of tissue will be seen attached to the head. This tag should contain the glistening, blue-stained salivary glands. If the glands do not come out with the head. they may be recovered by carefully tearing away tissues in the prothoracic region. Transfer the glands to a fresh drop of physiological saline, apply cover slip and remove excess saline with filter paper.

Examine the salivary glands as follows: Using the dissecting microscope, center the glands in a low-power field and crush them by pressing on the 
cover slip with a dissecting needle. Transfer the slide to a compound microscope and examine the crushed glands and adjacent fluid under high-dry magnification The sporozoites are minıte, glistening, slightly curved rods.

The technique of making permanent mounts of mid-guts and salivary glands is given by Wilcox and Logan (187).

\section{Collecting Mospuito Eggs}

It has become a generally accepted fact among mosquito workers that the study of mosquito eggs, in conjunction with other stages in the life cycle, is of much value. It may be of special importance when dealing with closelyrelated species. The importance of the study of mosquito eggs is clea:ly shown by Hackett and Missiroli (72) and Bates (12) in their works on the taxonomic status of the mosquitoes of the Anopheles malcupennis complex. Egg rafts of many species of Culicines are frequently encountered in the field and can be gathered and carried to the laboratory for hatching and rearing. Eggs of Anophelines may be collected in the field by placing a white muslin bag over the hand and sweeping the hand through the water in spots where oviposition is likely to have taken place, or water can be taken with a dipper and poured through the muslin. Muslin fitted on embroidery hoops may also be used for this purpose. The cloth should be carefully examined with a hand lens at frequent intervals for the detection of eggs. Eggs of certain species of Aedes and Psorophora may be obtained in soil scraping from likely breeding spots and hatched in the laboratory.

Gravid females may be captured and isolated for oviposition in small cotton-stoppered, flat-bottomed shell vials. Many species of Aedes, Psorophora and Anopheles will usually oviposit on cellucotton which has been tamped firmly into the bottom of the vial and saturated with water. When eggs are obtained from an ovipositing female in an isolation vial and are hatched and reared, one can study and correctly associate all stages of a species. In addition, this method of collection and study furnishes valuable information on the bionomics of the less common species.

\section{Collecting Larvae}

Mosquito larvae may occur in all types of waters, varying from large lakes and marshes to small collections of water in tree holes, leaf axils of plants and artificial containers; therefore in making an initial survey of mosciuito breeding in an area, every possible type of breeding place should be examined. A careful study of the different factors present in each breeding place should be made to determine, if possible, the breeding preferences and requirements of the different species. The association of a species of mosquito with certain characteristics of the breeding place is helpful to the collector and is of practical value to the control worker.

A uniform system of larval collections or inspections should be instituted as a part of every mosquito-control project to provide the following information: (1) The areas producing species of economic importance; (2) the 
need for larval control; (3) the effectiveness of the larvicidal program; and (4) any changes in breeding conditions that may occur.

Larval Stations.-The individual lakes, ponds, sections of streams, ditches, tree holes, groups of small adjacent pools and other specific larval habitats may be designated as larval stations from which regular collections are made, preferably at weekly intervals. In addition to the collections obtained from regular larval stations, frequent miscellaneous collections from other places should be made in order to obtain a more complete picture of the problem.

Equipment.-Larvae are usually collected with a white-enameled dipper having a hollow handle into which a round stick or cane may be inserted for convenience. Shallow, white-enameled rectangular pans are preferred by some collectors. A rubber suction bulb attached to 3 or 4 feet of rubber hose is a convenient device for collecting larvae from tree holes, crab holes, and similar inaccessible places. Other apparatus for collecting larvae include nets of various types and sizes, soup ladles, small dishes, spoons, and large glass pipettes with rubber bulbs attached.

Collecting Technique.-Dipping should be do:e in a systematic and uniform manner in order to provide data as to the comparative abundance of larvae at different seasons or in different areas. For Anopheles, the dips are made by skimming the surface of the water in places where aquatic vegetation or floating debris offer protection for the larvae. As it is usually desired to obtain a fair sample of the larvae present in a breeding place, the dips should be adequately distrituted over the station: the tendency to make most of the dips in a few spots should be avoided. The number of dips made when searching for larvae should mainly depend upon the size and type of breeding place and the abundance of larvae.

Larvae of some species sink to the bottom rapidly when disturbed and remain there for several minutes. If the collector will remain quiet near pools in which these mosquitoes breed, the larvae and pupae mav be picked up singly with a dipper or pipette as they come to the surface. When collecting Anopheles larvae from small pools, it is often advisable to remove the vegetation from a small area; then stir up the water, making it muddy. Larvae and pupae are very conspicuous against the muddy background and can be collected with a pipette or spoon as they appear on the surface. When collections are taken from tree holes, crab holes, and other specialized breeding places by means of a suction bulb and hose, the water should be emptied into a white-enameled pan to be examined for larvae. The suction bulb should be thoroughly rinsed after each collection, as specimens may cling inside it and appear to come from the next breeding place inspected.

Fourth instar larvae should be selected for identification, as immature specimens of most species cannot be accurately identified. It is often advisable to preserve the larvae in small vials at the time of collection, but if they are to be taken alive to a laboratory for studv, they mav be transferred bv means of a pipette from the dipper or other collecting device to a wide-mouth bottle or jar. Such containers should never be left in open sunlight, and during warm 
weather the corks or caps should be loosened occasionally to provide air.

Collection Data.-Complete observations and field notes on all collections provide valuable information on breeding and other habits of mosquitoes. The collector should not rely upon memory, but should make the notes at the time of collection. Bottles in which larvae are collected should each be given a collection number corresponding to a record kept in a field book. It is preferable to place penciled collection data inside the larval container rather than to use gummed outside labels which often become detached during transit.

\section{Rearing, Storing and Mounting Larvae}

Rearing.-Larvae collected for rearing should be brought into the laboratory as soon as possible and transferred to jars or pans with sufficient water surface for free movement and feeding. In rearing larvae, a large water surface is necessary to provide proper aeration of the water, adequate feeding surface for Anophelines, and to prevent overcrowding which may lead to cannibalism. If possible, each rearing pan or jar should contain some of the water and vegetation of the breeding place from which the larvae were collected. It is often necessary to supplement the food supply by addition of small portions of puiverized dog biscuit, Pablum, or yeast. If a considerable amount of evaporation takes place, fresh water should be added to the pans. Larvae of large, carnivorous species should be isolated in small jars and fed on larvae of some other species. Pupae should be transferred each day to containers in emergence cages.

When it is desired to associate larval and pupal exuviae with the adult, single larvae are isolated in rearing vials. Shell vials measuring approximately 1 inch by $31 / 2$ inches are satisfactory for this purpose and should be properly labeled and lightly plugged with cotton. The exuviae should be removed with a pipette and preserved in 70 to 80 per cent alcohol, or mounted on a slide and given a number corresponding to that of the emerged adult.

Killing and Storing.-Full-grown larvae may be killed and preserved in 70 to 80 per cent alcohol or 10 per cent commercial formalin. Larvae retain their form best if killed in hot, but not boiling water; but satisfactory specimens are usually obtained if they are placed alive directly into the preservative. Another convenient method is to place a larva on a clean slide in a drop of water and hold for a short period of time on the hot shade of a desk lamp.

For shipping, larval specimens may be placed in small vials filled with preservative and tightly corked. Large museum collections of larvae may be preserved in small stoppered vials which are in turn stored in a larger container filled with the preserving fluid. A label bearing the name of the species, collection number, locality, date of collection and name of collector written in pencil or India ink should be placed inside each vial with the larvae.

Another satisfactory method of packing specimens for storage or for shipping is to place them in dental procaine hydrochloride cartridges as shown in Fig. 6. The empty cartridges, which are usually discarded by 
dentists, can generally be obtained upon request. The specimens are packed as follows: Set the rubber plug at the middle of the cartridge; transfer the larvae to the cartridge and fill completely with preservative; insert a hypodermic needle into the rubber stopper, allowing the opening of the needle to come barely inside; push the rubber stopper, containing the inserted needle, into the cartridge (as the stopper is pushed into the cartridge, the excess preservative will escape through the needle); remove the needle. If the needle is set at the proper depth, the stopper can be inserted without trapping air in the tube. The collection data should be written on a small, flexible card, coiled and inserted in the open end of the cartridge below the rubber plug. This method of packing larvae reduces breakage of specimens and keeps evaporation of the preservative at a minimum.

Mounting Larvae.-Permanent slide mounts of larvae are often necessary for study of characters used in mosquito identification. Several reliable techniques for making mounts are discussed here.

Chloral gum, or Berlese's medium, may be conveniently used for mounting larvae since it has the advantage of being water-soluble. It has the disadvantages, however, of being very slow to harden and of discoloring and crystalizing within a few years. The modification of Berlese's medium given by King, Bradley and McNeel (96) is as follows:

Distilled water $8 \mathrm{cc}$.

Chloral hydrate $70 \mathrm{gm}$. Gum arabic (dry powdered) .......... 8 gm. Glycerin 5 cc.

Glacial acetic acid $3 \mathrm{cc}$.

Dissolve in the above order in a water bath and strain through muslin.

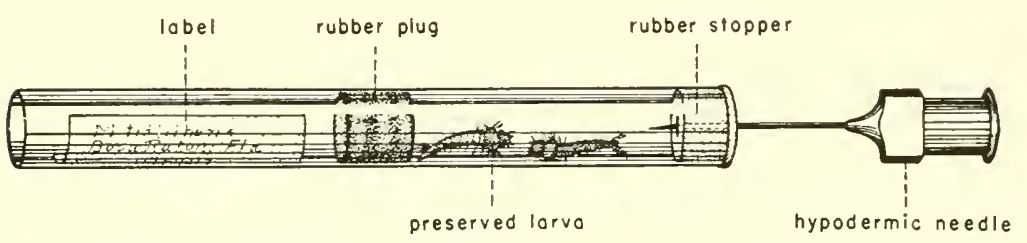

Fig. 6. Method of packing mosquito larvae for storage or shipment.

If the larva has been preserved in alcohol or formalin, it should be allowed to stand in water for a few minutes to rinse, then placed in chloral gum on a clean slide. An applicator stick, whittled to form a small spatula is convenient for lifting the larva without breaking hairs. The abdomen of a Culicine larva should be partially severed between the 7th and 8th segments with dissecting needles so that the siphon and anal segment will turn and lie flat. A similar procedure should be followed with Anopheline larvae when it is necessary to examine the pecten. After the larva has been properly oriented, a clean cover slip is applied. By gently touching the cover slip with a pair of forceps as it settles, shifting of the larva can usually be prevented. A little practice will soon enable the technician to know how much medium to 
use for the different-sized larvae. Small bubbles usually work out and do not spoil the mount. The finished slide should be kept in a horizontal position until the medium has hardened; then it may be sealed with ringing cement to prevent further evaporation.

Euparal is a very good alcohol-soluble mounting medium. Before mounting, the larva should be dehydrated by running it up through an alcohol series; 80 per cent, 95 per cent and absolute alcohol are all the graduations necessary. Allow the larva to stand about 5 minutes in each change. Cellosolve (ethylene glycol mono-ethyl ether) may be substituted for the 95 per cent and absolute alcohols. Place some Euparal on a clean slic'e, orient larva, and apply cover slip as described under the chloral gum mounting method. The drying is fairly rapid and no ringing is necessary. This method has an advantage over balsam in that there is no intermediate clearing step between the alcohol and mounting medium.

Canada balsam is a common mounting medium which, if properly used, makes excellent, permanent mounts of mosquito larvae. For mounting in balsam, dehydrate the specimen by running it up through an alcohol series as described for the Euparal mounting method. Clear in creosote U. S. P., clove oil, oil of wintergreen, xylene or toluene. Before xylene or toluene is used for clearing, an additional 5-minute step in absolute alcohol is necessary to insure complete dehydration. Specimens cleared in creosote, clove oil, or oil of wintergreen remain more pliable and undergo less shrinkage than those cleared in xylene or toluene. Leave the specimen in the clearing agent until it becomes transluscent; 5 to 10 minutes is generally sufficient. Place some Canada balsam on a clean slide, orient specimen and apply cover slip as described under the chloral gum mounting method. Allow to dry in a horizontal position for about 2 weeks. No ringing is necessary.

A creosote-balsam medium for mounting mosquito larvae is described by Wanamaker (185) and is used extensively by the authors. The procedure is briefly summarized as follows: Prepare the medium by gently heating Canada balsam at about $120-130^{\circ} \mathrm{F}$. until most of the solvent is driven off. Dilute this thick balsam to the proper mounting consistency with creosote U. S. P. The larva should be left in 80 per cent and 95 per cent alcohols for 5 minutes each, then in absolute alcohol for 1 to 2 minutes, depending upon the size of the specimen. It is then transferred directly to the creosote-balsam mixture on a clean slide and cover slip applied. Clearing takes place as the medium hardens. No ringing is necessary, but the slides should be left horizontal for 3 or 4 weeks since the creosote-balsam medium dries rather slowly.

A clearing and mounting medium composed of polyvinyl alcohol (Elvanol 90A-25), phenol and lactic acid is excellent for mounting mosquito larvae. The PVA-lacto-phenol medium contains 56 per cent PVA (stock solution), 22 per cent phenol, and 22 per cent lactic acid by volume. The PVA stock solution is prepared by adding PVA (Elvanol) slowly to cold water, stirring it in thoroughly until the solution becomes as viscous as cold molasses. Any undissolved lumps should be removed by straining. The larval specimen is 
taken from water and placed directly in the PVA-lacto-phenol medium for mountıng. It is often necessary in thick mounts to add more of the medium at intervals while drying. The mount will be cleared and the cover slip firmly glued in place within 2 or 3 days.

\section{Mosquito Identification}

The identification of adult and larval mosquito specimens requires a knowledge of morphology; suitable equipment, including microscopes and microscope lamps; and diagnostic keys or descriptions in which all the species in the area are included. This publication contains keys, descriptions, and illustrations dealing with the principal structures used for determining the identity of the different species occurring in the southern United States.

A wide-field dissecting microscope equipped with low, intermediate and high-power objectives is satisfactory for identifying adult mosquitoes and most larval specimens. Frequently a compound microscope with a higher magnification is necessary for more detailed larval study and for the examination of male terminalia.

The light from a flexible-arm desk lamp, condensed by a 1-liter Florence flask filled with water, is satisfactory for use with a dissecting microscope, although other types of microscope lamps may give good results. For use with a compound microscope, the writers prefer a flexible-arm desk lamp as described above but without the water-flask condenser.

An experienced technician will soon learn to identify adult mcsquito specimens in the pill box in which they were stored at the time the collection was made, providing they are not overcrowded. The transfer of individual specimens from pill boxes to the stage of the dissecting microscope requires considerable time and often results in breakage. Some workers prefer transferring all the specimens of a single collection to a petri cish lined with white paper; as each specimen is examined and identified, is is moved to one side with a pair of forceps. When identifying large numbers of specimens in this manner, much time can be saved if two persons work together, one identifying the specimens and the other recording the data.

When unmounted larvae are to be identified with a dissecting microscope, it is best to place them in a Svracuse watch glass in either water or the preserving fluid. Light from the flexible-arm desk lamp can be sharply focused by means of the water-flask condenser and the specimens may be manipulated as necessary with the aid of a dissecting needle or small camel's hair brush. All but the most minute structures are visible under the high power of the dissecting inicroscope when this method of examination is employed.

\section{Characters Used in Identification}

\section{Adult Characters}

The body of an adult mosquito, as in other insects, is divided into three well-marked regions, the head, the thorax, and the abdomen, each of which 
possesses variable characters useful in classification. The general appearance of an adult female mosquito is illustrated in Fig. 7.

HEAD. - The head of a mosquito is somewhat spherical in shape and is narowly joined to the thorax by a membranous connection. Most of the

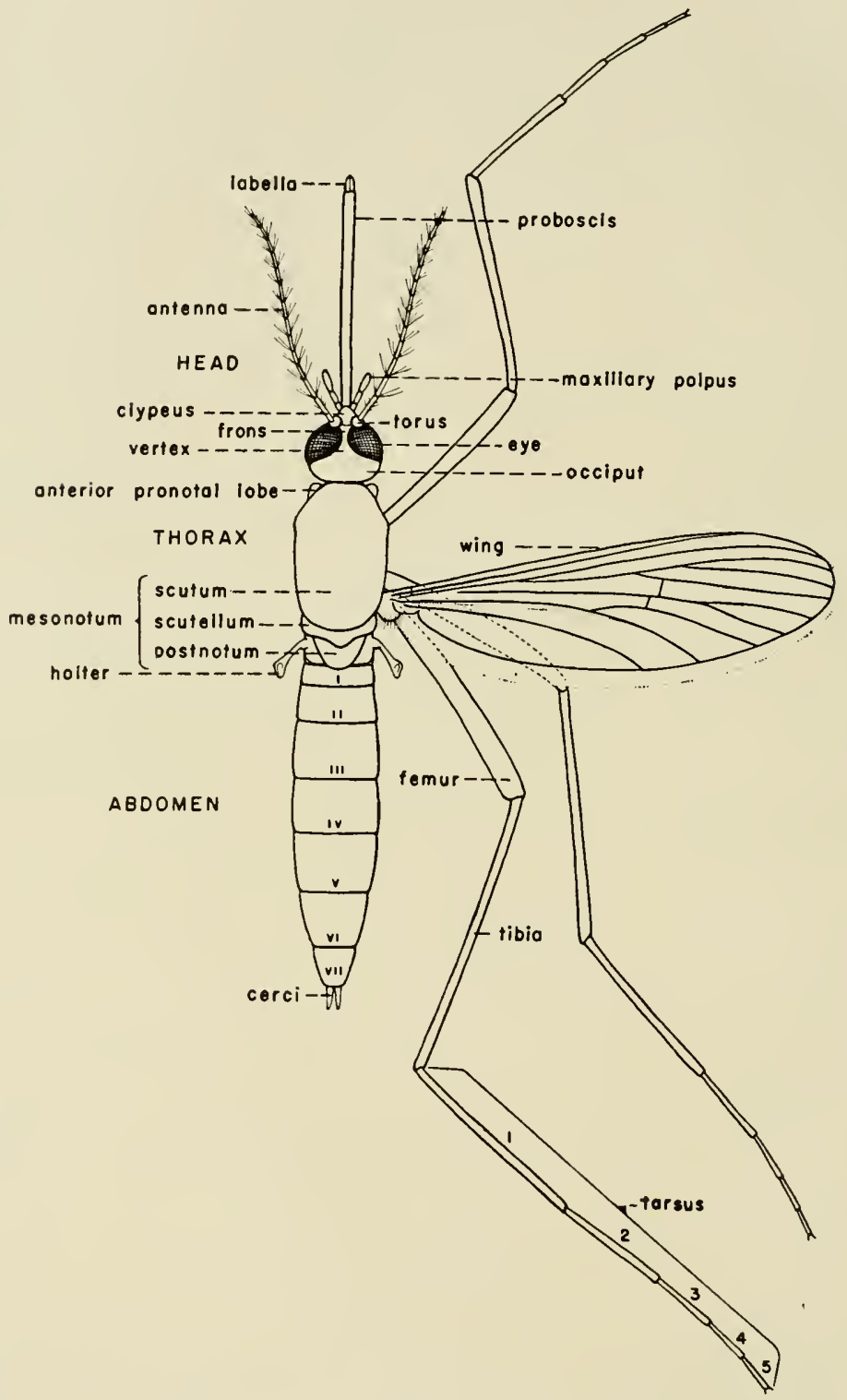

Fig 7 Generalized diagram of a female mosquito. 
lateral area of the head is occupied by the large compound eyes; ocelli are lacking. In the adult descriptions in this book the dorsal part of the head posterior to the eyes is termed the occiput, and that portion projecting forward between the eyes, the vertex. The frons lies between the bases of the antennae and joins the anterior margin of the vertex. The vestiture of the occiput and vertex consists of scales and setae of various types and colors and is often used in identification. The frontal tuft, a group of setae arising from the anterior part of the vertex and extending forward over the frons, is well developed in Anopheles, and its color differences can be used as an aid in separating some of our dark-winged sfecies.

The c!ypeus is a short, usually nude, snout-like projection just anterior to the frons. It is longer than broad, with distal margin rounded, in the tribes Anophelini and Culicini, but wider than long, with distal marg.n somewhat trilobed, in the tribe Megarhinini.

The antcnnae are a pair of long, slender, 15-segmented structures arising on either side of the frons between the eyes. The first segment, the scape, is small, cylinárical, and hidden beneath the globular second segment, the torus. The remaining 13 segments are filamentous and are collectively referred to as the flagellum or individually as the flagellar segments. Each flagellar segment bears a whorl of hairs, usually long and bushy in the males and much shorter and sparser in the females (Fig. 8).1

The maxillary palpi are 5-segmented (the first segment very short) and originate at the anterior margin of the head just below the clypeus. They are important to the taxonomist, not only because they may bear rings or patches of scales of contrasting color, but also because of sexual modification and striking variations in some genera, subgenera, and species. In Culicines the palpi of the female are more or less straight, much shorter than the proboscis, and usually smooth-scaled. The male Culicines usually have denselyhaired palpi longer than the proboscis, each with the last two segments angled upward and tapered to a point.2 These long, bushy palpi, together with the bushy antennee, usually make it comparatively easy to distinguish the sex with the naked eye. In the Anophelines the palpi of the fema!es are straight and, with the exception of the exotic genus Bironella, about as long as the proboscis. The palpi of male Anophelines are about as long or longer than the probonscis and usually less conspicuously haired than those of Culicines. The last two segments of each are somewhat ankylosed. flattened, anoled upward, and rounded at tip. Differences between the palpi of Culicines and of Anopinelines are illustrated in Fig. 8.

The mouthparts, consisting of a conspicuous, greatly elongated lower lip or labium, with its enclosed piercing and sucking structures, are collectively known as the proboscis (Fig. 9). The labium is a sheath-like organ which terminates in a pair of small, approximate lobes, the labella. As well as

1 Of the species occurring in the southern states, the males of Deinocerites cancer, Uranotaenia lowii and Wyeomyia spp. have antennae similar to those of the females.

2 In the southern states, the palpi of the males are similar to those of the females in Deinocerites, Uranotacnia, Wycomyia and Aedes cinereus. 
serving as a protective sheath, the labium guides and supports the enclosed piercing mouthparts during the feeding process. It never enters the wound, however, but is bent out of the way until feeding is completed.
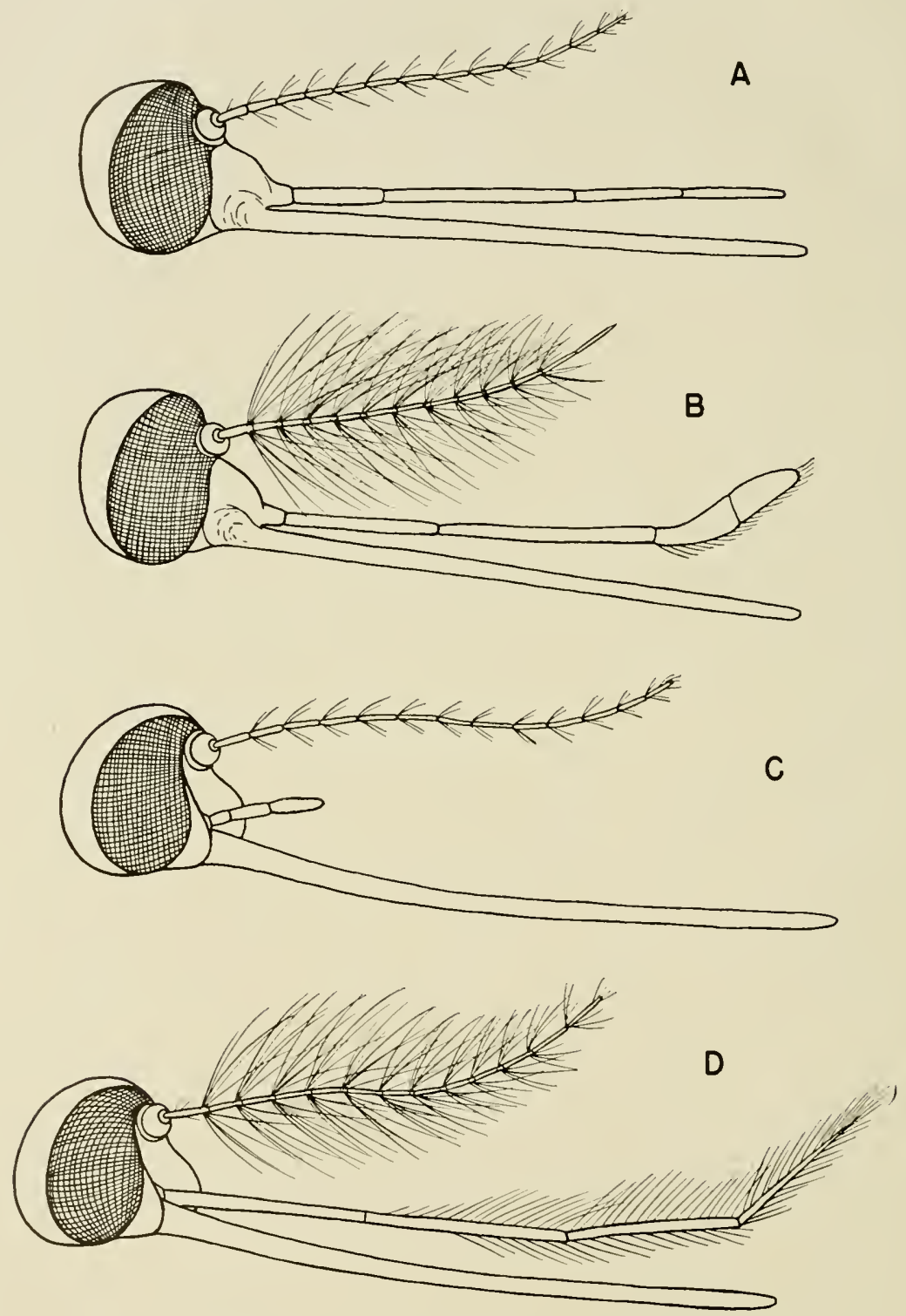

Fig. 8. Heads and appendages of mosquitoes. A, Female Anopheles. B, Male Anopheles. C, Female Culicine. D, Male Culicine. 
The mouthparts which lie within the labium of the female, listed in order with the most dorsal first, are as follows: (1) The upper lip or labrumepipharynx, an elongated organ pointed at its apex and inverted U-shape in cross-section; (2) the flat, blade-like hypopharynx, lying directly beneath the labrum-epipharynx and in close apposition to its ventral surface, forming a canal through which liquid food is drawn during ingestion (the hypopharynx itself is traversed by a minute salivary duct leading from the salivary glands); (3) the paired mandibles and distally dentate maxillae. The mandibles and maxillae are delicate linear-lanceolate organs, the former lying lateral to the labrum-epipharynx and the latter lying beneath and lateral to the hypopharynx.

The mouthparts of the male mosquito differ somewhat from those of the female in that the hypopharynx is apparently fused with the labium. The maxillae are delicate structures, greatly reduced in some species. The mandibles, when present, are also greatly reduced.

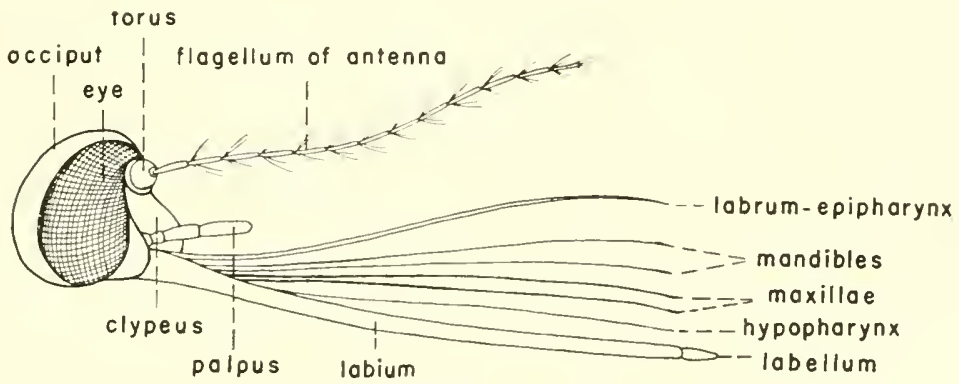

Fig. 9. Mouthparts of female mosquito.

THORAX (Fig. 10).-The thorax consists of three fused segments, the prothorax bearing the fore legs, the mesothorax bearing the second pair of legs and the wings, and the metathorax bearing the third or hind pair of legs and the halters or vestigial hind wings. The mesothorax is much more developed than the other segments due to the fact that it contains large, powerful wing muscles.

Prothorax (Fig. 10A, B).- -The prothorax is reduced and consists of the anterior pronotum, a lateral prominence just back of the head; the posterior pronotum, the region behind the anterior pronotum and in front of the spiracular area (small triangular area immediately before the anterior spiracle and set off from posterior pronotum by a definite ridge); the propleura, a small portion just above the fore coxa; and the prosternum, the region between the two fore coxae. The setae and scales of these regions serve as important taxonomic characters in some genera and species. In Uranotaenia, for instance, the propleural setae are reduced in number. Setae known as spiracular bristles arise from the spiracular area in Culiseta, but are absent in Culex. The posterior pronotum may bear setae or be covered with scales of various types. The anterior pronotum may be clothed with setae and scales, that of the genus Wyeomyia, for example, being shingled with broad, 
appressed scales, the hue of which is an important factor in separating the species occurring in the United States. Although the prosternum has been very little studied, Edwards (56) points out that the presence or absence of scales or bristles on its upper part might be used to good advantage since they are present in some mosquitoes and absent in others.

Mesothorax (Fig. 10A, B).-The mesothorax comprises the largest portion of the thorax, as in all Diptera, and bears many structures of prime
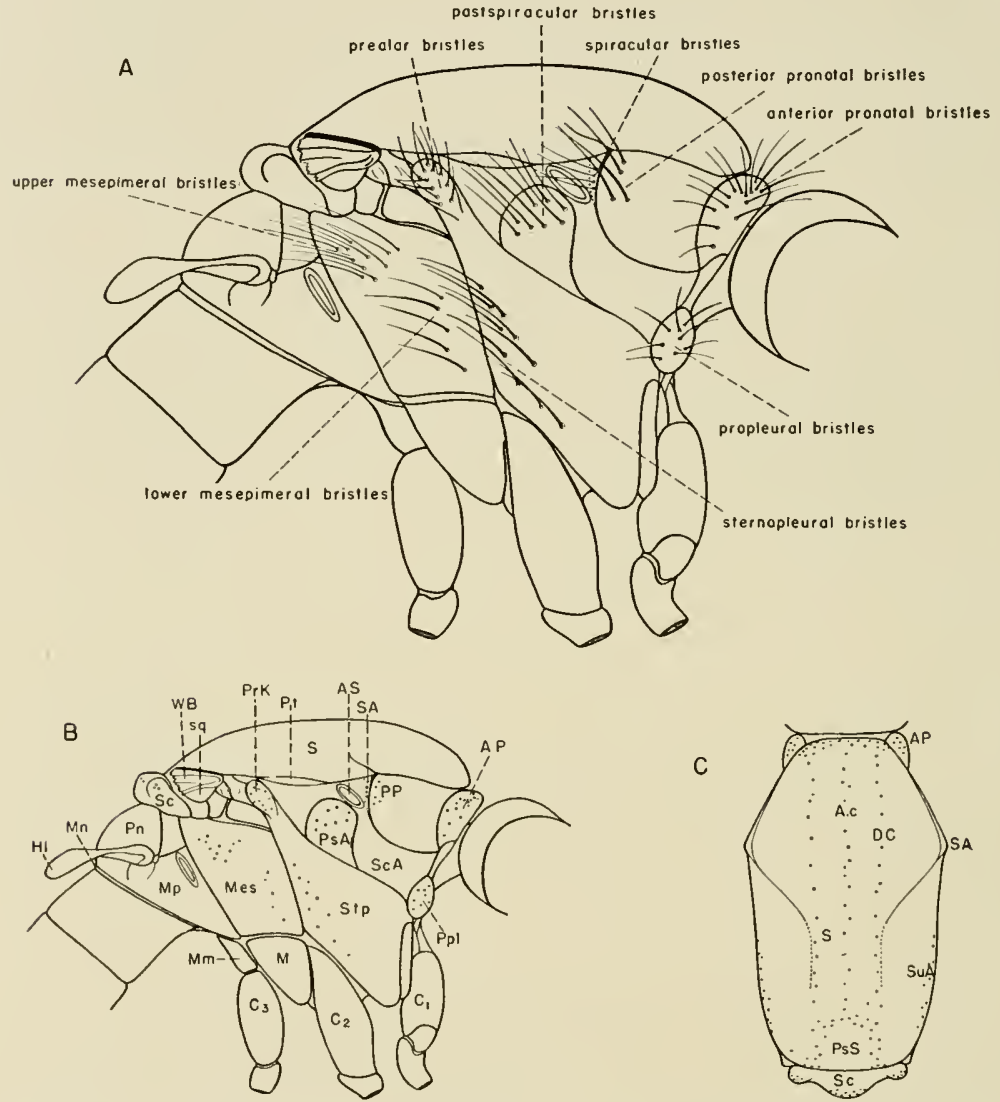

Fig. 10. Parts of thorax and pleural chaetotaxy of an adult Psorophora. (A) Pleural chaetotaxy.(B) Lateral view with sclerites and positions of bristles indicated. AP, anterior pronotum; $A S$, anterior spiracle; $C_{1}$, first coxa; $C_{2}$, second coxa; $C_{3}$, third coxa; Hl, halter; $M$, meron; Mes, mesepimeron; Mm, metameron; Mn, metanotum; $\mathrm{Mp}$, metapleura; $\mathrm{Pn}$, postnotum; PP, posterior pronotum; Ppl, propleura; PrK, prealar knob; $\mathrm{PsA}$, postspiracular area; $\mathrm{Pt}$, paratergite; $\mathrm{S}$, scutum; $\mathrm{SA}$, spiracular area; $\mathrm{Sc}$, scutellum; Sq. squama; SsA, subspiracular area; Stp, sternopleura; WB, wing base. (C) Dorsal view with positions of bristles indicated. Ac, acrostichal bristles; AP, anterior pronotal lobe; DC, dorso-central bristles; $\mathrm{PsS}$, prescutellar space; $\mathrm{S}$, scutum; SA. scutal angle; Sc, scutellum; SuA, supra-alar bristles. 
taxonomic importance. For discussion it may be conveniently divided into the mesonotum and the pleurae.

The mesonotum includes most of the dorsal surface of the thorax and consists of the scutum (combined praescutum and scutum), which makes up the largest share of the mesonotum; the paratergite, a small region cut off from the lateral margin of the scutum by a strong furrow just anterior to and above the prealar area; the scutellum, a trilobed or rourded section connected bumper-like to the posterior margin of the scutum; and the postnotum, the convex structure between the scutellum and metanotum $(45,56)$.

The pleural sclerites of the mesothorax occupy most of the side of the entire thorax, with the exception of the small areas comprising the prothorax and metathorax. The most important mesopleural divisions are the post spiracular area, the region immediately behind the anterior spiracle; the subspiracular area, below the anterior spiracle and adjacent to the posterior pronotum and propleura; the sternopleura, a large, ham-shaped sclerite posterior to the subspiracular area, projecting downward between the 1 st and 2nd coxae and extending dorso-posteriorly nearly to the lateral margin of the scutum; the pre-alar area, the neck-like upper portion of the sternopleura, terminating in the pre-alar knob anterior to the wing base; the mesepimeron, a large, well-defined, sub-rectangular sclerite adjacent to the posterior margin of the sternopleura; and the meron, a small, triangular sclerite slightly above and behind the 2nd coxa and immediately below the mesepimeron.

The vestiture of the scutum (commonly referred to in most literature as the mesnnotum) is of considerable taxonomic importance in specific determinations. The type of the scales and their coloration are characters commonly used, and in some mosquitoes, Aedes aegypti, for example, there can be little doubt as to the identity of the species once the definite bicolorous scale pattern is seen. In many other mosquitoes, such as most Culex, scutal scales are unicolorous, but their coarseness or fineness may be considered. Setae, as well as scales, are generally present to some extent on the scutum; and although not particularly uniformly distributed, considerable variation in number occurring even within members of the same species, they lie in fairly definite lines or areas (Fig. 10C). Those in the median longitudinal row are known as the acrostichal bristles. The submedian longitudinal row on each side of the acrostichals are dorso-central bristles. A supra-alar group on the scutum above and in front of the wing base is generally prominent. The anterior margin of the scutum, back of the head, may bear conspicuous setae, but the prescritellar space (mid-posterior area) has none.

The panatrigites are scaled in Aedes, but usually bare in other mosquitoes. The scutellum is definitely trilobed in all Culicines except Megarbinus, in which it is rounded as in most of the Anophelines. Setae are borne on the margins of the lobes of the scutellum, but are not as important in identification as the scales which are generally present dorsally. The shape and density of scutellar scales are often used in specific identifications of exotic Aedes. 
The postnotum is quite similar in all mosquitoes and therefore is of little taxonomic importance. In Wyeomyia, however, there is a small tuft of setae in the middle of the postnotum which helps distinguish the genus.

The pleural sclerites are not in themselves of great value in a systematic study of the mosquitoes since they are too nearly alike in shape throughout the subfamily and would be difficult to use. In general, the chaetotaxy and scaling of the mesopleural region are the most important. The presence or absence of setae in the post spiracular area (post spiracular bristles) in conjunction with the presence or absence of spiracular bristles is often a character of generic strength.

The genus Aedes, for example, has postspiracular bristles but no spiracular hristles, while Psorophora has both. The number and position of setae on the sternopleura may offer clues in some instances, since they may be sparse as in Wyeomyia and Uranotaenia, or abundant as in Aedes and Culex. Deinocerites cancer is quite readily distinguished from other mosquitoes in the southern states by the presence of bronze scales arranged shingle-like on the sternopleura.

The pre-alar and pre-alar knob may be densely or sparsely clothed, but are not of particular importance. The meron is always bare, and possesses no outstanding features in the mosquitoes of the southern United States. The mesepimeron furnishes two hair groups of value, the upper and lower mesepimeral bristles. Orthopodomyia may be conveniently separated from Mansonita on this basis, since lower mesepimerals are lacking on the former, but present on the latter.

Metathorax (Fig. 10A, B).-The metathorax is much reduced and is of no taxonomic interest. Dorsally the metanotum forms a narrow, usually indistinct, transverse band connecting the postnotum with the 1st abdominal tergite. Laterally a very small sclerite, the metameron, lies immediately above the hind coxa. Between the metameron and postnotum, and posterior to the mesepimeron, lies the metapleuron. It is separated by a diagonal suture into the metepisternum, with the posterior spiracle on its anterior margin, and the metepimeron, a narrow band bordering the 1st abdominal segment.

Legs.-Each of the thoracic segments bears a pair of long, slender legs; the fore-legs arising from the prothorax, the middle legs from the mesothorax, and the hind legs from the metathorax. Each leg is made up of a coxa, trochanter, femur, tibia, and a 5-segmented tarsus. The coxa is short, stout, conical, and connects with the ventral portion of the thorax. The trochanter is a small, hinge-like segment between the coxa and the long femur, which is the largest leg segment. The tibia is slender and about as long as the femur. The tarsus is slender and may be about as long as the femur and tibia combined, particularly on the hind or metathoracic pair of legs; segment 1 is the longest segment of the tarsus, often subequal to the tibia; segments $2,3,4$, and 5 are progressively shorter in most mosquitoes, although the length of one or more of the segments in relation to the others may occasionally vary enough to provide good taxonomic characters (in Ortho- 
podomyia segment 4 of the fore-tarsus is much shorter than segment 5).

The fifth or terminal tarsal segment of each leg bears a pair of smail claws or ungues, one of which may be much larger than the other on the fore-legs of the males of some genera. Arising between the bases of the claws is a small, hairy empodium, apparently always present but usually inconspicuous. A pair of small pad-like structures, the pulvilli, arise laterally near the base of the ungues in Culex and is a diagnostic feature of this genus. The ungues often exhibit generic and sexual differences, but since they are so small as to be inconvenient to examine, they are not discussed in detail.

The vestiture of the legs consists of scales, hairs and bristles, but the scales are by far the most important from a taxonomic standpoint. They are usually rather broad, appressed, and imbricate, but may occasionally be longer, more slender, and suberect, as in the subgenus Psorophora. Ornamentation due to the arrangement of contrasting dark and pale scales, particularly on the tarsal segments, forms the basis for some of the most useful specific characters. This ornamentation is commonly present as basal, medial, or apical bands of pale scales (often both apical and basal white bands present on the same segment). The presence or absence of pale markings, and the position and extent of these markings when present, are very frequently used as key differences between species.

Mesothoracic Wing.-The mesothoracic wings are well-developed orgars of flight; their scales and type of venation serve as infallible means of distinguishing the Culicinae from other Diptera. They are elongate-oval in shape, each with two indentations near the base on the posterior margin. The small, flap-like structure nearest the thorax and delimited by the first indentation is the squama. In most mosquitoes the squama is fringed with hairs, but this fringe is absent in a few genera, including Megarhinus. Wyeomyia, and Uranotaenia in the southern states. The area delimited by the second indentation, the small, lobate alula, is of little importance.

The nomenclature of wing venation adopted here (Fig. 11) is essentially that used by Barraud (11), Edwards (56), and Ross and Roberts (150),

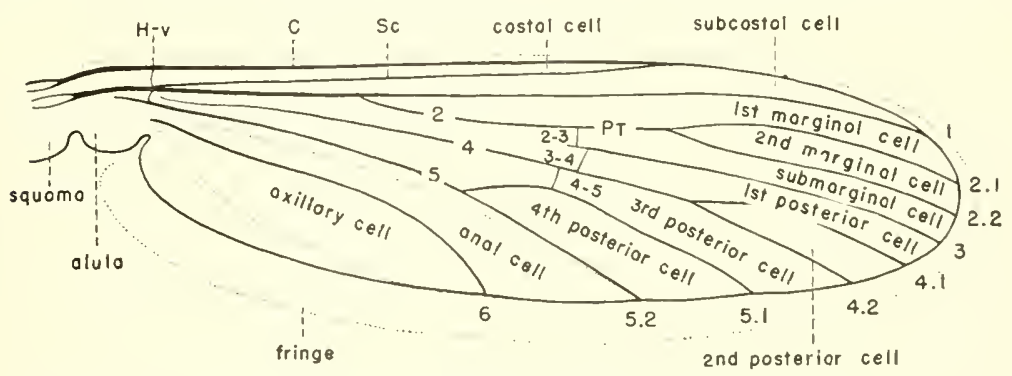

Fig. 11. Wing of mosquito, with venation shown. C, costa; Sc, subcosta; 1, first longitudinal vein; 2, second longitudinal vein (2.1, anterior branch; 2.2, posterior bıanch); 3, third longitudinal vein; 4 , fourth longitudinal vein $(4.1$, anterior branch; 4.2, posterior branch); 5, fifth longitudinal vein (5.1, anterior branch: 5.2 , posterior branch); 6 , sixth longitudinal or anal vein; $H-v$, humeral cross-vein; $2-3,3-4$ and $4-5$, cross-veins. Pt. petiole of vein 2 . 
The terminology of the Comstock-Needham system of wing venation is not included since it is rarely used by culicidologists.

The membranous areas bounded by the various veins and cross-veins are known as cells and are clothed with very fine hairs or microtrichia. The veins themselves are clothed with scales, generally of two types: Those which lie close to the veins and which are usually rather short and broad are termed squame scales; those which are suberect and usually narrow are known as outstanding or plume scales. The size, shape, and color of wing scales comprise important taxonomic characters.

The entire posterior margin of the wing, from the tip to the alula, bears a close-set row of long, slender fringe scales. Great variation in alternating spots of pigmentation on the wing-fringe is exhibited by many Anophelines; a characteristic fringe pattern helps to distinguish Psorophora signipennis in the United States.

With few exceptions, the wing venation is uniform in the Culicinae; the few differerces that do occur, however, are convenient and reliable characters for separating some groups. Uranotaenia and Megarbinus, for example, have the apical, forked portion of vein 2 much shorter than its petiole. The position of cross-vein 3-4 in relation to cross-vein 4-5, the length of the subcostal vein and the length of vein 6 comprise most of the remaining variations.

Halter.-In mosquitoes, as in other flies, the metathoracic wings are greatly reduced and not used in flight, except perhaps as balancing organs. These rudimentary structures, the halters, arise behind and slightly above the posterior spiracle from the dorsal region of the metapleuron. Each is made up of three portions: The scabellum, or base; the mid-halter, or stemlike portion; and the capitellum, or terminal knob. Coloration of scaling and integument, particularly of the capitellum, may occasionally be conveniently emploved in separating allied species.

ABDOMEN.-The abdomen is elongate, cylindrical, and made up of 10 segments, the first 8 of which are distinct. The 9th and 10th segments of both the males and females have undergone great modification for carrying out sexual functions. These modified terminal segments of the male, together with the eighth, are discussed separately under "Male Terminalia." These segmerits of the female, although possessing many generic and subgeneric characters, are not included in this work as they have been little studied and infrequently used by workers in this country.

Each of the distinct abdominal segments is divisible into a large dorsal tergite and a smaller, ventral sternite, connected laterally by a pleural $m \mathrm{~cm}$. brane. Successive segments are joined by intersegmental membranes. It is convenient to speak collectively of the tergites as the dorsum of the abdomen and of the sternites as the venter. Both the dorsum and the venter are shingled with scales in the Culicines, but are usually bare or with few scales in the Anophelines. The first tergite often shows differences in number and position of scales in various Culicine genera and subgenera, as well as differences in scale and integument color. Bicolorous patterns of scales, generally in the 
form of basal or apical bands or lateral patches, may be present on the tergites of many species. Although the shape and extent of these markings may vary somewhat on individuals within a species, they are nevertheless useful characters. Scale patterns are usually not so evident on the venter, but the predominating color of the scales may be important.

The shape of the female abdomen may be of diagnostic significance. In Aedes and Psorophora, for example, the abdomen is tapered apically, with the 8 th segment withdrawn into the 7 th. In the other genera occurring in the southern United States, the abdomen does not taper appreciably, being broadly truncate or bluntly rounded at the apex, although the 8th segment may be partially withdrawn in some cases.

MALE TERMINALIA.-The terminal abdominal segments of the male mosquito are greatly modified for copulation, exhibiting variations in structure which are of much taxonomic value. The term male terminalia is used here to include the anal and genital structures of the 8 th, 9 th and 10 th abdominal segments. The terminology followed is essentially the same as that used by Ross and Roberts (150) with slight modifications.

The male terminalia of the Culicinae undergoes a rotation of 180 on its longitudinal axis shortly after the adult emerges so that the structures which were dorsal become ventral, and vice versa. In referring to structures of the male terminalia, however, the original positions before rotation are used even though they appear opposite on the mature specimen.

The presence or absence of certain structures, as well as the modification of these structures, comprises the taxonomic characters found in the male terminalia. The structures include the eighth abdominal segment, ninth tergite, ninth sternite, proctiger, phallosome and supporting structures, basistyles, dististyles, and claspettes (Figs. 12 to 14). They are considered separately as follows :

Eighth abdominal segment.-Although this segment is included in the male terminalia, it is usually unmodified. It is relatively unimportant in Aedes, Psorophora and most Culex, although it may bear diagnostic setae or spines dorsally in some genera, particularly Mansonia and Wyeomyia.

Ninth tergite (IX.T).-The ninth tergite and the lobes of the ninth tergite borne postero-laterally or submedially on the transverse band of the ninth tergite, often provide good characters. The extent of sclerotization and the shape of the transverse band of the ninth tergite and the developmnt, position, and armature of its lobes are particularly significant.

Ninth sternite (IX.S).-The ninth sternite is usually unmodified in the mosquitoes of the southern United States and unimportant in classification.

Proctiger.-The proctiger, made up of elements of the tenth abdominal or anal segment, varies considerably in the different genera of mosquitoes. The tenth tergite is usually reduced. The tenth sternite (X-S) or paraproct is usually the most strongly developed portion and forms a pair of slender, sclerotized supports for the anal membrane (A-M) in most genera, as in 
Aedes, Psorophora, and Culiseta. It is vestigial or absent, however, in Anopheles and Uranotaenia, which have the anal lobe $(\mathrm{An}-\mathrm{L})$ or membrane unsupported. Although the tenth sternite may be characteristically knobbed or toothed apically in many species of several genera, the terminal armature has been found most valuable taxonomically in the genus $C u l e x$. This armature consists of a crown of spines in the subgenus Culex and a comb-like row of teeth in the subgenera Neoculex and Melanoconion.

Phallosome $(\mathrm{Ph})$.- The phallosome (mesosome) is a chitinous, tube-like structure surrounding the penis. It is situated just ventrad of the proctiger and is held in position by supporting structures, the basal plates (B-P) and parameres $(\mathrm{Pm})$, which articulate with the basal processes of the tenth sternite, the phallosome, and with each other. The phallosome furnishes some of the most reliable generic and subgeneric characters; its variations range from a long, slender tube with apical leaflets in Anopheles to complex toothed plates in Culex, Uranotaenia and Deinocerites.

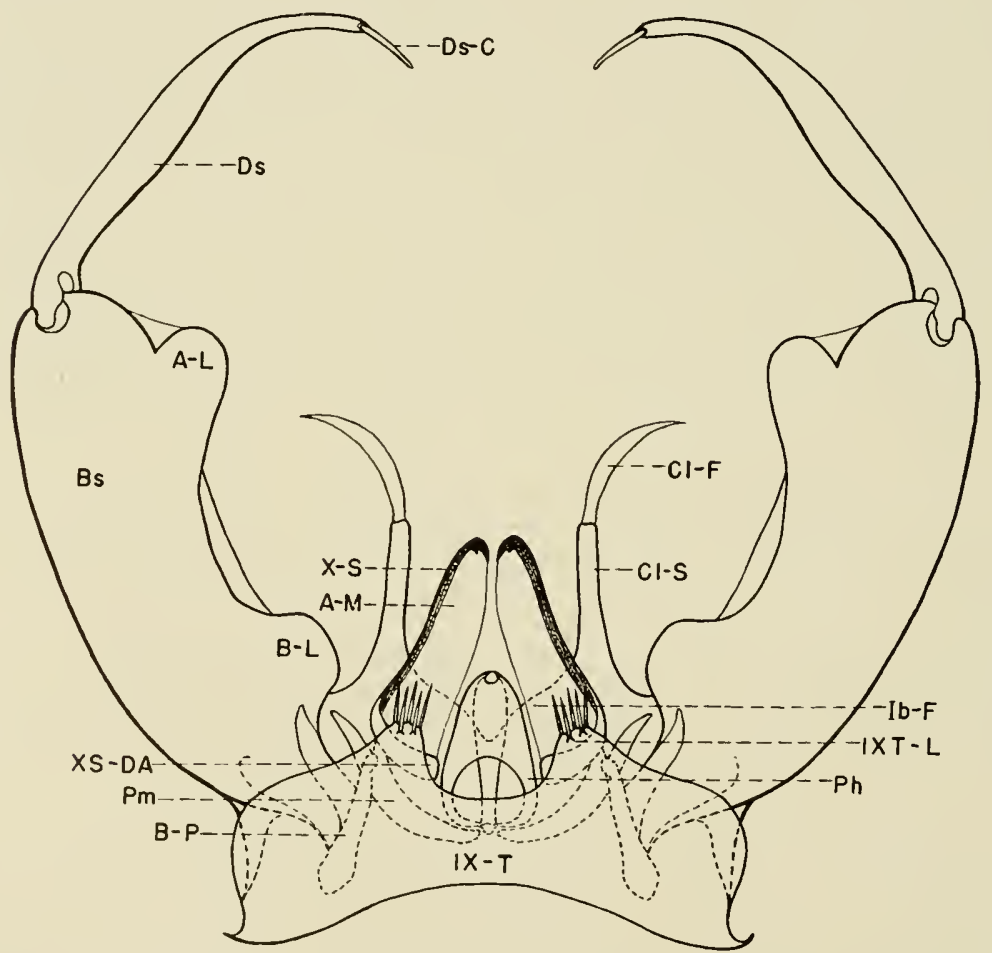

Fig. 12. Diagram of male terminalia of Aedes. A-L, apical lobe; A-M, anal membrane; B-L, basal lobe; B-P, basal plate; Bs, basistyle; Cl-F, filament of claspette; Cl-S, stem of claspette; Ds, dististyle; Ds-C, claw of dististyle; Ib-F, interbasal fold; IX-T, ninth tergite; IXT-L, lobe of ninth tergite; Ph, phallosome; Pm, parainere; X-S, tenth sternite; XS-DA. dorsal arm of tenth sternite. 
Basistyle (Bs).-The basistyles are a pair of large, hollow, thigh-like processes apparently arising from the ninth sternite. They usually bear long setae and scales, and may or may not possess apical, subapical, or basal lobes on the inner surfaces. A subapical lobe (S-L) is evident in Culex and bears important rods, spines, and leaf-like appendages. An apical lobe (A-L) is often present in Aedes, with varying shape and vestiture. A basal lobe (B-L) is present in several genera, but is best developed in some of the Aedes, where it may give rise to one or more large spines and characteristic hairs. The basal lobe is represented in Anopheles by only the large, parabasal spines (P. $\mathrm{S}$ ); it is always absent in Culex, which bears instead the subapical lobe, probably homologous with the basal lobe of Aedes.

Dististyle (Ds).-An articulated appendage, the dististyle, is borne on the

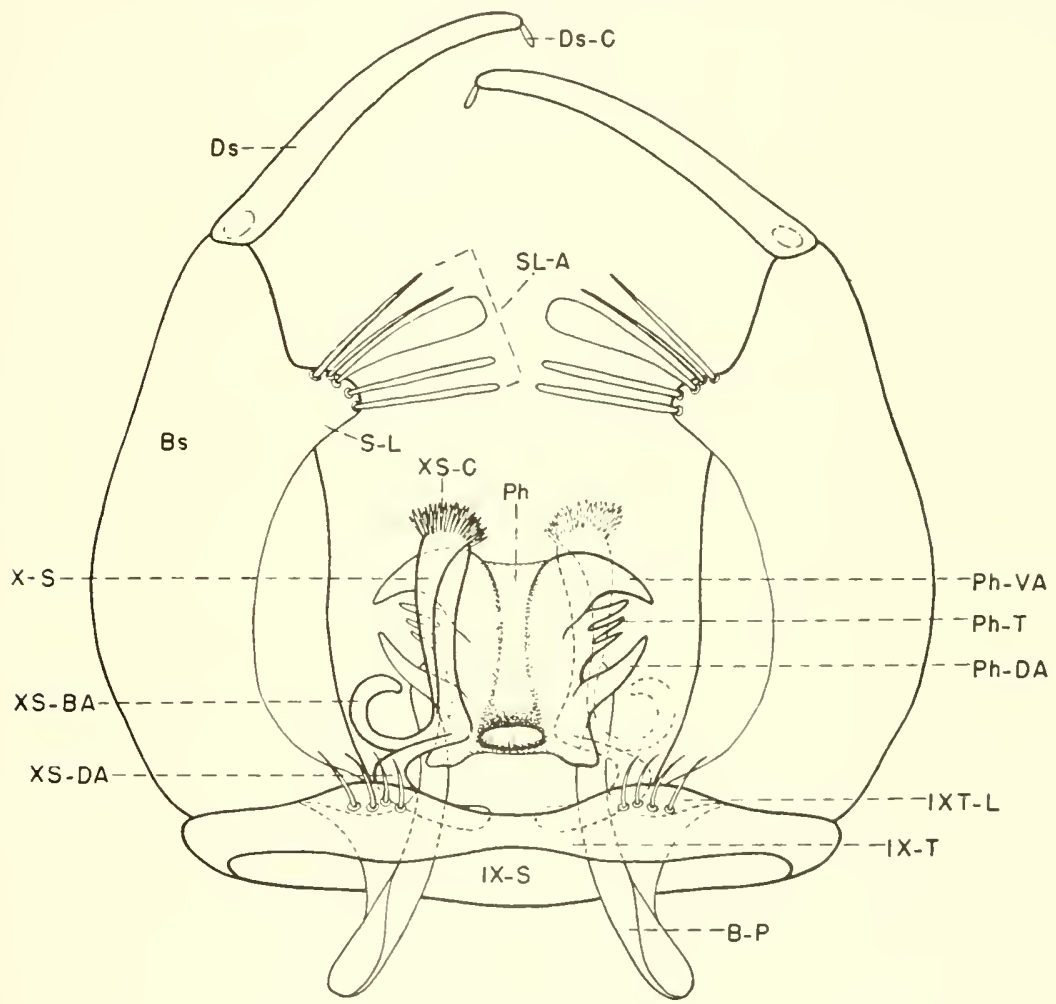

Fig. 13. Diagram of male terminalia of $C_{u l e x}$ (right plate of phallosome is shown in bold lines for clearness, although it lies beneath the tenth sternite). B-P, basal plate: Bs, basistyle; Ds, dististyle; Ds-C, claw of dististyle; IX-S, ninth sternite: IX-T, ninth tergite; IXT-L, lobe of ninth tergite; Ph, phallosome; Ph-DA, dorsal arm of phallosome; $\mathrm{Ph}-\mathrm{T}$, lateral teeth of phallosome; $\mathrm{Ph}-\mathrm{VA}$, ventral arm of phallosome; S-L, subapical lobe; SL-A, appendages of subapical lobe; X-S, tenth sternite; XS-BA. basal arm of tenth sternite; XS-C, crown of tenth sternite; XS-DA, dorsal arm of tenth sternite. 
apical portion of the basistyle. An articulated claw (Ds-C) is usually present at or near its apex. The shape of the dististyle, its vestiture, its place of origin from the apical portion of the basistyle, and the place of origin and type of claw are important characters. In Anopheles the dististyle is long, curved, and slender. In most Aedes it is slightly broader medially, somewhat pilose. In Psorophora great modification of the dististyle occurs. This is exhibited by the medially swollen type found in $P$. ferox, and $P$. confinnis (Figs. 73, 80), the truncate type of $P$. varipes with its claw subapical (Fig. 78 ), and the greatly modified type of $P$. howardii with its tremendously developed, lateral, hatchetshaped lobe (Fig. 69).

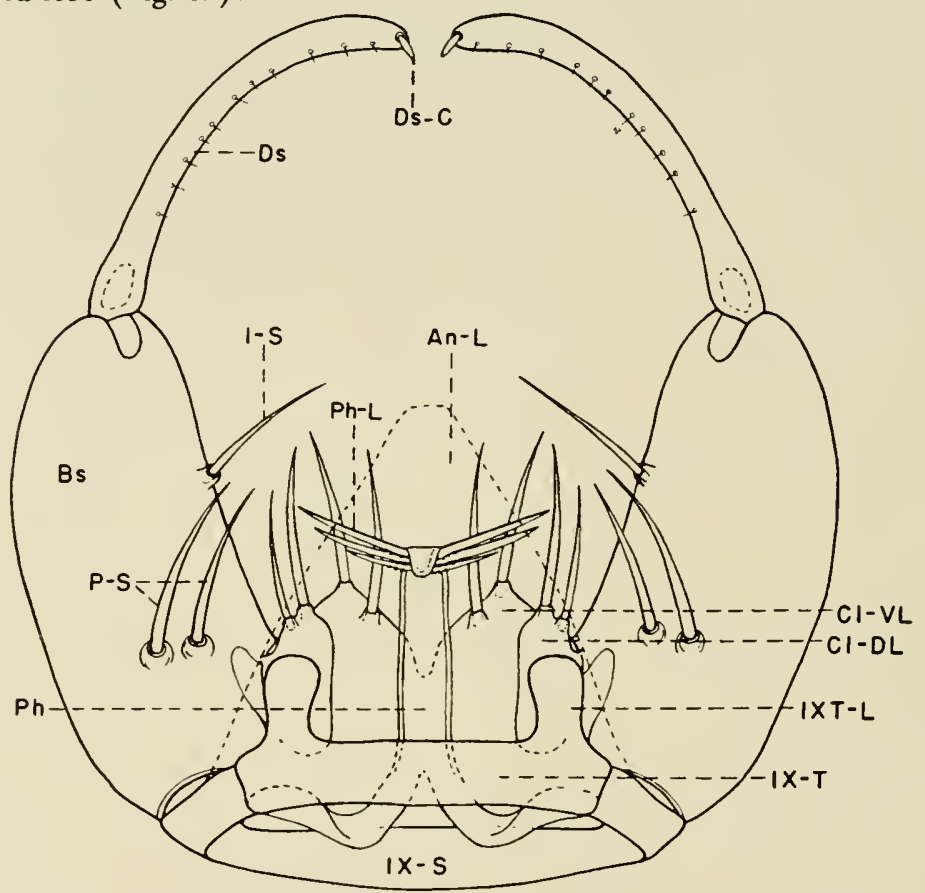

Fig. 14. Diagram of Male Terminalia of Anopheles. An-L, anal lobe; Bs, basistyle; Cl-DL, dorsal lobe of claspette; Cl-VL, ventral lobe of claspette; Ds, dististyle; Ds-C, claw of dististyle; I-S, internal spine; IX-S, ninth sternite; IX-T, ninth tergite; $\mathrm{Ph}$, phallosome; $\mathrm{Ph}-\mathrm{L}$, leaflets of phallosome; $\mathrm{P}-\mathrm{S}$, parabasal spines.

Claspette $(\mathrm{Cl})$.- The connecting membranous projections between the bases of the basistyles, the interbasal folds (Ib.F), may bear a pair of structures known as the claspettes. Their position is ventrad of the phallosome and in some Aedes can be seen projecting dorso-ventrally in a lateral view. Anopheles has the claspettes present as a pair of fleshy, spined, bilobed structures, each being incompletely divided into an outer or dorsal lobe (Cl-DL) and an inner or ventral lobe (Cl-VL). In Aedes there is but one lobe, presumably corresponding to the ventral one of Anopheles, developed into a well-defined 
stem (Cl-S) and a distal filament (Cl-F). Characters of the claspettes are generally clear-cut, reliable, and easy to use.

\section{Pupal Characters}

The pupae of both Culicines and Anophelines are comma-shaped and resemble each other in many respects. The enlarged anterior portion or cephalothorax is composed of the head and thorax. The slender tail-like portion is the eight-segmented abdomen which terminates in a pair of broad flattened plates or paddles (Fig. 15A).

The chief diagnostic characters used in the classification of pupae are to be found in the abdominal chaetotaxy, the paddles, and the respiratory trumpets, particularly in their shape and the relative length of the closed tubular portion, the meatus. To mount the pupa for examination, the cephalothorax can be split on the mid-ventral line, spread open and mounted dorsal side uppermost.

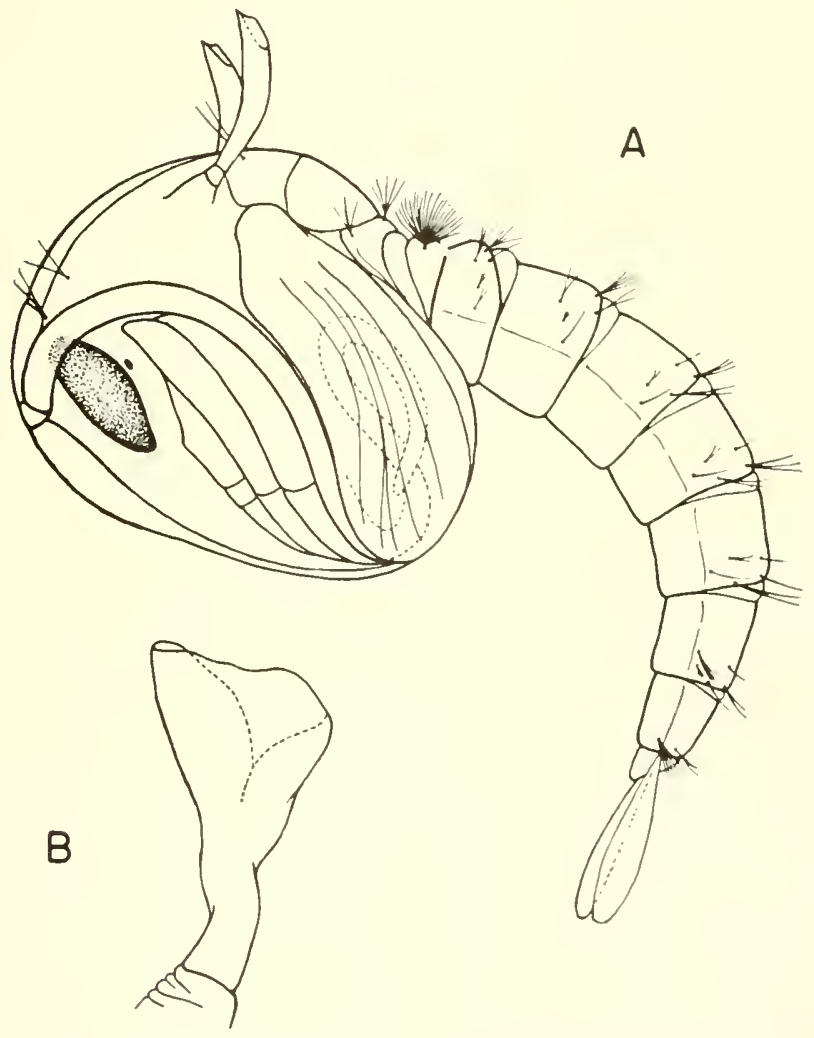

Fig. 15. Pupa of a mosquito. A, Culicine pupa (lateral view). B. Respiratory trumpet of Anopheles. 

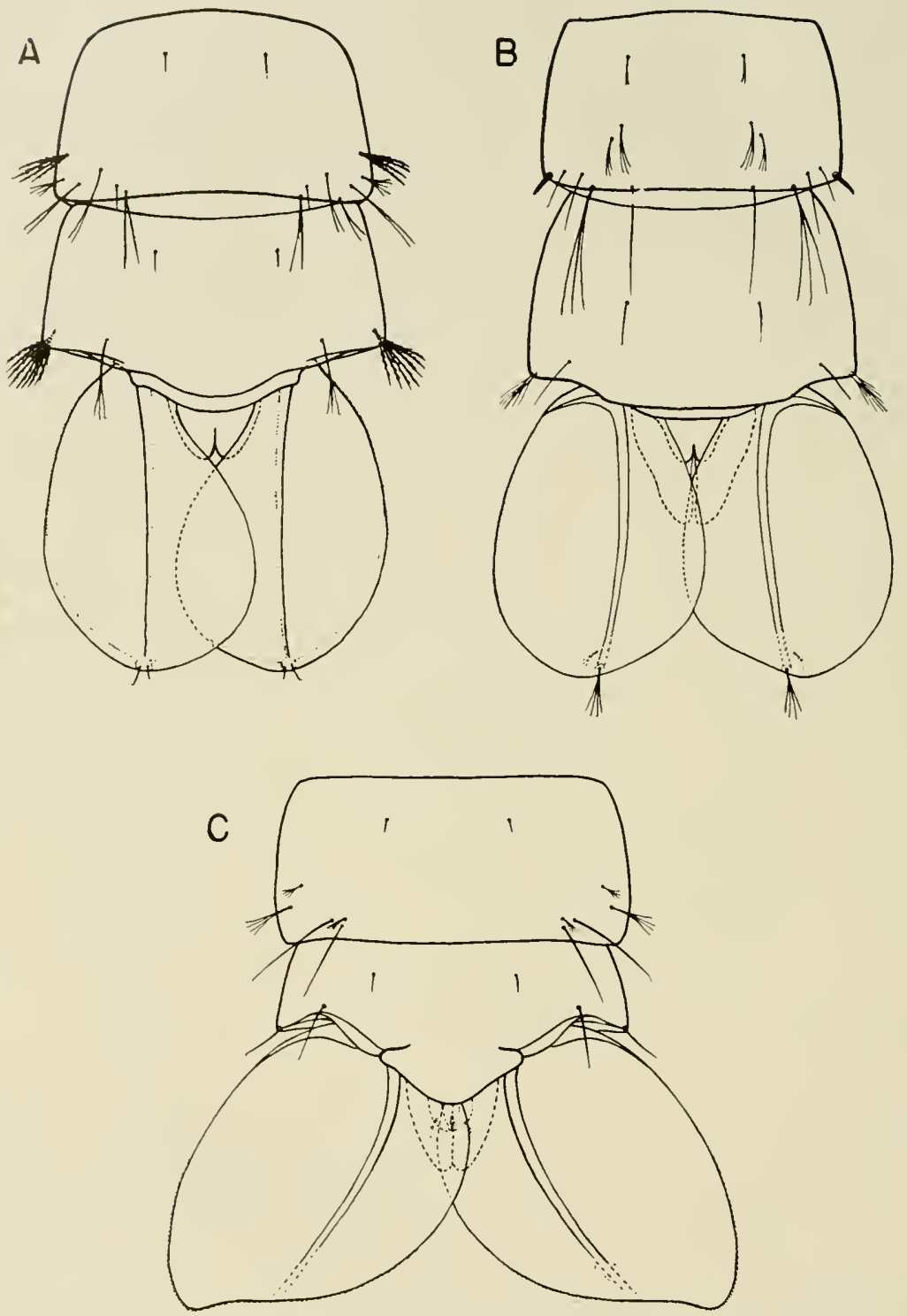

Fig. 16. Terminal abdominal segments of pupae (dorsal views). A, Culex. B, Anopheles. C, Megarhinus. 
RESPIRATORY TRUMPETS. (Fig. 15B).--Each thoracic respiratory trumpet consists of a closed tubular portion, the meatus, and an open portion, the pinna. The ratio of the length of the meatus to that of the entire trumpet has been much used in descriptions. The respiratory trumpets in the Anophelini are short, truncated at the distal end, and have a rather large oblique opening which terminates in a split. The respiratory trumpets in Culicines are variable, but are usually elongate or broadly conical and unsplit. Other characters of the cephalothorax are rarely used in classification.

ABDOMEN. (Fig. 16) -- The chaetotaxy of mosquito pupae has been studied by various workers, including Baisas (4), Christophers (40), Macfie (107) and Senevet (168). The bristles and hairs of the pupal abdomen are illustrated in the following general works: Christophers (40), Edwards (56) and Evans (57). The presence of simple spines on the posterior corners of abdominal segments III to VII and a fringed spine in the same position on segment VIII is characteristic of the Anophelini. In the Culicini the corresponding hairs are usually fine and branched and do not arise exactly at the posterior lateral corners. These hairs are very long in the Megarhinini and are placed well back from the corners of the segment.

PADDLES. (Fig. 16).-The shape, position or absence of hairs, and length and nature of the fringe of the paddles provide important diagnostic characters. In the Anophelini, there are two hairs near the posterior end of the paddle, the paddle hair arising near the hind margin, and the accessory paddle hair, on the ventral side, arising a little in front of the origin of the paddle hair. In the genus Culex there is a small accessory hair placed beside the small paddle hair. In other genera of the Culicini, the paddle hair is either single or absent. There is no paddle hair in the members of the tribe Megarhinini.

\section{Larval Characters}

The mosquito larva has three main body regions, head, thorax and abdomen, each of which possesses variable structures important in classification. The general appearance of larvae and their main morphological features are illustrated in Figs. 17 to 22.

The head capsule is more or less globular in shape, somewhat flattened dorso-ventrally, and formed of three large sclerites; a pair of latero-ventral ocular sclerites (epicranial plates) and a single dorsal plate, the clypeus (fronto-clypeus). A V-shaped epicranial suture is formed by the junction of these three sclerites. The ocular sclerites bear the antennae anteriorly and the imaginal and larval eyes mid-dorsally. The clypeus has attached to its median anterior border the narrow preclypeus, which bears the predypeal spines. Both the ocular sclerites and the clypeus possess several pairs of important hairs.

Although the mouth parts are ventral in position, parts of the labrum project anteriorly and are visible in a dorsal view. The labrum is composed of a median piece, the hairy palatum, and lateral lobes bearing the mouth brushes. The mouth brushes are little used in classification except for separating out the predacious forms, such as Psorophora ciliata, P. howardii, and Megurhinus spp. 


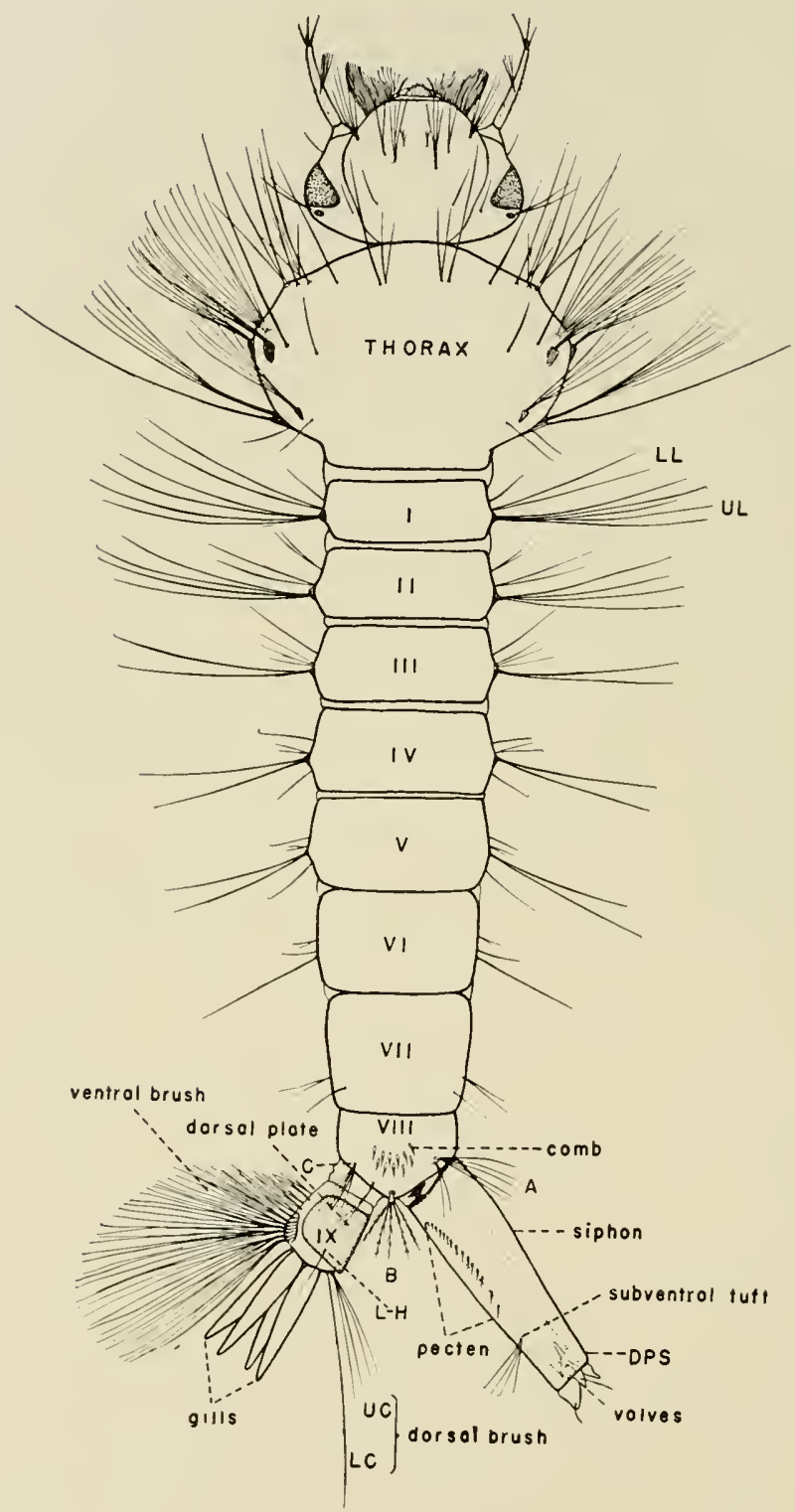

Fig. 17. Fourth instar larva of Aedes. A, siphonal hair; B, subsiphonal hair; C, anal hair; DPS, dorsal preapical spine; LC, lower caudal hair; LH, lateral hair of anal segment; LL, lower lateral abdominal hair; UC, upper caudal hair; UL, upper lateral abdominal hair. 
In addition to the labrum, the most prominent structures on the ventral side of the head are the large maxillae, occasionally armed with characteristic spines, and the heavily sclerotized mandibles, the teeth of which are used in conjunction with the sclerotized mentum for mastication. The labium forms the remainder of the floor of the mouth and is composed of a proximal prementum, an intermediate mentum and a distal submentum. The shape of the mentum and the size, shape and arrangement of its teeth provide useful diagnostic characters in some species.

The thorax consists of three completely fused segments, the pro-, meso-, and metathorax, distinguished only by hair groups, particularly the pleurals, which are present on each segment.

The abdomen is made up of nine segments, the first seven of which are somewhat similar. The eighth segment is modified to bear the breathing apparatus postero-dorsally. The modification of this respiratory organ is one of the most characteristic features in differentiating the larvae of Anophelines and Culicines. The ninth (anal) segment bears several structures of taxonomic importance, particularly in the identification of Culicines.

The larvae of Culicines and Anophelines, while possessing many morphological features in common as stated above, differ in numerous obvious respects. It is therefore convenient to discuss many of the diagnostic characters separately.

\section{CULICINE LARVAE}

Important characters used in classifying fourth instar Culicine larvae are shown in Figs. 17, 18. The terminology used here follows that employed by Hopkins (81), with modifications.

HEAD. (Fig. 18).-Each antenna is an elongate, cylindrical structure, generally curved inward. It bears an antennal tuft (often a single small hair) on the inner surface, and a pair of strong subterminal spines near the apex. A pair of strong terminal spines and a membranous papilla are located at the apex of the antenna. The principal taxonomic features of the antenna are its length, shape, the presence or absence of small spines on the shaft, and the position and nature of the antennal tuft.

The paired hairs of the head capsule commonly considered in specific descriptions are as follows: Preantennal (A), loner head hairs (B), upper head hairs (C), postclypeal hairs (d), suturals (e), trans-suturals (f), supraorbitals, basals and sub-basals. The position of these hairs, their size, and number of branches may vary greatly in the different genera and species.

THORAX.-The pleural hair groups (propleural, mesopleural and metapleural) are indicators of the segmentation of the thorax. The dorsal submedian prothoracic hairs (1-3) are useful in separating the two species of Uranotaenia occurring in this region (Figs. 52, 54). The lateral hairs (5-7) are of diagnostic value in separating Mansonia indubitans and $M$. titillans. The other hair groups of the thorax are seldom used in the classification of the Culicines. 
AвDOMEN. (Fig. 17).-Abdominal segments I to VII bear lateral abdominal hairs and subdorsal and dorsal paired tufts. Segment VIII is somewhat triangular in shape when seen from a lateral view and bears the siphon (airtube) postero-dorsally and three large hair tufts, the siphonal, the subsiphonal, and the anal, postero-laterally, with smaller, usually single, hairs between them. These large hair tufts are referred to by Hopkins (81) as tufts A, B, and C. The eighth segment also bears bilaterally a comb composed of a row or patch of tooth-like scales (absent in Megarhinini). The number, arrangement and shape of the comb scales are of considerable diagnostic value.

Extending bilaterally from the base of the siphon there is a subventral longitudinal row of spines or teeth forming the pecten (absent in Megarhinus, Orthopodomyia and Wyeomyia). The siphon also bears one or more pairs of subventral hair tufts (sometimes obsolete in Psorophora). Lateral and subdorsal tufts and a subapical spine may be present in some genera. The orifice of the siphon is surrounded by five valves; an unpaired median or dorsal valve, a pair of small lateral valves, and a pair of large ventral or posterior valves, each of which commonly bears a single small hair. A small, sclerotized, anchorlike projection, the acus, may be present at the base of the siphon. Features of the siphon which are of taxonomic importance are its shape, the relationship of

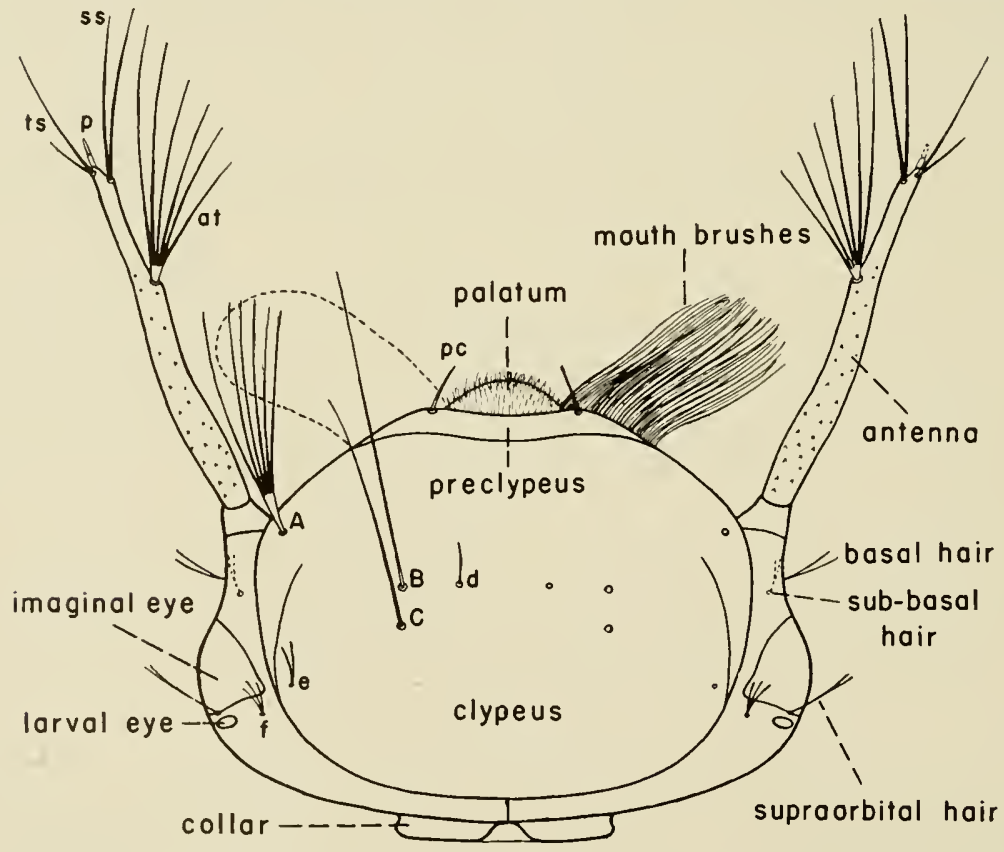

Fig. 18. Head of Culicine larva. A, preantennal hair; B, lower head hair; C, upper head hair; d, postclypeal hair; e, sutural hair; $f$, trans-sutural hair; pc, preclypeal spine; at, antennal tuft; p, papilla; ss, subterminal spines; ts, terminal spines. 
length to width, the nature, number and position of the pecten teeth, and the number, development and position of hair tufts. The proportion of length to width is usually obtained by comparing the length of the siphon, excluding the acus and the valves, with its width at the base or at its widest point. A siphon is referred to as "inflated" if it is considerably wider near the middle than at the base, as exhibited by Psorophora cyanescens (Fig. 72).

The ninth or anal segment possesses several important structures. A large sclerotized dorsal plate may or may not completely ring the anal segment and bears a lateral hair or tuft on either side near its posterior margin. From the dorso-apical angle of the anal segment arises the dorsal brush composed of the upper and lower caudal hairs. The ventro-apical margin has a ventral brush composed of a staggered row of hair tufts, the bases of which may be sclerotized to form what is known as the barred area or grid. The tufts arising from the barred area or grid may be referred to as the cratal tufts, and those arising before the grid as the precratal tufts. The anal segment bears posteriorly two

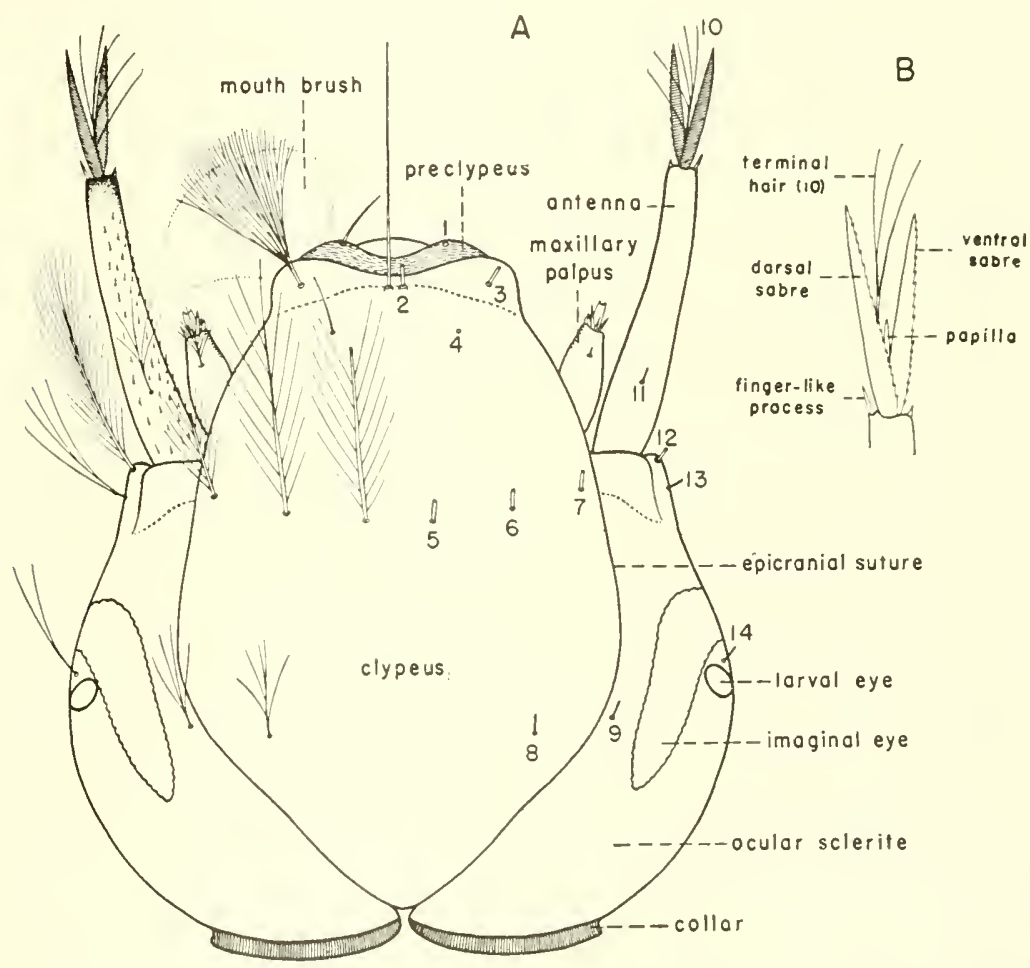

Fig. 19. Head of Anopheles larva. A. Dorsal view of head. (1), preclypeal spine; (2), inner clypeal hair; (3), outer clypeal hair: (4), postclypeal hair; (5), inner frontal hair: (6), middle frontal hair: (7), outer frontal hair; (8), sutural hair; (9). trans-sutural hair; $(10)$, terminal hair of antenna; (11), antennal hair; (12), hasal hair; (13), sub-basal hair; (14), supraorbital hair. B. Tip of antenna. 
to four cylindrical or tapering, thin-walled papilliform processes known as the anal gills. The number, length and shape of the gills are often used in classification.

\section{ANOPHELINE LARVAE}

Important characters used in classifying fourth instar Anopheline larvae are shown in Figs. 19-22. The designation of hairs by numbers follows rather closely that used by Christophers (40), with some modification.

HEAD. (Fig. 19).-The antenna is an elongate, cylindrical organ, usually somewhat spinose and possessing several structures of taxonomic value. The antennal shaft bears the antennal hair (11) on its basal half, and several prominent structures on its distal end; namely, a papilla, a finger-like process, a
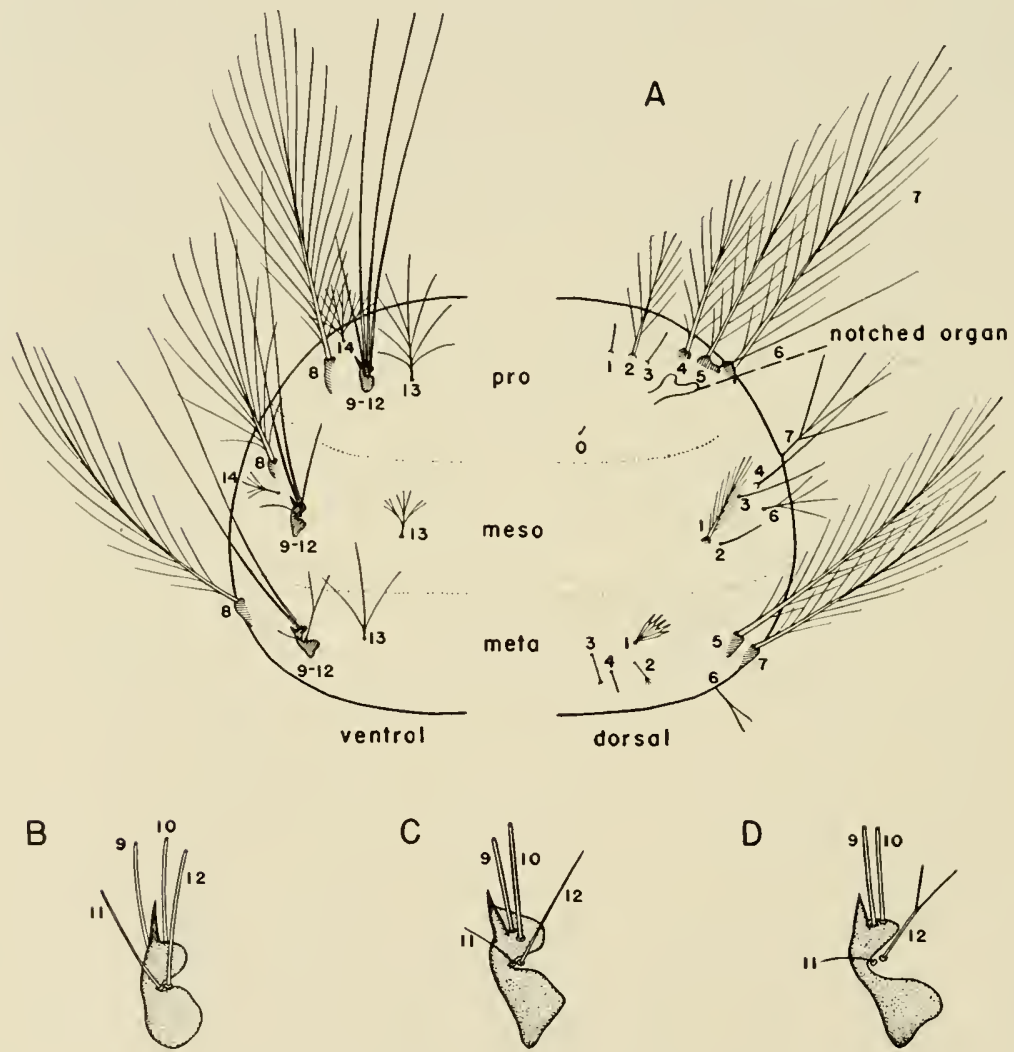

Fig. 20. Thorax of Anopheles larva. A, Ventral and dorsal views of thorax (pro $=$ prothorax; meso. = mesothorax; meta. = metathorax). Prothorax: (1-3), inner, middle and outer submedian prothoracic hairs; (4-8), lateral hairs; $(9-12)$, prothoracic pleural hairs; (13), ventral submedian hair. Mesothorax: (9-12), mesothoracic pleural hairs. Metathorax: (1), metathoracic palmate hair; (9-12), metathoracic pleural hairs. B, C, and $\mathrm{D}$, Bases of pleural hairs of pro-, meso-, and metathorax rsepectively. 


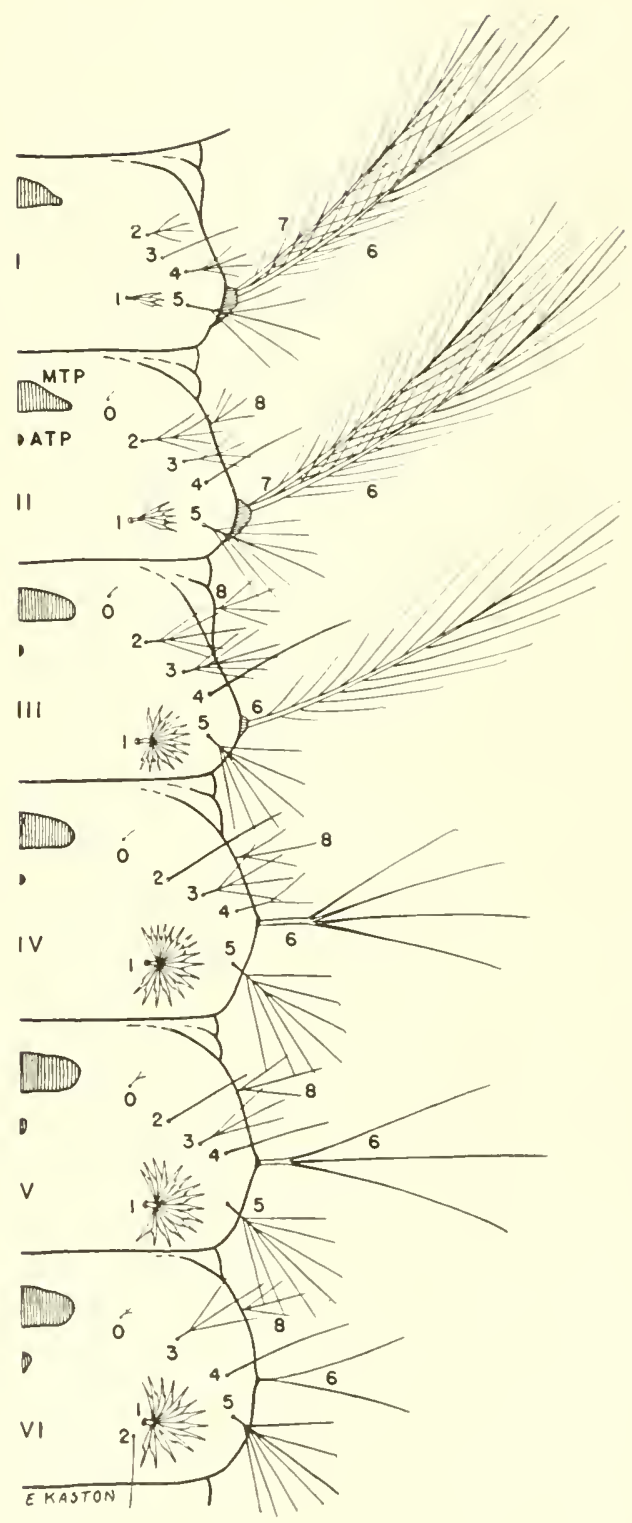

Fig. 21. Abdominal segments 1 to VI of Anopheles larva (dorsal view). MTP. main tergal plate; ATP, accessory plate; (O), dorsal anterior submedian hair; (1), palmate hair; (2), antepalmate hair; (3-5), sublateral hairs; (6-8), lateral hairs. 
terminal hair (10), and the dorsal and ventral sabres (Fig. 19B). Characters of the following paired head hairs are generally referred to in specific descriptions: Inner clypeals (2); outer clypeals (3); postclypeals (4); the frontal hairs, which are made up of the inner frontals (5), middle frontals (6), and the outer frontals (7); sutural hairs (8); trans-sutural hairs (9); basal hairs (12); sub-basal hairs (13); supraorbital hairs (14); and the infraorbital hairs (15). The infra-orbitals are not seen in a dorsal view.

THORAX. (Fig. 20).-Those hairs of the thorax which may be used in classifying the Anophelines are the inner, middle and outer submedian prothoracic hairs (1-3), sometimes referred to as the shoulder hairs; the lateral hairs (4-8); the groups of four hairs known as the pleural hairs (9.12) on the ventral surface of the pro-, meso-, and metathorax (Fig. 20B, C, D); and the ventral submedian hair (13). Most of the larger hairs are pinnately branched, a feature which is peculiar to Anophelines. Hair 1 of the metathorax is, in some species of Anopheles, a thoracic palmate hair similar to the palmate hair (1) on the abdominal segments. Hair 1 is similarly developed on the meso-

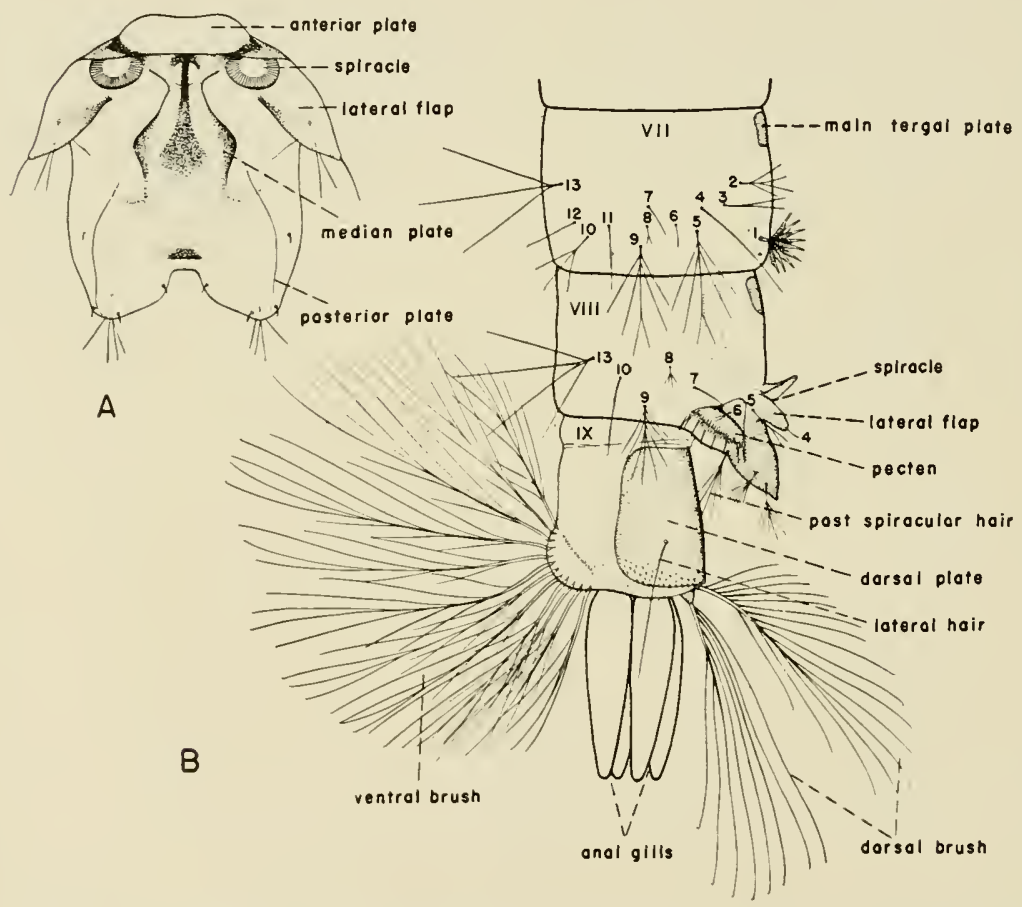

Fig. 22. Abdomen of Anopheles larva. A, Spiracular apparatus (dorsal view). B, Terminal segments VII to IX (lateral view). Segment VII: $(0-8)$, same as for segments I to VI in Fig. 21. Segment VIII : (4 and 5), hairs of lateral flap; (6), pecten hair; (7), subpecten hair; (8), small lateral hair; (9), ventro-lateral posterior hair; (10), small ventro-lateral hair; (13), ventral submedian hair. 
thorax in the genus Bironella. Located antero-dorsally on the thorax is a pair of transparent retractile notched organs of Nuttall and Shipley. When extended, these organs make contact with the surface film and probably help to support the larva. According to some authors these organs may prevent the thorax from rotating with the head before and after feeding.

ABDOMEN. (Figs. 21, 22).-The structures of most taxonomic importance on abdominal segments I to VII are the dorsal anterior submedian hair $(0)$, palmate hair (1), antepalmate hair (2), sublateral hairs (3-5), latcral hairs (6-8), the main tergal plate and the median accessory tergal plate. Segment VIII bears the spiracular structures postero-dorsally (a siphon is lacking), the pecten laterally, and hairs as illustrated (Fig. 22A, B). The pecten present on each side of segment VIII, is a sessile, sclerotized plate, bearing both long and short teeth, and according to Christophers (40) is homologous with the pecten of Culicine larvae. Segment IX (anal segment) is partially ringed by a sclerotized dorsal plate which has toward its posterior margin a lateral hair, usually single. From the dorso-apical angle of the anal segment arises the dorsal brush composed of the upper and lower candal hairs. The ventro-apical margin has a fan-shaped group of hairs known as the ventral brush. Four tapering papilliform processes, the anal gills, are borne posteriorly.

\section{Egg Characters}

The eggs of mosquitoes are composed of three distinct layers: The delicate vitelline nembrane surrounding the yolk; the endochorion, a more or less heavily sclerotized outer shell; and the exochorion, a thin hyaline layer covering the endochorion and patterned with small protuberances and reticulations. The endo- and exochorion together make up the chorion. The anterior pole of the egg bears the micropylar apparatus, which consists of a small, rosette-shaped membrane and a ring of exochorial bosses surrounding a minute opening, the micropyle. The micropyle permits entrance of a sperm cell from the spermathecae of the female during oviposition.

The eggs of Culicine mosquitoes are usually elongate-oval in sagittal section and circular in cross section. The larger, anterior end, containing the head of the developing embryo, is somewhat rounded, while the posterior end is bluntly pointed. The eggs are laid singly by some species while certain others lay them in rafts (Fig. 23). The shape of the individual egg is rather characteristic for various genera; the nature of the markings of the exochorion and the manner in which the eggs are laid are also useful in classification.

The eggs of Anopheline mosquitoes are usually boat-shaped, flattened or slightly concave dorsally, and decidedly convex ventrally (Fig. 23C, D). The larger, anterior end is somewhat rounded, while the slightly narrower posterior end is more or less bluntly pointed. The exochorion is modified to form a projecting frill, which partially or entirely surrounds the upper portion, and a pair of air-filled lateral floats. The character of these structures, together with the bosses and reticulations of the exochorion, are of considerable value in separating allied species and races in some parts of the world. 
A
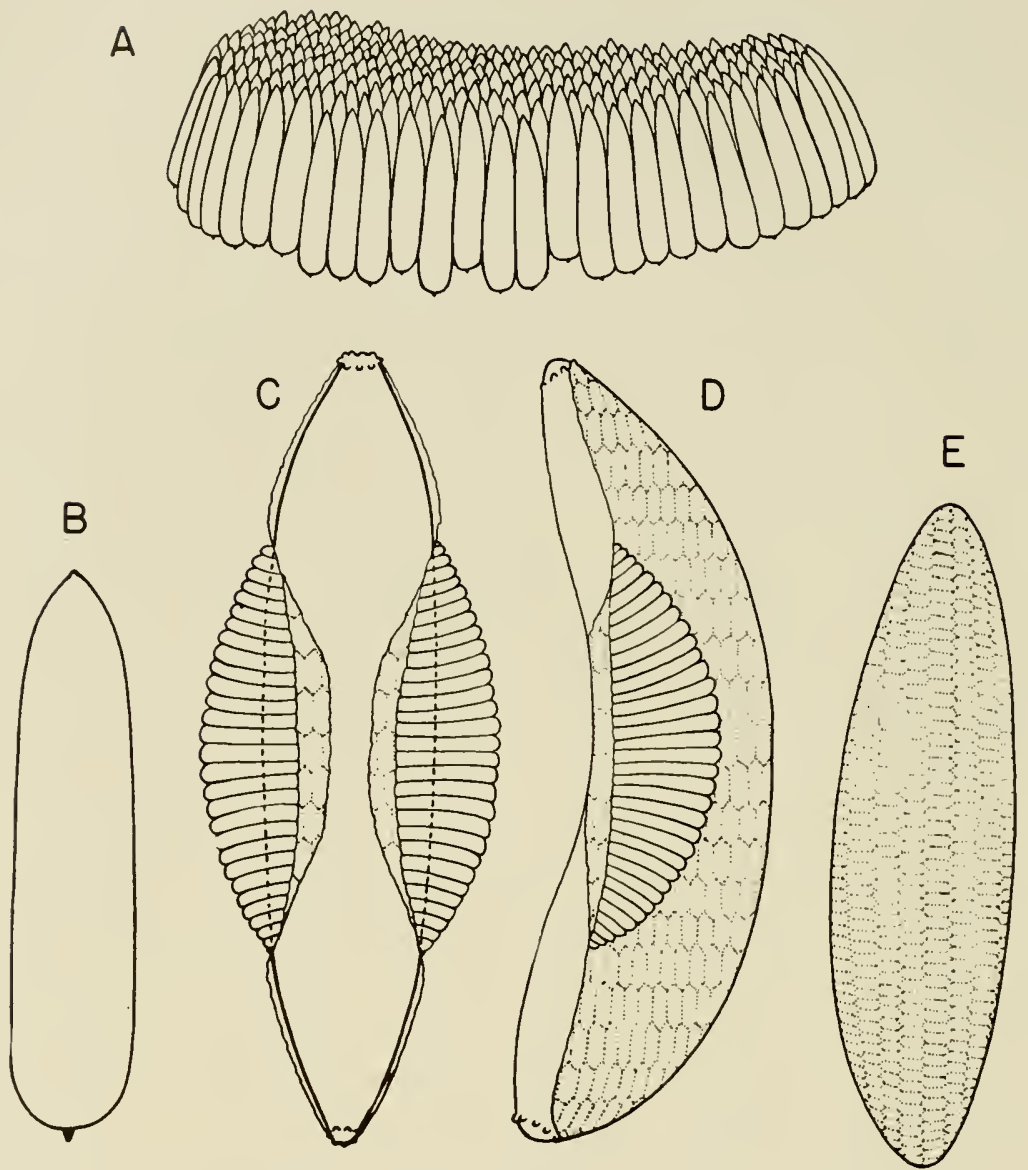

Fig. 23. Eggs of mosquitoes. A, Egg raft of Culex. B, Single egg of Culex. C, Egg of Anopheles (dorsal view). D, Egg of Anopheles (lateral view). E, Egg of Aedes aegupti.

\section{Family Culicidae}

Mosquitoes are two-winged insects belonging to the order Diptera, family Culicidae. The family Culicidae consists of three subfamilies, the Dixinae, the Chaoborinae and the Culicinae. Only species belonging to the subfamily Culicinae are included here. The main distinguishing characters of the three subfamilies are presented in the following keys:

\section{Keys to the Subfamilies}

\section{ADULTS}

1. Flagellum of antenna 14 segmented; wings without scales, vein Sc ending before or near the base of vein 2 ; mouth parts short, not fitted for piercing Dixinae 
Flagellum of antenna 13 segmented; wings with scales at least on fringe, vein Sc extending beyond base of vein 2

2. Mouth parts short; scales almost confined to wing fringe (veins clothed, for most part, with long hairs)

Chaoborinae

Mouth parts prolonged into the form of a proboscis; wing veins with scales

Culicinae

\section{PUPAE}

1. Swimming paddles fused and incapable of movement

Dixinae and Chaoborinae (in part)

Swimming paddles free and movable

2. Respiratory horn on thorax either almost closed, or if open, with the spiracular opening near its middle

Chaoborinae

Respiratory horn on thorax open at the tip and with the spiracular opening at its base

Culicinae

LARVAE

1. Thorax narrow and with distinct segmentation

Thorax distinctly broader than abdomen and without distinct segmentation

Dixinae

2. Antennae prehensile, with long and strong apical spines

Antenna not prehensile 2

Chaoborinae Culicinae

\section{Subfamily Culicinae}

The subfamily Culicinae is divided into three tribes: Anophelini, Megarhinini and Culicini. Important characters for separating the three tribes in the larval, pupal and adult stages are presented in the following keys:

\section{Keys to Tribes}

ADULTS

1. Abdomen unscaled. or at least the sternites largely bare; palpi of female nearly as long as proboscis (except in Bironella) Anophelini, p. 50

Abdomen densely scaled both dorsally and ventrally; palpi of female short (in some Megarhinini they may be nearly $2 / 3$ as long as proboscis)

2. Proboscis rigid, the basal half thickened, and apical half strongly downcurved; scutellum rounded Megarhinini, p. 83

Proboscis not rigid, of nearly uniform thickness, not down-curved at apical half; scutellum trilobed Culicini, p. 88

\section{PUPAE}

I. Lateral apical hairs spine-like on abdominal segments III to VI and placed

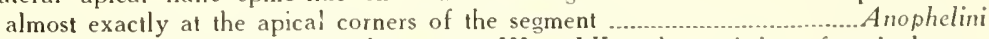

Lateral apical hairs on abdominal segments III to VI each consisting of a single hair or a branched hair and placed well before the apical corners of the segment ...2

2. Outer part of the swimming paddles lobe-like and extending beyond tip of the midrib Megarhinini

Outer part of the swimming paddles not lobe-like and not longer than midrib.... Culicini

\section{LARVAE}

1. Eighth abdominal segment without an elongate dorsal siphon …................... Anophelini

Eighth abdominal segment with an elongate dorsal siphon which is as long or longer than broad

2. Mouth brushes prehensile, each composed of ten stout rods ......................... Megarhinini Mouth brushes composed of 30 or more hairs (prehensile only in subgenus Psorophora) 


\section{Tribe Anophelini}

The tribe Anophelini consists of three recognized genera: Genus Chagasia, found in Central and South America; genus Bironella, found in New Guinea and nearby islands; and genus Anopheles, which is worldwide in its distribution.

SALIENT CHARACTERS.-Adult: Palpi long in both sexes except in the genus Bironella; the two terminal segments clavate in the male. Scutellum rounded posteriorly and with marginal hairs arranged in an unbroken row except in genus Chagasia which has the scutellum trilobed. Abdomen either without scales or with loosely applied scales; the sternites, at least, are largely bare in scaly species. Wings usually with distinct markings. While the adult is in the resting position, the proboscis, thorax and abdomen are held nearly in a straight line. Larva: While feeding, the head can be rotated through an arc of $180^{\circ}$ so that the ventral side is uppermost. Most of the larger hairs of the body are pinnately branched. The thorax has a pair of notched organs of Nuttall and Shipley. The eighth abdominal segment lacks a dorsal respiratory siphon. Many of the abdominal segments, and the thorax in some species, bear palmate hairs on the dorsal surface.

\section{Genus ANopheles Meigen 1}

Anopheles Meigen, 1818, Sys. Beschr. Zweifl. Ins. 1:10.

Edwards (55) divides the genus Anopheles into four subgenera, based largely on characters of the male terminalia: Anopheles, distributed throughout the Old and New World; Nyssorhynchus, found in the southern United States, Central and South America; Stethomyia, known from Central and South America; and Myzomyia, distributed throughout the tropical and subtropical regions of the Old World. Two subgenera, Anopheles and Nyssorhynchus, are represented in the Anopheline fauna of the United States.

The adult and larval characters given for the tribe Anophelini are applicable in general to the genus Anopheles. Important characters of the male terminalia are as follows: Phallosome tubular, tip bare or with one or more pairs of leaflets. Claspette usually bilobed, forming a dorsal and a ventral lobe. Basistyle cylindrical, conically tapered towards tip, the basal and apical lobes absent. The basistyle may bear parabasal spines, accessory spines, and internal spines. The subgenus Anopheles has two parabasal spines, no accessory spines and one internal spine. The subgenus $N y$ ssorbynchus has one parabasal spine, two accessory spines and a single internal spine. The dististyle is long, curved, and not swollen medially.

Keys to THE SPEcies

ADULT FEMALE

1. Wings with areas of pale scales

Wings entirely dark scaled

2. Tarsi, particularly on hind legs, with conspicuous white bands; costa with four or more spots of pale scales albimanus Wied., p. 80

1 Consult Dyar (1928) and Edwards (1932) for synonyms. 
Tarsi entirely dark; costa with no more than two pale spots

3. Costa with a pale spot at outer third opposite tip of subcosta; anal vein with one or two areas of dark scales

Costa dark except for pale spot at extreme tip; vein 6 with three areas of dark scales (two in male) crucians crucians Wied., p. 60 crucians bradlevi King, p. 58 crucians georgianus King, p. 64

4. Veins 3 and 5 entirely dark-scaled; apical half and basal fourth of vein 6 darkscaled punctipennis (Say), p. 69

Vein 3 and 5 with long areas of pale scales: apical half of vein 6 dark, basal half white ........................................................ 66

5. Wings without spots of dark scales; scutum with setae at least half as long as width of scutum; integument of scutum shiny on rubbed specimens; small

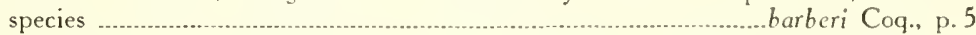

Wings with spots of dark scales. more or less disinct; scutum with setae rarely half as long as width of sculum; integument of scutum dull on rubbed specimens; medium sized species

6. Frontal tuft with some pale setae; wing with four distinct dark spots, palpi entirely dark a........ quadrimaculatus Say, p. 73

Frontal tuft entirely dark; wing usually with indistinct dark spots; segments of palpi with or without distinct white apical rings

7. Knob of halter pale-scaled; segments of palpi with narrow white apical rings; knee spots present ............................................................ Theob., 76

Knob of halter entirely dark scaled; segments of palpi with faint apical white rings or entirely dark; knee spots reduced or absent .........atropos D. and K.. p. 52

MALE TERMINALIA

1. Basistyle with one internal spine, two accessory spines, and one parabasal spine (Subgenus Nyssorhynchu*) …….... albimanus Wied., p. 1

Basistyle with one internal spine, no accessory spines, and two parabasal spines (Subgenus Anopheles)

2. Phallosome with leaflets; medium sized species

Phallosome without leaflets; small species barberi Coq., p. 55

3. Leaflets of phallosome serrated nearly to tips; dorsal lobe of claspette bearing three overlapping blade-like filaments on apex

p. pseudopunctipennis Theob., p. 66

Leaflets of phallosome smooth, although they may bear a few coarse basal teeth; dorsal lobe of claspette bearing spines or stout rods, not blade-like ....................... 4

4. Claspette with dorsal and ventral lobes fused to form a single fleshy conical lobe on each side crucians crucians Wied., p. 62 crucians bradlevi King. p. 58 crucians georgianus King, p. 64

Claspette with dorsal and ventral lobes distinct, at least apically 7 6 2 
Frontal hairs (5-7) small, simple; abdominal segments I-VI with hair 6 plumose

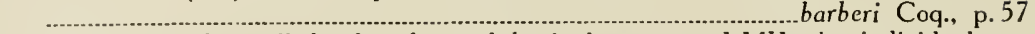

2. Palmate hair (1) well-developed on abdominal segments I-VII, the individual leaflets with smooth margins .................................................manus Wied., p. 81

Palmate hair (I) obsolete or greatly reduced on abdominal segments I and II ........... 3

3. Outer clypeal hair (3) simple; hair 9 of meso- and metathoracic pleural groups short and stout, no more than half as long as hair 10 p. pseudopunctipennis Theob., p. 66

Outer clypeal hair (3) feathered or branched; hairs 9 and 10 of meso- and metathoracic pleural groups nearly equal in length

4. Outer clypeal hair (3) sparsely branched (only 5-10 short branches)

Outer clypeal hair (3) strongly dichotomously branched (more than 25 branches)
(3)...

5. Inner clypeal hair (2) with sparse minute feathering toward tip; prothoracic hair 1 with 3-5 strong branches near base ..................................... Theri Theob., 77

Inner clypeal hair (2) simple; prothoracic hair I short, single or weakly branched at tip

6. Hair 0 and hair 2 on abdominal segments IV and $V$ both well-developed, each with 4-9 branches .................................... p. 62

Hair 0 absent or rudimentary

7. Palmate hair (1) on abdominal segments III-VII about equally developed (palmate on segment VII may be somewhat smaller); individual leaflets on palmates $\mathrm{II}$ and VII serrated on margins

Palmate hair (I) on abdominal segments IV-VI about equally developed, those on segments III and VII being rudimentary or only about one-half to twothirds as large; individual leaflets on palmates III and VII usually with smooth margins

8. Basal tubercles of inner clypeal hairs (2) separated by at least the diameter of one tubercle; hair 2 usually single, sometimes double, on abdominal segments IV and V; sutural hair (8) generally 8 to 10 -branched

quadrimaculalus Say, p. 75

Basal tubercles of inner clypeal hairs (2) close together, separated by less than the diameter of one tubercle; hair 2 usually double, occasionally single or triple, on abdominal segments IV and $V$; sutural hair (8) generally 5 to 7 branched punctipennis (Say), p. 71

9. Palmate hair (1) functional on abdominal segments IV-VI only, those on segments III and VII being rudimentary; hair 2 with 3-6 branches on segments IV and V (rarely with 2 branches) crucians georgianus King, p. 64

Palmate hair (1) functional on abdominal segmenis III-VII, although those on segment III and VII are reduced to about one-half to two-thirds the size of those on segments IV-VI; hair 2 single or double on segments IV and V (sometimes triple) crucians bradleyi King, p. 60

\section{Anopheles (Anopheles) atropos Dyar and Knab}

Anopheles atropos Dyar and Knab, 1906, Proc. Biol. Soc. Wash., 19:I60.

ADUlt Female.-Medium-sized species. Head: Proboscis long, black; palpi about as long as proboscis, entirely dark or with very narrow pale rings at apices of distal segments. Occiput clothed with dark erect forked scales; frontal tuft dark. Thorax: Integument of scutum dark brown to dark reddishbrown, clothed with short golden-brown hairs and larger black setae, the black setae more numerous laterally. Abdomen: Integument dark brown to black, clothed with dark hairs. Legs: Legs dark, knee spots reduced or absent. Wings: Scales narrow, entirely dark, some of the scales often arranged in four darker spots, usually indistinct. Halter: Knob of halter dark scaled. 
Adult male.-Coloration similar to that of female. TERminalia (Fig. 24).-Ninth tergite (IX-T) a narrow, moderately sclerotized band; lobes (IXT-L) lateral in position, slender, tapered, pointed or rounded at tip. Tenth sternite absent. Anal lobe (An-L) large, triangular, spiculate. Phallosome $(\mathrm{Ph})$ cylindrical, furcate at base; apex with three or four pairs of ligulate leaflets ( $\mathrm{Ph}-\mathrm{L})$, the apical pair usually about twice as long as the ante-apical pair; none of the leaflets with short, coarse, basal teeth. Claspette broad, fleshy, consisting of a dorsal lobe (Cl-DL) and a ventral lobe (Cl-VL). The dorsal lobe apically with one to several (usually 2 to 3) large, closely set, blunt, slightly capitate spines; the ventral lobe with a large, broad, pointed spine at apex and one or two smaller subapical spines. Basistyle (Bs) conical, nearly twice as long as broad, rourded apically, clothed with long setae and few or no scales; two parabasal spines (P-S) and one internal spine (I-S) present. Dististyle (Ds) about as long or slightly longer than basistyle,

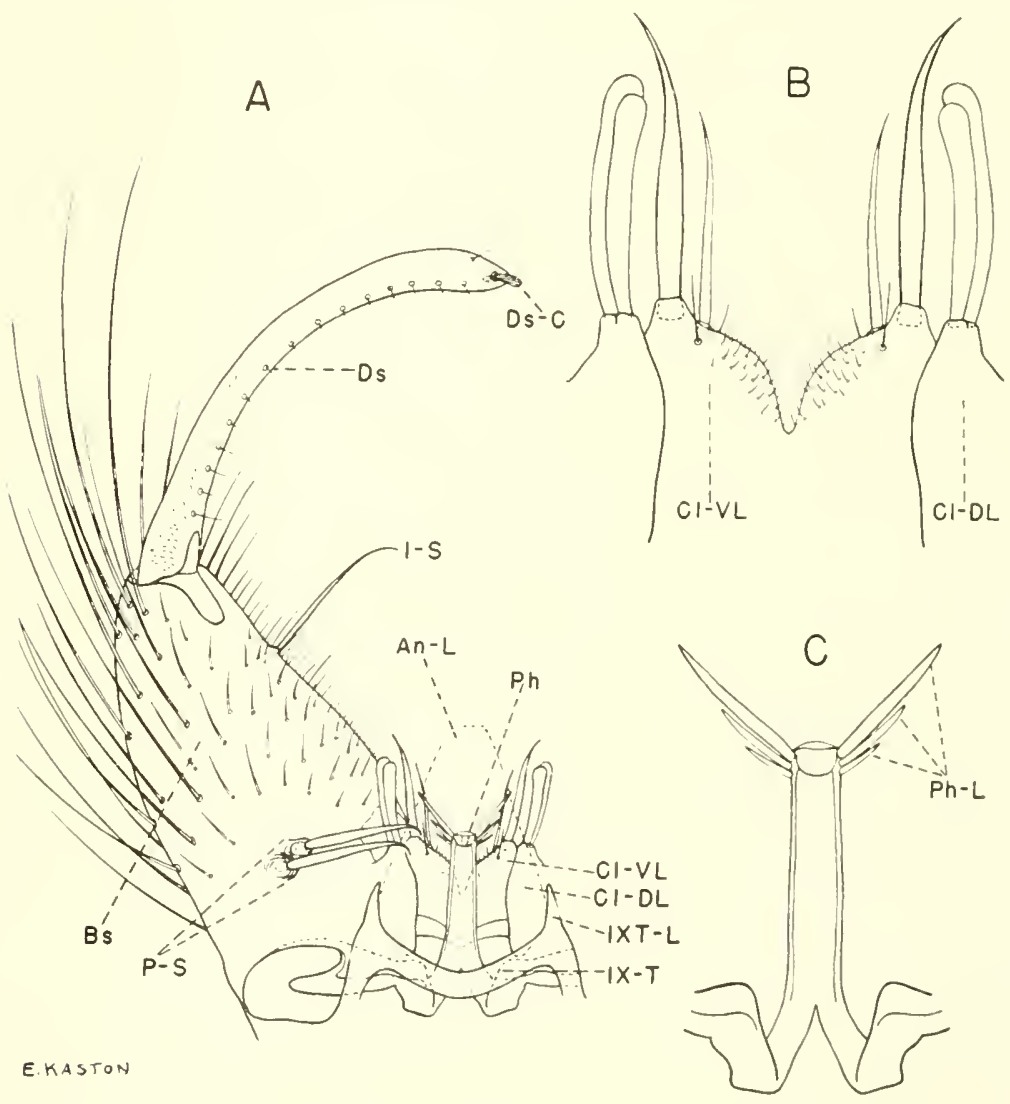

Fig. 24. Anopheles atropos Dyar and Knab. A. Male terminalia. B, Claspettes. C, Phallosome. 
curved, narrower medially, bluntly pointed at tip; numerous spicules (minute non-papillated hairs) present on basal third or basal half of dististyle; terminal claw (Ds-C) is short, blunt.

LARVA (Fig. 25).-Head: Inner clypeal hairs (2) sparsely feathered at tip (sometimes simple), basal tubercles may or may not be separated by more than the diameter of a single tubercle: outer clypeal (3) sparsely branched ( 5 to 10 short branches on distal half); postclypeal (4) simple or branched distally, reaching near base of inner clypeal hairs (2); frontal hairs

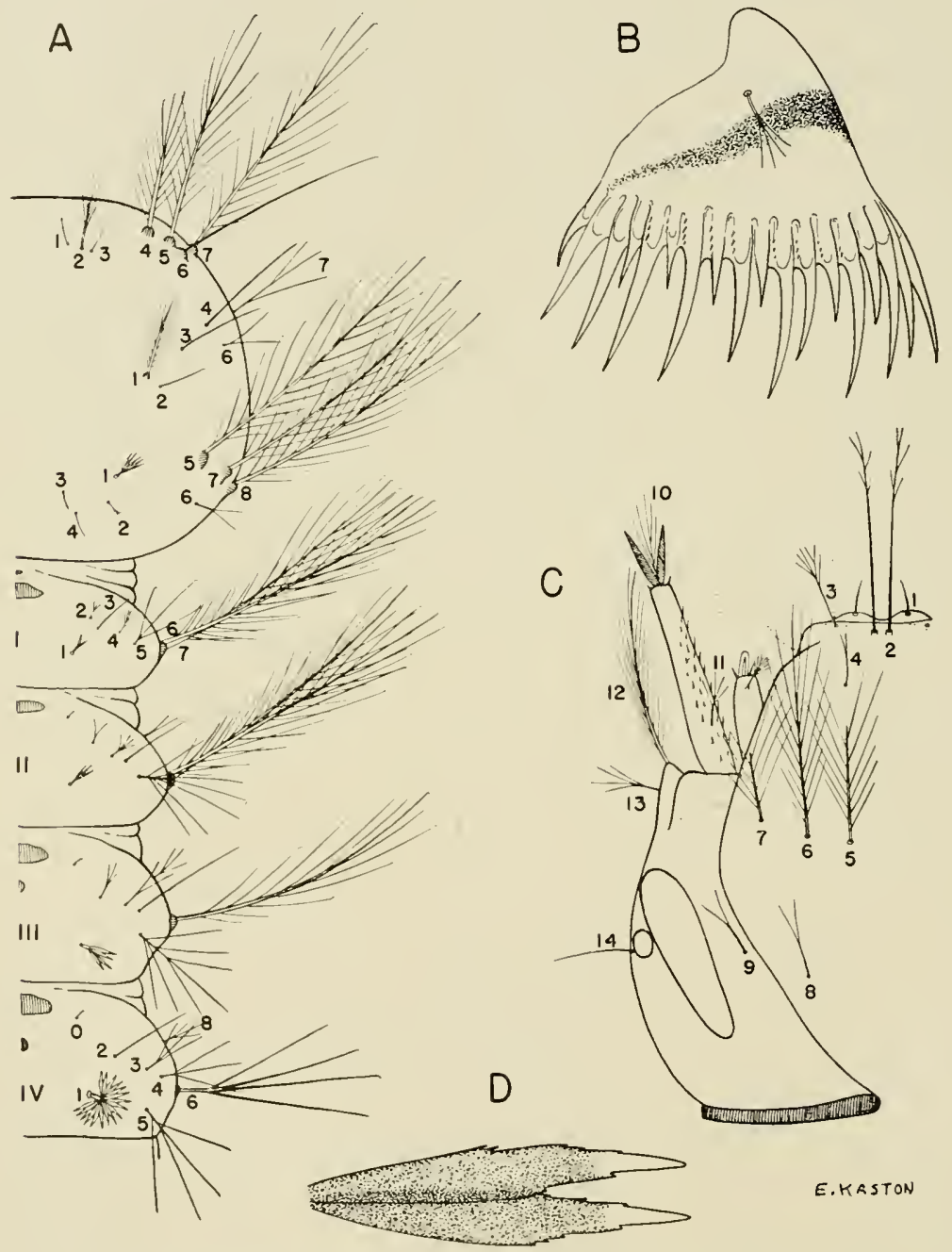

Fig. 25. Larva of Anopheles atropos Dyar and Knab. A, Thorax and abdomen. B, Pecten. C, Head. D, Leaflets of palmate hair. 
(5-7) large, plumose; sutural (8) and trans-sutural (9) single or 2- to 3-branched beyond base. Antenna sparsely spinose; terminal hair (10) with several branches, longer than the sabres; antennal tuft (11) inserted on basal third of shaft, several-branched, reaching near middle. Thorax: Prothoracic dorsal submedian group (1-3) with hairs 1 and 3 short, simple, hair 2 larger, variable (with two to many branches). Prothoracic pleural group (9-12) of four hairs; hairs 9, 10, and 12 long, simple; hair 11 about ene-fourth as long as 9, 10 or 12, simple or bifid. Mesothoracic pleural group (9-12) of four hairs; hairs 9 and 10 long, simple, subequal; hair 11 minute, simple; hair 12 simple, short (much longer than hair 11); metathoracic pleural group (9-12) of four hairs; hairs 9 and 10 long, simple, subequal; hair 11 minute, simple; hair 12 is 2- to 3-branched, short (much longer than hair 11); metathoracic palmate hair (1) small, with transparent leaflets. Abdomen: Submedian dorsal hair (0) obsolete; palmate hair (1) rudimentary on segments I and II, partially developed on segment III and well-developed on segments IV to VII; individual leaflets with serrations beyond middle. Antepalmate hair (2) long, single (tarely bifid) on segments IV and V. Upper lateral hair (6) on segments I to III long, plumose. Pecten as iliustrated.

Distribution.-Cuba (36); Atlantic and Gulf coastal regions of the United States from New Jersey to Texas. Southern States: Alabama and Florida (94); Georgia (125); Louisiana and Mississippi (94); North Carolina (166); South Carolina (94); Virginia (49). Other States: Maryland (19); New Jersey (77); Texas (108).

BIONOMICS. - The adults may be found in considerable numbers in saltmarshes near their breeding places where they occasionally attack men either during day or night. They rarely enter human habitations. Anopheles atropos breeds in permanent salt pools or in marshes where the salt content varies from approximately 1 to 12 per cent.

MEDICAL IMPORTANCE.-Mayne and Griffitts (120) were able to infect approximately 85 per cent of the specimens of $A$. atropos which had fed on suitable carriers of Plasmodium vivax. There are no data available on the infection rate of this species in nature. However, it is regarded as epidemiological $I_{y}$ unimportant, since its distribution is restricted to salt-matshes.

\section{Anopheles (Anopheles) Barberi Coquillett}

Anopheles barberi Coquillett, 1903, Can. Ent., 35:310.

Adult female.--Small species. Head: Proboscis long, dark; palpi about as long as proboscis, dark. Occiput clothed with numerous erect forked scales, the ones on the central portion and vertex pale. Thorax: Integument of scutum brown, shiny, clothed with long dark setae, at least half as long as width of scutum. Abdomen: Integument krown, clothed with dark-brown hairs. Legs: Legs entirely dark. Wings: Scales slightly broadened, uniformly dark. Halter: Knob of halter dark-scaled.

ADULT MALE.-Coloration similar to that of female. TERninalia (Fig. 26). - Ninth tergite (IX-T) moderately sclerotized laterally, membranous 
dorsally; lobes indefinite, but represented by the rounded, posterior portion of each lateral sclerotized area of the ninth tergite. Tenth sternite absent; anal lobe (An-L) large, triangular, finely spiculate. Phallosome $(\mathrm{Ph})$ cylindrical, broadened at base; apex rounded and lacking leaflets.

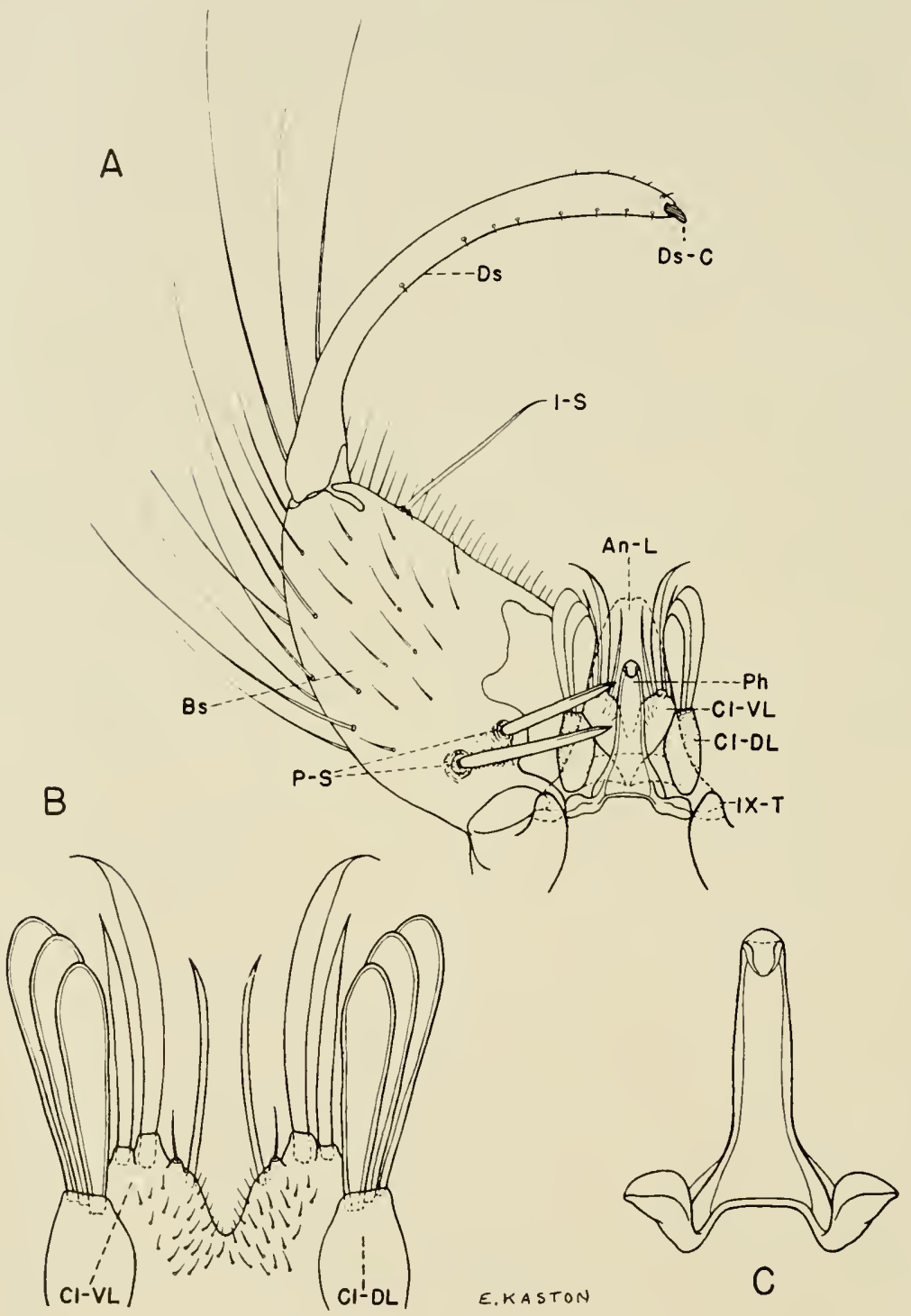

Fig. 26. Anopheles barberi Coquillett. A, Male terminalia. $\mathrm{B}$, Claspettes. C, Phallosome. 
Claspette broad, fleshy, consisting of a dorsal lobe (Cl-DL) and a verutral lobe (Cl-VL). The dorsal lobe apically with three overlapping spatulate filaments; the ventral lobe with a large, pointed, apical spine and two smaller subapical spines. Basistyle (Bs) conical, a little longer than broad, rounded apically, clothed with long setae; two parabasal spines (P.S) and one internal spine (I-S) present. Dististyle (Ds) longer than basistyle and lacking spicules (minute non-papillated hairs), curved, narrower medially, bluntly pointed at tip; terminal claw (Ds-C) short, blunt.

LARVA (Fig. 27).-Head: Inner clypeal hairs (2) simple, widely spaced; outer clypeal (3) reduced, usually simple; postclypeal (4) simple or bifid,

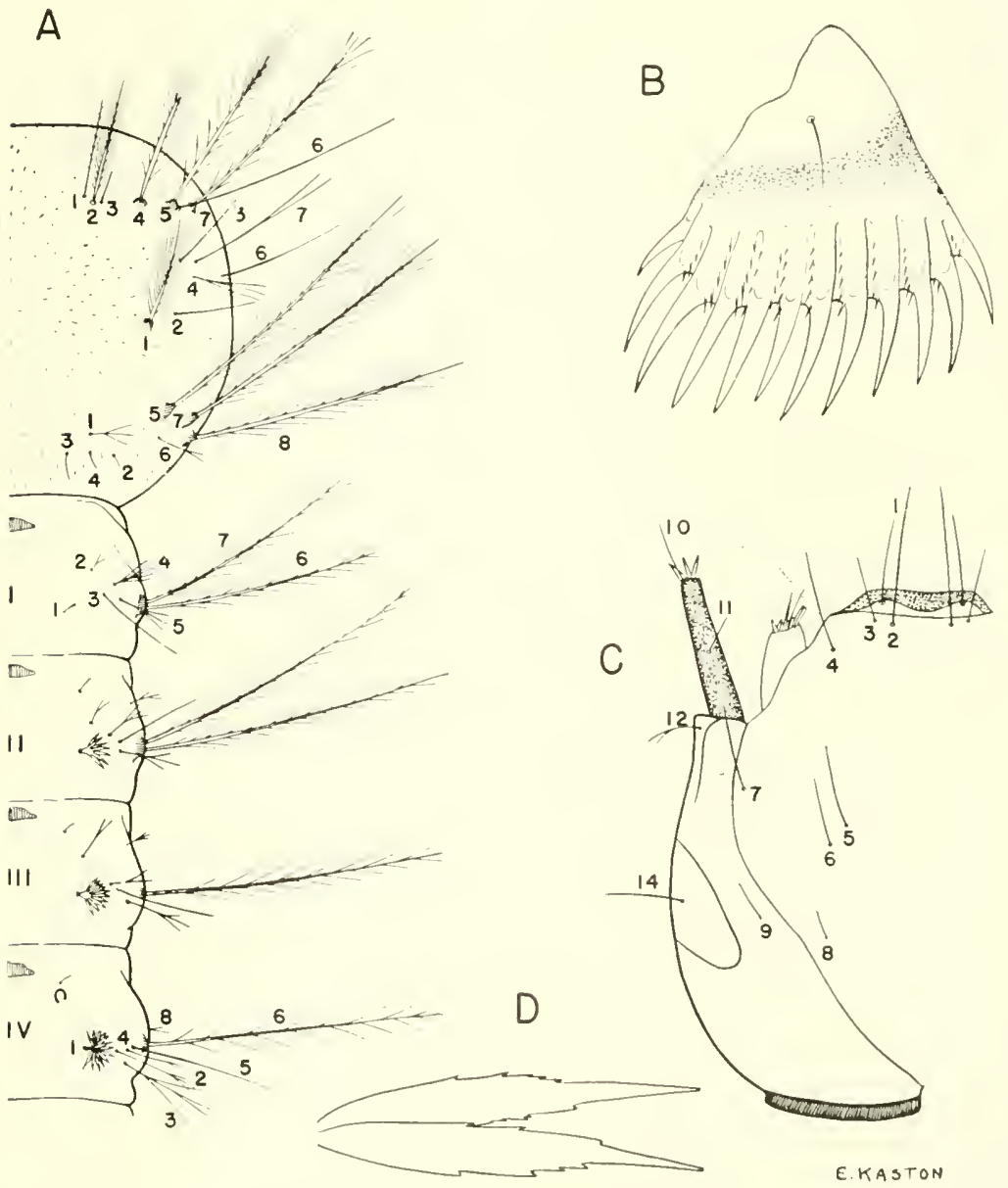

Fig. 27. Larva of Anopheles barberi Coquillett. A. Thorax and abdomen. B. Pecten. C. Head. D, Leaflets of palmate hair. 
lateral in position, larger than outer clypeal (3); frontal hairs (5-7) small, simple; sutural (8) and trans-sutural (9) small, simple. Antenna dark, rather short, glabrous; terminal hair (10) simple, more than twice the length of the sabres; antennal tuft (11) represented by a small, simple hair inserted near the middle of shaft. Thorax: Integument sparsely spiculate. Prothoracic dorsal submedian hairs (1-3) with inner hair (1) long, usually sparsely feathered (feathering may be weak or strong and may be distributed along shaft or restricted to apex); middle hair (2) somewhat longer than inner hair (1), with many branches; outer (3) short, simple. Prothoracic pleural group (9-12) of four hairs; hair 9 long, barbed; hairs 10 and 12 long, simple; hair 11 minute. Mesothoracic pleural group (9-12) of four hairs; hair 9 long, barbed; hair 10 long, simple; hair 11 obsolete; hair 12 short, simple or branched apically. Metathoracic pleural group (9-12) of four hairs; hair 9 long, barbed; hair 10 long, simple; hair 11 obsolete; hair 12 short, simple or branched apically. Metathoracic palmate hair (1) obsolete. Abdomen: Submedian dorsal hair (0) obsolete. Palmate hair (1) rudimentary on segment I, welldeveloped on segments II to VII; individual leaflets with serrations beyond middle. Antepalmate hair (2) long, branched apically, inserted lateral to palmate (1). Lateral hair (6) long, plumose on segments I to VI (lateral branches fine and short). Pecten as illustrated.

distribution.-Eastern United States, west to Kansas; Mexico (158). Southern States: Alabama (170); Arkansas (30); Florida and Georgia (96); Kentucky (140); Louisiana (96); Mississippi (52); Missouri (70); North Carolina and South Carolina (52); Tennessee (170); Virginia (49). Other States: Indiana (39); Maryland (52); New Jersey (94); New York (116); Oklahoma (159); Texas (108).

BIONOMICS.-Thibault (179) states that the adults enter dwellings and are persistent biters, but are easily disturbed while in the act of feeding. Adults of this species are frequently taken in resting station and light trap collections. Larvae occur in rot cavities in trees and occasionally in artificial wooden recentacles. The larvae usually dive when disturbed but soon rise to the surface where they are easily seen and recognized because of their shinyblack appearance.

MEDICAL IMPORTANCE.-This species has been infected in the laboratory with Plasmodium vivax but no data are available as to its infection in nature. It is a rare species and the available evidence indicates that it is unimportant as a natural vector of malaria.

\section{ANopheles (ANopheles) CRUCIANS bRAdLeyt King}

Anopheles crucians var. bradleyi King, 1939, Amer. Jour. Trop. Med., 19:468.

adult female.-Practically identical with $A$. crucians crucians, but frequently has stem of vein 5 entirely white-scaled (Fig. 28).

ADULT MALE.-Coloration similar to that of female, but with only two dark spots present on vein 6 . TERMinALIA.-The terminalia is very similar to that of $A$. crucians crucians (Fig. 30 ). 


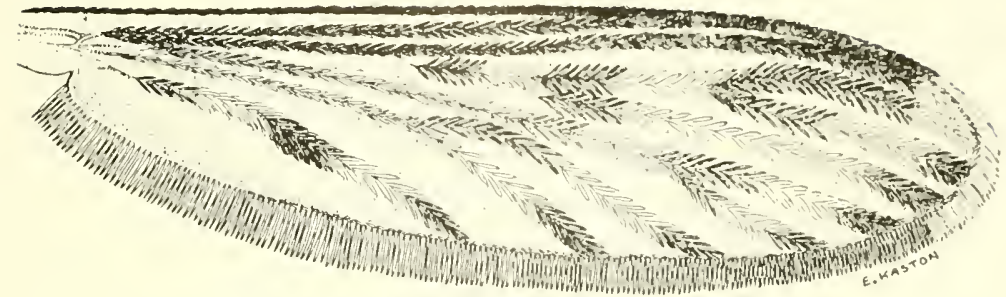

Fig. 28. Wing of Anopheles crucians bradleyi King.

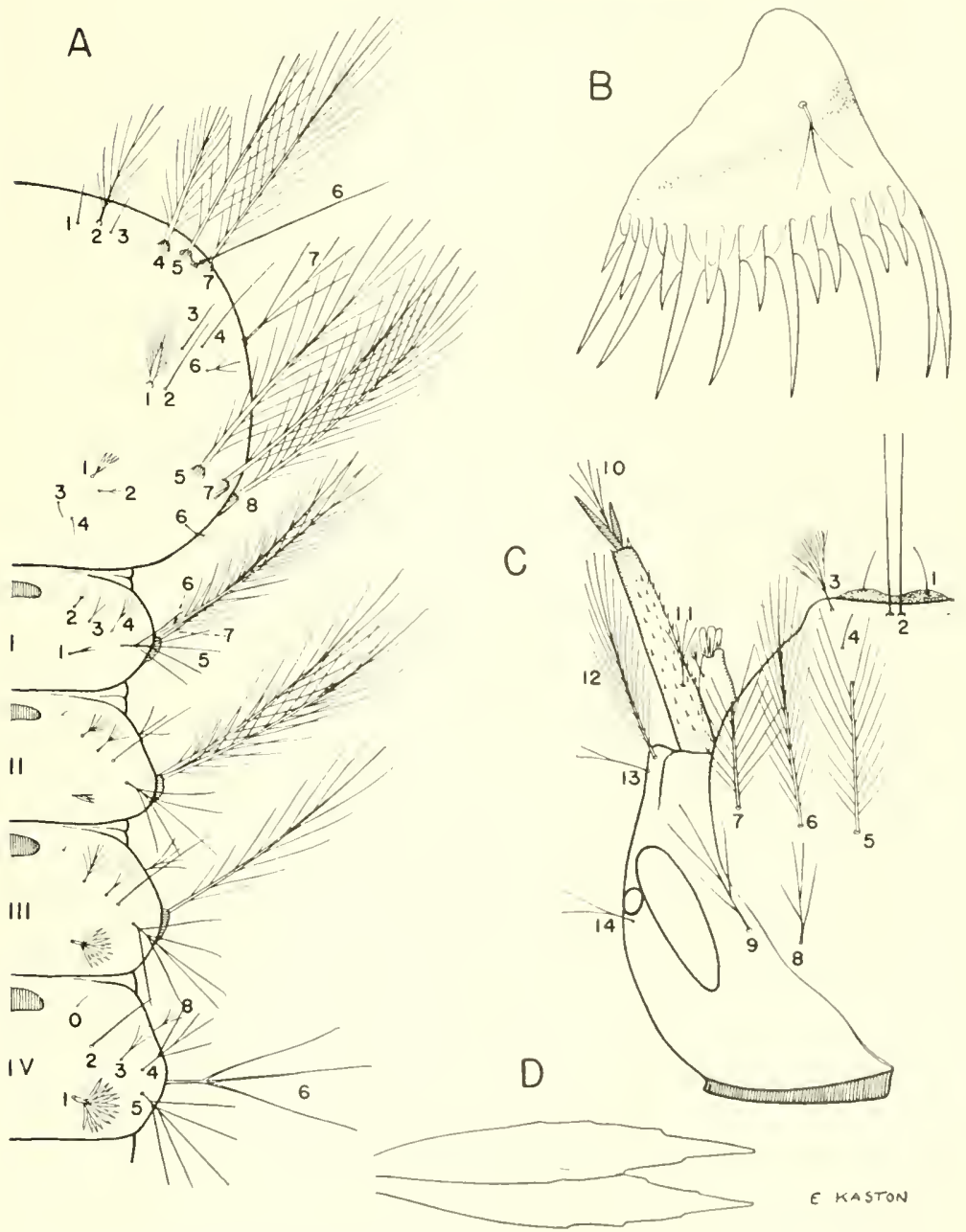

Fig. 29. Larva of Anopheles crucians bradleyi King. A, Thorax and abdomen. B, Pecten. C, Head. D, Leaflets of palmate hair. 
LARVA (Fig. 29).-Head: Inner clypeal hairs (2) simple, the distance between basal tubercles variable, usually less than the diameter of one of the tubercles; outer clypeal (3) densely, dichotomously branched, fan-shaped; postclypeal (4) small, single or double, rarely reaching preclypeus; frontal hairs (5-7) large, plumose; sutural (8) usually 3- to 5-branched; transsutural (9) usually 3- to 5-branched, reaching beyond base of frontal hair (7). Antenna spinose; terminal hair (10) with several branches, longer than the sabres; antennal tuft (11) inserted at basal third, several branched, reaching middle of shaft or slightly beyond. Thorax: Prothoracic dorsal submedian hairs (1-3) consisting of a short inner hair (1), simple or branched apically; a much longer stout middle hair (2), with many branches; and a short simple outer hair (3). Prothoracic pleural group (9-12) of four simple hairs; hairs 9,10 and 12 long, subequal; hair 11 short. Mesothoracic pleural group (9-12) of four simple hairs; hairs 9 and 10 long, subequal; hair 11 minute; hair 12 about one-third as long as hair 9 or 10. Metathoracic pleural group (9-12) of four hairs; hairs 9 and 10 long, simple; hair 11, minute, simple; hair 12 short, 2- or 3-branched. Metathoracic palmate (1) small, with transparent leaflets. Abdomen: Submedian dorsal hair $(0)$ obsolete on segments IV and V; palmate hair (1) well-developed and of equal size on segments IV to VI; individual leaflets serrated beyond middle; palmates usually about one-half to two-thirds as large on segments III and VII, individual leaflets usually smooth; palmates very weakly developed on I and II. Antepalmate hair (2) usually single on segments IV and V, sometimes double or triple. Hair 5 on segment $\mathrm{I}$ is 4 - or 5-branched (rarely more) and about twice the size of hair 4. Upper lateral hair (6) on segments I to III long, plumose. Pecten as illustrated.

Distribution.-Along the Atlantic and Gulf coasts from Maryland to Texas. Its range also extends south to Vera Cruz, Mexico (182). Southern States: Alabama and Florida (94); Georgia (97); Louisiana and Mississippi (96); North Carolina (166); South Carolina (94); Virginia (49). Other States: Maryland (94); Texas (130).

BIONomics.-Very little is known of the habits of the adults. The larvae occur in pools of brackish water near the coast. Water with a salt concentration of 1.5 per cent or less seems to be preferred. King et al. (96) state that larvae have been taken with $A$. atropos in waters of rather high salt concentration and with larvae of $A$. crucians crucians in nearly fresh water.

Medical importance.-Boyd et al. (23) were able to infect $A$. crucians bradleyi with Plasmodium falciparum, but nothing is known of its ability to transmit malaria in nature.

\section{Anopheles (ANopheles) CRUCIANS CRUCIANS Wiedemann}

Anopheles crucians Wiedemann (in part), 1828, Ausser. Zweif. Ins. $1: 12$.

Anopheles crucians var. crucians King, 1939, Amer. Jour. Trop. Med., 19:470.

adult female.-Medium-sized species. Head: Proboscis long, black; palpi a little shorter than proboscis, black, the third segment with a few 
white scales basally, the fourth segment white-ringed both basally and apically, the fifth segment entirely white (Fig. 30D). Occiput clothed with numerous erect forked scales, those of central portion white, the others dark; scales of vertex narrow, white; frontal tuft white. Thorax: Integument of scutum brownish-black, with a pair of dark-gray submedian longitudinal stripes; scutum clothed principally with numerous short yellowish hairs, but with
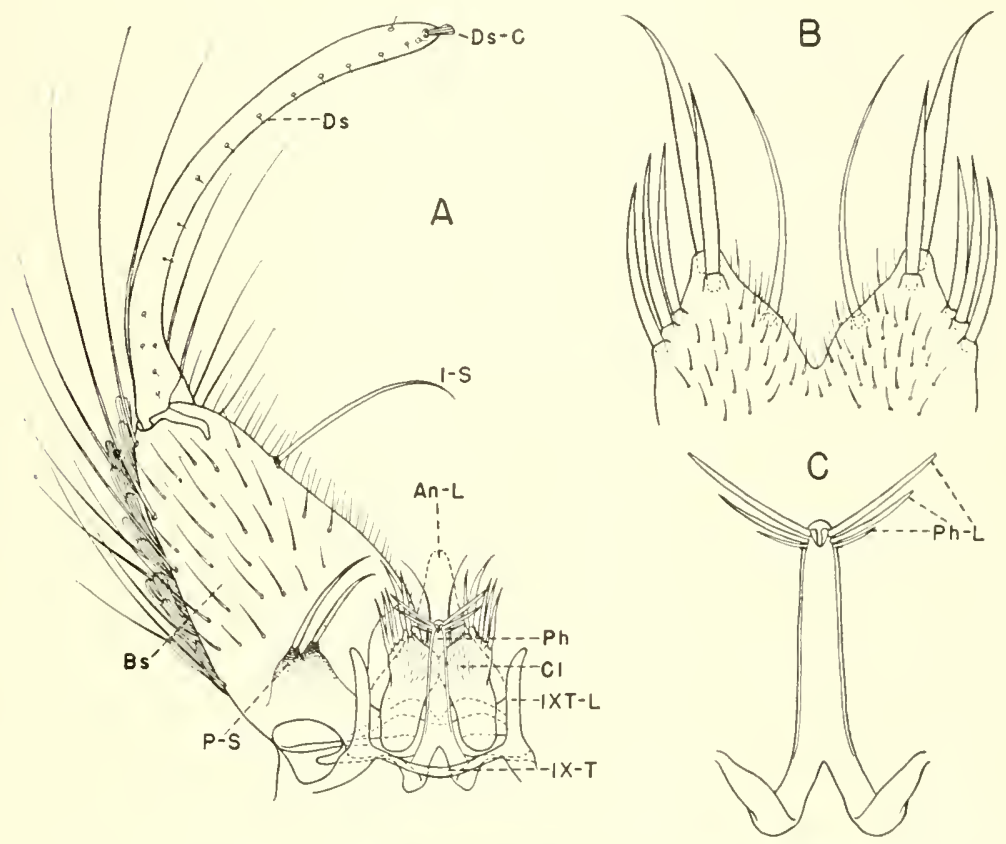

D

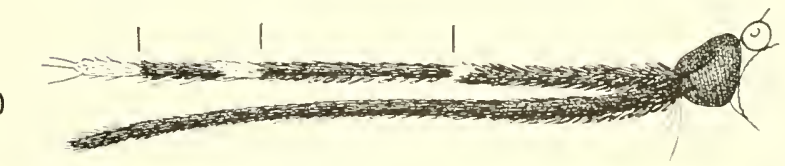

$E$

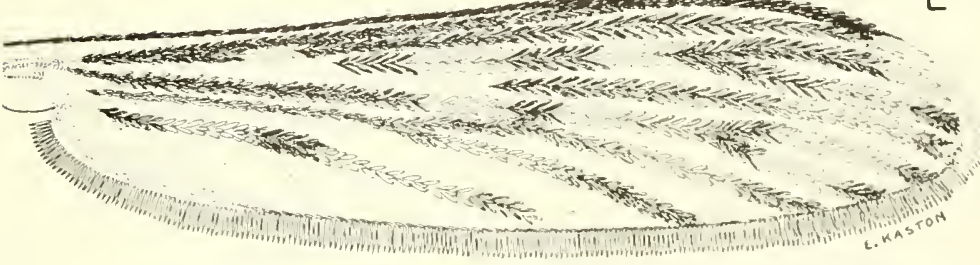

Fig. 30. Anopheles crucians crucians Wiedemann. A, Male terminalia. B. Claspettes. C, Phallosome. D, Palp and proboscis of female. E, Wing. 
a few narrow whitish scales on anterior promontory and black setae on lateral fossae. Abdomen: Integument of abdomen dark-brown to black, clothed with numerous yellow to dark-brown hairs. Legs: Legs dark, femora and tibiae tipped with white. Wings (Fig. 30E) : Scales black and white to yellowishwhite, arranged on the veins in contrasting lines and spots (costa dark-scaled except for pale spot at extreme tip; vein 6 with three spots of dark scales; stem on vein 5 apparently always partially or entirely dark-scaled). Halter: Knob of halter black-scaled.

ADULT MALE.-Coloration similar to that of female but only two dark spots present on vein 6. TERminalia (Fig. 30). Ninth tergite (IX-T) a narrow, sclerotized band; lobes (IXT-L) lateral in position, very long and slender (about 7 to 9 times as long as broad), rounded or bluntly pointed apically. Tenth sternite absent; anal lobe (An-L) large, triangular, spiculate. Phallosome $(\mathrm{Ph})$ cylindrical, furcate at base; apex with three or four pairs of ligulate leaflets ( $\mathrm{Ph}-\mathrm{L})$, the more distal pairs longer, one or more pairs of leaflets with a few short, coarse, basal teeth. Claspette $(\mathrm{Cl})$ with dorsal and ventral lobes fused to form a single fleshy, conical lobe on each side; armature variable but usually consisting of two large, pointed spines on apex (one slightly below the other), one long, slender spine arising from inner surface near middle, and one to three large pointed spines arising from outer surface near middle. Basistyle (Bs) conical, about twice as long as broad, rounded apically, clothed with long setae and many large scales on outer aspect; two parabasal spines (P-S) and one internal spine (I-S) present. Dististyle (Ds) longer than basistyle and lacking spicules (minute non-papillated hairs), curved, narrower medially, bluntly pointed at tip; terminal claw (Ds-C) short, blunt.

LARVA (Fig. 31).-Head: Inner clypeal hairs (2) simple, arising from basal tubercles separated by less than the diameter of one of the tubercles; outer clypeal (3) densely dichotomously branched, fan-shaped; postclypeal (4) small, single or branched, extending slightly beyond bases of inner and outer clypeals (2 and 3); frontal hairs (5-7) large, plumose; sutural (8) and trans-sutural (9) usually 4- to 5-branched; antenna spinose; terminal hair (10) with several branches, longer than the sabres; antennal tuft (11) inserted on basal third of shaft, several branched, reaching to or slightly beyond middle. Thorax: Prothoracic dorsal submedian hairs (1-3) consisting of a short inner hair (1), simple or sparsely branched apically; a much longer stout middle hair 2 with many branches; and a short simple outer hair (3). Prothoracic pleural group (9-12) of four hairs; hairs 9, 10, and 12 long, simple; hair 11 short, usually simple. Mesothoracic pleural group (9-12) of four hairs; hairs 9 and 10 long, simple; hair 12 about one-third the length of hair 9 or 10 , simple; hair 11 minute, simple. Metathoracic pleural group (9-12) of four hairs; hairs 9 and 10 long, simple; hair 12 short, branched beyond base; hair 11 minute, simple. Metathoracic palmate (1) small, with transparent leaflets. Abdomen: Submedian dorsal hair $(0)$ welldeveloped on segments IV and V, 4- to 9-branched; palmate hair (1) rudimentary on segments I and II, well-developed and nearly equal in size 
on III to VII (occasionally smaller on III and VII); individual leaflets with serrations on outer third. Antepalmate hair (2) 4- to 9-branched on segments IV and V, about equal in size to hair (0). Upper lateral hair (6) on segments I to III long, plumose. Pecten as illustrated.

Distribution.-Eastern United States, west to New Mexico. Its range also extends south along the Gulf Coast to northern Nicaragua (101), and occurs in the West Indies. Southern States: Alabama (96); Arkansas (30); Florida and Georgia (52); Kentucky (140); Louisiana and Mississippi (52);

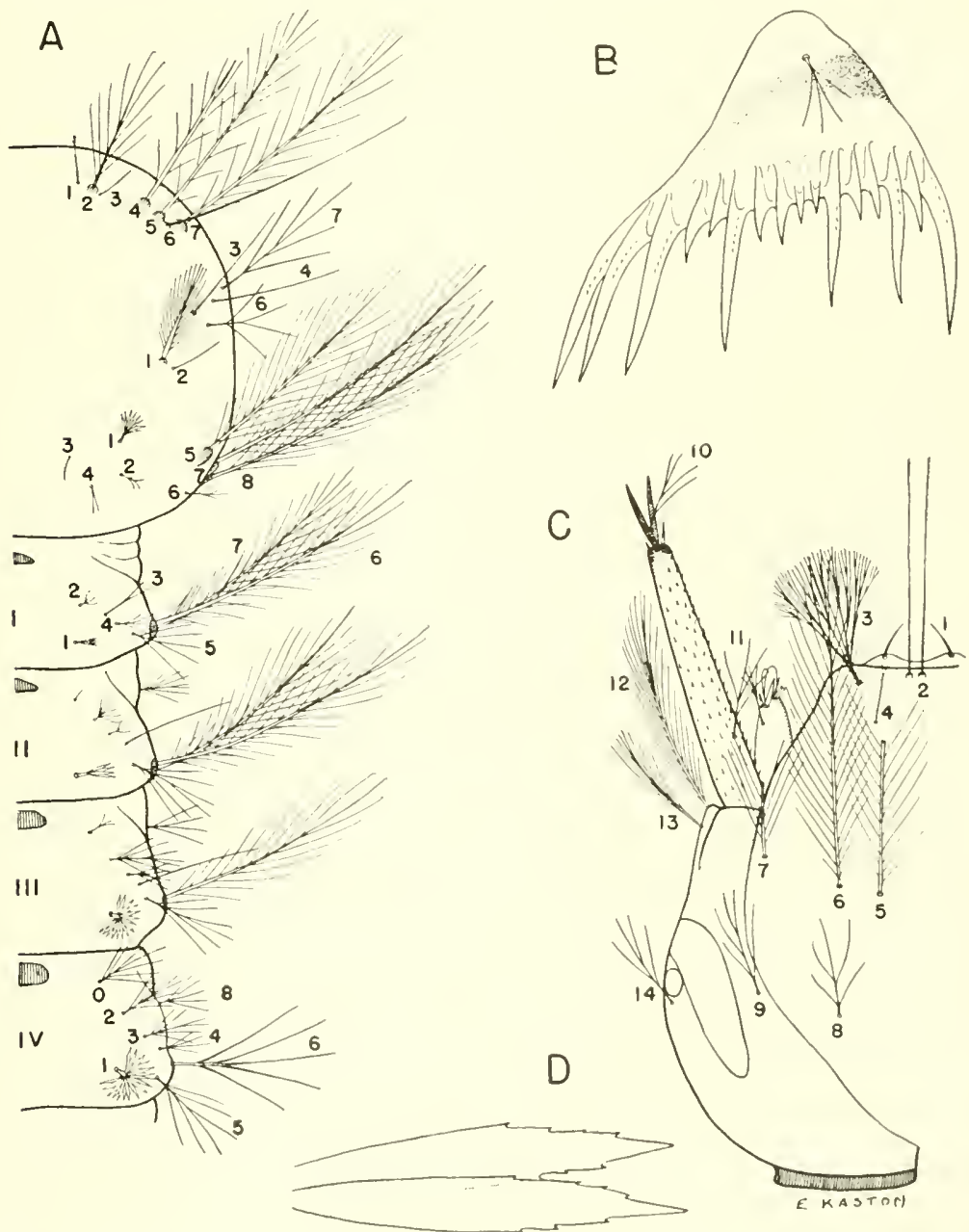

Fig. 31. Larva of Anopheles crucians crucians Wiedemanu. A, Thorax and abdomen. B, Peclen. C, Head. D. Leaflets of palmale hair. 
Missouri (70); North Carolina and South Carolina (52); Tennessee (96); Virginia (49). Other States: Connecticut (94); Delaware (106); District of Columbia (52); Illinois (94); Indiana (39); Maryland (19); Massachusetts (180); New Jersey (77); New Mexico (9); New York (94); Oklahoma (159); Pennsylvania (8); Texas (108).

BIONOMICS.-The adults ocassionally enter houses but are principally outdoor biters, attacking mostly at night. Adults are apparently able to migrate several miles from their breeding grounds. They are frequently taken in large numbers in light traps located on the coastal plains. It breeds principally in swamps, ponds and lakes. This mosquito seems to prefer slightly acid water and occurs in large numbers in the acid waters in cypress swamps in Georgia and Florida.

MEDICAL IMPORTANCE. - Several workers have been able to infect this mosquito with Plasmodium vivax and $P$. falciparum under experimental conditions, and stomach and salivary gland infections have been found in wild specimens $(172,173)$. However, most workers regard it as being relatively unimportant as a vector of malaria when compared with $A$. quadrimaculatus.

\section{Anopheles (ANopheles) crucians georgianus King}

Anopheles crucians var. georgianus King, 1939, Amer. Jour. Trop. Med., 19:462.

ADULT FEMALE.--Indistinguishable from $A$. crucians crucians.

ADULT MALE.-Coloration similar to that of $A$. crucians crucians. TERMiNALIA.-Similar to that of $A$. crucians crucians (Fig. 30 ).

LARVA (Fig. 32).--Head: Inner clypeal hairs (2) simple, arising from basal tubercles, rarely separated by more than the diameter of one of the tubercles; outer clypeal (3) densely, dichotomously branched, fan-shaped; postclypeal (4) small, single or branched apically, nearly reaching preclypeus; frontal hairs (5-7) large, plumose; sutural (8) 3- to 5-branched; trans-sutural (9) usually 3- or 4-branched, rarely reaching base of frontal hair (7). Antenna spinose; terminal hair (10) with several branches, longer than the sabres; antennal tuft (11) inserted at basal third of shaft, severalbranched, reaching middle or slightly beyond. Thorax: Prothoracic dorsal submedian hairs (1-3) consisting of a short inner hair (1), about 3- to 6branched apically; a much larger, stout middle hair (2) with many branches; and a short simple outer hair (3). Prothoracic pleural group (9-12) of four hairs; hairs 9, 10 and 12 long, simple, subequal; hair 11 short, simple or bifurcate. Mesothoracic pleural group (9-12) of four hairs; hairs 9 and 10 long, simple, subequal; hair 11 simple, minute; hair 12 about one-third as long as 9 or 10,2 - or 3-branched beyond middle. Metathoracic pleural group (9-12) of four hairs; hairs 9 and 10 long, simple; hair 11 simple, minute; hair 12 short, with several branches beyond base. Metathoracic palmate hair (1) small, with transparent leaflets. Abdomen: Submedian dorsal hair (0) obsolete on segments IV and V. Palmate hair (1) well-developed and of 
equal size on segments IV to VI, with individual leaflets serrated beyond middle; palmates very small and rudimentary on segments I to III, and VII, with individual leaflets smooth. Antepalmate (2) on segments IV and $\mathrm{V}$, 3- to 6-branched (rarely 2-branched). Hair 5 on segment I, 5- or 6branched (rately more) somewhat dichotomous, nearly twice as large as hair 4. Upper lateral hair (6) on segments I to III long, plumose. Pecten as illustrated.
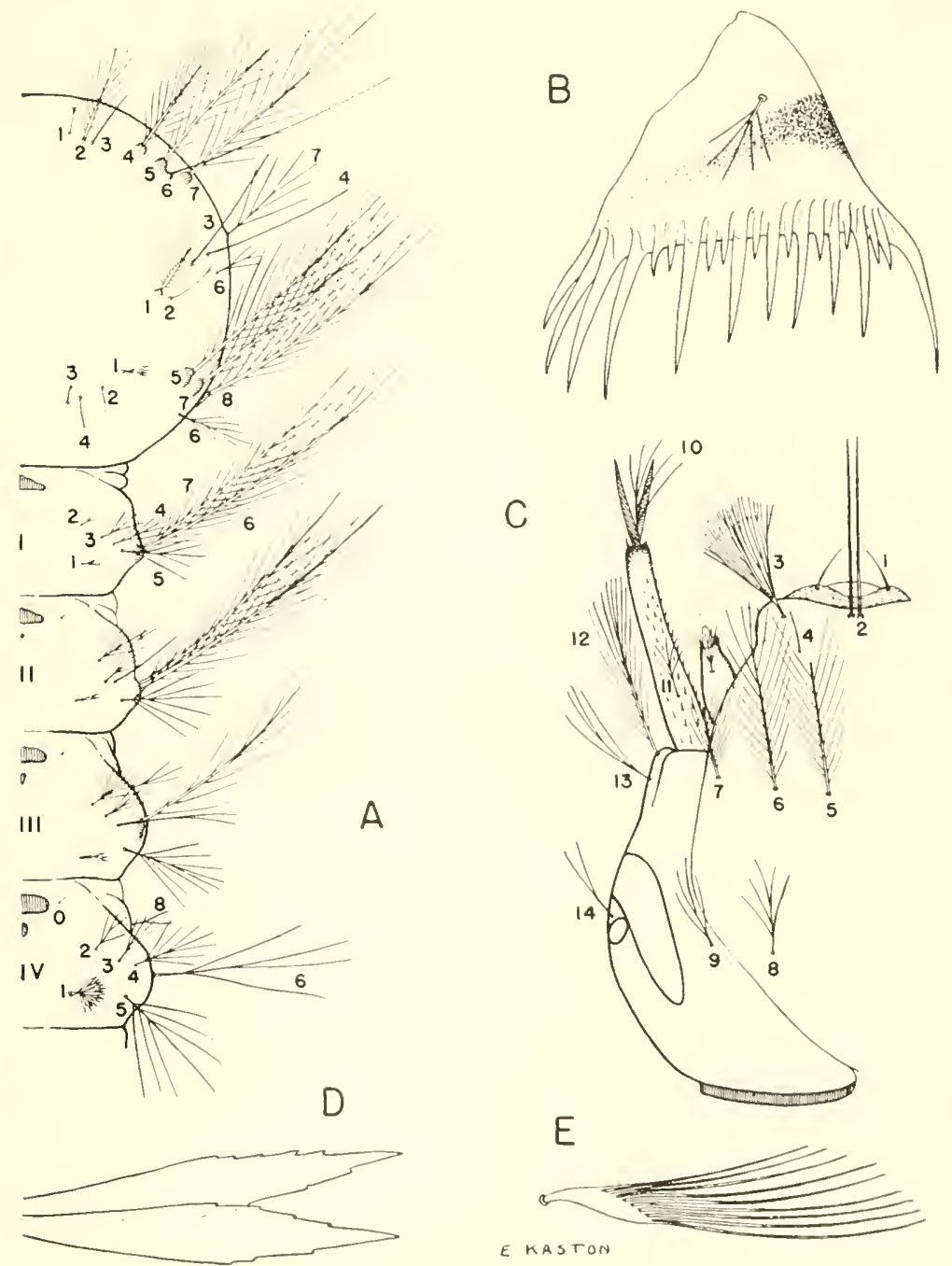

Fig. 32. Larva of Anopheles crucians georgianus King. A. Thorax and abdomen. B, Pecten. C. Head. D, Leaflets of palmate hair. E, palmate hair III. 
Distribution.-Southern States: Alabama and Florida (97); Georgia (96); Louisiana, Mississippi, North Carolina and South Carolina (97).

BIONOMICS.-Anopheles crucians georgianus cannot at this time be distinguished from $A$. crucians crucians in the adult stage; therefore nothing is known of the habits of the adults. The larvae occur mostly in seepage pools at the head of small streams and at the bases of hills.

MEDICAL IMPORTANCE.-No data on its relation to malaria.

\section{ANOPHELES (ANOPHELES) PSEUdopUNCTIPENNIS PSEUdOPUNCTIPENNIS}

\section{Theobald}

Anopheles pseudopunclipennis Theobald, 1901, Mon. Culic., 2:305.

adult fEMale.-Medium-sized species. Head: Proboscis long, dark; palpi about as long as proboscis, black, with the apices of segments 2, 3 and 4 narrowly ringed with white, the base of segment 4 more broadly ringed with white, the terminal segment entirely yellowish-white (Fig. 33D). Occiput clothed with numerous erect forked scales, those on central portion white, the others dark; scales of vertex white; frontal tuft white. Thorax: Integument with a broad longitudinal frosted stripe medially, dark-brown laterally; the frosted area clothed with white or pale yellow hairs, the darker lateral areas with larger dark setae. Abdomen: Integument dark-brown to black, clothed with numerous golden-brown hairs. Legs: Legs dark-scaled, tips of femora and tibiae pale. Wing (Fig. 33E): Scales black and pale yellow, arranged on the veins in contrasting lines and spots (costa with a pale spot at outer third opposite subcosta; vein 6 with apical half dark, basal half pale; veins 3 and 5 with long areas of pale scales). Halters: Knob of halter darkscaled.

ADUlt MALE.-Coloration similar to that of female. TERminalia (Fig. 33). Ninth tergite (IX-T) a narrow, sclerotized band; lobes (IXT-L) lateral in position, stout, about as long as broad, blunt or obtuse-angulate at tip. Tenth sternite absent; anal lobe (An-L) large, sub-triangular, spiculate. Phallosome $(\mathrm{Ph})$ cylindrical, furcate at base; apex with two or three pairs of slender, curved leaflets (Ph-L) serrated nearly to tips. Claspette broad, fleshly, consisting of a dorsal lobe (Cl-DL) and a ventral lobe (Cl-VL). Dorsal lobe bearing three overlapping, blade-like filaments on apex; ventral lobe with a long slender spine and a short weak spine apically, and a long, slender spine sub-apically. Basistyle (Bs) conical, about twice as long as broad, rounded apically, clothed with long setae; two parabasal spines (P-S) and one internal spine (I-S) present. Dististyle (Ds) a little longer than basistyle and lacking spicules (minute non-papillated hairs), curved, narrower medially, bluntly pointed at tip; terminal claw (Ds-C) short, blunt.

LARva (Fig. 34).-Head: Inner clypeal hairs (2) long, simple, with basal tubercles separated by at least the diameter of one of the tubercles; outer clypeal (3) simple, nearly as long as the inner clypeals (2); post- 
clypeal (4) long, simple, extending well beyond preclypeus; frontal hairs (5-7) large, plumose; sutural (8) and trans-sutural (9), with several branches arising from a stout shaft, extending to bases of frontal hairs. Antenna smooth on outer surface; terminal hair (10) 2- to 4-branched, slightly longer than the sabres; antennal tuft (11) usually represented by a single hair inserted near middle of shaft. Thorax: Prothoracic dorsal submedian hairs (1-3)
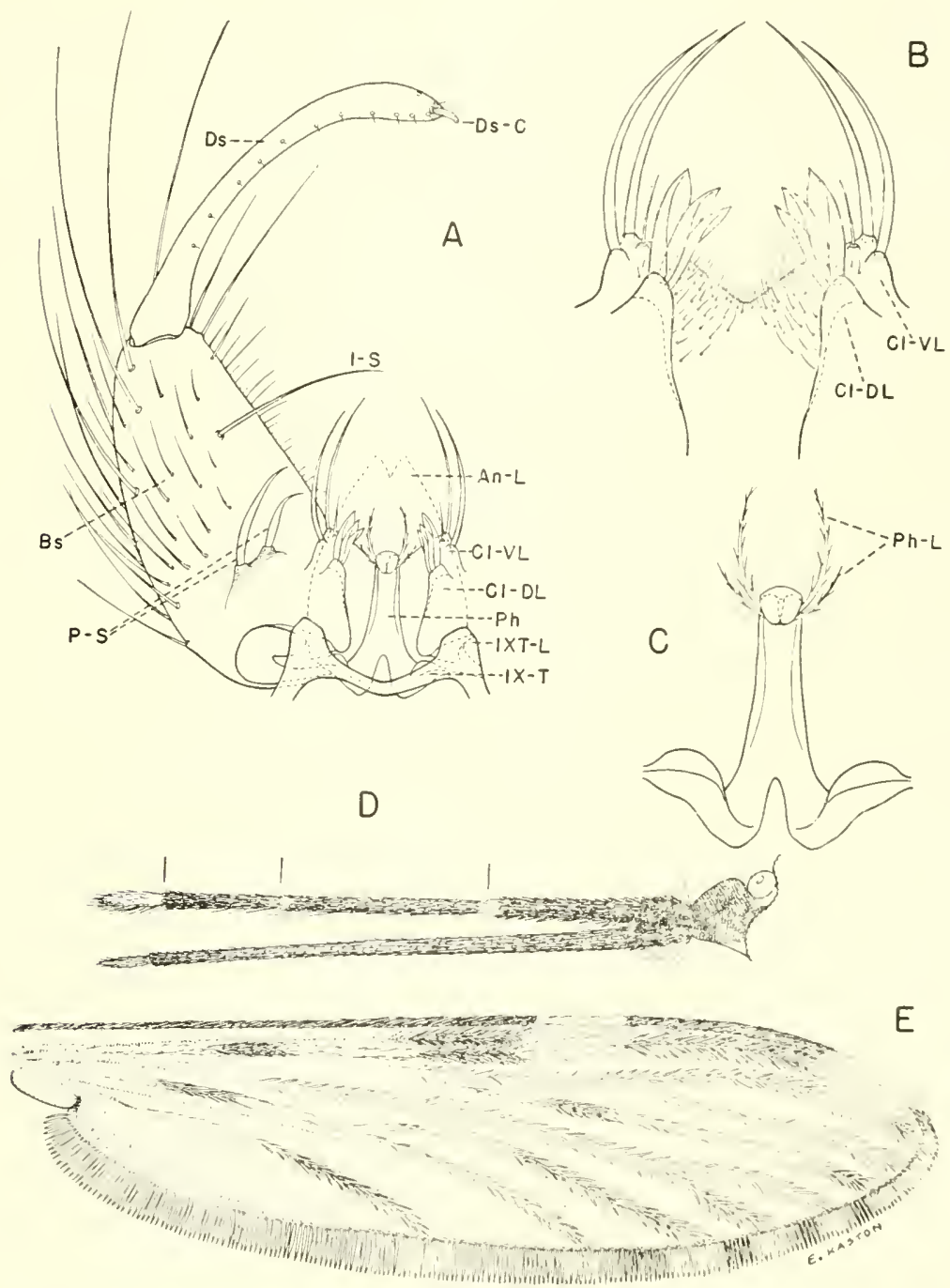

Fig. 33. Anopheles pseudopunctipennis pseudopunctipennis Theobald. A, Male terminalia. B, Claspettes. C, Phallosome. D, Palp and proboscis of female. E, Wing. 
consisting of a short inner hair (1) with about 2 to 4 branches beyond base, a much longer stouter middle hair (2) with many branches, and a simple outer hair (3) about twice as long as inner hair (1). Prothoracic pleural group (9-12) of four hairs; hairs 9, 10, and 12 long, simple; hair 11 much shorter, simple. Mesothoracic pleural hairs (9-12) of four hairs; hair 9 short, stout; hair 10 long, stout (about twice as long as hair 9); hair 11 minute; hair 12 weak, usually 2- to 3-branched (about two-thirds as long as hair 9). Metathoracic pleural group (9-12) of four hairs; hair 9 short,

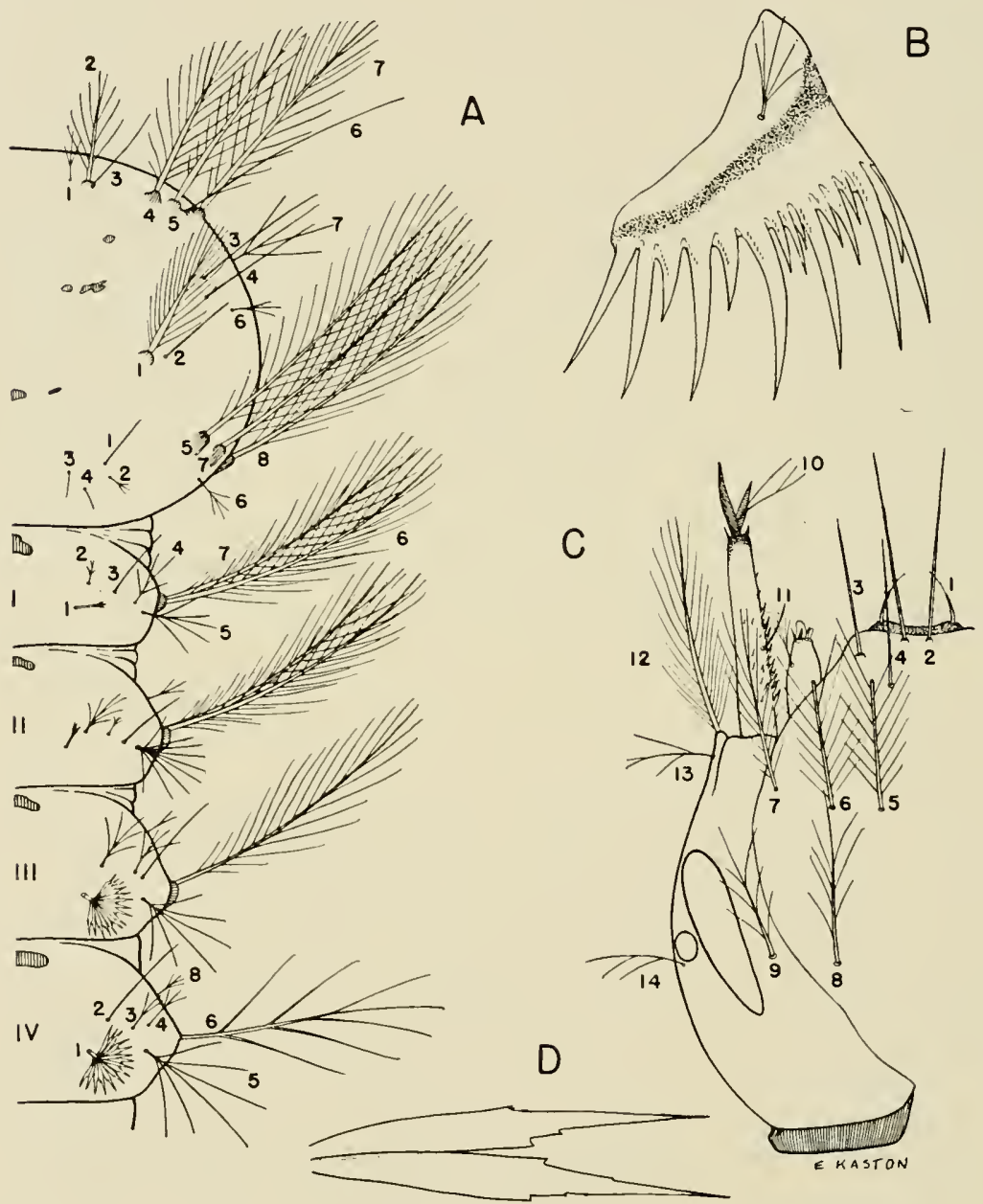

Fig. 34. Larva of Anopheles pseudopunctipennis pseudopunclipennis Theobald. A, Thorax and abdomen. B, Pecten. C, Head. D, Leaflets of palmate hair. 
stout; hair 10 long, stout (about twice as long as hair 9); hair 11 obsolete; hair 12 weak, usually 3- or 4-branched (about half as long as hair 9). Metathoracic palmate hair (1) absent. Abdomen: Submedian dorsal hair (0) absent on segments I to VII. Palmate hair (1) rudimentary on segments I and II, well-developed on segments III to VII; individual leaflets with serrations beyond middle, filament long and slender. Antepalmate hair (2) single on segments IV and V. Lateral hair (6) long, plumose on segments I-III. Posterior lobes of spiracular apparatus each with a long slender sclerotized projection arising from the inner angle. Pecten as illustrated.

Distribution.--Southern United States, south to Chile and Argentina. The distribution of this subspecies is not well known. Southern States: Arkansas (31); Louisiana and Mississippi (94); Missouri (128); Tennessee (94). Other States: Arizona, Kansas, New Mexico, Oklahoma, Texas and Utah (116).

BIONOMICS.- The adults enter houses and attack man. The larvae require a great deal of sunlight for their development and occur mostly in clear sunlit water, rich in algae.

MEDICAL IMPORTANCE.- It has been experimentally infected with $P$. vivax and $P$. falciparum and natural infections with malaria parasites have been reported by several workers $(172,173)$. It is regarded as an important vector of malaria in some areas within its range and relatively unimportant in others. It is not known to be of much importance in the United States.

\section{Anopheles (Anopheles) punctipennis (Say)}

Culex punctipennis Say, 1823, Jour. Acad. Nat. Sci. Phila., 3:9.

ADUlt feMale.-Medium-sized species. Head: Proboscis long, black; palpi about as long as proboscis, dark-scaled. Occiput clothed with numerous erect forked scales, those on central portion white, the others dark; scales of vertex white; frontal tuft white. Thorax: Integument with a broad longitudinal frosted stripe medially, dark-brown laterally; the frosted area clothed with short pale yellow hairs, the darker lateral areas with larger dark setae. Abdomen: Integument dark-brown to black, clothed with numerous pale and dark hairs. Legs: Legs dark-scaled, tips of femora and tibiae pale. IVing (Fig. 35D): Scales black and pale yellow, arranged on the veins in contrasting lines and spots (costa with a pale spot at outer third opposite tip of subcosta; vein 6 with apical half and basal fourth dark-scaled; veins 3 and 5 entirely dark-scaled). Halter: Knob dark-scaled.

ADULT MALE.-Coloration similar to that of female. Terminalia (Fig. 35). - Ninth tergite (IX-T) a narrow, sclerotized band; lobes (IXT-L) lateral in position, about three times as long as broad, often slightly constricted medially, rounded or noarly truncente apicallv. Tenth sternite absent: anal lobe (An-L) large, triangular, spiculate. Phallosome (Ph) cylindrical. furcate at base; apex with three or four pairs of ligulate leaflets ( $\mathrm{Ph}-\mathrm{L}$ ), the 
more distal pairs longer; one or more pairs of leaflets with a few short coarse basal teeth. Claspette broad, fleshy, consisting of a dorsal lobe (Cl-DL) and a ventral lobe (Cl-VL), fused basally but more or less distinct apically. Dorsal lobe with one or two long, stout, pointed or rounded spines at apex; ventral lobe bearing a long, stout, pointed spine at apex (spine broader than the apical spine of the dorsal lobe), and with a slender short and a slender long spine subapically. Basistyle (Bs) conical, nearly twice as long as broad, rounded apically, clothed with long setae and sparse scales; two parabasal spines (P-S) and one internal spine (I-S) present. Dististyle (Ds) a little longer than basistyle and lacking spicules (minute non-papillated hairs), curved, narrower medially, bluntly pointed at tip; terminal claw (Ds-C) short, blunt.

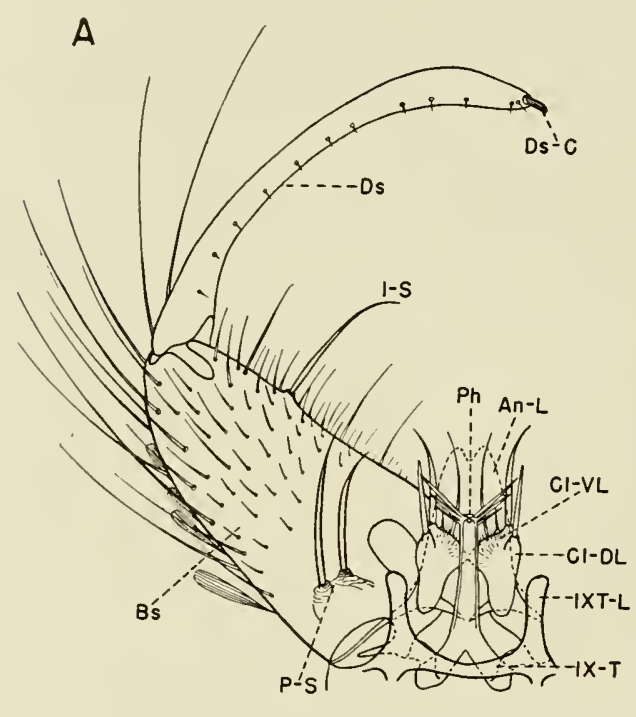

D

C
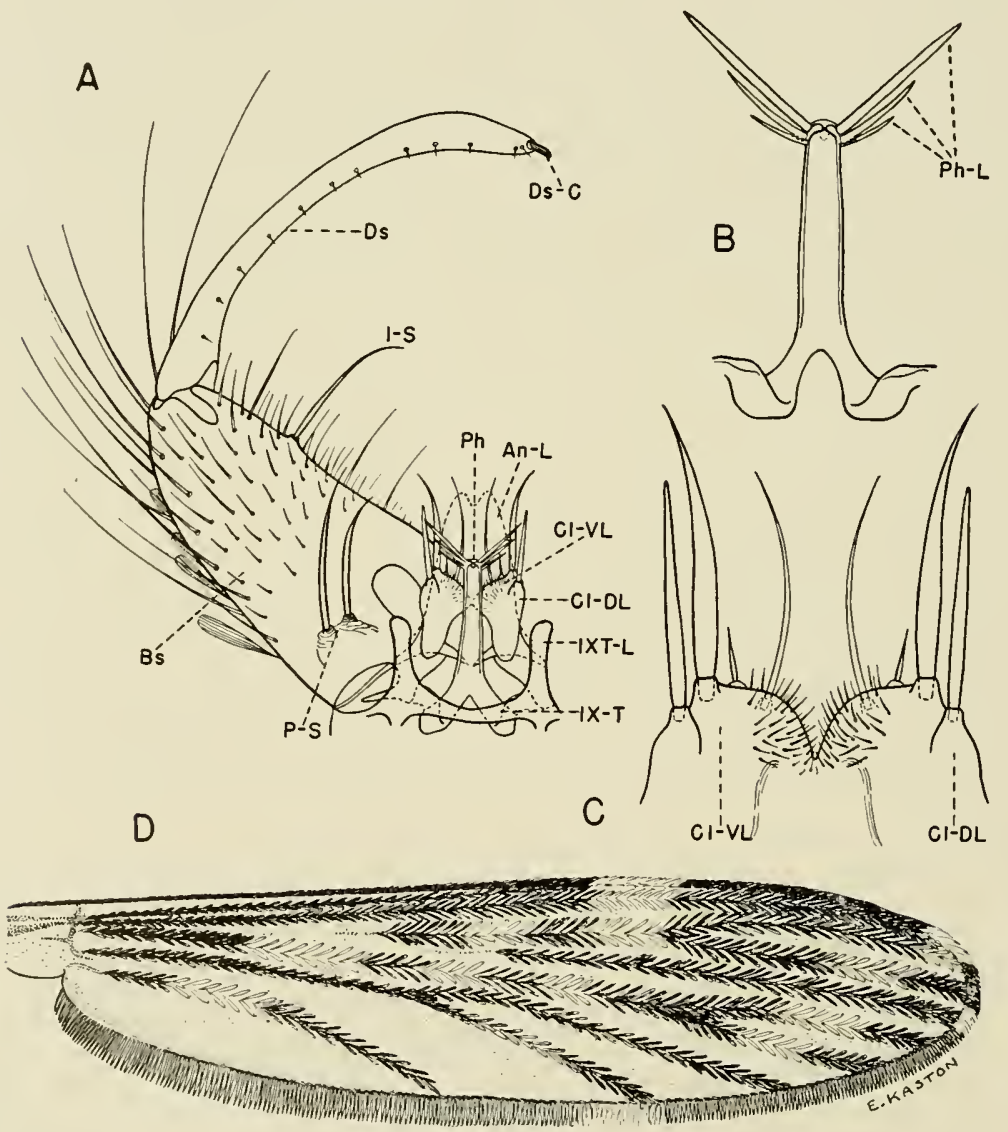

Fig. 35. Anopheles punctipennis (Say). A, Male Terminalia. B, Phallosome. C, Claspettes. D, Wing. 
LARVA (Fig. 36).- Head: Inner clypeal hairs (2) simple, arising from basal tubercles separated by less than the diameter of one of the tubercles; outer clypeal hair (3) densely, dichotomously branched, fan-shaped; postclypeal hair (4) small, branched, rarely reaching preclypeus; frontal hairs (5-7) large, plumose; sutural (8) and trans-sutural (9) usually 5- to 7branched (trans-sutural rarely reaching beyond base of outer frontal hair). Antenna spinose; terminal hair (10) with several branches, longer than the sabres; antennal tuft (11) inserted at basal third of shaft, several-branched,

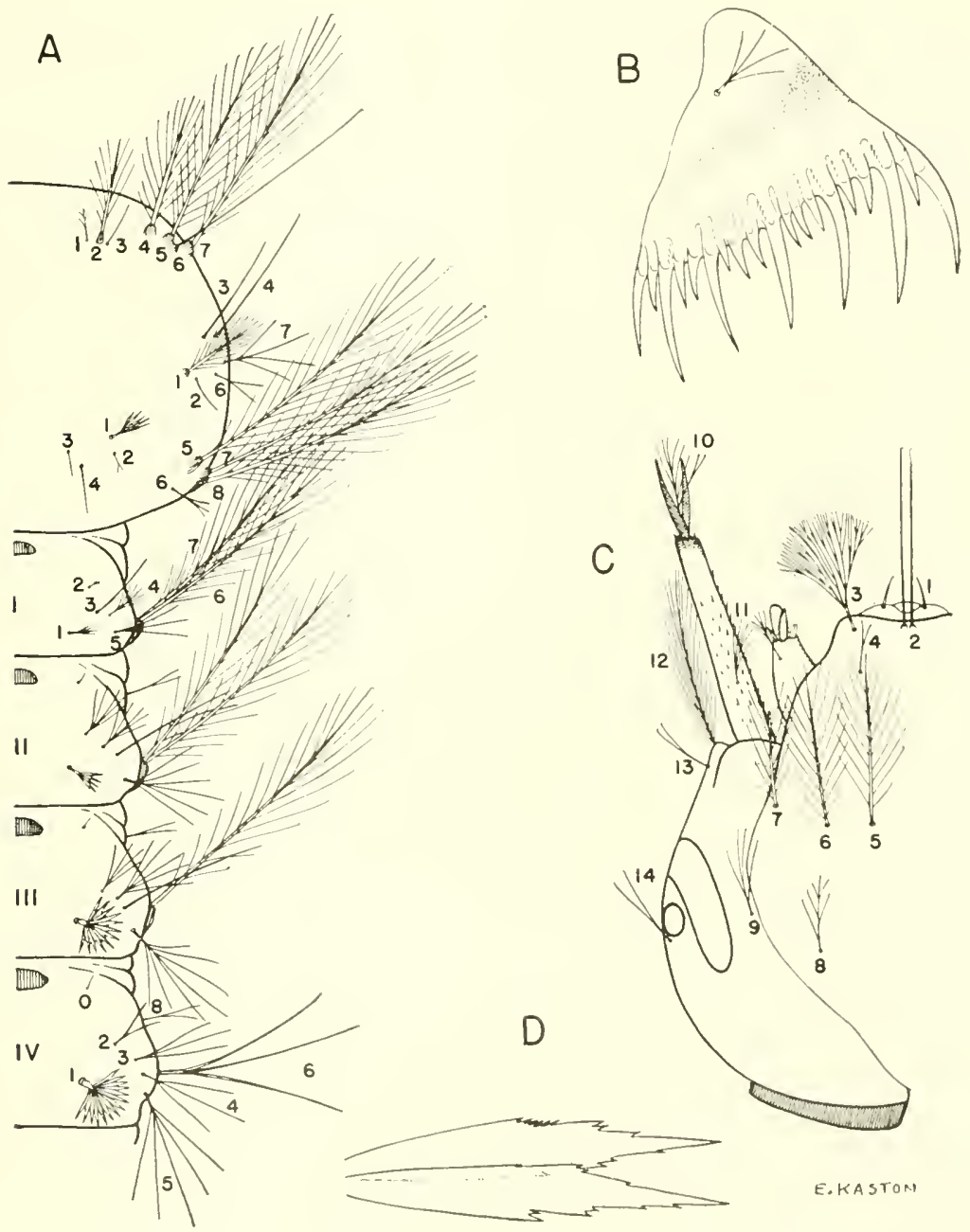

Fig. 36. Larva of Anopheles punctipennis (Say). A, Head and thorax.

B. Pecten. C. Head. D, Leaflets of palmate hair. 
reaching middle or slightly beyond. Thorax: Prothoracic dorsal submedian hairs (1-3) consisting of a short inner hair (1), with several weak branches beyond base; a much longer stout middle hair (2), with many branches; and a short simple outer hair (3). Prothoracic pleural group (9-12) of four hairs; hairs 9, 10, and 12 long, simple; hair 11 short, simple. Mesothoracic pleural group (9-12) of four hairs; hairs 9 and 10 long, simple; hair 11 minute, simple; hair 12 short, simple, about one-third as long as hair 9 or 10 . Metathoracic pleural group (9-12) of four hairs; hairs 9 and 10 long, simple; hair 11 minute, simple; hair 12 short, 2- or 3-branched. Metathoracic palmate (1) obsolete. Abdomen: Submedian dorsal hair (0) obsolete on segments IV and V; palmate hair (1) on segments I and II rudimentary, well-developed and of about equal size on III to VII (may be somewhat smaller on VII); individual leaflets with serrations beyond middle. Antepalmate hair (2) usually double, occasionally single or triple on segments IV and V. Hair 5 on segment $I$ is 3-branched (rarely 4-branched) and only slightly larger than hair 4. Upper lateral hair (6) on segments I to III long, plumose. Pecten as illustrated.

DISTRIBUTION.-Occurs throughout most of the United States (absent from Rocky Mountain region), north to the southern part of Canada and south to the tablelands of Mexico. Southern States: Alabama, Arkansas, Florida and Georgia (96); Kentucky (140); Louisiana and Mississippi (96); Missouri (1); North Carolina, South Carolina and Tennessee (96); Virginia (49). Other States: California (66); Colorado (128); Connecticut (21); Delaware (106); Illinois and Indiana (94); Iowa (155, 156); Kan. sas (79); Maine (103); Maryland (19); Massachusetts (96); Michigan (88); Minnesota (131); Montana (112); Nebraska (178); New Hampshire (105); New Jersey (77); New Mexico, New York and Ohio (94); Oklahoma (159); Oregon and Pennsylvania (94); Rhode Island (99); South Dakota (94); Texas (108); Vermont, Washington and West Virginia (94); Wisconsin (48); Wyoming (128).

BIONomics.--The females feed mostly after dusk but will bite during daylight hours in dense woodlands or in their daytime resting places. It is regarded as an outdoor species which seldom enters dwellings. Larvae are found along the margins of streams, also in temporary and permanent bodies of water of various types. It reaches its greatest abundance, in the southern states, in the early spring and late fall, becoming scarce in the summer.

MEDICAL iMPORTANCE.-Anopheles punctipennis has been infected experimentally with Plasmodium vivax, $P$. falciparum and $P$. malariae (172) but not a single gland infection has been reported in nature. Mayne (118) reports finding a sinole specimen with a stomach infection in nature but according to Simmons (172), there appears to be some doubt concerning the accuracy of this observation. Although available data indicate that this species is susceptible to infection with the three principal species of malaria plasmodia, it is not regarded as an important vector. 


\section{Anopheles (Anopheles) Quadrimaculatus Say}

Anopheles quadrimaculatus Say, 182\%. Keating's Narr. Exp. St. Peter's River, 2:356.

adult female.-Medium-sized species. Head: Proboscis long, dark; palpi about as long as proboscis, dark. Occiput clothed with numerous dark erect forked scales, those on the vertex pale; frontal tuft with some pale setae. Thorax: Integument of scutum brown; clothed with numerous pale-yellow to golden-brown hairs, more numerous medially. Abdomen: Integument of abdomen dark-brown to black, clcthed with pale-yellow to brown hairs. Legs:
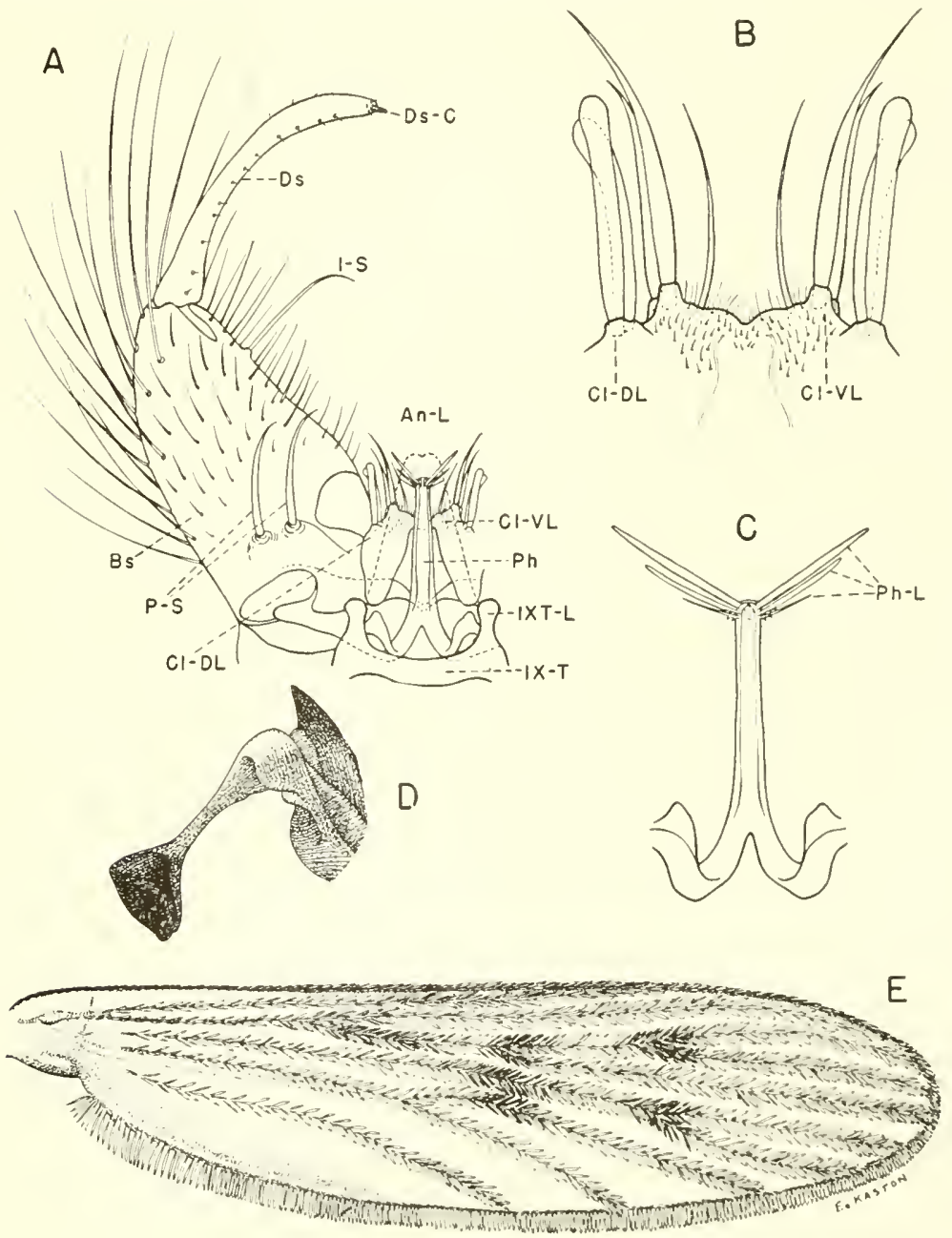

Fig. 37. Anopheles quadrimaculatus Say. A. Male terminalia. B. Claspettes. C. Phallosome. D. Halter, E, Wing. 
Legs entirely dark-scaled except for small whitish knee spots. Wing: Scales narrow, entirely dark; some of the scales arranged in four distinct darker spots (Fig. 37E). Halter: Knob of halter dark-scaled (Fig. 37D).

ADULT MALE.-Coloration similar to that of female. TERMinalia (Fig. 37). - Ninth tergite (IX-T) a narrow, sclerotized band; lobes (IXT-L) lateral in position, about two to three times as long as broad, usually somewhat constricted medially, rounded or truncate apically. Tenth sternite absent; anal lobe (An-L) large, triangular, spiculate. Phallosome (Ph) cylindrical,

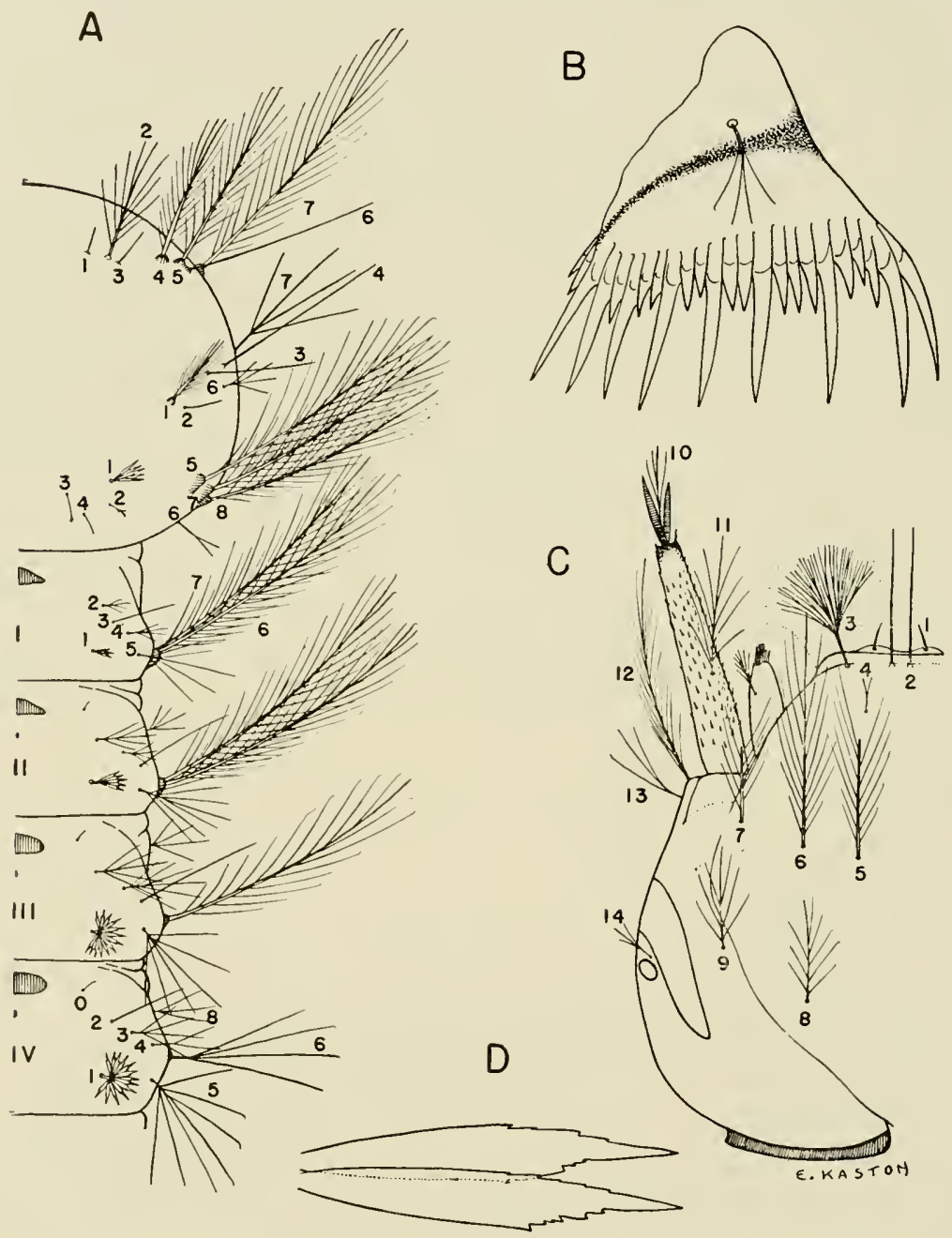

Fig. 38. Larva of Anopheles quadrimaculatus Say. A. Thorax and abdomen. B, Pecten. C, Head. D, Leaflets of palmate hair. 
furcate at base; apex with three or four pairs of ligulate leaflets (Ph-L), the more distal pairs longer; one or more pairs of the leaflets with a few short coarse basal teeth. Claspette broad, fleshy, consisting of a dorsal lobe (Cl-DL) and a ventral lobe (Cl-VL), fused basally but more or less distinct apically. Dorsal lobe apically with about one to five stout blunt spines (often fused to one another); ventral lobe usually bearing apically two large pointed spines of unequal length, though occasionally with one or three (largest spine rarely as broad as the blunt spine or fused spines of the dorsal lobe). Basistyle (Bs) conical nearly twice as long as broad, clothed with long setae and few or no scales; two parabasal spines (P.S) and one internal spine (I-S) present. Dististyle (Ds) about as long or slightly longer than basistyle and lacking spicules (minute non-papillated hairs), curved, narrower medially, bluntly pointed at tip; terminal claw (Ds-C) short, blunt.

LARVA (Fig. 38).- - Head: Inner clypeal hairs (2) simple, arising from basal tubercles separated by at least the diameter of one of the tubercles; outer clypeal hair (3) densely dichotomously branched, fan-shaped; postclypeal hair (4) small, branched, not reaching preclypeus; frontal hairs (5-7) large, plumose; sutural (8) usually 8- to 10-branched; trans-sutural (9) usually 6- to 8-branched. Antenna spinose; terminal hair (10) with several branches, longer than the sabres; antennal tuft (11) inserted near middle, several-branched, reaching base of sabres. Thorax: Prothoracic dorsal submedian hairs (1-3) consisting of a short inner hair (1), single or weakly branched at tip; a much longer stout middle hair (2), with many branches; and a short single outer hair (3). Prothoracic pleural group (9-12) of four hairs; hair 9 long, 2-branched before middle or simple; hairs 10 and 12 long, simple; hair 11 short, simple. Mesothoracic pleural group (9-12) of four hairs; hairs 9 and 10 long, simple; hair 11 minute, simple; hair 12 simple, about one-third as long as hairs 9 or 10; metathoracic pleural group (9-12) of four hairs: hairs 9 and 10 lon,, simple; hair 11 minute, simple; hair 12 short, 2- to 4-branched. Metathoracic palmate (1) small with transparent leaflets. Abdomen: Submedian dorsal hair (0) obsolete on segments IV and V; palmate hair (1) well-developed on abdominal segments III to VII, rudimentary on segments I and II; individual leaflets with serrations on outer third. Antepalmate hair (2) on segments IV and V single, sometimes dcublc. Upper lateral hair (6) on segments I to III long, plumose. Pecten as illustrated.

Distribution.-Eastern and central United States, north to the southern part of Canadr and south to Vern Crum. Mexico. It reaches its rreatest abundance in the southern United States. Southern States: Alabama (96); Arkansas (30); Florida and Georgia (96); Kentucky (140); Louisiana and Mississippi (96); Missouri (1); North Carolina, South Carolina and Tennessee (96); Virginia (49). Other States: Connecticut (94); Delaware (106); Illinois (94); Indiana (76); Iowa (155, 156); Kansas (79); Maryland (19); Massachusetts, Michigan and Minnesota (94); Nebraska (178); New Hampshire (94); New Jersey (77); New York (116); Ohio (94); 
Oklahoma (159); Pennsylvania (8); Rhode Island (99); South Dakota
(128); Texas (108); Wisconsin (48).

BIONOMics.- The females are active feeders on man and wild and domesticated animals. The adults are active principally at night and rest in dark corners in buildings, underneath houses, in hollow trees and other shelters during daylight hours. They are very active for a short period after dusk but their activities are probably limited to flights in search of a blood meal during the remainder of the night except for another active period at dawn when they shift to daytime resting places. The effective flight range of this species varies, depending on the proximity to suitable hosts upon which they can feed and the number produced, but it is usually regarded as approximately one mile under average conditions. Anopheles quadrimaculatus breeds mostly in fresh-water in sluggish streams, canals, ponds and lakes with surface-growing or emergent vegetation or floating debris.

MEDICAL IMPORTANCE.-The susceptibility of this species to experimental infection with Plasmodium vivax, $P$. falciparum and $P$. malariae has been well established and infections in nature have been found by numerous workers. This mosquito is regarded as the most important vector of malaria in the United States.

\section{ANopheles (ANOpHeles) WALKeRI Theobald}

Anopheles walkeri Theobald, 1901. Mon. Culic., $1: 199$.

adult female.-Medium-sized species. Head: Proboscis long, dark; palpi about as long as proboscis, dark-scaled except for a narrow white ring at apex of each segment (Fig. 39E). Occiput clothed with dark erect forked scales; the vertex with a few pale recumbent scales; frontal tuft dark. Thorax: Integument of scutum dark-brown, clothed with short golden-brown to darkbrown hairs. Abdomen: Integument dark-brown to black, clothed with yellow to brown hairs. Legs: Legs entirely dark-scaled except for white knee spots. Wing: Scales narrow, entirely dark; some of the scales arranged to form four darker spots, more or less distinct (Fig. 39F). Halter: Knob of halter pale scaled (Fig. 39D).

ADULT MALE.-Coloration similar to that of female. Terminalia (Fig. 39).-Ninth tergite (IX-T) a narrow, moderately sclerotized band; lobes (IXT-L) lateral in position, slender, tapered, pointed or rounded at tip. Tenth sternite absent; anal lobe (An-L) large, triangular, spiculate. Phallosome $(\mathrm{Ph})$ cylindrical, furcate at base; apex with 3 to 5 pairs of ligulate leaflets (Ph-L), the ante-apical pair more than half as long as the apical pair (usually about three-fourths as long); none of the leaflets with short, coarse basal teeth. Claspette broad, fleshy, consisting of a dorsal lobe (Cl-DL) and a ventral lobe (Cl-VL). Dorsal lobe apically with one or two large, blunt, often slightly capitate spines; ventral lobe with a large, pointed spine at apex and a long sub-apical spine. Basistyle (Bs) conical, about twice as long as broad, rounded apically, clothed with long setae and few or no scales; 
two parabasal spines (P.S) and one internal spine (I-S) present. Dististyle (Ds) a little longer than basistyle, curved, narrower medially, bluntly pointed at tip; a few spicules (minute non-papillated hairs) present on basal sixth of dististyle; terminal claw (Ds-C) short, blunt.

LARVA (Fig. 40).-Head: Inner clypeal hairs (2) arising from basal
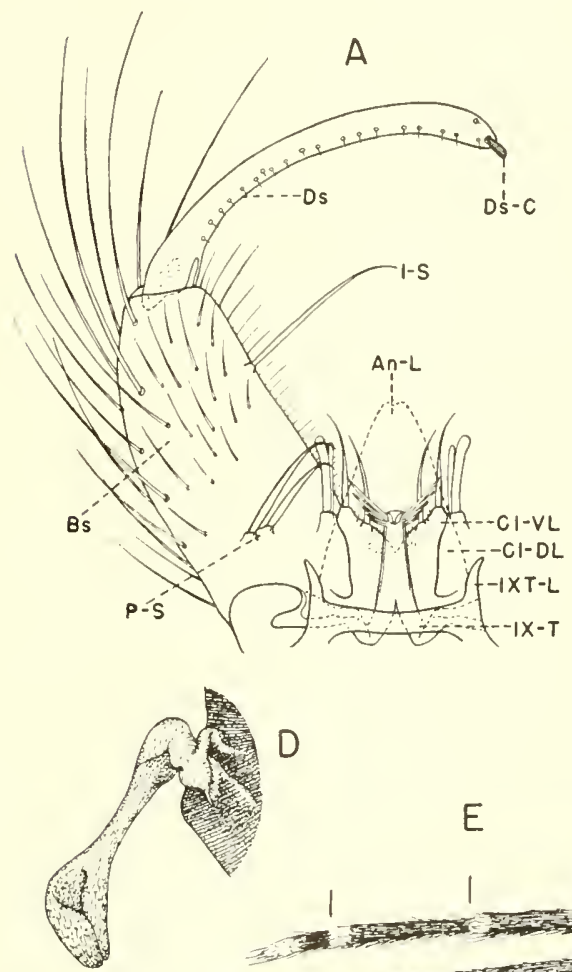

$E$

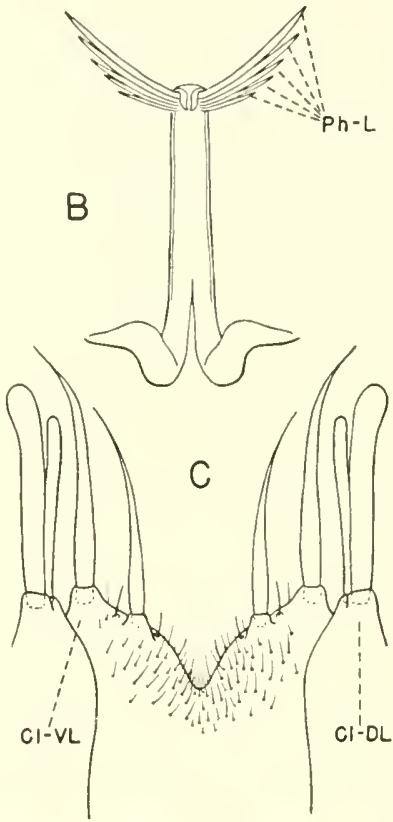

$\mathrm{F}$

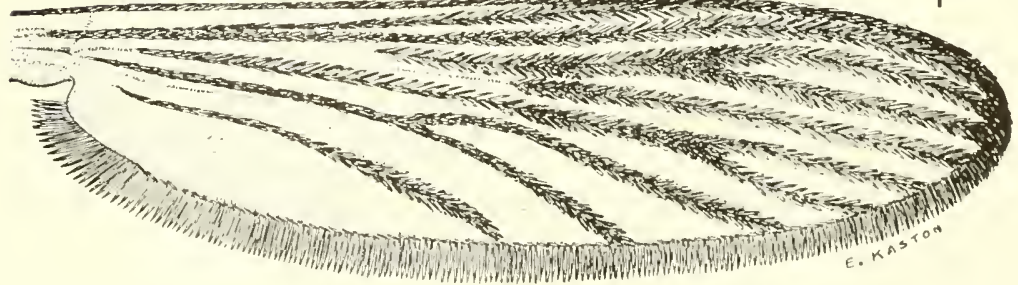

Fig. 39. Anopheles walheri Theobald. A, Male terminalia. B. Phallosome. C. Claspettes. D, Halter. E, Palp and proboscis of female. F, Wing. 
tubercles rarely separated by more than the diameter of one of the tubercles, with sparse, minute feathering toward tips; outer clypeal hair (3) densely " dichotomously branched, fan-shaped; postclypeal hair (4) small, branched; frontal hairs (5-7) large, plumose; sutural (8) and trans-sutural (9) with several branches. Antenna spinose; terminal hair (10) with several branches, longer than the sabres; antennal tuft (11) inserted near basal third of shaft, several-branched, reaching outer fourth. Thorax: Prothoracic dorsal submedian

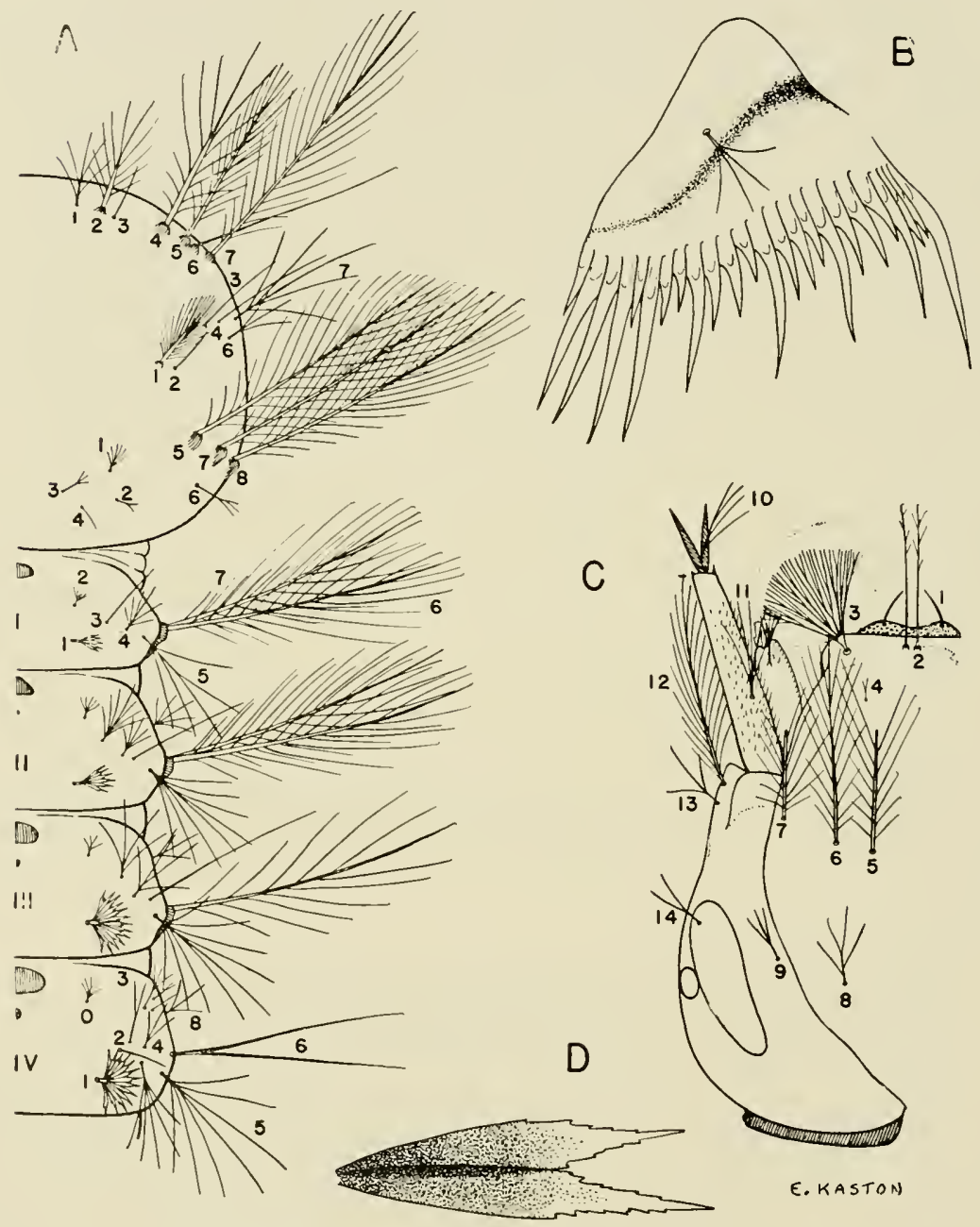

Fig. 40. Larva of Anopheles walkeri Theobald. A, Thorax and abdomen. B, Pecten. C, Head. D, Leaflets of palmate hair. 
hairs (1-3) consisting of an inner hair (1) with 3 to 5 strong branches near base; a longer stout middle hair (2) with many branches; and a short, simple outer hair (3). Prothoracic pleural group (9-12) of four hairs; hairs 9, 10 and 12 long, simple; hair 11, short, simple. Mesothoracic pleural group (9-12) of four hairs; hairs 9 and 10 long, simple; hair 11 minute, simple; hair 12 simple, about one-third as long as hair 9 or 10. Metathoracic pleural group (9-12) of four hairs; hairs 9 and 10 long, simple; hair 11 minute, simple; hair 12 short, usually simple. Metathoracic palmate hair (1) small, with transparent leaflets. Abdomen: Submedian dorsal hair (0) comparatively well-developed on segments IV and V (much smaller than antepalmate hair 2) with 3 to 7 branches. Palmate hair (1) well-developed, nearly equal in size on segments III to VII, individual leaflets serrated beyond middle; palmate hairs rudimentary on segments I and II. Antepalmate hair (2) on segments IV and V usually single, sometimes double or triple. Upper lateral hair (6) on segments I to III long, plumose. Pecten as illustrated.

distribution.-Eastern North America, from the Gulf of Mexico to Canada, west to Nebraska. It has been reported from Vera Cruz, Mexico: by Vargas (158). Southern States: Alabama (35); Arkansas (179); Florida and Georgia (94); Kentucky (140); Louisiana (94); Mississippi (97); North Carolina (166); South Carolina (27); Tennessee (7); Virginia (49); Other States: Connecticut, Delaware, District of Columbia, Illinois, Indiana, lowa and Maine (94); Maryland (19); Massachusetts, Michigan, and Minnesota (94); Nebraska (178); New Jersey (77); New York (116); Ohio (94); Rhode Island (99); Texas (130); Wisconsin (48).

BIONOMICs.- The females of this species are known to enter dwellings at night to feed on humans and then retire to secretive daytime resting places which are difficult to find. They will feed on man in the open, even at midday, in bright sunlight. Bang et al. (7) found that the adults rest in dark, extremely moist situations; their more favorite resting site was the shaded bases of cut-grass anci shoreline shrubbery. Engorged females were occasionally found in human dwellings near Reelfoot Lake in Tennessee. This species is readily attracted to the New Jersey light trap and is frequently taken in biting collections. Bang et al. (7) took specimens of Anopheles walkeri in light traps located $1 \mathrm{I} / 2$ to 2 miles from the nearest breeding places. The larvae occur in freshwater marshes containing aquatic vegetation.

MEDICAL IMPORTANCE. - This species has been shown under experimental conditions to be susceptible to infection with Plasmodium vivax (117) and P. falciparum (98). A single wild-caught specimen was found by Bang et al. (6) containing oocysts and a sporozoite infection. They state that the human origin of the parasites was probably indicated since $A$. walkeri prefers to feed on mammals, including man, rather than birds. This species appears to be a potential vector of malaria, but additional investigations will be required to determine its real importance in the transmission of the disease. 


\section{ANopheles (NySSORHYNCHUS) ALBIMANUS Wiedemann}

Anopheles albimanus Wiedemann, 1821, Dipt. Exot., p. 10.

Adult Female.-Medium-sized species. Head: Proboscis long, black, the labellae brown. Palpi about as long as proboscis dark, with the apices of segments 2 and 3 and all of segment 5 white (Fig. 41E). Occiput clothed dorsally with whitish erect forked scales, dark-scaled laterally; scales of verrex white; frontal tuft white. Thorax: Integument of scutum dark-gray to dark-
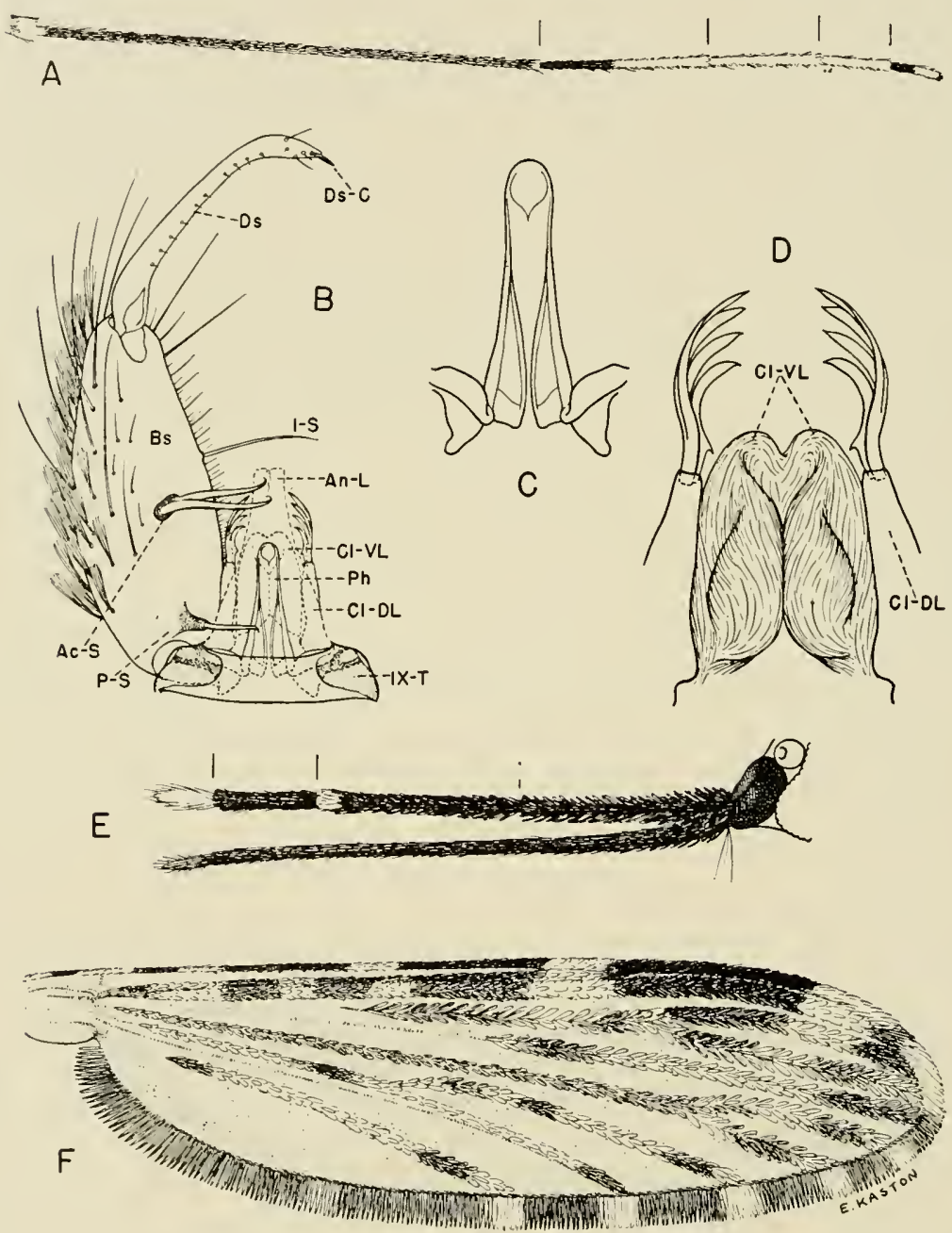

Fig. 41. Anopheles albimanus Wiedemann. A. Hind tarsus. B, Male terminalia.

C, Phallosome. D, Claspettes. E, Palp and proboscis of female. F, Wing. 
brown, with a pair of small submedian black spots slightly posterior to middle and a postero-median black spot on prescutellar space; vestiture of scutum consisting of gray scales and pale setae, the setae more numerous laterally. Abdomen: Integument dark-brown to black, clothed dorsally with long setae and pale scales, the scales more numerous on middle of the segments; ovate dark scales present laterally on segments III to VII. Legs: Femora dark, sparsely speckled with pale scales, II and III each with a palescaled subapical spot on one side. Tibiae dark, tipped with white. Tarsi marked as follows: Fore-tarsus with segment 1 narrowly white-ringed at apex, 2 and 3 with apical halves white, 4 and 5 entirely dark; mid-tarsus with segments 1 and 2 narrowly white-ringed at apices, 3 to 5 entirely dark; hindtarsus (Fig. 41A) with segment 1 entirely dark except for a few white scales at apex, segment 2 usually with basal half dark and apical half white, 3 and 4 entirelv white, 5 dark basallv and white apically. Wing: Scales black and yellowish-white, arranged on the veins in contrasting lines and spots (Fig. $41 F)$. Halter: Knob of halter dark-scaled.

ADULT MALE. - Coloration similar to that of female. TERminalia (Fig. 41). - Ninth tergite (IX-T) sclerotized laterally, membranous dorsally; lobes absent. Tenth sternite absent; anal lobe (An-L) sub-triangular, not spiculate, lateral margins darkly pigmented. Phallosome $(\mathrm{Ph})$ cylindrical, furcate at base; apex rounded and lacking leaflets. Ventral lobes of claspette (Cl-VL) fleshy, fused on the mid-line, each rounded apically and with a large, ovoid lobe ventrally. Dorsal lobes of claspette (Cl-DL) widely separated, cylindrical, apex of each with three or four sickle-shaped filaments set close together. Basistyle (Bs) conical, about two and one-half times as long as broad, bluntly rounded apically, clothed with scales and a few long setae; one stout, recurved parabasal spine (P-S), two stout, recurved accessory spines (Ac-S) and one slender, straight internal spine (I-S) present. Dististyle (Ds) a little shorter than the basistyle and without spicules (minute non-papillated hairs), curved, narrower medially, bluntly pointed at tip; terminal claw (Ds-C) bluntly pointed.

LARVA (Fig. 42).-Head: Inner clypeal hairs (2) and outer clypeal hairs (3) barbed, basal tubercles nearly equidistant; postclypeal (4) single or branched apically, extending slightly beyond bases of inner clypeals; frontal hairs (5-7) large, plumose; sutural (8) usually 2- to 4-branched; transsutural (9) usuallv 2- to 5-branched. Antenna spinose (spines on inner margin at middle of shaft much coarser); terminal hair (10) 2- to 4-branched beyond middle, longer than the sabres; antennal tuft (11) short, inserted on basal third of shaft, 2- to 4-branched. Thorax: Prothoracic dorsal submedian hairs (1-3) arising from a common sclerotized base; inner hair (1) and middle hair (2) with many branches on a stout shaft, inner hair (1) about half as long as middle hair (2); outer hair (3) small, simple. Prothoracic pleural group (9-12) of four hairs; hairs 10 and 12 long, simple; hair 9 long, with several lateral branches; hair 11 about one-half as long as hair 10 or 12, bifid or trifid. Mesothoracic pleural grouv (9-12) of four hairs; hairs 9 and 10 long, simple; hair 11 minute; hair 12 short (about one-fourth 
the length of 9 or 10), simple; Metathoracic pleural group (9-12) of four hairs; hair 9 long, with several weak lateral branches; hair 10 long, simple; hair 11 minute; hair 12 short, usually bifid. Metathoracic palmate hair (1) with numerous slender transparent leaflets. Abdomen: Submedian dorsal hair (0) present on segments II to VII, small, branched. Palmate hair (1) well-developed on segments I to VII (smaller on segment I and VII), individual leaflets slender, with smooth margins. Antepalmate hair (2) long,
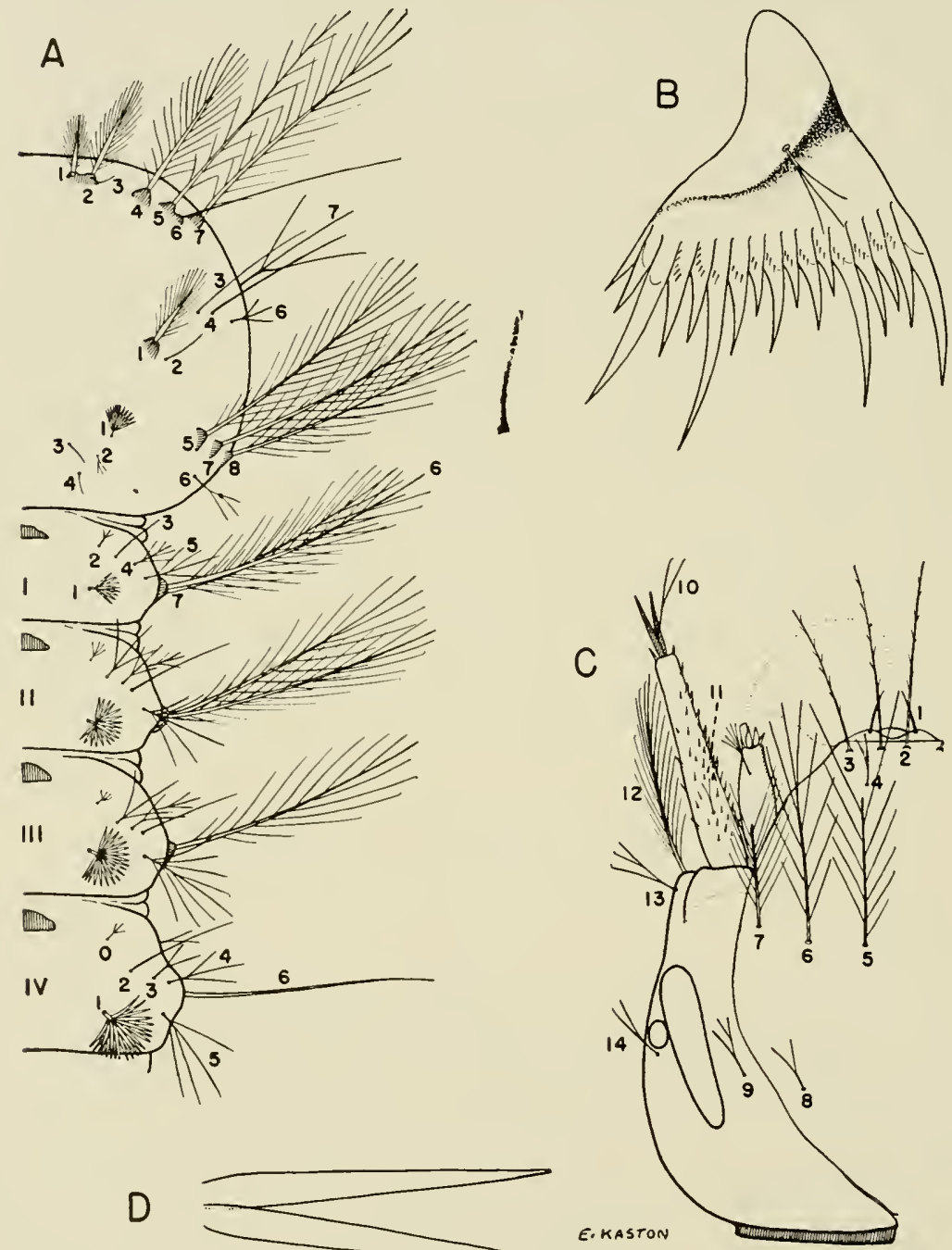

Fig. 42. Larva of Anopheles albimanus Wiedemann. A, Thorax and abdomen. B, Pecten. C, Head. D, Leaflets of palmate hair. 
simple on segments IV and V. Upper lateral hair (6) on segments I to III long, plumose. Pecten as illustrated.

Distribution.-Along the Rio Grande River in Texas; Mexico, the West Indies; Central America; and South America, south to Ecuador, Colombia, and Venezuela. A collection, consisting of 131 adult specimens, was taken at Key West, Florida by Dr. G. N. MacDonnell during 1904, and reported by King (91). One fourth instar larva was colletced by Ernest Erb at Boca Raton, Florida, during 1944 and reported by Carpenter et al. (35).

BIONOMICS. - The females are avid feeders on man and domestic animals. The adults are nocturnal and commonly invade houses in large numbers; however, it has been observed that they usually leave houses before sunrise. Larvae may occur in almost any collection of sunlit fresh or brackish water, whether it be fairly pure or stagnant. A surface matied with vegetation furnishes ideal conditions for its breeding in lakes and canals. It is occasionally found breeding in artificial containers.

MEDICAL IMPORTANCE.-Various workers have experimentally demonstrated the infectibility of $A$. albimanus with Plasmodium vivax and $P$. falciparum and it has been found infected in nature on several occasions. This species is regarded as the principal lowland vector of malaria in Central America and the West Indies.

\section{Tribe Megarhinini}

The tribe Megarhinini consists of large, usually brilliant-colored diurnal, non-bloodsucking mosquitoes. The tribe includes the single genus Megarhinus.

\section{Genus Megarhinus Robineau-Desvoidy}

Megarhinus Robineau-Desvoidy, 1827, Mem. Soc. d'Hist. Nat. 3:403.

The adults of the genus Megarhinus are daytime fliers and feed on plant juices. The larvae are found in tree holes and occasionally in artificial water containers where they feed upon other mosquitoes occupying the same breeding places. The genus is primarily a tropical one, but its distribution extends into the north temperate zone.

Edwards (55) divided the genus Megarhinus into three groups, A, B, and C. Groups $\mathrm{A}$ and $\mathrm{B}$ occur in the Americas, but group $\mathrm{C}$ is confined to the Old World. Two species, $M$. rutilus and $M$. septentrionalis, occur in the United States.

SAlient Characters.-Adult: Clypeus broader than long, front margin slightly trilobed. Proboscis rigid on basal half, flexible and strongly curved downward and backward on apical half. Palpi of females vary in length in different species from only about one-sixth to about two-thirds the length of the proboscis. Scutellum evenly rounded, with a continuous row of setae. All species large, usually with flat scales of metallic luster on head, thorax, ahdomen, and legs. Squamae without a fringe of hairs. Spiracular bristles present; 
posterior pronotal and postspiracular bristles absent. Wing scales sparse, short and broad. Second marginal cell of wing much shorter than its stem; a slight emargination present on hind margin of wing opposite vein 5.2, and a submarginal V-shaped thickening of the membrane between 5.1 and 5.2. Crossveins as in Anophelini, bent at right angles. Male Terminalia: Tenth sternite with not more than two or three terminal teeth. Phallosome consisting of two elongated plates, heavily sclerotized. Basistyle conical; basal lobe present, bearing spines; apical lobe absent. Claspette absent. Dististyle long, slender, not swollen medially. Larva: Larvae very large and predacious. Mouth brushes of strong, curved, flattened setae, about 10 in number, not serrated but with hooked tips. Head almost square; frontoclypeus divided by a transverse suture into an anterior part and a much larger posterior part which comprises most of the dorsal head surface. Mentum much broader than long. Antenna smooth, cylindrical, with a minute tuft beyond middle. Hairs of thorax and abdomen arising from heavily sclerotized plates; comb and pecten entirely absent. The fusion of the sclerotic plates of the upper and lower lateral hairs on abdominal segment VIII forms a large plate bearing strong serrated hairs in the position generally occupied by a comb in other mosquitoes.

\section{KeY TO THE SPECIES}

ADULT MALES

Fore-tarsus entirely dark scaled septentrionalis D. and K.. p. 85 Fore-tarsus with segment 2 and the basal portion of segment 3 white-scaled on one side rutilus Coq., p. 84

\section{Megarhinus rutilus Coquillett}

Megarhinus rutila Coquillett, 1896, Can. Ent., 28:44.

ADULT FEMALE.-Indistinguishable from $M$. septentrionalis.

ADULT MALE.-Similar to male of $M$. septentrionalis, but with segment 2 and basal portion of segment 3 of fore- and mid-tarsi white on one side. TERMiNALIA. The male terminalia of this species appears to be indistinguishable from that of $M$. septentrionalis (Fig. 43).

LARVA.-Unknown.

Distribution.-Florida and Georgia (96); South Carolina (4th Sv. C. Med. Lab. records, 1944, unpublished).

BIONOMICS.-Megarhinus rutilus breeds in rot cavities of trees, but it is a rare species and very little is known of its biology.

\section{Megarhinus septentrionalis Dyar and Knab}

Megarhimus seplentrionalis Dyar and Knab, 1907, Jour. N. Y. Ent. Soc., 15:12.

adult female.-Very large brilliantly ornamented species. Head: Proboscis long, black, tapered, the apical half strongly down-curved. Palpi about twothirds as long as proboscis, metallic dark blue to violet, with golden-yellow 
scales laterally on second and third segments. Occiput clothed dorsally with flat metallic blue scales, the eye margins and lateral portions of occiput with flat, silver to golden-yellow scales. Thorax: Anterior pronotal lobe covered with broad flat scales, those on dorsal portion brilliant metallic blue. Integument of scutum dark brown, with a narrow median longitudinal stripe and sides primarily gold scaled, and with blue reflections when viewed from certain angles; a broad longitudinal submedian stripe of fine dark purplish-brown scales present on either side between the median stripe and lateral pale-scaled areas. Pleurae and coxae shingled with flat golden-yellow scales. Abdomen: Abdomen primarily covered dorsally with metallic blue-green scales, laterally with goldenyellow scales. Legs: Femora golden-yellow on inner surfaces; tibiae primarily clothed with dark purple scales; fore- and mid-tarsi with apex of segment 1 and all of segments 2, 3 and 4 white; hind-tarsus with apex of segment 3, all of segment 4 and all but tip of segment 5 white. Wing: Scales rather broad, dark purple, sparse.

ADULT MALE.-Coloration similar to that of female but the fore- and midtarsi with all segments dark purple, the hind-tarsus with the fourth and occasionally the base or all of the fifth segment white. TERMinalia (Fig. 43). Ninth tergite (IX-T) a strongly convex band extending considerably dorsad of underlying structures; lobes (IXT-L) apico-lateral in position, poorly defined and broadly connected, each bearing numerous strong setae. Tenth sternite (X-S) prominent, heavily sclerotized apically. Phallosome (Ph) bottle-shaped, consisting of two separate lateral plates connected only subbasally on the dorsum by a narrow sclerotized bridge; apical neck-like portion slender, nearly as long as broadened base, heavily sclerotized, pointed, with a bilateral row of short teeth directed ventrally. Claspette absent. Basistyle (Bs) conical, a little more than twice as long as basal width, clothed with scales and long setae; basal lobe (B-L) prominent, rounded, bearing three or four large spines and several smaller ones; apical lobe absent. Dististyle (Ds) nearly as long as basistyle, slender, sides nearly parallel, rounded apically; clan. (Ds-C) slender, inserted a little before apex.

LARVA. (Fig. 44).--Large larva. Head quadrate, about as long as broad. Antenna smooth, about one-third as long as the head; antennal tuft short, multiple, inserted at outer fourth of shaft, preceded by a pair of single hairs at outer third. Mouth brusbes consisting of about ten stout, closely appressed. prehensile, smooth, curved rods. Head hairs: Preantemal (A), lower (B), and upper (C) single and of about equal length; postclypeal (d) short, multiple; (hairs A, B, C, and $d$ arranged more or less in a row on the anterolateral region of the clypeus); sutural (e) absent; trans-sutural (f) and supraorbital branched beyond bases. Abdomen with numerous stout, barbed hairs arising from sclerotized bases. Eighth abdominal segment without comb, but with a large sclerotized lateral plate bearing two long barbed hairs and two shorter weak hairs distally branched. Siphon one and one-half to two times as long as basal width; pecten absent; subventral tuft multiple, barbed, inserted near base of siphon. Anal segment about as long as wide; completely ringed by the dorsal plate; postero-lateral margin of dorsal plate fringed with a row 


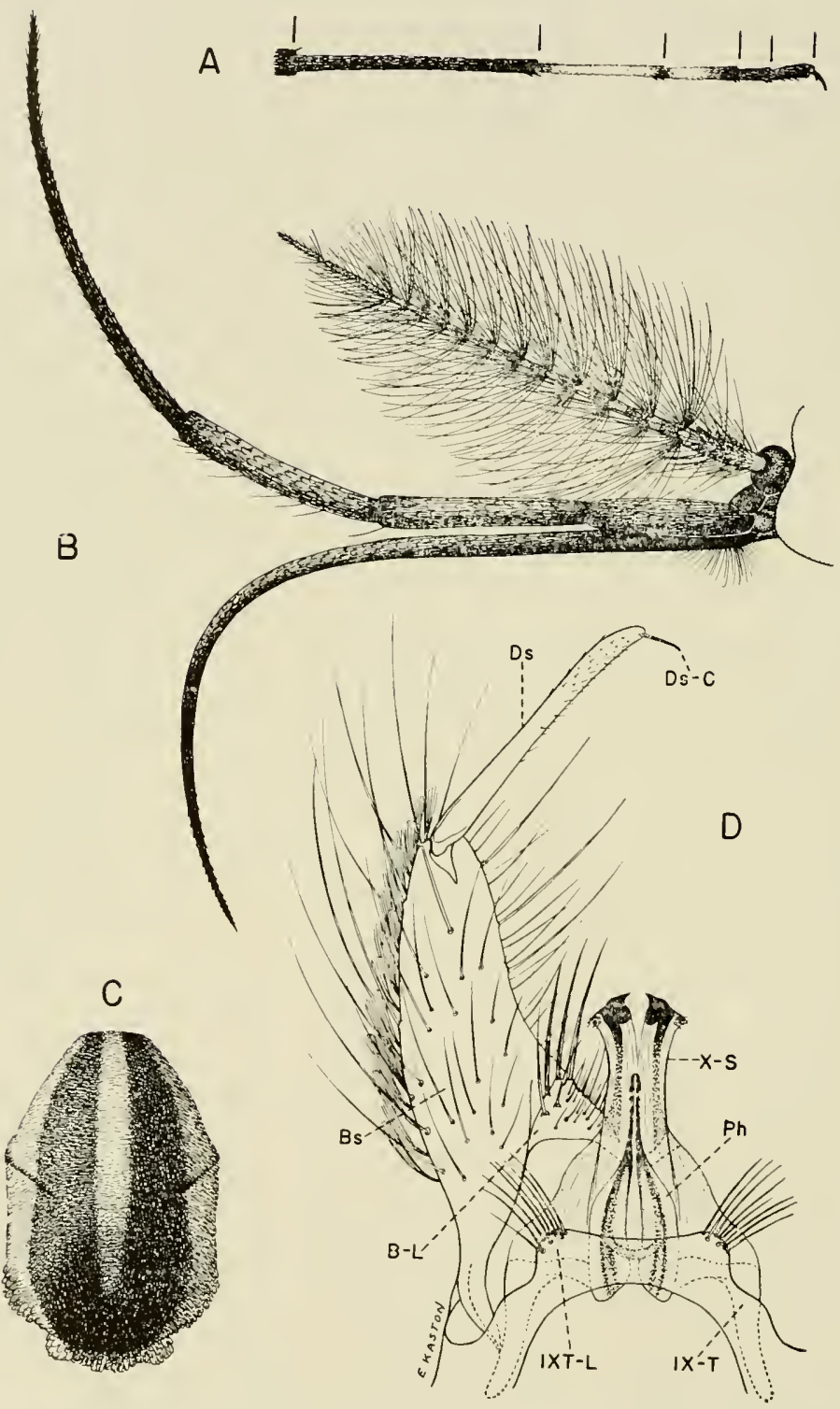

Fig. 43. Megarhinus rutilus Coquillett and $M$. septentrionalis Dyar and Knab. A, Fore tarsus of $M$. rutilus. B. Head of $M$. septentrionalis (male). C, Scutum and scutellum of $M$. septentrionalis. D, Male terminalia of $M$. septentrionalis. 
of inter-mixed short and long spines; lateral hair stout, barbed, inserted on distal margin of dorsal plate; dorsal brush consisting of a lower caudal tuft of about 4 branches and an upper caudal tuft of about 8 branches on either side; ventral brush well-developed, with individual hairs, feathered, confined to the barred area; gills 4 , short, bulbous.

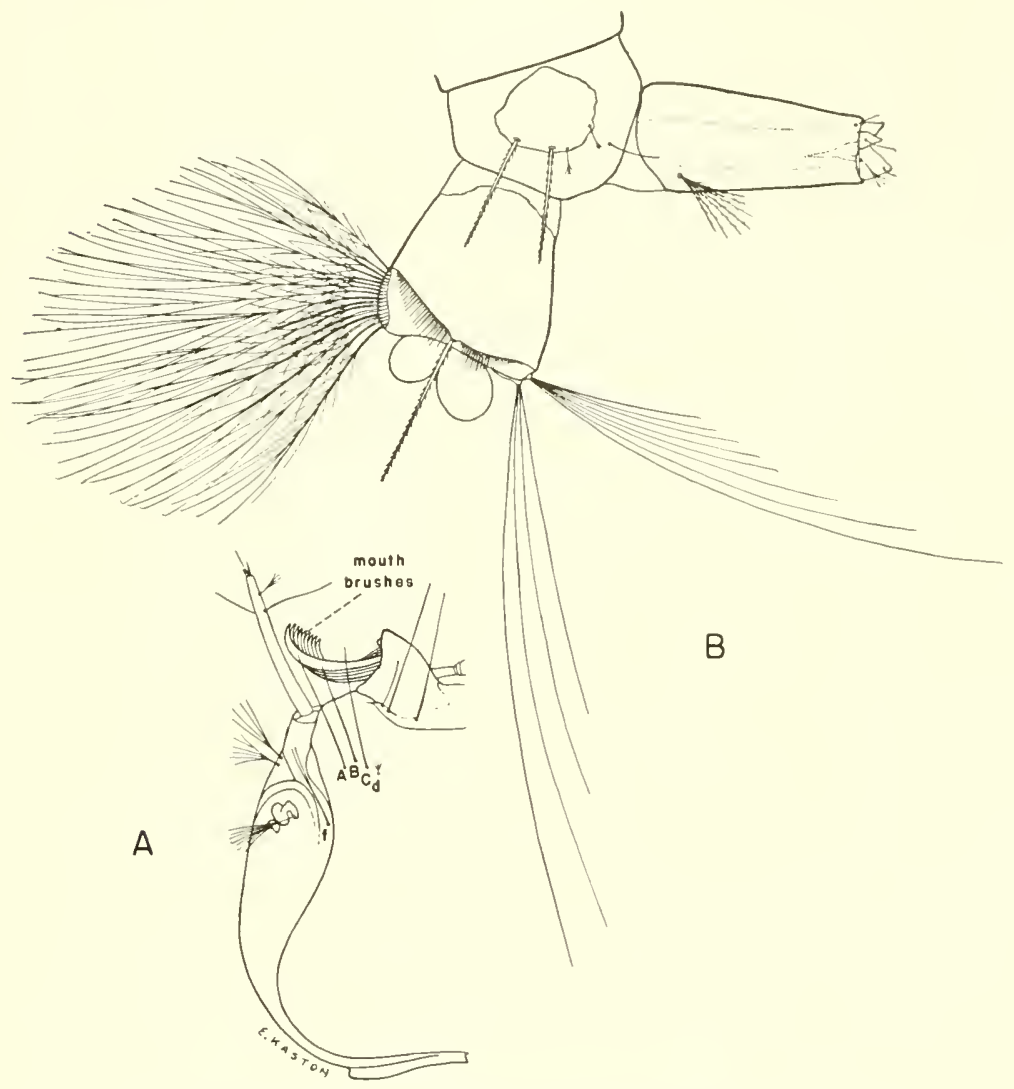

Fig. 44. Larva of Megarhinus septentrionalis Dyar and Knab.

A, Head. B, Terminal segments.

Distribution.-Eastern United States, north to New Jersey, west to Kansas and Texas. Southern States: Alabama (96); Arkansas (30); Georgia (52); Kentucky (140); Louisiana (96); Mississippi, Missouri and North Carolina (52); South Carolina (64); Tennessee and Virginia (52). Other States: District of Columbia (52); Kansas (79); Maryland (52); New Jersey (164); Oklahoma (159); Texas (108); West Virginia (52).

BIONOMics.-The adults fly by day and feed on nectar in flowers. The larvae are found principally in rot cavities in trees and occasionally in water 
in artificial wooden receptacles and in rock holes. The larvae are predacious, feeding on mosquito larvae and other aquatic animals with which they are associated in their breeding places.

\section{Tribe Culicini}

The tribe Culicini is represented in the Southern United States by nine genera, most of which are divided into subgenera. It is by far the largest of the three tribes of Culicinae, both in number of species and individuals.

SAliEnt Characters.-Adult: Clypeus longer than broad, rounded anteriorly. Proboscis slender, flexible, not hooked. Thorax arched above. Scutellum trilobed, each lobe bearing a distinct group of setae. Abdomen clothed with imbricate scales. Cross-vein 3-4 not bent at right angles, no spur extending basally from angle of vein 3. Wing without V-shaped thickening of membrane between veins 5.1 and 5.2. Second marginal cell about as long as its petiole (much shorter in Uranotaenia). Larva: Head rarely rotatable. Hairs of body never pinnately branched as in Anophelini. Thorax broad and without eversible organs. Abdomen without float-hairs. Eighth segment with a siphon. Lateral comb always present on eighth segment.

\section{Keys to the Genera}

ADULTS

1. 2nd marginal cell short, less than half as long as its petiole

2nd marginal cell at least as long as its petiole

Uranotaenia L. A., p. 100

2. Postnotum with a tuft of setae; squamae without a fringe of hairs

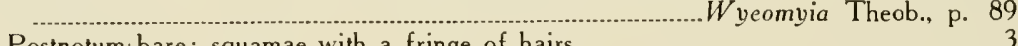

3. Spiracular bristles present

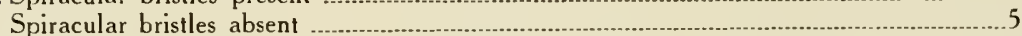

4. Postspiracular bristles present; tip of abdomen pointed...-..Psorophora R. D., p. 130

Postspiracular bristles absent; tip of abdomen blunt .......................... Fuliseta Felt, p. 107

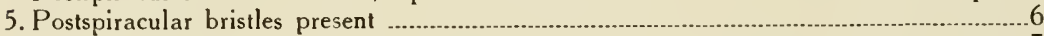

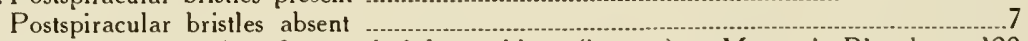

6. Wing scales very broad; tip of abdomen blunt (in part) .... Mansonia Blanch., p. 120

Wing scales narrow (rarely moderately broad); tip of abdomen pointed Aedes Meigen, p. 165

7. Antennae much longer than proboscis, first flagellar segment longer than the next three combined Deinocerites Theob., p. 274

Antennae not longer than proboscis or only slightly so, first flagellar segment about as long as each succeeding segment

8. Scutum bicolorous, with delicate longitudinal lines of white scales; penultimate segment of fore tarsi very short, only about as long as wide

Orthopodomyia Theob., p. 114

Scułum unicolorous, not marked with white longitudinal lines; penultimate segment of fore tarsi much longer than wide

9. Wing scales very broad, brown and white mixed ................................... 120

Wing scales narrow, uniformly dark Culex Linn., p. 237

\section{LARVAE (FOURTH INSTAR)}

1. Distal half of siphon strongly attentuated, adapted for piercing roots of aquatic plants. 
Siphon cylindrical or fusiform, not adapted for piercing roots of aquatic plants ..... . 2

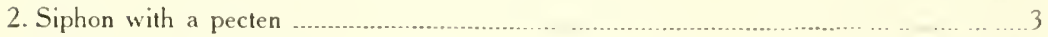

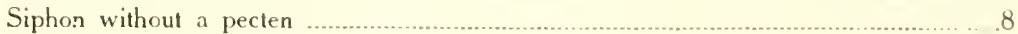

3. Lower and upper head hairs (B, C) very stout, spine-like, single; head longer than wide ............................. Uranotaenia L. A. 100

Lower and upper head hairs (B, C) slender, not spine-like, single, double or

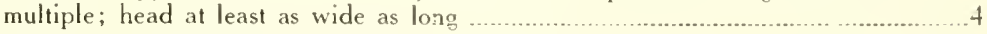

4. Head with a prominent triangular pouch on each side; anal segment with divided dorsal and venträ sclerotic plates, membranous laterally

Deinocerites Theob., p. 274

Head without a prominent trangular pouch on each side; anal segment completely ringed by the dorsal plate or membranous only ventrally ............................ 5

5. Siphon with a pair of large basal subventral hair tufts, or, if lufts are sinall,

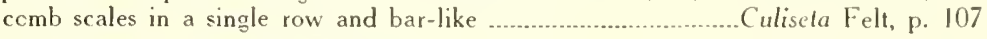

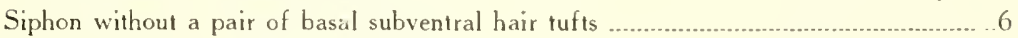

6. Siphon with several pairs of subventral hair tufts or single hairs...... Culex Linn., p. 237

Siphon with one median pair of subventral hair tufts (sometimes vestigial) or one pair of single hairs

7. Anal segment completely ringed by the dorsal plate and pierced on the midventral line by tufts of ventral brush _....... Psorophora R. D.. p.130

Anal segment not completely ringed by the dorsal plate, or if ringed, not pierced on midventral line by tufts of ventral brush …..............edes Meigen, p. 165

8. Anal segment with prominent median ventral brush consisting of a close-set row of tufts; comb with a row of short pointed scales and a second row of much longer pointed scales ............................................... 114

Anal segment without a median ventral brush, but with a pair of ventrolateral tufts; comb scales in a single row ...............................W yeomyia Theob., p. 89

\section{Genus Wyeomyia Theobald ${ }^{1}$}

Wyeomyia Theobald, 1901, Mon. Culic,, 2:267.

The genus Wyeomyia is confined mostly to the tropical and subtropical regions of the New World. One species, $W$. smithii, extends into the north temperate zone in North America. The larvae are found in water collections in plants. The adults are mostly diurnal; the females are known to bite warmblooded animals occasionally. The genus is represented in the United States by three species.

SALIENT CHARACTERs.-Adult: Postnotum always has a tuft of setae. Palpi short. Palpi and antennae of males and females are similar; however, the plumes are sometimes better developed in males than in females. Thorax dull colored, never conspicuously ornamented. Spiracular bristles always present. Abdomen blunt tipped. Squama without fringe, one to three small hairs rarely present. Male Terminalia: Basistyle and dististyle variable; dististyle usually with swollen head. Phallosome simple. Tenth sternites with 2 or 3 terminal teeth. Larva: Comb scales of eighth segment usually in a single regular row. Pecten absent or represented by a few teeth. Hairs of siphon single or branched, arising from all aspects, in no definite pattern. Ventral brush represented by bilateral ventro-lateral tufts.

1 See Edwards (1922) and Lane and Cerqueira (1942) for synonymy. 


\section{KeYs to THE SPECIES}

\section{ADULT FEMALES}

1. Scales of anterior pronotal lobe silvery-white; hind tarsal segments basally streaked with white on under side (breeds in bromeliads)

vanduzeei D. and K., p. 96

Scales of anterior pronotal lobe dark, with purplish reflection; hind tarsal segments either basally streaked with white on under side or entirely dark ..................2

2. Hind tarsal segments entirely dark (breeds in pitcher plants) ......smithii (Coq.), p. 9l

Hind tarsal segments (at least I to 3 ) each with a basal streak or spot of white scales on under side (breeds in bromeliads) mitchellii (Theob.), p. 90

\section{MALE TERMINALIA}

1. Basal stem-like portion of dististyle slender, longer than the apical capitate portion mitchellii (Theob.), p. 92

Basal stem-like portion of dististyle stout, shorter than the apical capitate portion

2. The branches of the capitate head about equal in length

vanduzeei D. and K., p. 97

The branches of the capitate head not equal in length, the outer being much shorter smithii (Coq.), p. 94

\section{LARVAE (FOURTH INSTAR)}

I. Lower head hair (B) double, upper head hair (C) multiple; ventro-lateral tufts of anal segment short, each with about 12 subequal branches mitchellii (Theob.), p. 93

Lower and upper head hairs ( $B$ and $C$ ) single; ventro-lateral tufts of anal segment each about 3 to 6 -branched

2. Siphon 4 or $5: 1$, with long single hairs on all aspects; ventro-lateral tufts of anal

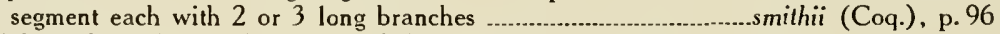

Siphon about $6: 1$, with several of the hairs double or triple; ventro-lateral tufts of anal segment each with 1 or 2 long and 3 or 4 shorter branches vanduzeei D. and K., p. 97

\section{WyEOMYiA (WYEOMYIA) MITCHELLII (Theobald)}

Dendromyia mitchellii Theobald, 1905, Mosq. or Cul. of Jamaica, 37.

Wyeomyia (Wyeomyia) mitchellii Lane and Cerqueira, 1942, Arq. de Zool., 3:556 (synonymy given).

adult female.-Very small species. Head: Proboscis long, black; palpi very short, dark. Occiput clothed dorsally with dark broad flat scales with metallic blue-green reflection; the vertex with broad silvery-white scales. Thorax: Anterior pronotal lobe covered with broad appressed dark violaceous scales; scales of posterior pronotum, pleurae and coxae broad, appressed, silvery white, often faintly tinted with blue. Scutum densely matted with large elliptical dark scales, appearing brown to metallic blue-green in different lights. Abdomen: Abdomen usually somewhat laterally compressed, the dorsum dark and the venter yellowish-white to silvery white, the dark and white scales separated laterally in a straight longitudinal line; the scales of the dorsum dark brown with coppery to metallic blue-green reflection. Legs: Knee spots absent. Femora and tibiae dark scaled on outer surfaces, with metallic blue-green reflection, the inner surfaces largely pale. Fore-tarsus entirely dark; 
mid-tarsus with segments 3 and 4 (occasionally 5) and apex of 2 white on one side; each segment of hind tarsus basally streaked with white on inner surface (white markings may be obsolete or absent on the more distal segments). Wing: Scales narrow, dark.

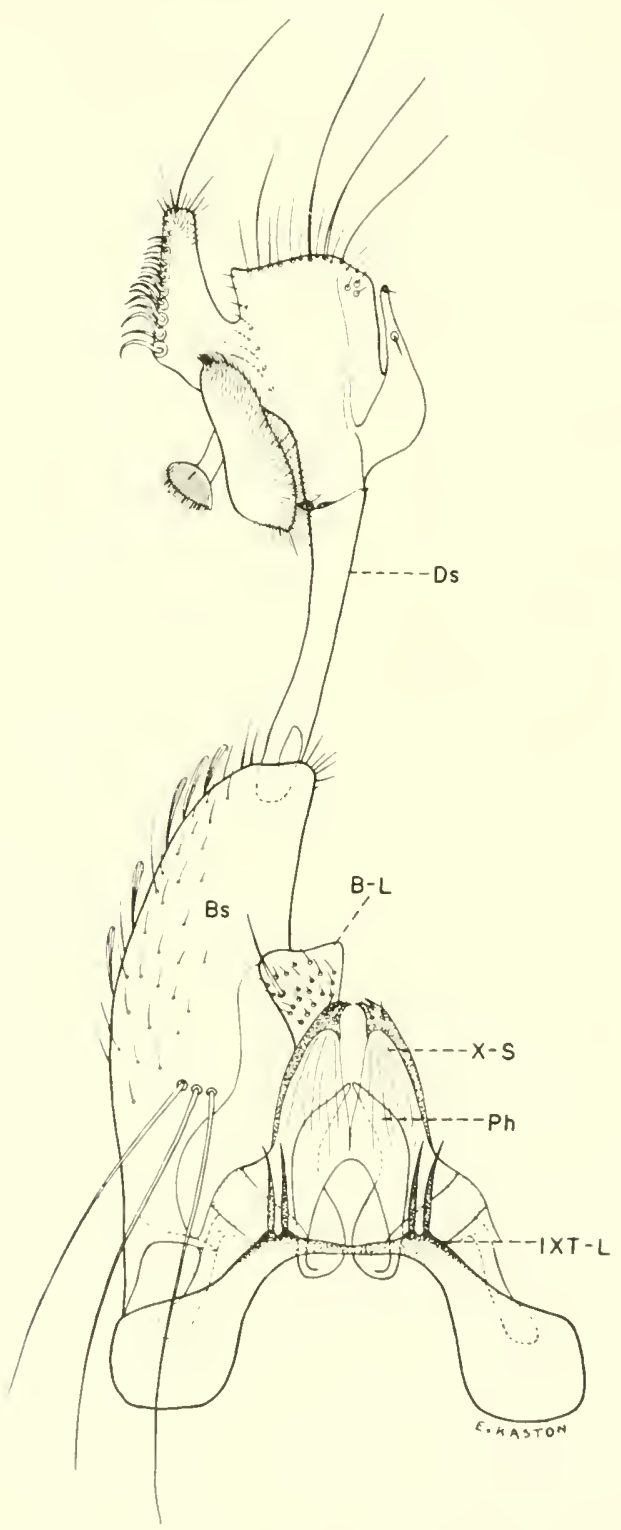

Fig. 45. Male terminalia of Weomyia mitchellii (Theobald). 
ADULT MALE.-Coloration, palpi and antennae similar to those of female. TERMiNALia (Fig. 45). Lobes of ninth tergite (IXT-L) small, sclerotized, widely separated, connected by a narrow, transverse band; each lobe arined with two short, stout spines. Tenth sternite (X-S) moderately sclerotized, with two or three small subapical setae. Phallosome (Ph) about two-thirds as long as broad, narrower at base, somewhat broadened hood-like toward apex, open ventrally, closed dorsally. Claspette absent. Basistyle (Bs) nearly three times as long as basal width, curved, a little narrower beyond middle; general vestiture consisting of scales and short setae; three very long, strong setae arising dorsally near base. Basal lobe (B-L) sub-triangular, bearing a spine and numerous small setae (this structure is probably not a true basal lobe, but a modification of the interbasal fold); apical lobe absent. Dististyle (Ds) as long as basi-

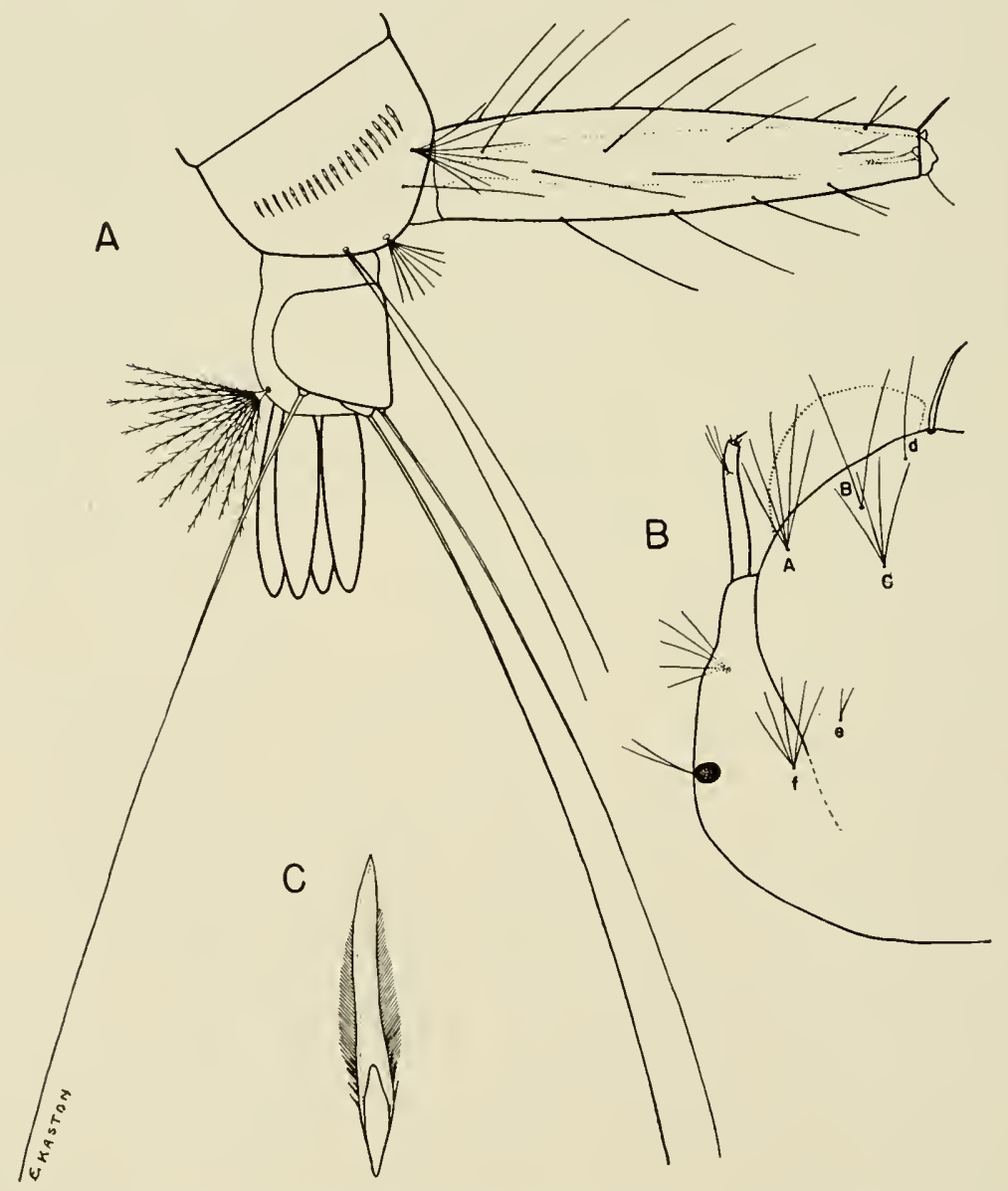

Fig. 46. Larva of Wyeomyia mitchellii (Theobald). A, Terminal segments. B, Head. C, Comb scale. 
style and consisting of a long, slender stem and a greatly enlarged, apical head; the head about two-thirds as long as the stem. Structural details of head of dististyle as illustrated.

LARva. (Fig. 46).-Head nearly as long as broad. Antenna small, about one-third as long as head, smooth; antennal tuft 3-branched, inserted on outer third of shaft, reaching beyond tip. Head hairs: Preantennal (A) usually 3 to 5-branched, about as long as antenna; lower (B) double; upper (C) usually 3 to 4-branched; postclypeal (d) single, about as long as upper (C); sutural (e) usually double; trans-sutural (f) multiple; supraorbital usually double. Upper lateral abdominal hairs long, usually triple on segment I and double on II to VI. Comb of eighth segment of numerous scales, variable in number, arranged in a single row, the scales becoming progressively somewhat smaller ventrally; individual scale elongate with numerous fine lateral spinules extending nearly to apex. Siphon about five times as long as wide; pecten absent; siphon with long single hairs on all aspects and a few small 2-branched tufts apically; dorsal preapical spine stout, about as long as apical width of siphon. Anal segment as long as wide, the dorsal plate reaching well down the sides; lateral hair very long, single; dorsal brush consisting of a long, upper and lower caudal hair on either side; a ventro-lateral tuft of about 12 short, subequal branches on either side; gills 4 , about as long as the segment, blunt.

Distribution.- Southern Florida (52); Central America and the West Indies.

BIONOMICS.- The females are frequently encountered in the jungle near their breeding places, and will occasionally feed on man. The larvae are found in water collections at the bases of the leaves of epiphytic Bromeliaceae. Breeding occurs throughout the year when the host plants contain water.

\section{WyeOMYia (WyeOMYia) SMITHI (Coquillett)}

Acdes smithii Coquillett, 1901, Can. Ent., 33:260.

Wyeomyia (Wyeomyia) smithi, Lane and Cerqueira, 1942, Arq. de Zool., 3:553 (synonymy given).

adult female.-Very small species. Head: Proboscis long, black; palpi very short, dark. Occiput clothed dorsally with dark broad flat scales with metallic blue-green reflection; the vertex with broad silvery-white scales. Scales of gena broad, appressed, silvery-white. Thorax: Anterior pronotal lobe covered with broad appressed violaceous scales; scales of posterior pronotum, pleurae and coxae broad, appressed, silvery-white, occasionally faintly tinted with blue. Scutum densely matted with large elliptical dark grey-brown scales, somewhat blue-green in certain lights. Abdomen: Abdomen usually laterally compressed, the dorsum dark and the verter yellowish-white to silvery-white, the dark and white scales separated laterally in a straight longitudinal line; the scales of the dorsum dark brown, with coppery to metallic blue-green reflection. Legs: Knee spots absent. Femora and tibiac dark scaled on outer surfaces, with metallic blue-green reflection; inner surfaces largely pale. Hind- and fore-tarsi entirely dark; mid-tarsus with segments 3 and 4 and apex of 2 white on one side. Wing: Scales narrow, dark. 
ADULT MALE.-Coloration, palpi and antennae similar to those of female. TERMinALIA (Fig. 47). Lobes of ninth tergite (IXT-L) short, truncate, moderately sclerotized, separated by about twice the width of one lobe, connected by a narrow, transverse band; each lobe armed with a few stout spines. Tenth sternite (X-S) moderately sclerotized, with two or three small subapical setae. Phallosome (Ph) about two-thirds as broad as long, narrowest at base, gradu-

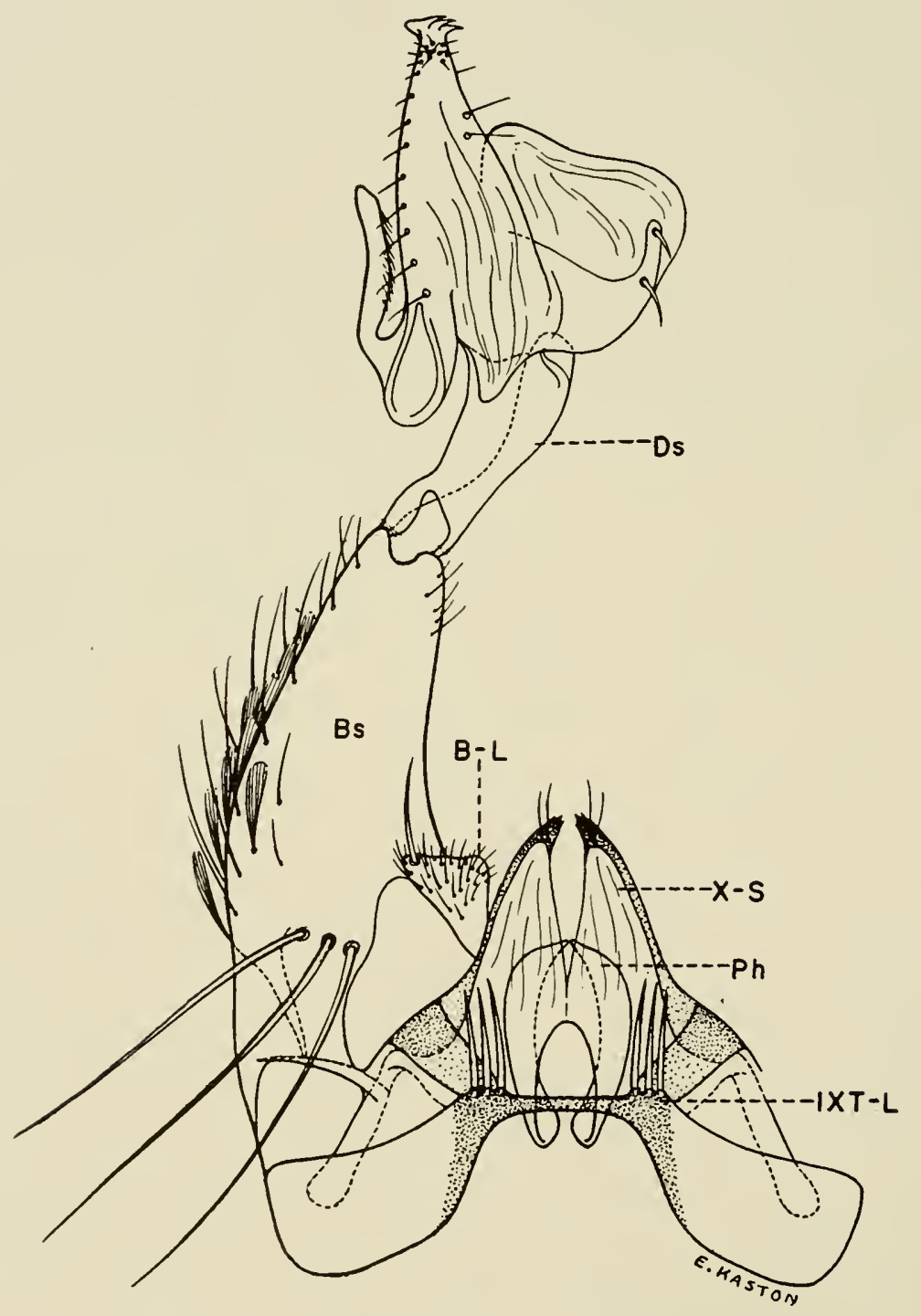

Fig. 47. Male terminalia of $W$ yeomyia smithii (Coquillett). 
ally broadened hood-like toward apex, open ventrally, closed dorsally. Claspette absent. Basistyle (Bs) about three times as long as basal width, curved, narrowed beyond middle; general vestiture consisting of scales and short setae; three very long, strong setae arising dorsally near base. Basal lobe (B-L) triangular, bearing a spine and numerous fine setae (this structure is probably not

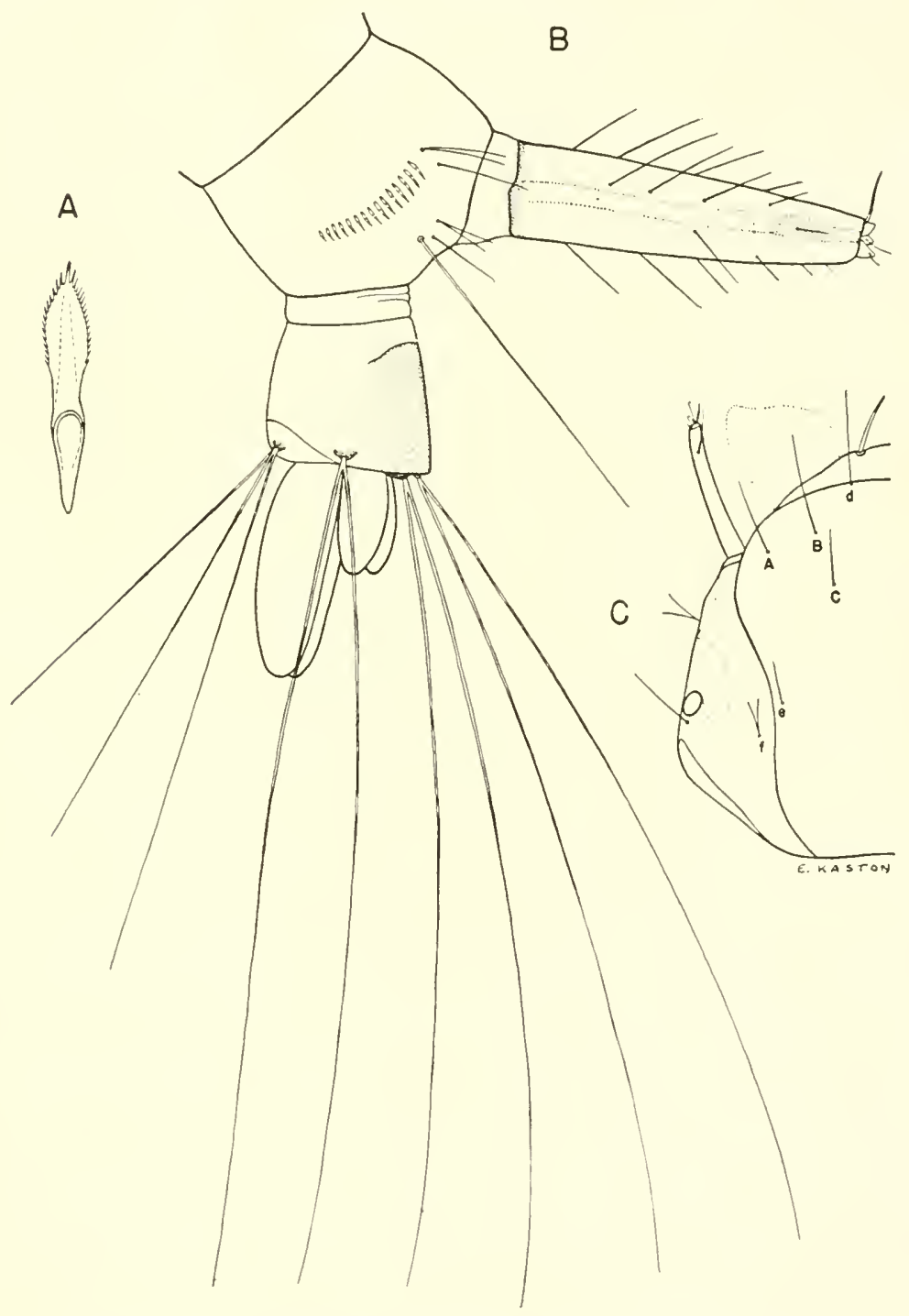

Fig. 48. Larva of Wyeomyia smithii (Coquillett). A, Comb scale.

B. Terminal segments. C, Head. 
a true basal lobe, but a modification of the interbasal fold); apical lobe absent. Dististyle (Ds) nearly as long as basistyle and consisting of a short, stout stem and a greatly enlarged, apical head; head about one and one-fourth times as long as stem and consisting of a large, quadrate inner lobe and a slender hook-like outer filament, both of which arise from the base of a larger, median, cone-shaped portion bearing three mesally directed teeth at apex.

Larva. (Fig. 47).- - Head nearly as long as broad. Antenna small, about one-third as long as head, smooth; antennal tuft represented by a single hair inserted on outer third and extending beyond tip of antenna. Head hairs: Preantennal (A) single, short; lower (B) single, extending beyond preclypeus; upper (C) single, short; postclypeal (d) single, about as long as lower (B), inserted near anterior margin of clypeus; sutural (e) small, single; transsutural (f) double or triple; supraorbital single or double. Upper lateral abdominal hairs long, 2 or more branched on segments I to VI. Comb of eighth segment of numerous scales, variable in number, in a single row, the scales becoming progressively somewhat smaller ventrally; individual scale somewhat variable in shape, usually elongate and evenly fringed with small delicate spinules, those on apex somewhat stronger. Siphon about four to five times as long as basal width, with long single hairs on all aspects; pecten absent; dorsal preapical spine stout, about as long as apical width of siphon. Anal segment about as wide as long; incompletely ringed by the dorsal plate; lateral hair very long, 2 to 3-branched; dorsal brush consisting of a long 2-branched upper and lower caudal tuft on either side; a ventro-lateral tuft of 2 to 3 branches cn either side; gills 4, bulbous, ventral pair as long or longer than the segment, dorsal pair much reduced, about one-third the size of the ventral pair.

Distribution.-Southern Canada and Eastern United States, south to South Carolina and Alabama. Southern States: Alabama, North Carolina, South Carolina (52). Other States: Connecticut (90); District of Columbia and Illinois (52); Maine (90); Maryland and Massachusetts (52); Michigan (88); Minnesota (131); New Hampshire, New Jersey and New York (52); Rhode Island (99); Wisconsin (48).

BIONOMics.- The female of this species is not known to bite humans. The larvae occur in the leaves of the pitcher plant, Sarracenia purpurea. The larvae may be found during any season of the year, even in winter, and are able to withstand freezing and thawing, as the water in the leaves of the plant freezes and thaws during the winter. The eggs are usually deposited by the female in the younger leaves before water collects in them; however, they may be deposited on the sides of older leaves above the water level.

\section{Wyeomyia (Wyeomyia) vanduzeei Dyar and Knab}

Wyeomyia vanduzeci Dyar and Knab, 1906, Proc. Biol. Soc. Wash., 19:138. $W$ yeomyia (Wyeomyia) vanduzeei Lane and Cerqueira, 1942, Arq. de Zool., 3:55C (synonymy given).

Adult female.-Very small species. Head: Proboscis long, black; palpi very short, dark. Occiput clothed dorsally with dark broad flat scales with metaliic blue green reflection; the vertex with broad silvery-white scales. Scales of gena broad, appressed, silvery-white. Thorax: Anterior pronotal lobe, posterior pronotum, pleurae and coxae covered with broad appressed silvery- 
white scales. Scutum densely matted with large elliptical scales appearing coppery-brown to metallic blue-green in different lights. Abdomen: Abdomen usually laterally compressed, the dorsum dark and the venter yellowish-white to silvery-white, the dark and white scales separated laterally in a straight longitudinal line; the scales of the dorsum dark brown, with coppery to metallic blue-green reflection. Legs: Knee spots absent. Femora and tibiae dark scaled on outer surfaces, with metallic blue-green reflections, the inner surfaces largely pale. Fore-tarsus entirely dark; mid-tarsus with segments 3 and 4 and apex of 2 white on one side; each segment of hind tarsus basally streaked with white on inner surface. Wing: Scales narrow, dark.

ADULT MALE.-Coloration, palpi and antennae similar to those of female. TERMINALIA (Fig. 49). Lobes of winth tergite (IXT-L) small, sclerotized, separated by about the width of one lobe; each lobe armed with a few short, stout spines. Tenth sternite (X-S) moderately sclerotized, with a few short, subapical setae. Phallosome (Ph) open ventrally, closed dorsally, about twothirds as long as broad, narrower at base, somewhat broadened hood-like apically; each plate with three small teeth at apex. Claspette absent. Basistyle (Bs) about three times as long as basal width, curved, narrower beyond middle; general vestiture consisting of scales and short setae; three very long, strong setae arising dorsally near base. Basal lobe (B-L) triangular, bearing a spine and numerous small setae (this structure is probably not a true basal lobe, but a modification of the interbasal fold); apical lobe absent. Dististyle (Ds) nearly as long as basistyle and consisting of a short, stout stem and a greatly enlarged apical head; the head about one and one-half times as long as the stem. Structural details of head of dististyle as illustrated.

LARVA. (Fig. 50).- - Head nearly as long as broad. Antenna about onethird as long as head, smooth; antennal tuft represented by a single hair on outer third of shaft, extending beyond tip. Head hairs: Preantennal (A) single, about as long as antenna; lower (B) and upper (C) single; postclypcal (d) single, nearly as long as upper (C); sutural (e) single; trans-sutural (f) 3 to 4-branched; supraorbital single or branched distally. Upper lateral abdominal hairs long, multiple on segments I and II, single on III to VI. Comb of eighth segment of numerous scales in a single row, the scales becoming progressively somewhat smaller ventrally, individual scale elongate, evenly fringed with minute spinules. Siphon about six times as long as basal width; pecten absent; a row of six small 1 to 2-branched tufts dorsally, a larger 2 to 3 branched tuft at basal fourth and two or three small 1 to 2-branched tufts apically. dorsal preapical spine stout, nearly as long as apical width of siphon. Anal segments about as wide as long; dorsal plate extending well down the sides; lateral hair long, double; dorsal brush consisting of a long 2-branched upper and lower caudal tuft on either side; a ventro-lateral tuft with one or two long and three or four shorter hairs on either side; gills 4, swollen, much longer than the segment, blunt at tips.

Distribution.- Southern Florida (52); Central America and the West Indies.

BIONOMICS.-The habits of $W$. vanduzeei are very similar to those of $W$. mitchellii with which it is commonly associated in its breeding places. 


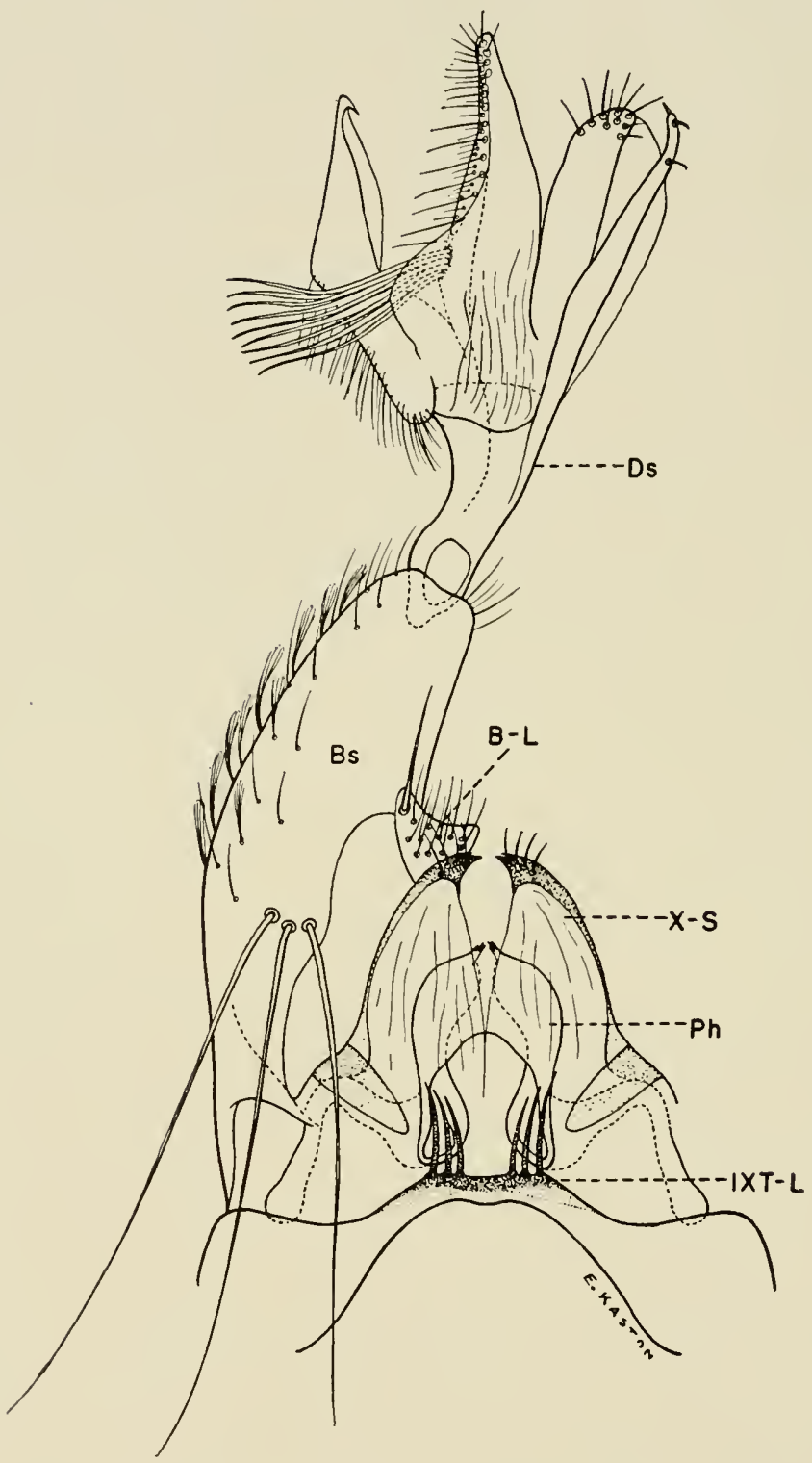

Fig. 49. Male terminalia of Wyeomyia vanduzeei Dyar and Knab. 


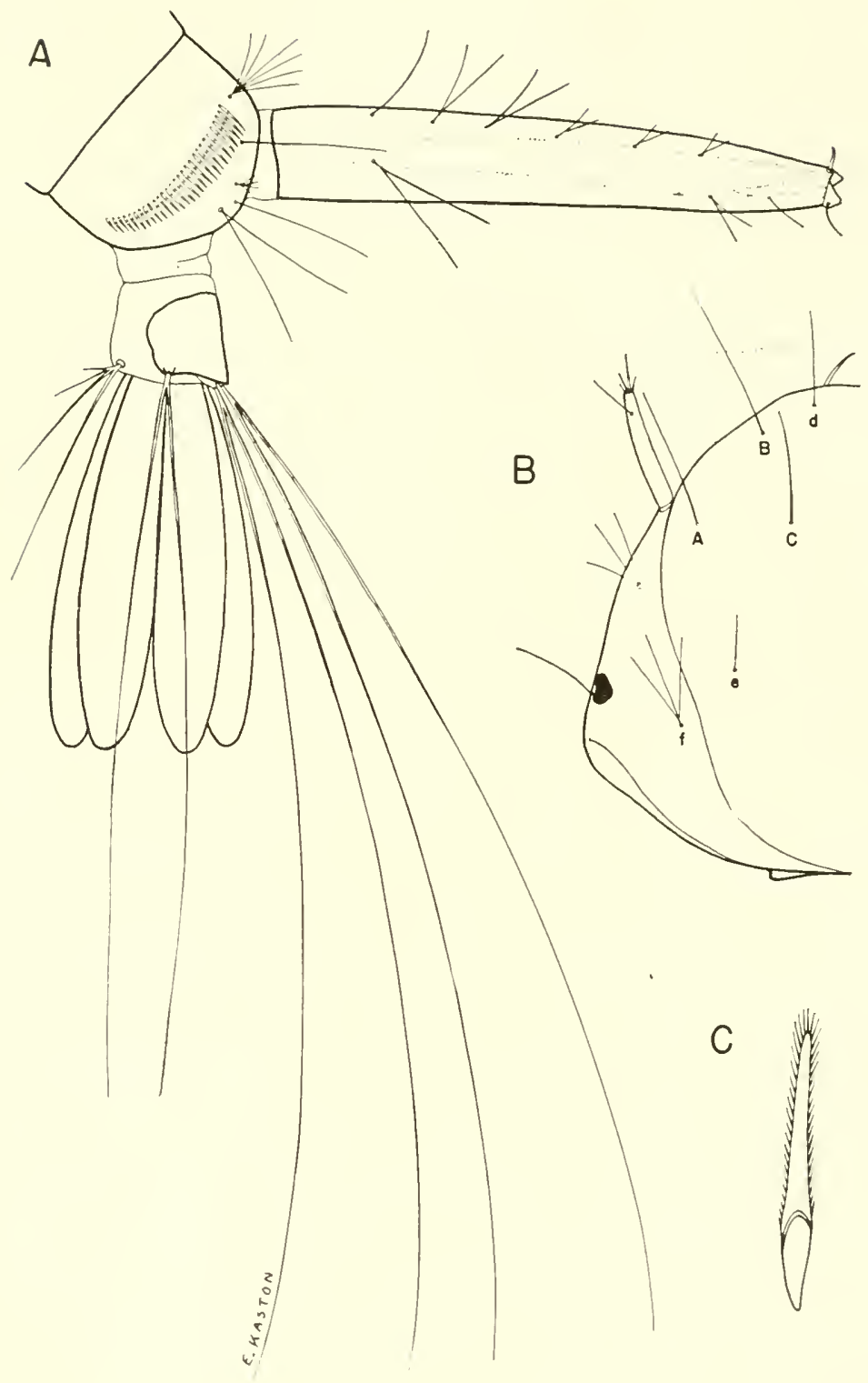

Fig. 50. Larva of Wyeomyia vanduzeei Dyar and Knab. A, Terminal segments. B, Head. C, Comb scales. 


\section{Genus Uranotaenia Lynch Arribalzaga}

Uranotaenia Lynch Arribalzaga, 1891, Rev. Mus. de La Plata, 1:375.

The genus Uranotaenia includes numerous species of small, usually highly ornamented, mosquitoes well distributed throughout the tropical and subtropical regions of the world. The members of this genus are of no economic importance and appear rarely to feed upon man. They are frequently found associated with larvae of Anopheles, with which they may be confused by the collector in the field, since they rest nearly horizontal with the surface of the water. Two species are indigenous in the southern states, $U$. lowii and $U$. sapphirina.

Salient Characters.-Adult: Pulvilli absent. Posterior pronotal and spiracular bristles reduced in numbers; postspiracular bristles absent. Squama without fringe; fork of vein 2 much shorter than its stem; microtrichiae minute, not visible under low magnification. Palpi very short in both sexes. Male Terminalia: Phallosome divided into a pair of heavily sclerotized, strongly toothed plates. Tenth sternite vestigial, not supporting the anal membrane. Claspettes absent. Basistyle short, stout. Larva: Anal segment completely ringed by the dorsal plate. Siphon with pecten and a single pair of subventral tufts. Comb of eighth segment consisting of a single row of scales on the posterior margin of a sclerotized plate. Head usually longer than broad, heavily sclerotized; antenna short, smooth or nearly so. Lower and upper head hairs (B and $C$ ) single, stout, spine-like in the species of the southern United States.

\section{Keys to THe Species}

\section{ADULT FEMALES}

Scutum without a narrow median longitudinal stripe of iridescent blue scales; hind tarsi with apex of 3rd and entire 4th and 5th segments white scaled; abdomen with prominent lateral apical patches of iridescent blue scales on segments III, $\mathrm{V}$, and VI lowii Theob., p. 101

Scutum with a narrow median longitudinal stripe of iridescent blue scales; tarsi entirely dark scaled; abdomen without lateral apical patches of iridescent blue scales ..sapphirina (O. S.), p. 104

\section{MALE TERMINALIA}

Dististyle strongly swollen, only about twice as long as wide, bluntly terminating in a crown of about 15 to 20 short, stout, curved spines; phallosome without a lateral thorn-like process subapically low ii Theob., p. 101

Dististyle not strongly swollen, about 4 or 5 times as long as wide, with apical third tapered and bearing a short subapical spine-like claw preceded by several short setae; phallosome with a large lateral thorn-like process subapically sapphirina (O. S.), p. 104

\section{LARVAE (FOURTH INSTAR)}

Upper lateral hairs of abdominal segment I and II double, the lower, single; prothoracic dorsal submedian hair group composed of about 7 long, heavy, serrated hairs, the longest extending to the bases of the upper head spines (C) lowii Theob., p. 101

Upper lateral hairs of abdominal segments I and II triple, the lower, single; prothoracic dorsal submedian hair group composed of 2 long, heavy serrated hairs 
extending to the bases of the upper head spines (C) and about 8 to 10 short. smooth hairs, less than half as long sapphirina (O. S.), p. 105

\section{URANOTAENIA LOWII Theobald}

Uranotaenia lowii Theobald, 1901, Mon. Culic., 2:339.

Adult female.--Very small species. Head: Proboscis dark scaled, long, broadened apically; palpi very short, dark scaled. Occiput shingled with dark broad appressed scales having a bronzy sheen, margined anteriorly and laterally with broad iridescent pale-blue scales. Thorax: Integument of scutum light brown, darker medianly, and with a darkly pigmented spot laterally just anterior to wing base; vestiture of scutum consisting of dark-brown lanceolate scales on dorsal surface and a row of broad pale-blup iridescent scales on lateral dark spot. Patches of similar iridescent scales present on anterior pronotal lobe, the bases of the coxae and the midportion of the sternopleura. A single spiracular bristle present; postspiracular bristles absent. Abdomen: Tergites dark-brown scaled with a metallic luster; segments III, V, and VI each with a rather large lateral apical patch of pale-blue iridescent scales, and lateral margin of segment II often similarly marked; sternites pale-yellow scaled. Eighth segment blunt, largely retracted within the seventh. Legs: Fore and middle legs entirely dark scaled; hind leg dark scaled except for tarsal segments 4 and 5 and apex of segment 3, which are white scaled (Fig. 51A). Wing: Scales rather broad, dark-brown except for a short row of iridescent pale-blue scales on base of vein 5 and another near base of vein 1; second marginal cell much shorter than its petiole.

ADULT MALE.-Coloration and antennae similar to that of fermale. TERMI NALIA (Fig. 51). Ninth tergite (IX-T) broad, deeply emarginate anteriorly, strongly convex and extending considerably dorsad of underlying structures; lobes (IXT-L) pointed, a little longer than basal width, separated by about twice the width of one lobe. (These prominent, triangular structures do not seem to be true lobes of the ninth tergite. They are probably the tips of either an enlarged ienth tergite or the dorsal arms of the tenth sternite. In a lateral view this pair of strong, rib-like structures can be seen to curve dorsally and fuse with the apical margin of the ninth tergite.) Tenth sternite vestigial, not supporting the anal membrane (A-M). Phallosome (Ph) consisting of two heavily sclerotized plates, open ventrally, closed dorsally; each plate with three large thorn-like dorsal projections and three very large sickle-shaped serrated ventro-lateral processes directed ventro-anteriorly. Claspette absent. Basistyle (Bs) about four-fifths as broad as long, pyriform, rounded apically, clothed with long setae; basal lobe (B-L) a rounded thickened area on the inner face of the basistyle, crowned with numerous stout setae; apical lobe absent. Dististyle (Ds) greatly swollen beyond base, twice as long as broad, half as lorg as basistyle; apex bluntly rounded, bearing 15 to 20 short broad heavily sclerotized spines.

LARVA (Fig. 52).-Head slightly longer than broad, darkly pigmented except in ocular region. Antenna about one-fourth as long as the head, with a few coarse spines; antennal tuft represented by a small single hair inserted 
outwardly near basal third. Head hairs: Preantennal (A) multiple, sparsely barbed, about as long as antenna; lower (B) and upper (C) stout, spine-like, darkly pigmented, barbed, as long or longer than antenna; postclypeal (d) hair-like, 3 to 4-branched, shorter than lower (B) and upper (C); sutural (e) single; trans-sutural (f) multiple; supraorbital single. Dorsal submedian pro-

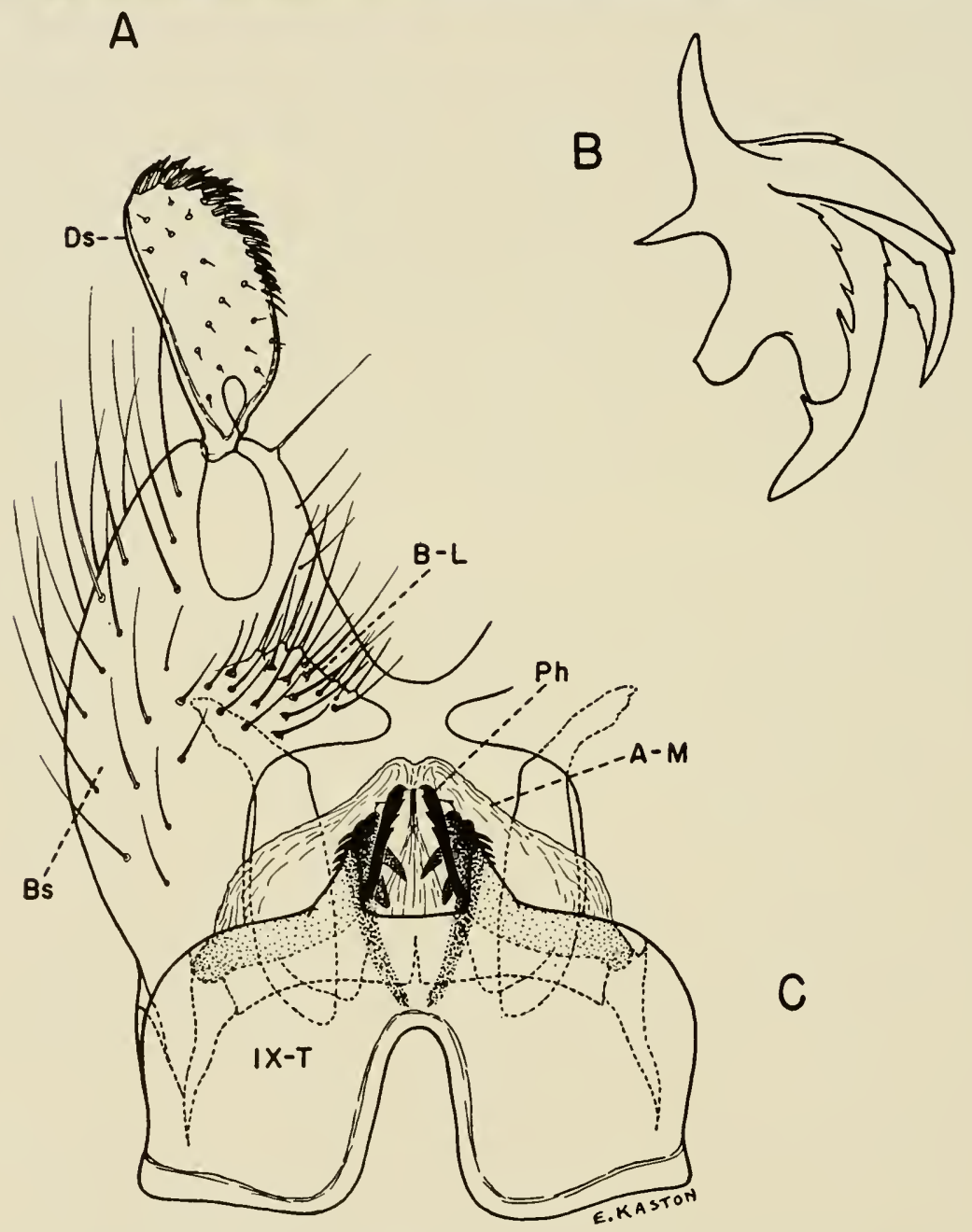

Fig. 51. Uranotaenia lowii Theobald. A, Hind tarsus. B, Phallosome (lateral view). C, Male terminalia. 
thoracic hair group (1 to 3) consisting of two long stout barbed hairs, reaching to or near the upper head hairs (C), and a multiple tuft of about five stout barbed hairs more than half as long as the two single hairs. Upper lateral abdominal hairs on segments I to II double, lower single. Comb of eighth segment consisting of a row of about 6 to 9 scales on the distal margin of a large transverse sclerotized plate; individual scale thorn-shaped, with minute lateral spinules on basal half. Siphon about four times as long as basal width, usually slightly upcurved; pecten of numerous evenly spaced teeth reaching near middle of siphon; individual tooth broad, fringed laterally and apically with fine spinules; subventral tuft multiple, inserted at or slightly beyond end of pecten. Anal segment longer than wide, completely ringed by the dorsal

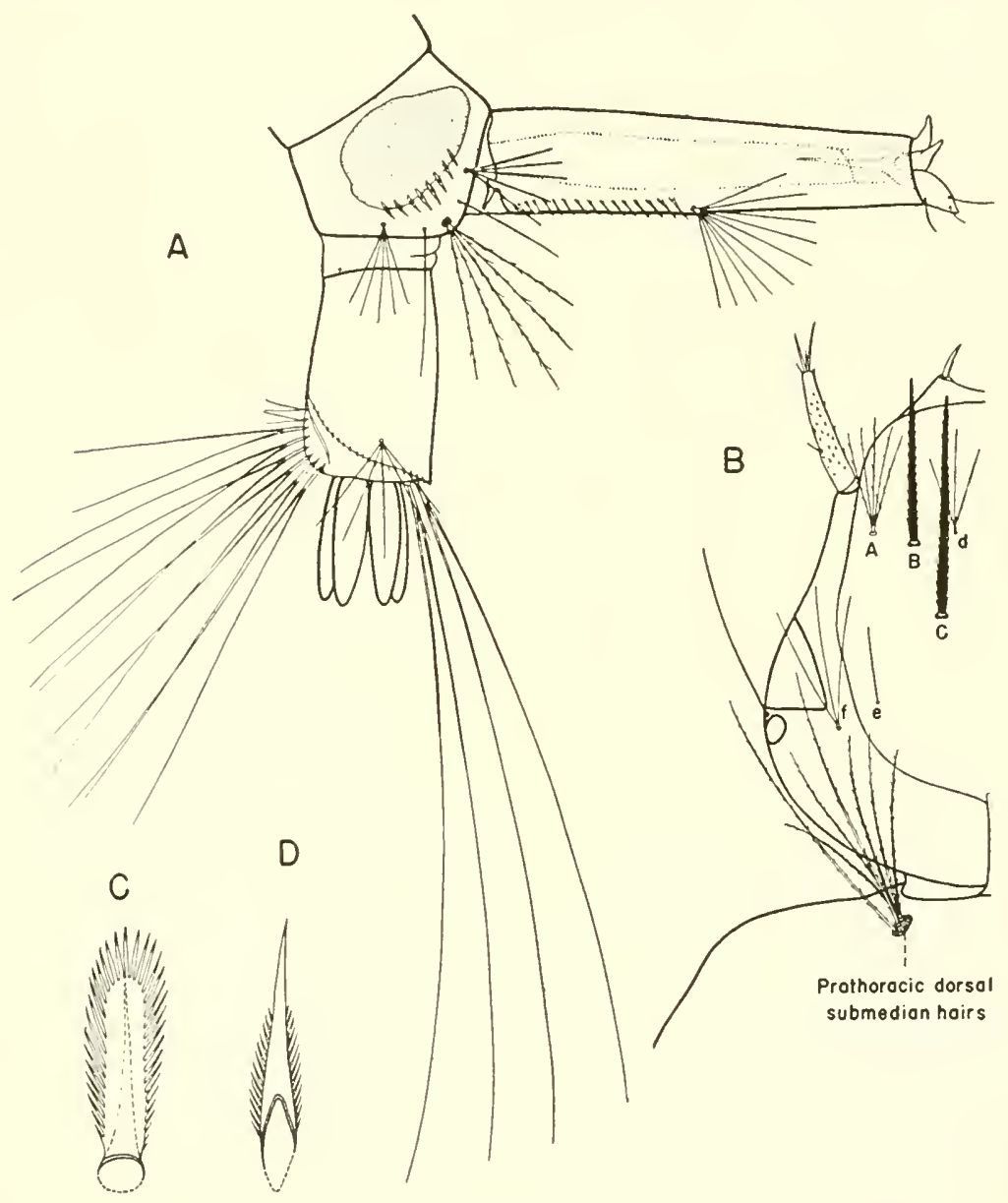

Fig. 52. Larva of Uranotaenia lowii Theobald. A, Terminal segments. B, Head. C. Pecten tooth. D, Comb scale. 
plate; lateral hair multiple; dorsal brush consisting of two long lower caudal hairs and a long 2 to 3-branched upper caudal tuft on either side; ventral brush sparse, confined to the barred area, the two proximal tufts very short; gills 4 , shorter than the segment, bluntly pointed.

distribution.-Southern United States, Mexico, West Indies, Central and South America. Southern States: Alabama (97); Arkansas (27); Florida (52); Georgia and Mississippi (97); North Carolina (125); South Carolina (64). Other States: Texas (108).

BIONOMICS.--Uranotaenia lowii is not known to bite man. Remington (146) found that they did not feed on man and reptiles but did feed on Amphibians. The breeding habits of this species are very similar to those of $U$. sapphirina. The larvae occur mostly along the grassy margins of ponds and lakes. Larvae are found throughout the year in Florida, but farther north breeding is usually restricted to the summer and early fall months (4th Sv. C. Med. Lab. records, 1942-1944, unpublished).

\section{Uranotaenia SAPphiRina (Osten Sacken)}

Aedes sapphirinus Osten Sacken, 1868, Trans. Amer. Ent. Soc., $2: 47$.

adult female.-Very small species. Head: Proboscis dark scaled, long, broadened apically; palpi very short, dark scaled. Occiput shingled with dark broad appressed scales having a bronzy sheen, margined anteriorly and laterally with broad iridescent bluish scales. Thorax: Integument of scutum light brown, clothed with dark-brown lanceolate scales; a narrow median, longitudinal line of broad iridescent bluish scales originating near anterior margin of scutum, extending entire length and covering most of middle lobe of scutellum; a line of similar iridescent bluish scales present on lateral margin of scutum between wing base and scutal angle. A patch of these iridescent scales also present on anterior pronotal lobe and on midportion of sternopleura. A single spiracular bristle present; postspiracular bristles absent. Abdomen: Tergites brown scaled with metallic luster; apices of third, fifth, and sixth segments usually with a patch of white scales; sternites clothed primarily with dingywhite and pale metallic scales. Eighth segment blunt, largely retracted within the seventh. Legs: Apices of femora and tibiae each with a small patch of white scales; remaining leg scales dark brown. Wing: Scales rather broad, brown except for a row of iridescent bluish scales on the stem of vein 5 and a much shorter row near the base of vein 1 ; second marginal cell much shorter than its petiole.

ADULT MALE.-Coloration and palpi similar to that of female. TERMinalia (Fig. 53). Ninth tergite (IX-T) broad, deeply emarginate anteriorly, strongly convex and extending considerably dorsad of underlying structures; lobes very large, quadrate, separated by less than the width of one lobe. (These prominent, quadrate structures do not seem to be true lobes of the ninth tergite. They are probably the greatly expanded tips of either an enlarged tenth tergite or the dorsal arms of the tenth sternite. In a lateral view this pair of strong rib-like structures can be seen to curve dorsally and fuse with the apical 
margin of the ninth tergite.) Tenth sternite vestigial, not supporting the and membrane (A-M). Phallosome ( $\mathrm{Ph})$ consisting of two heavily sclerotized plates, open ventrally, closed dorsally at base; each plate slender, bluntly pointed, with a large, subapical thorn-like projection directed ventro-laterally, and a row of about six or seven very long, stout, curved processes attached ventro-laterally near base and directed antero-ventrally. Claspette absent. Basistyle (Bs) about four-fifths as broad as long, pyriform, rounded apically, clothed with numerous long setae; basal lobe (B-L) conical, appressed to inner face of basistyle, crowned with two or three long, strong setae and several smaller ones; apical lobe absent. Dististyle (Ds) about three-fourths as long as basistyle, four or five times as long as broad, apical third tapered and bearing several very short, stout setae; claw (Ds-C) short, slender, inserted a little before apex.

Larva. (Fig. 54).- Head longer than broad. Antenna about one-fourth as long as head, with a few coarse spines; antennal tuft represented by a small, single hair inserted outwardly before middle. Head hairs: Preantennal (A)
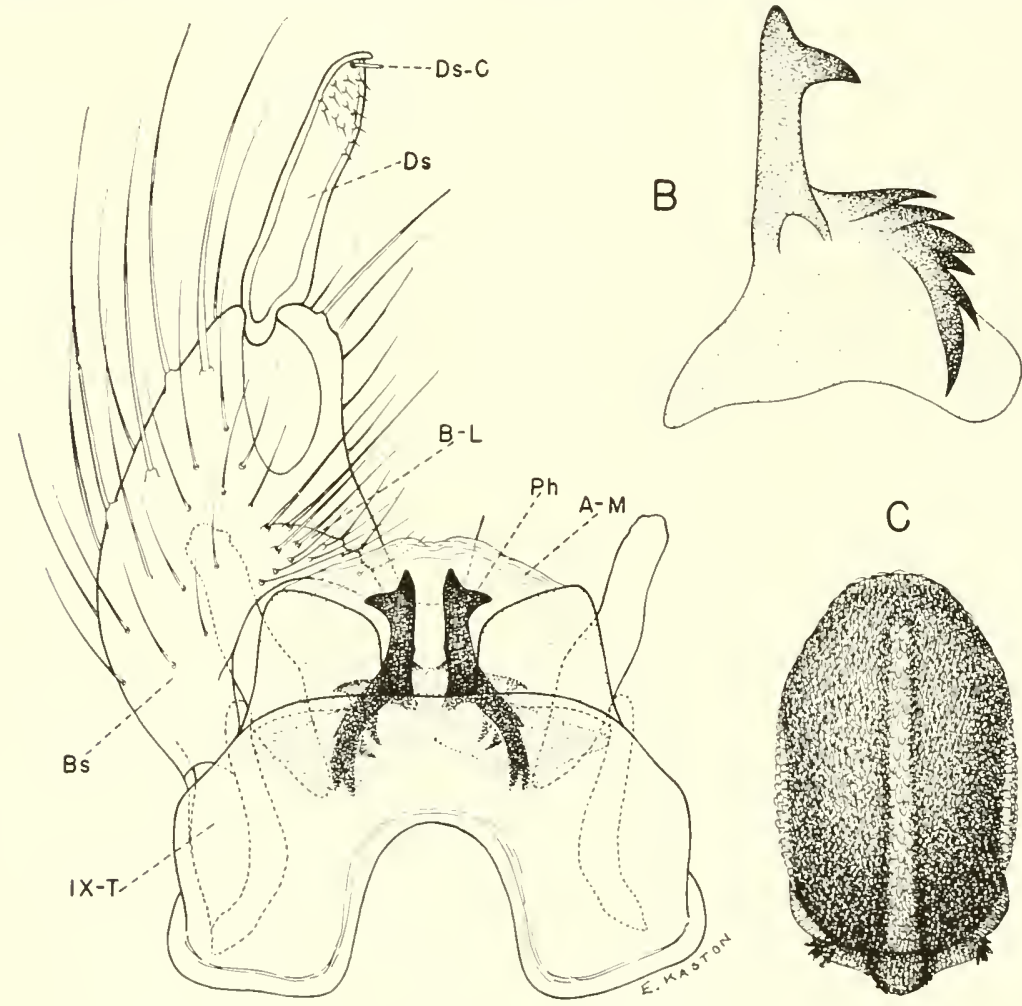

A

Fig. 53. Uranotaenia sapthirina (Osten Sacken). A. Male terminalia. B. Phallosome (lateral view). C, Scutum and scutellum. 
multiple, sparsely barbed, about as long as antenna; lower (B), and upper (C) stout, spine-like, darkly pigmented, barbed, as long or longer than antenna; postclypeal (d) double, hair-like, about as long as lower (B) and upper (C); sutural (e) single; trans-sutural (f) multiple; supraorbital, single. Prothoracic dorsal submedian hair group consisting of two long stout barbed hairs reaching to or near upper head hairs $(C)$ and a short, 8 to 10-branched tuft of fine smooth hairs less than half as long as the stout ones. Upper lateral hairs of abdominal segments I and II, triple; lower, single. Comb of eighth segment consisting of a row of 7 to 10 scales on the distal margin of a large transverse sclerotized plate; individual scale thorn-shaped with minute lateral spinules on basal half. Siphon about four to five times as long as basal width, slightly upcurved; pecten of numerous evenly spaced teeth not reaching middle

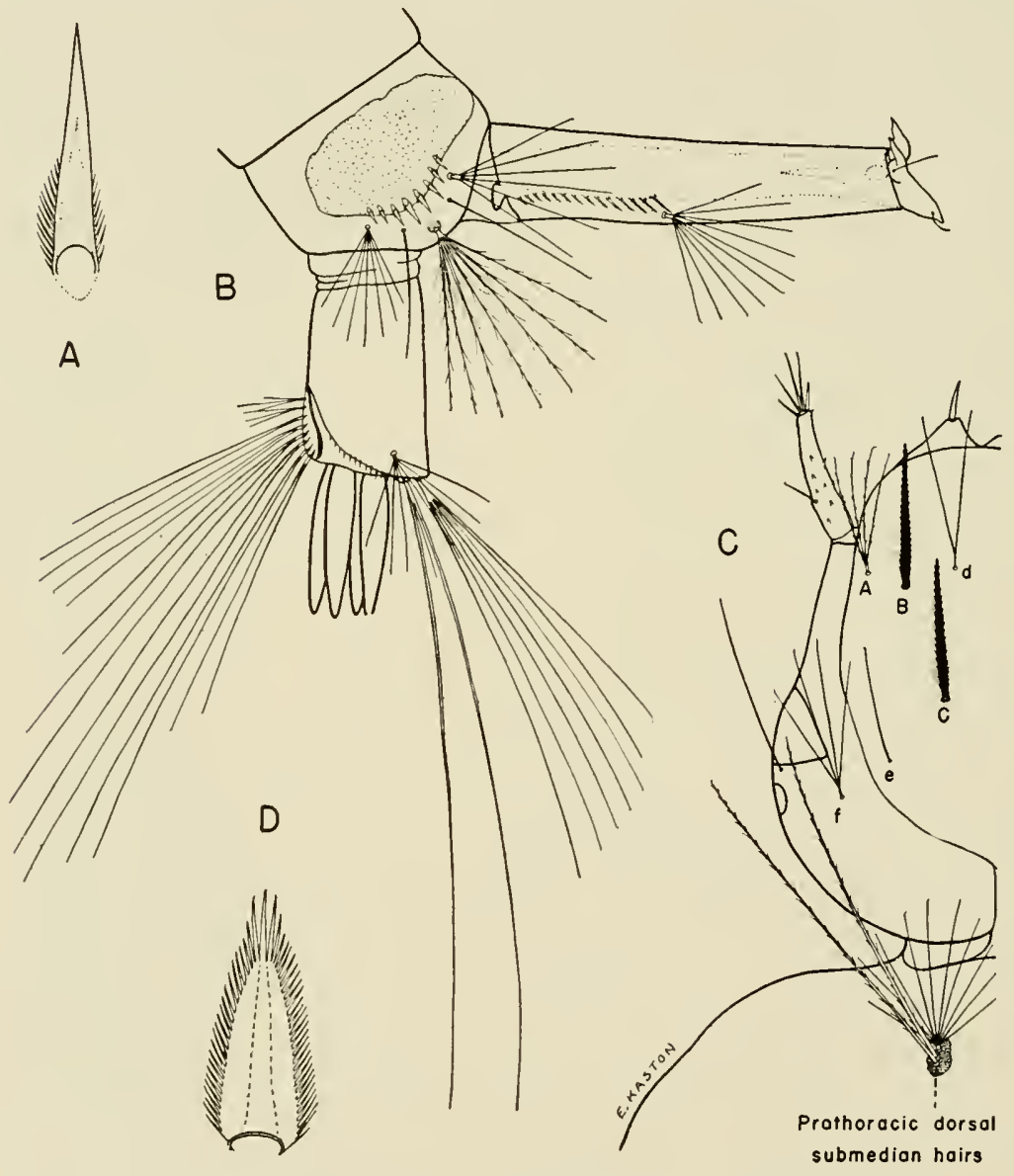

Fig. 54. Larva of Uranotaenia sapphirina (Osten Sacken). A, Comb scale. B, Terminal segments. C, Head. D, Pecten tooth. 
of siphon; individual tooth broad, fringed on both sides with fine spinules, coarser apically; subventral tuft multiple, inserted at or slightly beyond end of pecten. Anal segment longer than wide, completely ringed by the dorsal plate; lateral hair multiple; dorsal brush consisting of two long lower caudal hairs and a shorter, multiple upper caudal tuft on either side; ventral brush sparse, confined to the barred area, the two proximal tufts very short; gills 4 , shorter than the anal segment, pointed.

distribution.-Eastern United States, Mexico, West Indies and Central America. Southern States: Alabama (96); Arkansas (30); Florida (52); Georgia (96); Kentucky (140); Louisiana and Mississippi (52); Missouri (1); North Carolina (97); South Carolina (64); Tennessee (96); Virginia (49). Other States: Delaware (106); Illinois (42); Indiana (76); Iowa (155, 156); Kansas (128); Maryland (52); Massachusetts (180); Michigan (88); Minnesota (131); Nebraska (178); New Hampshire (52); New Jersey (77); New York (52); North Dakota (127); Ohio (52); Oklahoma (159); Pennsylvania (8); Rhode Island (99); Texas (108); Wisconsin (48).

BIONOMICS. - It is the opinion of most workers that the females of this species rarely if ever bite humans. The adults generally rest during the daytime in damp situations in culverts, hollow trees, and amongst vegetation near their breeding places. Uranotaenia sapphirina shows a preference in its breeding for permanent pools, ponds and lakes containing emergent or floating vegetation. It is commonly associated with $A$. quadrimaculatus in its breeding places in the southern states. Larvae occur throughout the year in the extreme South, but farther north breeding is usually restricted to the months of May through November.

\section{Genus Culiseta Felt}

Theobaldia Neveu-Lemaire, 1902, C. R. Soc. Biol., $54: 1331.1$

Culiseta is a rather small genus, but is widely distributed throughout the Palearctic and Nearctic regions of the world. Two subgenera, Culiseta and Climicura, are found in the southern states, represented by one species each. The generic characters included in the following paragraph are based mainly upon the species occurring in the United States.

SAlient CHARACTERS.-Adult: Similar to Culex in general appearance. Pulvilli absent. Spiracular bristles present; postspiracular bristles absent. Squama fringed. Tuft of setae present on under side of wing at base of subcosta. Tip of abdomen of female bluncly rounded, the eighth segment not retractile. Male Terminalia: Tenth sternites with a few terminal teeth. Phallosome usually divided. Basistyle rather long, basal lobe present, apical lobe absent. Dististyle simple and with terminal claw. Claspette absent. Larva: Head wider than long. Comb of eighth segment present. A pair of subventral hair tufts inserted near base of siphon; pecten present.

1 Freeborn, 1943, states. "Theobaldia N-L. 1902 is invalidated by the prior use of Theobaldia Fischer 1887 as an emendation of Theobaldius Nevill 1878. As both available synonyms, Culicella and Culiseta, were proposed in the same publication by Felt in 1904. Culiseta takes priority because it was used by the first reviewer (Dyar, 1921) to designate the groups now included in the genus." 


\section{Keys to the Species}

\section{ADULT FEMALES}

Large species with very broad, sparsely scaled wings; costa with mixed dark and white scales; cross veins arising from vein 4 separated by less than the length of either cross vein; spiracular bristles yellow, numerous (approximately 10 to 15) inornata (Will.), p. 108

Medium sized species with wings normal in shape and densely scaled; costa entirely dark scaled; cross veins arising from vein 4 separated by more than the length of either cross vein; spiracular bristles dark, few in number (rarely more than 5) melanura (Coq.), p. 112

\section{MALE TERMINALIA}

Lobes of the ninth tergite prominent, dome-like, heavily sclerotized, crowned with numerous short stout spines; plates of phallosome long, slender, heavily sclerotized except for hyaline portion at apex inornata (Will.), p. 109

Lobes of ninth tergite only slightly elevated, not dome-like, weakly sclerotized, bearing several setae; phallosome short, broad, lightly sclerotized throughout melanura (Coq.), p. 112

\section{LARVAE (FOURTH INSTAR)}

Lower and upper head hairs ( $\mathrm{B}$ and $\mathrm{C}$ ) multiple, long; pecten followed by an even row of long hairs; the basal tuft of siphon large; siphon about $31 / 2: 1$; comb consisting of many scales in a patch inornata (Will.), p. 111

Lower head hair (B) single, upper head hair (C) much shorter and multiple; pecten followed by a row of about 12 short subequal tufts; the basal tuft of siphon small; siphon about 6 or $7: 1$; comb consisting of a single row of about 25 long bar-like scales melanura (Coq.), p. 112

\section{Culiseta (Culiseta) inornata (Williston)}

Culex inornatus Williston, 1893, U. S. Dept. Agr. Div. Ornith. and Mam., N. Amer. Fauna, No. 7:253.

adult female.-Large species. Head: Proboscis long, dark scaled, speckled with inconspicuous pale scales; palpus short, dark, speckled with white scales. Broad dorsal region of occiput clothed with white to pale yellow lanceolate scales and numerous dark erect forked scales; lateral region of occiput clothed with broad whitish scales. Thorax: Integument of scutum brown; scales of dorsal surface narrow, golden-brown and pale yellow intermixed; anterior and lateral margins and prescutellar space pale-yellow scaled. Numerous yellow spiracular bristles present; postspiracular bristles absent. Abdomen: First tergite whitish-yellow scaled; tergites II to VII each with a whitish-yellow basal band which broadens on either side to clothe the entire lateral margin of the segment, the remaining scales dark brown; eighth tergite entirely pale scaled. Venter clothed with pale yellow scales. Legs: Legs dark brown, with the femora and tibiae, and often the tarsi, speckled with pale scales. Wing: Wing very large and broad, with the points of origin of cross veins 3-4 and 4-5 separated by less than the length of either cross vein; scales small, narrow, dark and rather sparse on all veins but costa, subcosta and vein 1 , where they are broader, more numerous and intermixed with white scales. A dense tuft of yellowish setae present at base of subcosta on under side of wing (Fig. 55A). 
ADULT MALE.-Coloration similar to that of female; but the wings are smaller and narrower, and cross veins 3-4 and 4.5 have their points of origin on vein 4 separated by about the length of one cross-vein. The palpi differ from those of most male Culicines in lacking long hairs and having the terminal segment broad and flattened. TERminalia (Fig. 55B). Lobes of ninth tergite (IXT-L) prominent, heavily sclerotized, dome-like, separated by less
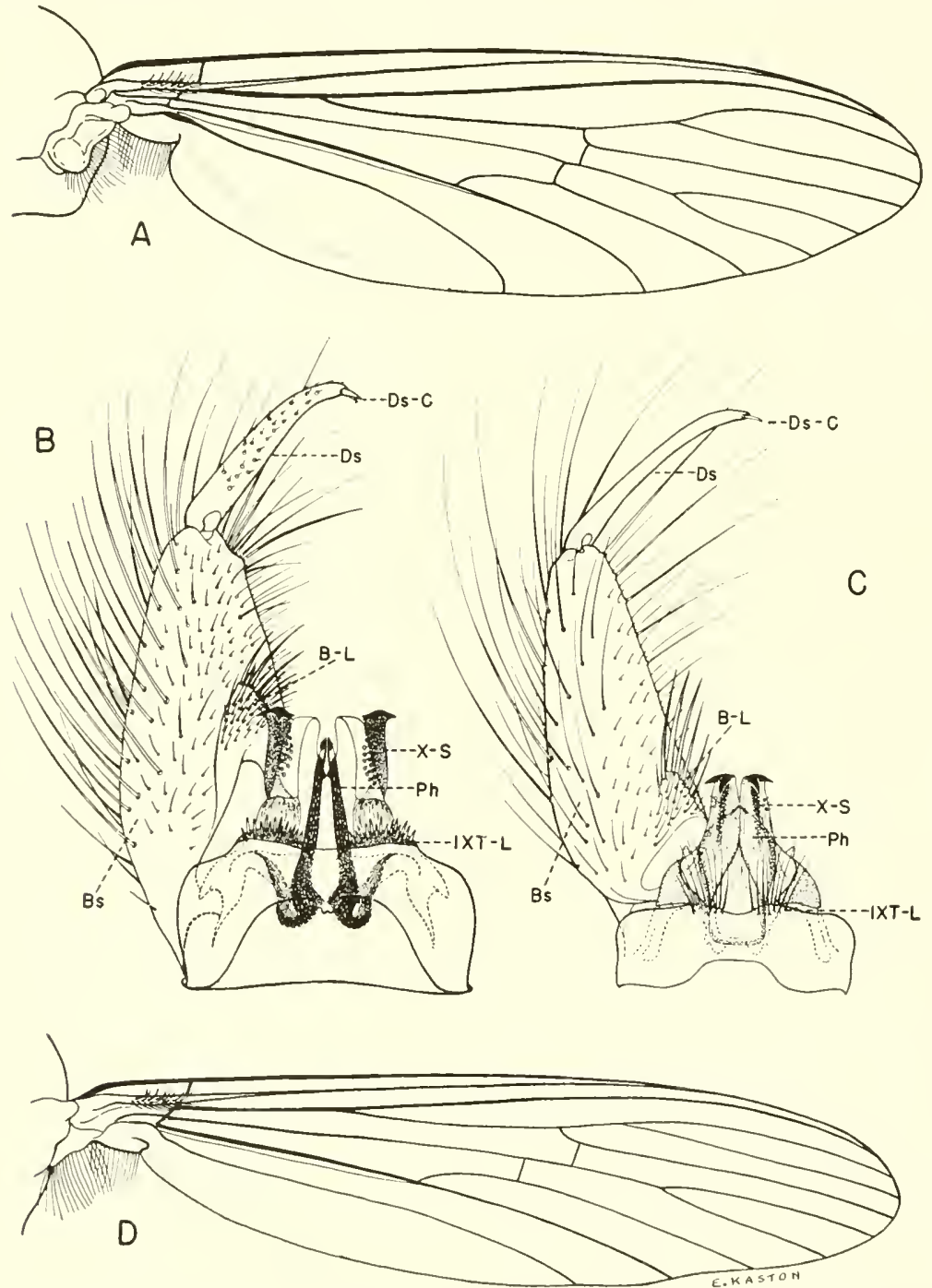

Fig. 55. Culiseta inornala (Williston) and C. mclanura (Coquillett). A. Under side of left wing of C. inornata. B, Male terminalia of C. inornata. C, Male terminalia of C. melanura. D, Under side of left wing of C. melanura. 
than the width of one lobe and crowned with numerous short stout spines. Tenth sternite (X-S) heavily sclerotized, with several strong teeth on apex and a broad sclerotized striated structure near base (this striated structure is probably the basal portion of the tenth tergite). Phallosome $(\mathrm{Ph})$ broadest at base, tapered to a pointed apex, open dorsally and ventrally, formed of two separated elongated plates which are heavily sclerotized except for an apical hyaline portion; hyaline portion with tooth-like divisions subapically; heavily sclerotized portion with a short subapical projection directed dorsally. Claspette absent. Basistyle (Bs) conical, a little more than twice as long as basal width, rounded at apex, clothed with long setae; basal lobe (B-L) conical, appressed to basistyle, bearing several spine-like setae apically and numerous smaller setae on sides; apical lobe absent. Dististyle (Ds) about half as long as basistyle, stout,

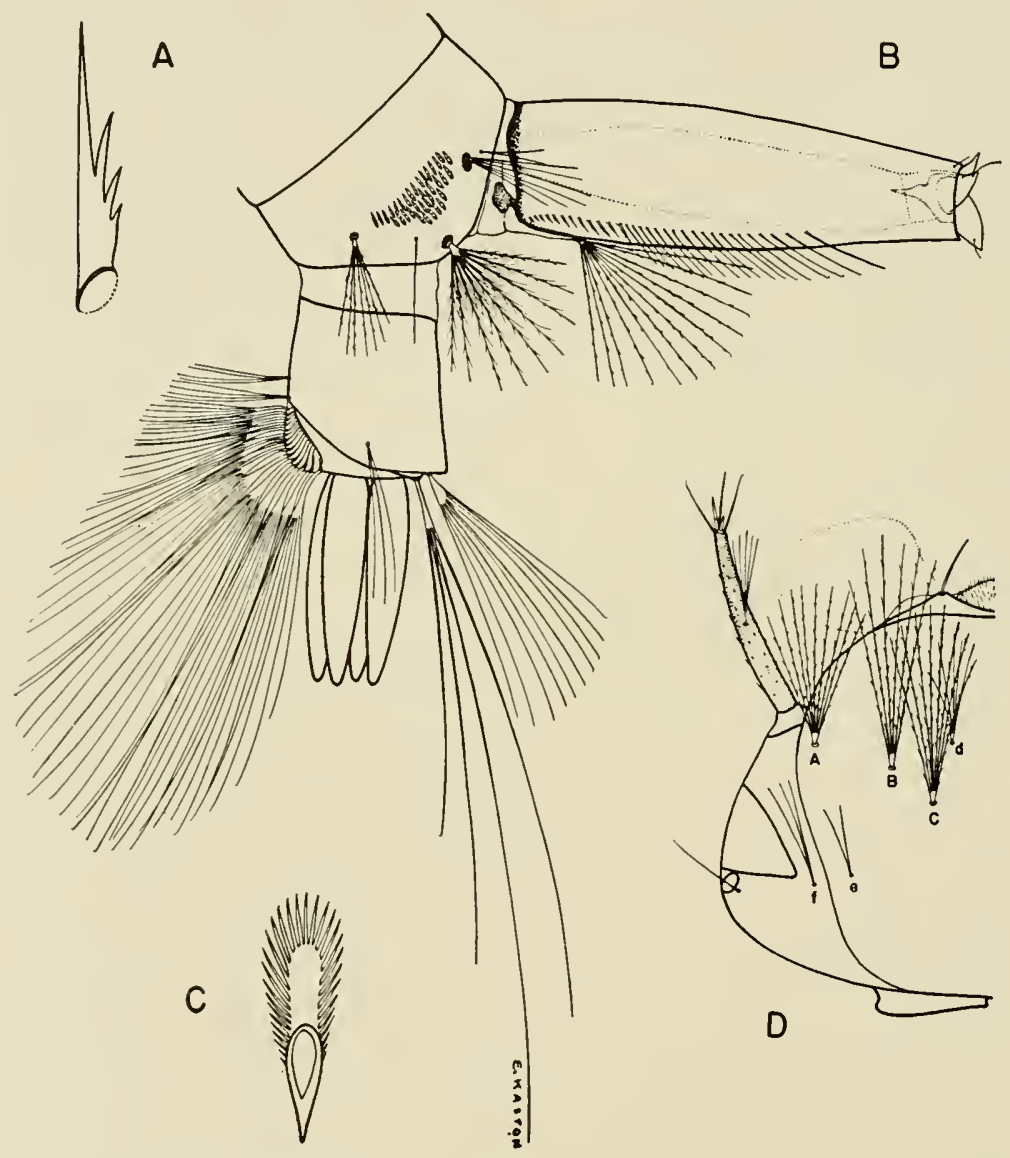

Fig. 56. Larva of Culiseta inornata (Williston). A, Pecten tooth.

B, Terminal segments. C, Comb scale. D, Head. 
tapered and slightly curved beyond middle, bearing numerous small papillated hairs; terminal claw (Ds-C) short, stout, bifurcate.

Larva. (Fig. 56). - Head broader than long. Antenna less than half as long as head, sparsely spined. Antennal tuft multiple, inserted near middle of shaft, reaching near tip. Head hairs: Preantennal (A) multiple, barbed, reaching insertion of antennal tuft; lower (B) multiple, barbed, extending slightly beyond anterior margin of head; upper (C) multiple, barbed, about as long as B; postclypeal (d) small, multiple, barbed; sutural (e) double; trans-sutural (f) usually 3 to 4-branched; supraorbital single or double. Upper lateral abdominal hairs multiple on segments I and II, double on III to VI; subdorsal hairs prominent on segments III to VII. Comb of eighth segment of numerous scales in a patch; individual scale rounded apically and fringed with subequal spinules. Siphon about three and one-half times as long as basal width; pecten of about 12 to 14 evenly spaced teeth on basal fifth of siphon, closely followed by an even row of numerous long hairs extending to apical fourth of siphon; subventral tuft laige, multiple, barbed, inserted within pecten near the base of siphon. Anal segment slightly longer than wide, completely ringed by the dorsal plate; lateral hair long, double or triple; dorsal brush bilaterally consisting of a lower caudal tuft of 3 or 4 unequal branches and a shorter multiple upper caudal tuft; ventral brush well-developed and with one or two small tufts preceding the barred area; gills 4 , as long or longer than the segment, bluntly pointed.

Distribution.-Widely distributed in the United States and southern Canada. Southern States: Alabama, Arkansas and Florida (52); Georgia (97); Kentucky (140); Louisiana, Mississippi, Missouri, North Carolina and South Carolina (52); Tennessee (97); Virginia (49, 50). Other States: Arizona, California and Colorado (52); Delaware (106); District of Columbia, Idaho and Illinois (52); Indiana $(39,76)$; Iowa $(155,156)$; Kansas (52); Maryland (19); Massachusetts (52, 180); Michigan (88); Minnesota (131); Montana (112); Nebraska (178); Nevada (52); New Jersey (77); New Mexico (9); New York (52); North Dakota (127); Oklahoma (159); Oregon and South Dakota (52); Texas (52, 108); Utah (142); Wisconsin (48); Wyoming (129).

BIONOMICS. - The adults are frequently taken in large numbers in both light trap and resting station collections in the southern states during the winter. They are often taken in light traps during cold weather when no other night-flying insects are captured. The females are not troublesome biters in this region, but some observers have reported that they frequently bite man in other localities. Larvae occur in pools, ditches, and ocasionally in artificial containers. Larval records extend from October to April in the South.

MEDICAL IMPORTANCE.-Culiseta inornata has been found naturally infected with the virus of western equine encephalitis (75) and is a proven laboratory vector (74). 


\section{Culiseta (Climacura) melanura (Coquillett)}

Culex melanurus Coquillett, 1902, Jour. N. Y. Ent. Soc., 10:193.

ADULT FEMALE.-Medium sized species. Head: Proboscis very long, slender, dark scaled; palpi short, dark. Occiput clothed with narrow yellowish scales and numerous dark erect forked scales; a patch of broad whitish scales present laterally. Thorax: Integument of scutum dark brown to reddish brown, clothed with fine dark bronzy-brown scales, paler on prescutellar space. A few dark spiracular bristles present; postspiracular bristles absent. Abdomen: Tergites dark brown to black scaled, with bronze to purplish reflection, and with small yellowish-white basal patches laterally, usually not visible from above; faint narrow yellowish-white dorsal transverse bands sometimes present on some segments. Venter primarily dingy-white to yellowish scaled, usually blended with a few dark scales. Legs: Legs entirely dark scaled except for pale inner surfaces of femora. Wing: Wing moderate in size with points of origin of cross veins 3-4 and 4-5 from vein 4 separated by more than the length of either cross vein; longitudinal veins densely clothed with dark slightly broadened ligulate scales; a tuft of dark setae arising from base of subcosta on under side of wing (Fig. 55D).

ADUlt MALE.-Coloration similar to that of female. Palpi long, the terminal segments slender, cylindrical, bearing numerous long hairs. TERMINALIA (Fig. 55C). Lobes of ninth tergite (IXT-L) only slightly raised, weakly sclerotized, separated by the width of one lobe, each bearing several setae. Tenth sternite (X-S) extending to near apex of basal lobe, heavily sclerotized, with two or three strong recurved teeth at tip. Phallosome (Ph) large, less than twice as long as broad, subcylindrical (wider across apical third than across base), open ventrally, closed and carinate dorsally beyond middle, pointed at apex. Claspette absent. Basistyle (Bs) two and one-half times as long as basal width, distinctly cone-shaped, rounded at apex, clothed with numerous long setae; basal lobe (B-L) conical, appressed to basistyle, bearing four or five stout spines apically and numerous smaller setae on sides; apical lobe absent. Dististyle (Ds) two-thirds as long as basistyle, glabrous, slender, a little broader basally than apically, terminating in a short, pointed claw (Ds-C).

LARVA. (Fig. 57).- Head broader than long. Antenna nearly as long as head, slender, spinose; antennal tuft large, multiple, barbed, inserted on a projection at outer fourth of shaft and extending beyond tip. Head hairs: Preantennal (A) multiple barbed; lower (B) long, single, sparsely barbed, extending well beyond anterior margin of head; upper (C) multiple, sparsely barbed, about one-third as long as B; postclypeal (d) single, nearly as long as C; sutural (e) and tran-sutural (f) double or triple; supraorbital long, single. Upper lateral abdominal hairs multiple on segments I and II, double on III to V, and usually single on VI; subdorsal hairs small on segments III to VII. Comb of eighth segment of about 25 bar-like scales in a single row; individual scale long, slender, pointed basally, rounded and fringed apically with subequal spinules. Siphon slender, sides nearly parallel, 6 to 7 times as long as 
basal width; pecten of numerous teeth on basal third of siphon, progressively more widely spaced; a small 2 to 3 -branched subventral tuft at base of siphon; a median ventral row of about 10 to 16 short multiple tufts beginning slightly within the pecten and extending to the outer fourth of siphon; a row of 5 or 6 minute 2-branched tufts inserted dotso-laterally. Anal segment longer than wide, completely ringed by the dorsal plate; lateral hair long, single or double; dorsal brush bilaterally consisting of a lower caudal tuft of three branches (one long and two short) and a shorter multiple upper caudal tuft of about 8 branches of unequal length; ventral brush well-developed, with one or two small tufts preceding the barred area; gills 4 , usually somewhat shorter than the anal segment, pointed.

Distribution.- Eastern and central United States from Canada to the Gulf of Mexico. Southern States: Alabama (170); Arkansas (83); Florida

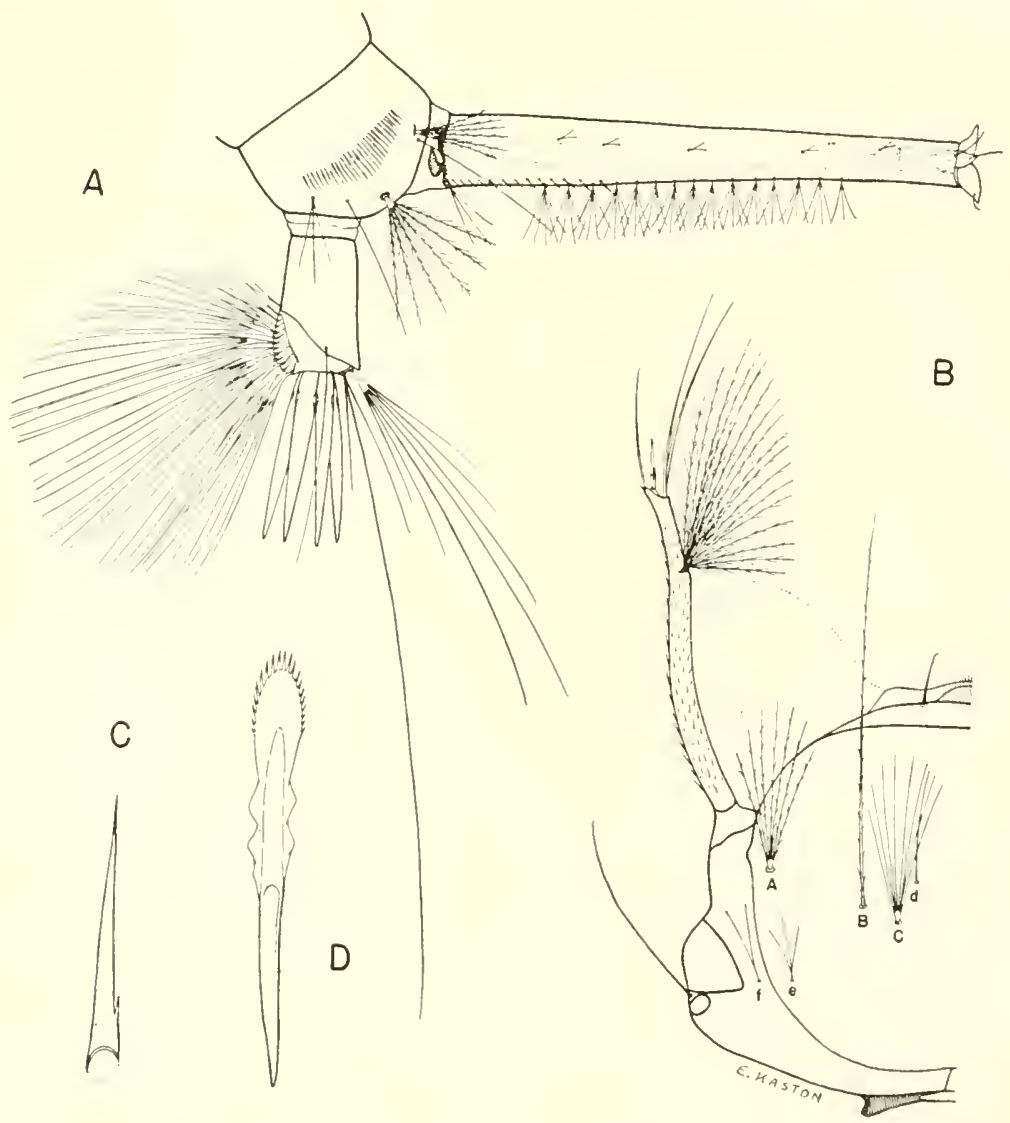

Fig. 57. Larva of Culiseta melanura (Coquillett). A, Terminal segments. B, Head. C, Pecten tooth. D. Comb scale. 
(96); Georgia (52); Kentucky (140); Louisiana (96); Mississippi (97); Missouri (31); North Carolina (166); South Carolina (64); Tennessee (125); Virginia (49). Other States: Colorado (128); Delaware (106); Iowa (128); Maryland (19); Massachusetts (180); Michigan (88); Nebraska (128); New Hampshire (105); New Jersey (77); New York (52); Oklahoma (159); Pennsylvania (8); Rhode Island (99); Texas (130); Wisconsin (48).

BIONOMICS.-The adults of this species have been collected in considerable numbers in the southern states with New Jersey light traps and to a lesser extent in diurnal resting stations. The larvae occur mostly in small permanent bodies of water, particularly in swamps. This species is said to overwinter as larvae under the ice farther north. Both larvae and adults occur throughout the year in the South (4th Sv. C. Med. Lab. records, 1942-1944, unpublished).

\section{Genus Orthopodomyia Theobald}

Orthopodomyia Theobald, 1904, Entomologist, $37: 236$.

Orthopodomyia is a small genus widely distributed throughout the tropical and temperate regions of the world. It is rarely found in large numbers due to its restricted types of breeding habitats, consisting chiefly of water in tree-holes, bamboo stems and leaf bases of Bromeliads. The adults of most species are distinctly ornamented. This genus is represented in the United States by two species, signifera and alba, which can be separated with certainty only in the larval stage.

SALIENT CHARACTERS.-Adult: Fourth segment of fore-tarsus very short, only about as long as wide. Spiracular and postspiracular bristles absent. Postnotum without setae. Squama fringed. Fork of vein 2 much longer than its petiole. Tip of abdomen of female bluntly rounded, the eighth segment not retractile. Male Terminalia: Apical margin of eighth tergite with a rounded, median projection. Tenth sternite with several teeth at tip. Claspette absent. Basal lobe small, armed with spines and setae; apical lobe absent. Larva: Siphon without pecten; a single pair of subventral tufts present, never inserted near base. Comb of eighth segment usually consisting of two rows of thornlike scales, those of the posterior row much longer.

\section{Key to THe SPEcies}

LARVAE (FOURTH INSTAR)

Abdominal segment VIII with a large dorsal sclerotic plate; anal segment completely ringed by the dorsal plate; lateral hair of anal segment single signifera (Coq.), p. 118

Abdominal segment VIII without a sclerotic plate; anal segment not completely ringed by dorsal plate; lateral hair of anal segment 2 to 3 -branched. alba Baker, p. 116

\section{Orthopodomyia alBa Baker}

Orthopodomyia alba Baker, 1936, Proc. Ent. Soc. Wash., 38:1.

ADULT FEMALE.-Very similar to $O$. signifera. The only apparent difference in the females of the two species is found in the scales of the second 
abdominal tergite. King et al. (96), state that this tergite is almost entirely pale scaled in $O$. alba, the pale scales extending to the apex in the middle of the segment (Fig. 58F).

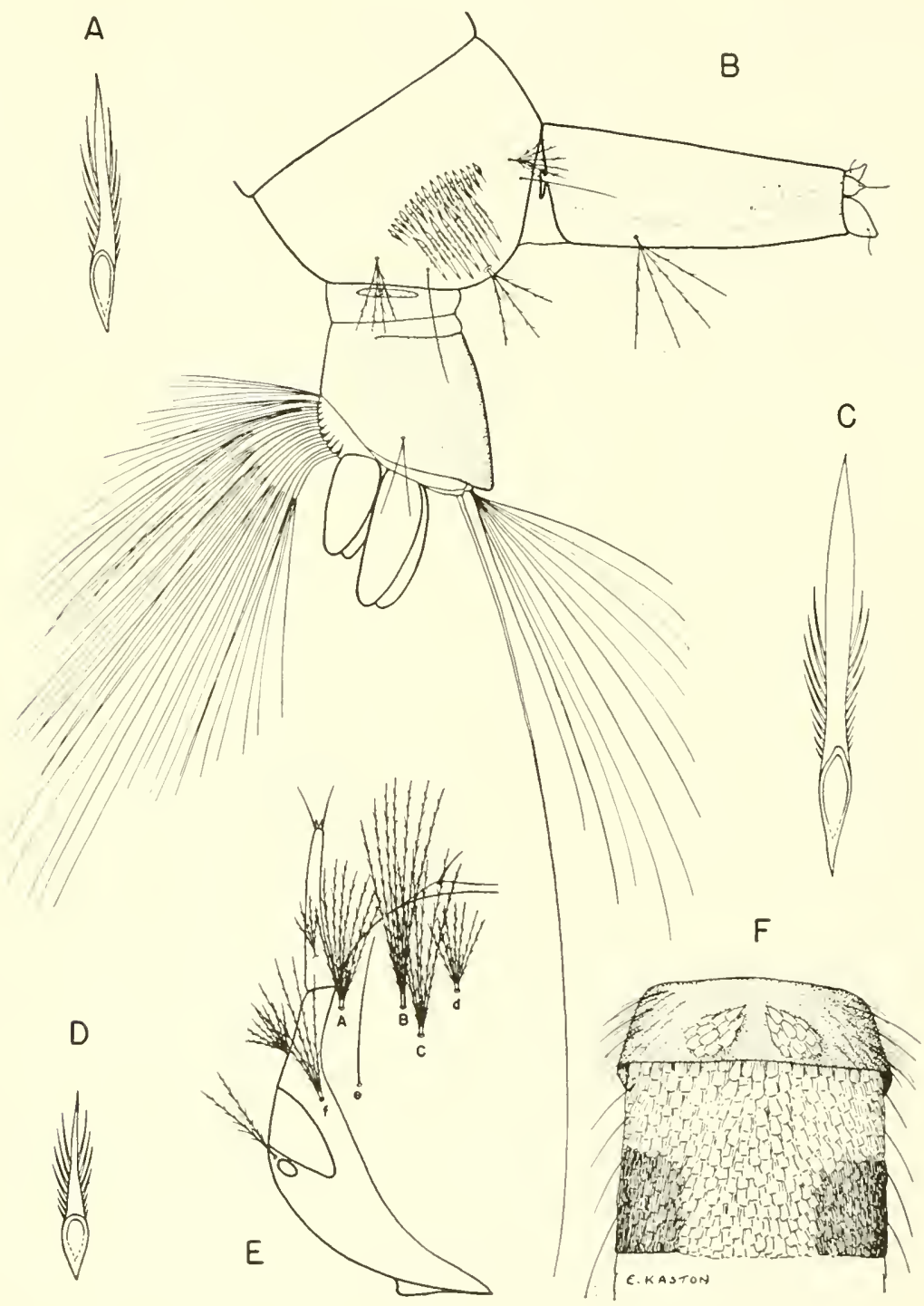

Fig. 58. Orthopodomyia alba Baker. A, C, and D, Comb scales of larva. B, Terminal segments of larva. E. Head of larva. F, First and second abdominal segments of female (tergites). 
ADUlt MALE.-Coloration similar to that of female. Terminalia. The male terminalia of this species appears to be indistinguishable from that of O. signifera (Fig. 59C).

LARva. (Fig. 58).-Head slightly broader than long. Antenna less than half as long as head, smooth; antennal tuft multiple, barbed, inserted at basal fourth of shaft, short. Head hairs: Preantennal (A) multiple, barbed, nearly as large as upper (C); lower (B) and upper (C) multiple, barbed, extending to or beyond the preclypeus; postclypeal (d) multiple, barbed, shorter than upper (C); sutural (e) long, single; trans-sutural (f) large, multiple, barbed; supraorbital single or double, lightly barbed; sub-basal conspicuous, 7 to 11branched, barbed. Upper lateral abdominal hairs long, 2 to 3-branched. Abdominal segment VI to VIII without dorsal sclerotized plates. Comb of eighth segment consisting of two rows of thorn-shaped scales, the anterior row with about 14 to 17 short scales and the posterior row with about 9 to 11 long scales (scales of anterior row become longer medially and overlap the bases of those of the posterior row); individual scale long, pointed and fringed on basal half with small spines (small scales on ventral portion of anterior row are often fringed apically with subequal spines); subsiphonal tuft small, barbed, 3 to 5 . branched. Siphon about two to two and one-half times as long as basal width; pecter absent; subventral tuft with 3 to 5 branches rarely longer than the width of the siphon, barbed, inserted at basal third of siphon. Anal segment longer than wide, dorsal plate fading ventrally and not meeting on midventral line; a small linear sclerotized area near base of segment; lateral hair 2 to 3 . branched; dorsal brush consisting of a long lower caudal hair and a shorter multiple upper caudal tuft on either side; ventral brush well developed, confined to the barred area; gills 4 , blunt, shorter than the segment, dorsal pair longer than the ventral pair.

Distribution. ${ }^{1-E a s t e r n}$ and central United States. Southern States: Alabama (171); Florida (35); Georgia (4th Sv. C. Med. Lab. records, 1945, unpublished); Louisiana (Hathaway, 1944, unpublished); Mississippi (125); Missouri (70); North Carolina (166); Virginia (50). Other States: Kansas (128); Nebraska (129); New York (116); Texas (141).

BIONOMICS.-Nothing is known of the habits of the adults of this species. It breeds in rot cavities of trees and occasionally in artificial containers. The larvae are usually found associated with the larvae of $O$. signifera.

\section{ORTHOPODOMYIA SIGNIFERA (Coquillett)}

Culex signifer Coquillett, 1896, Can. Ent. 28:43.

adult female.-Medium sized species. Head: Proboscis long, clothed with black and white scales, the white scales arranged so as to form longitudinal striae dorsally. Palpi about one-third as long as proboscis, dark scaled, streaked with white scales dorsally. Occiput clothed dorsally with numerous narrow white scales and long dark erect forked scales; eye margins and lateral portions of occiput white scaled. Thorax: Integument of scutum dark brown, clothed

1 The following state records are based on adult specimens: Georgia, Florida, Kansas, Nebraska, and Texas. 
with numerous long dark hairs and small reddish-brown scales, except for six narrow longitudinal lines of white scales (Fig. 59D). Abdomen (Fig. 59B): Tergite I whitish scaled; tergites II and III, and occasionally IV, dark with white basal bands, the basal band of II wide and often projecting medially nearly to the rear margin of the segment; remaining tergites dark scaled dorsally. Venter clothed with mixed dark and pale scales, the dark ones predominant apically. Legs: Vestiture of dark-brown and white scales; fore tarsi all dark; mid-tarsi with base and apex of first segment and base of second segment narrowly ringed with white; hind tarsi with segment 5 entirely white on one side, all other segments broadly ringed with white apically and basally (rings broadest on first and second segments, narrow on third and fourth). Wing: Scales, broad, ovate, intermixed dark brown and white.

ADUlt Male.--Coloration similar to that of female. Terminalia (Fig. 59C). Eighth tergite (VIII-T) with posterior margin medially produced tongue-like, overlapping the roundly emarginate anterior margin of the ninth

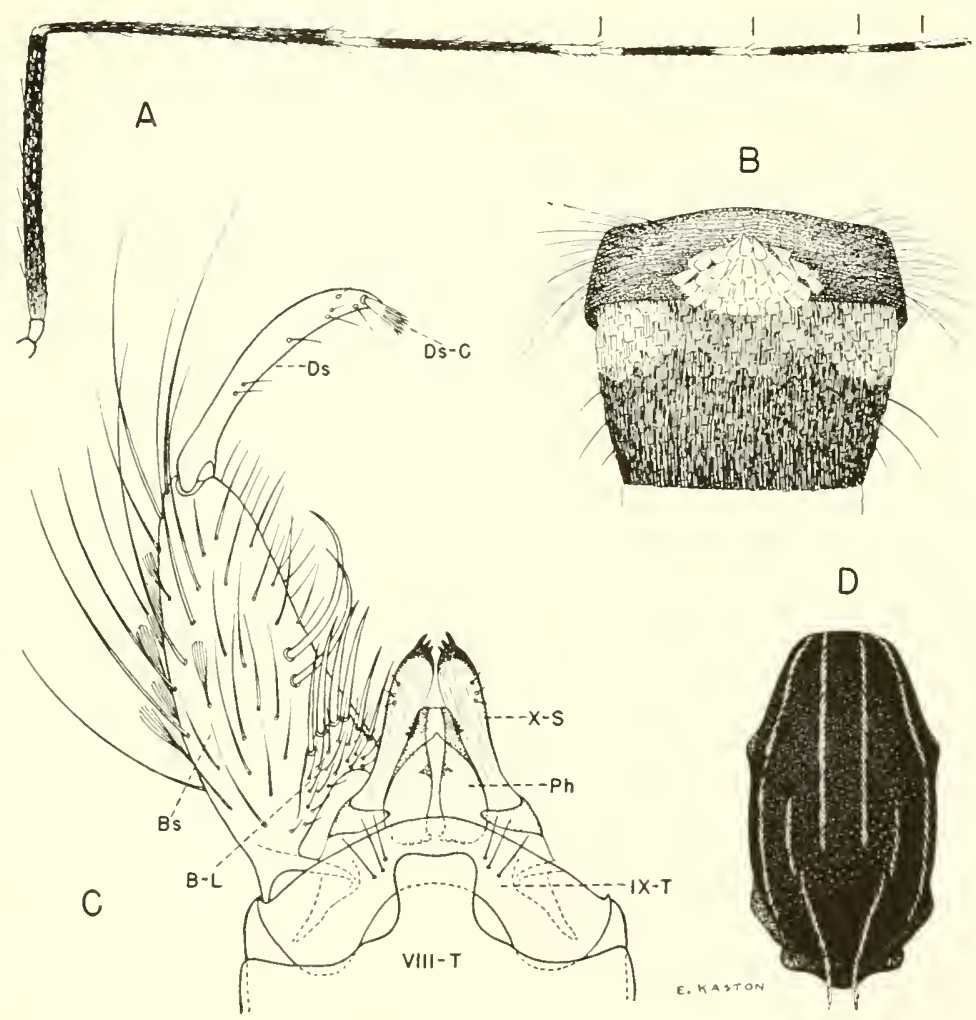

Fig. 59. Orthopodomyia signifera (Coquillett). A. Hind leg. B. First and second abdominal segments of female (tergites). C, Male terminalia. D. Scutum and scutellum. 
tergite (IX-T); lobes of ninth tergite not developed, represented only by a few setae. Tenth sternite (X-S) prominent, heavily sclerotized apically. Phallosome $(\mathrm{Ph})$ broadly pyriform, truncate at apex, closed dorsally beyond middle by a membranous connection, open ventrally; each plate broad on basal half, narrowed distally, with a small but distinct medial triangular projection turned ventrally and laterally from the inner margin and with several short heavily sclerotized subapical teeth directed dorsally. Claspette absent. Basistyle (Bs) conical, slightly curved, about three times as long as basal width, rounded at apex, clothed with scales and long setae and with one or two large curved spines arising from inner face near middle; basal lobe (B-L) conical, appressed to basistyle, bearing a few large spines apically and numerous smaller setae on sides; apical lobe absent. Dististyle (Ds) about three-fifths as long as basistyle, slightly curved, bluntly pointed at tip; terminal claw (Ds-C) broader apically, truncate, longitudinally divided beyond middle into numerous comblike teeth.

LARVA. (Fig. 60).- - Head slightly broader than long. Antenna about half as long as head, smooth; antennal tuft multiple, barbed, inserted at basal third of shaft, not reaching tip. Head hairs: Preantennal (A) multiple, barbed, extending near insertion of antennal tuft; lower (B), and upper (C) multiple, barbed, extending beyond preclypeus; postclypeal (d) multiple, barbed, shorter than upper (C); sutural (e) long, single; trans-sutural (f) and supraorbital long, single or branched distally; sub-basal inconspicuous, about 1 to 3 branched. Upper lateral abdominal hairs short, multiple on segments I and II. A large dorsal sclerotized plate present on abdominal segment VIII, often present on VI and VII. Comb of eighth segment consisting of two rows of thorn-shaped scales, the anterior row with about 17 to 23 short scales and the posterior row with about 6 to 10 long scales (scales of anterior row become larger medially and overlap the bases of those of the posterior row); individual scale long, pointed and fringed on basal half with small spines (small scales on ventral portion of anterior row are fringed apically with subequal spines); subsiphonal tuft large, barbed, 5 to 12-branched. Siphon about three and onehalf times as long as basal width; pecten absent; subventral tuft with 5 to 12 branches as long or longer than the width of the siphon, barbed, inserted beyond basal third. Anal segment longer than wide, completely ringed by the dorsal plate; a small linear sclerotic plate at base of segment; lateral hair single, often feathered at tip; dorsal brush consisting of a long lower caudal hair and a shorter multiple upper caudal tuft on either side; ventral brush well developed, confined to the barred area; gills 4 , about as long as the segment, the dorsal pair longer than the ventral pair.

Distribution.-Southern United States, north to Massachusetts and west to California. It also occurs in Jamaica (54) and Puerto Rico (136). Southern States: Alabama (170); Arkansas (30); Florida (96); Georgia (52); Kentucky (140); Louisiana (96); Mississippi, Missouri, North Carolina and South Carolina (52); Tennessee (170); Virginia (49). Other States: California (67); Delaware (106); District of Columbia (52); Illinois (42); Indiana (76); Iowa (156); Kansas (128); Maryland (19); Massachusetts (180); 
Nebraska (178); New Jersey (77); Oklahoma (159); Pennsylvania (8); Rhode Island (99); Texas (108).

BIONOMiCs. - Adults of this species are occasionally found resting near tree holes in which they breed but are apparently of little or no economic importance. The larvae are found principally in rot cavities of trees and on occasions in artificial containers. Breeding occurs throughout the year in the South.

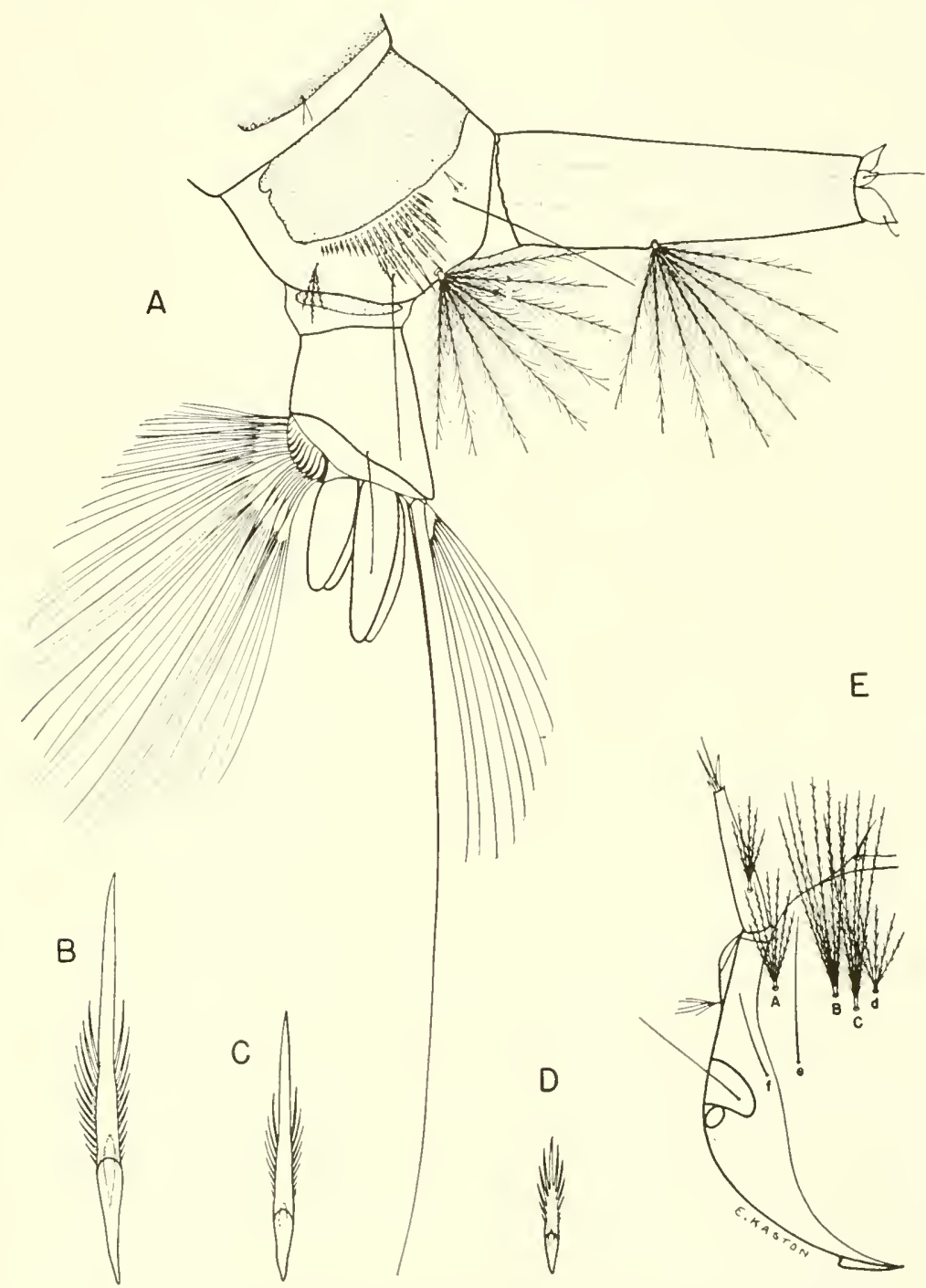

Fig. 60. Larva of Orthopodomyia signifera (Coquillett). A. Terminal segments. B, C, and D, Comb scales. E, Head. 


\section{Genus Mansonia Blanchard1}

Mansonia Blanchard, 190I, C. R. Soc. de Biol., 53:1045.

The genus Mansonia is represented in the United States by three species belonging to two subgenera. Shortly after hatching, the larvae of Mansonia attach themselves by means of the modified siphon, to roots or stems of plants from which they obtain their air supply. The females are troublesome biters.

SALIENT CHARACTERS.-Adults: The adults are difficult to separate from other genera of Culicini. Palpi of male long, female palpi short. Male antenna plumose. Postnotum without setae. Spiracular bristles absent; postspiraculars present or absent. Pulvilli absent. Wings with many or all the scales broad. Squama fringed. Male Terminalia: Variable in structure. Basal lobe either appressed to basistyle or long and slender, usually bearing blunt spines or modified filaments. Apical lobe absent. Dististyle variable in shape. Larva: Two long filaments on antenna before tip. Anal segment long, completely ringed by the dorsal plate. Comb of eighth segment of a few teeth in a single row. Siphon short, without pecten. Siphon attenuated and modified to form a saw-like apparatus for piercing underwater stems and roots of aquatic plants.

\section{Keys to THE SPECIES}

ADULT FEMALES

1. Postspiracular bristles absent; segment $\mathrm{I}$ of hind tarsus with a pale ring near middle perturbans (Walker), p. 127

Postspiracular bristles present; segment $l$ of hind tarsus without a pale ring near middle

2. Palpus a little more than $1 / 3$ as long as proboscis, with 4 th segment about twice as long as 3rd; apical margin of 7 th tergite bearing a close-set row of minute, pointed spines (visible on pinned specimen with dissecting microscope); spines of 8th tergite clumped posteriorly (visible only when softened in $\mathrm{KOH}$ and dissected) titillans (Walker) p. 124

Palpus less than $1 / 3$ as long as proboscis, with 4 th segment about $11 / 2$ times as long as 3rd; apical margin of 7 th tergite without minute spines; spines of 8 th tergite more or less uniformly spaced indubitans Dyar and Shannon, p. 121

\section{MALE TERMINALIA}

1. Dististyle without a tooth-like lateral branch on inner margin

Dististyle with a tooth-like lateral branch on inner margin perturbans (Walker), p. 127

Dististyle not convoluted titillans (Walker), p. 125

Dististyle somewhat convoluted indubitans Dyar and Shannon, p. 121

\section{LARVAE (FOURTH INSTAR)}

1. Lateral spine of maxilla smooth; anal segment about twice as long as wide perturbans (Walker), p. 128

Lateral spine of maxilla strongly serrated on one side; anal segment about three times as long as wide

2. Comb scale long, slender, thorn-like, with a few minute spinules on basal portion titillans (Walker), p. 125

Comb scale rather broad, fringed apically with several stout subequal spines indubitans Dyar and Shannon, p. 121

1 See Edwards (1932) or Dyar (1928) for synonymy. 


\section{Mansonia (Mansonia) indubitans Dyar and Shannon}

Mansonia indubitans Dyar and Shannon, 1925, Jour. Wash. Acad. Sci., $15: 41$.

ADUlt Female.-Medium sized to rather small species. Head: Proboscis dark, speckled with pale scales and with a narrow white ring near apical third; palpi a little less than one-third as long as proboscis (segment 4 one and onehalf times as long as segment 3), dark scaled, speckled with white, and with small terminal segment white. Occiput clothed with pale lanceolate scales and numerous black erect forked scales. Thorax: Scutum covered by dark-brown lanceolate scales intermingled with light golden-brown lanceolate scales (the paler scales more numerous anteriorly and on prescutellar space). Postspiracular bristles present. Abdomen: First tergite pale scaled; remaining tergites predominately dark scaled, with a few scattered whitish scales laterally and apically; sternites with intermixed white and brown scales, the white scales more numerous apically. Eighth segment blunt, largely retracted within the seventh; eighth tergite (Fig. 61D) with numerous short stout spines more or less regularly spaced and arranged in a curved posterior row and a somewhat irregular anterior row. Apical margin of seventh tergite without a close-set row of small spines. Legs: Femora and tibiae of fore, middle, and hind legs dark-brown scaled, speckled with pale scales; inner surfaces of femora II and III predominantly pale scaled. Tarsal segments 1 to 4 of fore and middle legs each with a narrow basal white band; all segments of hind tarsus with white basal bands. Wing: Scales mixed brown and white, very broad, intermingled with a few narrower scales, particularly on veins 3,4 , and 5 .

ADUlt MALE.-Coloration similar to that of female. TERMinalia (Fig. 61). Ninth tergite (IX-T) unsclerotized, with lobes indistinct. Tenth sternite (X-S) prominent, heavily sclerotized beyond middle; dorsal arm long, curved dorsally, weakly sclerotized. Phallosome (Ph) large, lightly sclerotized, open ventrally, closed dorsally near apex, expanded laterally and apically, broadly rounded at apex. Claspette absent. Basistyle (Bs) about three times as long as broad, narrower at middle than across apical fourth due to concavity of inner margin, rounded at apex; outstanding vestiture consisting of a group of strong setae at apex and several very long strong setae near base. Basal lobe (B-L) very long and slender, extending a little beyond apical third of basistyle; basal two-thirds of lobe narrow, stem-like, fused to basistyle; apical third swollen, free, bearing a short stout bluntly pointed terminal rod. Apical lobe absent. Dististyle (Ds) about two-fifths as long as basistyle, with basal third very broad and thick, giving off a slender, inner branch; remainder of dististyle becoming a little narrower and abruptly much thinner beyond origin of inner branch, curved, slightly convoluted, tapered to a narrow tip. Terminal claw (Ds-C) short, stout.

LARvA. (Fig. 62).-Head much broader than long. Antenna about twice as long as head, whip-like, sparsely spined basally; antennal tuft multiple, barbed, arising from a notch on basal third; a pair of long stout setae inserted at middle of shaft and extending to tip of antenna; apex of antenna bearing a short spine, seta, and membranous papilla. Head hairs: Preantennal (A) mul. 
tiple, barbed; lower (B), upper (C), and postclypeal (d) short, multiple; sutural (e) and trans-sutural (f) multiple; supraorbital double or triple. Lateral spine of maxilla strongly serrated on one side. Prothoracic lateral hairs (5 to 7) as follows: 5 , three or four-branched; 6, single; 7, double or triple. Upper lateral abdominal hairs single on segments I to VI. Comb of eighth segment of about 6 to 8 subequal scales in a single row; individual scale rather short, broad, fringed apically with subequal spines. Subsiphonal hair long, 3 or 4-branched, weakly barbed. Siphon short, sharply conical, strongly attenuated beyond middle; attenuated portion of siphon heavily sclerotized, bearing saw-like projections dorsally and stout hooks apically; a long stout recurved dorsal spine, a

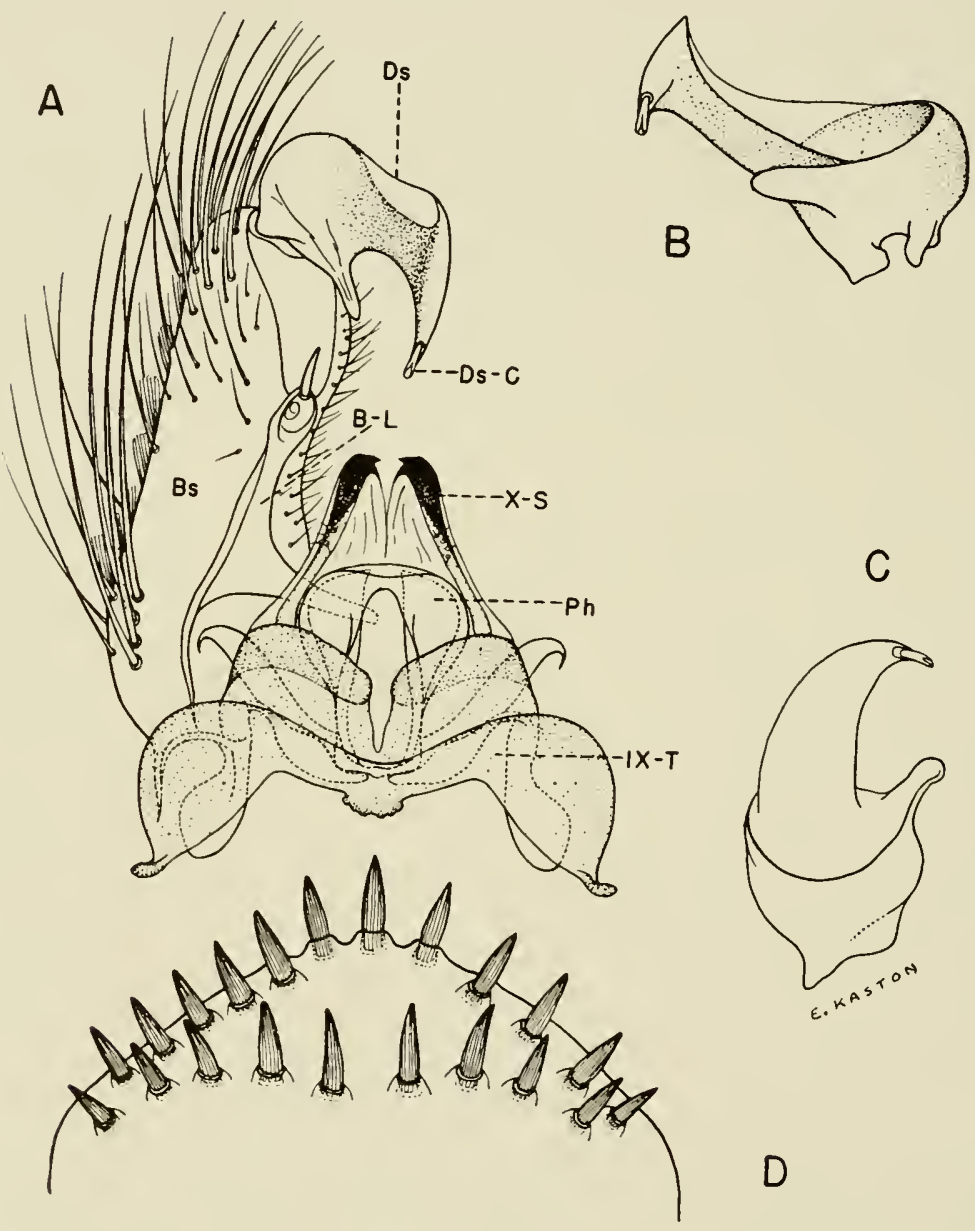

Fig. 61. Mansonia indubitans Dyar and Shannon. A, Male terminalia. B, C, Dististyle (different aspects). D, Eighth abdominal tergite of female. 
single stout dorso-lateral hair, and a multiple subventral tuft arising before the heavily sclerotized portion; pecten absent. Dorsal plate about three times as long as broad and completely ringing the anal segment; lateral hair multiple, inserted well before the posterior margin of the dorsal plate; dorsal brush consisting of a long multiple lower caudal tuft and a moderately long multiple upper caudal tuft on either side; ventral brush well developed, with about four small precratal tufts piercing the dorsal plate; gills 4 , shorter than the segment, bluntly pointed.

Distribution.-Recorded from the West Indies, Florida and northern South America (135). As pointed out by Pratt (135), many of the records of $M$. titillans in the literature for the United States may ba based on $M$. in. dubitans. Definite locality records for this species in Florida are given by Chamberlain and Duffey (38).

BIONomics.-The females are probably as troublesome biters as $M$. titillans in regions where they are numerous. Adults are frequently taken in light trap collections. The larva and pupae attach themselves to the roots of water lettuce (Pistia) from which they obtain their air supply.

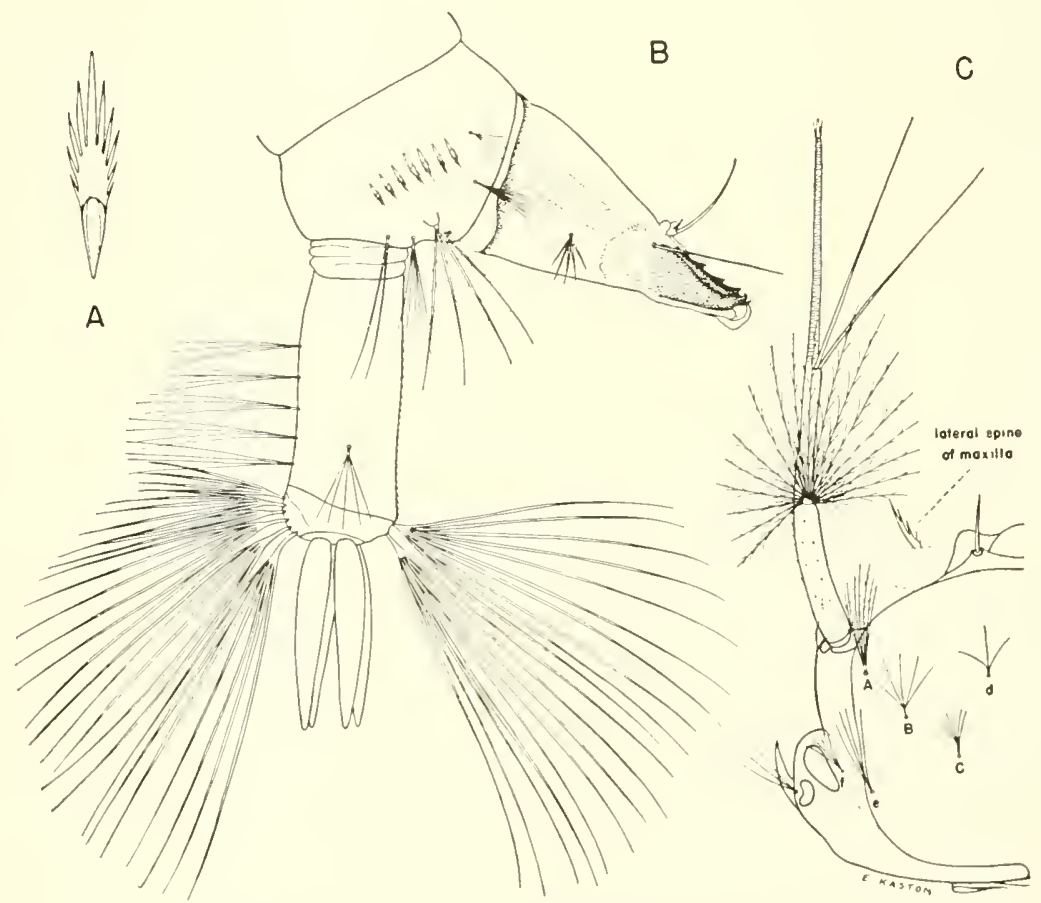

Fig. 62. Larva of Mansonia indubitans Dyar and Shannon. A. Comb scale. B. Terminal segments. C. Head. 


\section{Mansonia (Mansonia) titillans (Walker)}

Culex titillans Walker, 1848, List Dipt. Brit. Mus., 1:5.

adult female.-Medium sized species. Head: Proboscis dark, speckled with pale scales and with a narrow white ring near apical third; palpi a little more than one-third as long as proboscis (segment 4 twice as long as segment 3), dark scaled, speckled with white, and with small terminal segment white. Occiput clothed with pale lanceolate scales and numerous black erect forked scales. Thorax: Scutum covered by dark-brown lanceolate scales intermingled with light golden-brown lanceolate scales (the paler scales more numer-

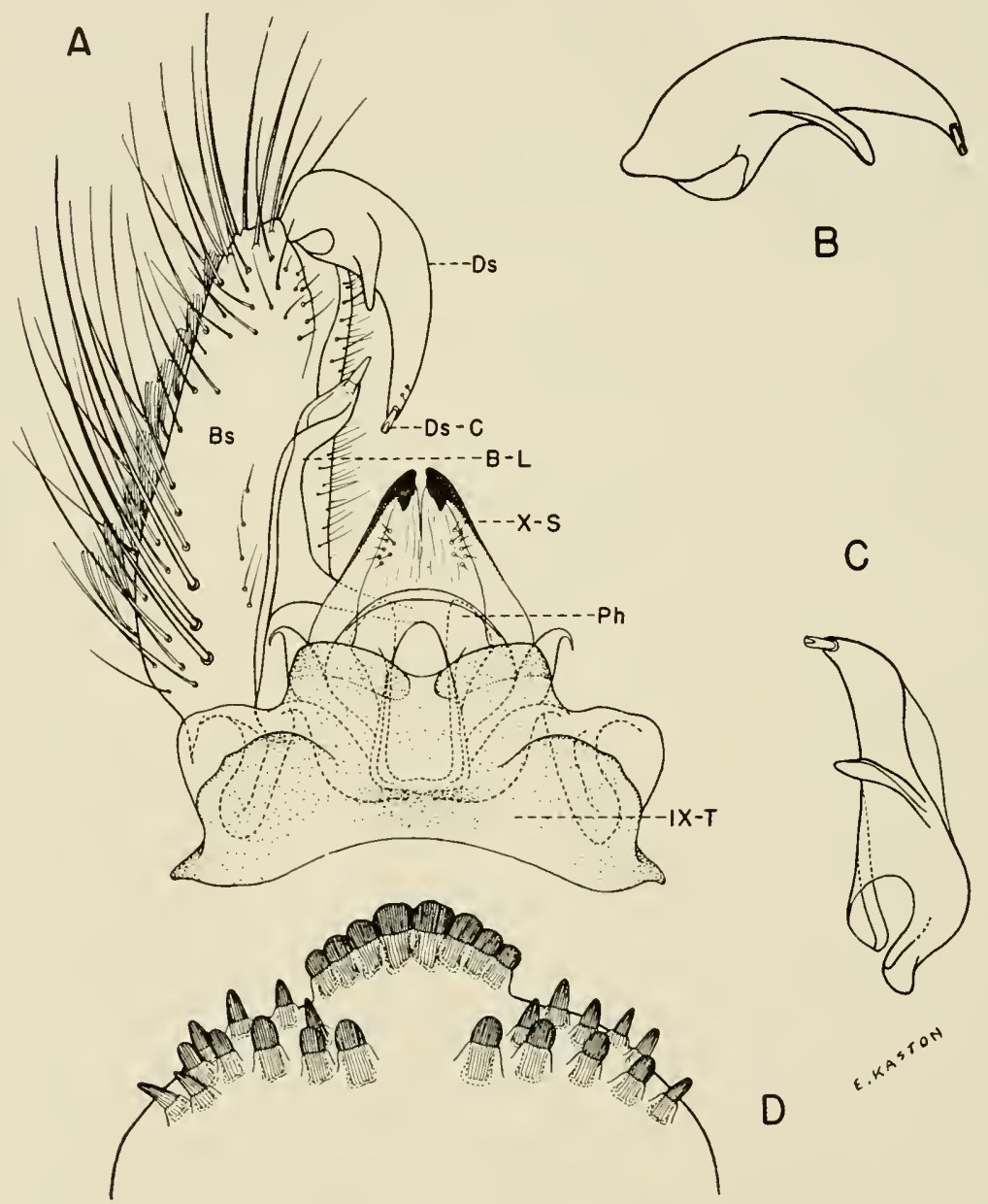

Fig. 63. Mansonia titillans (Walker). A, Male terminalia. B, C, Dististyle (different aspects). D. Eighth abdominal tergite of female. 
ous anteriorly and on prescutellar space). Postspiracular bristles present. Abdomen: First tergite pale scaled; remaining tergites predominantly dark scaled, with scattered yellow scales laterally, and yellow and white scales apically; sternites with intermixed white, yellow, and brown scales. Eighth segment blunt, largely retracted within the seventh; eighth tergite (Fig. 63D) with numerous short stout spines arranged in a curved posterior row and an irregular anterior row; middle of posterior row with six to nine closely set spines distinctly separated from the others in the row. Apical margin of seventh tergite with a close-set row of short stout pointed spines (much smaller than those of 8th tergite). Legs: Femora and tibiae of fore, middle and hind legs darkbrown scaled, speckled with pale scales; inner surfaces of femora II and III predominantly pale scaled. Tarsal segments 1 to 4 of fore and middle legs each with a narrow basal white band; all segments of hind tarsus with white basal bands. Wing: Scales mixed brown and white, very broad, intermingled with a few narrower scales, particularly on veins 3,4 , and 5 .

AdUlt Male.-Coloration similar to that of female. TERminalia (Fig. 63). Ninth tergite (IX-T) moderately sclerotized medially and laterally, remainder hyaline, with lobes indistinct. Tenth sternite (X-S) prominent, heavily sclerotized beyond middle; dorsal arm long, curved dorsally, weakly sclerotized. Phallosome (Ph) large, lightly sclerotized, open ventrally, closed dorsally near apex, expanded laterally and apically, broadly rounded at apex. Claspette absent. Basistyle (Bs) about three times as long as basal width, subcylindrical (width at apical fourth about equal to that across middle), rounded at apex; outstanding vestiture consisting of a group of strong setae at apex and several very long strong setae near base. Basal lobe (B-L) very long and slender, extending to apical fourth of basistyle; basal two-thirds of lobe narrow, stem-like; apical third swollen, bearing a short stout bluntly pointed terminal rod. Apical lobe absent. Dististyle (Ds) nearly half as long as basistyle, broad and stout at base, evenly curved and narrowed to tip, not convoluted, with a short stout pointed inner branch arising a little before middle; terminal claw (Ds-C) short, stout.

LARva. (Fig. 64).-Head much broader than long. Antenna about twice as long as head, whip-like, sparsely spined basally; antennal tuft multiple, barbed, arising from a notch on basal third; a pair of long stout setae inserted at middle of shaft and extending to tip of antenna; apex of antenna bearing a short spine, seta, and membranous papilla. Head hairs: Preantennal (A) mul. tiple, barbed; lower (B), upper (C), and postclypeal (d) short, multiple; sutural (e) double or triple; trans-sutural (f) multiple; supraorbital double or triple. Lateral spine of maxilla strongly serrated on one side. Prothoracic lateral hairs ( 5 to 7 ) as follows: 5 , double or triple; 6 , single; and 7 , single or double. Upper lateral abdominal hairs single on segments I to VI. Comb of eighth segment of about 7 or 8 scales in a single row, the scales becoming progressively much smaller ventrally; scales on dorsal portion of row long, spine-like, each with small lateral spinules near base. Subsiphonal hair long, 2 or 3-branched, barbed. Siphon short, sharply conical, strongly attenuated beyond middle; attenuated portion of siphon heavily sclerotized, bearing saw-like projections 
dorsally and stout hooks apically; a long stout recurved dorsal spine, a single stout dorso-lateral hair, and a multiple subventral tuft arising before the heavily sclerotized portion; pecten absent. Dorsal plate about three times as long as broad and completely ringing the anal segment; lateral hair multiple, inserted well before the posterior margin of the dorsal plate; dorsal brush consisting of a long multiple lower caudal tuft and a moderately long multiple upper caudal tuft on either side; ventral brush well developed, with about four small precratal tufts piercing the dorsal plate; gills 4 , shorter than the segment, bluntly pointed.

Distribution.-The distribution of this species is not well known. As pointed out by Pratt (135), many of the records of $M$. titillans recorded in the literature for the United States may be based on M. indubitans. Shannon (169) has indicated that many of the South American records were not $M$. titillans. Definite locality records for this species in Florida are given by Chamberlain and Duffey (38). It has also been recorded from Texas by McGregor and Eads (108). A single record of $M$. titillans from Alabama was reported by Middlekauff and Carpenter (125), but the specimen has been lost, thus making it impossible to determine its true identity.

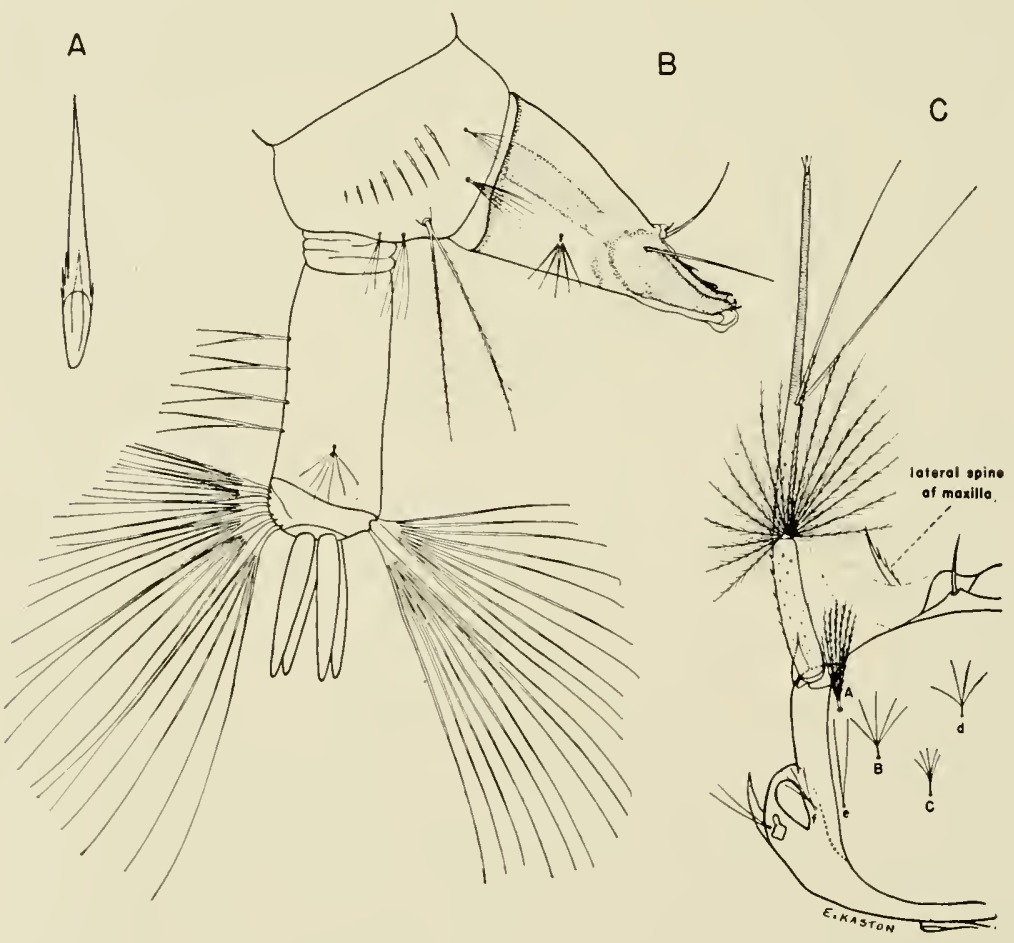

Fig. 64. Larva of Mansonia titillans (Walker). A, Comb scale. $B$, Terminal segments. C, Head. 
BIONOMICS. - The females are troublesome biters. The adults are frequently taken in light traps located near important sources of breeding. The eggs are laid on the leaves of water lettuce (Pistia), and the larvae attach themselves by means of their siphons to the roots of these plants, from which they obtain their air supply. The pupae also remain attached to the roots until time for emergence of the adults.

\section{Mansonia (Coquillettidia) perturbans (Walker)}

Culex perturbans Walker, 1856, Ins. Saund., Dipt., p. 428.

Adult female.-Moderately large species. Head: Proboscis dark, with a broad ring of pale scales medially; palpi abcut one-fifth as long as proboscis, dark scaled, speckled with a few pale scales (Fig. 65B). Occiput clothed with pale-gold lanceolate scales and numerous dark erect forked scales (a few forked scales on anterior portion of occiput pale). Thorax: Scutum covered by darkbrown lanceolate scales intermingled with light golden-brown lanceolate scales (the paler scales more numerous anteriorly, laterally, and on prescutellar space). Spiracular and postspiracular bristles absent. Abdomen: First tergite dark scaled; remaining tergites predominantly dark scaled, with white or paleyellow basal lateral patches and occasionally with narrow basal transverse bands of pale scales; sternites with intermixed dark and pale scales (the pale scales more numerous basally). Eighth segment bluntly rounded, not largely retracted within the seventh; eighth tergite lacking short, stout spines. Legs: Femora of fore, middle and hind legs dark, speckled with pale scales, with apices almost entirely dark scaled; femur III with a narrow subapical ring of pale scales, often indefinite; inner surfaces of femora II and III predominantly pale scaled on basal two-thirds. Tibiae I and II dark scaled, speckled with white, the apices narrowly ringed with white scales; tibia III dark scaled, speckled with white, ringed with white scales at outer third and at apex. First tarsal segment of all legs with a narrow white ring basally and a broader white ring a little beyond middle; each of the remaining tarsal segments with basal half white, apical half dark (Fig. 65A). Wing: Scales very broad, mixed dark and white, the dark scales predominating.

ADULT MALE.-Coloration similar to that of female. TERMinalia (Fig. 65C). Ninth tergite (IX-T) with dorsal band rather narrow; lobes (IXT-L) narrow, about as long as wide, sclerotized, widely separated, each bearing several short, stout setae. Tenth sternite (X.S) prominent, heavily sclerotized; dorsal arm (XS.DA) large, curving considerably dorsad to a position just beneath lobe of ninth tergite. Phallosome ( $\mathrm{Ph}$ ) large, heavily sclerotized, twothirds as broad as long, open dorsally and ventrally, constricted at middle, and slanting suddenly from shoulder at apical third to a toothed apex; each plate with a sinuous longitudinal ventral row of short, heavily sclerotized teeth directed ventrally. Claspette absent. Basistyle (Bs) about one and one-half times as long as wide, bluntly conical, clothed with numerous broad scales and long setae; basal lobe (B-L) flat, triangular, fused to basal half of basistyle, and with a thick, blunt, sclerotized rod and a smaller stout spine, arising from its apex; apical lobe absent. Dististyle (Ds) curved, about two-thirds as long 
as basistyle, enlarged at base, constricted and slender to middle, broadly expanded beyond middle, then tapering to a point and terminating in a short stout claw (Ds-C).

Larva. (Fig. 66).- - Head much broader than long. Antenna about twice as long as head, whip-like, sparsely spined basally; antennal tuft multiple, barbed, arising from a notch on basal third; a pair of short, stout setae inserted at middle of shaft; a short spine, seta, and membranous papilla at tip. Head hairs: Preantennal (A) multiple, barbed; lower (B) multiple, barbed, reaching anterior margin of head; upper (C) small, multiple, smooth; postclypeal (d) small, multiple; sutural (e), trans-sutural (f) and supraorbital multiple. Lateral spine of maxilla smooth. Upper lateral abdominal hairs single on seg-

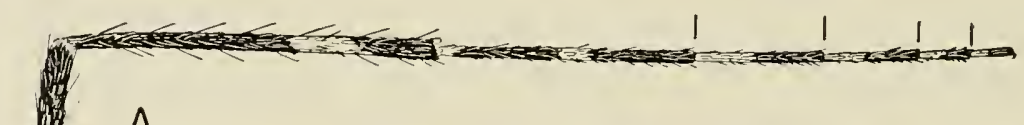

A

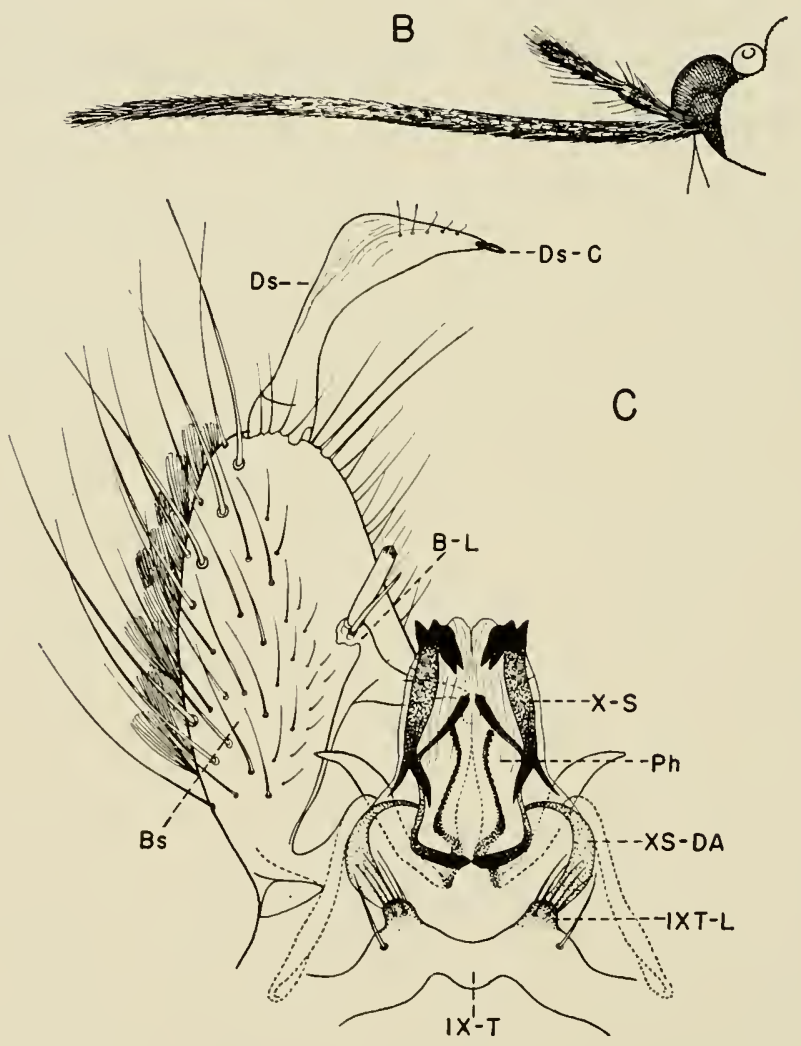

Fig. 65. Mansonia perturbans (Walker). A, Hind leg. B, Palp and proboscis of female. C. Male terminalia. 
ments I to VI. Comb of eighth segment of about 9 to 15 thorn-shaped scales in a single irregular row; individual scale elongate, pointed, fringed on basal half with short spinules. Subsiphonal hair long, 2-branched, barbed. Siphon short, sharply conical, strongly attenuated beyond middle; pecten absent, attenuated portion of siphon heavily sclerotized, bearing saw-like projections dorsally and stout hooks apically; arising before the heavily sclerotized portion of the siphon are a long stout recurved dorsal spine, a single stout dorso-lateral hair, and a multiple subventral tuft. Dorsal plate one and one-half to two times as long as broad and completely ringing the anal segment; lateral hair multiple, inserted well before the posterior margin of dorsal plate; dorsal brush consisting of a multiple lower caudal tuft and a shorter multiple upper caudal tuft on either side; ventral brush well developed, usually restricted to the barred area, but often with one or two very small precratal tufts piercing the dorsal plate; gills 4 , shorter than the segment, bluntly pointed.

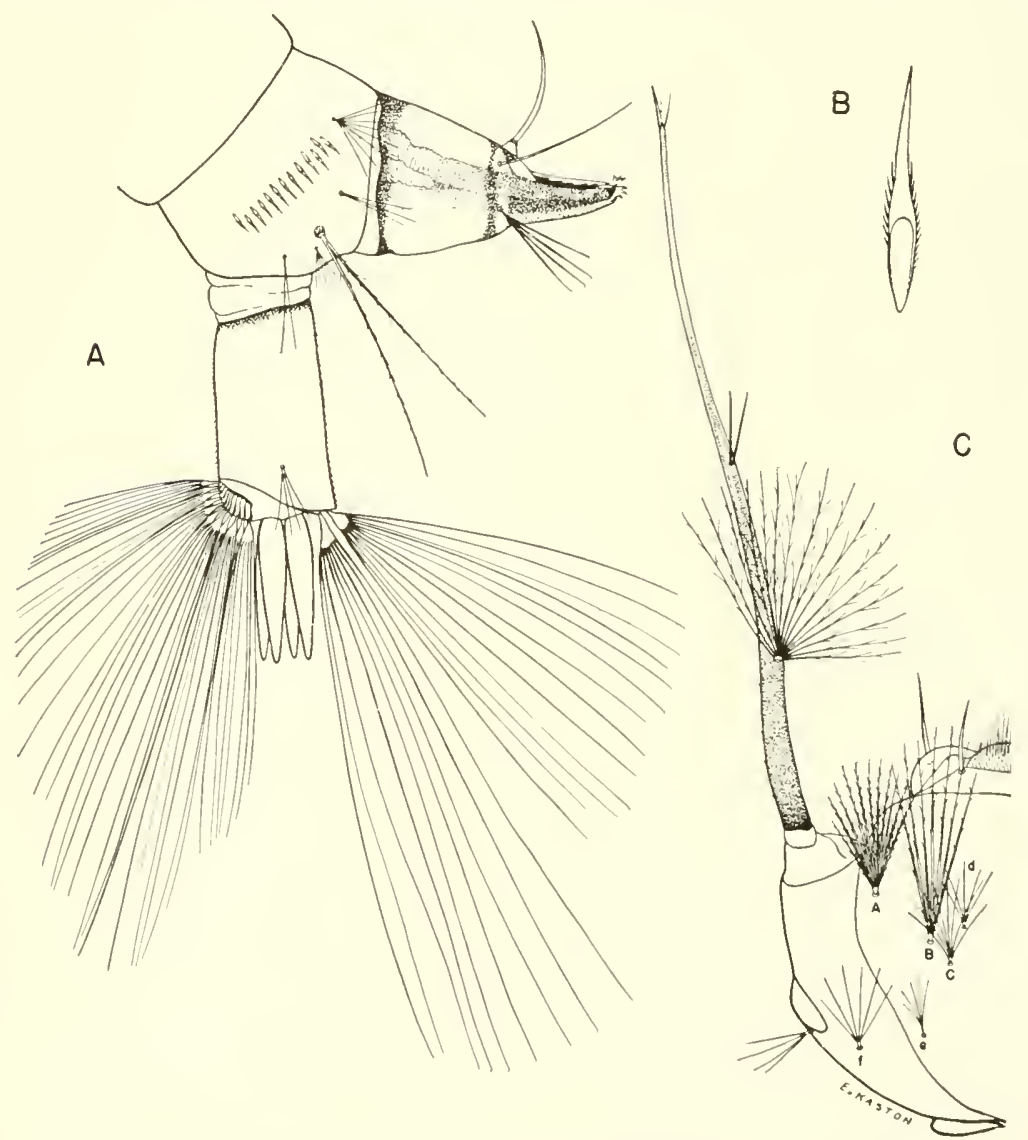

Fig. 66. Larva of Mansonia perturbans (Walker). A, Terminal segments. B, Comb scale. C. Head. 
Distribution.-Throughout the United States and Southern Canada. It also occurs in Mexico (114) and in Europe. Southern States: Alabama (170); Arkansas (30); Florida and Georgia (52); Kentucky (140); Louisiana (52); Mississippi (97); Missouri (70); North Carolina (166); South Carolina and Tennessee (96); Virginia (49). Other States: California (67); Connecticut (52); Delaware (106); District of Columbia (52); Illinois (42); Indiana (39); Iowa $(155,156)$; Kansas (128); Maine, Maryland and Massachusetts (52); Michigan (88); Minnesota (131); Montana (112); Nebraska (178); New Hampshire, New Jersey, New York, and Ohio (52); Oklahoma (159); Pennsylvania (8); Rhode Island (99); Texas (108); Utah (143); Vermont (90); Wisconsin (48).

BIONOMICs.-Mansonia perturbans is primarly a night-biting mosquito, most active during the early part of the night. Females occasionally feed during daylight hours in shady places when their haunts are invaded. The adults are strong fliers. They are troublesome pests in some localities and readily enter human habitations. The females lay their eggs on the surface of the water in areas containing a heavy growth of aquatic vegetation. After hatching, the larvae descend to the bottom and attach themselves to the roots or stems of plants by means of the modified siphon. The pupae also remain attached to the plants by means of their respiratory trumpets until the adults are ready to emerge, when they detach themselves and rise to the surface. The larvae develop slowly and the winter is passed as immature or mature larvae, thus emergence of the adults takes place in the spring and summer. The larvae usually drop off their host plants when disturbed.

Methods of collecting larvae of $M$. perturbans are described by McNeel (109) and may be briefly summarized as follows: Pull up the host plant from a small area and lay them aside, scoop up muck and debris from the area from which the plants were removed and pour through a strainer. Transfer the material in small quantities from the strainer to clear water in a white enamel tray and examine carefully for the presence of larvae.

\section{Genus Psorophora Robineau-Desvoidy 1}

Psorophora Robineau-Desvoidy, 1827, Mem. Soc. d'Hist. Nat., 3:412.

The genus Psorophora is widespread throughout North, Central, and South America and nearby islands, and although it is more common in the tropics, it extends well into the temperate regions. The females are vicious biters, but little is known of their importance as vectors of human and animal diseases. The larvae are found in temporary rain-filled and floodwater pools. The development of the larvae is usually rapid. In general the eggs are able to withstand long periods of drying. The genus is represented in the United States by eleven species, belonging to the subgenera Psorophora, Janthinosoma and Grabhamia.

SALIENT CharaCters. - Adult: Spiracular and postspiracular bristles present. Postnotum without setae. Squama fringed. Fork of vein 2 much shorter than

1 Consult Dyar (1928), Edwards (1932) or Matheson (1944) for synonymy. 
its petiole. Tip of abdomen more or less pointed, the eighth segment retractile. Male Terminalia: Phallosome variable in the different subgenera. Claspette and dististyle very diversified in form. Tenth sternite similar to that of Aedes. Basal and apical lobes of basistyle absent. Larva: Anal segment completely ringed by the dorsal plate; the dorsal plate pierced on the mid-ventral line for nearly its entire length by tufts of the ventral brush. Siphon with a single pair of subventral tufts, never inserted basally, often reduced in size. Comb of eighth segment present. Mouth brushes prehensile in the subgenus Psorophora. Antenna long in the subgenera Janthimosoma and Grabhamia.

\section{Keys to THE SPECIES}

\section{ADULT FEMALES}

1. Wings with mixed dark and white scales; hind femur with a narrow subapical ring of white scales, more or less distinct (subgenus Grabhamia)

Wings entirely dark scaled or with only a few inconspicuous white scales distributed on costa and subcosta; hind femur without a subapical ring of white scales.... 5

2. Segment I of hind tarsus with a white ring at middle or largely pale

Segment I of hind tarsus entirely dark scaled, except for a few pale scales in a narrow basal ring pygmaca (Theob.), p. 160

3. Wings speckled with white scales in no definite arrangement; segment 1 of hind tarsus ringed at base and at middle with white scales ....confinnis (L. Arr.), p. 153

Wings with definite areas of white and dark scales; segment I of hind tarsus largely pale scaled

4. Apical half of costa with two conspicuous spots of dark scales separated by a longer area of white scales; apical third of vein 6 white-scaled; scales of wing fringe arranged in alternately dark and pale groups

Costa with a streak of white scales at base and at poin! of union with subcostal vein, the remainder of costa dark, speckled with white; apical third of vein 6 dark scaled; scales of wing fringe uniformly dark ............. discolor (Coq.), p. 156

5. Hind legs, including apices of femora, very shaggy, with long erect scales; 5 th segment of hind tarsus never entirely white; scutum with smooth nude areas anteriorly and submedially; very large species (subgenus Psorophora)

Hind legs not markedly shaggy, the apices of femora without erect scales (if tibiae are somewhat shaggy, the 5 th segment of hind tarsus is entirely white scaled); scutum without smooth nude areas, although sometimes sparsely scaled; moderate sized species (subgenus Janthinosoma)

6. Scutum with a narrow median longitudinal stripe of golden scales; proboscis yellow scaled at outer third, dark at tip ............................iliata (Fabr.), p. 133

Scutum with only dark scales medially, without a longitudinal stripe of golden scales; proboscis entirely dark scaled howardii Coq., p. 136

7. Hind tarsi enturely dark scaled; abdominal segments apically with submedian triangular patches of golden scales ...................................escens (Coq.), p. 139

Hind tarsi with white markings on apical segments; pale scaling of abdominal segments restricted to lateral apical corners or narrow apical margin, never in submedian triangular patches egmert 4 of hind tarsus white scaled, at least on one side, segment 5 dark; scutum with a broad median longitudinal stripe of dark scales, dingy white or yellowish scaled laterally ................................. 15

Both segments 4 and 5 of hind tarsus white scaled, and often the tip of segment 3; scutum either with or without a median longitudinal dark stripe ......................9

9. Scutum uniformly covered with mixed dark-brown and golden-yellow scales ....... ferox (Humb.), p. 142

Scutum with a broad median longitudinal stripe of dark bronzy-brown scales, broadening posteriorly, pale-yellow or dingy-white scaled laterally 
10. Pale knee spots present; palpus less than $1 / 3$ as long as proboscis, with segment 4 about equal in length to the first three segments combined

horrida (D. and K.), p. 145

Pale knee spots absent; palpus a little more than $1 / 3$ as long as proboscis, with segment 4 more than $11 / 2$ times as long as the first three segments combined....

longipalpis Roth, p. 147

\section{MALE TERMINALIA}

1. Apical half of phallosome with a pair of submedian longitudinal toothed ridges dorsally and a pair of thorn-like projections laterally

Phallosome cylindrical or conical, without teeth or lateral thorn-like projections .......3

2. Dististyle stout, with a very large lateral hatchet-shaped lobe projecting mesad

Dististyle slender, with a small dorsal angular projection at distal fifth howardii Coq., p. 137 ciliata (Fabr.), p. 134

3. Phallosome conical, the apical half with a lateral flange and a mid-dorsal basally divaricated carina; crown of claspette with about nine elongated flattened pointed filaments and several feathered setae ........cyanescens (Coq.), p. 139

Phallosome conical or cylindrical, without a lateral flange or divaricated carina; crown of claspette not as above

4. Dististyle broadly truncate at apex; claw of dististyle inserted subapically varipes (Coq.), p. 151

Dististyle not truncate at apex; claw of dististyle inserted apically

5. Basistyle large, subspherical, about $11 / 2$ times as long as wide; dististyle constricted basally, strongly swollen and rounded apically ......longipalpis Roth, p. 148

Basistyle at least twice as long as broad, cylindrical; dististyle tapered apically to a narrow tip

6. Claspette slender, about $3 / 4$ as long as basistyle, with two contorted pointed leaflike filaments at tip and numerous feathered setae on inner margin of apical fourth

Claspette stem slender, expanded into a broad crown apically, not extending beyond middle of basistyle; crown bearing 5 to 7 narrow pointed blades or setae

7. Dististyle expanded beyond middle, broadest at apical third

Dististyle broadest at middle

pygmaea (Theob.), p. 161

8. Claspette with about 5 or 6 seta-like filaments on crown

signipennis (Coq.), p. 164

Claspette with 5 to 7 long pointed apically feathered blades and a single apically feathered seta

9. Inner ventral margin of each plate of phallosome with a distinct triangular projection turned ventro-laterally; claspette bearing 5 or 6 blades and a single apically feathered seta ...................................................... p. 159

Inner ventral margin of each plate of phallosome with only a narrow rounded portion turned ventrad lacking a distinct triangular projection; claspette bearing 6 or 7 blades and a single apically feathered seta

confinnis (L. Arr.), p. 154

\section{LARVAE（FOURTH INSTAR)}

1. Pecten teeth numerous (about 18 or more), each terminating in a hair-like filament; subventral tuft of siphon represented by a long single hair; mouth brushes of very stout filaments strongly pectinate on one side

Pecten teeth few (less than 10), not prolonged into hair-like filaments; subventral tuft of siphon multiple, either large, small or obsolete, never long and single; mouth brushes normal hair-like, smooth or very weakly pectinate .............. 3

2. Lateral hair of anal segment 3 or 4-branched near base .........ciliata (Fabr.), p. 135

Lateral hair of anal segment single or forked beyond middle ....howardii Coq..p. 138 
3. Subventral tuft of siphon very large, multiple, as long as siphon; siphon small, not inflated, with a pair of strong dorsal reflexed spines subapically; antennae inflated discolor (Coq.), p. 159

Subventral tuft of siphon multiple, very small or obsolete; siphon large, more or less inflated medally, without a pair of strong dorsal reflexed spines subapical$\mathrm{ly}$; antennae not inflated

4. Upper head hair (C) and lower head hair (B) multiple confinmis (L. Arr.), p. 155

Upper head hair (C) single or double, lower head hair (B) single, double or triple

(B) single, double or

5. Upper head hair (C) and lower head hair (B) single; antennae no longer than the head

Upper head hair (C) double, lower (B) double or triple, antennae usually longer than head.

air of lateral valve of siphon more than half as long as apical width of siphon

Hair of lateral valve of siphon less than half as long as apical width of siphon

7. Antennal and preantennal tufts strongly multiple (antennal tuft generally 8 to 15-branched, preantennal tuft about 6 to 8-branched), conspicuously barbed; antenna apically with a short spine, a moderately long spine and a membranous papilla, and subapically with 2 longer spines; comb with about 6 scales signipennis (Coq.), p. 164

Antennal and preantennal tufts only about 2 to 4 -branched, occasionally rebranched toward tip, sparsely barbed; antennal tip with 3 long spines, one short spine and a membranous papilla, all apical in position; comb with 4 scales, rarely 5 ........................... 140

8. Siphon only slightly inflated; subapical spine of largest comb scale only about $1 / 5$ to $1 / 4$ as long as apical spine ....................................... p. 152

Siphon strongly inflated; subapical spines of largest comb scale about $1 / 3$ as long as apical spine

9. Lower head hair (B) triple, sometimes double, not reaching preclypeus, the branches nearly equal; upper head hair (C) double, not reaching preclypeus, the branches nearly equal .................... 146

Lower and upper head hairs (B and $C$ ) double, reaching to or beyond preclypeus

10. Lateral abdominal hairs single or double on segments IV to VI; branches of lower head hair (B), as well as upper head hair (C), nearly equal

ferox (Humb.), p. 143

Lateral abdominal hairs multiple on segments IV to VI; branches of lower head hair (B), as well as upper head hair (C), not equal, one branch of each being shorter and weaker than the other longipalpis Roth, p. 150

\section{PSOROPHORA (PSOROPHORA) CILIATA (Fabricius)}

Culex ciliata Fabricius, 1794, Ent. Syst., 4:401.

ADULT FEMALE.-Very large species. Head: Integument of proboscis yellow, becoming dark at apical fourth; scales on basal half long, dark, suberect; scales beyond middle of proboscis appressed, yellow, speckled with dark, those at tip entirely dark. Palpi about one-third as long as proboscis, clothed with dark suberect scales. Occiput white scaled, with a narrow longitudinal nude stripe. Thorax: Integument of scutum dark brown; vestiture consisting of a narrow median longitudinal stripe of bright golden-yellow lanceolate scales, a submedian longitudinal line of golden-yellow lanceolate scales adjacent to a line of slender black scales, and a large lateral area of broad appressed pale-yellow to 
white scales; a narrow nude stripe present between the median stripe and submedian lines; a broader nude area present on either side of the prescutellar space (prescutellar space white-scaled, bisected partially or entirely by a median gold stripe). Abdomen: Tergites pale yellow to brown scaled, the pale scales predominating dorsally, the brown laterally; sternites primarily white scaled. Legs: Femora yellow scaled, speckled with dark, except for apical portion of each, which is densely clothed with long dark erect scales; tibiae each with a narrow ring of yellow appressed scales basally, remainder clothed with long dark erect scales. Tarsi of fore and middle legs clothed with appressed scales; segments 1 to 3 pale basally, dark apically; segments 4 and 5 entirely dark. Each segment of hind tarsus with a ring of pale appressed scales basally. Scales beyond basal ring long, dark, erect on segments 1 and 2, dark, recumbent on segments 3 to 5 (Fig. 67A). Wing: Scales narrow, dark; a few inconspicuous pale scales distributed on costa and subcosta.

ADULT MALE.-Coloration similar to that of female. TERMinalia (Fig. 67B). Ninth tergite (IX-T) with well-separated, rounded lobes (IXT-L) bearing numerous setae. Tenth sternite (X-S) prominent, heavily sclerotized.
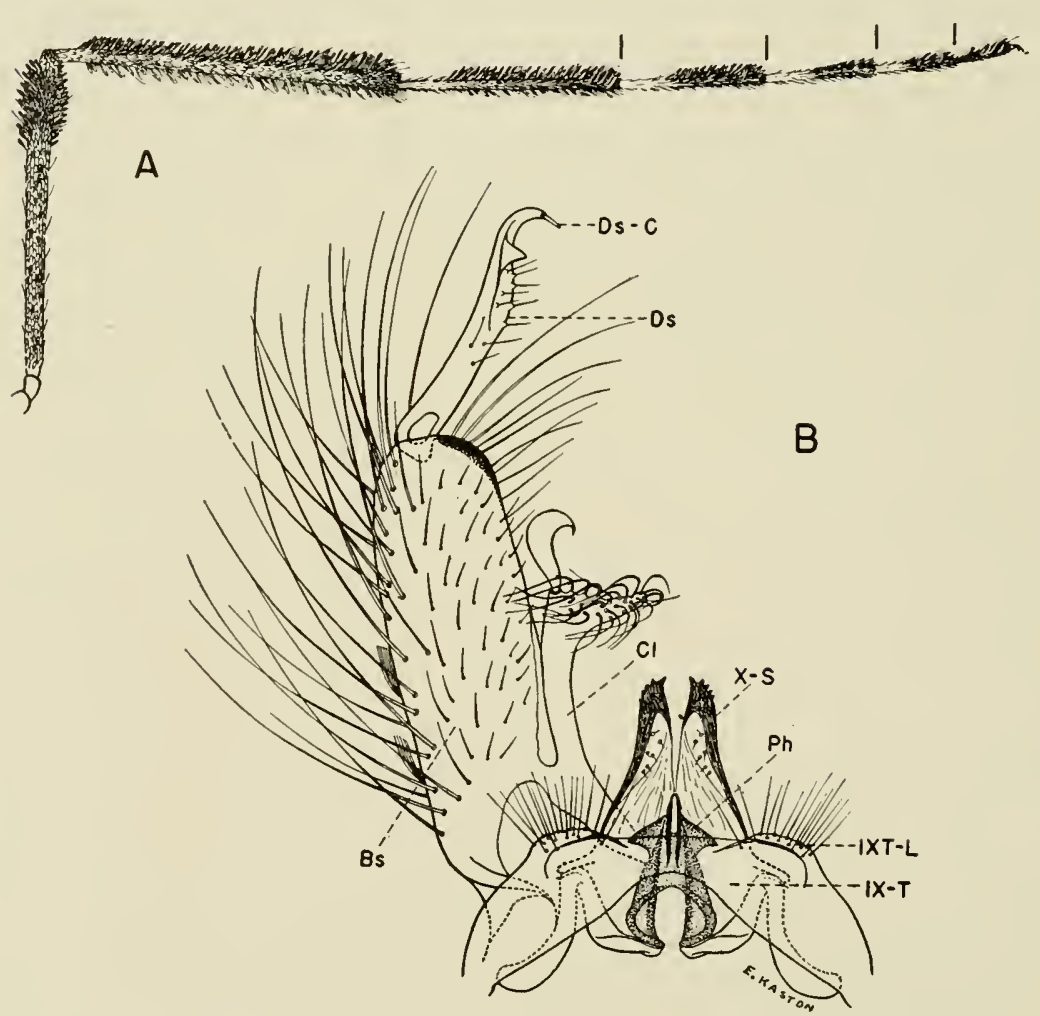

Fig. 67. Psorophora ciliata (Fabricius). A, Hind leg. B, Male terminalia. 
Phallosome $(\mathrm{Ph})$ heavily sclerotized, conical, open ventrally, closed dorsally on basal half; apical half of each plate with a broad lateral thorn-like projection and a dorsal longitudinal toothed ridge. Claspette $(\mathrm{Cl})$ extending to apical third of basistyle, stem slender; apex with a broad glabrous sickle-shaped filament inserted on outer angle, and a strong fleshy thumb-like projection directed mesad, clothed with numerous flexible setae. Basistyle (Bs) cylindrical, rounded apically, about two and one-half times as long as broad, clothed with sparse scales and numerous long setae; basal and apical lobes absent. Dististyle (Ds) about two-thirds as long as basistyle, somewhat bowed inward medially, tapering at outer third, with short stout setae arising from inner surface; a dorsal angular projection present at outer fifth; tip curved and terminating in a short, strong claw (Ds-C).

LARVA. (Fig. 68).-Large larva. Head quadrate, concave anteriorly, broader than long. Antenna about one-third as long as head, extending near anterior margin of head, sparsely spined; antennal tuft represented by a single short hair on outer third. Mouth brushes formed of stout prehensile hairs, each

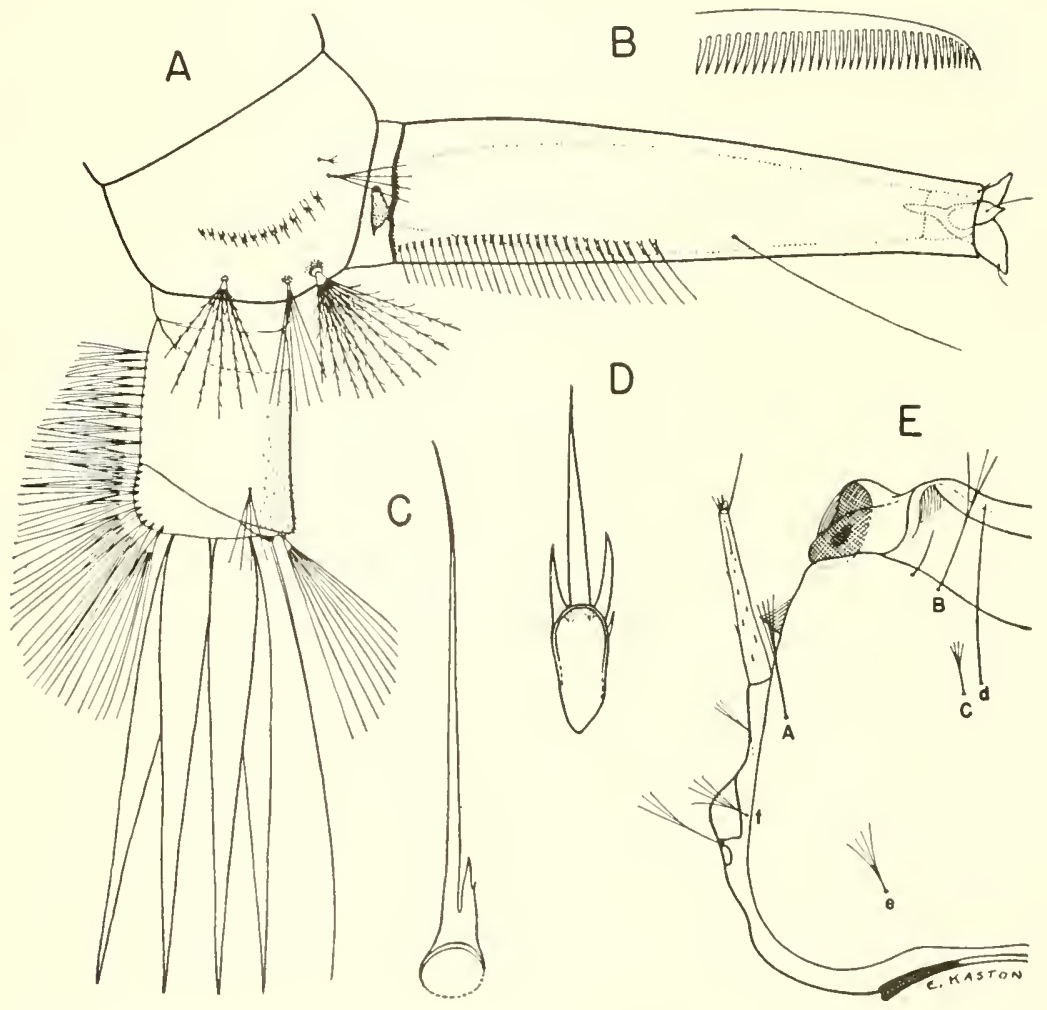

Fig. 68. Larva of Psorophora ciliata (Fabricius). A, Terminal segments. B, Mouth Brush. C, Pecien spine. D, Comb scale. E. Head. 
hooked at tip and with a row of comb-like teeth along the side. Head hairs: Hair $A$ single, branched distally; hair $B$ single, branched distally, inserted near anterior margin of clypeus, expanding beyond anterior margin of head; hair $C$ short, branched distally; hair $d$ long, single, inserted mesad and slightly anterior to hair $\mathrm{C}$, extending beyond anterior margin of head; sutural (e) and trans-sutural (f) multiple; supraorbital branched distally. Comb of eighth segment of about 12 to 16 thorn-shaped scales in a single curved row; individual scale trifid, with basal spines about one-third the length of the median spine. Siphon three to four times as long as basal width; pecten of numerous teeth on basal half of siphon; individual tooth long, hair-like, with one or more short stout basal teeth; subventral tuft represented by a long single hair inserted beyond pecten. Anal segment longer than wide, completely ringed by the dorsal plate; lateral hair 3 to 4-branched near base; dorsal brush consisting of a long lower caudal hair and a shorter multiple upper caudal tuft on either side; ventral bush extending almost the entire length of the anal segment; gills 4, longer than the segment, sharply pointed.

Distribution.-Southern Canada; Eastern United States west to Nebraska and Texas. Southern States: Alabama (170); Arkansas (30); Florida and Georgia (52); Kentucky (140); Louisiana and Mississippi (52); Missouri (1); North Carolina (166); South Carolina (64); Tennessee (96); Virginia (49). Other States: Connecticut (52); Delaware (106); District of Columbia (52); Illinois (42); Indiana (76); Iowa $(155,156)$; Kansas (79); Maryland (19); Massachusetts (52); Michigan (88); Nebraska (178); New Hampshire (105); New Jersey (77); New York (52); Oklahoma (159); Pennsylvania (8); Rhode Island (99); Texas (108); West Virginia (52); Wisconsin (48).

вгоNомics.-The females are vicious biters, attacking any time during the day. The larvae occur in temporary rain-filled pools where they feed on the larvae of other associated species, particularly $P$. confinnis. Psorophora ciliata breeds from March to October in the extreme South and from May to September farther north (4th Sv. C. Med. Lab. records, 1942-1944, unpublished).

\section{PSOROPHORA (PSOROPHORA) HOWARdil Coquillett}

Psorophora howardii Coquillett, 1901, Can. Ent., 33:258.

ADULT FEMALE.-Very large species. Head: Integument of proboscis brown, becoming darker apically; all scales dark, recumbent. Palpi nearly half as long as proboscis; scales dark, recumbent. Occiput white scaled, with a narrow longitudinal nude stripe. Thorax: Integument of scutum shiny, dark brown (often nearly black); vestiture consisting of a narrow median longitudinal stripe of slender black scales, a narrow submedian longitudinal stripe of similar scales, and a large lateral area of broad appressed white scales; a narrow nude stripe present between the median and submedian stripes; a broader nude area present on either side of the white-scaled prescutellar space. Abdomen: First tergite white scaled; remaining segments blue-black scaled dorsally, each with an apical transverse line of white scales; lateral margin and venter white scaled. Legs: Femora yellow scaled, speckled with dark, except for apical portion of each which is clothed with long dark erect or suberect scales (most conspicuous 
on middle and hind legs); tibiae mainly covered by blended yellow and purple recumbent scales, with those of apices dark purple, suberect, particularly on hind legs. Tarsi primarily dark scaled blended with yellow; second segment and often first segment of each tarsus with a narrow basal pale ring; segments 3, 4, and 5 dark. Wings: Scales narrow, dark.

ADULT MALE.-Coloration similar to that of female. TERminalia (Fig. 69). Ninth tergite (IX-T) with dorsal band narrow, heavily sclerotized; lobes (IXT-L) narrow, heavily sclerotized, widely separated, each bearing a variable number of stout spine-like setae. Tenth sternite (X-S) prominent, heavily sclerotized. Phallosome $(\mathrm{Ph})$ heavily sclerotized, conical, open ventrally, closed dorsally on basal half; apical third of each plate with a broad lateral thorn-like projection and a dorsal longitudinal toothed ridge. Claspette $(\mathrm{Cl})$ extending near apical third of basistyle, consisting of a short broad stem fused with basistyle, and a very large subspherical crown; crown entirely covered with short curved setae and bearing subapically a large, erect sickle-shaped filament on outer margin. Basistyle (Bs) about twice as long as basal width, curved, narrower beyond middle, and bluntly rounded at apex, clothed with sparse scales and long setae; basal and apical lobes absent. Dististyle (Ds) about half as

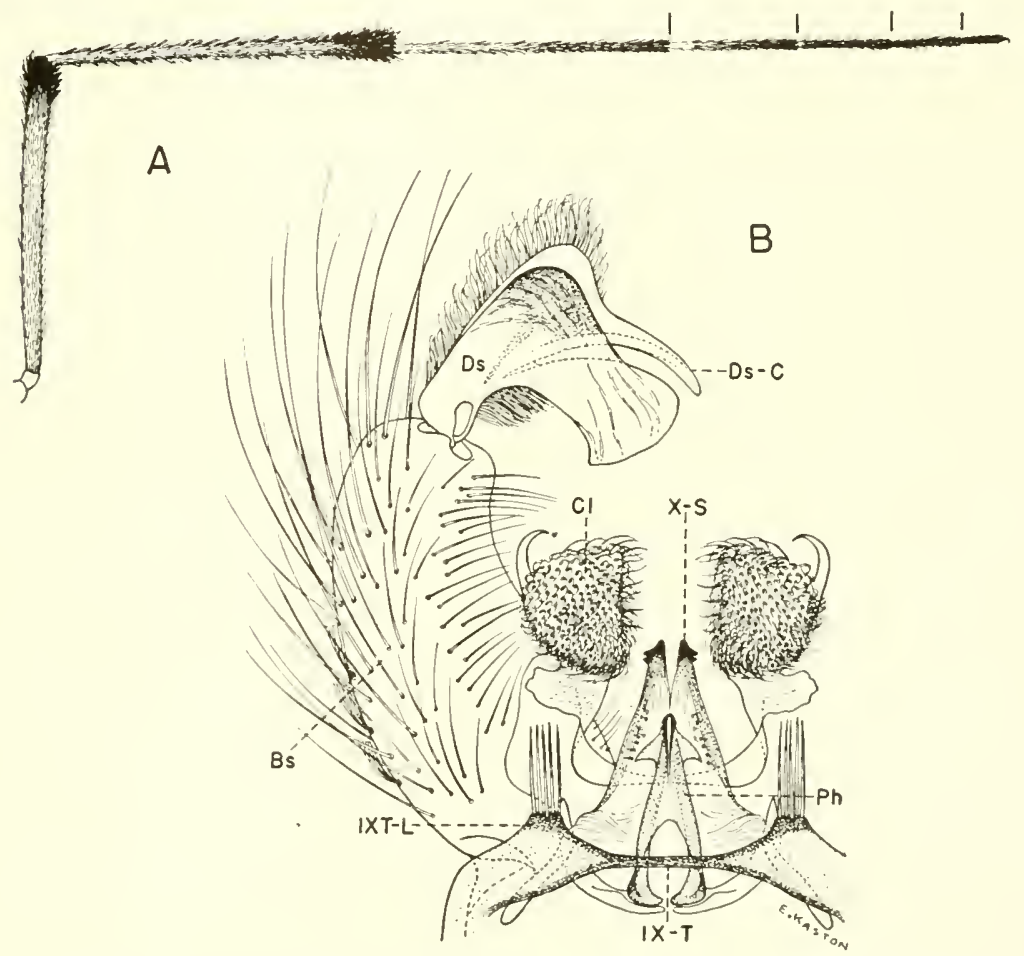

Fig. 69. Psorophora howardii Coquillett. A, Hind leg. B, Male terminalia. 
long as basistyle, densely setiferous ventrally, with a very large lateral hatchetshaped lobe projecting mesad, closely followed by a long slender subapical projection, also directed mesad; claw (Ds-C) inconspicuous, inserted on this latter structure slightly before apex.

LARVA (Fig. 70).-Large larva. Head quadrate, concave anteriorly, broader than long. Antenna about one-third as long as head, extending near anterior margin of head, sparsely spined; antennal tuft represented by a single hair on outer third. Mouth brushes formed of stout prehensile hairs, each hooked at tip and with a row of comb-like teeth along the side. Head hairs: Hair $A$ single, branched distally; hair $B$ single, branched distally, inserted near anterior margin of clypeus, extending beyond anterior margin of head; hair $C$ short, branched distally; hair $d$ long, single, inserted mesad and slightly anterior to hair $\mathrm{C}$, extending beyond anterior margin of head; sutural (e) and transsutural (f) multiple; supraorbital branch :d distally. Comb of eighth segment

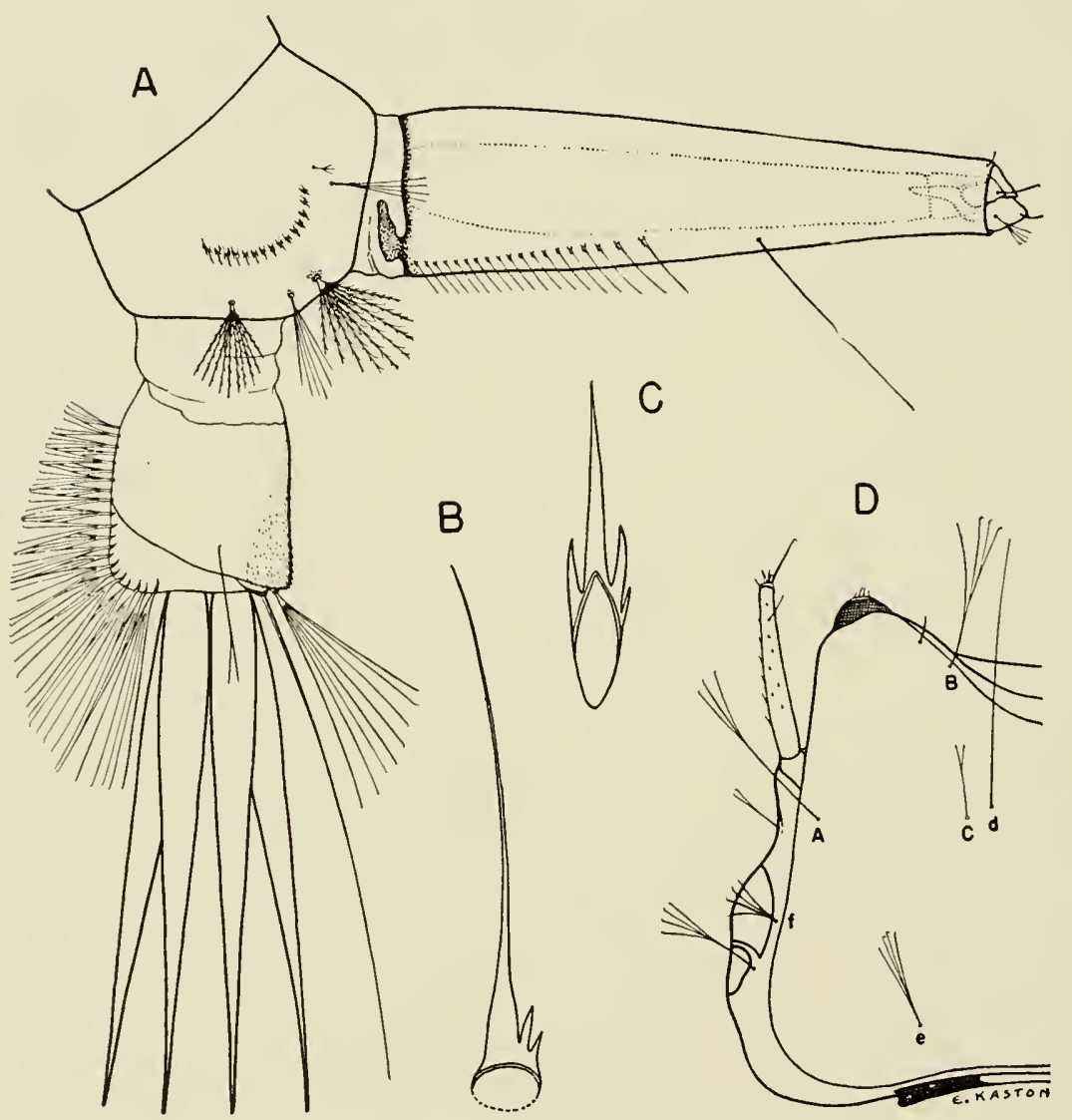

Fig. 70. Larva of Psorophora howardii Coquillett. A, Terminal segments. B, Pecten spine. C, Comb scale. D. Head. 
of about 15 to 18 thorn-shaped scales in a single curved row; individual scale trifid with basal spines about one-third the length of the median spine. Siphon about four times as long as basal width; pecten of numerous teeth on basal half of siphon; individual tooth long, hair-like, with one or more short stout basal teeth; subventral tuft represented by a single long hair inserted beyond pecten. Anal segment longer than wide, completely ringed by the dorsal plate; lateral hair single or branched beyond middle; dorsal brush consisting of a long lower caudal hair and a shorter multiple upper caudal tuft on either side; ventral brush extending almost the entire length of the anal segment; gills 4, longer than the segment, pointed.

distribution.-Southern United States; Mexico, West Indies and Central America. Southern States: Alabama (125); Arkansas (30); Florida (52); Georgia (148); Kentucky (140); Louisiana and Mississippi (96); Missouri (1) ; North Carolina (166); South Carolina (64); Tennessee (170); Virginia (49). Other States: District of Columbia (52); Illinois (42); Indiana (76); Kansas (128); Maryland (52); Oklahoma (159); Texas (108).

BIONOMICS. - The females are fierce biters and will attack any time during the day when their haunts are invaded. The larvae of $P$, howardii occur in temporary rain-filled pools. They are predacious, feeding on other mosquito larvae with which they are associated. This species breeds from March to October in the extreme South and from May to September farther north (4th Sv. C. Med. Lab records, 1942-1944, unpublished).

\section{Psorophora (Janthinosoma) Cyanescens (Coquillett)}

Culex cyanescens Coquillett, 1902, Jour. N. Y. Ent. Soc., 10:137.

Adult female.-Medium sized to rather large species. Head: Proboscis slender, blue-black; palpi short, black. Occiput clothed dorsally with ovate curved white to pale-gold scales and numcrous pale erect forked scales; occiput laterally with a small blue-black scaled patch, followed by broad yellowish scales. Thorax: Integument of scutum black, rather evenly clothed with small scales, those of the median regior intermixed lanceolate and ovate, mostly pale yellow to gold or golden-brown, those of the lateral areas ovate, gold to pale yellow or white. Scales of posterior pronotum similar to those of the anterolateral portion of scutum. Abdomen: First tergite whitish scaled; remaining tergites black scaled, with blue or purplish reflection, and with apical submedian triangular patches of golden-yellow scales; these apical patches narrow laterally, broadened toward the mid-dorsal line, those of the anterior segments usually joined medially. Sternites II to VI whitish-yellow to yellow scaled, VII dark. Legs: Small spots present on middle and hind legs; inner surfaces of all femora pale scaled, darker near apices; outer surface of femur I pale on basal half, femur III pale on basal three-fourths. Scales of tibiae and tarsi entirely black with blue to purple reflection, appressed or recumbent. Wing: Scales narrow, dark.

ADUlT MALE.-Coloration similar to that of female. TERMinalia (Fig. 71). Ninth tergite (IX-T) with lobes (IXT-L) broadly rounded, widely sep- 
arated, each bearing several scattered setae. Tenth sternite (X-S) prominent, heavily sclerotized. Phallosome $(\mathrm{Ph})$ heavily sclerotized, conical, broad at base, narrowly rounded at apex, closed dorsally, open ventrally; apical half with a lateral flange and a mid-dorsal longitudinal basally divaricated carina. Claspette $(\mathrm{Cl})$ (exclusive of the apical filaments) reaching middle of basistyle or slightly beyond; stem slender, curved, its mid-portion fused to the basistyle, expanded apically into a broad triangular crown. Crown with about nine elongated pointed flattened filaments (filaments nearest basistyle larger, curved), and several feathered setae. Basistyle (Bs) nearly two and one-half times as long as broad, cylindrical, bluntly rounded at apex, clothed with sparse scales and numerous long setae; basal and apical lobes absent. Dististyle (Ds) reticulated, about three-fifths as long as basistyle, moderately expanded a little beyond middle (expanded inner margin pubescent and with four or five short spines), tapering distally, slender and curved near tip and terminating in a peg-like claw (Ds-C).

LARVA. (Fig. 72).-Head broader than long. Antenna stout, shorter than head, coarsely spined; antennal tuft small, 2 to 4-branched, sparsely barbed, inserted slightly beyond middle of shaft; tip of antenna with three long spines, one short spine and a membranous papilla, all apical in position. Head hairs:

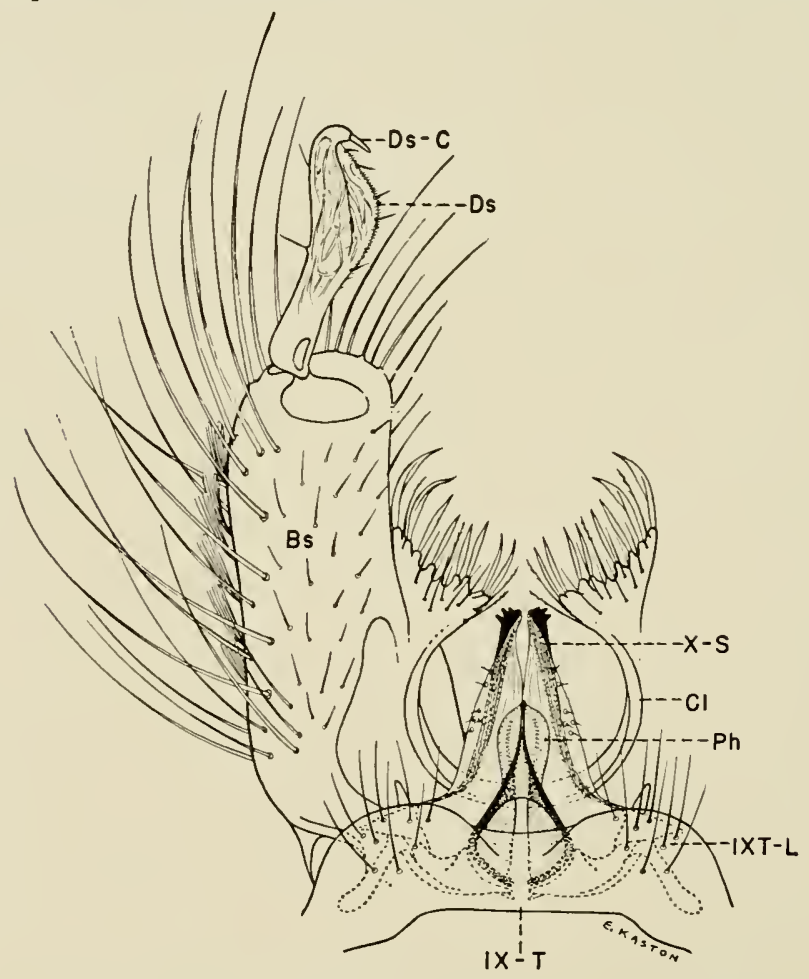

Fig. 71. Male terminalia of Psorophora cyanescens (Coquillett). 
Preantennal (A) double or triple, sparsely barbed; lower (B) and upper (C) long, single; postclypeal (d) small, branched; sutural (e) and trans-sutural (f) 2 to 4-branched; supraorbital, single or double. Comb of eighth segment of four, rarely five, thorn-shaped scales; individual scale trifid, with long central tooth. Siphon strongly inflated, about two and one-half to three times as long as basal width; pecten of three to five teeth on basal third of siphon; subventral tuft minute, multiple, inserted laterally beyond middle of siphon; hair of lateral valve longer than apical width of siphon. Anal segment somewhat longer than wide, completely ringed by the dorsal plate; lateral hair small, multiple; dorsal brush consisting of a long lower caudal hair and a shorter multiple upper

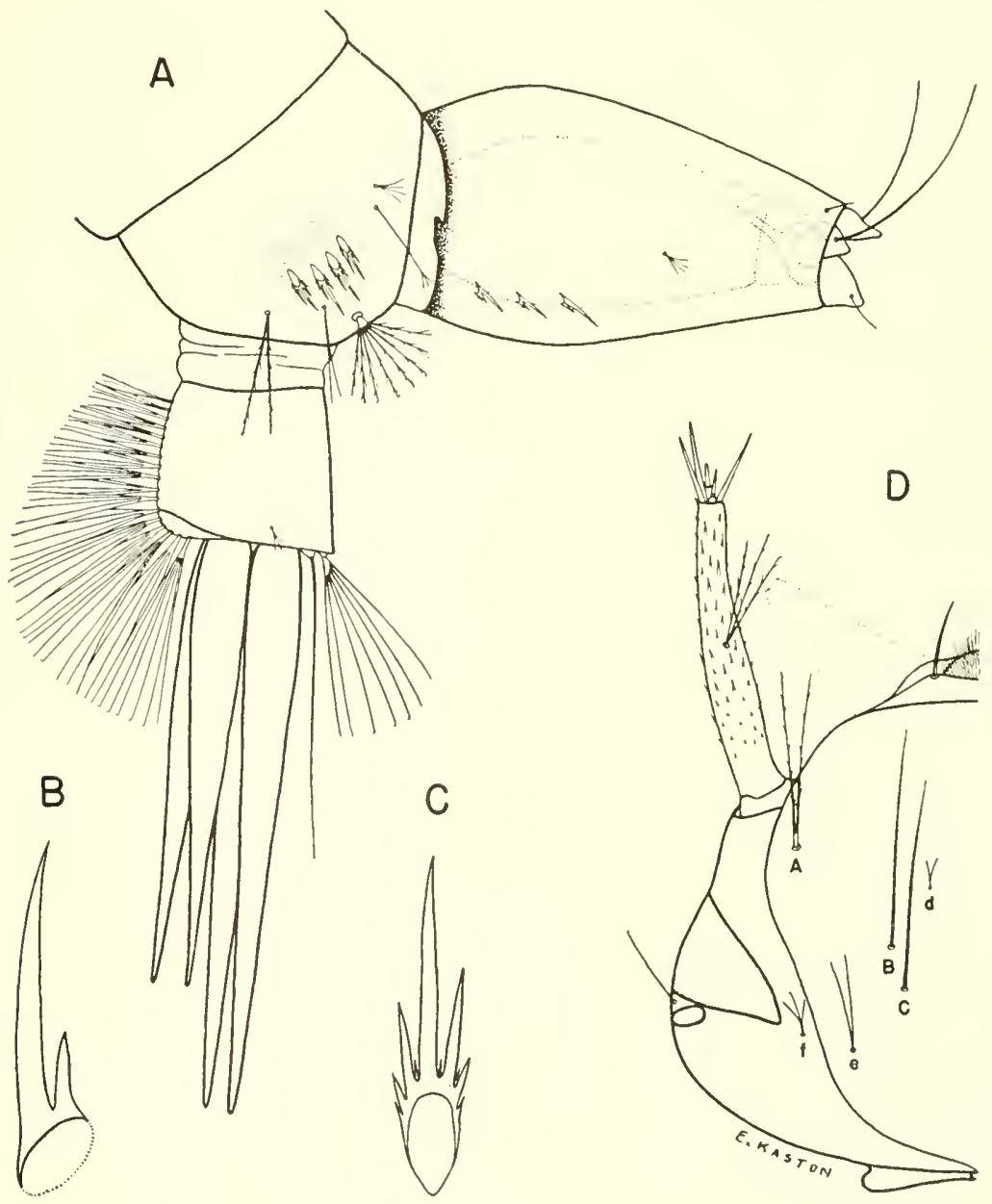

Fig. 72. Larva of Psorophora cyanescens (Coquillett). A, Terminal segments. B, Pecten tooth. C, Comb scale. D. Head. 
caudal tuft on either side; ventral brush extending almost the entire length of the anal segment; gills 4 , longer than the anal segment, pointed.

Distribution.-Southern United States, north to Indiana and Kansas; Mexico; Central and South America. Southern States: Alabama (170); Arkansas (30); Florida (35); Georgia (97); Kentucky (140); Louisiana and Mississippi (52); Missouri (1); North Carolina (166); South Carolina and Tennessee (125); Virginia (49). Other States: Indiana (76); Kansas (79); Oklahoma (159); Texas (108).

BIONOMICs.-This species is more abundant in certain areas of Alabama, Mississippi, Louisiana and Arkansas than in the Atlantic and Gulf coastal plains. The females are vicious biters and will engorge themselves until they are barely able to fly. The adults are often so plentiful in certain areas following prolonged summer rains, that they seriously annoy livestock and interfere with farm work. They are more commonly encountered in woodlands and thickets but will come out into open fields to attack. The larvae are found in temporary rain-filled pools in the southern states from May to October (4th Sv. C. Med. Lab. records, 1942-1944, unpublished).

\section{PSOROPHORA (JANTHINOSOMA) FEROX (Humboldt)}

Culex ferox Humboldt, 1820, Voy. Reg. Equin., 7:119.

adult female.-Medium sized species. Head: Proboscis dark scaled; palpi short, dark, about one-fifth as long as proboscis. Occiput clothed with numerous broad recumbent whitish-yellow to golden-yellow scales, paler anteriorly; many yellow erect forked scales present on posterior half. Thorax: Scutum covered by rather broad dark-brown and golden-yellow (often whitishyellow) scales, intermingled and in no definite pattern, the dark scales more abundant. Abdomen: All tergites (first included) dark scaled with purplish reflections, except for prominent apico-lateral triangular patches of whitishyellow to golden-yellow scales; sternites yellow scaled on segments II to VI, mainly dark on segment VII. Legs: Knee spots present on middle and hind legs; inner surfaces of all femora primarily pale scaled, darker near apices; outer surfaces of femora I and III usually pale scaled on basal third or half. Tibiae and tarsi of fore and middle legs entirely dark scaled; segments 4 and 5 of hind tarsus, and frequently the apex of segment 3 , white scaled, other segments dark (Fig. 73A); segments 1 and 2 of hind tarsus and apical portion of hind tibia with scales suberect, appearing rather shaggy. Wing: Scales dark, narrow.

ADULT MALE.-Coloration similar to that of female. TERMinalia (Fig. 73B). Ninth tergite (IX-T) with lobes (IXT-L) widely separated, broadly rounded, each bearing many scattered setae. Tenth sternite (X-S) prominent, heavily sclerotized. Phallosome $(\mathrm{Ph})$ heavily sclerotized, conical, broad basally and pointed apically, open ventrally, closed dorsally. Claspette $(\mathrm{Cl})$ slender, cylindrical, about three-fourths as long as basistyle, becoming abruptly broader at apical fourth, then tapering to tip; apical fourth with numerous slender, feathered filaments on inner margin; a large leaf-like contorted pointed fila- 
ment at apex, a similar one and a shorter, straight blunt filament subapically. Basistyle (Bs) about three times as long as broad, sides nearly parallel, somewhat curved, nearly truncate at apex, clothed with scales and numerous long setae. Dististyle (Ds) reticulated, about three-fourths as long as basistyle, slender basally, strongly inflated medially, broadest slightly beyond middle, tapering distally, slender and curved near tip, terminating in a strong, peglike claw (Ds-C).

LARVA. (Fig. 74).-Head broader than long. Antenna longer than head, usually as long as the width of the head across the eyes, spinose; antennal tuft multiple, barbed, inserted near middle. Head hairs: Preantennal (A) multiple, barbed, not reaching insertion of antennal tuft; lower (B) and upper (C) long, 2-branched, barbed, extending beyond preclypeus (branches of B as well as C nearly equal); postclypeal (d) small, branched; sutural (e), trans-sutural (f) and supraorbital usually double or multiple. Upper lateral abdominal hairs multiple on segments I and II, usually triple on III and single or double on IV to VI. Comb of eighth segment of 6 to 8 scales; indi-

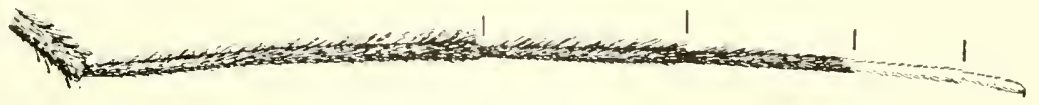

A

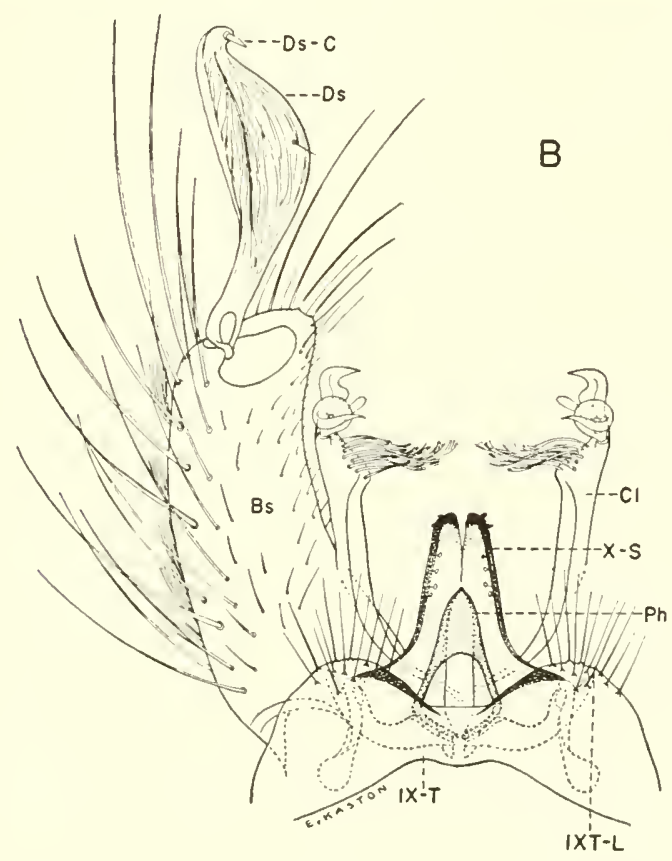

Fig. 73. Psorophora ferox (Humboldt). A, Hind tarsus. B, Male terminalia. 
vidual scale trifid, with subapical spines about one-third as long as apical spine. Siphon strongly inflated, about four times as long as basal width; pecten of 3 to 5 widely spaced teeth on basal fourth of siphon; subventral tuft minute, multiple, inserted laterally beyond middle of siphon. Anal segment longer than wide, completely ringed by dorsal plate; lateral hair small, usually multiple, branched near base; dorsal brush consisting of a long lower caudal hair and a shorter multiple upper caudal tuft on either side; ventral brush extending almost the entire length of the anal segment; gills 4, longer than the segment, each gradually tapering to a sharp point.

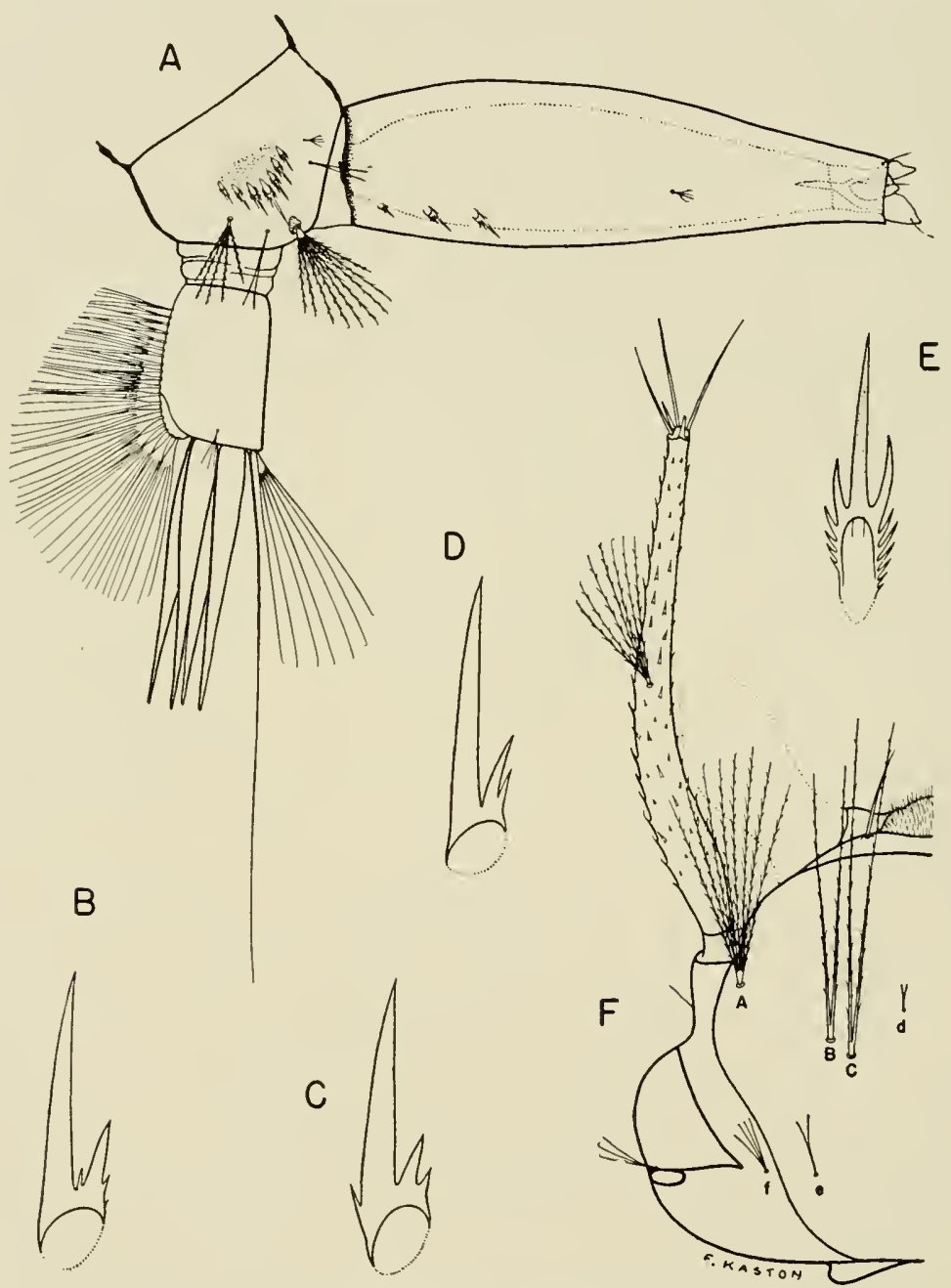

Fig. 74. Larva of Psorophora ferox (Humboldt). A, Terminal segments. B, C, and D, Pecten teeth. E, Comb scale. F, Head. 
Distribution.-Southern Canada; eastern United States westward to Nebraska and Texas; Mexico; West Indies; Central and South America. Southern States: Alabama (170); Arkansas (30); Florida and Georgia (52); Kentucky (140); Louisiana and Mississippi (52); Missouri (1); North Carolina (166); South Carolina (64); Tennessee (52); Virginia (49). Other States: Delaware (106); District of Columbia (52); Illinois (42); Indiana (76); Iowa (155, 156); Kansas (128); Massachusetts (52); Michigan (88); Minnesota (147); Nebraska (178); New Jersey (77); New York (160); Oklahoma (159); Pennsylvania (121); Texas (108); Wisconsin (48).

Bionomics.-The adults of $P$. ferox are usually encountered in or near thickets or forests following rainy periods during the summer months. The females are persistent and painful biters. Larvae are found in temporary rainfilled pools in thickets and woodlands and occasionally in pot holes in stream beds. Larvae and adults occur from March to November in the extreme South, but are more common from May to September farther north (4th Sv. C. Med. Lab. reports, 1942-1944, unpublished).

\section{PSOROPHORA (JANTHINOSOMA) HORRIDA (Dyar and Knab)}

Aedes horridus Dyar and Knab, 1908, Proc. U. S. Nat. Mus., 35:56 (in part).

Psorophora (Janthinosoma) horrida, Roth, 1945, Proc. Ent. Soc. Wash., 47:1 (synonymy given).

Adult female.-Medium sized species. Head: Proboscis long, dark scaled; palpi dark, less than one-third as long as proboscis, with the fourth segment usually curved, and about equal in length to the first three segments combined. Occiput dorsally with a large median patch of broad recumbent whitish to pale-yellow scales; a patch of broad appressed purplish scales present laterally, margined below by a patch of broad appressed yellowish scales. Pale erect forked scales numerous on central portion of occiput. Thorax: Integument of scutum black, with a broad longitudinal median stripe of lanceolate dark bronzy-brown scales; the longitudinal median stripe margined laterally with broad whitish to pale yellow scales, which extend down over the posterior pronotum anteriorly; prescutellar space margined with broad pale scales. Abdomen: First tergite entirely or primarily pale scaled; remaining tergites dark purplish scaled dorsally, with apical patches of pale yellow scales laterally (often absent on VII). Venter yellowish scaled, except for segment VII and bases of IV to VI, dark scaled. Legs: White knee spots present. Femora I and II dark purplish scaled except for pale inner surfaces (outer surface of femur I pale at base); femur III with the basal half to two-thirds pale on all aspects. Tibiae and tarsi of fore and middle legs entirely dark scaled; segments 4 and 5 of hind tarsus, and occasionally the apex of segment 3, white scaled, other segments dark (segment 4 rarely with dark scales as well as white); scales of segments 1 and 2 of hind tarsus and apical portion of hind tibia somewhat suberect, appearing rather shaggy. Wing: Scales narrow, dark.

ADULT MALE.-Coloration similar to that of female. Proboscis slender, of about equal width for its entire length, not swollen apically. Palpus with the 
last two segments much stouter than the preceding segments. Antenna extending to the apex of the third palpal segment or only slightly beyond; the last two antennal segments comprising less than half the length of the entire antenna. TERMINALIA. The terminalia of this species is very similar to that of Psorophora ferox (Fig. 73B).

Larva. (Fig. 75).- - Head much wider than long. Antenna variable in length (may be shorter or slightly longer than head), spinose; antennal tuft multiple, barbed, inserted near middle of shaft, not reaching tip or barely so. Head hairs: Preantennal (A) multiple, barbed, not reaching insertion of antennal tuft; lower (B) triple, sometimes double, barbed, short, not reaching

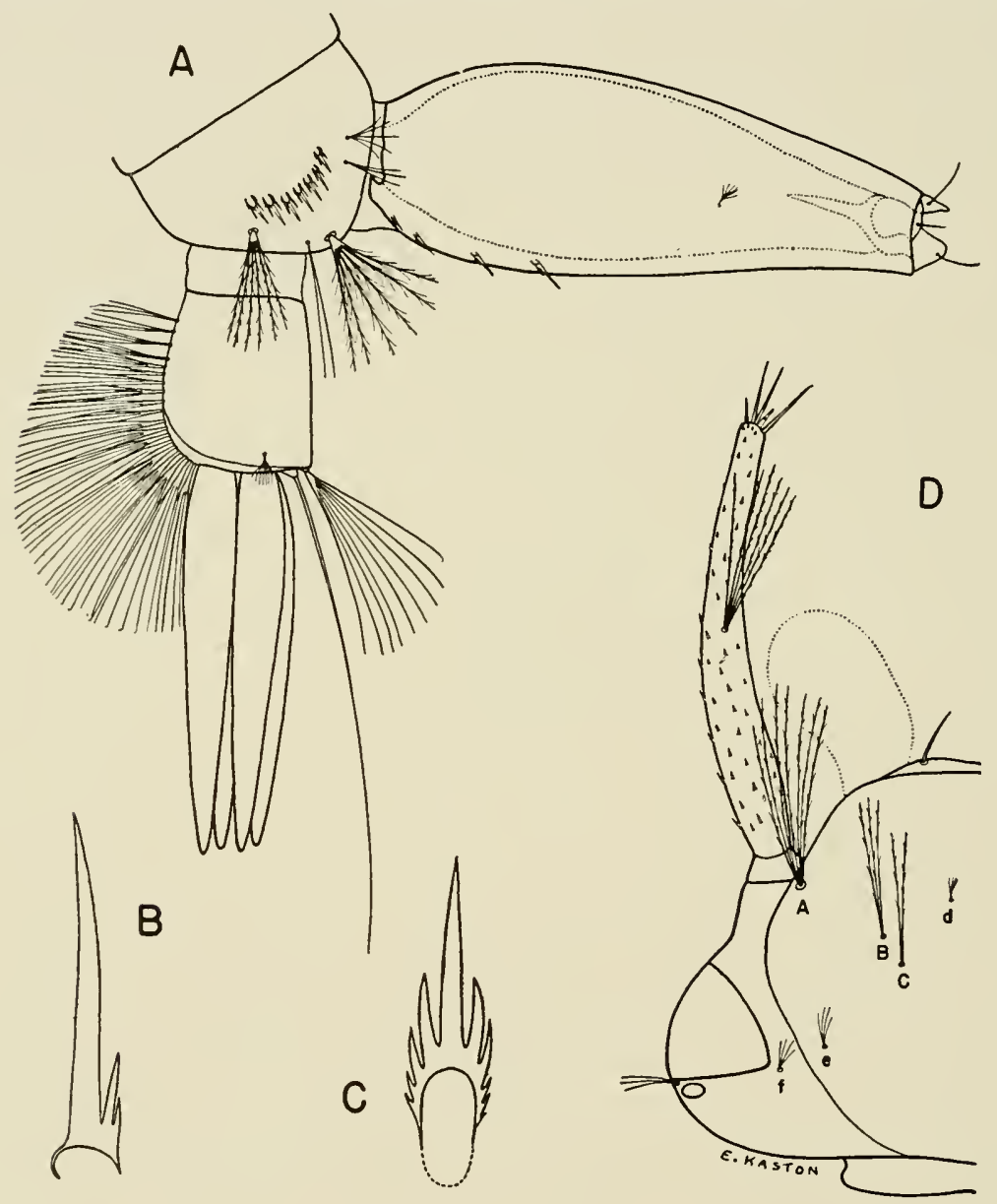

Fig. 75. Larva of Psorophora horrida (Dyar and Knab). A, Terminal segments. B, Pecten tooth. C, Comb scale. D, Head. 
preclypeus; upper (C) double, barbed, short, not reaching preclypeus (branches of $B$ as well as $C$ nearly equal); postclypeal (d) small, multiple; sutural (e), trans-sutural (f), and supraorbital multiple. Upper lateral abdominal hairs multiple and longer on segments I and II, 3 to 4-branched on III, usually double or triple on IV to VII. Comb of eighth segment of 6 to 8 scales in a curved row; individual scale trifid, with subapical spines about one-third as long as apical spine. Siphon strongly inflated, about four times as long as basal width; pecten of about 3 to 5 widely spaced teeth on basal fourth of siphon; individual tooth with one or more large coarse teeth on one or both sides; subventral tuft minute, multiple, about as long as apical pecten tooth, inserted laterally at apical third of siphon; hair of dorsal valve shorter than apical width of siphon. Anal segment longer than wide, completely ringed by the dorsal plate; lateral hair small, usually 6 to 8 -branched near base; dorsal brush consisting of a long lower caudal hair and a short multiple upper caudal tuft on either side; ventral brush extending almost the entire length of the anal segment; gills 4, longer than the anal segment, each gradually tapering to a point.

Distribution.-Eastern United States. Southern States: Alabama and Arkansas (153); Florida (4th Sv. C. Med. Lab. records, 1945, unpublished); Georgia, Kentucky, Louisiana, Mississippi, Missouri, North Carolina, South Carolina, Tennessee and Virginia (153). Other States: District of Columbia, Illinois, Indiana, Iowa, Kansas, Maryland, Ohio, Oklahoma and Texas (153).

BIONOMICS.-The females are fierce biters, attacking any time during the day in shady places. The larvae have been taken by Eaton in Georgia in partially shaded temporary pools following prolonged rains. Adults have been collected from April to September in the southern states (4th Sv. C. Med. Lab. records, 1942-1944, unpublished).

\section{PSOROPHORA (JANTHINOSOMA) LONGIPALPIS Roth 1}

Psorophora longipalpis Roth, 1945. Proc. Ent. Soc. Wash., 47:1 (synonymy given).

adult female. (Adapted from Roth, 1945).-Medium sized species. Head: Proboscis long, dark scaled; palpi dark, a little more than one-third as long as proboscis, with the fourth segment straight, more than one and onehalf times as long as the other segments combined. Occiput dorsally with a large median patch of both broad and narrow recumbent white scales; occiput laterally with a patch of broad appressed violet scales, margined with broad appressed white scales above and broad yellowish-white scales below. White erect forked scales numerous on central portion of occiput. Thorax: Integument of scutum black, with a broad longitudinal median stripe of narrow dark bronzy brown scales; the longitudinal median stripe margined laterally with broad white to yellowish scales; lanceolate pale scales intermixed with broad ones on the posterior third of the scutum; prescutellar space margined by broad pale scales. Posterior pronotum with a few broad pale scales. Abdomen: First tergite yellow scaled; remaining tergites violet scaled dorsally, with small apical patches of yellowish scales laterally on IV to VI (sometimes VII).

1 This species has been confused with $P$. horrida. 
Venter yellowish scaled, except for segment VII, base of VI, and occasionally base of V, dark scaled. Legs: Pale knee spots absent. Femora I and II violet scaled except for pale inner surfaces (outer surface of femur I pale at base); femur III with basal two-thirds pale on all aspects. Tibiae and tarsi of fore and middle legs entirely dark scaled; segments 4 and 5 of hind tarsus, and sometimes the apex of segment 3, white scaled, other segments dark (segment 4 rarely with a few dark scales); scales of hind tibia and of segments 1 to 3 of hind tarsus somewhat suberect. Wing: Scales narrow, dark.

ADULT MALE. (Head characters adapted from Roth, 153).-Coloration similar to that of female. Proboscis slender, distinctly swollen apically. Palpus with the last two segments not appreciably stouter than preceding segments. Antenna extending well beyond apex of the third palpal segment; the last two antennal segments comprising at least half the length of the entire antenna. TERMiNALIA (Fig. 76). Ninth tergite (IX-T) greatly modified, with lateral portions separated by a strongly chitinized median structure; this structure is narrow basally (anteriorly), broadened beyond middle into a pair of elongate lateral projections, constricted subapically, broadly truncated at tip and often with a median terminal tooth. Tenth sternite (X-S) heavily sclerotized beyond middle; dorsal arm large, curved. Phallosome $(\mathrm{Ph})$ ovoid, bluntly pointed at apex, open ventrally, closed dorsally, the opening at base small and with a wide lip. Claspette $(\mathrm{Cl})$ slender, unequally bifurcate; the shorter branch armed with a long slender curved spine at tip and a strong seta arising from a tubercle near base; the longer branch bearing a long slender curved spine at tip and two spine-like setae arising from a short stout projection at basal third. Basistyle

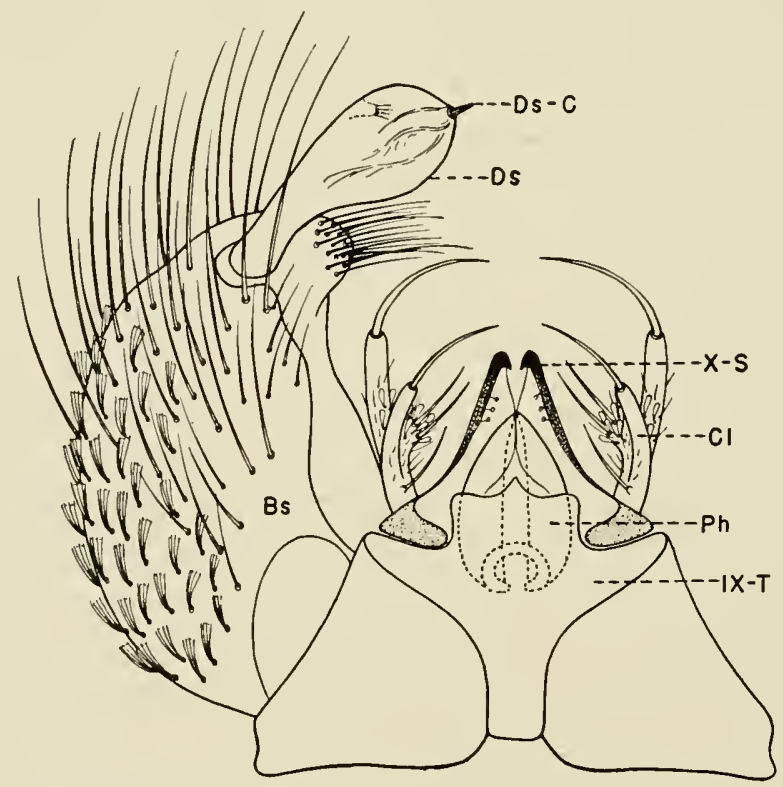

Fig. 76. Male terminalia of Psorophora longipalpus Roth. 
(Bs) about one and one-half times as long as wide, nearly hemispherical in shape with outer margin strongly curved; apex lobiform, directed mesad, bearing a dense patch of setae; vestiture of basistyle consisting primatily of numerous scales on basal half and long and short setae on apical half. Dististyle (Ds) about half as long as basistyle, the basal half constricted, the apical half strongly swollen, the tip rounded; a minute sub-ventral lobe bearing a few small setae present at apical fourth near outer margin; terminal claw (Ds-C) short, peg-like, inserted at tip of dististyle.

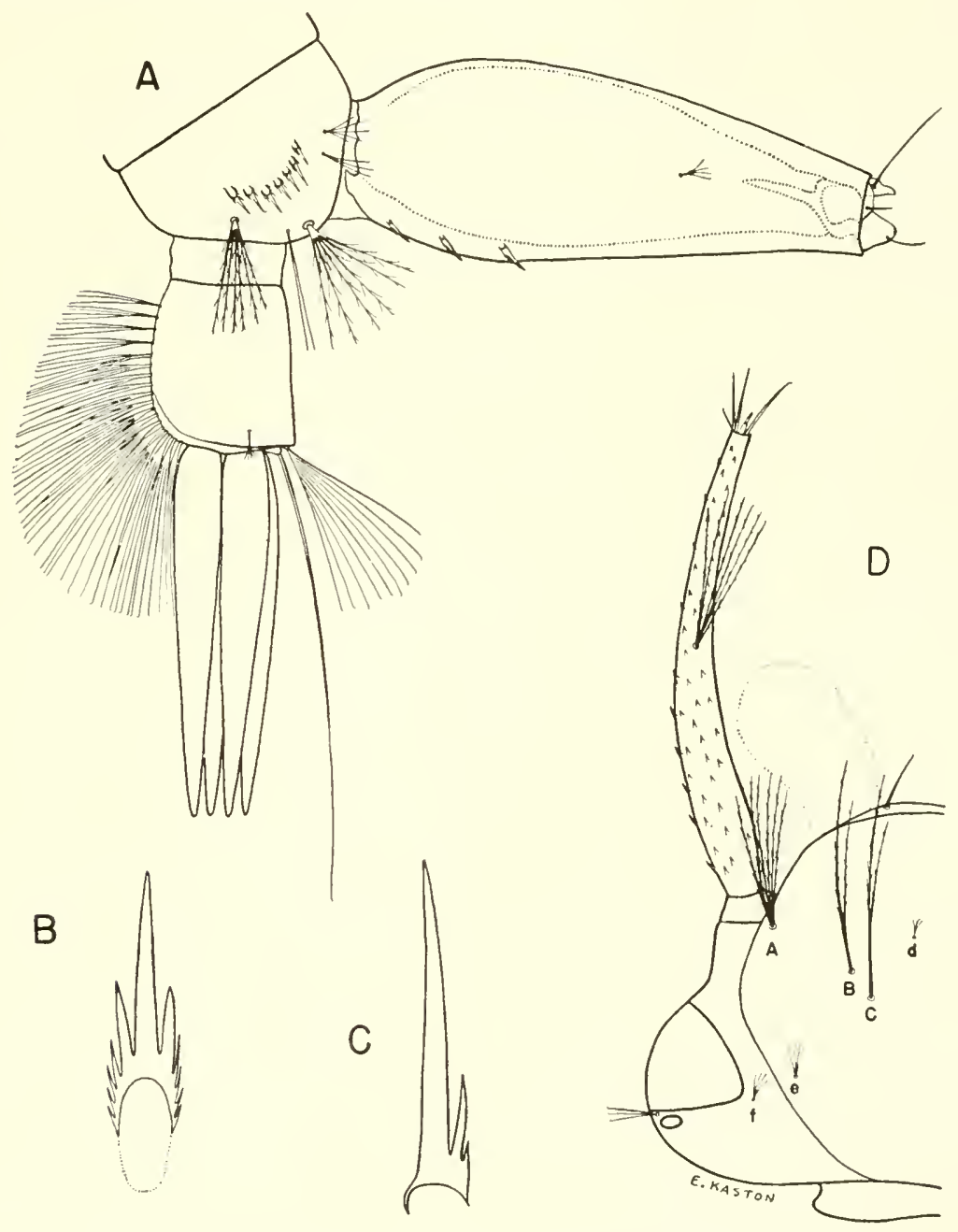

Fig. 77. Larva of Psorophora longipalpus Roth. A. Terminal segments. B, Comb scale. C, Pecten tooth. D, Head. 
LARv.A. (Fig. 77).-Head much wider than long. Antenna longer than head, spinose; antennal tuft multiple, barbed, inserted a little beyond middle of shaft and not reaching tip. Head hairs: Preantennal (A) multiple, barbed, not reaching insertion of antennal tuft; lower (B) and upper (C) double, barbed, extending to or beyond preclypeus (one branch of each tuft a little shorter and weaker than the other); postclypeal (d) small, multiple; sutural (e), trans-sutural (f) and supraorbital usually multiple. Upper lateral abdom. inal hairs multiple on segments I to VII, those on segments I and II longer. Comb of eighth segment of seven scales in a curved row; individual scale trifid with subapical spines about one-third as long as apical spine. Siphon strongly inflated, about four times as long as basal width; pecten of 3 to 4 widely spaced teeth on basal fourth of siphon; individual pecten tooth with a coarse basal side tooth; subventral tuft minute, multiple, about as long as apical pecten tooth, inserted laterally at apical third of siphon; hair of dorsal valve longer than apical width of siphon. Anal segment longer than wide, completely ringed by the dorsal plate; lateral hair small, usually multi-branched beyond middle; dorsal brush consisting of a long lower caudal hair and a short multiple upper caudal tuft on either side; ventral brush extending almost the entire length of the anal segment; gills 4 , longer than the segment, each gradually tapering to a point.

DISTRIBUTION.-Midwestern United States. The distribution of this species is not fully known. Southern States: Arkansas and Missouri (153). Other States: Kansas, Oklahoma, South Dakota and Texas (153).

BIONOMICS.-Rozeboom (157) states that the larvae are found in heavily shaded, temporary, rain-filled pools. Rozeboom (159) reports that the males can be identified, even while resting, by their bulbous terminalia.

\section{PSOROPHORA (JANTHINOSOMA) VARIPES (Coquillett)}

Conchiliastes varipes Coquillett, 1904, Can. Ent., 36:10.

Psorophora discrucians Howard, Dyar and Knab (not Walker), 1917, Mosq. N. and Cent. Amer. and W. I., 4:569.

ADUlt Female.-Medium sized species. Head: Proboscis dark scaled; palpi short, dark, about one-fifth as long as proboscis. Occiput with pale erect forked scales centrally; broad appressed yellow or cream-colored scales dorsally, extending forward to rear margin of eyes; and broad appressed dark and purplish scales laterally. Thorax: Scutum with a broad longitudinal stripe of dark lanceolate scales; this longitudinal stripe margined laterally with rather broad yellowish-white scales extending down over the posterior pronotum anteriorly (Fig. 78C). Abdomen: First tergite dingy-white scaled; remaining tergites dark scaled with purplish reflections except for whitish or pale yellow scales on apico-lateral angles; sternites primarily white scaled on segments II to VI, dark on segments VII. Legs: Knee spots present on middle and hind legs; basal two-thirds of femora I and III pale scaled on both inner and outer surfaces; femur II mainly dark scaled on outer surface, pale scaled at basal one-third on inner surface. Tibiae and tarsi of fore and middle legs entirely 
dark scaled; 4th tarsal segment of hind leg (Fig. 78A) white, at least on one side, other segments dark. Wing: Scales dark, narrow.

ADUlt MaLe.-Coloration similar to that of female. TERminalia (Fig. 78B). Ninth tergite (IX-T) with lobes (IXT-L) broadly rounded, bearing scattered setae. Tenth sternite (X-S) prominent, heavily sclerotized. Phallosome $(\mathrm{Ph})$ heavily sclerotized, conical, broad at base and pointed apically, open ventrally, closed dorsally. Claspette $(\mathrm{Cl})$ nearly straight, narrow, flattened dorso-ventrally, about four-fifths as long as basistyle; inner surface at tip bearing a single strong down-curved claw-like appendage; inner margin near middle of stem with a pair of long smooth setae, followed by a few smaller setae, some of which are feathered. Basistyle (Bs) nearly two and one-half times as long as broad, cylindrical, bluntly rounded at apex, clothed with sparse scales and numerous long setae; basal and apical lobes absent. Dististyle (Ds) nearly as long as claspette, natrow basally, expanded beyond basal third, constricted somewhat at apical sixth, broadly truncate at apex; claw (Ds-C) short, peglike, inserted on inner margin of dististyle immediately preceding apical constriction.

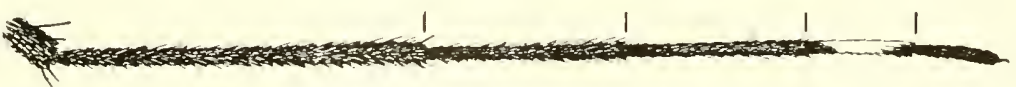

A

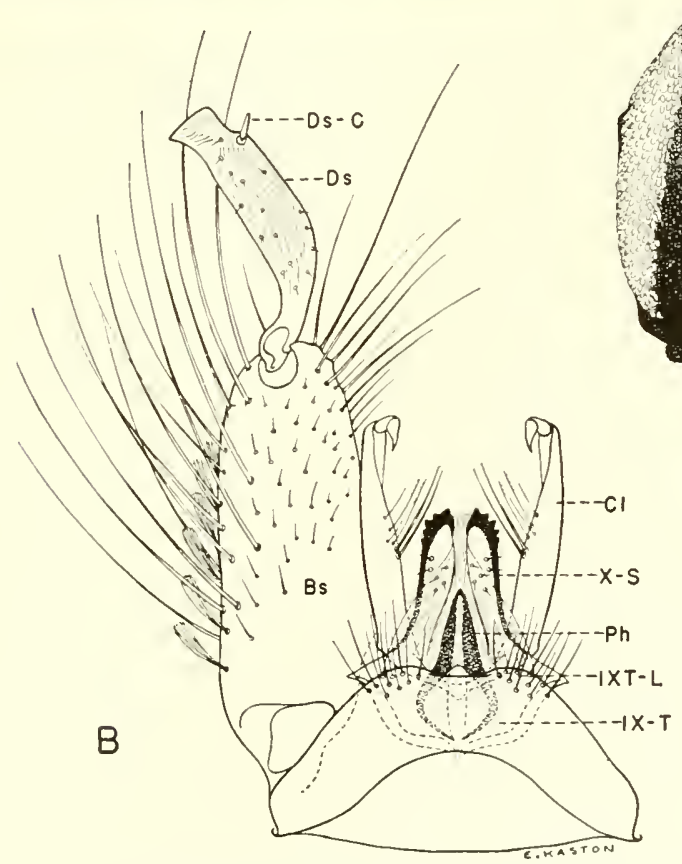

Fig. 78. Psorophora varipes (Coquillett). A, Hind tarsus.

B, Male terminalia. C. Scutum and scutellum. 
LARVA. (Fig. 79).- - Head broader than long. Antenna about as long as head, spinose; antennal tuft barbed, 5-branched, inserted a little beyond middle of shaft and reaching tip. Head hairs: Preantennal (A) multiple, barbed, reaching to insertion of antennal tuft; lower (B) double or triple, sparsely barbed, extending slightly beyond preclypeus; upper (C) double, sparsely barbed, extending to or slightly beyond preclypeus; postclypeal (d) small, multiple; sutural (e), trans-sutural (f), and supraorbital usually double or multiple. Upper lateral abdominal hairs multiple on segments I and II, variable on III to VI. Comb of eighth segment of about seven thorn-shaped scales in a $V$-shaped row, the median scales much larger and longer than the outer scales; individual scale with subapical spines only about one-fifth to one-fourth as long as apical spine. Siphon only slightly inflated, about four times as long as basal

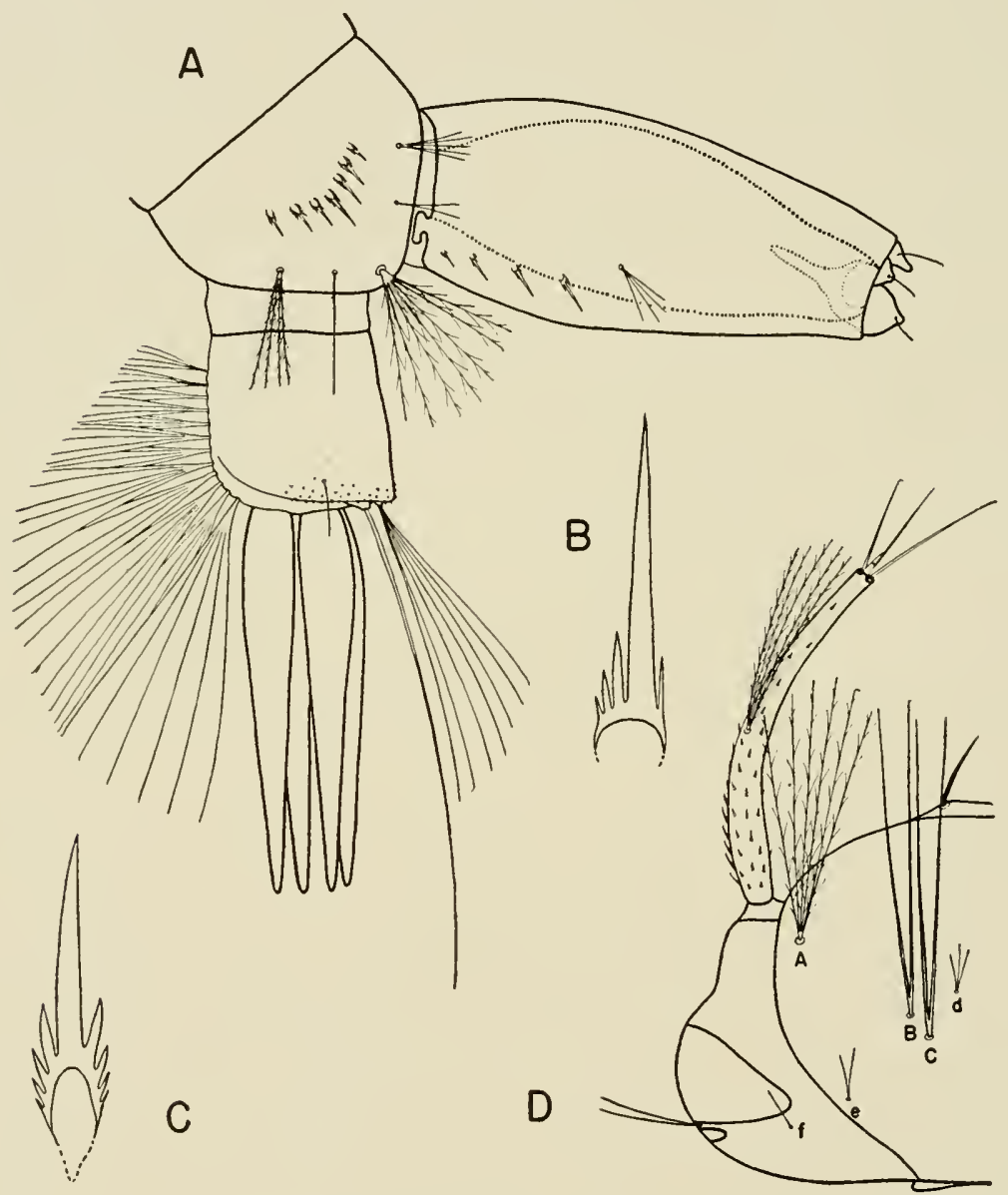

Fig. 79. Larva of Psorophora varipes (Coquillett). A, Terminal segments. B, Pecten tooth. C, Comb scale. D, Head. 
width; pecten of 3 to 4 widely spaced teeth on basal third of siphon; subventral tuft small, 4 to 6-branched, inserted beyond middle of siphon. Anal segment at least one and one-half times as long as wide, completely ringed by the dorsal plate; lateral hair single, about 4-branched apically; dorsal brush consisting of a long lower caudal hair and a shorter multiple upper caudal tuft on either side; ventral brush extending almost the entire length of the anal segment; gills 4, longer than the segment, pointed.

Distribution.--Southern United States notth to Indiana and west to Texas; Mexico; Central and South America. Southern States: Alabama (170); Arkansas (30); Florida and Georgia (96); Kentucky (140); Louisiana and Mississippi (96); Missouri (1); North Carolina (166); South Carolina (125); Tennessee (96); Virginia (49). Other States: Indiana (76); Oklahoma (159); Texas (130).

BIONOMICS.-The females are vicious biters, attacking any time during the day. Adults of this species have been reported by Horsfall (83) and Carpenter (30) as occurring in swarms in woodlands along creeks in Arkansas following flooding in the spring. Larvae develop in temporary floodwater pools. This species has been collected from April to September in the southern states (4th Sv. C. Med. Lab. records, 1942-1944, unpublished).

\section{Psorophora (Grabhamia) confinnis (Lynch Arribalzaga)}

Taeniorhynchus confinnis Lynch Arribalzaga, 1891, Rev. Mus. de la Plata, 2:149.

Psorophora (Grabhamia) confinnis. Aitken, 1940, Rev. de Ent., 11:677 (Synonymy given).

adult female.-Medium sized to rather large species. Head: Proboscis dark scaled, except for a very wide median whitish-yellow band (width about equal to half the length of the proboscis); palpi short, dark, with apical half of fourth segment primarily white scaled. Occiput clothed dorsally with narrow white to pale violet scales and numerous black erect forked scales; a patch of broad flat dark scales usually present laterally, followed by broad dingy-white to light brown scales. Thorax: Integument of scutum dull black, clothed with fine narrow bronzy-brown to blackish scales, except for the prescutellar space, a lunate patch on antero-lateral angle of scutum, a streak on scutal angle, a lateral patch above wing base, and a small submedian spot near middle of scutum, each whitish-scaled, usually tinted with lavender. Abdomen: First tergite whitish scaled. Remaining tergites dark, with whitish to pale-yellow scaled apical markings in the form of paired submedian patches; these apical submedian patches triangular on the anterior segments, becoming subquadrate on the posterior segments; the patches on II and III frequently joined medially, those on III to VII usually well separated. Venter clothed with intermixed dark and pale scales. Legs: White knee spots present. Femora largely pale scaled on inner surfaces, the outer surfaces dark-brown to black scaled, liberally speckled with white scales; each femur with a narrow subapical white-scaled ring. Tibiae black, with numerous small white-scaled spots on outer surfaces. Segments of hind tarsus each with a broad white basal ring, the first segment with a pale median ring as well. Fore- and mid-tarsi similarly marked, but with white rings reduced or lacking on segment 4, absent on 5. Wing: Scales rather 
broad, intermixed dark brown and white, the white scales in no definite arrangement; fringe entirely dark.

ADULT MALE.-Coloration similar to that of female, but with the ring of the proboscis narrower. TERMINALIA (Fig. 80B). Ninth tergite (IX-T) with lobes (IXT-L) broadly rounded, widely separated, each bearing many scattered setae. Tenth sternite (X-S) prominent, heavily sclerotized. Phallosome $(\mathrm{Ph})$ subcylindrical (slightly narrower across apical third than across base), bluntly pointed or rounded at apex, open ventrally, closed dorsally; middle of each plate with a narrow rounded portion of its inner ventral margin turned ventrad (not a distinct triangular projection as in $P$. discolor.) Claspette $(\mathrm{Cl})$ with stem curved, slender, expanded apically into a triangular crown; lower portion of crown attached to basal third of basistyle; apical portion free and bearing a row of six or seven long pointed apically feathered blades and a single apically feathered seta. Basistyle (Bs) nearly two and one- half times as

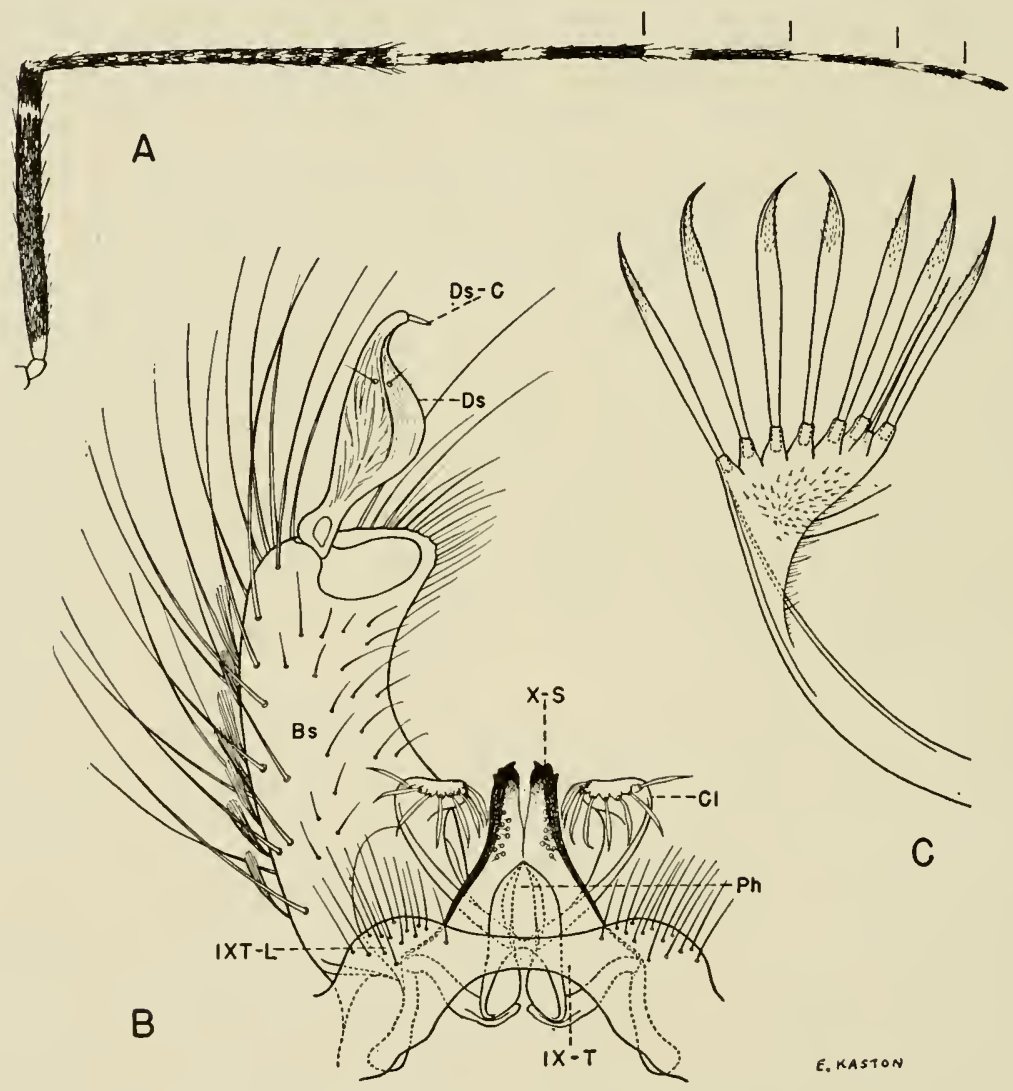

Fig. 80. Psorophora confinnis (Lynch Arribalzaga). A, Hind leg. B, Male terminalis. C, Crown of claspette. 
long as broad, cylindrical, curved, nearly truncate at apex, clothed with scales and numerous long setae; basal and apical lobes absent. Dististyle (Ds) reticulated, about three-fifths as long as basistyle, strongly inflated medially, tapering distally, slender and curved near tip, terminating in a peg-like claw (Ds-C).

LARva. (Fig. 81).-Head broader than long. Antenna shorter than head, spinose; antennal tuft multiple, barbed, inserted near middle of shaft, extending to tip. Head hairs: Preantennal (A), lower (B) and upper (C) multiple, strongly barbed, inserted in a straight line; postclypeal (d) small, multiple; sutural (e) usually single or double; trans-sutural (f) and supraorbital usually double or triple. Upper lateral abdominal hairs multiple on segments I and II,

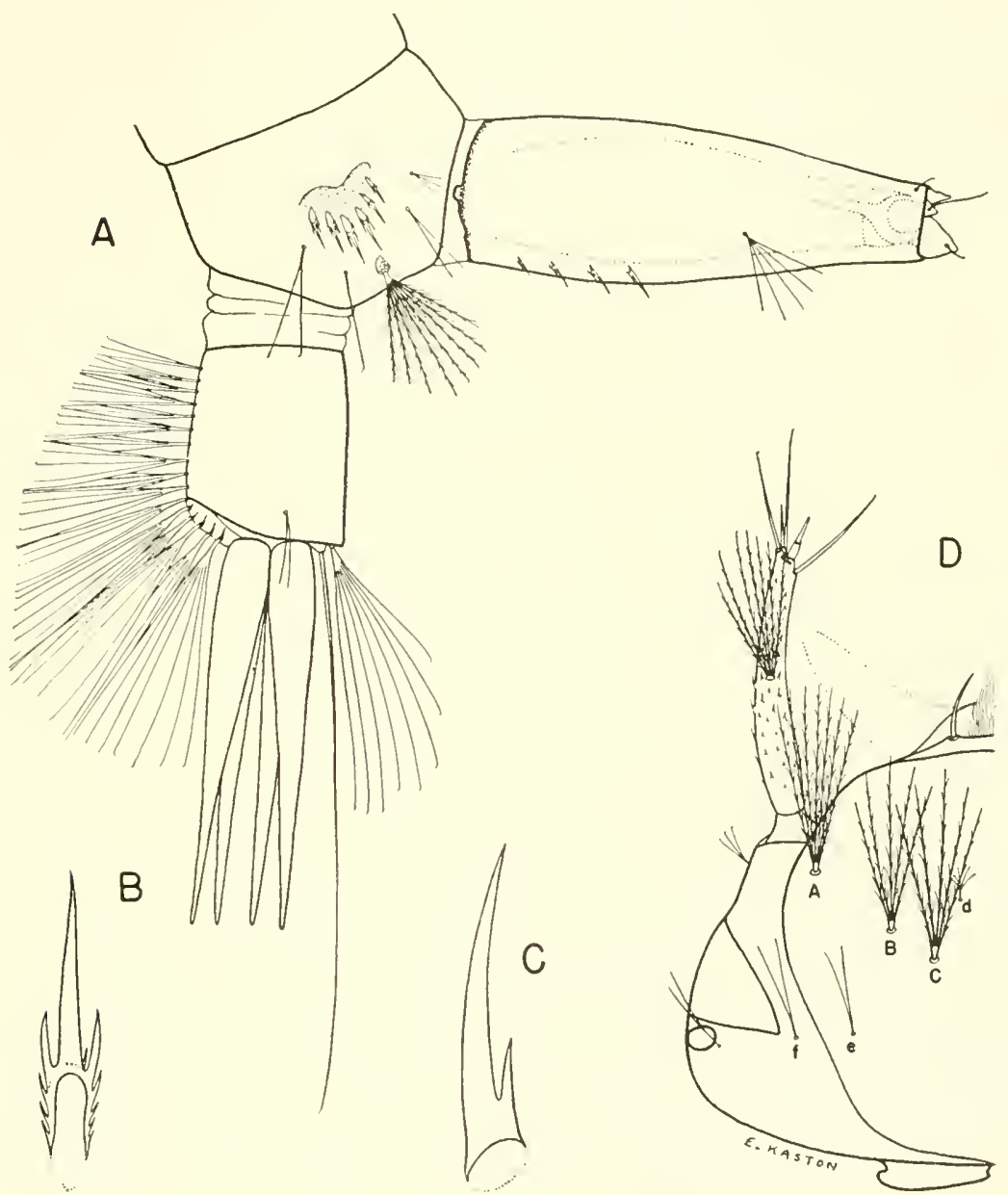

Fig. 81. Larva of Psorophora confunis (Lynch Arribalzaga). A, Terminal segments. B, Comb scale. C. Pecten tooth. D, Head. 
double on III to V. Comb of eighth scgment of six thorn-shaped scales; individual scale with a long apical spine about three times the length of the stout subapical spines. Siphon slightly inflated, about three times as long as basal width; pecten of 3 to 6 widely spaced teeth, not reaching middle of siphon; subventral tuft small, multiple, inserted at outer third of siphon. Anal segment longer than wide, completely ringed by dorsal plate; lateral hair double or triple; dorsal brush consisting of a long lower caudal hair and a shorter multiple upper caudal tuft on either side; ventral brush extending almost the entire length of the anal segment; gills, 4 longer than the anal segment, pointed.

distribution.-United States, Mexico, West Indies, Central and South America. Southern States: Alabama (96); Arkansas (30); Florida and Georgia (52); Kentucky (140); Louisiana and Mississippi (52); Missouri (1); North Carolina (52); South Carolina (64); Tennessee (52); Virginia (49). Other States: Arizona and California (2); Colorado (128); Delaware (106); District of Columbia and Illinois (52); Indiana (76); Iowa (155, 156); Kansas (79); Maryland (19); Massachusetts (180); Nebraska (178); New Jersey (77); New York (52); Oklahoma (159); Pennsylvania (8); Texas (108); West Virginia (52).

BIONOMICs. - The females are fierce biters, attacking any time during the day or night. This species reaches its greatest abundance in the Florida Everglades and in the rice fields of Arkansas. In these areas it occasionally kills livestock and at times makes it almost unbearable for people to remain outdoors at night, or in shaded areas during the day. Horsfall (86) found that the flight range of this species may be as great as nine miles in the rice fields in Arkansas. The females lay their eggs on damp soil in depressions subject to flooding by rainfall or overflow, particularly from irrigation canals in the rice fields. Soil with rank low-growing vegetation seems to provide ideal oviposition sites. The larval period is relatively short, usually requiring only 4 to 10 days. The winter is passed in the egg stage. Breeding occurs throughout most of the year in the extreme South, but is more common from May to October farther north (4th Sv. C. Med. Lab. records, 1942-1944, unpublished).

\section{Psorophora (Grabhamia) discolor (Coquillett)}

Culex discolor Coquillett, 1903, Can. Ent., 35:256.

adult female.-Medium sized species. Head: Proboscis dark scaled, except for a very wide median whitish-yellow band (width of band about equal to half the length of the proboscis); palpi short, dark, the apices pale scaled. Occiput clothed dorsally with narrow golden-yellow scales and erect forked scales, the forked scales mostly dark but those of central region pale; occiput clothed laterally with broad flat yellowish scales. Thorax: Integument of scutum dark brown, clothed with fine narrow pale-yellow to pale golden-brown scales. Abdomen: First tergite whitish scaled; remaining tergites almost entirely whitish to pale-yellow scaled dorsally, more or less speckled with dark scales; basal dark-scaled patches present laterally, each pair sometimes connected dorsally by a narrow basal dark band. Venter pale scaled, frequently 
speckled with dark scales. Legs: Pale knee spots present. Femora largely pale on inner surfaces, the outer surfaces clothed with intermixed dark brown and pale scales; each femur with a narrow subapical pale-scaled ring (often indefinite). Tibiae predominantly pale, sparsely speckled with dark scales, the apices usually dark. Hind tarsus with segments 1 mostly pale, usually speckled with dark scales, blending into a dark ring at apex (apical dark ring variable in width); segment 2 to 5 variably marked, but usually with basal halves pale, apical halves dark. Fore- and mid-tarsi similarly marked, but with basal pale rings lacking or greatly reduced on segment 4, absent on 5. Wing: Scales rather broad, dark-brown and white, arranged more or less in definite patterns.
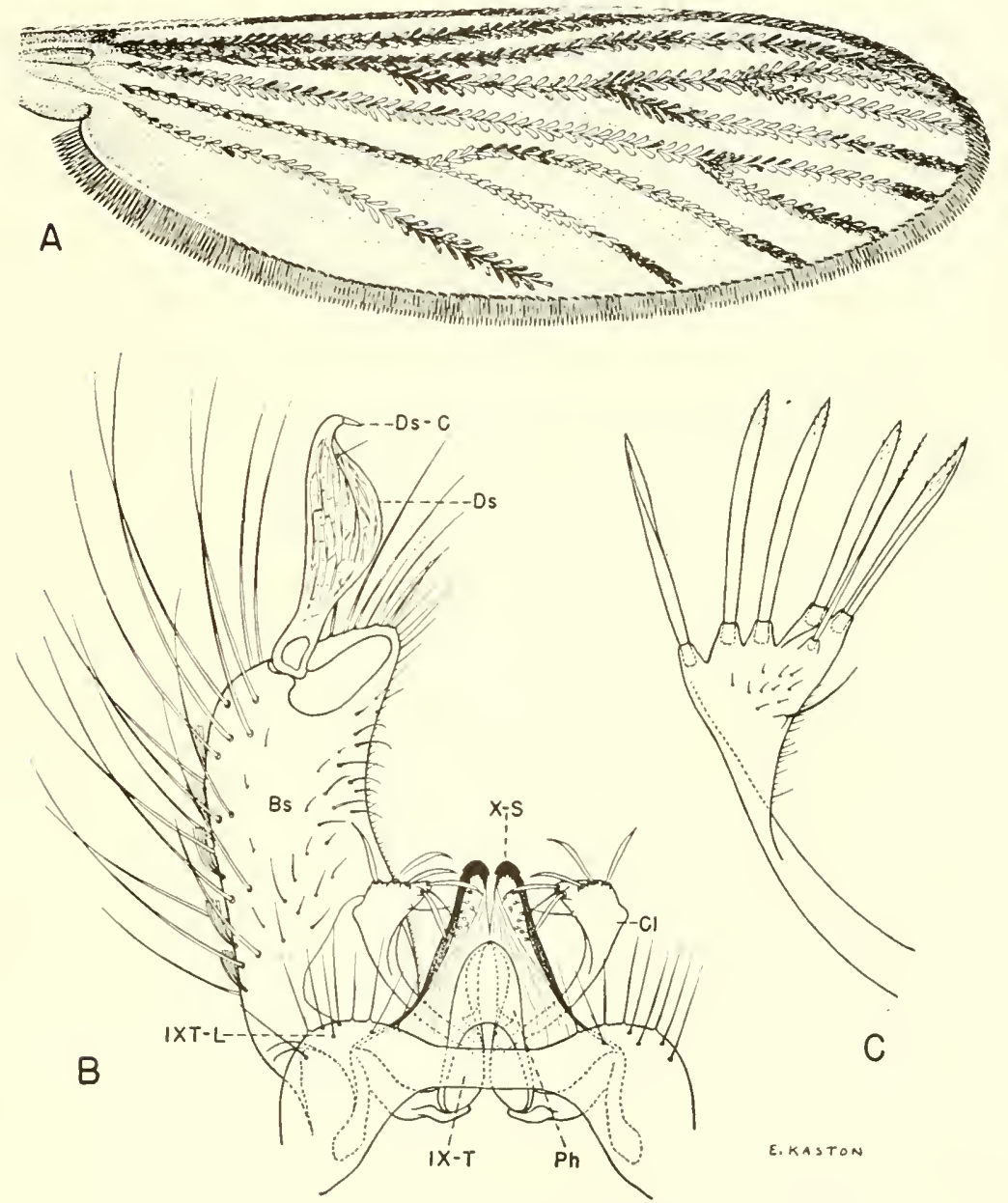

Fig. 82. Psorophora discolor (Coquillett). A, Wing. B, Male terminalia. C. Crown of claspette. 
Costa with a streak of white scales at base and at point of union with subcosta, the remainder speckled with dark scales. Vein 6 with basal two-thirds white scaled, apical third dark. Remaining veins clothed with intermixed dark and white scales and with definite areas of all-dark and all-white scales. Fringe scales uniformly rather dark.

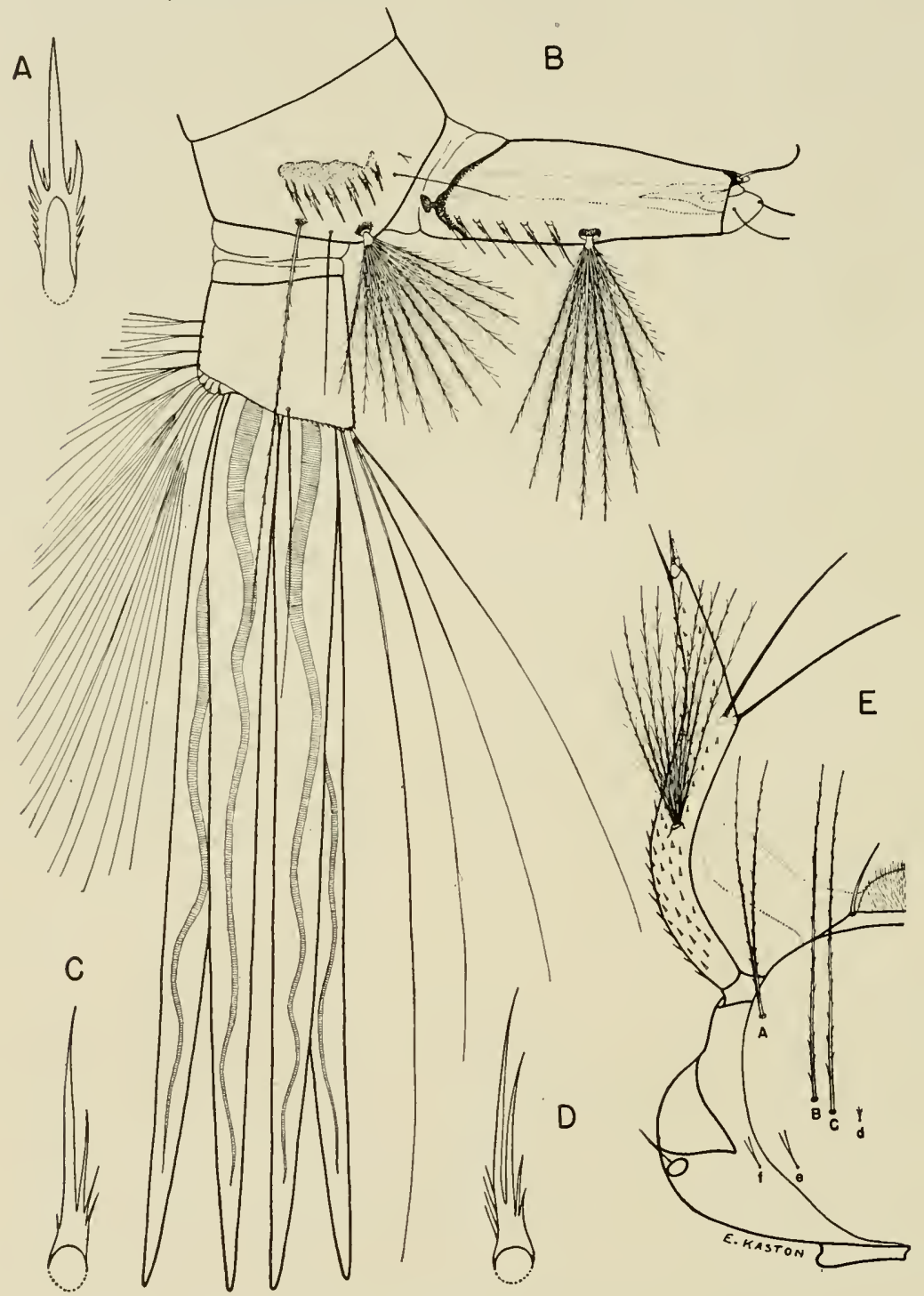

Fig. 83. Larva of Psorophora discolor (Coquillett). A, Comb scale. B, Terminal segments. C, D, Pecten teeth. E, Head. 
ADULT MALE.- Coloration similar to that of female, but with the ring of the proboscis narrower. TERMINALIA (Fig. 82B). Ninth tergite (IX-T) with lobes (IXT-L) broadly rounded, widely separated, each bearing many scattered seta. Tenth sternite (X-S) prominent, heavily sclerotized. Phallosome $\mathrm{Ph}$ ) subcylindrical (slightly narrower across apical third than across base), bluntly pointed or rounded at apex, open ventrally, closed dorsally; middle of each plate with a distinct triangular projection turned ventro-laterally from its inner ventral margin. Claspette $(\mathrm{Cl})$ with stem curved, slender, expanded apically into a triangular crown; lower portion of crown attached to basal third of basistyle; apical portion free and bearing a row of five or six long pointed apically feathered blades and a single apically feathered seta. Basistyle (Bs) nearly two and one-half times as long as broad, cylindrical, curved, nearly truncate at apex, clothed with scales and numerous long setae; basal and apical lobes absent. Dististyle (Ds) reticulated, about three-fifths as long as basistyle, strongly inflated medially, tapering distally, slender and curved near tip, terminating in a peg-like claw (Ds-C).

LARVA. (Fig. 83).-Head broader than long. Antenna longer than head, inflated, sinuate, spinose; antennal tuft multiple, barbed, reaching apex of antenna; a pair of long spines inserted on the inner projection of the antennal shaft at distal fourth; tip of antenna with a short subapical spine, a short apical spine and a membranous papilla. Head hairs: Preantennal (A) long, double, slightly barbed; lower (B) and upper (C) very long, single, slightly barbed; postcly. peal (d) small, branched, inserted posterior to upper (C); sutural (e) and trans-sutural (f) usually double or triple; supraorbital single, or branched toward tip. Upper lateral abdominal hairs multiple on segments I and II, usually double on segments III to V. Comb of eighth segment of six large thorn-shaped scales; individual scale trifid, with long central tooth. Siphon small, not inflated, about three times as long as basal width (much narrower than anal segment); pecten of about 4 to 8 long teeth on basal half of siphon; subventral tuft multiple, as long as siphon, strongly barbed, inserted slightly beyond middle; dorsal preapical spine strong, reflexed. Anal segment about as long as wide, completely ringed by the dorsal plate; lateral hair very long, single; dorsal brush consisting of a long lower caudal hair and a shorter 3 to 4 branched upper caudal tuft on either side; ventral brush sparse, with about three tufts piercing the dorsal plate on the mid-ventral line; gills 4, three or four times as long as the anal segment, each conspicuously tracheate.

DISTRIBUTION.-Southern United States, north to New Jersey and west to Nebraska and Texas. Southern States: Alabama (170); Arkansas (30); Florida (96); Georgia (148); Kentucky (140); Louisiana and Mississippi (96); Missouri (1); North Carolina (166); South Carolina (97); Tennessee (96); Virginia (52). Other States: District of Columbia (52); Iowa, Kansas and Nebraska (128); New Jersey (77); Oklahoma (159); Texas (108).

BIONOMICS.-This species reaches its greatest abundance in Arkansas, Oklahoma and Texas. Horsfall (86) reports that it constituted about 36 per cent of the total specimens collected in light traps located in the Arkansas rice fields from 1939 to 1941 . The females are troublesome biters when abundant. 
It breeds in temporary rain-filled pools and flooded areas, and is known to occur from April to October in the southern states (4th Sv. C. Med. Lab. records, 1942-1944, unpublished).

\section{Psorophora (Grabhamia) pygmaea (Theobald)}

Gramhamia pygmaea Theobald, 1903, Mon. Culic., 3:245.

Adult Female.-Rather small species. Head: Proboscis dark scaled, except for a very broad median whitish band; palpi short, dark, with a few white scales at apices. Occiput clothed dorsally with narrow pale-yellow scales and numerous erect forked scales; the forked scales of central region usually pale, others dark. Occiput clothed laterally with broad flat whitish scales surrounding a small black-scaled patch near eye margin. Thorax: Integument of scutum dark brown to blackish, clothed with fine narrow pale-yellow to golden-brown scales. Abdomen: First tergite white scaled. Remaining tergites dark basally, white apically, the apical white bands wider medially than laterally; VI and VII often entirely white-scaled. Venter primarily whitish scaled, speckled or spotted with dark scales. Legs: Pale knee spots present. Femora largely pale on inner surfaces, the outer surfaces dark, speckled with pale scales; each femur with a

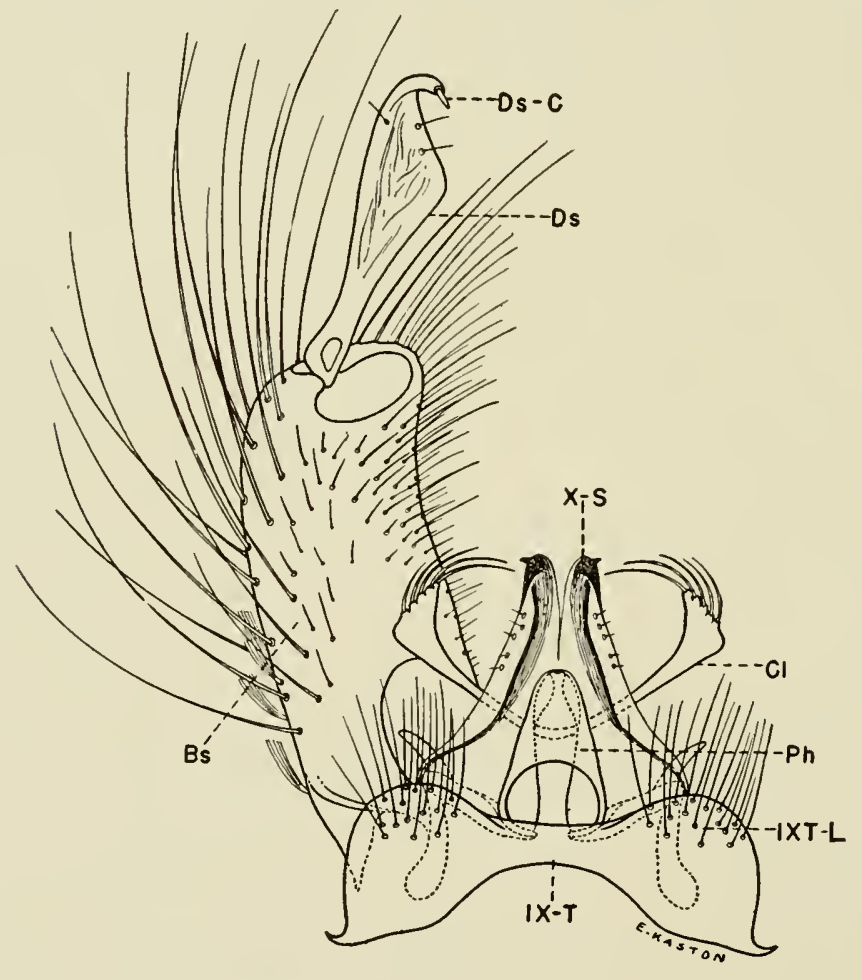

Fig. 84. Male terminalia of Psorophora pygmaea (Theobald). 
narrow subapical white-scaled ring. Tibiae dark scaled, speckled with white. Each segment of hind tarsus with a narrow white-scaled ring at base; segments 1 to 3 of fore- and mid-tarsi each with a very narrow white-scaled ring at base, absent on segments 4 and 5. Wing: Scales rather broad, intermixed white and dark-brown to black, the dark scales predominating, not arranged in a particular pattern; fringe entirely dark.

ADULT MALE. - Coloration similar to that of female, but the ring of proboscis narrower, as well as the apical white bands of the terminal abdominal segments. TERminalia (Fig. 84). Ninth tergite (IX-T) with lobes (IXT.

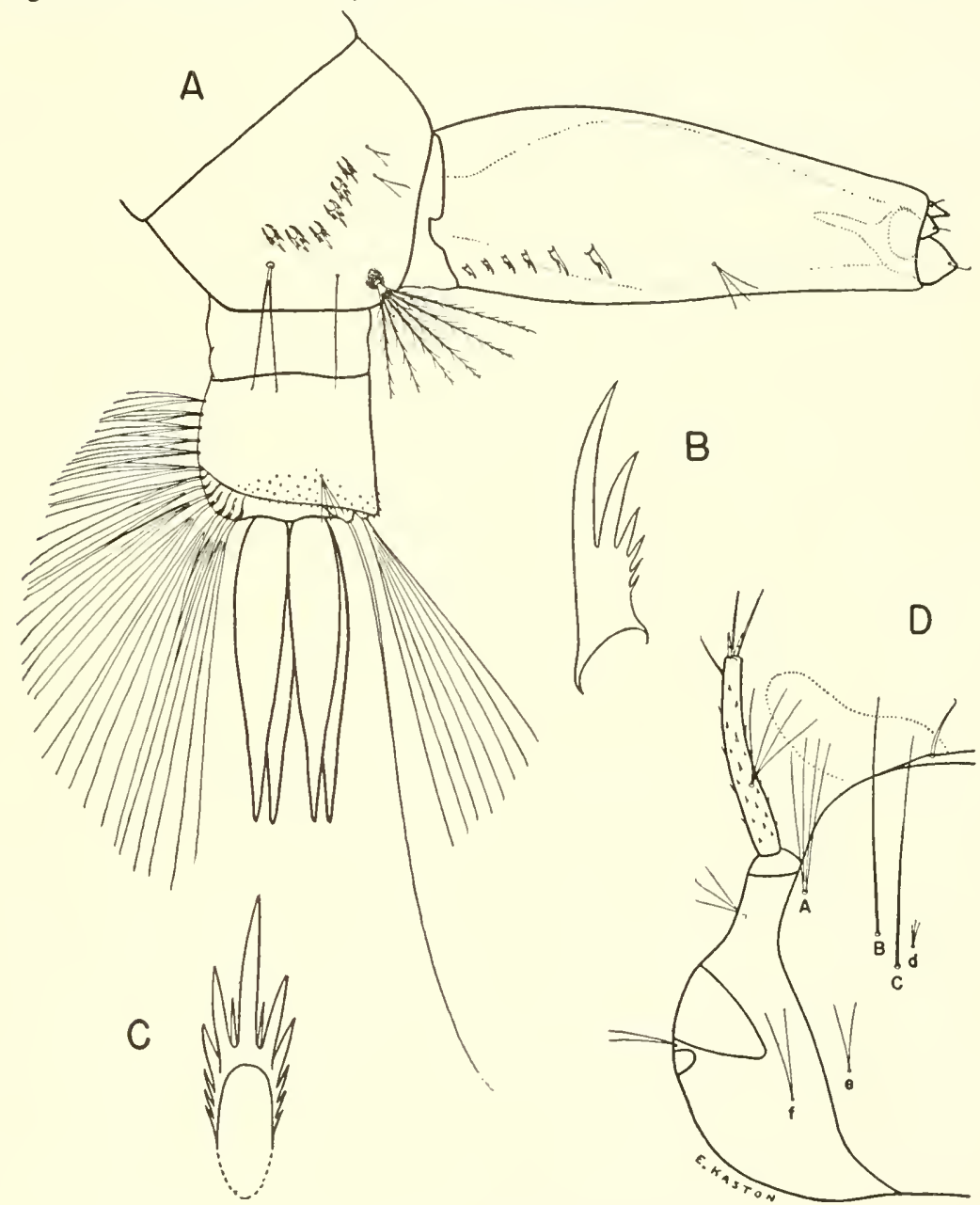

Fig. 85. Larva of Psorophora pugmaea (Theobald). A, Terminal segments. B, Pecten tooth. C, Comb scale. D, Head. 
L) broadly rounded, widely separated, bearing numerous scattered setae. Tenth sternite (X-S) prominent, heavily sclerotized beyond middle. Phallosome (Ph) bluntly conical, open ventrally, closed dorsally. Claspette $(\mathrm{Cl})$ with stem slender, triangular expanded apically, the expanded portion weakly attached to basistyle before middle, with a row of about five long slender filaments on crown, generally directed dorsad. Basistyle (Bs) slightly more than twice as long as broad, cylindrical, nearly truncate at apex, clothed with sparse scales and numerous long setae; basal and apical lobes absent. Dististyle (Ds) reticulated, about two-thirds as long as basistyle, narrow basally, expanded beyond middle and broadest at apical third, then tapering to a slender curved tip, terminating in a peg-like claw (Ds-C).

LARva. (Fig. 85).-Head broader than long. Antenna shorter than head, spinose; antennal tuft multiple, inserted before middle of shaft, not reaching tip. Head hairs: Preantennal (A) multiple, extending beyond insertion of antennal tuft; lower (B) and upper (C) long, single; postclypeal (d) small, branched; sutural (e), trans-sutural (f), and supraorbital usually double. Comb of eighth segment of six scales; individual scale with apical spine about twice as long as the stout subapical spines. Siphon somewhat inflated, about three times as long as basal width; pecten of about six teeth on basal third of siphon; subventral tuft small, usually 3-branched, inserted beyond middle of siphon; hair of lateral valve minute, less than half as long as the apical width of siphon. Anal segment longer than wide, completely ringed by the dorsal plate; lateral hair small, multiple; dorsal brush consisting of a long lower caudal hair and a shorter multiple upper caudal tuft on either side; ventral brush extending almost the entire length of the anal segment; gills 4, longer than the anal segment, pointed.

Distribútion.-Florida Keys (52); Bahamas and West Indies. The species was recorded from Key West, Florida, in 1901, but has not since been found in the United States.

BIONOMICS.-Dyar (54) states that the larva occur in temporary rain-filled pools.

\section{Psorophora (Grabhamia) signipennis (Coquillett)}

Taeniorhynchus signipennis Coquillett, 1904, Proc. Ent. Soc. Wash., 6:167.

adult female.-Medium sized species. Head: Proboscis dark scaled, except for a very wide median whitish-yellow band (width of band about equal to half the length of the proboscis); palpi short, dark, speckled with a few pale scales. Occiput clothed dorsally with narrow golden-yellow scales and erect forked scales; the forked scales of central region pale, others dark. Occiput clothed laterally with a patch of broad flat yellowish scales; this pale-scaled patch usually either speckled with dark scales or partially enclosing a small dark-scaled area near eye margin. Thorax: Integument of scutum dark brown, clothed with fine narrow pale-yellow to golden-brown scales. Abdomen: First tergite white scaled; remaining tergites primarily white scaled, more or less speckled or spotted with dark scales. Venter mostly pale scaled, speckled or 
spotted with dark scales. Legs: White knee spots present. Femora largely pale on inner surfaces, the outer surfaces dark, liberally speckled with pale scales; each femur with a narrow subapical whitish-scaled ring (often indefinite). Tibiae dark, somewhat mottled with pale scales. Hind tarsus with segment 1 white-ringed basally, dark ringed sub-basally and apically; the area between the dark rings comprises about half the segment and is clothed with intermixed dark and pale scales, the pale predominating; segments 2 to 5 pale scaled on basal third or basal half, the remaining portions dark. Fore- and mid-tarsi similarly marked, but with the white scaling reduced on segment 4 , absent on 5. Wing: Scales rather broad, intermixed white and dark-brown to black. Apical half of costa with two conspicuous spots of dark scales separated by a longer area of white scales; scales of vein 6 intermixed dark and white on basal
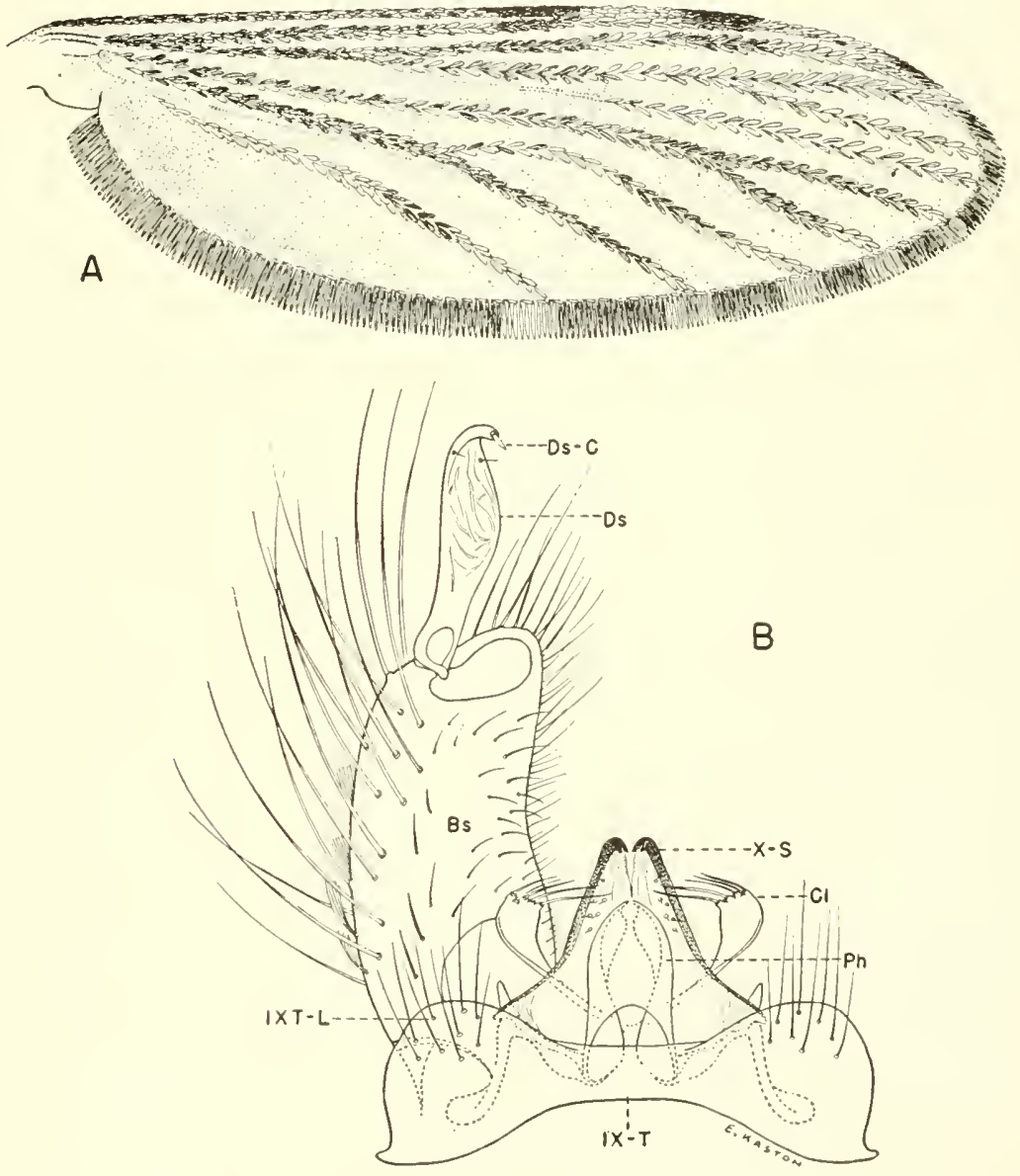

Fig. 86. Psorophora signipennis (Coquillett). A, Wing. B. Male terminalia. 
two-thirds, entirely white on apical third. Fringe scales arranged in alternately dark and pale groups.

ADUlT MALE.-Coloration similar to that of female. Terminalia (Fig. 86B). Ninth tergite (IX-T) with lobes (IXT-L) broadly rounded, widely separated, each bearing several scattered setae. Tenth sternite (X-S) prominent, heavily sclerotized. Phallosome $(\mathrm{Ph})$ cylindrical, rounded apically, open ventrally, closed dorsally. Claspette (Cl) with stem curved, slender, expanded apically; expanded portion attached to basal third of basistyle and bearing about five seta-like filaments on crown. Basistyle (Bs) about twice as long as broad, cylindrical, nearly truncate at apex, clothed with sparse scales and numerous long setae; basal and apical lobes absent. Dististyle (Ds) reticulated, a little more than half as long as basistyle, moderately inflated medially, tapering distally, slender, and curved near tip, terminating in a peg-like claw (Ds-C).

LARVA. Fig. 87),--Head broader than long. Antenna shorter than head, moderately spinose; antennal tuft generally 8 to 15 -branched, barbed, inserted near middle of shaft; tip of antenna apically with a short spine, a moderately

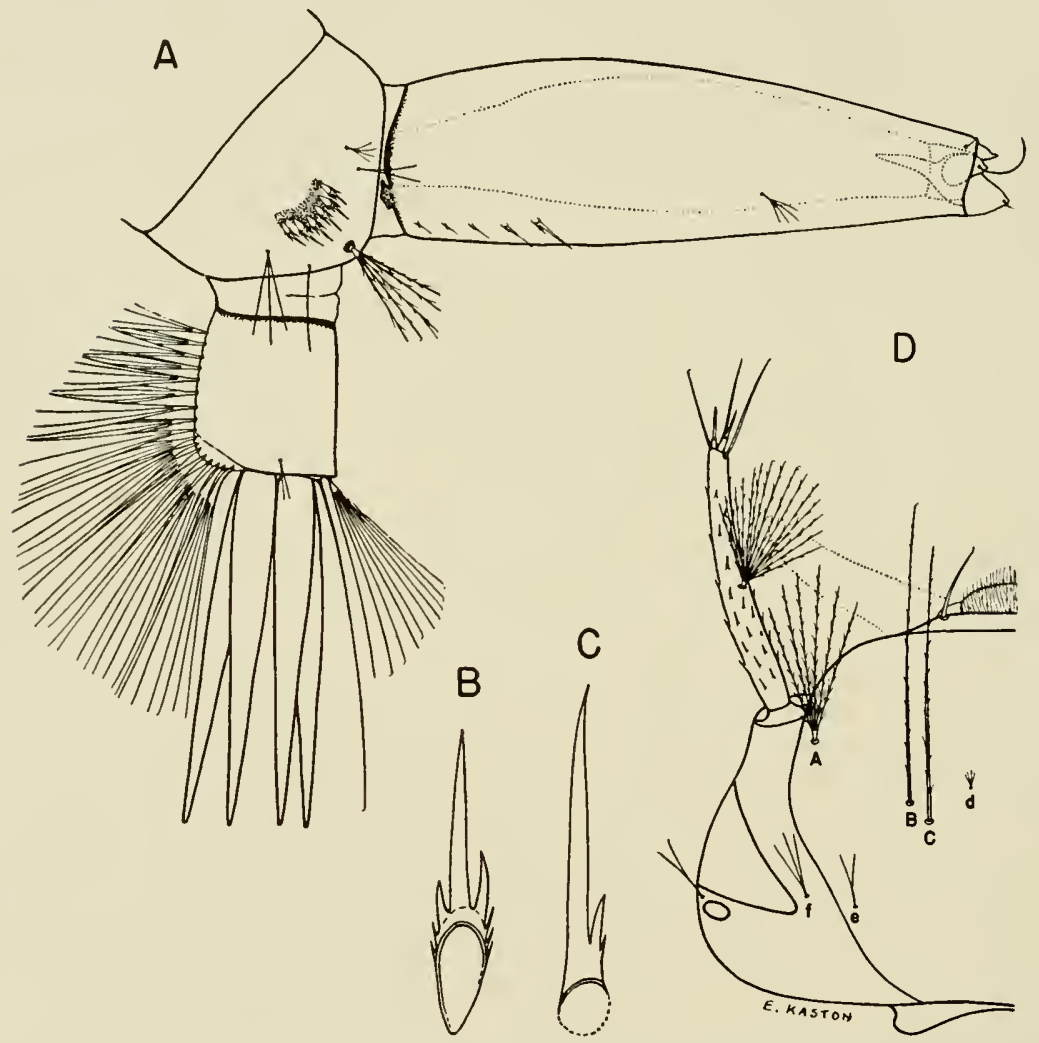

Fig. 87. Larva of Psorophora signipenuis (Coquillett). A, Male terminalia. B, Comb scale. C, Pecten tooth. D, Head. 
long spine and a membranous papilla, subapically with two longer spines. Head hairs: Preantennal (A) about 6 to 8-branched, conspicuously barbed; lower (B) and upper (C) single, barbed; postclypeal (d) small, multiple; sutural (e) 2-branched; trans-sutural (f) 3 to 4-branched; supraorbital 2branched. Comb of eighth segment of about six thorn-shaped scales; individual scale trifid, with long central tooth. Siphon somewhat inflated medially, about three times as long as basal width; pecten of 4 to 6 progressively longer teeth on basal third of siphon; subventral tuft minute, multiple, inserted at apical third of siphon; hair of lateral valve more than half as long as apical width of siphon. Anal segment longer than wide, completely ringed by the dorsal plate; lateral hair small, usually 2 to 3-branched; dorsal brush consisting of a long lower caudal hair and a shorter multiple upper caudal tuft on either side: ventral brush extending almost the entire length of the anal segment; gills 4 . longer than the anal segment, pointed.

Distribution.-Central United States, south to Mexico. Southern States: Arkansas (30); Kentucky (140); Missouri (70). Other States: Arizona (52); Colorado (89); Iowa (155, 156); Kansas (79); Montana (112); Nebraska (178); New Mexico (52); North Dakota (127); Oklahoma (159); South Dakota (129); Texas (108).

BIONOMics.-According to Rozeboom (159), the females of this species are extremely annoying biters in Oklahoma when their haunts are invaded. Rowe (155) claims that they are also troublesome in Iowa. Mail (112) states that $P$. signipennis is well adapted to breeding in temporary ground pools in arid regions and that it can pass from the egg to adult stage in five days under favorable conditions.

\section{Genus Aedes Meigen ${ }^{1}$}

Aedes Meigen, 1818, Syst. Beschr. Bek. Eur. Zweill. Ins., $1: 13$.

The genus Aedes is large, including a great many species distributed throughout the world. The members of this genus are frequently of considerable economic importance, since the majority are vicious biters and many are vectors of diseases of man and animals. The eggs of most species are laid on damp soil in depressions subject to flooding by rains, melting snow or floodwater from streams, although a few species breed in more restricted habitats, such as rot cavities in trees and in artificial containers. In general, the eggs are able to withstand long periods of drying. The genus is represented in the southern states by 23 species belonging to the following subgenera: Ochlerotatus, Finlaya, Stegomyia, Aedimorphus and Aedes.

SALIENT CHARACTERS.-Adult: Pulvilli absent or hair-like. Tarsal claws of female usually toothed. Spiracular bristles absent; postspiracular bristles present. Paratergites usually scaled. Postnotum without setae. Squama fringed. Fork of vein 2 much longer than its petiole. Tip of abdomen of female more or less pointed, the eighth segment retractile. Male Terminalia: The structure of the male terminalia varies greatly in different subgenera, providing useful characters for classification. The extent of variation in the species of the southern

1 Consult Dyar (1928) or Edwards (1932) for synonymy. 
states will be noted in the individual descriptions. Larva: The head hairs vary greatly in their position and branching. The siphon is usually short, with a single pair of subventral tufts never inserted near base; pecten present. Anal segment always with a barred area, the ventral brush rarely reduced. Comb scales variable in shape and number.

\section{Keys to the Species}

\section{ADULT FEMALES}

1. Proboscis with a white ring near middle 2

Proboscis without a white ring near middle

2. Abdomen with a white to yellow dorsal median longitudinal stripe or dorsal median longitudinal row of disconnected spots; wing scales either intermixed dark brown and white or entirely dark

Abdomen with transverse basal bands of white scales, but lacking a pale median longitudinal stripe; wing scales entirely dark .......taeniorhynchus (Wied.), p. 209

3. Wing scales entirely dark; first segment of hind tarsus without a pale median ring; last segment of hind tarsus entirely white ................mitchellae (Dyar), p. 195

Wing scales intermixed dark-brown and white; first segment of hind tarsus with or without a pale median ring; last segment of hind tarsus either entirely white or dark on apical half

4. Lateral pale markings of abdomen white, the dorsal pale markings yellowish; first segment of hind tarsus with a definite yellow median ring; last segment of hind tarsus entirely white sollicitans (Walker), p. 199

Lateral and dorsal pale markings of abdomen both white; first segment of hind tarsus variably marked (if a median pale ring is present, it is white, not yellow); last segment of hind tarsus white basally, dark apically, rarely all white

nigromaculis (Ludlow), p. 197

5. Integument of scutum bright yellow, except for a pair of large dark brown to black postero-lateral spots; proboscis yellow scaled, dark at tip fulvus pallens Ross, p. 185

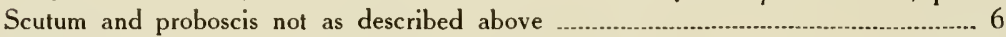

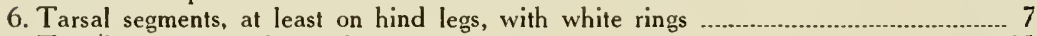

Tarsal segments without white rings

7. Tarsal segments white-ringed only basally ................................................................. 8

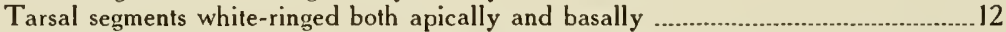

8. Scutum with a very conspicuous lyre-shaped marking of white scales against a dark background aegypti (Linn.), p. 227

Scutum without such markings

9. Wing scales entirely dark, narrow; basal white rings of hind tarsus usually very narrow, no broader than the diameter of the tarsal segments (basal rings sometimes rather broad, however, on segments 1 and 2)

Wing scales intermixed dark and whie, either narrow or very broad; basal white rings of hind tarsus broad (several times broader than diameter of tarsal segments)

10. Lower mesepimeral bristles absent; seventh abdominal tergite mostly dark scaled dorsally except for white scaling at apex vexans (Meigen), p. 230

A few lower mesepimeral bristles present; seventh abdominal tergite entirely pale scaled cantator (Coq.), p. 176

11. Wing scales very large, broad, deltoid; scutum with a broad median longitudinal stripe, pale golden-brown on anterior half, dark bronzy-brown on posterior half ..grossbechi D. and K., p. 188

Wing scales moderate in size, narrow, ligulate; scutum with a broad median longitudinal stripe, bronzy-brown for its entire length ..stimulans (Walker), p. 206

12. Wing scales intermixed dark brown and white, the white scales predominating; abdomen entirely white scaled dorsally, except for a pair of submedian black patches on each segment (segments VI and VII often entirely white) 
Wing scales entirely dark or with only a small white-scaled patch at base of costa; abdomen entirely dark scaled dorsally or with only narrow basal white bands (apices of the terminal segments may be white scaled)

13. Scutum with a wide median longitudinal stripe of dark bronzy-brown scales, broadly margined laterally by pale-yellow to golden-yellow scales, these pale scales indenting the longitudinal stripe along the prescutal-scutal suture. atropalpus (Coq.), p. 2

Scutum without a wide median longitudinal stripe of dark bronzy-brown scales broadly margined with pale scales

14. Hind tarsus with broad white rings present basally and apically on all segments except the last, which is entirely white; scutum clothed dorsally with goldenbrown scales canadensis (Theob.), p. 173

Hind tarsus with narrow white rings present basally and apically on segments ] and 2 and basally on segment 3 , the remaining segments entirely dark; scutum clothed dorsally with bronzy-black scales except for a very narrow median longitudinal line of golden-brown scales .................mathesoni Middk., p. 194

15. Scutum without a bicolorous pattern, being evenly clothed with golden-brown scales (a little paler on anterior and lateral margins and on prescutellar space); palpi short in both sexes ...................................... Meigen, p. 234

Scutum with a definite pattern of bicolorous scales; palpi of males longer than proboscis

16. Scutum with a median longitudinal stripe of silvery-white to pale-yellow scales

Scutum without a median longitudinal stripe of pale scales

17. Median longitudinal stripe extending the full length of the scutum, generally narrower than the dark-scaled area on either side (variable in $A$. dupreei)

Median longitudinal stripe extending from the anterior margin of the scutum to a little beyond middle, much broader than the dark scaled area on either side infirmatus D. and K., p. 19

18. Small species (wing about $2.5 \mathrm{~mm}$.) ; occiput dorsally with a narrow median stripe of white lanceolate scales bounded submedially by a patch of broad white scales; the median longitudinal stripe of scutum silvery-white, about equal in width to the dark-scaled area on either side (very broad in males, covering most of the dorsal surface) …….........................upreei (Coq.), p. 182

Medium sized species (wing about $3.5 \mathrm{~mm}$.); occiput dorsally with a median stripe of white lanceolate scales bounded submedially by a patch of broad dark scales; the median longitudinal stripe of scutum usually pale yellow, a little narrower than the dark-scaled area on either side (similar in males) atlanticus D. and K., p. 170 tormentor D. and K., p. 214

19. Scutum with a pair of broad submedian stripes of white to whitish-yellow scales, dark-brown scaled medially and laterally .............trivittatus (Coq.), p. 217

Scutum without a pair of pale submedian stripes, but with a single broad dark stripe medially, pale scaled laterally

20. Scutum media!ly with a golden-brown scaled longitudinal stripe about as wide as the areas of pale yellow scales on either side; dark portions of femora speckled with pale scales; tibiae and segment 1 of tarsi pale scaled on one side

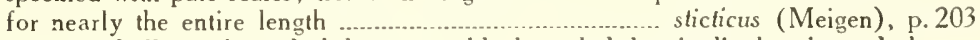

Scutum medially with a dark-brown to black scaled longitudinal stripe; dark portions of femora not speckled with pale scales; tibiae and segment I of tarsi not pale scaled on one side or only slightly so

21. Median longitudinal dark stripe of scutum broadly margined on anterior half by white lanceolate scales; posterior pronotum clothed with broad appressed white triseriatus (Say), p. 224

Median longitudinal dark stripe of scutum broadly margined on anterior half by gold lanceolate scales; posterior pronotum clothed with gold lanceolate scales not appressed thibaulti D. and K., .21 
MALE TERMINALIA

I. Dististyle furcate near base, inserted well before apex of basistyle cinereus Meigen, p. 235

Dististyle not furcate, inserted at apex of basistyle 2

2. Claspettes absent; basistyle very short (about $11 / 2$ times as long as wide) aegypti (Linn.), p. 227

Claspettes present; basistyle elongate ( $2 \frac{1}{2}$ to 4 times as long as wide)

3. Claspette stem crowned with a dense tuft of setae, the filament lacking; claw of dististyle subapical in position, arising from a small thumb-like projection at apical fifth of dististyle vexans (Meigen), p. 231

Claspette with a distinct filament; claw of dististyle apical in position

4. Claspette filament broadly expanded, contorted, leaf-like, arising from a short lateral branch near middle of claspette stem ihibaulti D. and K., p. 213

Claspette filament ligulate to blade-like, arising at apex of claspette stem

5. Apical lobe of basistyle absent; basal lobe without an enlarged spine 5

Apical lobe of basistyle present; basal lobe either with or without an enlarged spine

6. Basistyle with a small dense patch of long hairs arising on inner face at apical third; terminal claw about half as long as dististyle .......-triserialus (Say). p. 225

Basistyle without a dense patch of long hairs arising on inner face at apical third; terminal claw never more than one-third as long as dististyle ................... 7

7. Claspette filament sharply pointed, with a prominent sharp retrorse projection

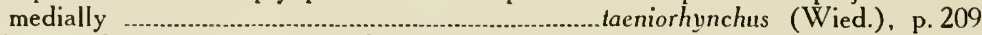

Claspette filament slender, curved, without a retrorse projection

8. Dististyle of nearly equal width throughout, glabrous except for a few short setae near apex; basistyle distinctly conical in shape; lobes of 9th tergite inconspicous, without spines or setae atropalpus (Coq.), p. 221

Dististyle distinctly broadened before middle, pilose; basistyle cylindrical in shape; lobes of 9 th tergite small but distinct, armed with short spine-like setae

9. Basal lobe of basistyle conical, rounded at apex, prominent mitchellae (Dyar), p. 197

Basal lobe of basistyle only slightly rounded, not prominent sollicitans (Walker), p. 200 nigromaculis (Ludlow), p. 199

10. Basal lobe of basistyle without an enlarged spine; apical lobe clothed with numerous short flattened setae ...canadensis (Theob.), p. 174 mathesoni Middk., p. 194

Basal lobe of basistyle with an enlarged spine; apical lobe clothed with short slender setae

11. Basal lobe of basistyle with a short stout spine, in addition to a long stout spine and numerous setae dorsalis (Meigen), p. 180

Basal lobe without a short stout spine, bearing only a single long stout spine and numerous setae

12. Claspette filament with a median retrorse pointed projection

infirmatus D. and K., p. 191

trivittatus (Coq.), p. 218

Claspette filament without a median retrorse pointed projection

13. Claspette stem stout and sinuous, broad medially, constricted basally and apically, the median portion much broader than the claspette filament

allanticus D. and K., p. 171

Claspette stem slender, never more than slightly broader than the claspette filament

14. Claspette stem sinuous; enlarged spine of basal lobe arising from a stout elongated tubercle near base tormentor D. and K., p. 214

Claspette stem straight or curved, not sinuous; enlarged spine of basal lobe arising from a short tubercle

15. Filament of claspette ligulate, curved, not expanded blade-like on convex side 
Filament of claspette expanded blade-like on convex side

dupreei (Coq.), p. 183

16. Enlarged spine of basal lobe with tip flattened, broadened, and usually slightly retrorse fulvus pallens Ross, p. 186

Enlarged spine of basal lobe sharply pointed, usually somewhat recurved …..........17

17. Basal lobe with a dense subapical tuft of fine long setae adjacent to the enlarged spine, distinct from the other shorter setae on the basal lobe

sticticus (Meigen), p. 203

Basal lobe without a distinct subapical tuft of fine long setae adjacent to the enlarged spine

18. Basal lobe bearing a few large setae, in addition to numerous small setae and an enlarged spine; claspette filament more than one one one-half times as long as claspette stem cantator (Coq.), p. 178

Basal lobe without a few large setae, bearing only small setae and an enlarged spine: claspette filament less than one and one-half times as long as claspette stem

19. Margin of blade on convex side of claspette filament angulate near middle; claspette filament about as long as claspette stem or slightly longer

stimulans (Walker), p. 206

Margin of blade on convex side of claspette flament rounded; claspette filament about two-thirds to three-fourths as long as claspette stem

\section{LARVAE (FOURTH INSTAR)}

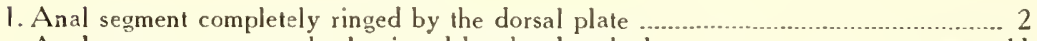

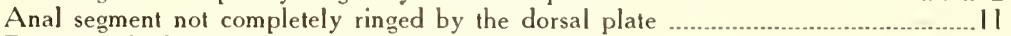

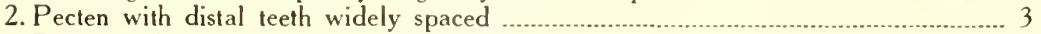

Pecten with all teeth more or less evenly spaced .................................................... 4

3. Subventral tuft of siphon inserted beyond pecten; lower head hair (B) single nigromaculis (Ludlow), p. 199

Subventral tuft of siphon inserted within pecten; lower head hair (B) double, sometimes triple fulvas pallens Ross, p. 187

4. Comb scales arranged in a single (sometimes irregular) row usually less than 12 in number

Comb scales arranged in a triangular patch, usually more than 14 in number ........ 7

5. Subventral tuft of siphon inserted within pecten ..........tormentor D. and K., p. 217

Subventral tuft of siphon inserted beyond pecten

6. Anal gills more than 5 times as long as anal segment, with conspicuous darkly pigmented tracheae; lower head hair (B) double or triple; antennal hair usually double ................................................................... 185

Anal gills never more than three times as long as anal segment, lacking conspicuous darkly pigmented tracheae; lower head hair (B) single; antennal hair multiple

allanticus D. and K., p. 172

7. Individual comb scale rounded apically, fringed with subequal spinules

laeniorhynchus (Wied.), p. 209

Individual comb scale thorn-like

8. Dorsal preapical spine of siphon as long as apical pecten tooth; upper lateral abdominal hair usually double on segments III to $\mathrm{V}$.

Dorsal preapical spine of siphon no more than half as long as apical pecten tooth; upper lateral abdominal hair single on segments III to $\mathrm{V}$

9. Siphon 3 to $31 / 2: 1$; pecten not quite reaching middle of siphon; lower and upper head hairs ( $B$ and $C$ ) well barbed ..................mitchellae (Dyar), p. 197

Siphon 2 to $21 / 2: 1$; pecten reaching middle of siphon or slightly beyond; lower and upper head hairs ( $B$ and $C$ ) smooth or very finely barbed sollicitans (Walker), p. 200

10. Apical spine of individual comb scale 3 or 4 times as broad and about twice as long as subapical spinules infirmatus D. and K. p. 192

Apical spine of individual comb scale about twice as broad and generally about 
$11 / 3$ times as long as subapical spinules trivillatus (Coq.), p. 219

11. Subventral tuft of siphon inserted within pecten; pecten with three or four distal teeth widely spaced alropalpus (Coq.), p. 222

Subventral tuft of siphon inserted beyond pecten; pecten either with distal teeth widely spaced or all teeth rather evenly spaced

12. Upper head hair (C) multiple

Upper head hair (C) single or double

13. Pecten with distal teeth widely spaced

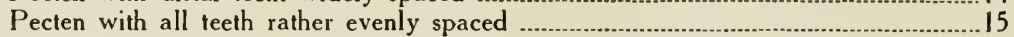

14. Preantennal hair, lower head hair, and upper head hair (A, B, and C) inserted in a straight line; upper lateral abdominal hair single on segments 111 to $\mathrm{V}$ cinereus Meigen, p. 236

Preantennal hair, lower head hair, and upper head hair (A, B, and C) not inserted in a straight line; upper lateral abdominal hair double or triple on segments III to $\mathrm{V}$ vexans (Meigen), p. 232

15. Lower head hair (B) double (sometimes single or triple)

Lower head hair (B) with 3 or more branches

16. Comb scales each with a long apical spine and short lateral spinules

Comb scales each with lateral spines nearly as long as apical spine ticticus (Meigen), p. 204 grossbechi D. and K., p. 190

17. Inner preclypeal spines separated by a little more than the length of one spine, lightly pigmented; siphon about $4 \frac{1}{2}$ to $5: 1$; upper lateral abdominal hair 3 to 4 -branched on segments I and II thibaulti D. and K., p. 214

Inner preclypeal spines not separated by more than the length of one spine, darkly pigmented; siphon about $21 / 2$ to $4: 1$; upper lateral abdominal hair usually double on segments I and II

18. Anal gills usually about as long as the anal segment; siphon 3 to $4: 1$

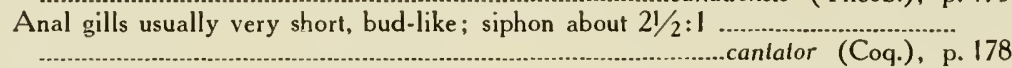
canadensis (Theob.), p. 175

19. Comb of many scales (usually more than 20) arranged in a sub-triangular patch; antennal hair multiple

Comb of about 7 to 15 scales in a single or partly double row; antennal hair single (rarely forked)

20. Upper head hair (C) double; anal gills about as long as the anal segment; subventral tuft of siphon 3 to 4 -branched ....................stimulans (Walker), p. 207

Upper head hair (C) single (rarely double); anal gills usually very short, budlike; subventral tuft of siphon usually with 5 to 9 branches dorsalis (Meigen), p. 182

21. Lower head hair (B) 2 to 4-branched; preantennal hair (A) multiple; individual comb scale long, gradually tapered, evenly fringed with fine short spinules Iriseriatus (Say), p. 225

Lower head hair (B) single; preantennal hair (A) single; individual comb scale with a strong apical spine and several shorter stout thorn-like lateral spines aegypli (Linn.), p. 229

\section{Aedes (Ochlerotatus) atlanticus Dyar and Knab}

Aedes allanticus Dyar and Knab, 1906, Jour. N. Y. Ent. Soc., 14:198.

Adult female.-Medium sized species. Head: Proboscis dark scaled; palpi short, dark. Occiput dorsally with a rather broad longitudinal stripe of white lanceolate scales, extending forward between the eyes; this median stripe bounded on either side by a large submedian patch of broad dark scales; lateral region of occiput clothed with broad appressed dingy-white scales. Erect forked scales numerous, those on central portion of occiput pale. Thorax: Integument 
of scutum dark brown, densely clothed with fine dark bronzy-brown scales, except for a silvery-white to pale-yellow scaled median longitudinal stripe extending the full length of the scutum and covering the middle lobe of the scutellum (this longitudinal stripe a little less than one-third as wide as scutum). Dorsal half of posterior pronotum covered with narrow dark brown scales. Abdomen: Tergites dark scaled, with conspicuous basal triangular patches of white scales laterally; venter primarily whitish scaled, often speckled with dark scales. Legs: Legs dark scaled except for pale inner surfaces of femora. Wing: Scales narrow, dark.

ADULT MALE. - Coloration similar to that of female, but with pale scales usually more prevalent on occiput. TERMINALIA. (Fig. 88A). Lobes of ninth tergite (IXT-L) about as long as broad, separated by a little less than the width of one lobe, each bearing several short spines. Tenth sternite (X-S) prominent, heavily sclerotized beyond middle. Phallosome $(\mathrm{Ph})$ stoutly conical, rounded apically, open ventrally, closed dorsally. Claspette stem (Cl-S) pilose, large, stout, sinuous, broader medially, constricted basally and apically, extending a little beyond middle of basistyle; claspette filament (Cl-F) about one- third as long as stem but much narrower than expanded middle portion of stem, striated, curved and tapered at apical third. Basistyle (Bs) about three times as long as width across middle, narrower distally, rounded at apex,

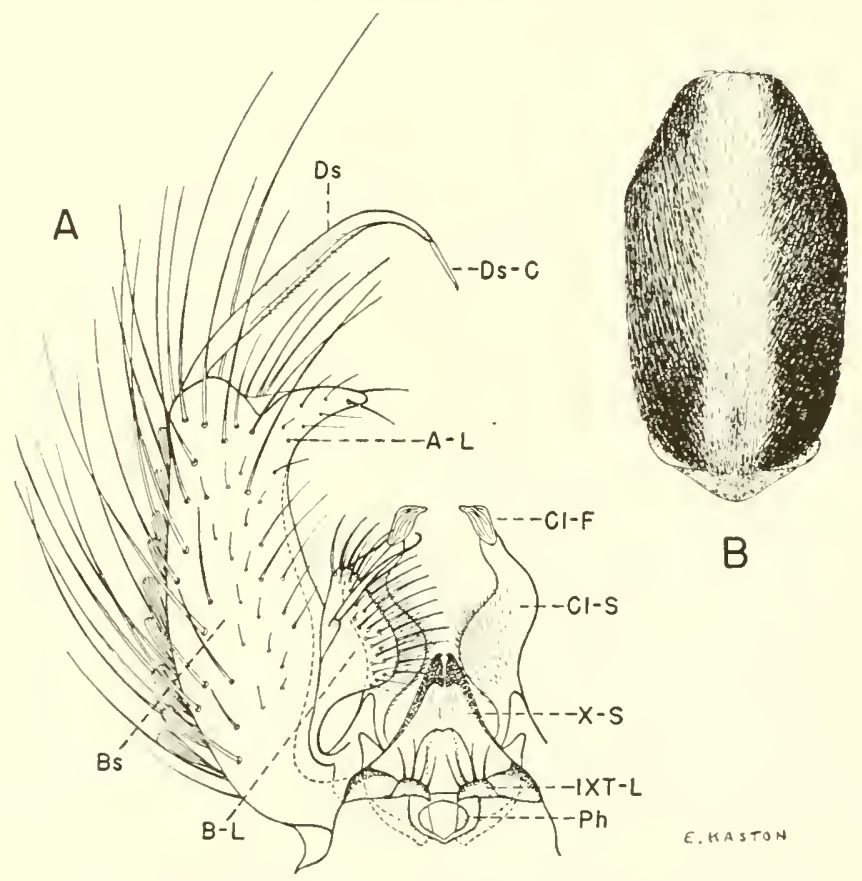

Fig. 88. Aedes atlanticus Dyar and Knab. A, Male terminalia. $B$, Scutum and scutellum. 
clothed with scales and long setae on outer aspects; basal lobe (B-L) large, narrow at base, broadly expanded apically, bearing numerous short stout setae on apex and a large stout recurved spine near middle of outer margin; apical lobe (A-L) a prominent thumb-like projection bearing several short setae. Dististyle (Ds) approximately two-thirds as long as basistyle, broader medially; terminal claw (Ds-C) slender, about one-fifth as long as dististyle.

LARva. (Fig. 89).-Head broader than long. Antenna less than half as long as head, slightly curved, sparsely spined; antennal tuft small, multiple,

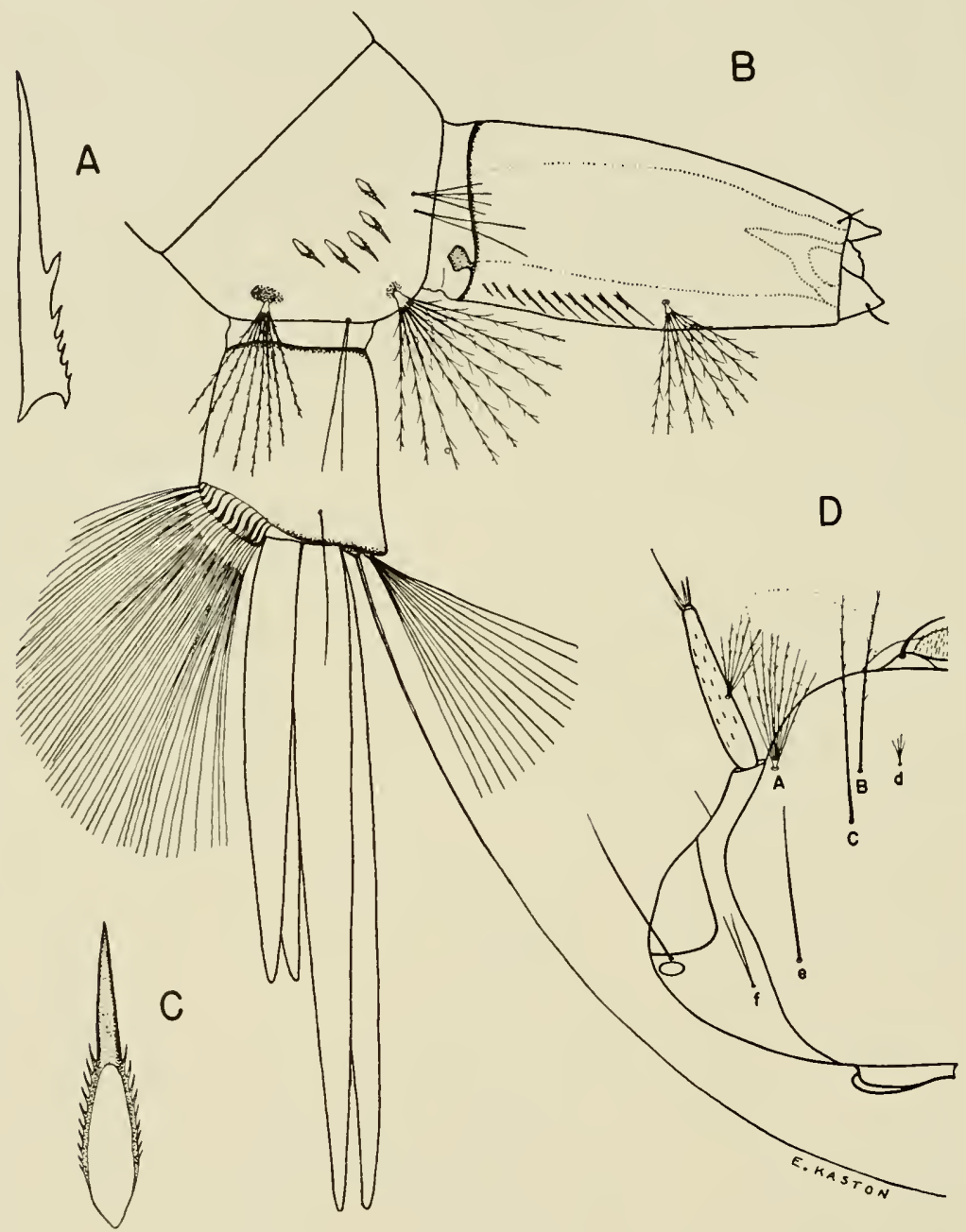

Fig. 89. Larva of Aedes allanticus Dyar and Knab. A, Pecten tooth. B, Terminal segments. C, Comb scale. D, Head. 
slightly barbed, inserted at middle of shaft, not reaching tip. Head hairs: Preantennal (A) multiple, sparsely barbed, projecting slightly beyond insertion of antennal tuft; lower (B) and upper (C) single, sparsely barbed; postclypeal (d) small, multiple; sutural (e) single; trans-sutural (f) usually double; supraorbital single. Lateral abdominal hairs double on segments I and II, single on segments III to VI. Comb of eighth segment of about 5 or 6 large thorn-shaped scales in a single curved row; individual scale with minute lateral spinules. Siphon about twice as long as wide; pecten of numerous evenly spaced teeth extending nearly to middle of siphon; subventral tuft multiple, barbed, inserted beyond pecten. Anal segment slightly longer than wide, completely ringed by a heavily sclerotized dorsal plate; lateral hair single or double; dorsal brush consisting of a long lower caudal hair and a shorter multiple upper caudal tuft on either side; ventral brush well-developed, confined to the barred area; gills 4, longer than the anal segment, the upper pair longer than the lower pair.

Distribution.-Florida to New Jersey and west to Oklahoma and Texas. Southern States: Alabama (71); Arkansas (83); Florida, Georgia and Louisiana (52); Mississippi (125); Missouri (70); North Carolina (52); South Carolina (64); Virginia (49). Other States: Delaware (106); District of Columbia (52); Maryland (19); New Jersey (77); Oklahoma (159); Texas (108).

BIONOMiCs.- -King et al., (96) state that the females are vicious biters. No characters are known for separating the females of $A$. atlanticus from those of $A$. tormentor; therefore adult records are based on structures of male terminalia. Larvae occur in temporary pools in both open fields and woodlands. Breeding extends from March to November in the South (4th Sv. C. Med. Lab. records, 1942-1944, unpublished).

\section{Aedes (Ochlerotatus) canadensis (Theobald)}

Culex canadensis Theobald, 1901, Mon. Culic., 2:3.

adult female.-Medium sized species. Head: Proboscis dark scaled; palpi short, dark, with a few white scales at tip and at base of segment 4 . Scales of postero-dorsal region of occiput narrow, whitish-yellow, arranged in a sub-triangular patch projecting anteriorly on the mid line; scales dorsally margining eyes narrow, whitish-yellow; scales of submedian areas between the postero-dorsal patch and eye margins narrow, golden brown. Lateral regions of occiput clothed with broad whitish scales, often surroundnig a small darkscaled patch near eye margin. Erect forked scales numerous on dorsal surface of occiput, those on central portion pale. Thorax: Integument of scutum reddish brown; vestiture of small narrow golden-brown scales, paler on anterior and lateral margins and on prescutellar space; prescutellar space frequently bordered on either side by a narrow submedian line of pale scales extending anteriorly to near middle of scutum. Posterior pronotum clothed on dorsal half with narrow brown scales. Abdomen: Tergites dark scaled, with narrow transverse basal bands (frequently lacking) and conspicuous basal lateral 
patches of white scales; the seventh segment, and often the sixth, white scaled on dorso-apical margin. Venter entirely whitish scaled or with apices of segments dark. Legs: Legs dark scaled except for white knee spots, pale inner surfaces of femora and tibiae, a few white scales at tips of tibiae, and basal and apical white rings on tarsal segments. Hind tarsi with the white rings of segments 1 to 4 broader than the diameter of the segments and with segment 5 entirely white scaled. Fore- and mid-tarsi with white rings of segments 1 to 3 narrower than those of hind-tarsi, and with white rings entirely lacking or much reduced on segments 4 and 5. Wing: Scales narrow, dark.

ADULT MALE. - Coloration similar to that of female, but the dorsal abdominal bands much broader and whitish occipital scales more prevalent. TERMINALIA (Fig. 90B). Lobes of ninth tergite (IXT-L) as long as broad, rounded, separated by about the width of one loba, each bearing several short stout spines. Tenth sternite (X-S) prominent, heavily sclerotized beyond middle.

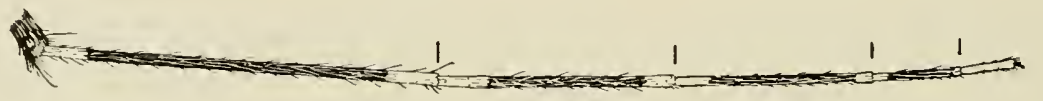

A

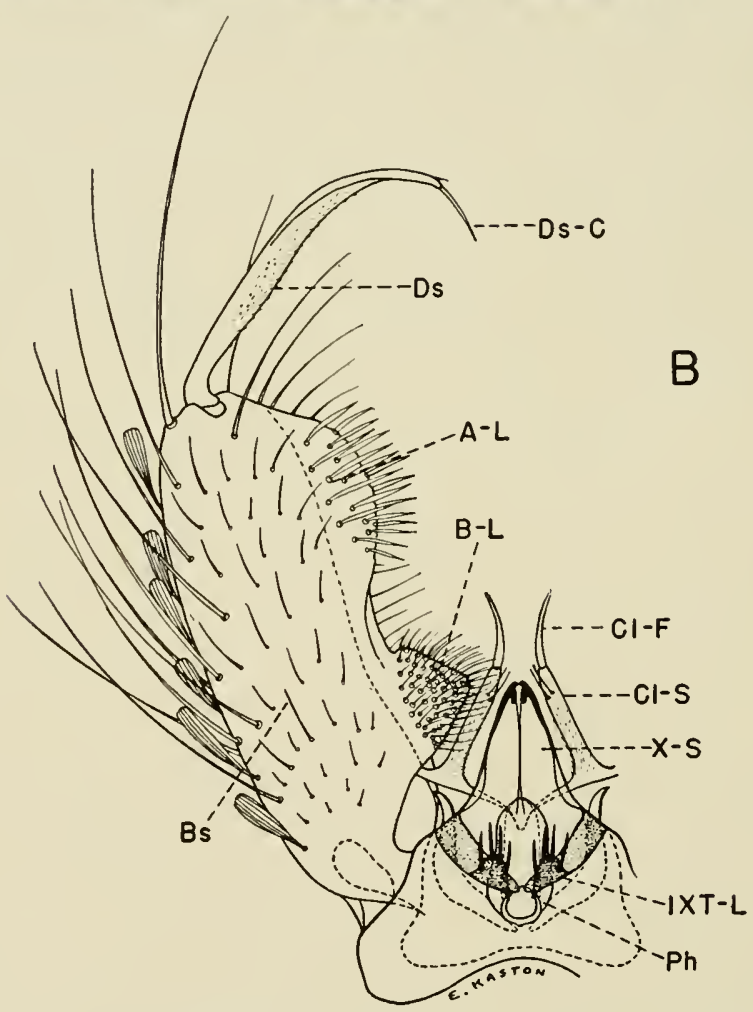

Fig. 90. Aedes canadensis (Theobald). A, Hind tarsus. B, Male terminalia. 
Phallosome $(\mathrm{Ph})$ cylindrical, about twice as long as broad, rounded apically, open ventrally, closed dorsally, lightly sclerotized. Claspette stem (Cl-S) slender, cylindrical, pilose, reaching near apex of basal lobe; claspette filament (Cl-F) ligulate, about two-thirds as long as stem, gradually tapering to a blunt point. Basistyle (Bs) about three times as long as width actoss middle, rounded at apex, clothed with large scales and long setae on outer aspect; basal lobe (B-L) large, quadrate, densely clothed with short setae arising from small tubercles; apical lobe (A-L) large, broadly rounded, covered with numerous short flattened setae. Dististyle (Ds) about two-thirds as long as basistyle, broader medially; terminal claw (Ds-C) slender, about one-sixth as long as dististyle.

LARVA. (Fig. 91).-Head broader than long. Antenna shorter than head,

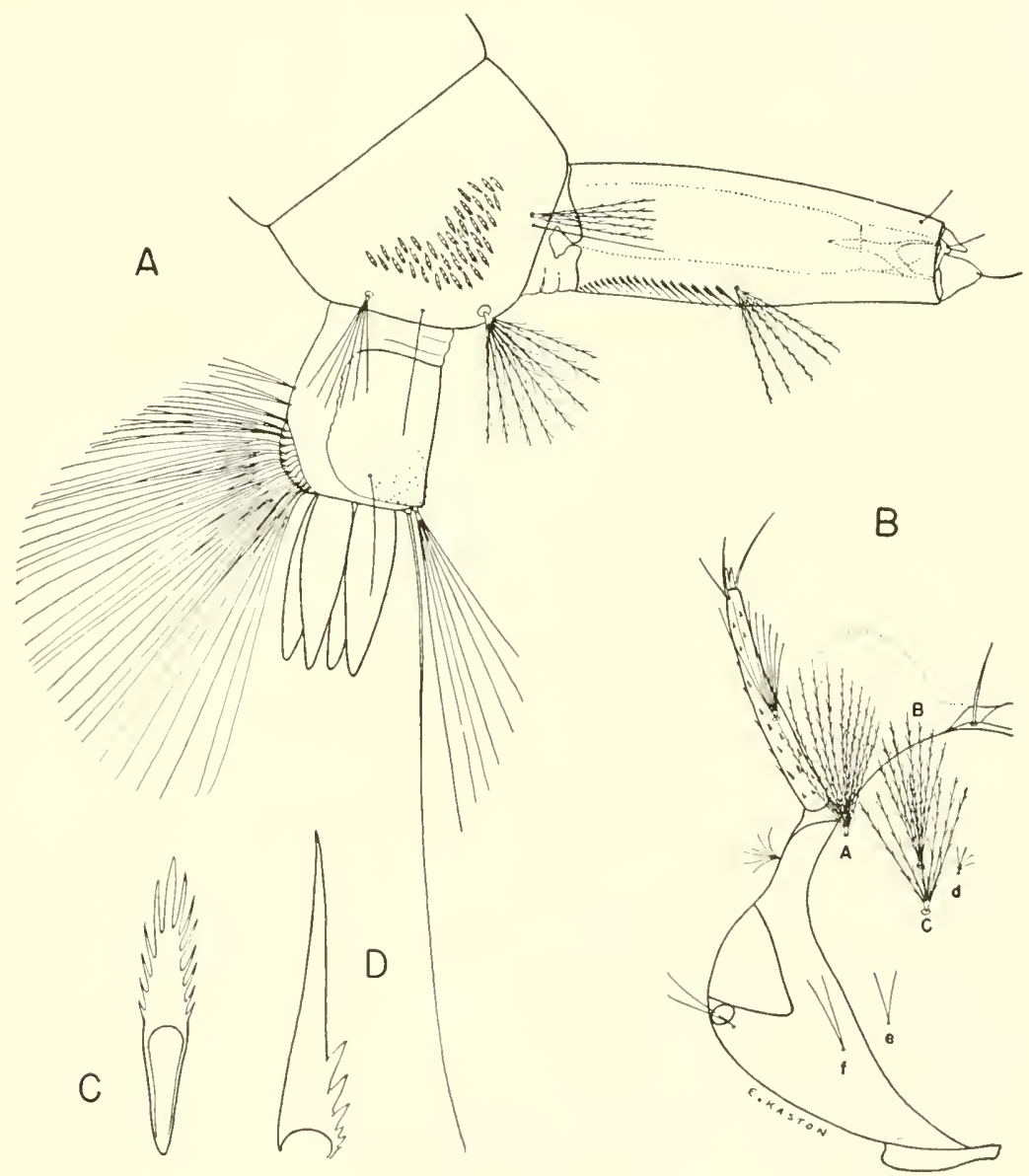

Fig. 91. Larva of Aedes canadensis (Theobald). A, Terminal segments. B, Head. C, Comb scale. D. Pecten tooth. 
slightly curved, spinose; antennal tuft multiple, inserted before middle of shaft, reaching nearly to tip. Inner preclypeal spines darkly pigmented, not separated by more than the length of one spine. Head hairs: Preantennal (A), lower (B), and upper (C) large, multiple, barbed, not inserted in a straight line; postclypeal (d) small, multiple; sutural (e), trans-sutural (f), and supraorbital usually 2 to 3-branched. Upper lateral abdominal hairs usually double on segments I and II. Comb of eighth segment of many scales in a patch; individual scale pointed and fringed with rather slender subequal spinules. Siphon three to four times as long as wide; pecten of numerous evenly spaced teeth not reaching middle of siphon; subventral tuft multiple, barbed, inserted beyond pecten. Anal segment longer than wide, with dorsal plate reaching twothirds of the way down the sides; lateral hair single; dorsal brush consisting of a long lower caudal hair and a shorter multiple upper caudal tuft on either side: ventral brush well-developed, with about two short tufts preceding the barred area; gills 4 , variable in length, generally about as long as the anal segment.

Distribution.-Widely distributed in North America. Southern States: Alabama (97); Arkansas (30); Florida (52); Georgia (97); Kentucky (140); Louisiana and Mississippi (96); Missouri (1); North Carolina and South Carolina (52); Tennessee (96); Virginia (49). Other States: Connectcut (21); Delaware (106); Illinois (42); Indiana (76); Iowa (155, 156); Maine (52); Maryland (19); Massachusetts (180); Michigan (88); Minnesota (131); Montana (112); Nebraska (129); New Hampshire (52); New Jersey (77); Oklahoma (159); Pennsylvania (52); Rhode Island (99); Texas (130); Vermont (90); Wisconsin (48).

BIONOMics.- The adults are often encountered in considerable numbers in woodlands near their breeding places but are seldom troublesome biters and rarely enter dwellings. This species shows preference in its breeding for woodland pools containing decaying leaves but is found to a lesser extent in small streams, pools, and ditches adjacent to woodland areas. It overwinters in the egg stage and hatches in large numbers in the South following late winter and early spring flooding. The larvae also occur in small numbers, following rains, during the summer, fall, and winter months in the Southern States (4th Sv. C. Med. Lab. reports, 1942-1944 unpublished). It is not known whether these late-season larvae are from eggs laid during the previous year or from recently deposited eggs.

\section{Aedes (Ochlerotatus) CANTATor (Coquillett)}

Culex cantator Coquillett, 1903, Can. Ent., 35:255.

adult female.-Medium sized to rather large species. Head: Proboscis long, slender, dark scaled; palpi short, dark. Broad dorsal region of occiput clothed with narrow whitish-yellow to pale golden brown scales and numerous dark erect forked scales; lateral region of occiput covered with broad dingyyellow scales, usually speckled with dark or surrounding a small dark patch near margin of eye. Thorax: Integument of scutum reddish brown; vestiture consisting of narrow golden-brown scales, with those of anterior and lateral 
margins and of prescutellar space paler. Scales of posterior pronotum similar to those of scutum. A few lower mesepimeral bristles present. Abdomen: First tergite predominately pale scaled; tergites II to $\mathrm{V}$ dark scaled, each with a yellowish band basally (bands narrow medially, broadened laterally) and a

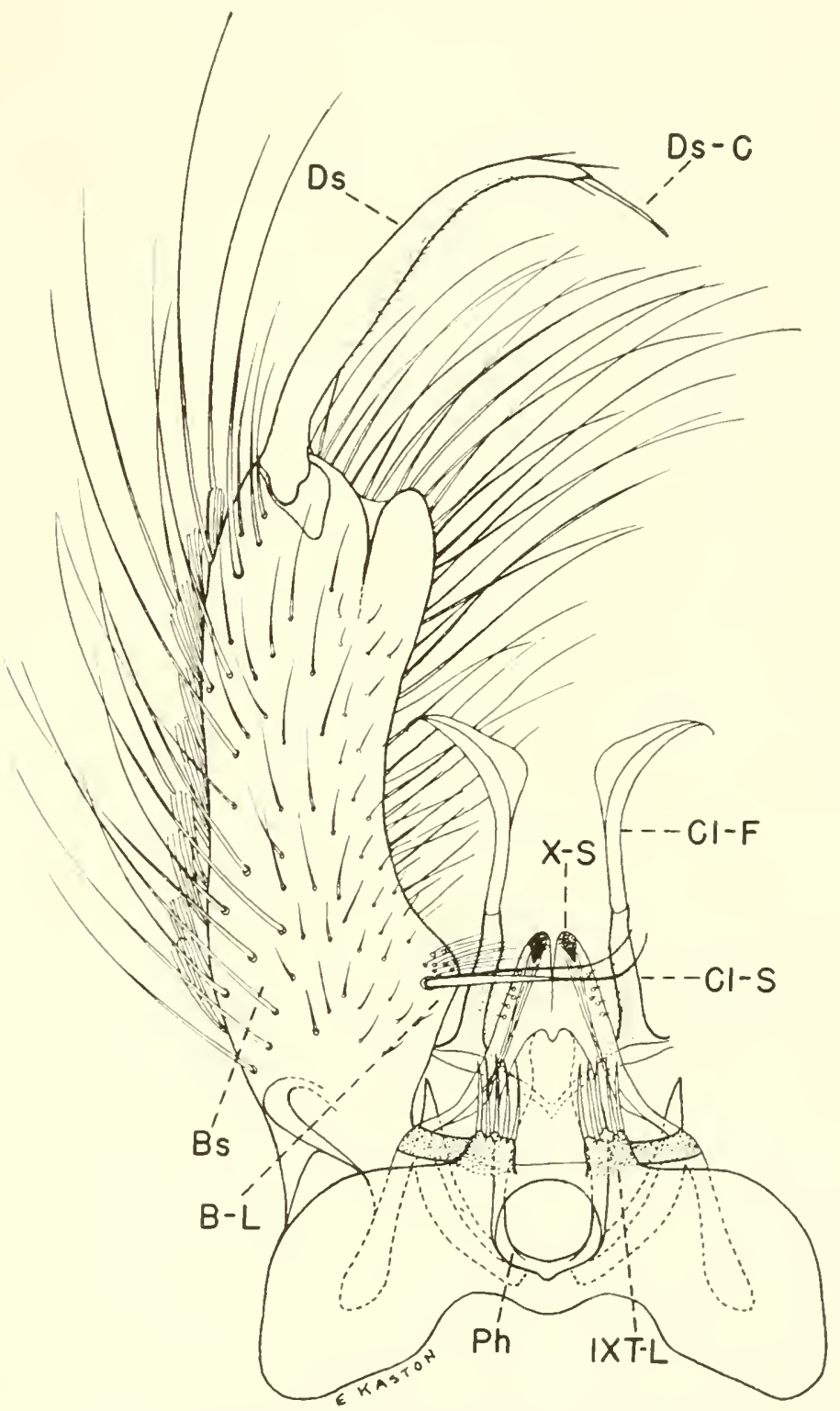

Fig. 92. Male terminalia of Aedes cantator (Coquillett). 
narrow margin of pale scales apically; sixth tergite mostly pale scaled, seventh entirely pale scaled. Venter yellowish-white scaled. Legs: Femora and tibiae brown scaled, yellowish on inner surface; knee joints pale. Tarsi usually darker, with first segment often streaked on one side with pale scales; each segment of hind tarsus with a narrow basal pale ring; fore- and mid-tarsi similarly marked but with basal rings of segments 4 and 5 much reduced or lacking. Wing: Scales rather narrow, brown.

ADULT MALE.-Coloration similar to that of female. TERMinalia (Fig. 92). Lobes of ninth tergite (IXT-L) about as long as broad, usually separated by a little more than the width of one lobe, each bearing several short spines. Tenth sternite (X-S) prominent, heavily sclerotized beyond middle. Phallosome $(\mathrm{Ph})$ cylindrical, about twice as long as broad, rounded apically, open ventrally, closed dorsally. Claspette stem (Cl-S) pilose, curved, rather slender, extending a little beyond basal lobe; claspette filament (Cl-F) more than one and one-half times as long as stem, slender, curved, sharply pointed, with a blade-like expansion medially on the convex side. Basistyle (Bs) about three and one-half times as long as width across middle, bluntly rounded at apex, clothed with scales and long setae (setae very numerous and long on inner ventral margin); basal lobe (B-L) short, bluntly conical, bearing a stout recurved spine subapically on dorsal side and numerous short and two or three rather long setae apically; apical lobe (A-L) rounded, prominent, clothed with a few shott setae. Dististyle (Ds) about two-thirds as long as basistyle, a little broader medially; terminal claw (Ds-C) slender, approximately one-fourth as long as dististyle.

LARva. (Fig. 93).-Head broader than long. Antenna shorter than head, spinose; antennal tuft multiple, finely barbed, inserted before middle of shaft, reaching nearly to tip. Inner preclypeal spines darkly pigmented, not separated by more than the length of one spine. Head hairs: Preantennal (A) large, multiple, barbed; lower (B) and upper (C) rather large, multiple, smooth or weakly barbed (hairs A, B, and C not inserted in a straight line); postclypeal (d) small, usually 3-branched; sutural (e), trans-sutural (f), and supraorbital small, usually double. Upper lateral abdominal hairs on segments I to $\mathrm{V}$ double, occasionally triple. Comb of eighth segment of many scales in a patch; individual scale fringed laterally with small spinules and apically with a few larger subequal spines, the middle spine somewhat longer and stouter. Siphon about two and one-half times as long as wide; pecten of numerous evenly spaced teeth, not reaching middle of siphon; subventral tuft multiple, barbed, inserted beyond pecten. Anal segment longer than wide, with dorsal plate reaching about two-thirds of the way down the sides; lateral hair single; dorsal brush consisting of a long lower caudal hair and a shorter multiple upper caudal tuft on either side; ventral brush well-developed, with about two short tufts preceding the barred area; gills 4 , variable in length, but generally very short and bud-like.

Distribution.-Occurs along the Atlantic Coast, from Virginia to Canada. Southern States: Virginia (49). Other States: Connecticut (52); Delaware (106); Maine (52); Maryland (19); Massachusetts (52); New Hampshire 
(90); New Jersey (77); New York (52); Pennsylvania (8); Rhode Island (99).

BIONOMICs.-The females are fierce biters, attacking principally in the open, but also freely entering houses. Adults frequently migrate considerable distances from their breeding places. Larvae occur in coastal marshes, including both fresh and salt water, but less brackish water seems to be preferred.

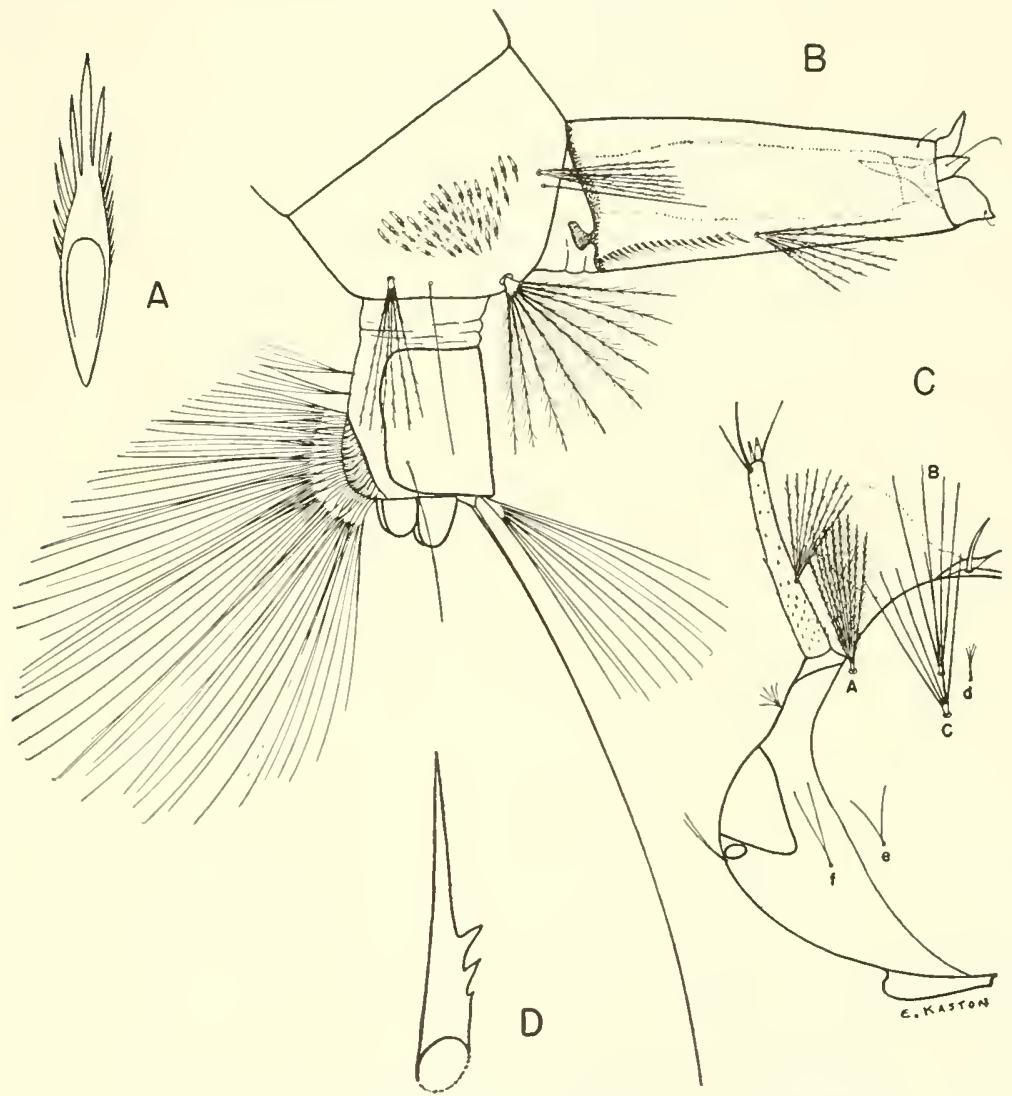

Fig. 93. Larva of Aedes cantator (Coquillett). A, Comb scale.

B, Terminal segments. C, Head. D, Pecten tooth.

MEDICAL IMPORTANCE.-According to Feemster and Getting (60) this species ranks high as a vector of equine encephalitis under laboratory conditions and may be an imporatnt vector of this disease in nature.

\section{Aedes (Ochlerotatus) dorsalis (Meigen)}

Culex dorsalis Meigen, 1830, Syst. Beschr. Zweifl. Ins., 6:242.

adult female.-Medium sized species. Head: Proboscis dark with a few scattered white scales near base; palpi short, dark, speckled with white scales. 
Broad median area of occiput clothed with whitish lanceolate scales and erect pale forked scales; a small submedian patch of narrow brown scales and a few black erect forked scales bordering the median pale area on either side; lateral portion of occiput clothed with broad appressed scales, mostly pale but often sprinkled with dark. Thorax: Integument of scutum blackish; a narrow longitudinal stripe of brown lanceolate scales present medially (this stripe often variable in width); remainder of scutum, including prescutellar space, clothed with whitish-yellow lanceolate scales. Scales of dorsal half of posterior pronotum golden brown, lanceolate. Abdomen: First tergite white scaled; remaining tergites each white basally, laterally and medially, with a pair of submedian black patches (last two segments often entirely white). Venter white scaled. Legs: Femora and tibiae principally white scaled, speckled with dark on outer aspects; first segment of each tarsus dark, liberally streaked with white and with white basal and apical rings. Hind tarsus with segments 2, 3 and 4 dark, ringed basally and apically with white; segment 5 almost entirely white. Midtarsus with segments 2 and 3 dark, narrowly white-ringed basally and apically; segment 4 dark except for a few white scales at base; segment 5 entirely dark. Fore-tarsus with segment 2 narrowly white-ringed basally and apically; segment 3 with a few white scales at base; segments 4 and 5 entirely dark. Wing: Scales narrow, mixed dark brown and white, the white scales predominating.

Adult Male.-Coloration similar to that of female. Terminalia. (Fig. 94). Lobes of ninth tergite (IXT.L) nearly as long as broad, separated by a

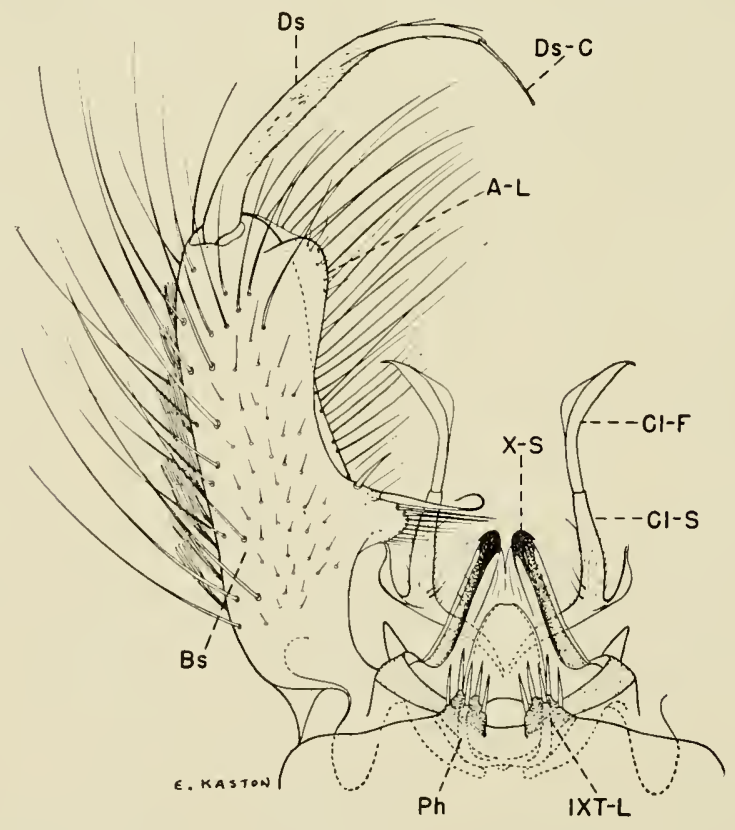

Fig. 94. Male terminalia of Aedes dorsalis (Meigen). 
little less than the width of one lobe, each bearing several short stout spines. Tenth sternite (X-S) prominent, heavily sclerotized beyond middle. Phallosome $(\mathrm{Ph})$ conical, about two-thirds as broad as long, rounded apically, open ventrally, closed dorsally. Claspette stem (Cl-S) rather slender, pilose, somewhat curved, extending to or a little beyond middle of basal lobe; claspette filament $(\mathrm{Cl} \cdot \mathrm{F})$ about as long as stem, broad, blade-like, tapering to a slightly recurved tip. Basistyle (Bs) cylindrical, about three and one-half times as long as width across middle, rounded at apex, clothed with scales and long setae on outer aspect and numerous long setae near inner ventral margin; basal lobe (B-L) prominent, conical or sub-quadrate, bearing numerous fine setae, a short stout spine, a long strong recurved spine, two or three long stout setae, and

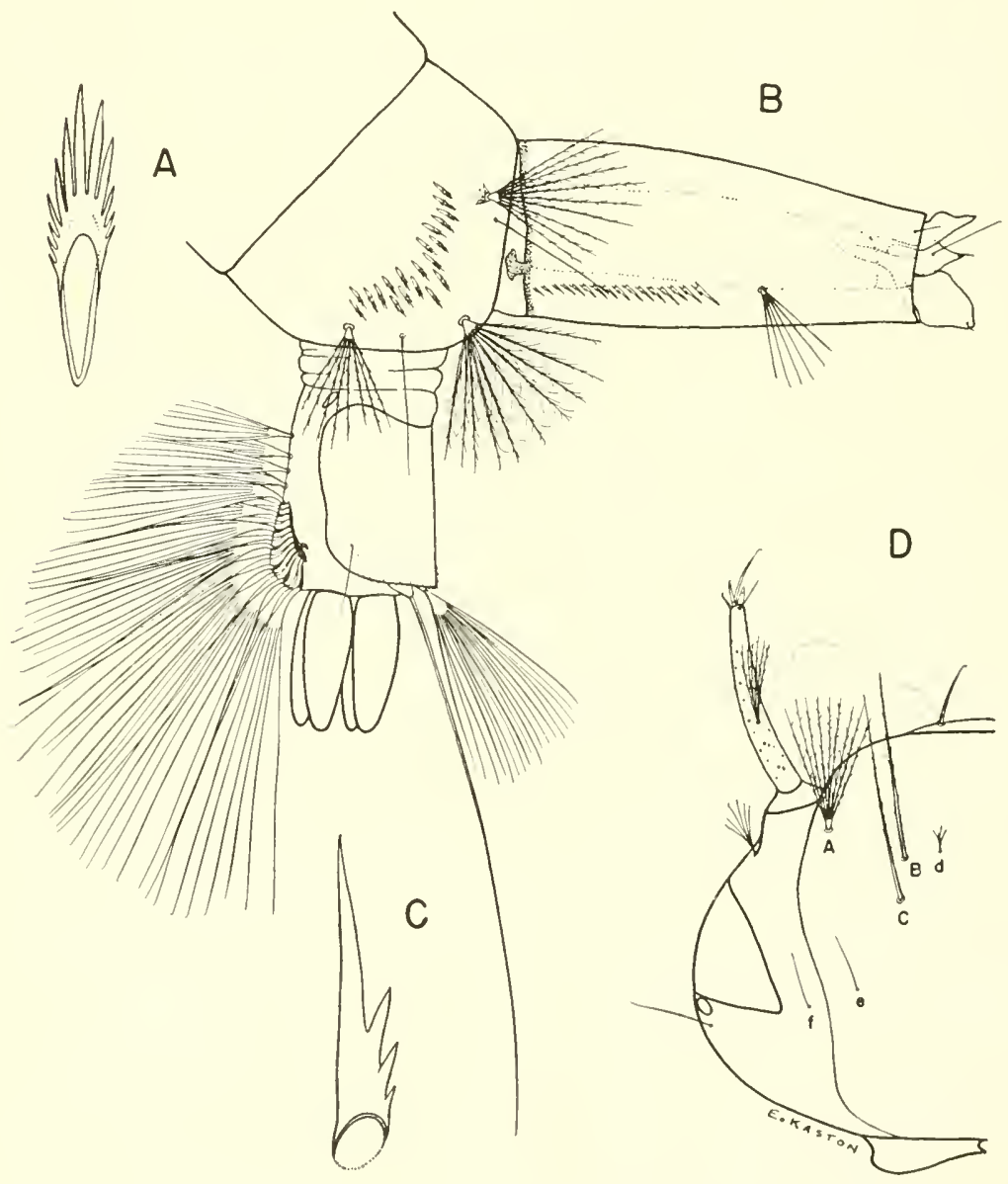

Fig. 95. Larva of Aedes dorsalis (Meigen). A. Comb scale.

B, Terminal segments. C, Pecten tooth. D, Head. 
several other progressively weaker setae; apical lobe (A-L) short, rounded, bearing a number of short curved setae. Dististyle (Ds) about three-fifths as long as basistyle, slightly broader medially; terminal claw (Ds-C) slender, nearly one-fourth as long as dististyle.

LARva. (Fig. 95).- - Head broader than long. Antenna less than half the length of the head, spinose; antennal tuft multiple, finely barbed, arising near middle of shaft. Head hairs: Preantennal (A) multiple, barbed, reaching insertion of antennal tuft; lower (B) and upper (C) single (hair $C$ rarely double); postclypeal (d) small, multiple; sutural (e), trans-sutural (f) and supraorbital single or double. Upper lateral abdominal hairs usually 2 to 3 . branched on segments I to IV, double on V and single on VI. Comb of eighth segment of numerous scales in a patch; individual scale fringed with subequal spines, the apical one a little longer and stouter. Siphon about two and onehalf to three times as long as wide; pecten of numerous evenly spaced teeth not reaching middle of siphon; subventral tuft multiple, inserted beyond pecten. Anal segment longer than wide, with dorsal plate reaching well down the sides; lateral hair single; dorsal brush consisting of a long lower caudal hair and a shorter multiple upper caudal tuft on either side; ventral brush large, with several tufts preceding the barred area; gills 4 , variable in length, usually very short and bud-like but occasionally as long or longer than the anal segment.

distribution.-United States, Canada, Europe and Asia. Southern States: Kentucky (140); Louisiana (52); Mississippi (4th Sv. C. Med. Lab. records, 1945, unpublished); Missouri (1). Other States: California and Colorado (52); Connecticut (90); Idaho and Illinois (52); Iowa $(155,156)$; Kansas (128); Massachusetts (52); Minnesota (131); Montana (112); Nebraska (178); Nevada, New Mexico, New York and North Dakota (52); Oklahoma (159); Oregon and Pennsylvania (52); Texas (130); Utah (142); Washington (52); Wisconsin (48); Wyoming (129).

віоNомісs.-The females are vicious biters, attacking any time during the day or night, but are particularly active toward the evening, or on calm, cloudy days. They are strong fliers and occasionally migrate considerable distances from their breeding places. The breeding places may vary from coastal salt marshes to fresh inland flood and irrigation waters. It is a common species in the rice fields of California. There appears to be considerable variation in this species, possibly due to different types of larval habitats.

\section{Aedes (Ochlerotatus) Dupreei (Coquillett)}

Culex dupreei Coquillett, 1904, Can. Ent., 36:10.

Adult FEMAle.-Rather small species. Head: Proboscis dark scaled; palpi short, dark. Occiput dorsally with a longitudinal median stripe of lanceolate silvery-white scales extending the length of the occiput and projecting forward between the eyes; this median stripe bounded submedially by a patch of broad white scales which blend on either side with a large lateral patch of broad appressed brown scales. Erect forked scales on central portion of occiput pale. Thorax: Integument of scutum dark brown, clothed with narrow bronzy-brown 
scales except for a broad longitudinal stripe of silvery-white scales extending the full length of the scutum (this longitudinal pale stripe usually slightly more than one-third as wide as scutum). Posterior pronotum with narrow bronzy-brown scales on dorsal half. Abdomen: First tergite clothed with dark scales, usually mixed with pale scales; remaining tergites dark, with white basal patches laterally. Venter white scaled. Legs: Legs dark scaled except for pale inner surfaces of femora and tibiae. Wing: Scales narrow, brown.

ADULT MALE.-Coloration similar to that of female, but with pale scales more prevalent on occiput, the longitudinal stripe on scutum very broad and covering entire dorsal surface, and the lateral spots of abdomen extending to, or nearly to, the mid-dorsal line. TERMINALIA (Fig. 96). Lobes of ninth tergite (IXT-L) as long as broad, rounded or truncate apically, separated by less than the width of one lobe, each bearing approximately four or five stout spines. Tenth sternite (X-S) prominent, heavily sclerotized beyond middle. Phallosome $(\mathrm{Ph})$ broadly conical, nearly twice as long as broad, rounded apically, open ventrally, closed dorsally, moderately sclerotized. Claspette stem (Cl-S) slender, cylindrical, curved, pilose, reaching a little beyond apex of basal lobe; claspette filament (Cl-F) ligulate, curved, about as long as stem, gradually tapering to a point. Basistyle (Bs) about two and one-half times as long as broad, rounded at apex, clothed with large scales and long setae on outer

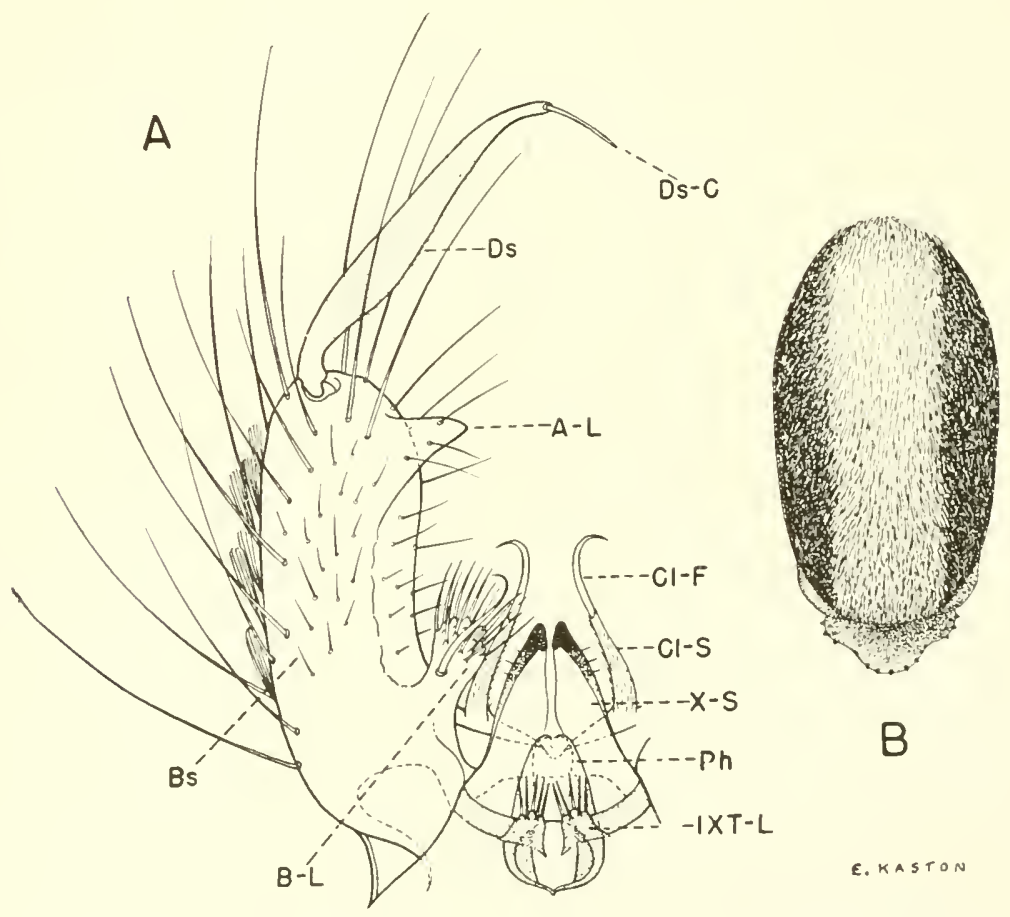

Fig. 96. Aedes dupreei (Coquillett). A, Male terminalia. B, Scutum and scutellum. 
aspect; basal lobe (B-L) longer than broad, expanded and truncate distally, bearing a large recurved basal spine and many apical setae; apical lobe (A-L) distinct, slender, conical, bearing a few small setae. Dististyle (Ds) about

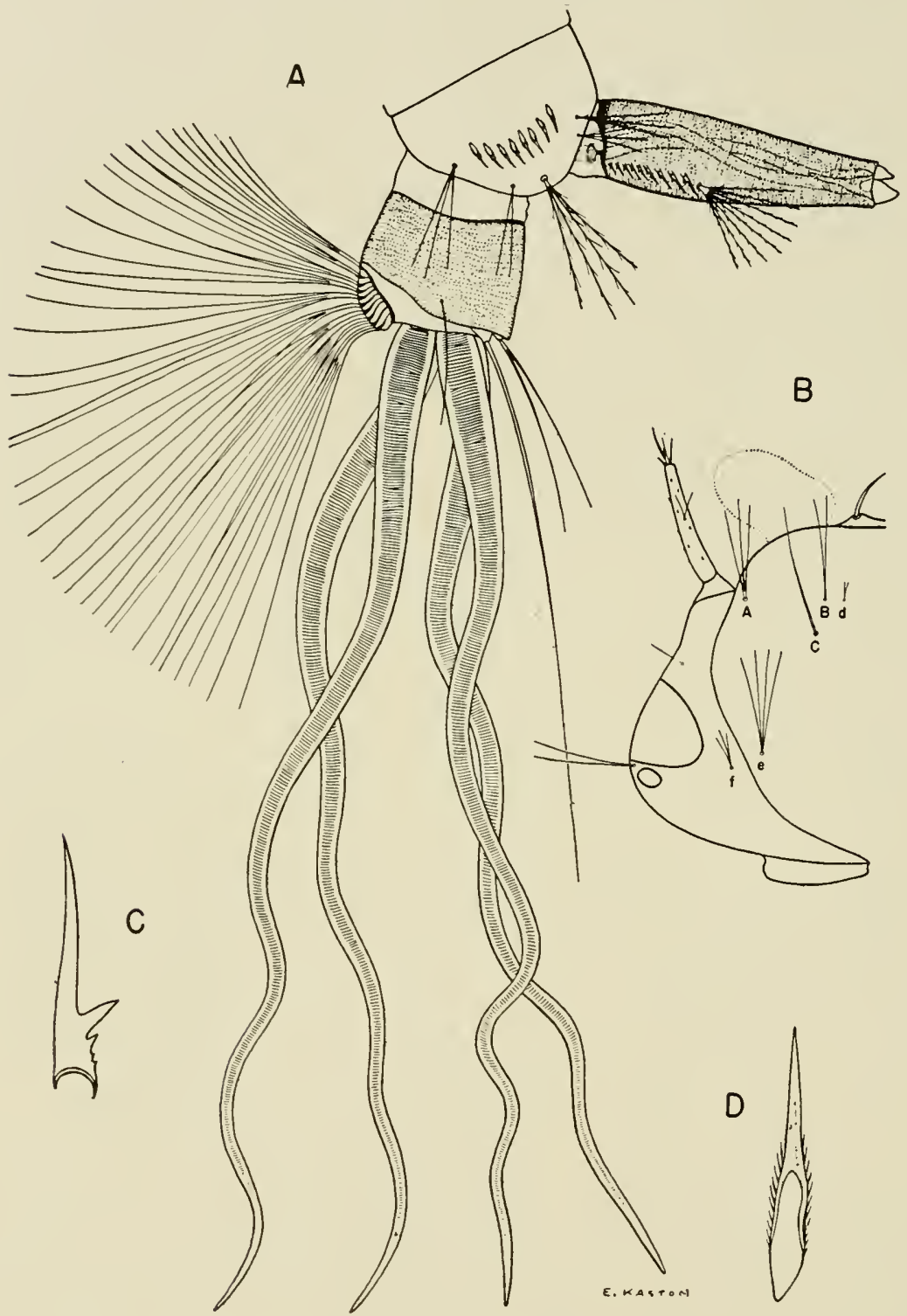

Fig. 97. Larva of Aedes dupreei (Coquillett). A, Terminal segments.

B, Head. C, Pecten tooth. D, Comb scale. 
three-fourths as long as basistyle, broader medially; terminal claw (Ds-C) slender, about one-fifth to one-fourth as long as dististyle.

LARva. (Fig. 97).-Head broader than long. Antenna less than half as long as head, slightly curved, sparsely spined; antennal hair usually double, arising a little beyond middle of shaft. Head hairs: Preantennal (A) small, double or triple barbed; lower (B) double or triple, usually feathered toward tip; upper (C) single; postclypeal (d) small, branched; sutural (e) 2 to 4 branched; trans-sutural (f) small, multiple; supraorbital single or double. Comb of eighth segment consisting of a single curved row of 7 to 10 thornshaped scales; individual scale with strong apical spine and minute lateral spinules. Siphon about four times as long as wide, much narrower than the anal segment; pecten of numerous evenly spaced teeth reaching basal third of siphon; subventral tuft large, multiple, strongly barbed, inserted beyond pecten. Anal segment shorter than wide, completely ringed by the dorsal plate; lateral hair single; dorsal brish consisting of a long lower caudal hair and a smaller double or triple upper caudal tuft on eicher side; ventral brush large, posterior to the dorsal plate; gills 4, very long, more than five times the length of the anal segment, tapered, with prominent darkly pigmented tracheae.

Distribution.-From Florida to New York and west to Iowa. Southern States: Alabama (170); Arkansas (31); Florida (96); Georgia (97); Kentucky (140); Louisiana (96); Mississippi (97); Missouri (46); North Carolina (166); South Carolina (64); Tennessee (125); Virginia (49). Other States: Iowa (156); New Jersey (77); New York (162).

BIONOMICS.-Adults are frequently taken in light traps in the southern states. The larvae are found in temporary rain-filled pools and have a habit of hiding among the leaves and debris on the bottom, making it difficult to collect them. They are known to occur from June to October in the southern states (4th Sv. C. Med. Lab. records, 1942-1944, unpublished).

\section{Aedes (Ochlerotatus) fulvus pallens Ross ${ }^{1}$}

Aedes fulvus pallens Ross, 1943. Proc. Ent. Soc. Wash., 45:148.

ADULT FEMALE.-A medium sized to rather large bright orange-yellow species. Head: Proboscis long, yellow scaled, dark at tip; palpi about one-fifth as long as proboscis, yellow scaled, with dark-scaled tips. Integument of occiput yellow to light yellowish-brown, clothed dorsally with narrow bright-yellow scales, yellow setae, and yellow erect forked scales; clothed laterally with broad flat yellow scales. Thorax: Integument of scutum yellow except for a pair of large dark-brown to black postero-lateral spots, separated by the width of the prescutellar space. Vestiture of scutum consisting of sparse narrow yellow scales on the anterior half and sparse narrow dark-brown scales on the posterior half, particularly on the lateral dark patches. Anterior pronotum, posterior

1 This name was proposed by Ross for "bimaculatus" of the southern United States which had been confused with Aedes bimaculatus (Coquillett) and Aedes fulvus (Wiedemann). 
pronotum and pleura yellow; a dark spot present beneath anterior spiracle. Abdomen: Tergites yellow scaled basally and laterally, dark scaled apically; the last two segments usually entirely yellow scaled. Venter yellow scaled. Legs: Femur, tibia and first tarsal segment of each leg yellow to orange-yellow, tipped with dark scales; remaining tarsal segments primarily dark scaled, blended with yellow, the amount of yellow being variable. Wing: Scales yellow and rather broad on costa, subcosta and vein 1, narrow and darker on remaining veins.

ADUlt MALE.-Coloration similar to that of female. TERminalia (Fig. 98). Lobes of ninth tergite (IXT-L) about as long as broad, rounded or truncate apically, separated by a little less than the width of one lobe, each bearing several spines. Tenth sternite (X-S) prominent, heavily sclerotized beyond middle. Phallosome $(\mathrm{Ph})$ stout, conical, narrowly rounded or bluntly pointed apically, open ventrally, closed dorsally, lightly sclerotized. Claspette stem (ClS) slender, cylindrical, moderately pilose, extending to or slightly beyond the most posterior portion of the basal lobe; claspette filament (Cl-F) nearly as long as stem, broad, blade-like, gradually tapering to a recurved tip. Basistyle

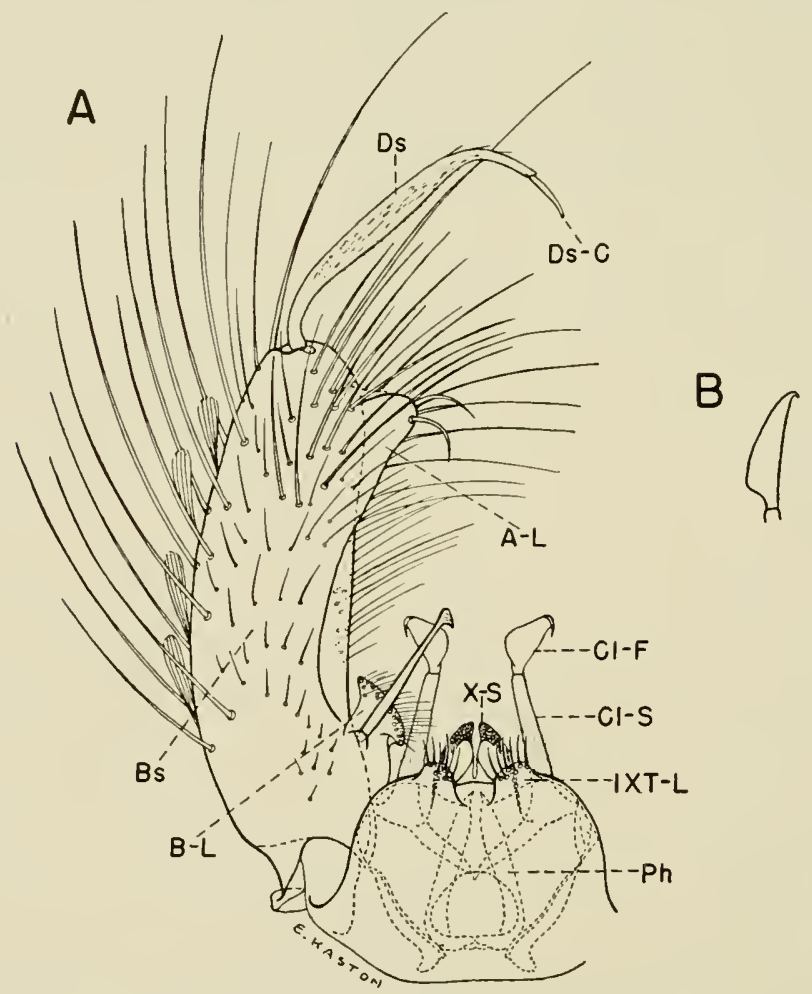

Fig. 98. Aedes fulvus pallens Ross. A, Male terminalia. B. Filament of claspette (flattened). 
(Bs) about three times as long as width across middle, rounded at apex, clothed with scales and long setae (setae more numerous distally on dorsal surface and on inner margin of ventral surface); basal lobe (B-L) prominent, constricted laterally, expanded and truncate apically, bearing numerous small apical setae (each arising from a distinct tubercle) and a large basal rod with tip flattened, broadened, and usually slightly retrorse; apical lobe (A-L) a short thumb-like projection bearing a few stout curved setae on apex. Dististyle (Ds) a little more than half as long as basistyle, broader medially; terminal claw (Ds-C) slender, about a fifth as long as dististyle.

Larva. (Fig. 99).-Head much broader than long. Antenna less than half as long as head, sparsely spined; antennal tuft about 4 to 6-branched, inserted at middle of shaft. Head hairs: Preantennal (A) multiple; lower (B) usually

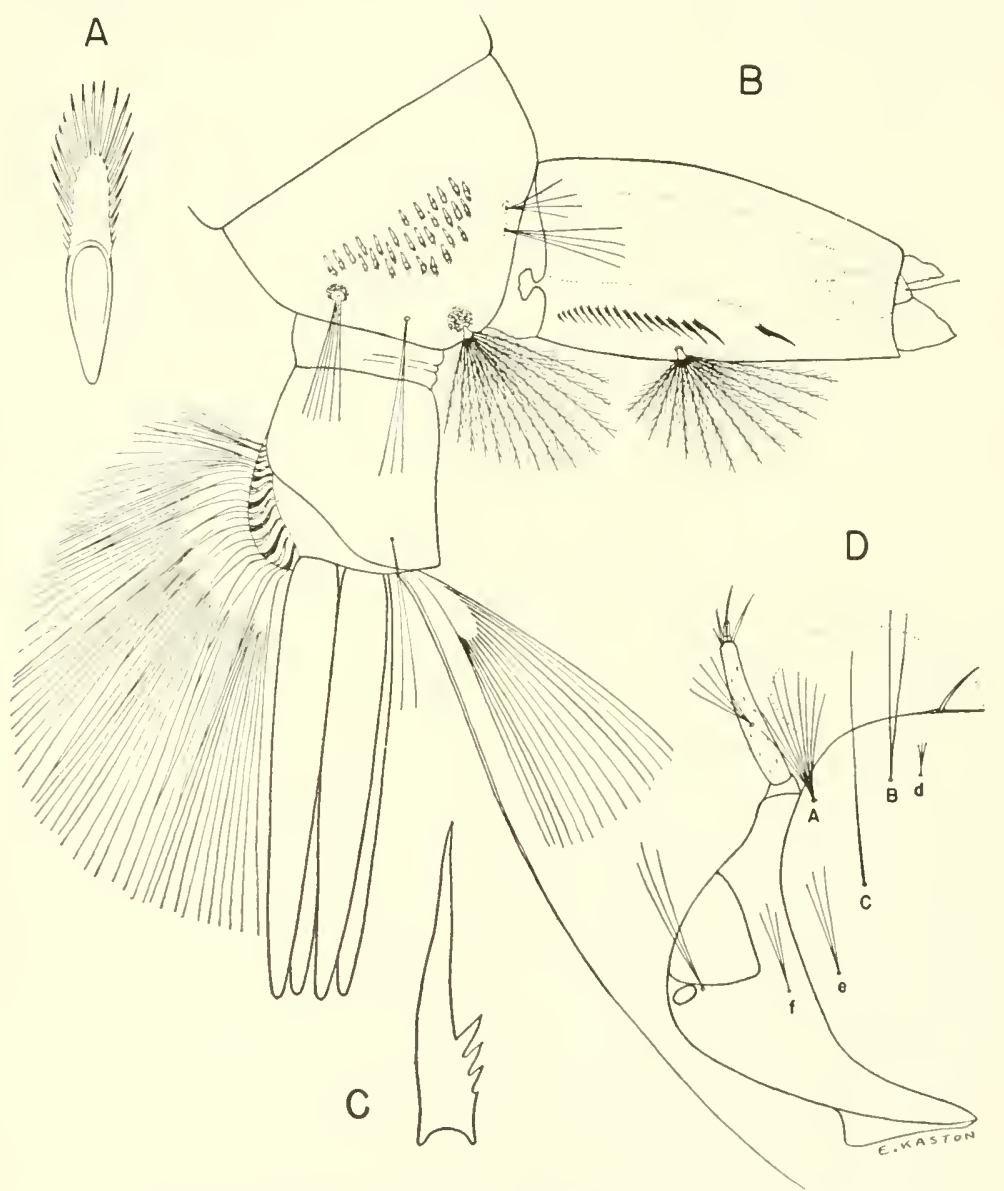

Fig. 99. Larva of Aedes fulvus pallens Ross. A. Comb scale.

B, Terminal segments. C, Pecten tooth. D. Head. 
double, sometimes triple; upper (C) single; sutural (e), trans-sutural (f), and supraorbital usually triple. Upper lateral abdominal hairs usually single on segments III to V. Comb of eighth segment consiting of about 30 scales in a patch; individual scale rounded apically and fringed with subequal spinules. Siphon about twice as long as wide; pecten reaching beyond middle of siphon, last tooth widely spaced; subventral tuft large, multiple, heavily barbed, inserted within the pecten. Anal segment slightly longer than wide, completely ringed by the dorsal plate; lateral hair usually single or double; dorsal brush consisting of a long lower caudal hair and a shorter multiple upper caudal tuft on either side; ventral brush large, heavy, restricted to the barred area; gills 4, longer than the segment, pointed.

Distribution.-Southern States: Alabama (125); Arkansas (179); Florida (96); Georgia (148); Kentucky (140); Louisiana (149); Mississippi (52); North Carolina (166); South Carolina (96); Virginia (49). Other States: Oklahoma (159); Maryland (149); Texas (141).

віоломісs.-The females are fierce biters. Adults are occasionally taken in biting, resting and light trap collections. Larvae occur in temporary pools following rains throughout the summer months.

\section{Aedes (Ochlerotatus) grossbecki Dyar and Knab}

Aedes grossbecki Dyar and Knab, 1906, Jour. N. Y. Ent. Soc., 14:201.

Adult Female.-Medium sized to rather large species. Head: Proboscis long, dark, with a few white scales; palpi short, dark, speckled with white. Occiput clothed dorsally with white lanceolate scales arranged in a broad median patch and projecting forward between the eyes; this median patch bounded dorso-laterally by dark scales. Lateral region of occiput clothed with broad appressed white scales enclosing a dark-scaled patch. Numerous erect forked scales present, those on central portion of occiput white. Thorax: Integument of scutum dark brown to black, broadly margined on either side by white lanceolate scales; a wide median longitudinal stripe of narrow darker scales present, originating near anterior margin of scutum and extending to the white-scaled prescutellar space; the anterior half of this longitudinal stripe of nearly uniform width, pale golden-brown; the posterior half of this longitudinal stripe widening slightly, dark bronzy-brown; a small patch of goldenbrown to dark-brown scales usually present in submedian position above and slightly anterior to wing bases. Posterior pronotum clothed with pale goldenbrown and white lanceolate scales. Abdomen: First tergite white-scaled; remaining tergites each with a broad basal band of white to pale-yellow scales; apical half of each tergite black scaled, speckled with pale scales. Venter primarily white scaled, spotted with dark. Legs: Femora, tibiae, and first segment of tarsi clothed with intermixed black and pale scales, the pale scales usually predominating on the femora; white knee spots present. Segments of hind tarsus each with a broad basal white ring; segments 1 to 4 of fore- and mid-tarsi with nartower basal white rings than hind tarsus; segment 5 of fore- and mid-tarsi entirely dark or with white ring greatly reduced. Wing: Scales very broad, deltoid, intermixed dark and white. 
ADUlt MALE.-Coloration similar to that of female. TERminalia (Fig. 100B). Lobes of ninth tergite (IXT-L) about as long as broad, separated by the width of one lobe, each bearing several short spine-like setae. Tenth sternite (X-S) prominent, heavily sclerotized beyond middle. Phallosome (Ph) subcylindrical (a little narrower across apical third than across base), about twice as long as basal width, open ventrally, closed dorsally, and with a notch at apex. Claspette stem (Cl-S) pilose, slender, curved, extending beyond basal lobe; claspette filament (Cl-F) about two-thirds to three-fourths as long as stem, broad, blade-like (the blade narrow or absent on basal third of filament, broadest near middle of filament and with margin rounded), tapering to a pointed curved tip. Basistyle (Bs) cylindrical, about three and one-half times as long as width across middle, rounded at apex, clothed with setae and scales
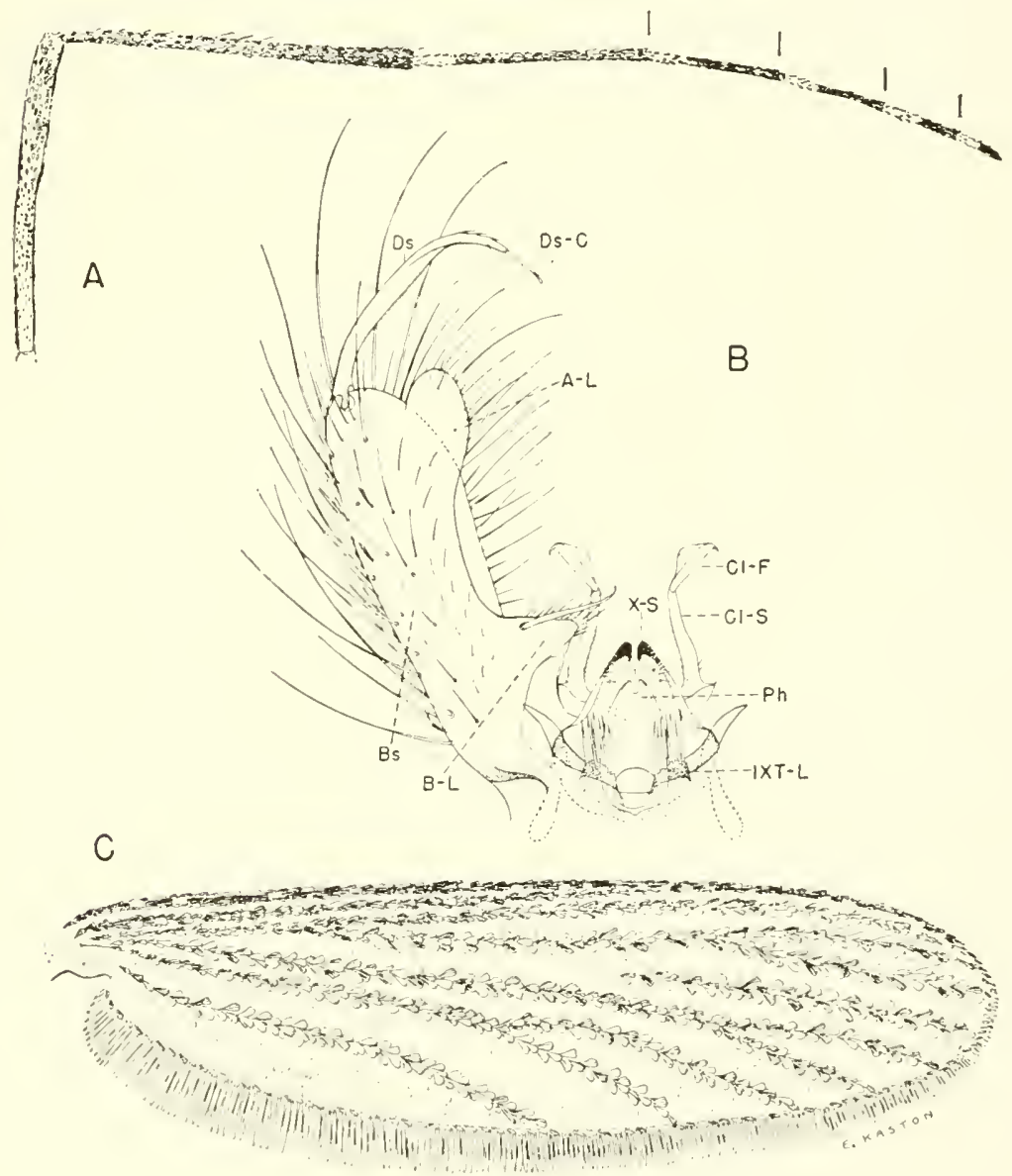

Fig. 100. Aedes grossbecki Dyar and Knab. A, Hind leg.

B, Male terminalia. C, Wing. 
on outer aspect and numerous long sctae on inner ventral margin; basal lobe (B-L) prominent, conical, directed mesad, with numerous small apical setae and a stout slightly curved lateral spine; apical lobe (A-L) a prominent thumblike projection bearing fine setae. Dististyle (Ds) a little broader medially, approximately two-thirds as long as basistyle; terminal claw (Ds-C) slender, nearly one-fourth as long as dististyle.

LARva. (Fig. 101). Head broader than long. Antenna moderate in length, spinose; antennal tuft multiple, inserted a little before middle of shaft. Head hairs: Preantennal (A) long, multiple, nearly reaching tip of antenna; lower (B) double, sometimes triple; upper (C) triple, postclypeal (d) small, branched; sutural (e) trans-sutural (f) and supraorbital usually double. Upper lateral abdominal hairs usually double on segments III to VI. Comb of eighth segment of numerous scales in a patch; individual scale with lateral spines nearly as long as apical spine. Siphon a little more than three times as long as wide; pecten of numerous evenly spaced teeth not reaching middle of siphon; subventral tuft large, multiple, barbed, inserted beyond pecten. Anal

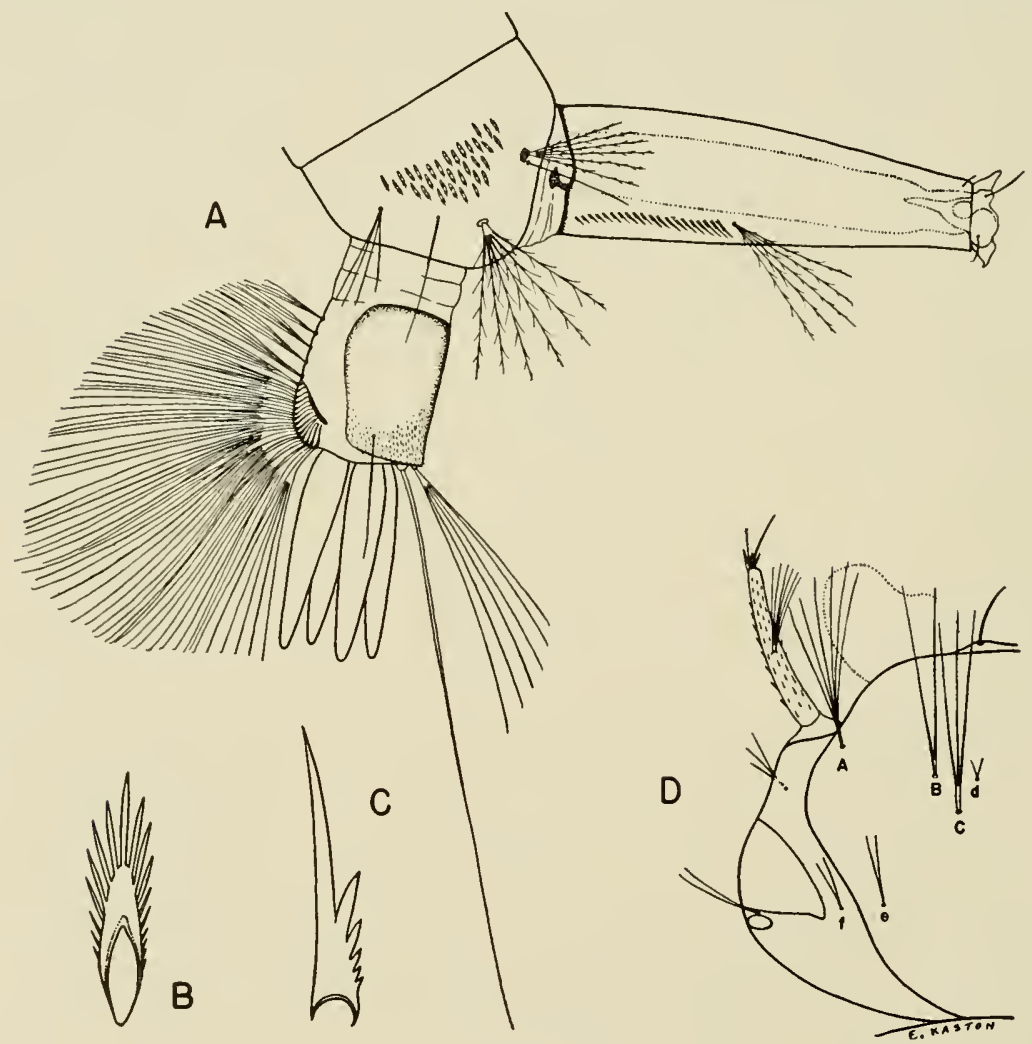

Fig. 101. Larva of Aedes grossbechi Dyar and Knab. A, Terminal segments. B, Comb scale. C, Pecten tooth. D, Head. 
segment longer than wide, with dorsal plate finely spinose apically and extending a little more than half way down the side; lateral hair single; dorsal brush consisting of a long lower caudal hair and a shorter multiple upper caudal tuft on either side; ventral brush large, with several small tufts preceding the barred area; gills 4, about as long as the anal segment, tapered.

Distribution.-A rare species occurring in the Eastern United States. Southern States: Arkansas (31); Kentucky (140); Louisiana and Mississippi (96); Missouri and Virginia (54). Other States: Delaware (106); Illinois (42); Maryland and New Jersey (52); New York (116); Ohio (Vernard, 1943, unpublished).

BIONOMics. - Very little is known of the habits of the adults of this species. Dr. Carl Vernard, who collected a good series of male and female specimens during May, 1943, in Ohio, states that the females are fierce biters. Aedes grossbecki breeds in early spring pools and is apparently single brooded.

\section{Aedes (Ochlerotatus) infirmatus Dyar and Knab}

Aedes infirmatus Dyar and Knab, 1906, Jour. N. Y. Ent. Soc. 14:197.

Adult female.-Medium sized species. Head: Proboscis long, dark scaled; palpi short, dark. Occiput dorsally with a rather broad longitudinal stripe of white lanceolate scales, extending forward between the eyes; this median stripe bounded on either side by a large patch of broad brownishyellow scales surrounding a dark-scaled patch; lateral region of occiput clothed with broad appressed dingy-yellow to brownish-yellow scales. Erect forked scales numerous, those on central portion of occiput pale. Thorax: Integument of scutum dark brown, clothed with fine dark bronzy-brown scales, except for a few pale scales on prescutellar space and a broad silvery-white to pale-yellow scaled median longitudinal stripe extending from the anterior margin of scutum to a little beyond the middle (this longitudinal stripe about half as wide as scutum). Dorsal half of posterior pronotum covered with narrow dark-brown scales. Abdomen: Tergites dark scaled, with conspicuous basal triangular patches of white scales laterally; venter white scaled, occasionally speckled with a few dark scales. Legs: Legs entirely dark scaled except for femora I and II having the inner surfaces pale and III having all aspects of basal two-thirds pale. Wing: Scales narrow, dark.

ADULT MALE.-Coloration similar to that of female, but with narrow basal white bands usually present on some segments of abdomen. TERMINALIA (Fig. 102A). Lobes of ninth tergite (IXT-L) about as long as broad, separated by about the width of one lobe, or a little less, each bearing several short spines. Tenth sternite (X-S) prominent, heavily sclerotized beyond middle. Phallosome (Ph) stoutly conical, nearly one and three-fourths times as long as broad, rounded apically, open ventrally, closed dorsally. Claspette (Cl-S) pilose, slender, curved, extending well beyond basal lobe. Claspette filament (Cl-F) about four-fifths as long as stem, expanded blade-like, tapered and curved beyond middle; the blade-like portion with a large sharp retrorse projection arising from its basal margin; one or more smaller accessory retrorse 
spines usually present on inner margin of the large projection and between the large projection and non-expanded basal portion of the filament. Basistyle (Bs) nearly four times as long as width across middle, bluntly rounded at apex, clothed with scales and long setae (setae very numerous and long on inner ventral margin); basal lobe (B-L) prominent, bluntly conical, bearing numerous short setae apically and a large, curved spine on dorsal side; apical lobe (A-L) rounded, not prominent, its vestiture consisting of a few short fine setae. Dististyle (Ds) nearly two-thirds as long as basistyle, slightly broader medially; terminal claw (Ds-C) slender, about one-fifth as long as dististyle.

LARva. (Fig. 103) - - Head broader than long. Antenna slightly less than half as long as head, sparsely spined; antennal tuft small, multiple, inserted near middle of shaft, not reaching tip. Head hairs: Preantennal (A) multiple, barbed, extending beyond insertion of antennal tuft; lower (B) and upper (C) long, single, slightly barbed apically; postclypeal (d) small, multiple; sutural (e) long, single; trans-sutural (f) shorter, double or triple; supraorbital single or double. Body spiculate. Upper lateral abdominal hairs single on segments III to V. Comb of eighth segment of about 15 to 22 thorn-shaped scales in a patch; individual scale with apical spine three or four times as broad and about twice as long as subapical spinules. Siphon about two and one-half times as long as wide; pecten of numerous long slender evenly spaced teeth extending

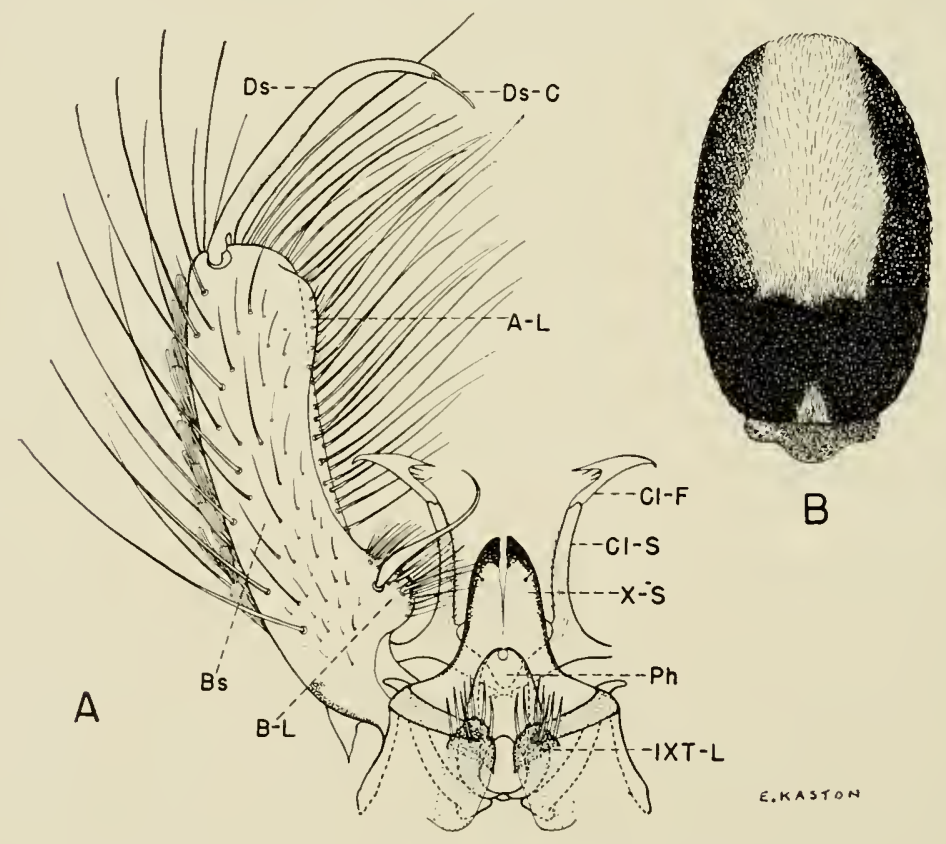

Fig. 102. Aedes infirmatus Dyar and Knab. A, Male terminalia. B. Scutum and scutellum. 
slightly beyond middle of siphon; subventral tuft multiple, inserted beyond pecten; dorsal preapical spine not more than half as long as apical pecten tooth. Anal segment longer than wide, completely ringed by the dorsal plate; lateral hair single; dorsal brush consisting of a long lower caudal hair and a shorter multiple upper caudal tuft on either side; ventral brush confined to the barred area; gills 4 , longer than the segment, each tapering to a point.

Distribution.--Southern States: Alabama (125); Arkansas and Florida

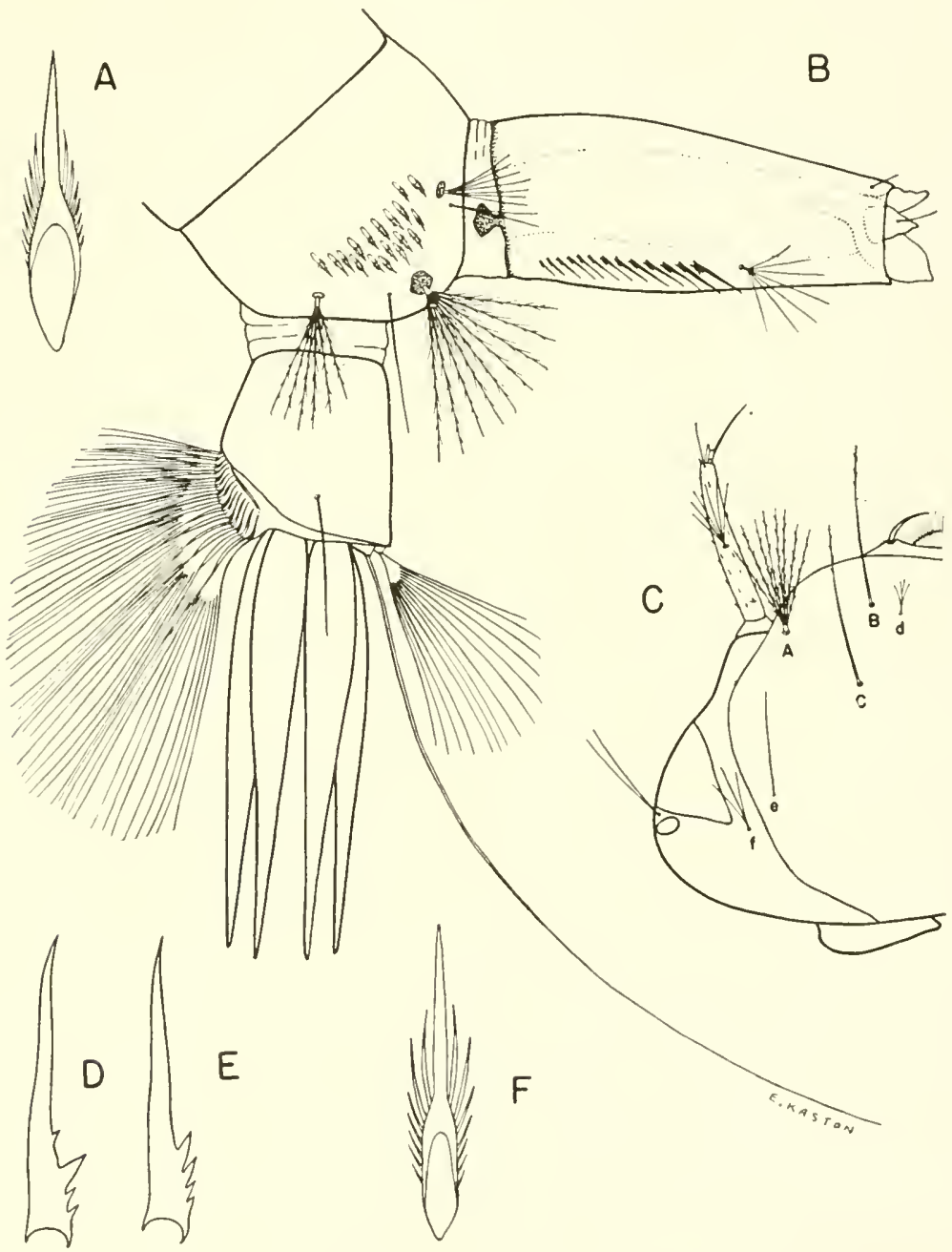

Fig. 103. Larva of Acdes infirmatus Dyar and Knab. A and F. Comb scales. B, Terminal segments. C. Head. D, and E, Pecten teeth. 
(52); Georgia (96); Kentucky (140); Louisiana (52); Mississippi (96); Missouri (128); North Carolina (166); South Carolina (64). Other States: Texas (108).

BIONOMICS.- The females are vicious biters, attacking during the day in or near wooded areas. They are occasionally encountered at night near dwellings, but seldom enter houses. Larvae are found in temporary pools following rains. Larval and adult collections have been recorded from March to December in the South (4th Sv. C. Med. Lab. records, 1942-1944, unpublished).

\section{Aedes (Ochlerotatus) mathesoni Middlekauff}

Aedes mathesoni Middlekauff, 1944, Proc. Ent. Soc. Wash., 46:42.

adult female.-Medium sized species. Head: Proboscis long, slender, black with faint purplish iridescence; palpi short, black, with purplish iridescence. Scales of postero-dorsal region of occiput white, lanceolate, arranged in a sub-triangular patch projecting anteriorly on the mid-line; scales of submedian areas between the postero-dorsal patch and eye margins black, lanceolate; lateral region of occiput clothed with broad appressed yellowish-white scales surrounding a small patch of broad appressed dark scales. Dark erect forked scales numerous on central portion of occiput. Thorax: Integument of scutum dark brown, clothed with narrow bronzy-black scales except for the following markings: A very narrow median longitudinal line of fine slender golden-brown scales; a narrow elongate patch of lanceolate yellow-white scales on the anterior angles and a similar patch laterally near middle; a few lanceolate yellowishwhite scales laterally just above wing base; a few lanceolate yellowish-white scales on prescutellar space. Abdomen: Tergites uniformly purplish-black dorsally, with small triangular basal white-scaled patches laterally. Sternites white scaled basally, black scaled apically. Legs: Hind leg black scaled with purplish reflection except for pale inner surface of femur; a white knee spot; narrow white rings basally and apically on tarsal segments 1 and 2, basally on segment 3 . Fore and middle legs each similarly marked, but with pale area on inner surface of femur usually less extensive and with tarsal rings restricted to the base and apex of segment 1 and base of segment 2. Wings: Scales narrow, purplish-black.

ADUlT MALE.-Coloration similar to that of female. TERminalia. The male terminalia appears to be identical with that of $A$. canadensis (Fig. 90).

LARVA.-Unknown.

Distribution.-Known only from a few specimens collected in Florida (123).

BIONOMICS.-Nothing is known of the habits of this species. Melanistic forms of $A$. canadensis nearly as dark as $A$. mathesoni have been recently collected in Florida. It may be possible that $A$. mathesoni represents an extreme in melanism of $A$. canadensis; therefore its validity is questionable until additional specimens are available. 


\section{Aedes (Ochlerotatus) Mitchellae (Dyar)}

Culex mitchellae Dyar, 1905, Jour. N. Y. Ent. Soc., 13:74.

Adult female.-Medium sized species. Head: Proboscis dark scaled, with a rather broad white-scaled ring at middle; palpi short, dark, with tips white scaled and frequently with a few white scales at basal third. Occiput dorsally with a rather broad longitudinal stripe of pale yellow to golden-yellow lanceolate scales, the stripe broader posteriorly, narrowed anteriorly and projecting forward between the eyes; a submedian patch of narrow dark bronzy-brown scales present on either side of the median pale-scaled area; lateral region of

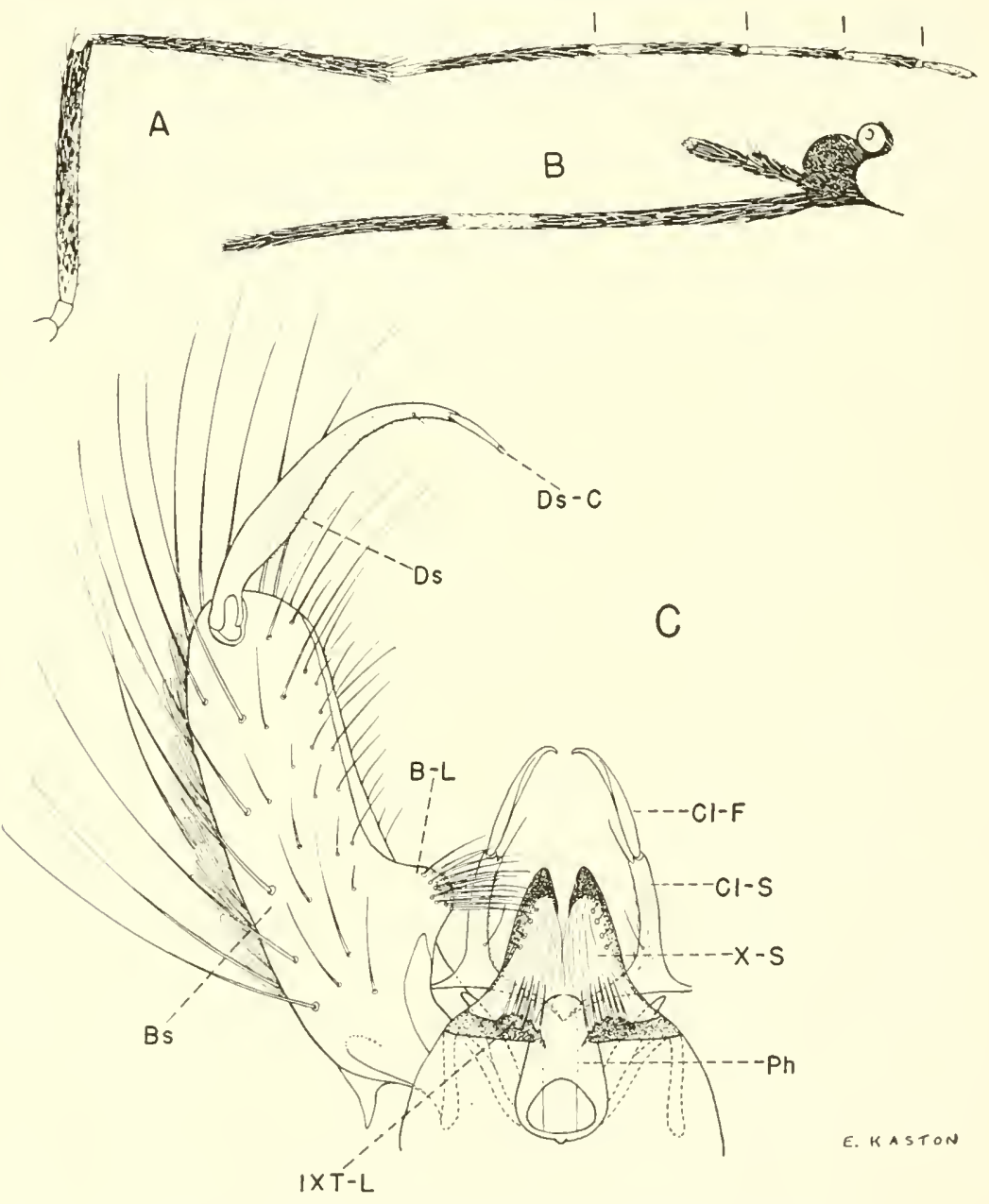

Fig. 104. Aedes mitchelli (Dyar). A. Hind leg. B, Palp and proboscis of female. C, Male terminalia. 
occiput clothed with broad appressed whitish scales surrounding a patch of broad appressed dark scales. Erect forked scales numerous, those on central portion of occiput pale. Thorax: Integument of scutum black, clothed with narrow yellow-orange scales dorsally, becoming dark bronzy-brown laterally; anterior margin and prescutellar space golden-yellow scaled; frequently a pair of narrow, rather indefinite, longitudinal submedian lines of golden-yellow scales originating at the outer margins of the prescutellar space and extending forward nearly the full length of the scutum. Posterior pronotum clothed with narrow dark bronzy-brown scales. Abdomen: Tergites dark scaled, with narrow basal bands of white to yellowish-white scales and central patches of yellowishwhite scales, the central patches frequently extending to the apical margins of the segments; a broad patch of white scales present on each tergite laterally. Venter primarily white scaled, with intermingled dark scales. Legs: Femora and tibiae black scaled, speckled with white; white knee spots present. Hind tarsi black scaled, with broad white basal rings on segments 1 to 4 and with segment 5 entirely white; fore- and mid-tarsi black scaled, with white basal

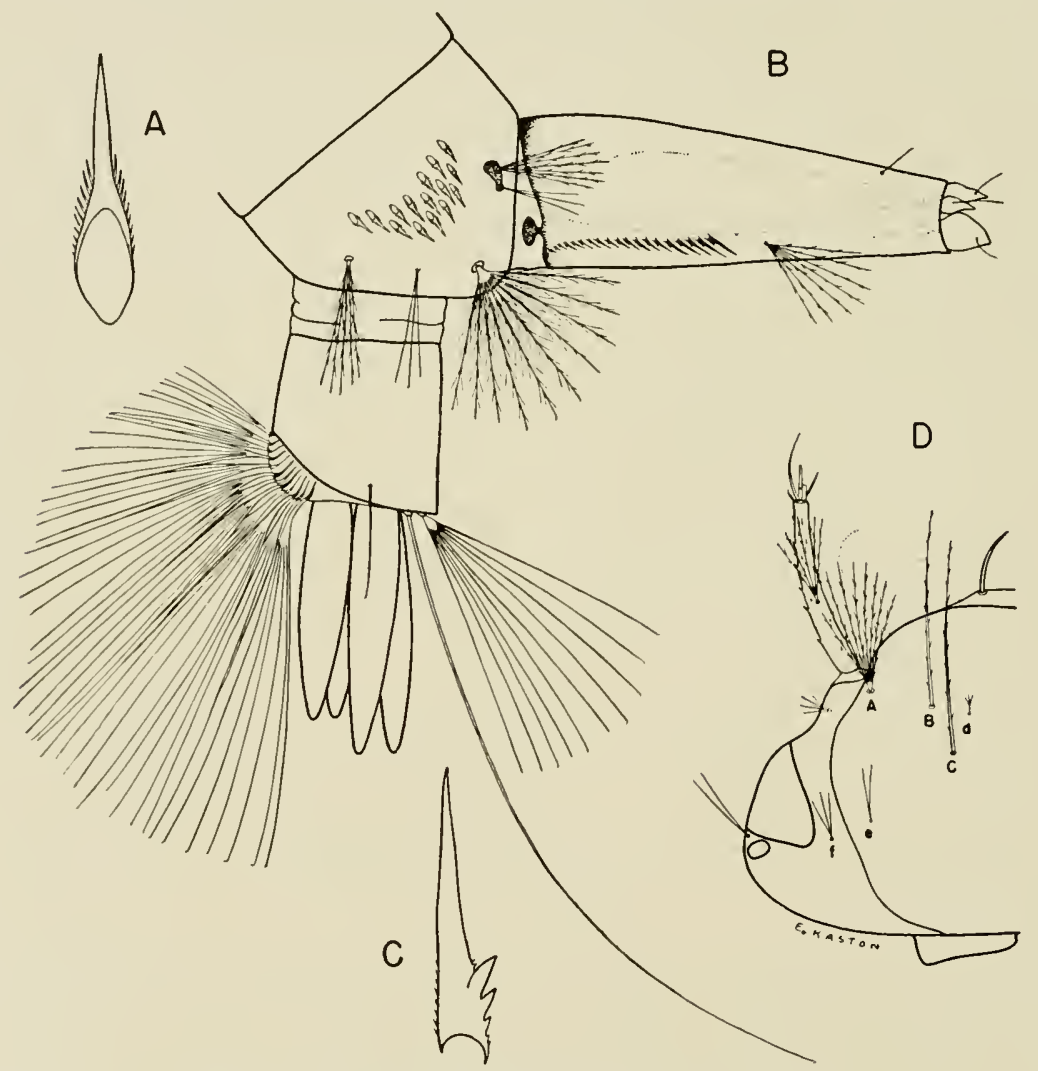

Fig. 105. Larva of Aedes mitchellae (Dyar). A, Comb.

B, Terminal segments. C, Pecten tooth. D, Head. 
rings much narrower and on segments 1 to 3 only. Wing: Scales narrow, dark.

ADUlt MALE.-Coloration similar to that of female. Terminalia (Fig. 104C). Lobes of ninth tergite (IXT-L) about two-thirds as long as wide, separated by a little less than the width of one lobe, each bearing several short stout setae. Tenth sternite (X-S) prominent, heavily sclerotized. Phallosome $(\mathrm{Ph})$ stoutly conical, about two-thirds as broad as long, rounded apically, open ventrally, closed dorsally. Claspette stem (Cl-S) rather stout, pilose, somewhat curved, extending slightly beyond basal lobe and bearing a short seta near apex arising from a prominent tubercle; claspette filament (Cl-F) as long as stem, slender, curved. Basistyle (Bs) about three and one-half times as long as width across middle, cylindrical, rounded at apex, clothed with scales and numerous long setae; basal lobe (B-L) distinct, conical, bluntly rounded at apex and bearing numerous short setae; apical lobe absent. Dististyle (Ds) about two-thirds as long as basistyle, broader at basal third; terminal claw (DsC) slender, one-fifth as long as dististyle.

LARVA. (Fig. 105).-Head broader than long. Antenna about half as long as head, slightly curved, spinose; antennal tuft multiple, inserted near middle of shaft, not reaching tip. Head hairs: Preantennal (A) multiple, barbed, extending beyond insertion of antennal tuft; lower (B) and upper (C) long, barbed, usually single; postclypeal (d) small, branched; sutural (e) transsutural (f) and supraorbital usually double or triple. Upper lateral abdominal hairs usually double on segments III to V. Comb of eighth segment of about 15 to 18 thorn-shaped scales in a patch; individual scale with long apical spine and smaller lateral spinules. Siphon three to three and one-half times as long as wide; pecten of numerous, evenly-spaced teeth not quite reaching middle of siphon; subventral tuft multiple, inserted beyond pecten; dorsal preapical spine as long as apical pecten tooth. Anal segment a little longer than wide, completely ringed by the dorsal plate; lateral hair single; dorsal brush consisting of a long lower caudal hair and a shorter multiple upper caudal tuft on either side; ventral brush large, confined to the barred area; gills 4, variable in length, but usually about as long as the anal segment, each tapering to a blunt point.

Distribution.-Along the coastal plain from Florida to Delaware and west to Texas. Southern States: Alabama, Florida and Georgia (52); Louisiana and Mississippi (97); North Carolina (166); South Carolina (97); Virginia (49). Other States: Delaware (106); Maryland (19) New Jersey (164); Texas (108).

BIONOMICS.-King et al. (96) state that the females are rather severe biters. The larvae are found in temporary rain-filled pools. Both adults and larvae occur throughout the year in the extreme South (4th Sv. C. Med. Lab. records, 1942-1944, unpublished).

\section{Aedes (Ochlerotatus) nigromaculis (Ludlow)}

Grabhamia nigromaculis Ludlow, 1907, Geo. Wash. Univ. Bull., 5:85.

AdUlt FEMALE.-Medium sized species. Head: Proboscis dark scaled, ringed with white near middle; palpi short, dark. Broad median area of occiput clothed with pale yellow lanceolate scales and erect pale forked scales; a sub- 
median patch of narrow brown scales and a few brown erect forked scales bordering the median pale area on either side; lateral portion of occiput clothed with broad appressed dingy-yellow scales, often surrounding a small patch of broad dark scales. Thorax: Integument of scutum blackish; a broad longitudinal stripe of golden-brown lanceolate scales present medially; most of remainder of dorsal surface of scutum, including prescutellar space, clothed with yellow lanceolate scales; lateral margins dark bronzy-brown scaled, particularly on anterior half of scutum. Scales of posterior pronotum brown, lanceolate. Abdomen: First tergite white scaled, remaining tergites each basally, laterally and medially white, with a pair of large quadrate submedian patches of dark scales. Venter white scaled. Legs: Femora and tibiae dark on outer aspects,

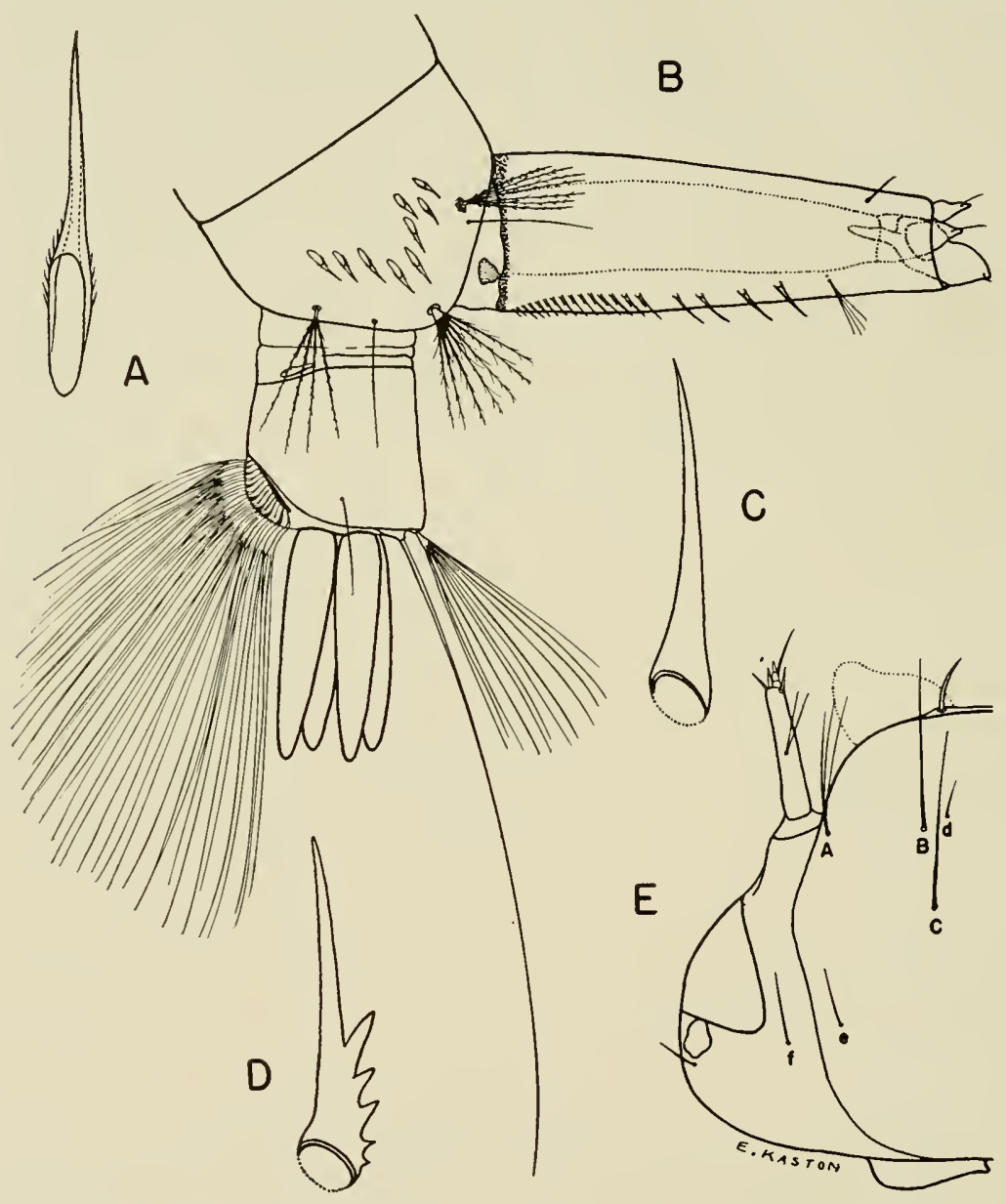

Fig. 106. Larva of Aedes nigrcmaculis (Ludlow). A, Comb scale. B, Terminal segments. C, Apical pecten tooth. D, Median pecten tooth. E, Head. 
liberally speckled with pale scales; inner surfaces pale. Hind tarsal segments each broadly white-banded at base (the white markings of segment 1 quite variable, ranging from a rather narrow basal band to one occupying most of the segment); fore-and mid-tarsi with segments 4 and 5 entirely dark; basal white bands of other segments narrower than the corresponding ones of hind tarsus. Wing: Scales narrow, dark, sprinkled with a few pale ones, especially on costa and subcosta.

ADUlt MALE. - Coloration similar to that of female. Terminalia. The terminalia appears to be identical with that of A. sollicitans (Fig. 107C).

LARva. (Fig. 106).- - Head much broader than long. Antenna less than half the length of the head, nearly smooth; antennal tuft 1 to 3-branched, smooth, inserted near middle of shaft, extending nearly to tip. Head hairs: Preantennal (A) multiple, smooth or very finely barbed, extending beyond insertion of antennal tuft; lower (B) and upper (C) single, smooth or weakly barbed, usually a little longer than antennae; postclypeal (d) small, single or branched; sutural (e) trans-sutural (f) and supraorbital small, usually single. Upper lateral abdominal hairs usually 2 to 3-branched on segments I to V. Comb of eighth segment of about 6 to 12 scales in either an uneven single row or an irregular triangular patch; individual comb scale thorn-shaped, with minute spinules on basal portion. Siphon two to two and one-half times as long as wide; pecten of numerous teeth extending to or slightly beyond apical third of siphon, with about 2 to 4 of the distal teeth widely spaced; subventral tuft small (much shorter than width of siphon), smooth, 3 to 5-branched, inserted beyond pecten at apical fourth of siphon; dorsal preapical spine about as long as apical pecten tooth. Anal segment a little wider than long, ringed by the dorsal plate; lateral hair short, single; dorsal brush consisting of a long lower caudal hair and a shorter, multiple upper caudal tuft on either side; ventral brush well developed, confined to the barred area; gills 4, longer than the segment, bluntly pointed.

Distribution. - Central and western United States, southern Canada and Mexico. Scuthern States: Kentucky (140); Missouri (1). Other States: California (67); Colorado and Idaho (52); Iowa (155, 156); Kansas (79); Minnesota (131); Montana (112); Nebraska (178); New Mexico and North Dakota (52); Oklahoma (159); Oregon (116); South Dakota (52); Texas (108); Utah (142); Wyoming (52).

BIONOMICS.-The females bite readily during the day but are more active in the evenings. They are strong fliers and have been taken several miles from their breeding places. Larvae occur mostly in alkaline waters in rain-filled depressions and irrigation ditches.

\section{Aedes (Ochlerotatus) sollicitans (Walker)}

Culex sollicitans Walker, 1856, Ins. Saund. Dipt., p. 427.

adult female.-Medium sized to rather large species. Head: Proboscis dark scaled, rather broadly ringed with white near middle; palpi short, dark, with a few white scales at tips. Occiput dorsally clothed with a broad longi- 
tudinal stripe of narrow golden-yellow scales, the stripe broader posteriorly, narrowed anteriorly and projecting forward between the eyes; a submedian patch of narrow dark bronzy-brown scales present on either side of the median pale-scaled area; lateral region of occiput clothed with broad appressed yellowish scales surrounding a patch of broad appressed dark scales. Erect forked scales numerous, those on central portion of occiput pale. Thorax: Integument of scutum black, clothed with narrow golden to golden-brown scales dorsa!ly, becoming dark bronzy-brown laterally; anterior margin and prescutellar space somewhat paler; frequently a pair of narrow, rather indefinite, longitudinal submedian lines of pale yellow to golden-yellow scales originating at the outer margins of the prescutellar space and extending forward nearly the full length of the scutum. Posterior pronotum clothed with narrow dark bronzy-brown scales. Abdomen: First tergite whitish-yellow scaled; remaining tergites each laterally white, basally and medially pale yellow, and with a pair of large quadrate dark-scaled submedian patches. Venter whitish to pale-yellow scaled, speckled with dark scales. Legs: Femora and tibiae dark on outer aspects, liberally speckled with pale scales; inner surfaces pale; white knee spots present. Hind tarsus with segment 1 white-ringed at base and yellow ringed at middle, segments 2 to 4 broadly white-ringed at bases, and segment 5 entirely white. Fore-and mid-tarsi similarly marked, but with bands narrower on segments 1 to 3 and absent on 4; segment 5 of fore-tarsus usually entirely dark scaled; segment 5 of mid-tarsus usually principally white, blended with dark scales. Wing: Plume scales narrow; squame scales rather broad, mixed dark-brown and white.

AdUlT MALE.-Coloration similar to that of female. TERMinalia (Fig. 107C). Lobes of ninth tergite (IXT-L) about two-thirds as long as wide, separated by a little less than the width of one lobe, each bearing several short stout setae. Tenth sternite (X-S) prominent, heavily sclerotized. Phallosome $(\mathrm{Ph})$ stoutly conical, about two-thirds as broad as long, rounded apically, open ventrally, closed dorsally. Claspette stem (Cl-S) rather stout (usually slightly stouter than in $A$. mitchellae), pilose, somewhat curved, extending to or slightly beyond basal lobe and bearing a short seta near apex arising from a prominent tubercle; claspette filament (Cl-F) as long as stem, slender, curved. Basistyle (Bs) about three times as long as width across middle, cylindrical, rounded at apex, clothed with scales and numerous long setae; basal lobe (B-L) only slightly raised, bearing numerous short setae; apical lobe absent. Dististyle (Ds) about two-thirds as long as basistyle, broader at basal third; terminal claw (Ds-C) slender, one-fifth as long as dististyle.

The terminalia of this species is very similar to that of $A$. mitchellae. The most reliable character for separation is found in the basal lobe, which is more prominent in $A$. mitchellae. After carefully comparing terminalia of both species, it is usually possible to identify subsequent specimens with reasonable certainty.

LARVA. (Fig. 108).-Head broader than long. Antenna about half as long as head, curved, spinose; antennal tuft multiple, finely barbed, inserted near middle. Head hairs: Preantennal (A) multiple, barbed, extending to insertion 
of antennal tuft; lower (B) and upper (C) long, usually single, smooth or very finely barbed; postclypeal (d) small, branched; sutural (e), trans-sutural (f) and supraorbital single, or double. Upper lateral abdominal hairs usually double on segments III to V. Comb of eighth segment of about 15 to 18 thorn-shaped scales in a patch; individual scale with a long apical spine and smaller lateral spinules. Siphon two to two and one-half times as long as wide; pecten of numerous evenly spaced teeth reaching middle of siphon or slightly beyond; subventral tuft multiple, inserted beyond pecten; dorsal preapical spine as long as apical pecten tooth. Anal segment about as wide as long, completely ringed by the dorsal plate; lateral hair single; dorsal brush consisting of a long
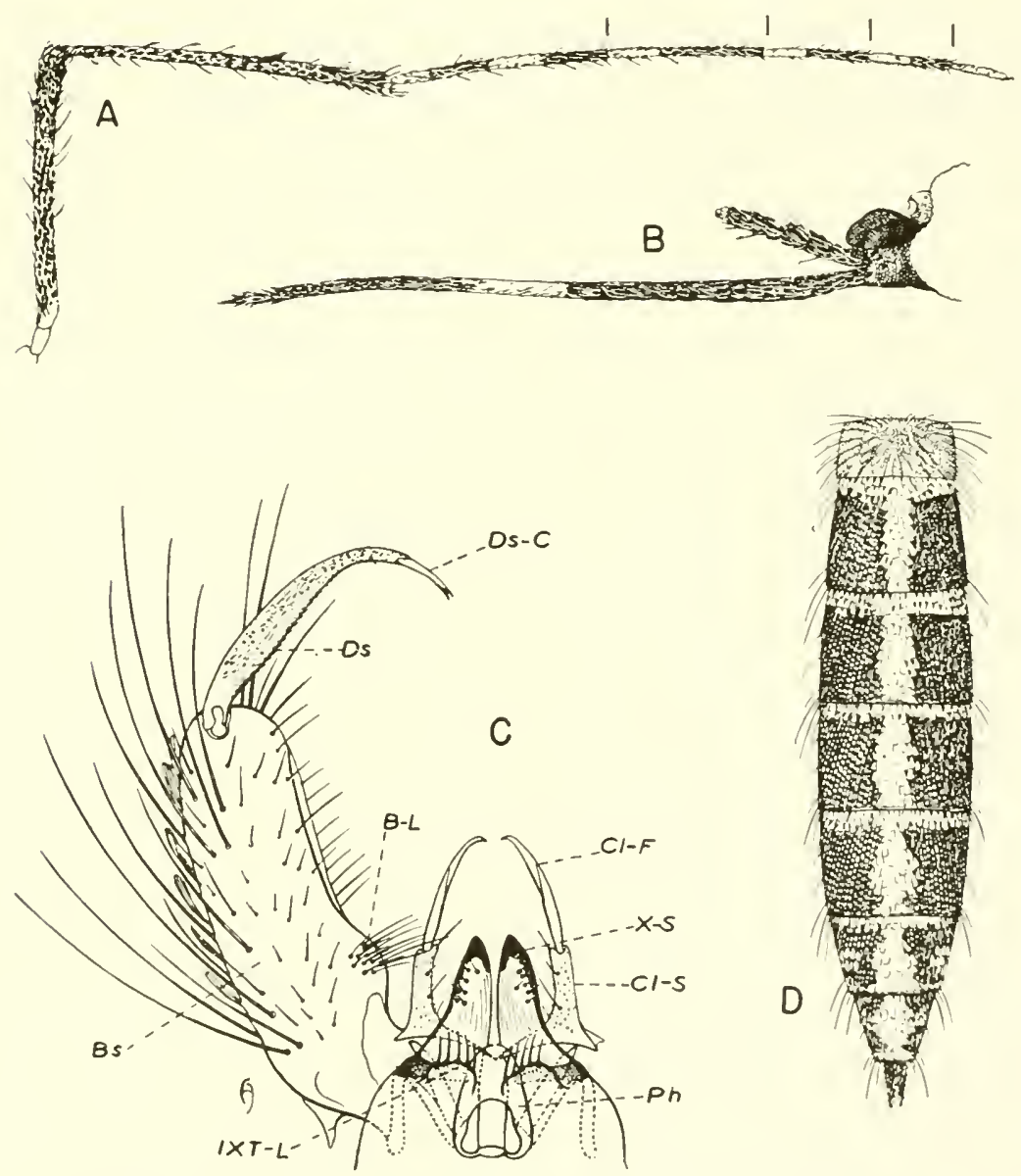

Fig. 107. Aedes sollicilans (Walker). A, Hind leg. B. Palp and proboscis of female. C, Male terminalia. D, Abdomen of female. 
lower caudal hair and a shorter, multiple upper caudal tuft on either side; ventral brush large, confined to the barred area; gills 4, variable in length, usually short, but may be as long or longer than the anal segment, bluntly rounded.

Distribution.-The Bahamas; the Antilles; and Atlantic and Gulf coasts from Maine to Texas. It is also found in several inland areas where brackish water is available. Southern States: Alabama (96); Arkansas (30); Florida, Georgia, Louisiana and Mississippi (52); Missouri (1); North Carolina, South Carolina and Virginia (52). Other States: Arizona (61); Connecticut and Delaware (52); Illinois and Indiana (61); Maine, Maryland and Massa-

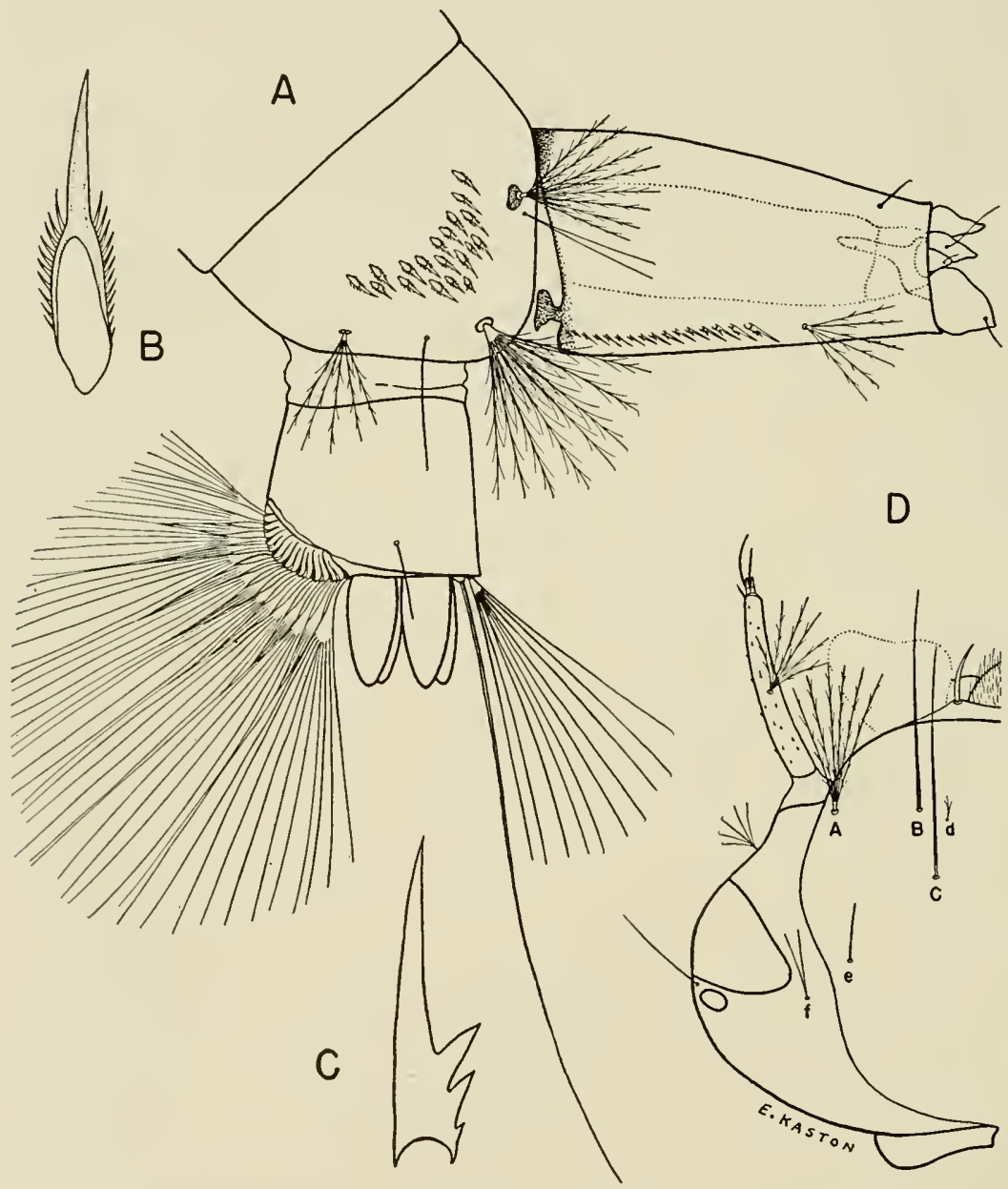

Fig. 108. Larva of Aedes sollicitans (Walker). A, Terminalia segments. B, Comb scale. C. Pecten tooth. D, Head. 
chusetts (52); Nebraska (128); New Hampshire, New Jersey and New York (52); New Mexico (9); Oklahoma (159); Pennsylvania, Rhode Island and Texas (52).

BIONOMICS. - The adults are strong fliers and often migrate in large numbers to communities located many miles from their breeding places. The females are fierce biters and will attack any time during the day or night. Aedes sollicitans breeds in saltmarshes in coastal areas but also occurs in inland brackish water swamps, particularly in oil fields.

\section{Aedes (Ochlerotatus) sticticus (Meigen)}

Culex sticticus Meigen, 1838, Syst. Beschr. Eur. Zweifl. Ins., 7:1.

Aedes hirsuteron Dyar, 1928, Mosq. of Americas, 175.

Adult female.-Medium sized species. Head: Proboscis long, dark scaled; palpi short, dark. Occiput clothed dorsally with narrow pale yellow scales and yellowish erect forked scales; occiput clothed laterally with broad appressed yellowish scales surrounding a dark-scaled patch. Thorax: Integument of scutum dark-brown to black, clothed with pale yellow lanceolate scales, except for a broad median longitudinal stripe of fine narrow goldenbrown scales; this median stripe about one-third as wide as scutum, originating near anterior margin and extending to the pale-scaled prescutellar space; a pair of short submarginal stripes of fine golden-brown scales present on either side of the prescutellar space and extending forward to near middle of scutum. Posterior pronotum with golden-brown lanceolate scales on dorsal half. Abdomen: Tergites dark scaled, each with a narrow basal white band (occasionally lacking) which broadens on either side into a basal triangular white patch. Venter primarily white scaled, the apices of the terminal segments usually speckled with dark scales. Legs: Femora I and II dark scaled on outer aspects, speckled with pale scales, the inner surfaces pale; femur III mostly pale scaled, dark apically. White knee spots present. Legs beyond femora dark scaled, but with a pale streak extending down one side of tibiae and first segment of tarsi. Wing: Scales narrow, dark.

ADULT MALE.-Coloration similar to that of female, but with dorsal abdominal white bands broader and dark scales usually more prevalent on the venter. TERMINALIA (Fig. 109A) Lobes of ninth tergite (IXT-L) about as long as broad, rounded or truncate apically, separated by a little less than the width of one lobe, each bearing several stout spines. Tenth sternite (X-S) prominent, heavily sclerotized beyond middle. Phallosome (Ph) broadly conical, about twice as long as broad, rounded apically, open ventrally, closed dorsally, lightly sclerotized. Claspette stem (Cl-S) rather stout, cylindrical, slightly curved, pilose, extending to posterior margin of basal lobe; claspette flament (Cl-F) about two-thirds as long as stem, broad, blade-like, tapering to a recurved tip. Basistyle (Bs) about three times as long as width across middle, rounded at apex, clothed with scales and long setae on outer aspect; basal lobe (B-L) large, quadrate, bearing numerous short setae on apical portion and a dense tuft of longer setae subapically, this subapical tuft adjacent to a large recurved basal 
spine; apical lobe (A-L) large, broadly rounded, bearing numerous short weak setae on inner margin. Dististyle (Ds) about three-fifths as long as basistyle, slightly broader medially; terminal claw (Ds-C) slender, nearly one-fourth as long as dististyle.

LARVA. (Fig. 110) - - Head broader than long. Antenna about half as long as head, moderately spined; antennal tuft multiple, inserted before middle of shaft, not reaching tip. Head hairs: Preantennal (A) multiple, extending beyond base of antennal tuft; lower (B) usually double, sometimes single or triple; upper (C) usually 3 to 4-branched; postclypeal (d) small, branched; sutural (e) double; trans-sutural (f) small, single; supraorbital long, single. Lateral abdominal hairs double on segments I to V. Comb of eighth segment of about 20 thorn-shaped scales in a patch; individual scale with a long apical spine and short lateral spinules. Siphon two and one-half to three times as long as wide; pecten of numerous, evenly spaced teeth, reaching to middle of siphon or nearly so; subventral tuft 4 to 6-branched, rather small, inserted beyond pecten. Anal segment longer than wide, with the dorsal plate reaching almost to the mid-ventral line; lateral hair single; dorsal brush consisting of a long lower caudal hair and a shorter multiple upper caudal tuft on either side; ventral brush well-developed, with a few shorter tufts preceding the barred area; gills 4 , longer than the anal segment, pointed.

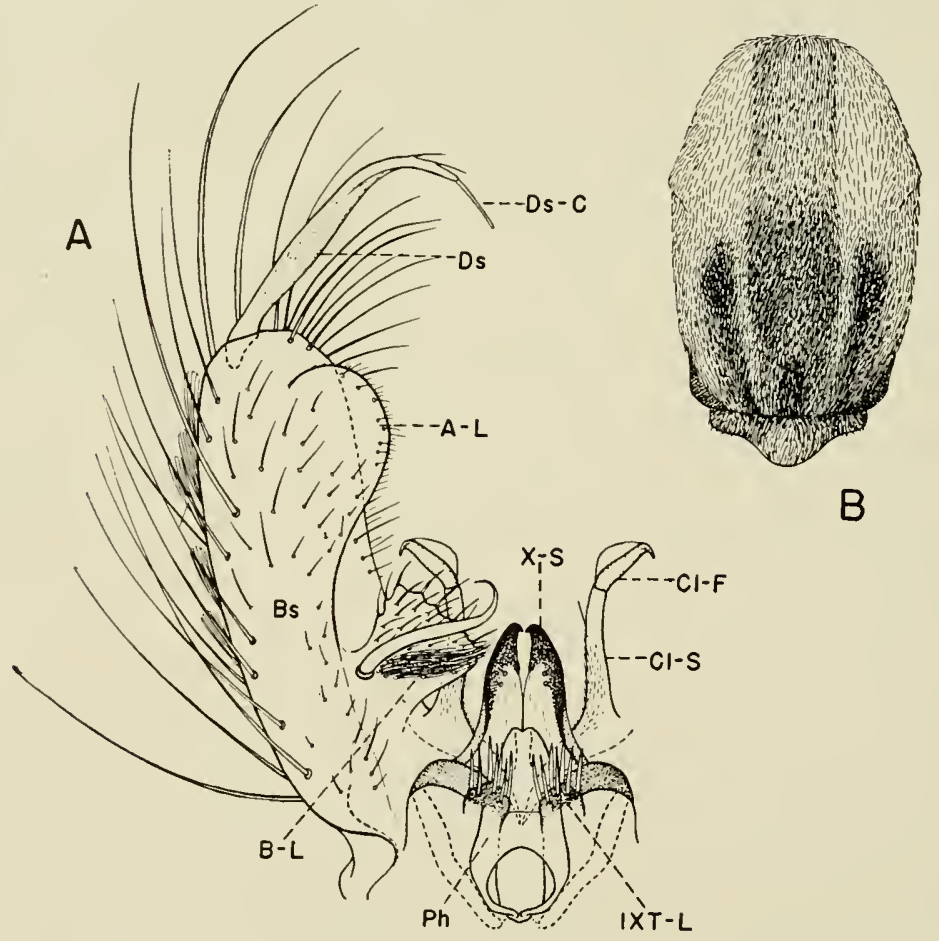

Fig. 109. Aedes sticticus (Meigen). A, Male terminalia. B, Scutum and scutellum. 
Distribution.-Canada to Florida, west to Utah and Montana. Southern States: Alabama (97); Arkansas (30); Florida (96); Georgia (148); Kentucky (140); Louisiana (96); Mississippi (97); Missouri (1); North Carolina (166); South Carolina (97); Tennessee and Virginia (52). Other States: Connecticut (52); Indiana (76); Iowa (155, 156); Kansas (128); Maine (90); Maryland and Massachusetts (52); Michigan (88); Minnesota (131); Montana (112); Nebraska (178); New Hampshire and New Jersey (52); New York (161); Oklahoma (159); South Dakota (52); Texas (108); Utah (142).

BIONOMICS.-The females are fierce biters, attacking during daylight hours and in early evening in woodlands and thickets near their breeding places. Matheson (116) says that the females are known to migrate several miles. The larvae of $A$. sticticus are found in flood pools in river valleys, especially in

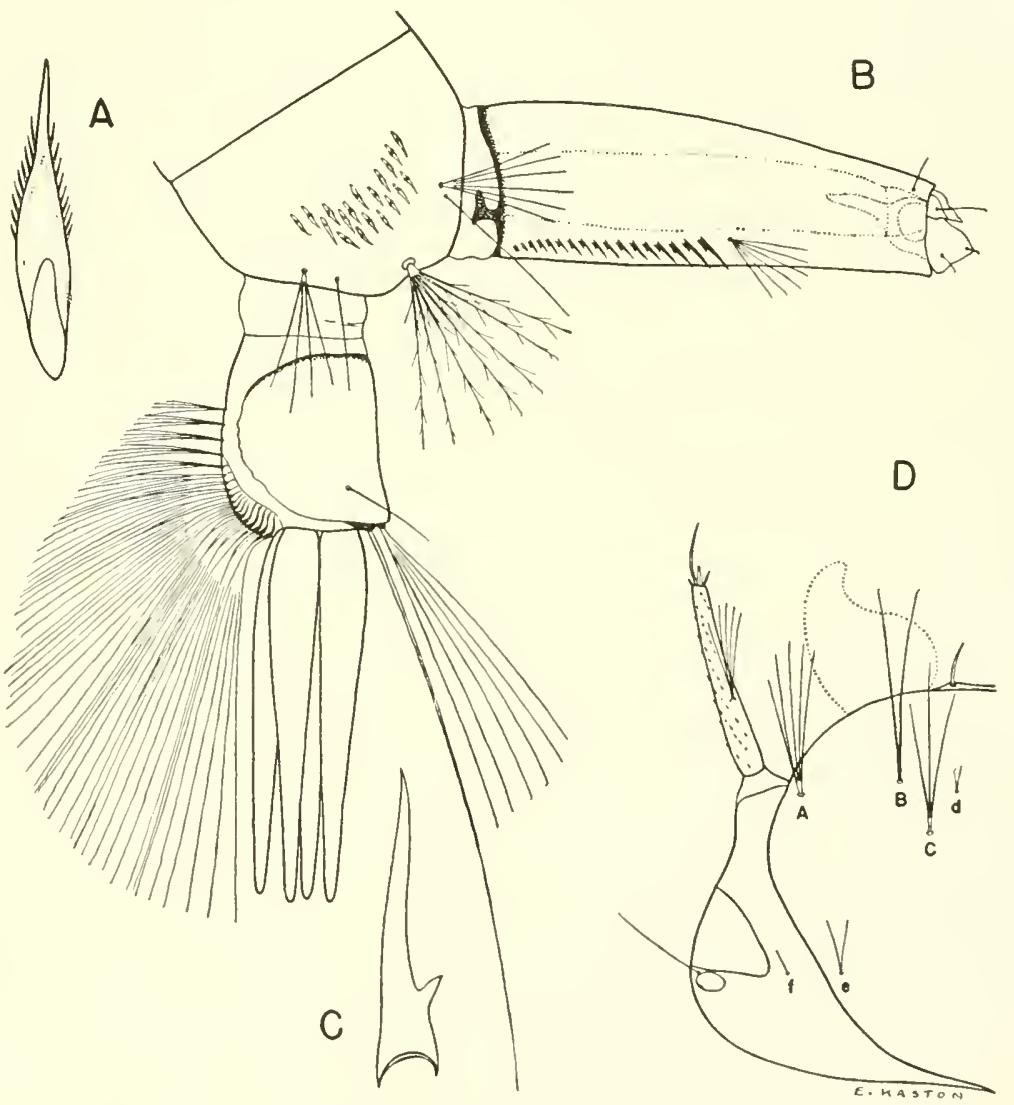

Fig. 110. Larva of Aedes sticticus (Meigen). A, Comb scale.

B, Terminal segments. C, Pecten tooth. D, Head. 
depressions containing vegetable matter. They develop from overwintering eggs, and there is but a single brood annually. Observations made by Rees (142) in Utah indicate that the eggs will remain viable for at least three seasons and probably longer in the absence of flooding. Similar observations have been made elsewhere by Twinn (181) and Dyar (54). Adults have been collected from February to August in the South but are more common from April to June farther north (4th Sv. C. Med. Lab. records, 1942-1944, unpublished).

\section{Aedes (Ochlerotatus) stimulans (Walker)}

Culex stimulans Walker, 1848, List. Dipt. Brit. Mus., 1 :4.

adult female.-Medium sized species. Head: Proboscis long, dark, inconspicuously speckled with pale scales; palpi short, dark, speckled with pale scales. Occiput dorsally with a median triangular patch of pale yellow lanceolate scales, the patch broad posteriorly, narrowed anteriorly and projecting forward between the eyes; scales of the submedian area adjacent to the margin of the eye narrow, golden-brown; lateral region of occiput clothed with broad appressed yellowish-white scales surrounding a small dark-scaled patch. Erect forked scales numerous, those on central portion of occiput pale. Thorax: Integument of scutum dark brown, covered with narrow bronzy-brown scales, except for anterior and lateral margins, prescutellar space, and a pair of submedian longitudinal lines, all of which are pale yellow to whitish scaled. Dorsal half of posterior pronotum with golden-brown lanceolate scales. Abdomen: First tergite white scaled; remaining tergites each with a broad basal band of white to pale-yellow scales; apical half of each tergite dark scaled, frequently speckled with a few pale scales; apices of terminal segments pale scaled. Venter primarily white scaled, frequently spotted with dark. Legs: Femora, tibiae, and first segment of tarsi clothed with intermixed dark and pale scales, the pale scales usually predominating on the femora; pale knee spots present. Segments of hind tarsus each with a broad basal white ring; segments 1 to 4 of fore- and mid-tarsi with narrower basal white rings than hind tarsus; segment 5 of foreand mid-tarsi usually entirely dark. Wing: Scales narrow, ligulate, intermixed dark and dingy-white.

ADULT MALE.-Coloration similar to that of female. TERMinAlia (Fig. 111B). Lobes of ninth tergite (IXT-L) about as long as broad, separated by the width of one lobe, each bearing several short spine-like setae. Tenth sternite (X-S) prominent, heavily sclerotized beyond middle. Phallosome (Ph) subcylindrical (a little narrower across apical third than across base), about twice as long as basal width, open ventrally, closed dorsally, and with a notch at apex. Claspette stem (Cl-S) pilose, slender, curved, extending beyond basal lobe; claspette filament (Cl-F) about as long as stem or slightly longer, broad, blade-like (the blade very thin, broadest and angulate near middle of filament), gradually tapering to a pointed curved tip. Basistyle (Bs) cylindrical, about three and one-half times as long as width across middle, rounded at apex, clothed with setae and scales on outer aspect and numerous long setae near inner ventral margin; basal lobe (B-L) short, bluntly conical, with numerous small apical setae and a stout curved, subapical spine; apical lobe (A-L) a prom- 
inent thumb-like projection bearing short setae. Dististyle (Ds) a little broader medially, approximately two-thirds as long as basistyle; terminal claw (Ds-C) slender, about one-fifth as long as dististyle.

LARva. (Fig .112). - Head broader than long. Antenna less than half as long as head, spinose; antennal tuft multiple, arising near middle of shaft. Head hairs: Preantennal (A) multiple, weakly barbed, barely reaching base of antennal tuft; lower (B) single; upper (C) double; postclypeal (d) small, branched; sutural (e), trans-sutural (f), and supraorbital single or double. Upper lateral abdominal hairs double or multiple on segment I, double on II to V and single on VI. Comb of eighth segment of numerous scales in a patch; individual scale with apical spine almost one and one-half times as long as subapical spines. Siphon about three times as long as wide; pecten of numerous evenly spaced teeth on basal third of siphon; subventral tuft 3 to 4-branched, inserted beyond pecten; anal segment longer than wide, with the dorsal plate reaching well down the sides; lateral hair single; dorsal brush consisting of a long lower caudal hair and a shorter multiple upper caudal tuft on either side; ventral brush large, with several small tufts preceding the barred area; gills 4 , about as long as the anal segment, each tapering to a blunt point.

Distribution.-Mostly restricted to Northern United States, Canada and Alaska. Southern States: Mississippi (52). Other States: Colorado (129);

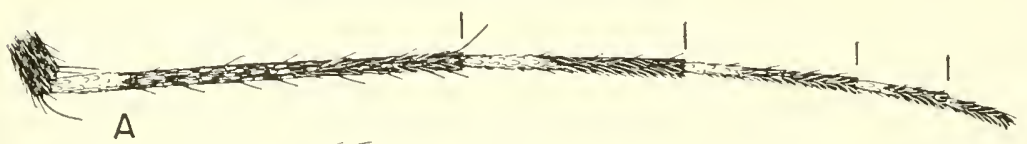

A
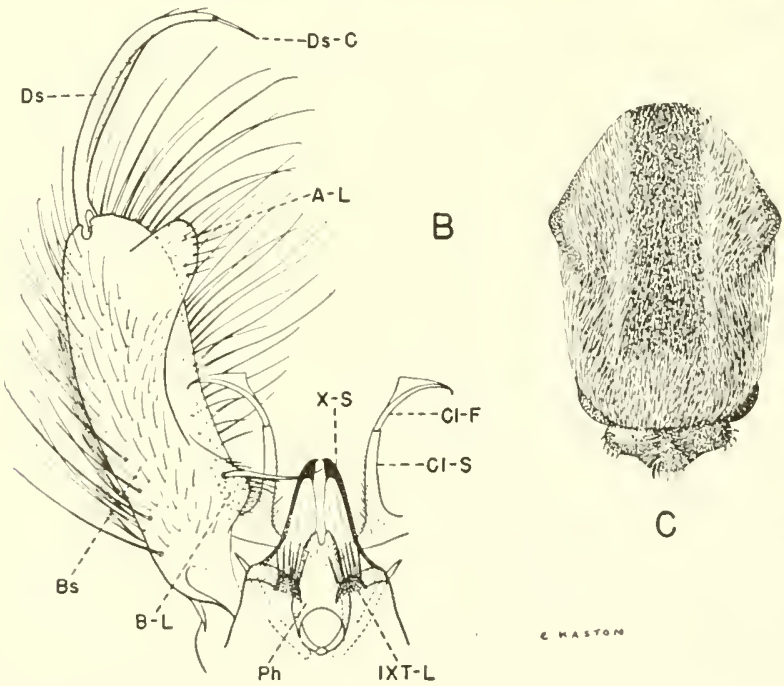

Fig. 111. Aedes stimulans (Walker). A, Hind tarsus. B, Male terminalia. C, Scutum and scutellum. 
Connecticut (90); Delaware (106); Illinois (42); Iowa (155, 156); Maine (137); Massachusetts (52); Michigan (88); Minnesota (131); Montana (112); Nebraska (178); New Hampshire (105); New Jersey (77); New York (52); Pennsylvania (8); Rhode Island (99); Utah (142); Vermont (90); Wisconsin (48); Wyoming (129).

BIONOMICS.-The females are vicious biters and are often annoying in woodlands when abundant. The larvae are found mostly in rain-filled woodland pools, and floodwater pools along rivers.

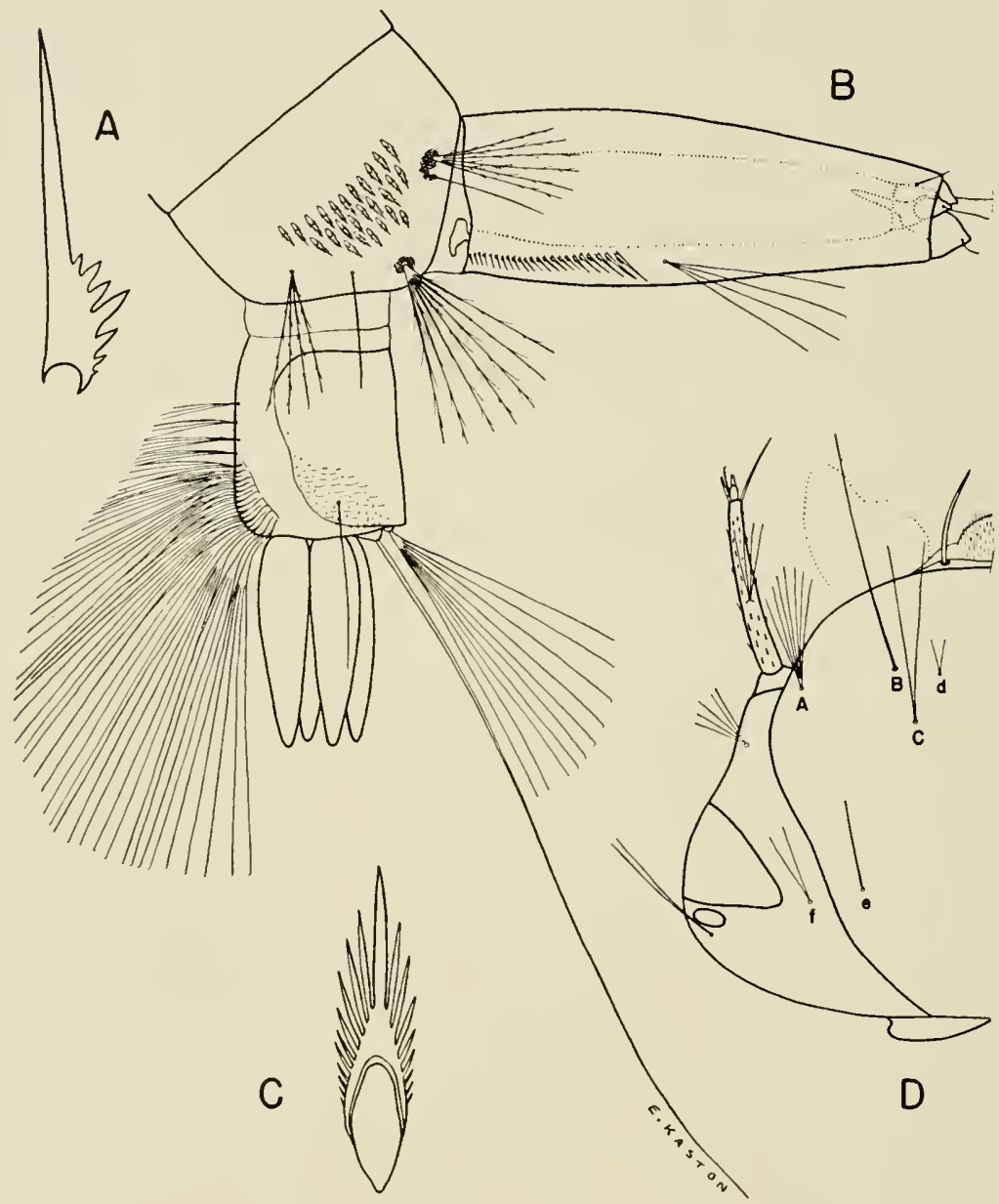

Fig. 112. Larva of Aedes stimulans (Walker). A, Pecten tooth.

B. Terminal segments. C, Comb scale. D. Head. 


\section{Aedes (Ochlerotatus) TAeniorhynchus (Wiedemann)}

Culex taeniorhynchus Wiedemann, 1821. Dipt. Exot., p. 43.

AdUlt female.-Medium sized to rather small species. Head: Proboscis long, dark scaled, with a white ring near middle; palpi short, dark, white scaled at tips. Occiput dorsally with a broad longitudinal stripe of goldenyellow to pale golden-brown lanceolate scales, the stripe narrowed anteriorly and projecting forward between the eyes; this broad median stripe bounded on either side by a few dark scales, followed laterally by a large patch of broad appressed white scales enclosing a small dark-scaled area. Erect forked scales numerous, those on central portion of occiput pale. Thorax: Integument of scutum dark-brown, clothed dorsally with narrow golden-brown scales becoming dark-brown on lateral margins; the scales on the anterior margin, on prescutellar space, and immediately above the wing bases pale yellow to nearly silvery white. Posterior pronotum with narrow dark-brown scales. Abdomen: First tergite dark scaled; remaining tergites dark scaled, with narrow basal white bands dorsally and conspicuous white patches laterally; apices of the terminal tergites with a few pale scales. Sternites white scaled basally, dark scaled or speckled with white apically. Legs: Femora and tibiae dark brown to black scaled, pale on inner surfaces; femora usually tipped with a few white scales. Tarsi black; segments of hind tarsi each with a broad white basal ring, segment 5 occasionally entirely white; segments 1 to 3 of fore- and mid-tarsi with basal rings narrower than those of hind tarsi; segments 4 and 5 of fore- and midtarsi entirely dark. Wing: Scales narrow, dark.

ADULT MALE.-Coloration similar to that of female, but with occiput usually rather indefinitely marked. TERMINALIA (Fig. 113B). Lobes of ninth tergite (IXT-L) about as broad as long, moderately sclerotized, separated by the width of one lobe, each bearing several short stout setae. Tenth sternite (X-S) prominent, sclerotized. Phallosome $(\mathrm{Ph})$ a stout cylinder, rounded apically, open ventrally, closed dorsaily, lightly sclerotized. Claspette stem (Cl-S) slender, cylindrical, curved, pilose, extending a little beyond apex of basal lobe; claspette filament (Cl-F) nearly as long and broad as stem, curved, tapering to a point and bearing a prominen simple sharp retrorse projection medially. Basistyle (Bs) about three and one-half times as long as broad, cylindrical, rounded apically, clothed with large scales and numerous long setae; basal lobe (B-L) a broadly conical projection having many slender setae on apex; apical lobe absent. Dististyle (Ds) about half as long as basistyle, broadened medially, slender and curved apically; terminal claw (Ds-C) slender, curved, a little more than one-fourth as long as dististyle.

LARva. (Fig. 114).-Head broader than long. Antenna less than half as long as head, slightly curved, sparsely spined; antennal tuft small, double or triple, inserted slightly before middle of shaft, not reaching tip. Head hairs: Preantennal (A) short, multiple, barbed; lower (B) and upper (C) long, single; postclypeal (d) small, branched; sutural (e), trans-sutural (f) and supraorbital single. Upper lateral abdominal hairs on segments III to $\mathrm{V}$ with three or more branches. Comb of eighth segment of numerous scales in a 
patch, usually more than 15 ; individual scale small, rounded apically, fringed with subequal spinules. Siphon less than twice as long as wide; pecten of numerous short evenly spaced teeth reaching middle of siphon or slightly beyond; subventral tuft multiple, inserted beyond pecten; dorsal preapical spine as long as apical pecten tooth. Anal segment wider than long, completely ringed by the dorsal plate; lateral hair single; dorsal brush consisting of a long lower caudal hair and a shorter multiple upper caudal tuft on either side; ventral brush large, confined to the barred area; gills 4, very short, bluntly rounded.

Distribution.-Occurs along the Atlantic coast from Brazil to New England and along the Pacific coast from Peru to California. It is also found in several inland communities where brackish water is available for breeding. Southern States: Alabama (96); Arkansas (30); Florida, Georgia, Louisiana, Mississippi, North Carolina and South Carolina ((96); Virginia (49). Other States: California (66); Connecticut (52); Delaware (106); Maryland (19);

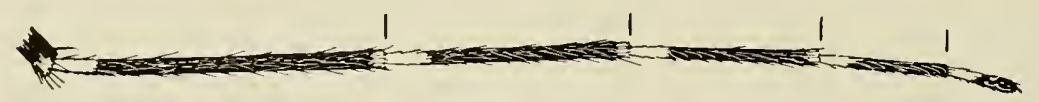

A

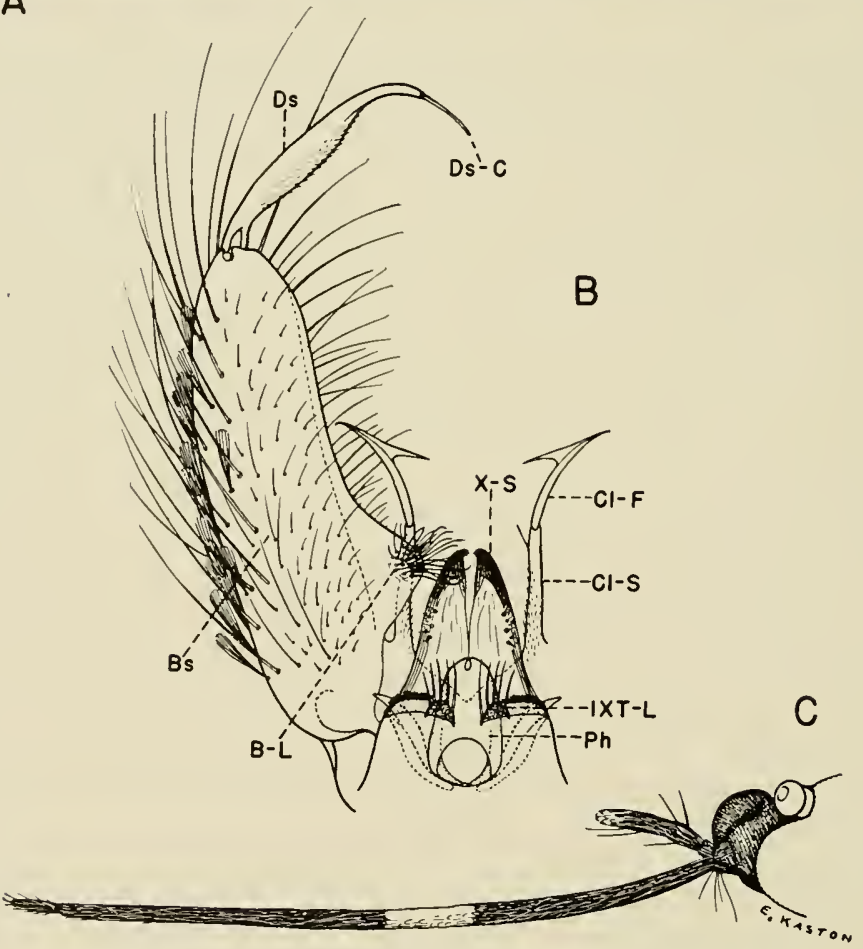

Fig. 113. Aedes taeniorhynchus (Wiedemann). A, Hind tarsus. B, Male terminalia. C, Palp and proboscis of female. 
Massachusetts (90); New Jersey (77); New York (52); Pennsylvania (8); Rhode Island (90); Texas (108).

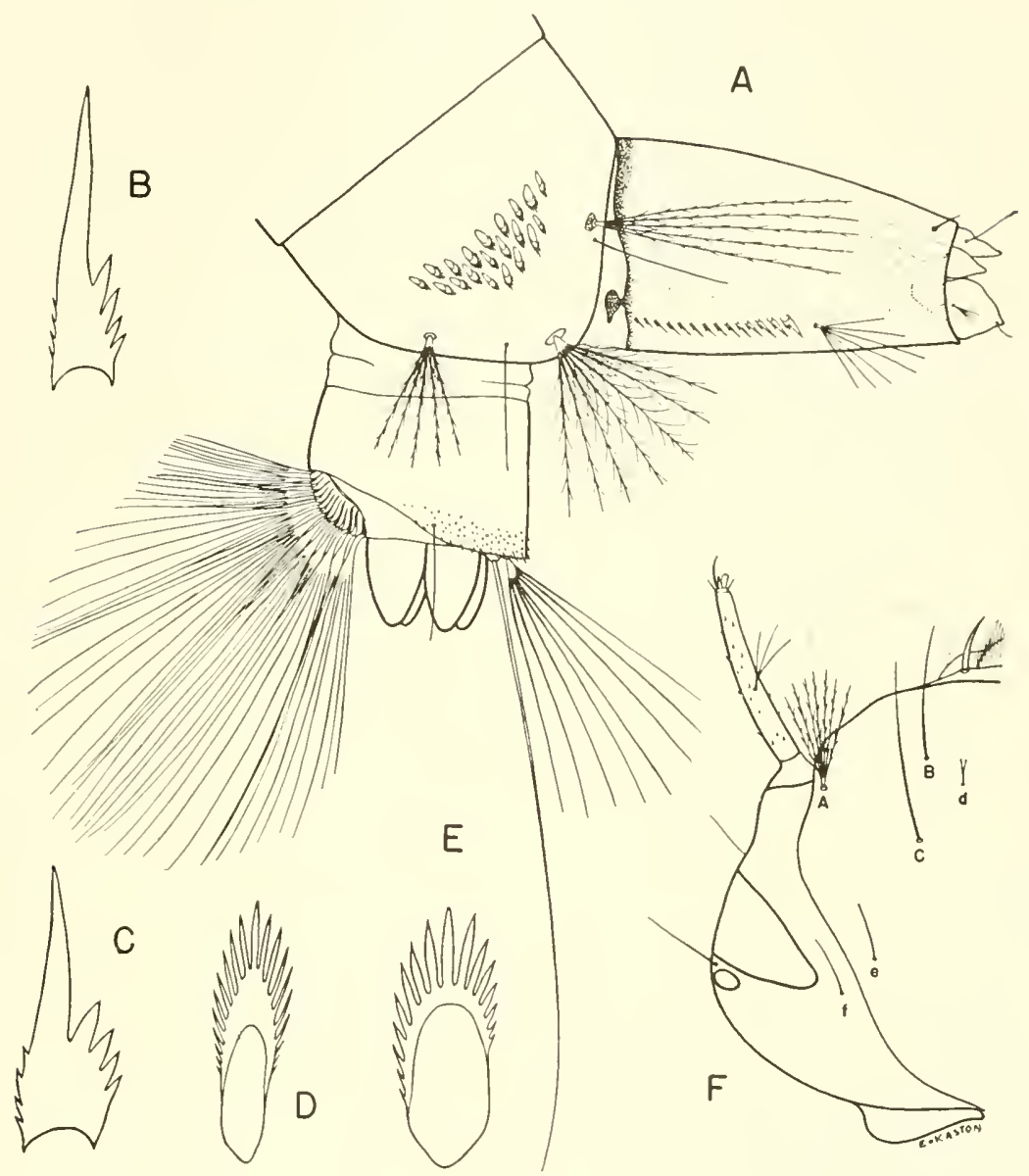

Fig. 114. Larva of Aedes taeniorhynchus (Wiedemann). A, Terminal segments. B, and C, Pecten teeth. D, and E, Comb scales. F, Head.

вIоNOMICs.-The females are fierce biters, attacking any time during the day or night. The adults are strong fliers and often migrate considerable distances from their breeding places. This species breeds in saltmarshes flooded by tides or rains, and is occasionally found in freshwater pools.

\section{Aedes (Ochlerotatus) thibaulti Dyar and Knab}

Acdes thibaulti Dyar and Knab, 1910, Proc. Ent. Soc. Wash., 11:174.

adult female.-Medium sized species. Head: Proboscis long, black scaled; palpi short, black. Occiput dorsally with a longitudinal stripe of 
narrow yellow scales, the stripe broad posteriorly, narrowed anteriorly and extending forward between the eyes; a small submedian patch of broad dark scales, bordered anteriorly by a line of narrow yellow scales margining the eye present on either side of the median pale-scaled area; lateral region of occiput clothed with broad appressed pale scales. Pale erect forked scales numerous on dorsal surface of occiput. Thorax: Integument of scutum black, with a wide longitudinal stripe of narrow bronzy-black scales, the anterior half of which is broadly margined laterally by golden lanceolate scales; the longitudinal dark stripe originating near anterior margin of scutum, remaining about constant in width to near middle, abruptly broadening beyond middle to cover entire postero-dorsal surface of scutum. A few golden scales present on the margins of the prescutellar space. Posterior pronotum clothed with golden lanceolate scales. Abdomen: Tergites blue-black scaled, with conspicuous basal patches of white to whitish-yellow scales. Venter primarily whitish scaled, with the apices of the terminal segments dark. Legs: Legs blue-black scaled except for white knee spots and pale inner surfaces of femora. Wing: Scales narrow, dark.

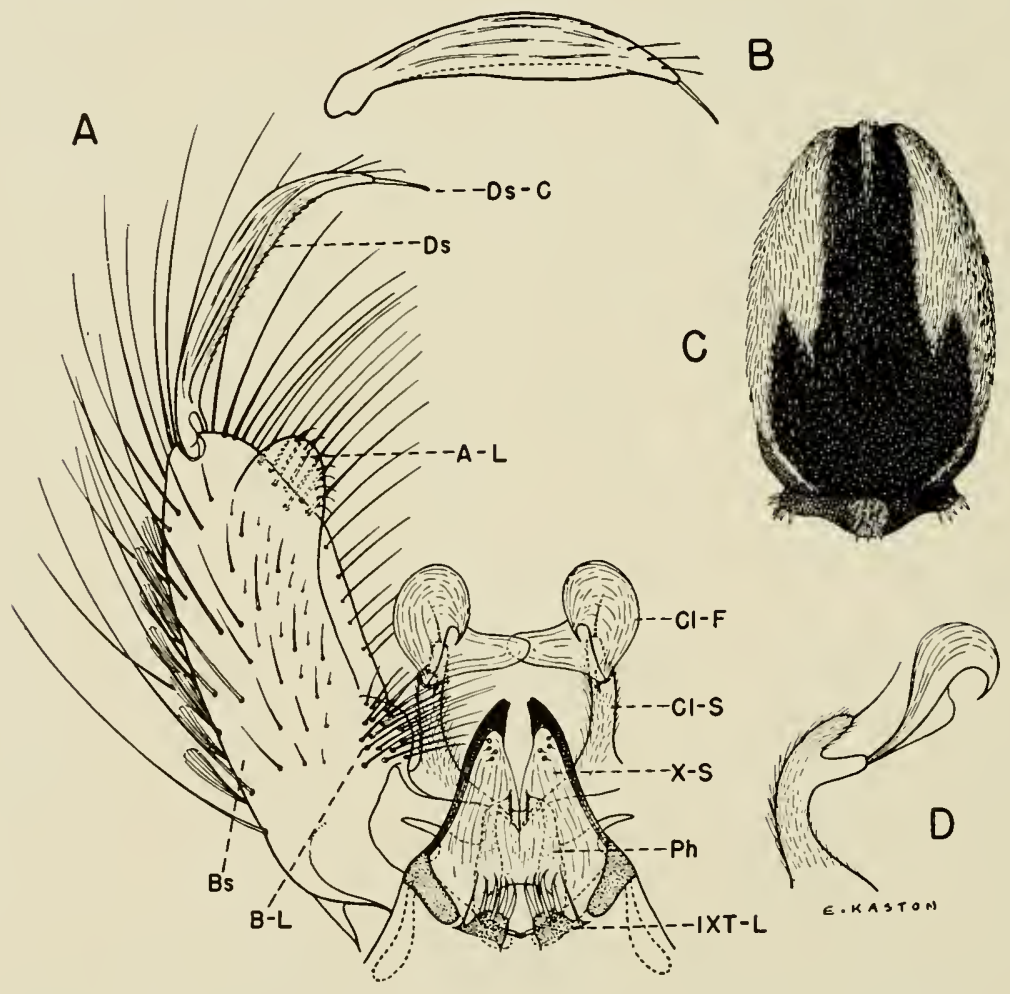

Fig. 115. Aedes thibaulti Dyar and Knab. A, Male terminalia. B, Dististyle.

C, Scutum and scutellum. D. Claspette (side view). 
ADULT MALE.-Coloration similar to that of female. TERminalia (Fig. 115A). Lobes of ninth tergite (IXT.L) about as long as broad, separated by a little less than the width of one lobe, each bearing several short spines. Tenth sternite (X-S) prominent, heavily sclerotized beyond middle. Phallosome (Ph) cylindrical, about two-thirds as broad as long, strongly constricted just before apex, open ventrally and at tip, closed dorsally. Claspette stem (Cl-S) rather stout, pilose, bearing a short seta at tip and a short stout branch near middle; claspette filament ( $\mathrm{Cl}-\mathrm{F}$ ) a large broadly expanded contorted leaf-like structure about as long as claspette stem and arising from the tip of the short median branch of the stem. Basistyle (Bs) cylindrical, nearly three times as long as broad, rounded at apex, clothed with scales and long setae (setae more numerous apically and near inner ventral margin); basal lobe (B-L) rounded, covered with numerous moderately long setae arising from distinct tubercles; apical lobe (A-L) a stout thumb-like projection bearing short curved setae on margin. Dististyle (Ds) approximately two-thirds as long as basistyle, broader

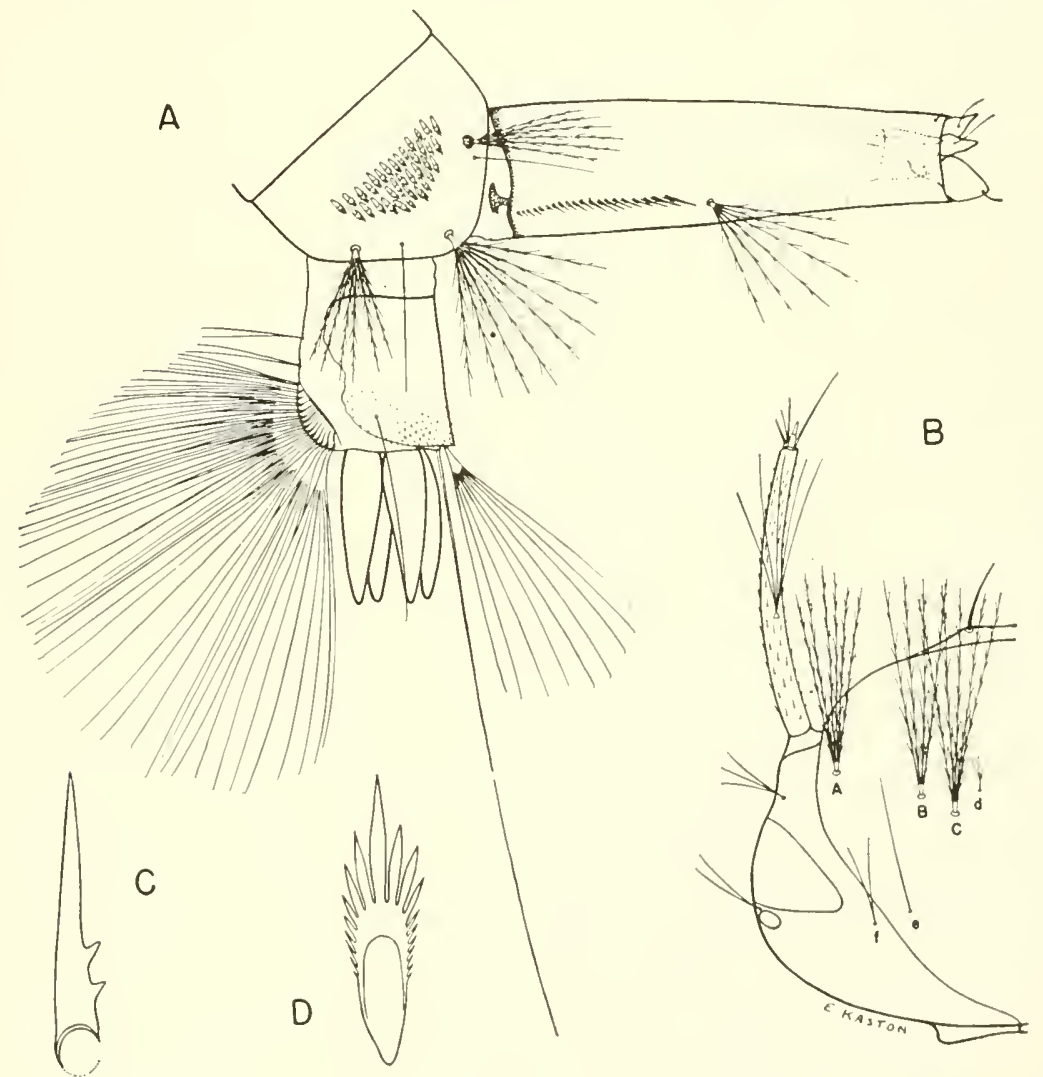

Fig. 116. Larva of Aedes thibaulti Dyar and Knab. A. Terminal segments. B. Head. C. Pecten tooth. D. Comb scale. 
medially; terminal claw (Ds-C) slender, about one-sixth as long as dististyle.

LARVA. (Fig. 116).-Head broader than long. Antenna nearly as long as head, slender, curved, spinose; antennal tuft multiple, inserted near middle of shaft, reaching tip. Inner preclypeal spines long, slender, lightly pigmented, separated by more than the length of one spine. Head hairs: Preantennal (A), lower (B), and upper (C) multiple, barbed, inserted nearly in a straight line; sutural (e) long, single; trans-sutural (f) and supraorbital 2-branched. Upper lateral abdominal hairs 3 to 4-branched on segments I and II, double on segments III to VI. Comb of eighth segment of more than 20 scales in a patch; individual scale fringed with fairly stout spines, the apical spine somewhat longer and stouter. Siphon about four and one-half to five times as long as wide; pecten of numerous evenly spaced teeth not reaching middle of siphon; subventral tuft 5 to 7-branched, inserted beyond pecten. Anal segment longer than wide, nearly ringed by the dorsal plate; lateral hair single; dorsal brush consisting of a long lower caudal hair and a shorter multiple upper caudal tuft on either side; ventral brush well-developed, with two or three small tufts preceding the barred area; gills 4 , somewhat shorter than the anal segment.

Distribution.-Southern United States west to Texas. Southern States: Alabama (170); Arkansas (30); Florida (125); Georgia (96); Kentucky (140); Louisiana (24); Mississippi (54); Missouri (52); North Carolina (166); South Carolina (125); Tennessee (35). Other State: Texas (141).

BIONOMICS.- The adults of this species are found during the spring in thickets and woodlands near their breeding places and are fierce biters, attacking even at midday. Hollow stumps and tree cavities are favorite resting places for the adults. Larvae are found in the hollow bases of gum trees following the flooding of lowland areas. Carpenter (30) states that he has found larvae of A. thibaulti in other kinds of trees on only two occasions. It is believed that very few trees, other than gum, have the type of cavities favorable for their breeding. Larvae of $A$. thibaulti have been collected from December to May in the South (4th Sv. C. Med. Lab. records, 1942-1944, unpublished).

\section{Aedes (Ochlerotatus) tormentor Dyar and Knab}

Aedes tormentor Dyar and Knab, 1906, Jour. N. Y. Ent. Soc., 14:191.

ADULT FEM.ALE. - The female cannot be separated at this time from Aedes atlanticus.

ADULT MALE.-Coloration similar to that of female. TERMINALIA (Fig. 117). Lobes of ninth tergite (IXT-L) about three-fourths as broad as long, separated by slightly less than the width of one lobe, each bearing several short spines. Tenth sternite (X-S) prominent, heavily sclerotized beyond middle. Phallosome $(\mathrm{Ph})$ about three-fifths as broad as long, stoutly conical, rounded apically, open ventrally, closed dorsally. Claspette stem (CI-S) pilose basally, glabrous toward apex, slender, sinuous, extending a little beyond middle of basistyle; claspette filament (Cl-F) about two-fifths as long as stem, striated, curved and tapered at apical third. Basistyle (Bs) about four times as long as 
width across middle, slightly curved, rounded at apex, clothed with scales and long setae on outer aspects (numerous moderately long setae present on inner ventral margin); basal lobe (B.L) cylindrical, nearly four times as long as wide, bearing numerous short stout setae on apex and a long curved spine near base, the spine arising from a large elongated tubercle; apical lobe (A-L) a

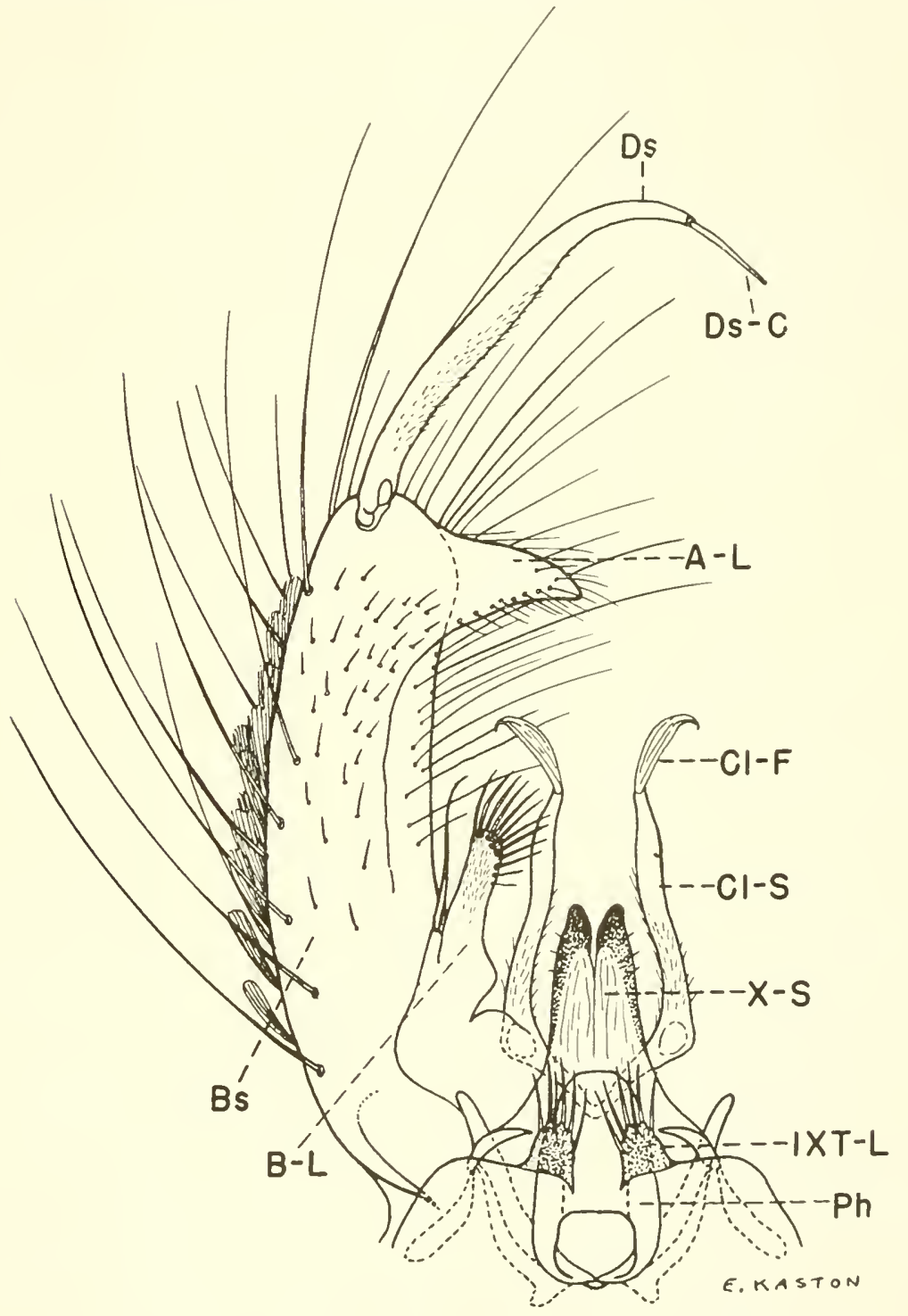

Fig. 117. Male terminalia of Acdes tormentor Dyar and Knab. 


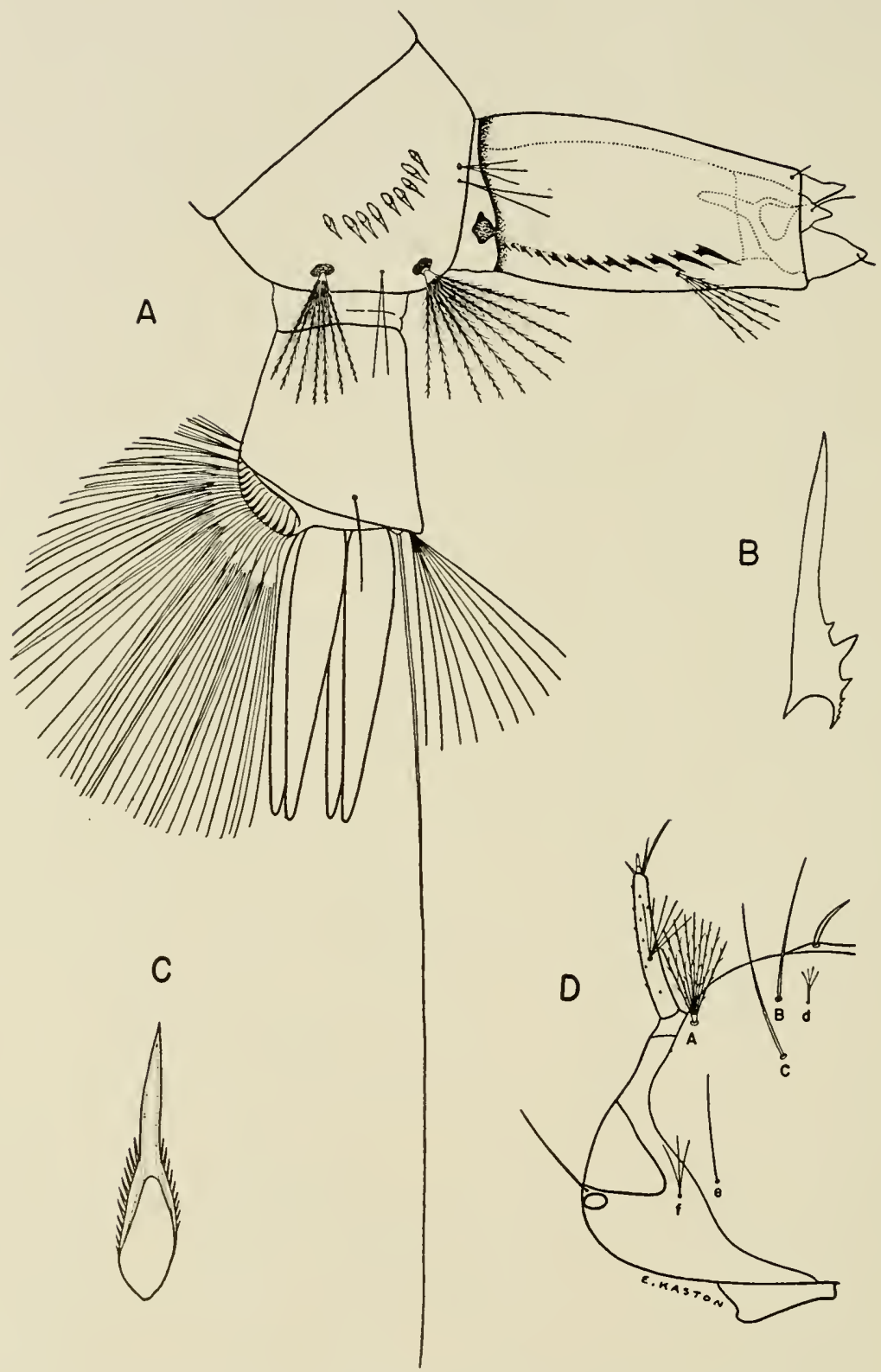

Fig. 118. Larva of Aedes tormentor Dyar and Knab. A, Terminal segments. B, Pecten tooth. C, Comb scale. D, Head. 
prominent thumb-like projection bearing numerous short setae. Dististyle (Ds) approximately three-fifths as long as basistyle, broader medially; terminal claw (Ds-C) slender, about one-fourth as long as dististyle.

LARVA. (Fig. 118).- - Head broader than long. Antenna less than half the length of the head, slightly curved, sparsely spined; antennal tuft multiple, inserted before middle of shaft, not reaching tip. Head hairs: Preantennal (A) multiple, sparsely barbed, extending beyond insertion of antennal tuft; lower (B) and upper (C) single; postclypeal (d) small, 3 to 4-branched; sutural (e) long, single; trans-sutural (f) double or triple; supraorbital single. Lateral abdominal hairs usually single on segments III to V. Comb of eighth segment of about 9 to 12 thorn-shaped scales in a single curved, sometimes irregular row; individual scale with a long apical spine and short lateral spinules. Siphon about twice as long as wide; pecten of numerous evenly spaced teeth, extending to near distal fourth of siphon; subventral tuft multiple, barbed, inserted within the pecten. Anal segment longer than wide, completely ringed by the dorsal plate; lateral hair single; dorsal brush consisting of a very long lower caudal hair and a shorter multiple upper caudal tuft on either side; ventral brush well developed and with one of two small tufts preceding the barred area; gills 4, as long or longer than the anal segment, each tapering to a point.

Distribution.-Southern States: Alabama (96); Arkansas and Florida (52); Georgia (148); Louisiana (96); Mississippi (52); North Carolina (125); South Carolina (64). Other State: Texas (108).

BIONOMICS.- Very little is known of the habits of the adults of $A$. tormentor, since they cannot be separated from $A$. atlanticus except by examination of the male terminalia. The larvae are rare and occur in temporary rainfilled pools during the summer months.

\section{Aedes (Ochlerotatus) trivittatus (Coquillett)}

Culex trivittatus Coquillett, 1902, Jour. N. Y. Ent. Soc., 10:193.

ADUlt FEMAle.-Medium sized species. Head: Proboscis dark scaled; palpi short, dark. Narrow median area of occiput clothed with white lanceolate scales and erect pale forked scales; remainder of occiput with a few dark erect forked scales and numerous broad appressed dingy-white scales (often a small patch of broad brown scales present laterally, surrounded by the broad pale scales). Thorax: Integument of scutum dark brown, with a pair of conspicuous longitudinal submedian stripes of narrow white to whitish-yellow scales; the submedian stripes uniting on anterior margin and often joined posteriorly on prescutellar space; remaining portions of scutum and dorsal half of posterior pronotum clothed with bronzy-brown lanceolate scales. Abdomen: First tergite dark scaled; other tergites dark scaled with basal patches of white scales laterally. Scales of venter white to whitish-yellow. Legs: Scales dark except for pale inner surfaces of femora and tibiae and a white streak usually on inner surface of segment 1 of each tarsus. Wing: Scales narrow, dark brown. 
ADULT MALE. - Coloration similar to that of female, except that the abdomen usually has narrow white basal bands dorsally, at least on some segments.

TERMINALIA (Fig. 119A). Lobes of ninth tergite (IXT-L) about as long as broad, separated by approximately the width of one lobe, each bearing several short spines. Tenth sternite (X-S) prominent, heavily sclerotized beyond middle. Phallosome $(\mathrm{Ph})$ stoutly conical, about one and three-fourths times as long as broad, rounded apically, open ventrally, closed dorsally. Claspette stem (Cl-S) pilose, slender, curved, extending beyond basal lobe. Claspette filament (Cl-F) nearly as long as stem, expanded blade-like, tapered and curved beyond

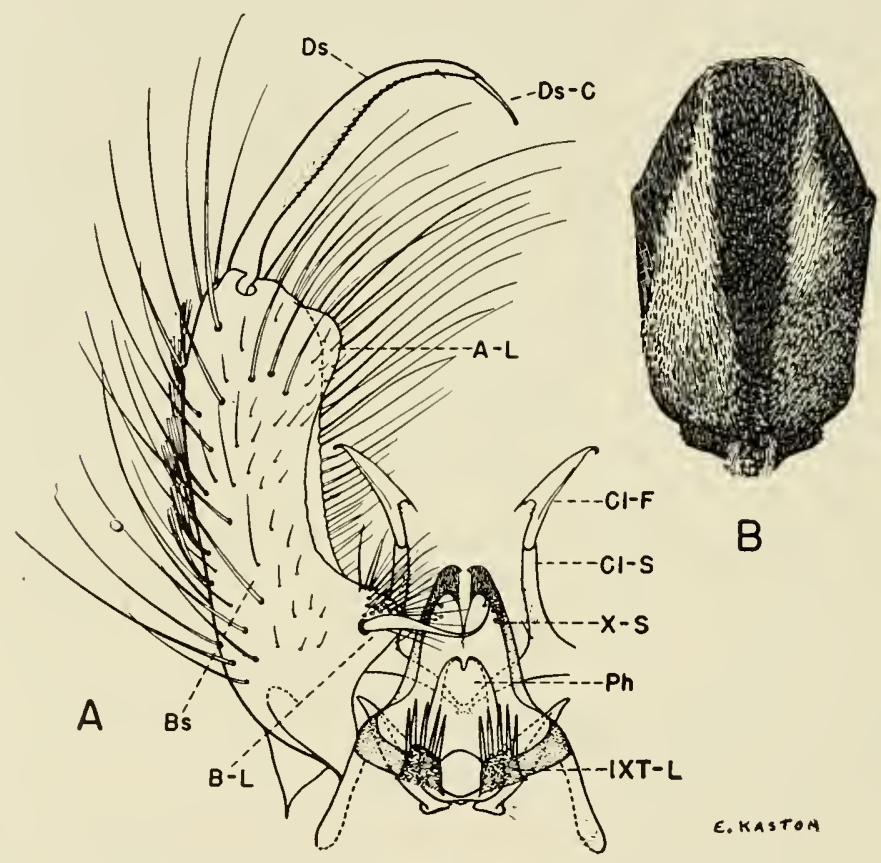

Fig. 119. Aedes trivittatus (Coquillett). A, Male terminalia.

B. Scutum and scutellum.

middle; the blade-like portion with a large sharp retrorse projection arising from its basal margin; one or more minute accessory retrorse spines occasionally present on inner margin of the large projection or between the large projection and the non-expanded basal portion of filament. Basistyle (Bs) nearly four times as long as width across middle, bluntly rounded at apex, clothed with scales and long setae (setae very numerous and long on inner ventral margin); basal lobe (B-L) prominent, bluntly conical, bearing numerous short setae apically and a large curved spine on dorsal side; apical lobe (A-L) rounded, not prominent, its vestiture consisting of a few fine short setae. 
Dististyle (Ds) about two-thirds as long as basistyle, slightly broader medially; terminal claw (Ds-C) slender, approximately one-fifth as long as dististyle.

LARva. (Fig. 120)-Head broader than long. Antenna a little less than half as long as head, curved, sparsely spined; antennal tuft multiple, inserted near middle of shaft, not reaching tip. Head hairs: Preantennal (A) multiple, sparsely barbed, extending to insertion of antennal tuft; lower (B) and upper (C) single, sparsely barbed; postclypeal (d) small, branched; sutural (e)

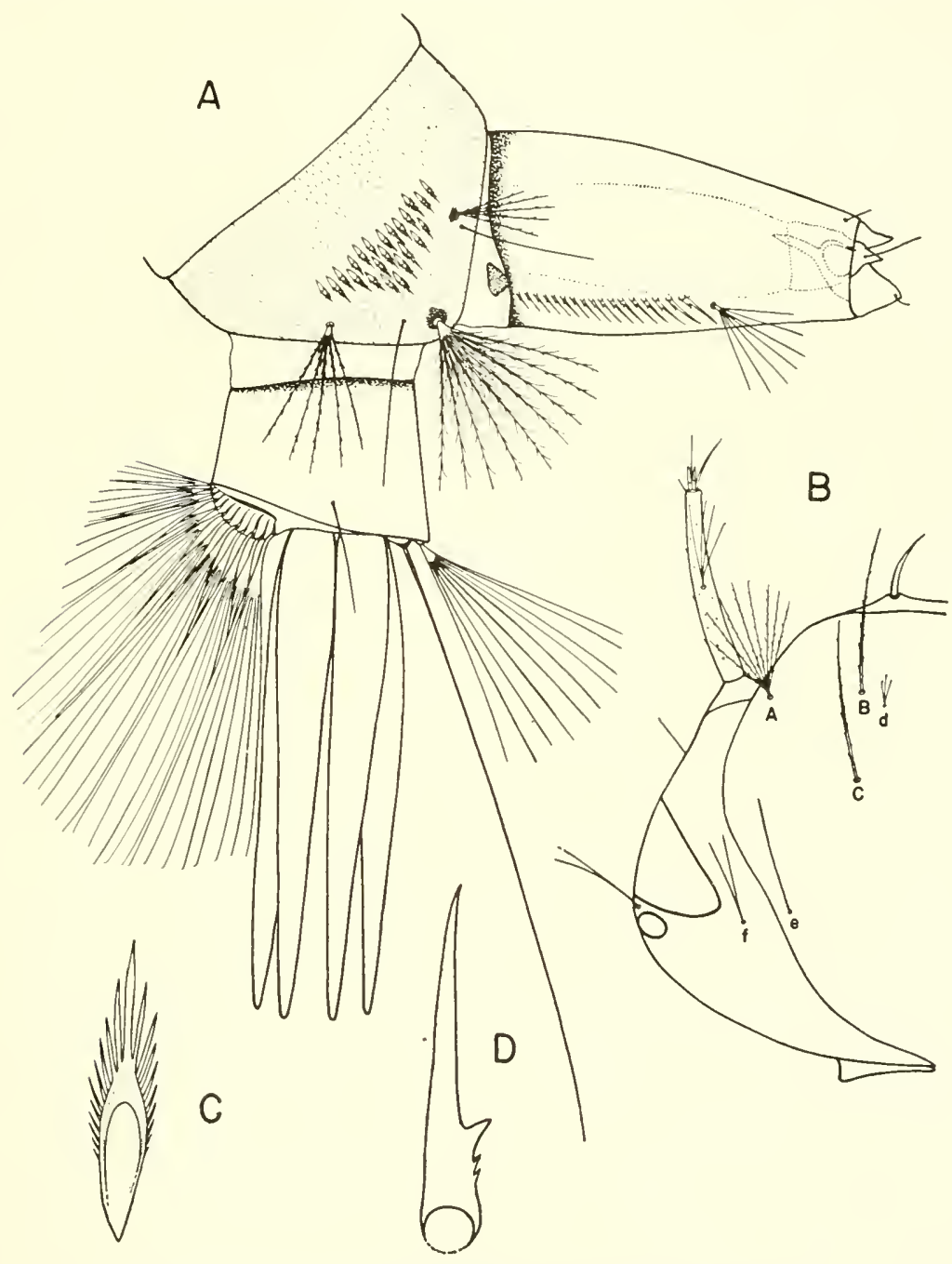

Fig. 120. Larva of Aedes trivitlatus (Coquillett). A. Terminal segments. B, Head. C, Comb scale. D, Pecten tooth. 
single; trans-sutural (f) double or triple; supraorbital single or double. Body spiculate. Upper lateral abdominal hairs single on segment III to V. Comb of eighth segment of about 18 to 25 thorn-shaped scales in a patch; individual scale with apical spine about twice as broad and generally about one and onethird times as long as subapical spinules. Siphon about twice as long as wide; pecten of numerous evenly spaced teeth, usually extending beyond middle of siphon; subventral tuft multiple, inserted beyond pecten; dorsal preapical spine less than half as long as apical pecten tooth. Anal segment wider than long, completely ringed by the dorsal plate; lateral hair single; dorsal brush consisting of a long lower caudal hair and a shorter multiple upper caudal tuft on either side; ventral brush well-developed, confined to the barred area; gills 4 , longer than the anal segment, each tapering to a point.

Distribution.--Southern Canada; United States, south to South Carolina and Louisiana, west to Colorado and Montana. Southern States: Arkansas (31); Georgia (148); Kentucky (140); Louisiana (54); Missouri (1); North Carolina (97); South Carolina (27); Tennessee (97); Virginia (49). Other States: Colorado (89); Connecticut (21); District of Columbia (52); Illinois (42); Indiana (76); Iowa (155, 156); Kansas (128); Maine, Maryland and Massachusetts (52); Minnesota (131); Montana (112); Nebraska (178); New Jersey (77); New Mexico (52); New York (162); North Dakota (127); Oklahoma (159); Pennsylvania (8); Rhode Island (99); Texas (108); West Virginia (54); Wisconsin (48); Wyoming (129).

BIONOMICS.--The females are fierce biters, attacking during daylight hours when their haunts are invaded. Owen (131) states that the bite of this mosquito is the most painful he has experienced from any species in Minnesota. Larvae of $A$. trivittatus occur mostly in flood-water pools along rivers and in woodland pools. This species passes the winter in the egg stage and larvae may be found any time during the summer following rains.

\section{Aedes (Finlaya) atropalpus (Coquillett)}

Culex atropalpus Coquillett, 1902, Can. Ent., 34 :292.

adult female.-Medium sized species. Head: Proboscis long, slender, dark scaled; palpi short, dark. Occiput dorsally with a median patch of yellow lanceolate scales, the patch broad posteriorly, narrowed anteriorly and projecting forward between the eyes; this dorso-median patch bounded submedially by a large area of broad appressed white scales which extend far down the sides, enclosing a lateral patch of broad dark scales. Pale erect forked scales numerous on central portion of occiput. Thorax (Fig. 121C) : Integument of scutum black, clothed dorsally with a very wide irregular longitudinal stripe of fine dark bronzy-brown scales; this longitudinal dark stripe broadly margined and partially interrupted by a lateral area of narrow pale-yellow to goldenyellow scales; the lateral area of pale scales extends from the anterior margin of the scutum to near the wing base and projects medio-posteriorly from the region of the scutal angle along the prescutal-scutal suture. Prescutellar space 
margined with pale yellow scales; posterior pronotum clothed with narrow dark scales dorsally, broader pale scales ventrally. Abdomen: First tergite dark scaled; remaining tergites dark, all except the last with narrow basal white bands. Venter white scaled basally, speckled or entirely covered with dark scales apically. Legs: Hind leg dark scaled except for pale inner surface of femur, prominent white knee spot, white tip of tibia, and rather broad basal and apical rings on tarsal segments, the last segment entirely white. Fore and middle legs similarly marked, but with tarsal rings narrower on segments 1 and 2, entirely lacking or greatly reciuced on 3 and 4; segment 5 always entirely dark. Wing: Scales narrow, all dark except for a small white-scaled patch at base of costa.

A
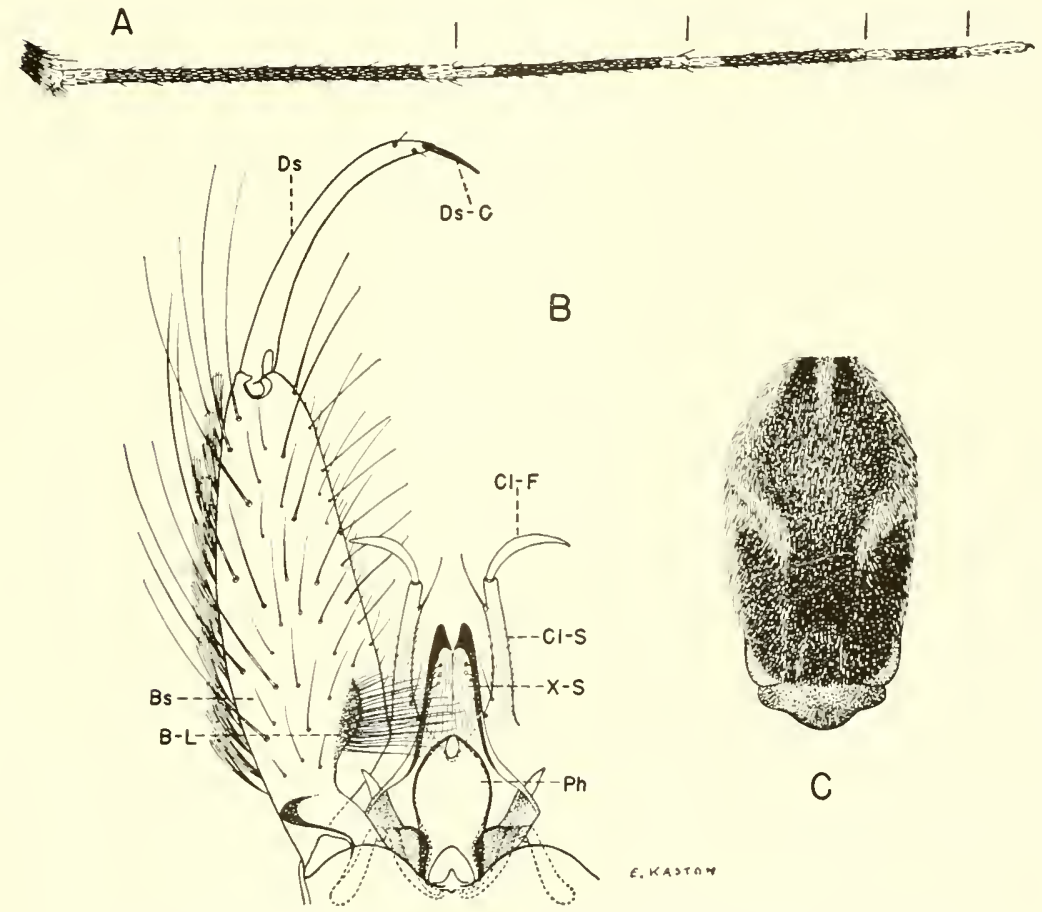

Fig. 121. Aedes atropalpus (Coquillett). A, Hind tarsus. B, Male terminalia. C, Scutum and scutellum.

ADULT MALE.-Coloration similar to that of female. TERminalia (Fig. 121B). Lobes of ninth tergite inconspicuous, without spines or setae. Tenth sternite (X-S) prominent, heavily sclerotized beyond middle. Phallosome (Ph) nearly twice as long as broad, subcylindrical (slightly constricted at basal third and somewhat bulbous at apical third), bluncly pointed at tip, open ventrally, closed dorsally. Claspette stem (Cl-S) slender, extending a little beyond basal third of basistyle, pilose, slightly curved, and with a subapical seta arising from 
a prominent tubercle; claspette filament (Cl-F) about three-fourths as long as stem, slender, sickle-shaped. Basistyle (Bs) about three and one-half times as long as broad, nearly straight, conical, narrowly rounded at apex, clothed with long and short setae and numerous large scales; basal lobe (B-L) inconspicuous, represented by a small slightly raised darkly pigmented area covered by a dense patch of setae; apical lobe absent. Dististyle (Ds) about two-thirds as long as basistyle, evenly curved, not expanded medially, glabrous except for a few setae near apex; terminal claw (Ds-C) slender, about one-fifth as long as basistyle.

LARva. (Fig. 122).-Head slightly broader than long. Antenna less than half as long as head, slightly curved, spinose; antennal tuft small, 2 to 3 branched, inserted at middle of shaft. Head hairs: Preantennal (A) 3 to 4 branched; lower (B) and upper (C) single; postclypeal (d) small, usually 2 to 3-branched; sutural (e), trans-sutural (f), and supraorbital single. Comb of eighth segment of many scales in a patch; individual scale fringed apically with subequal spinules. Siphon slightly less than twice as long as wide; pecten of numerous teeth extending nearly to tip of siphon, the last 3 or 4 teeth widely spaced; subventral tuft multiple, barbed, inserted within the pecten. Anal segment about as long as wide, with the dorsal plate extending halfway down the sides; lateral hair single or double; dorsal brush consisting of a long lower caudal hair and a shorter multiple upper caudal tuft on either side; ventral brush well-developed, confined to the barred area; gills 4, about three times as long as the anal segment.

Distribution.-Southern Canada; United States; Mexico (87); and Central America (54). Southern States: Arkansas (30); Missouri (70); North Carclina (166); Tennessee (170); Virginia (52). Other States: Arizona, Connecticut and District of Columbia (52); Maine (137); Maryland and Massachusetts (52); Minnesota (131); New Hampshire (52); New Jersey (77); New Mexico and New York (52); Oklahoma (159); Pennsylvania (52); Rhode Island (99); Texas (108); Vermont (90); Wisconsin (48).

BIONOMICS.-The females of $A$. atropalpus are active biters and frequently annoying near rocky streams along which they breed. Carpenter $(30)$ found adults, both males and females, resting during the daytime under rock ledges near their breeding places on Petit Jean Mountain in Arkansas in 1938, and several females were taken biting during daylight hours in the same area. Owen (131) states that females of this species feed freely on man in Minnesota when encountered but were never observed far away from their breeding places. Owing to the limited number of suitable breeding places, the species is never extremely abundant and only troublesome locally.

The larvae are usually found throughout the summer in overflow pools in rock holes along mountain streams, but may also be taken on occasions in rainfilled depressions in rocks some distance from such streams. Although the larvae are slow in development, the species is several-brooded in warmer climates. The eggs are laid singly above the water level and are apparently firmly attached to the rock so as to withstand winter floods. Larvae were collected 
by Carpenter (30) in Arkansas during February, March, and September through December.

MEdiCAL importanCE.-During 1939, workers at the Harvard Medical School demonstrated in the laboratory that $A$. atropalpus can transmit the eastern variety of equine encephalitis. They concluded, however, that it is probably not an important natural vector of the disease due to its peculiar geographical distribution and to the small number of specimens ordinarily encountered.

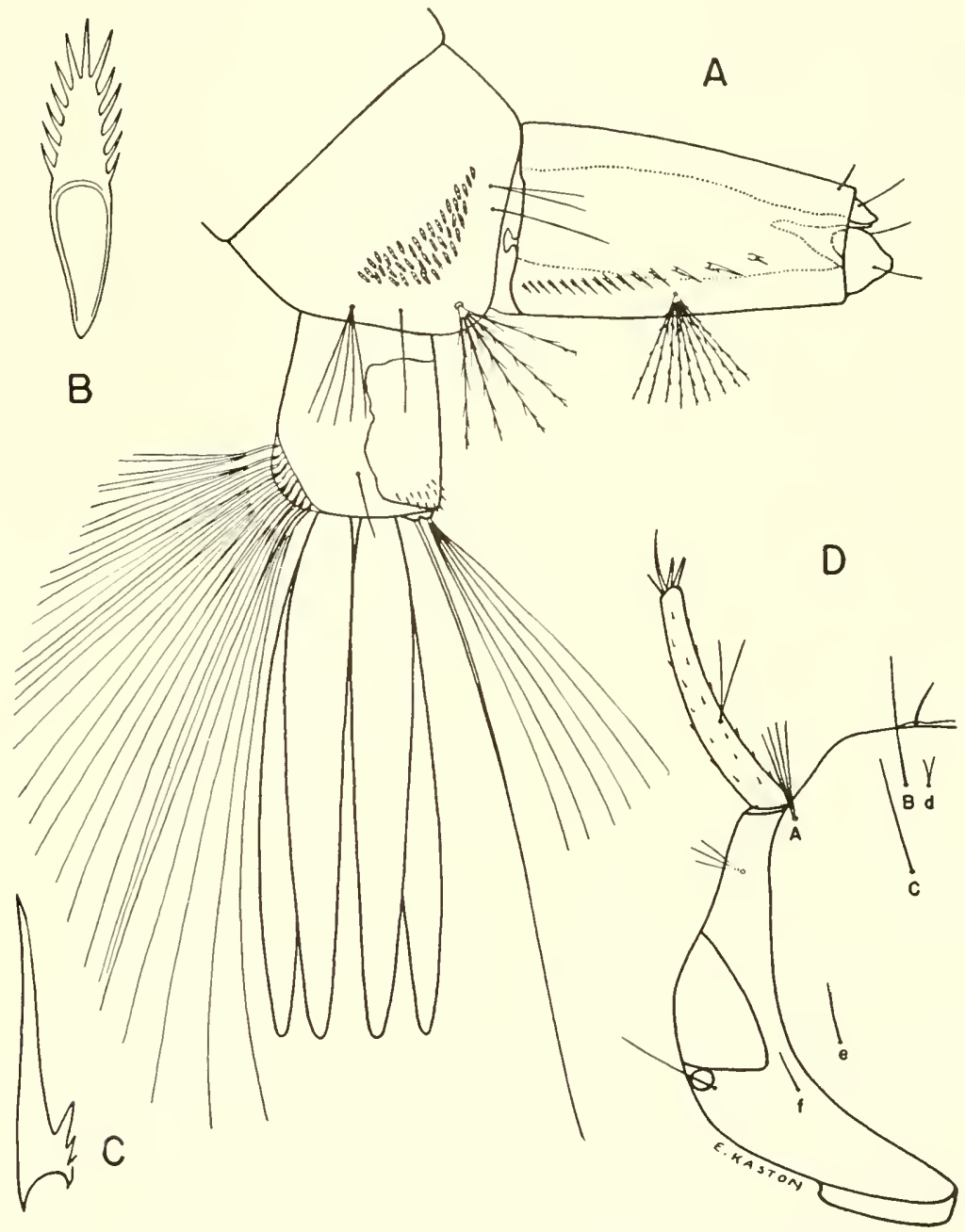

Fig. 122. Larva of Aedes atropalpus (Coquillett). A. Terminal segments. B, Comb scale. C. Pecten tooth. D. Head. 


\section{Aedes (Finlaya) TRiseriatus (Say)}

Culex triseriatus Say, 1823, Jour. Acad. Nat. Sci. Phila., 3:12.

AdUlt Female.-Medium sized species. Head: Proboscis long, black; palpi short, black. Occiput clothed dorsally with curved silvery-white lanceolate scales and white erect forked scales; occiput clothed laterally with broad appressed white scales. Thorax: Integument of scutum black, with a wide longitudinal stripe of narrow dark-brown scales, broadly margined on either side by white lanceolate scales; the longitudinal dark stripe of scutum originating near the anterior margin, becoming gradually broader to middle, much

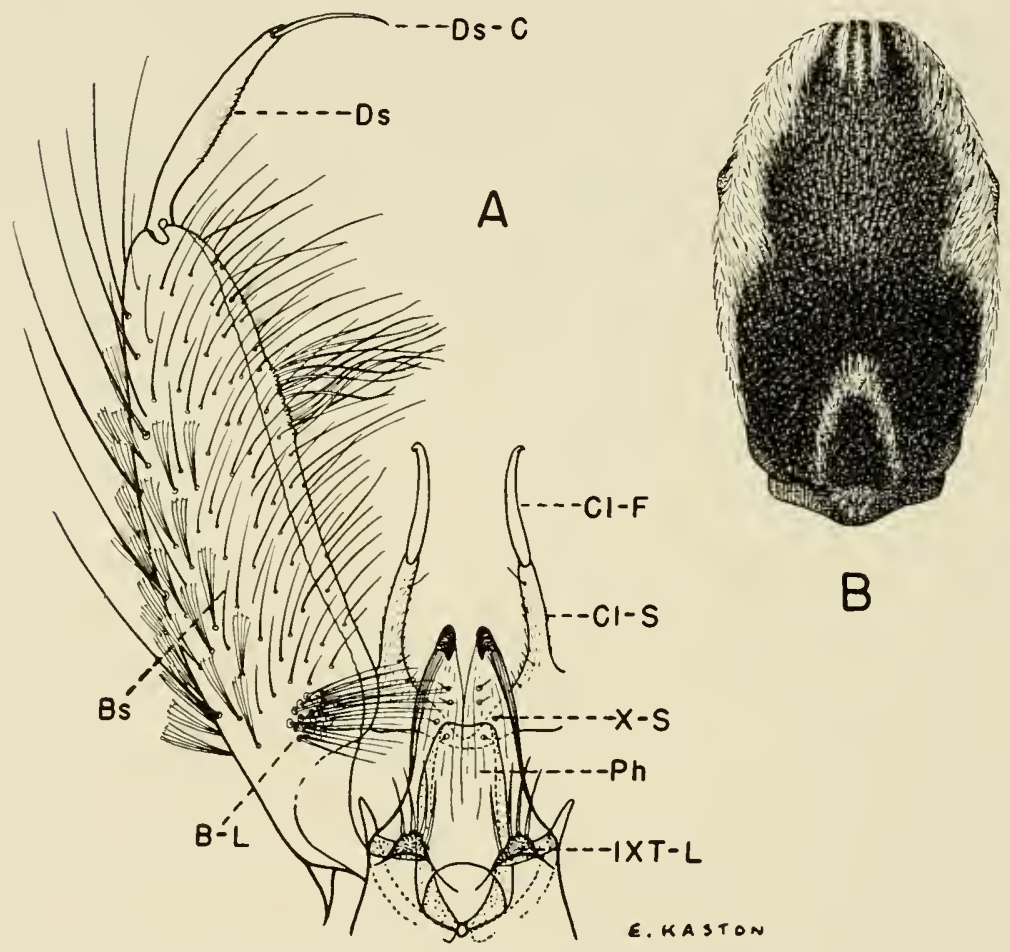

Fig. 123. Aedes triseriatus (Say). A, Male terminalia. $B$, Scutum and scutellum.

broader beyond middle (covering most of dorsal surface), and extending to posterior margin on either side of the white-bordered presuctellar space. Posterior pronotum clothed with broad appressed white scales. Abdomen: Tergites blue-black scaled, with conspicuous basal patches of white scales laterally. Venter white scaled, with the apices of the terminal segments black. Legs: Fore and middle legs black scaled except for femora, which are pale on inner sur- 
faces and usually have a few white scales at tips. Hind legs black scaled except for femora, which have the basal one-half to two-thirds whitish scaled on all aspects and the tips conspicuously white scaled. Wing: Scales narrow, dark.

ADUlt MALE.-Coloration similar to that of female. TERMinalia (Fig. 123A). Lobes of ninth sternite (IXT-L) distinct but not as long as broad, moderately sclerotized, well separated, each bearing a row of stout setae. Tenth sternite (X-S) prominent, sclerotized apically. Phallosome $(\mathrm{Ph})$ a stout cylinder, truncate apically, open ventrally, closed dorsally, lightly sclerotized. Claspette stem (Cl-S) slender, cylindrical, curved, pilose, reaching basal third of basistyle; claspette filament (Cl-F) ligulate, slightly longer and narrower than stem, gradually tapering to a pointed, somewhat recurved tip, and extending a little beyond middle of basistyle. Basistyle (Bs) nearly three times as long as broad, rounded apically, clothed with large scales and moderately long setae; basal lobe (B-L) ill-defined, represented by a dense patch of setae near base of basistyle; apical lobe also ill-defined, but probably represented by a small dense patch of long setae on inner face of basistyle at apical third. Disti. style (Ds) less than half as long as basistyle, broader basally and medially; termnial claw (Ds-C) slender, curved, about half as long as dististyle.

LARVA. (Fig. 124).-Head slightly broader than long. Antenna about half as long as head, slightly curved, smooth; antennal tuft represented by a single hair inserted near middle of shaft, nearly reaching tip. Head hairs: Preantennal (A) short, multiple; lower (B) 2 to 4-branched; upper (C) long, single; postclypeal (d) well-developed, multiple; sutural (e) and trans-sutural (f) single or double; supraorbital single. Lateral abdominal hairs on segments I and II triple, sometimes double; double on III to VI. Comb of eighth segment of about 9 to 15 scales in a single or partially double row; individual scale long, gradually tapered, evenly fringed with short spinules. Siphon two and one-half to three times as long as wide; pecten of numerous evenly spaced teeth extending to near middle of siphon; subventral tuft represented by a single, sometimes double, barbed hair inserted beyond pecten. Anal segment about as wide as long, with dotsal plate extending a little more than half-way down the sides; lateral hair large, multiple, inserted near ventro-lateral margin; dorsal brush consisting of a long lower caudal hair and a shorter multiple upper caudal tuft on either side; ventral brush well developed, consisting of long rather sparse tufts and with two or three smaller tufts preceding the barred area; gills 4 , small, blunt, shorter than the anal segment, the dorsal pair somewhat longer than the ventral pair.

Distribution.-Southern Canada; eastern United States west to Montana. Southern States: Alabama (170); Arkansas (30); Florida and Georgia (52); Kentucky (140); Louisiana, Mississippi, Missouri, North Carolina, South Carolina, Tennessee and Virginia (52). Other States: Colorado (134); Connecticut (52); Delaware (106); District of Columbia (52); Illinois (42); Indiana (76); Iowa and Kansas (52); Maine (90); Maryland (19); Massachusetts (52); Michigan (88); Minnesota (131); Montana (52); Nebraska (178); New Hampshire (52); New Jersey (77); New York (52); Oklahoma (159); Rhode Island (99); Texas (108); Wisconsin (48). 
BIONOMICS.-The adults of this species frequent woodlands near their breeding places. The females attack man during the day or early evening in woodlands or around homes located in or near wooded areas. The bite of this mosquito is painful and lasting. The larvae occur in water in rot cavities of trees and occasionally in artificial containers, particularly wooden tubs, barrels, and troughs, and may be found throughout the year in the southern states (4th Sv. C. Med. Lab, reports, 1942-1944, unpublished).

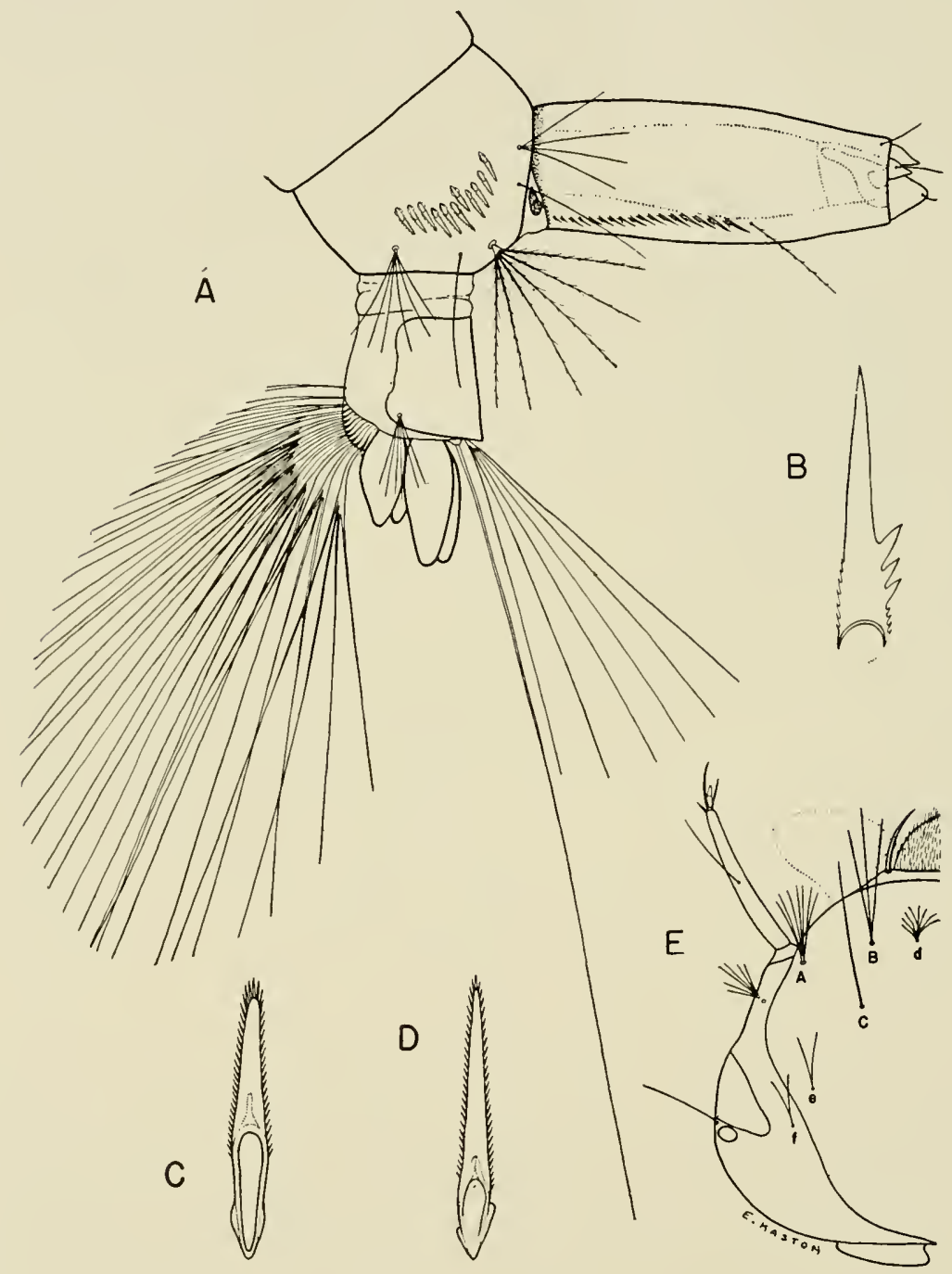

Fig. 124. Larva of Aedes triseriatus (Say). A, Terminal segments.

B, Pecten tocth. C, and D, Comb scales. E, Head. 
MEDICAL IMPORTANCE.--Laboratory experiments performed by the Harvard Medical School during 1939 showed that $A$. triseriatus can transmit the eastern variety of equine encephalitis. Bennett et al. (16) were able to transmit the virus of yellow fever experimentally with this species, but it is unimportant as a vector since its range of distribution is outside the present endemic zones of the disease.

\section{Aedes (Stegomyia) aEgypti (Linnaeus) 1}

Culex aegypti Linnaeus, 1762, Hass. Pal. Reise, p. 470.

adult female.-Medium sized species. Head: Proboscis dark scaled; palpi short, dark, tipped with silvery-white scales. Clypeus and tori with broad appressed silvery-white scales. Occiput clothed dorsally with a rather narrow longitudinal stripe of broad white scales, extending anteriorly between the eyes; this median stripe bounded on either side by a large submedian patch of broad dark scales (the anterior margin of the submedian dark patch with a line of narrow silvery-white scales bordering the eye); lateral region of occiput clothed with a patch of broad white scales, the patch interrupted near its middle by a smaller patch of dark scales. Erect forked scales pale, restricted to the dorsoposterior portion of occiput. Thorax: Integument of scutum dark brown to black, covered with narrow dark bronzy-brown scales except for a small patch of narrow white scales at the middle of the anterior margin and a conspicuous lyre-shaped pattern of pale scales. (The outer frame-work of the "lyre" consisting of a pair of strong lines of rather broad silvery-white scales; these lines broader, curved outward and crescent-shaped on the anterior half of scutum; these lines nartower, submedian in position and straight on posterior half of scutum and extending to posterior margin. The "strings" of the "lyre" consisting of a pair of narrow straight submedian lines of slender yellowish-white scales originating at the anterior margin of scutum and extending nearly to prescutellar space). Anterior pronotal lobe, posterior pronotum and lobes of scutellum clothed with broad appressed white scales. Abdomen: Tergites dark scaled, with narrow basal white bands dorsally and silvery-white basal patches laterally. Venter white scaled except for the last two segments, which are principally dark. Legs: Legs dark scaled, but with white knee spots, white streaks on femora, and white-scaled basal rings on tarsal segments. Basal rings of hind tarsi broad on segments 1 to 4 ; segment 5 entirely white. Basal rings of foreand mid-tarsi narrower than those of hind tarsi, present on only segments 1 and 2. Wing: Scales rather narrow, dark.

ADULT MALE.-Coloration similar to that of female. TERMinalia (Fig. 125E). Lobes of ninth tergite (IXT-L) large, triangular, clothed apically with a few very small setae. Tenth sternite (X-S) prominent, moderately sclerotized, blunt apically, bearing a short ventral branch at basal third. Phallosome (Ph) conical in dorsal view, a little more than twice as long as basal width, open ventrally, closed dorsally at apical third; each plate with a row of strong ventral teeth beyond middle, those at tip more numerous and projecting dorsally to form a crown at the apex of the phallosome. Claspette absent; inter-

1 Stegomyia fasciata of authors. The reader is referred to Dyar (1928) and Edwards (1932) for synonymy and references to this species. 
basal fold (Ib-F) prominent; pilose. Basistyle (Bs) conical, short, about one and one-half times as long as wide, clothed on outer and ventral aspects with setae and numerous large scales; basal lobe (B-L) indistinct, represented by a few stout, apically bent spines and a dense patch of setae covering most of the inner face of the basistyle (this may not be a true basal lobe); apical lobe

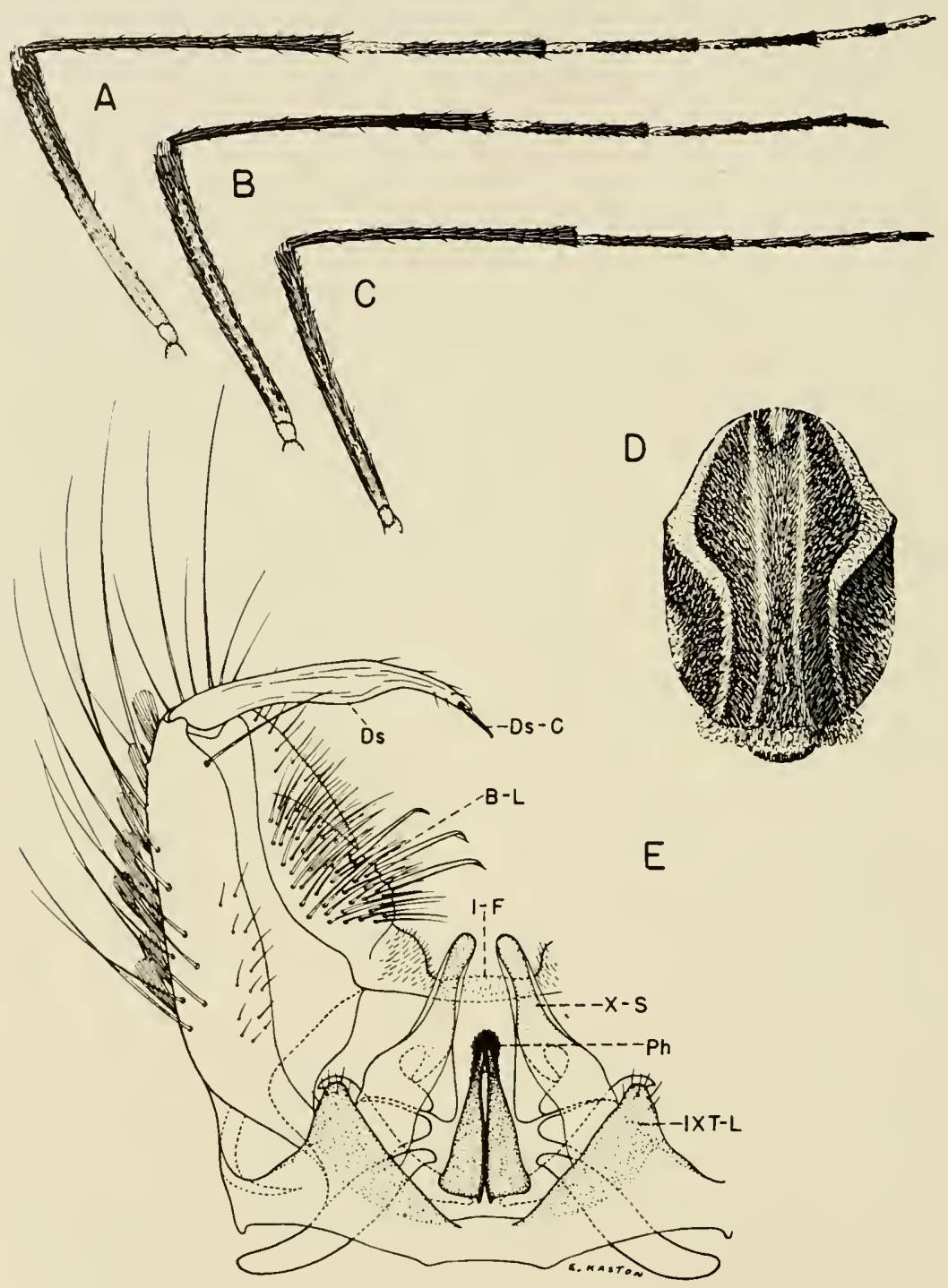

Fig. 125. Aedes aegypti (Linnaeus). A, B, and C, Hind-, mid-, and forelegs, respectively. D, Scutum and scutellum. E, Male terminalia. 
absent. Dististyle (Ds) about five-eighths as long as basistyle, slightly expanded a little beyond middle, tapered and curved distally; terminal claw (Ds-C) pointed, about one-seventh as long as dististyle.

LARva. (Fig. 126).-Head slightly broader than long. Antenna about onethird as long as head, slightly curved, smooth; antennal tuft represented by a single hair inserted at middle of shaft. Head hairs: Preantennal (A), lower (B), and upper (C) single; postclypeal (d) about 3 to 5-branched; sutural (e) trans-sutural (f), and supraorbital single. Upper lateral abdominal hairs

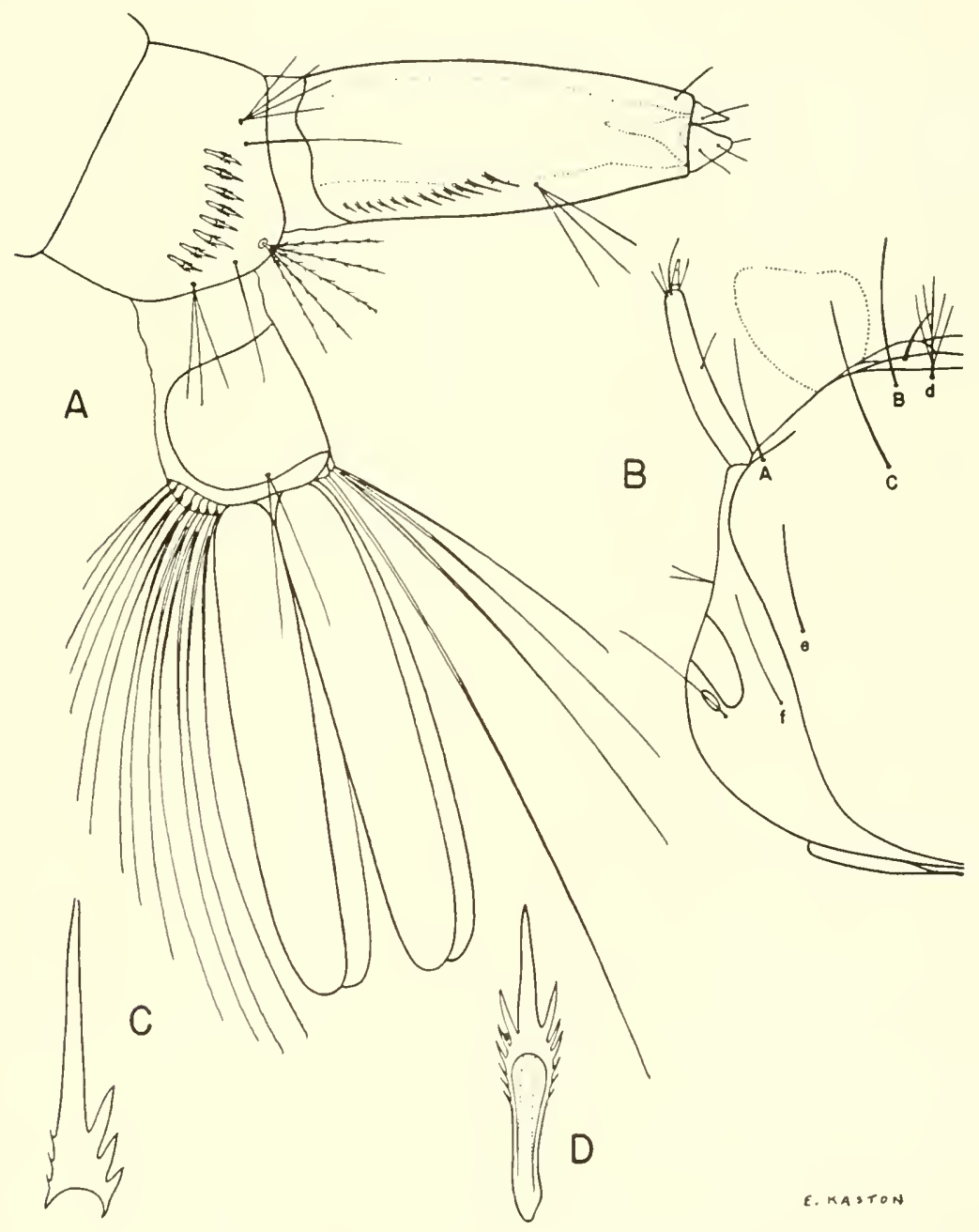

Fig. 126. Larva of Aedes aegypti (Linnaeus). A, Terminal segments.

B, Head. C, Pecten tooth. D. Comb scale. 
on segments I to V variable, usually double or triple. Comb of eighth segment of 7 to 12 thorn-shaped scales in a single curved row; individual scale with a strong apical spine and several shorter stout thorn-like lateral spines. Siphon about twice as long as wide; pecten of numerous more or less evenly spaced teeth reaching middle of siphon; subventral tuft about 3-branched, inserted beyond pecten. Anal segment about as long as wide, almost ringed by the dorsal plate; lateral hair single or double; dorsal brush consisting of a long lower caudal hair and a 2 to 4-branched upper caudal tuft on either side; ventral brush composed of about 7 to 10 long double hairs arising posterior to the dorsal plate; gills 4, each more than twice as long as the anal segment, broad, bluntly rounded at tip.

DisTRIBUTION.-Occurs throughout most of the tropical and subtropical regions of the world. Southern States: Alabama, Arkansas, Florida, Georgia, Kentucky, Louisiana, and Mississippi (52); Missouri (1); North Carolina (166); South Carolina and Tennessee (52); Virginia (49). Other States: District of Columbia (52); Indiana (76); New Mexico (9); Oklahoma (159); Texas (108).

BIONOMICS.-This species is frequently abundant and troublesome in dwellings or near human habitations, especially in towns and cities. It is closely associated with man and seems to prefer the blood of man over that of animals. The females are quite wary and more commonly bite around the ankles; they may even crawl under clothing to reach a favorable spot. Feeding takes place mostly during daylight hours or under bright lights at night. The adults are frequently found resting inside houses in clothes closets, in cupboards, behind pictures, underneath beds, and in other similar places. Specimens are rarely taken in light trap collections but are frequently found in diurnal resting shelters. They remain near human habitations and probably do not fly more than a few hundred feet from their breeding places.

The eggs are deposited almost exclusively in artificial containers around dwellings, either just above the water level or on the surface of the water. The species is able to pass the winter in the egg stage, but breeding may be continuous throughout the year in the extreme southern United States. It frequently breeds during the winter months in heated buildings throughout most of its range.

MEDICAL IMPORTANCE.-Aedes aegypti is an important vector of yellow fever and dengue. Control of this species requires frequent inspections of premises and the elimination or screening of water containers in which breeding may occur.

\section{Aedes (Aedimorphus) vexans (Meigen)}

Culex vexans Meigen, 1830, Syst. Beschr. Zweifl. Ins., 6:241.

AdUlt females.-Medium sized species. Head: Proboscis dark scaled; palpus short, dark, with fourth segment bearing a few white scales at base and tip. Broad dorsal region of occiput clothed with narrow pale yellow to goldenbrown scales and numerous dark and pale erect forked scales; lateral region of occiput covered with broad whitish or pale-yellow scales adjacent to a dark- 
scaled patch near margin of eye. Thorax: Integument of scutum brown; vestiture consisting of narrow golden-brown scales, with those of prescutellar space, postero-lateral and anterior margins paler; a pair of indefinite submedian spots or streaks of pale scales occasionally present anteriorly on scutum. Scales of posterior pronotum similar to those of scutum. Lower mesepimeral bristles absent. Abdomen: First tergite predominantly dark scaled, with a few white scales near base. Tergites I to VI dark scaled, each with a conspicuous white band basally and basal white patches laterally (basal bands usually not joining the lateral patches; mid-posterior margin of each band indented); white scales present on apical margins of the last three segments; seventh tergite mostly dark dorsally except for the apical white scaling. Venter whitish scaled, with each sternite marked medially with dark scales, often in a V-shaped patch with the base of the $\mathrm{V}$ directed anteriorly. Legs: Legs dark scaled, with inner surfaces of femora, tibiae, and often segment 1 of tarsi pale scaled; knee joints pale. Each segment of hind tarsus with a narrow basal white ring; fore- and mid-tarsi similarly marked but with the basal rings of segments 4 and 5 much reduced or lacking. $W$ ing: Scales narrow, dark.

ADULT MALE.-Coloration similar to that of female. TERminalia (Fig. 127B). Lobes of ninth tergite (IXT-L) indistinct, widely separated, only

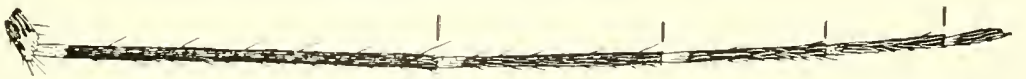

A
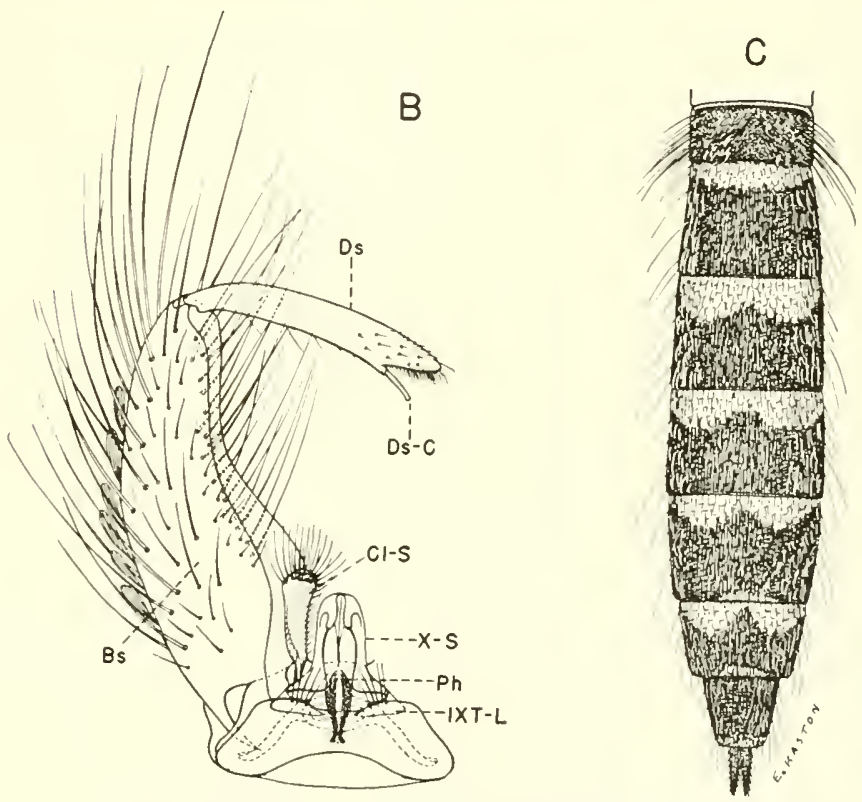

Fig. 127. Aedes vexans (Meigen). A, Hind tarsus. B, Male terminalia. C, Abdomen of female. 
slightly elevated, each bearing several weak setae. Tenth sternite (X-S) lightly sclerotized. Phallosome $(\mathrm{Ph})$ small, narrowest near base, open dorsally and ventrally, consisting of two heavily sclerotized plates bearing strong teeth apically. Claspette stem (Cl-S) stout, pilose, capitate, fused to basistyle and crowned with numerous short setae; claspette filament absent. Basistyle (Bs) about three times as long as basal width, curved, becoming narrower beyond basal third, rounded at apex, clothed with scales and long setae (setae more numerous on outer aspects and along inner ventral margin); basal and apical lobes absent. Dististyle (Ds) broad, flattened, about two-thirds as long as basistyle, with tip densely pilose and bluntly pointed; claw (Ds-C) stout, borne on a small thumb-like projection at apical fifth of dististyle.

LARva. (Fig. 128).-Head broader than long. Antenna half as long as head, curved, spinose; antennal tuft multiple, inserted near middle of shaft, nearly reaching tip. Head hairs: Preantennal (A) multiple, reaching beyond insertion of antennal tuft; lower (B) 2 to 3-branched; upper (C) 3 to 5 . branched (hairs $A, B$, and $C$ not inserted in a straight line); postclypeal (d) small, multiple; sutural (e) single; trans-sutural (f) and supraorbital single or double. Upper lateral abdominal hairs double or triple on segments I to V. Comb of eighth segment of about 9 to 12 thorn-shaped scales in an irregular single or double row; individual scale with a long apical spine and short lateral spinules. Siphon about three to three and one-half times as long as wide; pecten reaching to middle of the siphon or beyond, with the last two or three teeth somewhat longer and widely spaced; subventral tuft small, multiple, inserted beyond pecten. Anal segment longer than wide, with dorsal plate extending ventrally nearly to mid-line; lateral hair single; dorsal brush consisting of a long lower caudal hair and a shorter upper caudal tuft of 4 or 5 branches on either side; ventral brush large, with 4 or 5 smaller tufts preceding the barred area; gills 4 , longer than the segment.

DISTRIBU.TION.-A widespread species occurring throughout the Palearctic, Nearctic and Oriental regions. It is common in southern Canada and throughout most of the United States, but less abundant in the extreme South. Southern States: Alabama (170); Arkansas (30); Florida and Georgia (52); Kentucky (140); Louisiana and Mississippi (52); Missouri (1); North Carolina (166); South Carolina (64); Tennessee (170); Virginia (49). Other States: Arizona, California, Colorado and Connecticut (52); Delaware (106); District of Columbia, Idaho, Illinois and Indiana (52); Iowa (155, 156); Kansas (79); Maine (137); Maryland and Massachusetts (52); Michigan (88); Minnesota and Montana (52); Nebraska (178); New Hampshire, New Jersey, New Mexico, New York, North Dakota and Ohio (52); Oklahoma (159); Pennsylvania (8); Rhode Island (99); South Dakota (129); Texas (108); Utah (142); Vermont (90); West Virginia (52); Wisconsin (48); Wyoming (129).

BIONOMICS. - The females are troublesome biters, feeding in shady places during the day and becoming especially annoying at dusk and after dark. Adults are able to migrate long distances from their breeding places. Rees (142) found that this species has a flight range of 5 to 8 miles in Utah, while Hearle (78) claims that in British Columbia it can migrate a distance of ten miles with ease. The larvae occur in rain-filled depressions and flood waters. 
Breeding occurs throughout the year in the extreme South but is more common from May to October farther north.

MEDICAL IMPORTANCE.-Aedes vexans has been shown through laboratory experiments to transmit both the eastern and western varieties of equine encephalitis. Feemster and Getting (60) state that it is not unlikely that this species may prove to be the most important natural vector of this disease. The virus of St. Louis encephalitis has also been successfully transmitted by Aedes vexans in the laboratory (74).

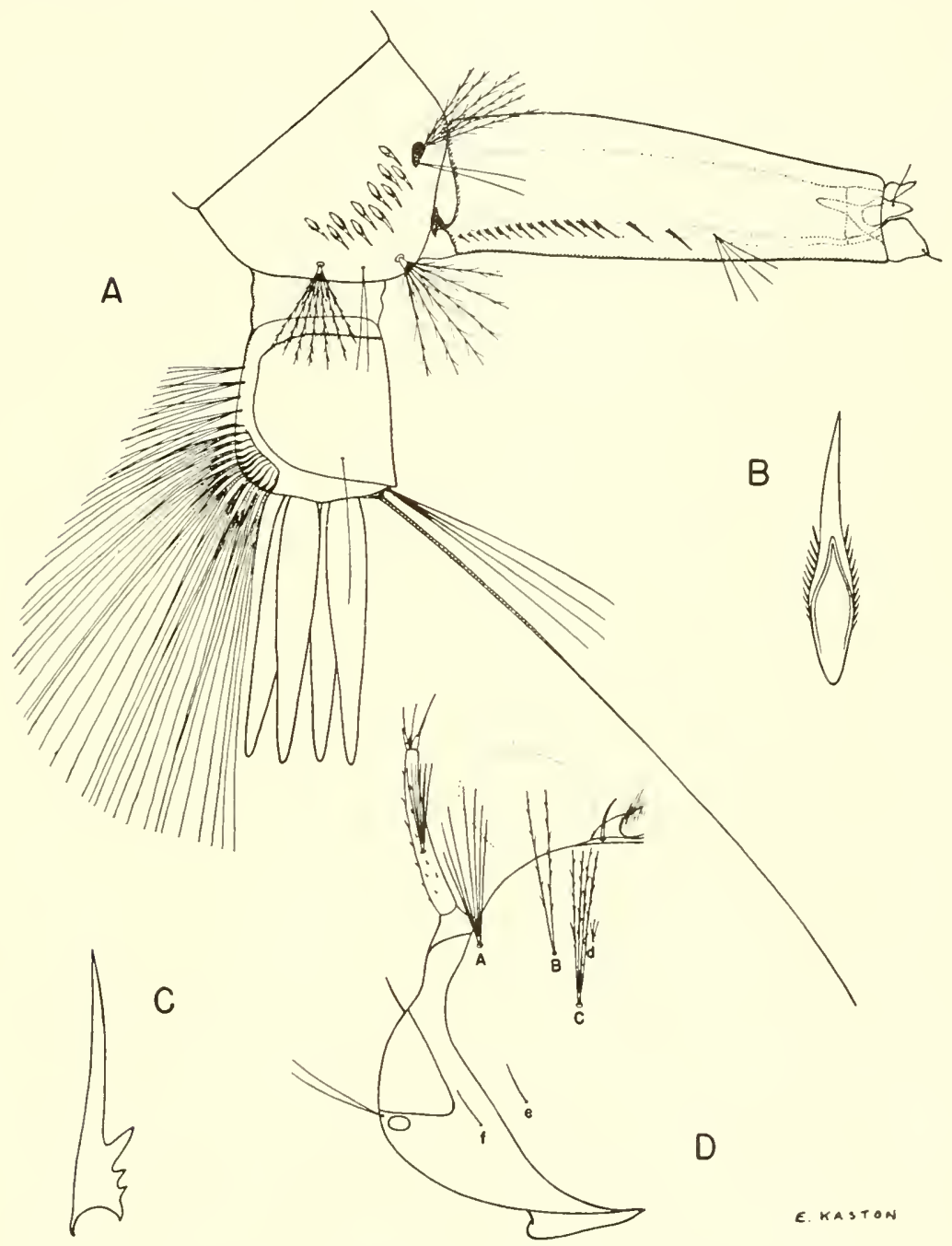

Fig. 128. Larva of Aedes vexans (Meigen). A, Terminal segments.

B, Comb scale. C, Pecten tooth. D, Head. 


\section{Aedes (Aedes) Cinereus Meigen}

Aedes cinereus Meigen, 1818, Syst. Beschr, Eur. Zweifl. Ins., 1:13.

adult female.-Medium sized to rather small species. Head: Proboscis brown scaled; palpi short, brown. Occiput dorsally with a median longitudinal stripe of narrow pale-yellow to light golden-brown scales, the stripe broader posteriorly, narrowed anteriorly and projecting forward between the eyes; median stripe bounded on either side by a large submedian patch of broad appressed brown scales, these submedian patches margined anteriorly along eye margin by a line of narrow pale scales. Lateral region of occiput clothed with broad brownish-white dingy-yellow scales. Erect forked scales numerous on dorsal surface of occiput, pale brown to brown. Thorax: Integument of scutum

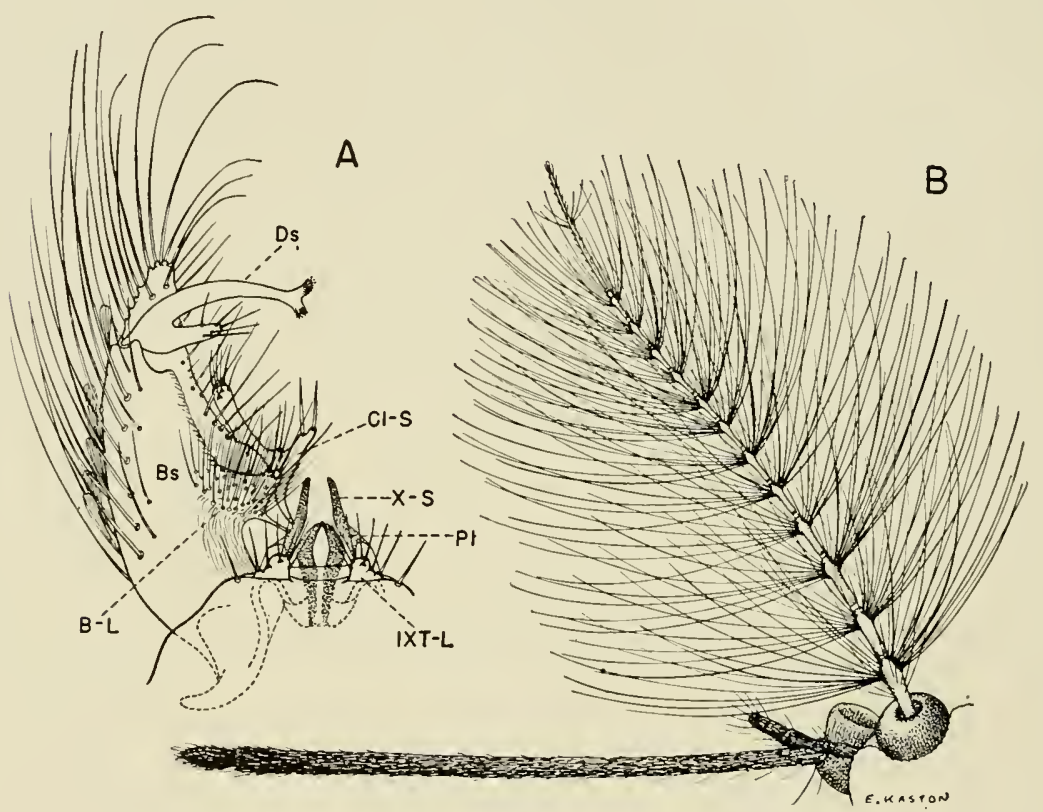

Fig. 129. Acdes cinereus Meigen. A, Male terminalia.

$B$, Head appendage of male.

reddish brown, evenly clothed with fine narrow golden-brown scales, paler on anterior margin, lateral margin above wing base, and on prescutellar space. Posterior pronotum with narrow golden-brown scales dorsally, paler ventrally. Abdomen: Tergites each with a narrow basal whitish-scaled band dorsally, abruptly broadened on either side to cover the entire lateral margin; remaining surface brown scaled. Venter whitish-yellow scaled. Legs: Legs brown scaled except for pale inner surfaces of femora and tibiae. Wing: Scales narrow: brown. 
ADUlT MALE.-Coloration similar to that of female. Palpi minute, even shorter than those of female. Antennae extremely bushy. TERminalia (Fig. 129A). Lobes of ninth tergite (IXT-L) as long as broad, rounded or truncate apically, separated by about twice the width of one lobe, each bearing numerous setae, arising from distinct tubercles. Tenth sternite (X-S) heavily sclerotized, slender, rod-like; tip rounded, no broader than shaft. Phallosome $(\mathrm{Ph})$ heavily sclerotized, closed at apex and base, open dorsally and ventrally; basal half narrow, apical half swollen, apex rounded or bluntly pointed. Claspette stem (Cl-S) unequally bifurcate; the shorter (inner) branch with one or two apical and one or two subapical spines; the longer branch generally with two apical and two to four subapical spines. Basistyle (Bs) about twice as long as basal width, shaped like a curved cone, tapered to a narrowly rounded apex bearing many long setae; lateral and ventral aspects of basistyle with scales and moderately long setae. Basal lobe (B-L) broad at base and with narrow conical apical

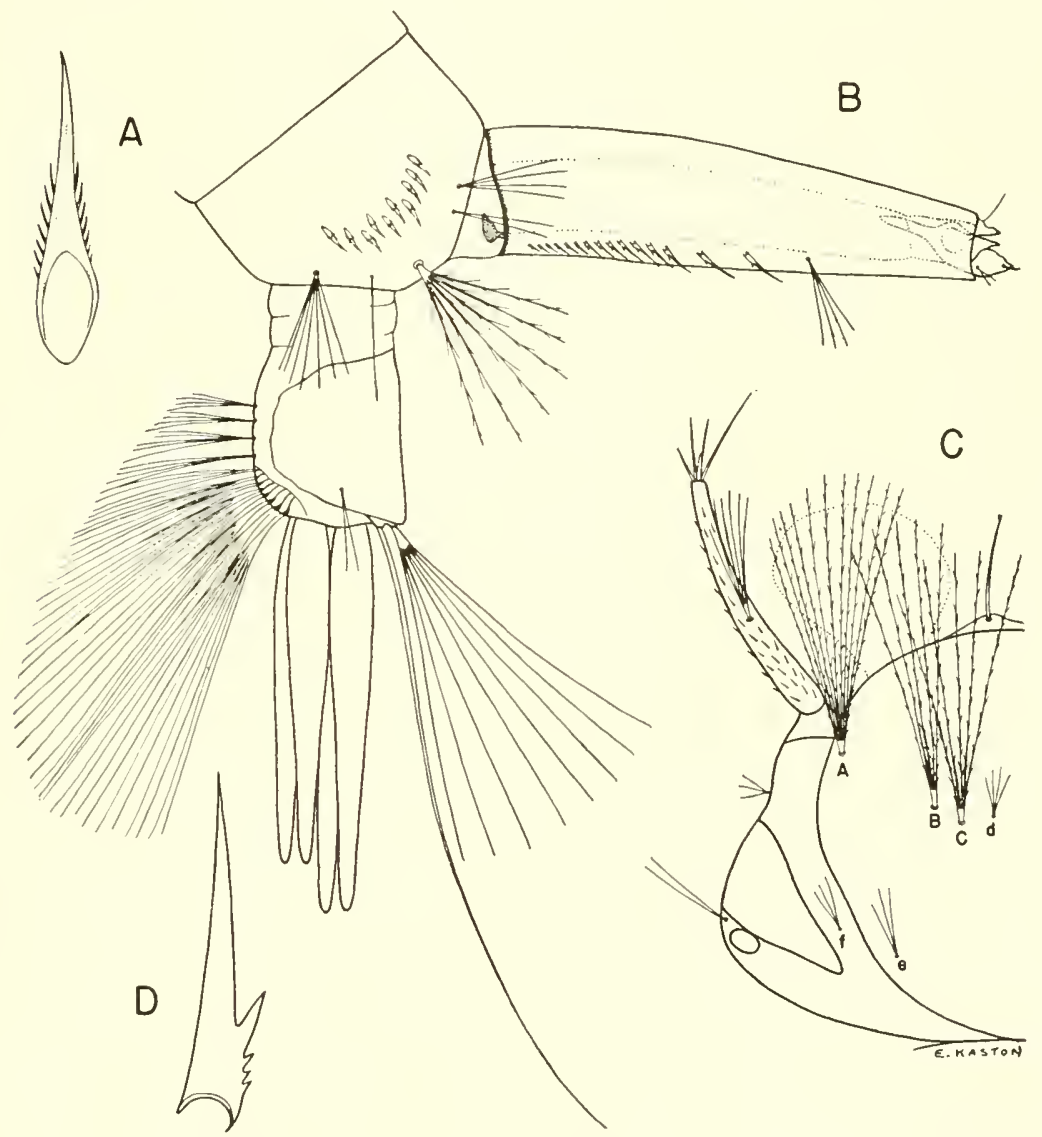

Fig. 130. Larva of Aedes cinereus Meigen. A, Comb scale.

B, Terminal segments. C, Head. D. Pecten tooth. 
portion directed mesad, densely covered with setae arising from distinct tubercles, the setae stouter on apex; apical lobe absent. Dististyle (Ds) inserted well before apex of basistyle, unequally bifurcate near base; the shortened (inner) branch bearing small setae distally; the longer branch bifurcate at apex, with each small branch blunt and bearing numerous small tooth-like spines.

LARVA. (Fig. 130).- Head broader than long. Antenna more than half as long as head, slender, slightly curved, spinose; antennal tuft multiple, inserted before middle of shaft. Head hairs: Preantennal (A), lower (B) and upper (C) long, multiple, barbed, and inserted in a straight line; postclypeal (d) small, multiple; sutural (e) and trans-sutural (f) usually triple; supraorbital usually double. Upper lateral abdominal hairs on segments I and II double, single on segments III to V. Comb of eighth segment consisting of about 10 to 14 thorn-shaped scales in a partially double row; individual scale with a strong apical spine and very fine lateral spinules. Siphon approximately four times as long as wide; pecten of about 15 teeth extending beyond middle of siphon, the last two or three teeth somewhat longer and widely spaced; subventral tuft about 4-branched, inserted beyond pecten. Anal segment longer than wide, about three-fourths ringed by the dorsal plate; lateral hair double; dorsal brush consisting of a long lower caudal hair and a shorter multiple upper caudal tuft on either side; ventral brush well developed, with several small tufts preceding the barred area; gills 4, long, narrowly lanceolate, the dorsal pair slightly longer than the ventral pair.

Distribution.--United States, Canada, Europe and Asia. Southern States: Alabama (4th Sv. C. Med. Lab. records, 1945, unpublished); Arkansas (30); Florida (35); Georgia (97); Mississippi (35); North Carolina (125); South Carolina (97); Tennessee (125). Other States: California (66); Connecticut (52); Illinois (42); Iowa $(155,156)$; Maine (103); Maryland (19); Massachusetts (52); Michigan (88); Minnesota (131); Montana (112); Nebraska (178); New Hampshire (52); New Jersey (77); New York and Oregon (52); Pennsylvania (121); Rhode Island (99); Utah (142); Washington (52); Wisconsin (48).

вiоNomics.-Adults are rarely taken in the southern states except in light trap collections; therefore very little is known about their habits in this region. Owen (131), Hearle (78) and Marshall (113) report that the fernales are troublesome biters on occasions. Larvae occur in woodland pools, unshaded temporary rain-filled pools, and occasionally in marshes. This species is apparently single-brooded, overwintering in the egg stage and hatching mostly in the late spring, although some larvae may be found any time during the summer following rains.

\section{Genus Culex Linnaeus ${ }^{1}$}

Culex Linnaeus, 1758, Syst. Nat., Ed. 10:602.

The genus Culex includes approximately 300 species, most of which are restricted to the tropical and subtropical regions of the world. The genus is

1 Consult Dyar (1928) or Edwards (1932) for synonymy. 
represented in the southern United States by comparatively few species belonging to three subgenera, Culex, Melanoconion, and Neoculex.

SALIENT CHARACTERS.-Adult: A pair of pad-like pulvilli at the bases of the tarsal claws. Tarsal claws of female without teeth. Pharyngeal bar of female distinctly toothed. Eyes contiguous above antennae. Spiraculat and postspiracular bristles absent. Squama fringed. Tip of abdomen of female bluntly rounded, the eighth segment not retracted. Male Terminalia: Phallosome formed of a pair of heavily sclerotized, variously divided plates. Tenth sternite crowned with numerous short spines or a comb-like row of teeth. Claspette absent. Subapical lobe of basistyle present and armed with modified spines and setae. Larva: Head wider than long. Comb of eighth segment usually consisting of many scales in a triangular patch. Siphon with pecten and several pairs of subventral tufts. Anal segment completely ringed by the dorsal plate.

\section{Keys to THE SPECIES}

\section{ADULT FEMALES}

1. Proboscis and tarsi ringed with white

Proboscis and tarsi dark scaled

2. White tarsal rings brozd; femora and tibiae each with a longitudinal line of white scales on outer surface; a few white scales present on costa and subcosta tarsalis Coq., p. 256

White tarsal rings very narrow; femora and tibiae dark on outer surfaces; wing scales entirely dark ..._...................... 240

3. Abdominal segments each with an apical triangular patch of white scales on either side, usually joined by a narrow dorso-apical band of similar scales apicalis Adams, p. 271

Abdominal segments with white scaling basal when present

4. Occiput dorsally with broad appressed scales, sometimes limited to a narrow border back of eyes; wing scales slightly or distinctly broadened, at least on vein 2 (Subgenus Melonoconion) ..................erraticus (D. and K.). p. 262 pilosus (D. and K.), p. 268 peccator D. and K., p. 265 atratus Theob., p. 260

Occiput dorsally without broad, appressed scales; wing scales narrow ................... 5

5. Abdominal segments each with a broad basal band of whitish scales dorsally ….... 6

Abdominal segments without broad basal whitish bands dorsally; pale scaling, if present, in very narrow bands or restricted to lateral angles

6. Abdominal bands broadly rounded on posterior margins, constricted laterally, rather narrowly joining or entirely disconnected from the lateral patches: scales of scutum somewhat coarse, golden; scutum lacking a pair of smal] pale-scaled submedian spots near middle quinquefasciatus Say, p. 247

pipiens Linn., p. 245

Abdominal bands with posterior borders nearly straight across, broadly joining the lateral patches (particularly on segments 3-5): scales of scutum fine, golden-brown; scutum usually with a pair of small pale-scaled submedian spots near middle restuans Theob., p.

7. Lateral basal patches of abdomen dingy-yellow, rather poorly defined; abdominal segment usually with very narrow dingy-yellow basal bands dorsally, and with the apices of the segments more or less blended with yellowish scales, VII either primarily or entirely clothed with dingy-yellow scales; scales of scutum golden brown; pleura with several groups of broad pale scales, each group usually comprised of more than 6 scales

\section{(n)}


Lateral basal patches of abdomen white, sharply defined; abdominal segments entirely dark dorsally (rarely with very narrow white basal bands), VII entirely dark scaled except for white lateral patches; scales of scutum dark brown; pleura with few or no scales (if scales present, rarely more than 5 or 6 in any single group) nigripalpus Theob., p. 242

\section{MALE TERMINALIA}

1. 10th sternite crowned apically with a comb-like row of blunt teeth 2

10th sternite crowned apically with a dense tuft of short spines

2. Subapical lobe of basistyle not divided into two main trunks; lateral plates of phallosome columnar, blunt and denticulate at apices, connected at apical fourth by a narrow transverse bridge (Subgenus Neoculex)

apicalis Adams, p. 272

Subapical lobe of basistyle deeply divided into two main trunks; lateral plates of phallosome not columnar, without a narrow subapical connecting bridge (Subgenus Melanoconion)

3. Lobes of 9th iergite slender, finger-like, widely separated, the tips slightly convergent pilosus (D. and K.), p. 268

Lobes of 9 th tergite inflated, the bases contiguous or separated by no more than the width of one lobe

4. Basistyle very large, subspherical; dististyle quadrately expanded apically peccator D. and K., p. 265

Basistyle moderate in size, only about half as broad as long; dististyle not quadrately expanded apically

5. Lobes of 9 th tergite large, ovate, contiguous at bases, widely divergent apically, clothed on all aspects with numerous long smooth setae

Lebes of 9ih tergite large, pyriform, separated basally by nearly the width of one lobe, divergent apically, clothed on basal half with numerous long feathered and smooth setae, the apical halves bare and tapered

atratus Theob., p. 260

6. Subapical lobe arising near middle of basistyle, stout, thumb-like, about twice as long as wide; apical armature consisting of three stout rods, shorter than the lobe bahamensis D. and K., p. 240

Subapical lobe arising near apical third of basistyle, little if any longer than wide; apical armature consisting of a variable number of appendages, all longer than the lobe

7. Subapical lobe with 8 appendages, one of which is a broadly expanded leaflike filament; basal arm of 10 th sternite represented by a short protuberance, never long and curved

ubapical lobe with 5 or 6 appendages, one of which may or may not be a broadly expanded leaf-likê filament; basal arm of 10th sternite long, curved ...... 9

8. Ventral arm of phallosome large, weakly sclerotized, broadly wing-like, curved outwardly, tapered to a point; dorsal arm of phallosome long, slender, straight, pointed or narrowly rounded at tip, directed posteriorly and crossing over the ventral arm nearly at right angle to its wing-like outward extension quinquefasciatus Say, p. 247

Ventral arm of phallosome large, moderately sclerotized, narrowly wing-like, curved outwardly, tapered to a point; dorsal arm of phallosome long, slender, straight, truncate or bluntly rounded at tip, directed postero-laterally and obliquely crossing over the wing-like lateral extension of the ventral arm........ pipiens Linn., p. 245

9. Leaf-like filament of subapical lobe narrow; crown of 10 th sternite with outer spines blunt tarsalis Coq., p. 257

Leaf-like filament of subapical lobe very broad; crown of 10th sternite either with all spines pointed or with the outer spines blunt (1) 
Each plate of phallosome with a single short triangular tooth between the dorsal and ventral arms; crown of 10 th sternite with all spines pointed; basal arm of 10 th sternite weakly sclerotized restuans Theob., p. 250

11. Dorsal arm of phallosome bent medially at right angle; crown of 10th sternite with all spines pointed; lobes of 9 th tergite widely separated, only slightly raised; apical margin of 8 th tergite bearing numerous, rather long slender setae (longer than those on lobes of 9th tergite) ...............salinarius Coq., p. 253

Dorsal arm of phallosome nearly straight; crown of 10 th sternite with outer spines blunt; lobes of 9th tergite separated by no more than the width of one lobe, prominent; apical margin of 8 th tergite bearing numerous short stout setae (shorter than those on lobes of 9 th tergite) ...........nigripalpus Theob., p. 243

\section{LARVAE (FOURTH INSTAR)}

1. Siphon spinose; anal gills 2, thick, bulbous; pect=n teeth progressively more widely spaced ................................................. 242

Siphon not spinose; anal gills 4, normal; pecten teeth usually evenly spaced .......... 2

2. Antenna of nearly uniform shape, with antennal tuft inserted near middle of shaft: siphon with 4 to 6 long single irregularly placed hairs and a pair of small 2 to 3 -branched subapical tufts restuans Theob., p. 251

Antennal tuft placed in a distinct constriction at outer third of shaft, the portion beyond the tuft more slender; siphon with hair tufts multiple (occasionally double or single), not irregularly placed although the subapical pair may be laterally out of line

3. Both lower and upper head hairs (B and C) multiple, long .............................. 4

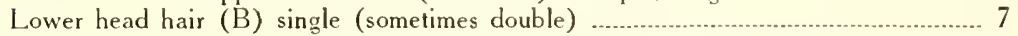

4. Siphon 4 or $5: 1$; lower head hair (B) usually with 5 or more branches ............... 5 Siphon 6 or $7: 1$; lower head hair (B) usually with 3 or 4 branches _................. 6

5. Siphon with subventral tufts in a straight line .............................tarsalis Coq., p. 259 Siphon with subapical subventral tuft laterally out of line.

quinquefasciatus Say, p. 248 pipiens Linn., p. 246

6. Thorax spicular; lateral hair of anal segment usually single

nigripalpus Theob., p. 244

Thorax glabrous; lateral hair of anal segment usually double ..salinarius Coq.. p. 254

7. Pecten teeth each with I to 4 long coarse side teeth; upper head hair (C) usually single, occasionally double, nearly as stout and about two-thirds as long as the lower head hair (B); siphon very long and slender, about $7: 1$ apicalis Adams, p. 272

Pecten teeth each fringed on one side nearly to tip; upper head hair (C) single. double, or multiple, much shoster and weaker than the lower head hair

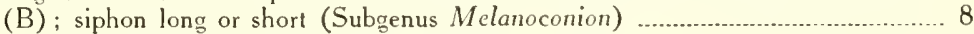

8. Comb scales long, thorn-shaped, arranged in a curved or irregular single or double row

Comb scales rounded apically and fringed with subequal spinules, arranged in a triangular pat:h

9. Siphon short, 3 or $4: 1$, with 8 pairs of very long subventral tufts, the proximal pairs about as long as siphon; upper head hair (C) single, occasicn ?lly double, about half as long as the lower head hair (B) and much weaker pilosus (D. and K.), p. 269

Siphon long and slender, 6 or $7: 1$, with 5 or 6 pairs of subventral tufts, none of which are more than one-third the length of the siphon: upper head hair (C) $t$ or more branched, very short, weak ….......erraticus (D. and K.), p. 263

10. Upper head hair (C) short, weak, 5 or 6 -branched; siphon about 7 or $8: 1$ atratus Theob., p. 261

Upper head hair (C) short, weak, 2 or 3-branched, occasionally single: siphon about $6: 1$ peccator D. and K., p. 266 


\section{Culex (Culex) bahamensis Dyar and Knab}

Culex bahamensis Dyar and Knab, 1906, Jour. N. Y. Ent. Soc., 14:210.

adult female.-Medium-sized species. Head: Proboscis dark scaled, with a rather broad white band medially; palpi short, dark, pale scaled at tips. Occiput clothed dorsally with pale-gold lanceolate scales and dark erect forked scales; clothed laterally with broad white scales. Thorax: Integument of scutum brown; scutal scales narrow, curved, golden-brown to brown. Pleura with a few broad white scales, particularly on sternopleura. Abdomen: Tergites darkbrown scaled, each with a basal band of white scales; sternites predominantly pale scaled. Legs: Each leg dark scaled except for the following markings: Pale inner surface of femur; white line on inner side of tibia and often on inner side of first tarsal segment; narrow white ring at tip of tibia; a very narrow basal and apical white ring on each segment of tarsus (apical rings may be absent on tarsi of fore and middle legs). Wing: Scales narrow, dark.

ADULT MALE.-Coloration similar to that of female. TERMinalia (Fig. $131 \mathrm{C})$. Lobes of ninth tergite (IXT-L) only slightly raised, each bearing
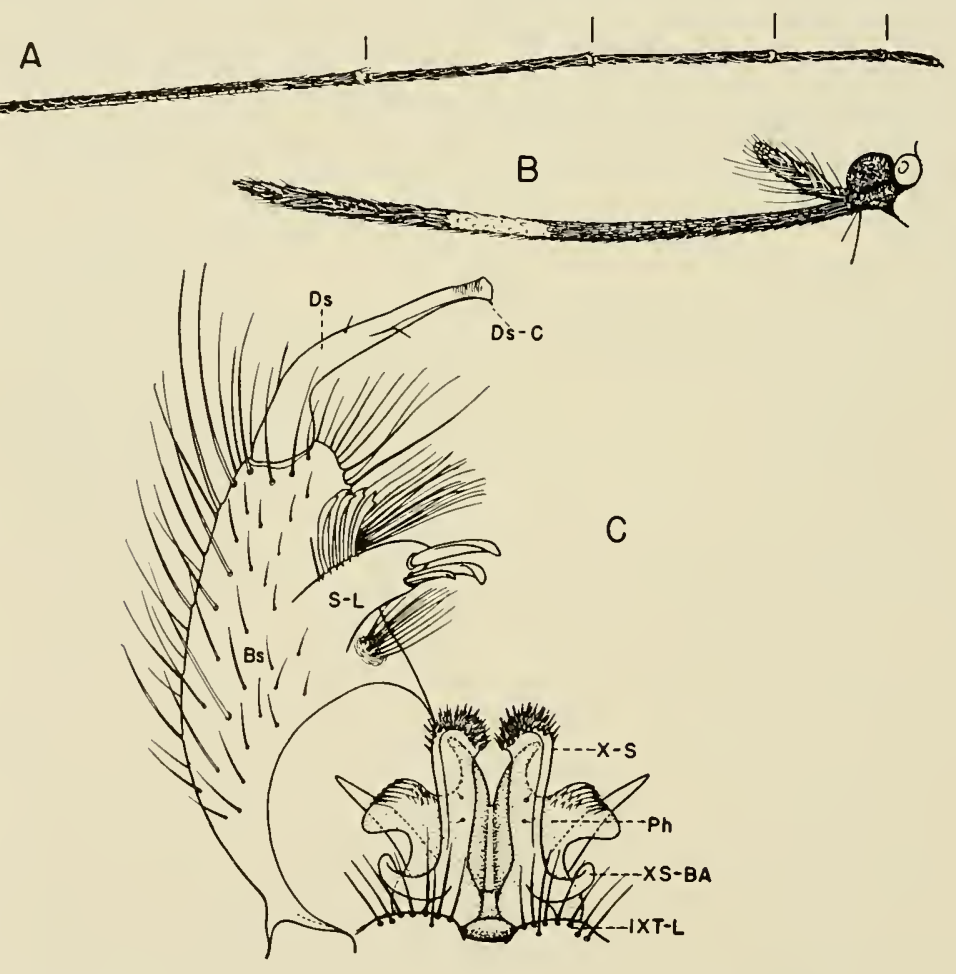

Fig. 131. Culex bahamensis Dyar and Knab. A, Hind tarsus. B, palp and proboscis of female. C. Male terminalia. 
several short setae. Tent'́ sternite (X-S) crowned with numerous short, pointed spines; basal arm (B-A) stout, slightly curved. Phallosome (Ph) formed of two plates, each with a slender outwardly-curved apical process and a large hatchet-shaped lateral lobe; apical portion of lateral lobe laminate, being overlaid by numerous flattened appressed teeth. Claspette absent. Basistvle (Bs) conical, about twice as long as basal width, clothed with numerous setae (longer ventrally and apically); two dense patches of moderately long setae present on inner surface, one patch arising near base of subapical lobe and the other just posterior to subapical lobe. Subapical lobe (S-L) arising near middle of basistyle, stout, thumb-like, a little more than twice as long as wide, bearing two long and one short, slightly flattened rods on apex (the longest rod shorter than the subapical lobe) and a row of several flattened setae on posterior side. Dististyle (Ds) about two-thirds as long as basistyle, broad at base, becoming more slender and bending sharply at basal third, gradually

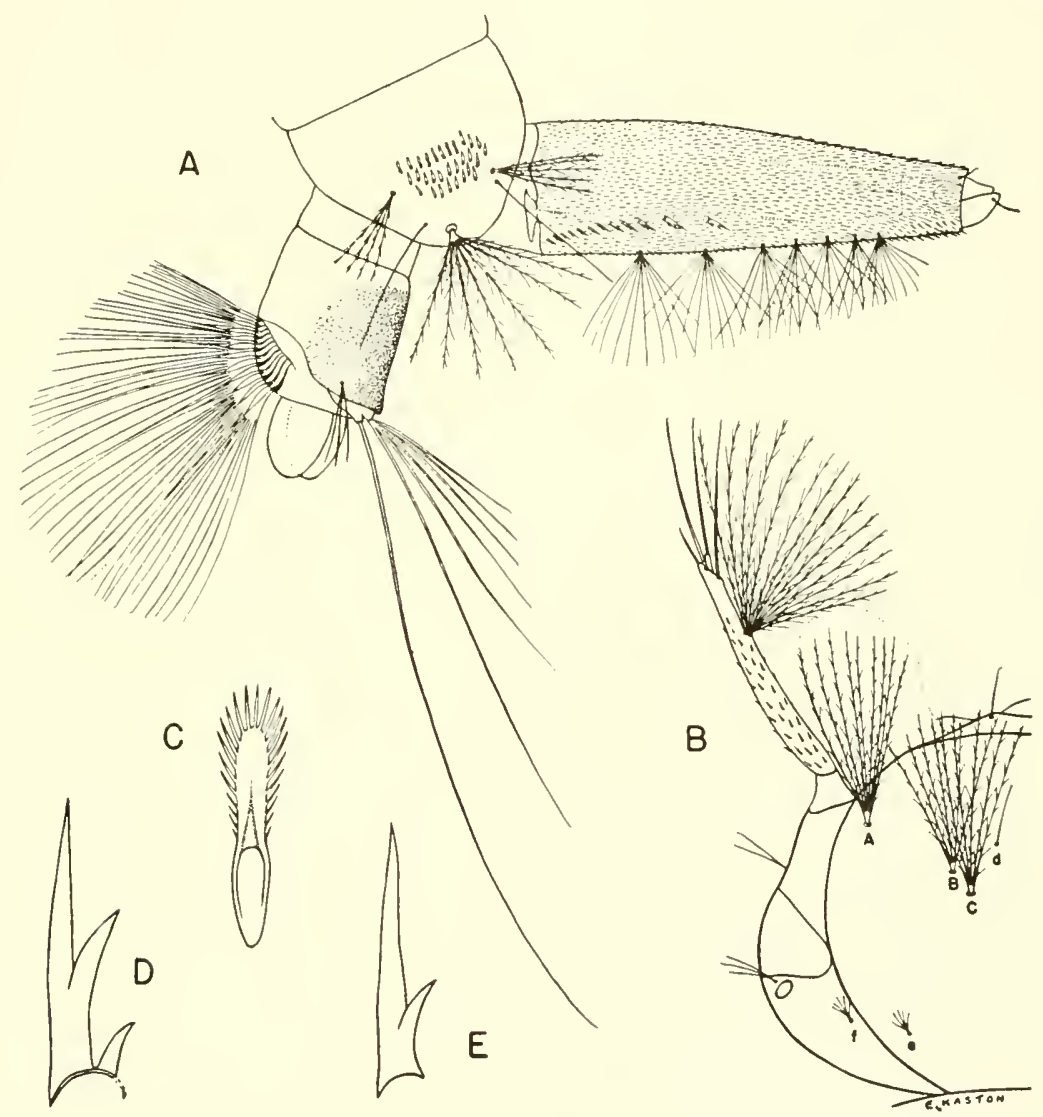

Fig. 132. Larva of Culex bahamensis Dyar and Knab. A, Terminal segments. B, Head. C, Comb scale. D, and E. Pecten teeth. 
tapering distally, finely crenulate near apex, truncate at tip; terminal claw (DsC) minute, blunt, inserted slightly before apex of dististyle.

LARva. (Fig. 132).- Head broader than long. Antenna shorter than head, constricted beyond antennal tuft, with portion before constriction spinose, portion beyond constriction more sparsely spined; antennal tuft large, multiple, barbed, inserted at outer third of shaft, extending beyond tip. Head hairs: Preantennal (A) multiple, barbed, reaching nearly to insertion of antennal tuft; lower (B) and upper (C) multiple, barbed, about as long as preantennal tuft (A); postclypeal (d) single, rather long; sutural (e), trans-sutural (f), and supraorbital small, multiple. Body finely and sparsely spicular. Lateral abdominal hairs double or triple on segments III to VI. Comb of eighth segment of numerous scales in a patch; individual scale rounded apically and fringed with subequal spinules. Siphon four to five times as long as basal width, finely and densely spinose basally, growing progressively more coarsely spined toward apex; pecten of numerous teeth progressively more widely spaced, extending slightly beyond basal third of siphon; individual pecten tooth with coarse teeth on one side; six to seven multiple, smooth subventral tufts present, two or three of the basal pairs inserted within the pecten and with branches little or no longer than the diameter of siphon. Anal segment slightly longer than wide, completely ringed by the dorsal plate; pigmentation of dorsal plate darker dorsally; lateral hair double or triple; dorsal brush consisting of a long lower caudal hair and a shorter 6-branched upper caudal tuft on either side; ventral brush large, restricted to the barred area; gills 2, short, bulbous.

Distribution.-Florida (63); Puerto Rico (136); Bahamas and Virgin Islands.

BIONOMICS.-This species was collected by Fisk (63) from several breeding places, including a cistern containing brackish water at Key West, Florida. Additional recent collections are from Elliotts Key (96) and Boca Chica Key (4th Sv. C. Med. Lab. reports, 1942-1944, unpublished).

\section{Culex (Culex) Nigripalpus Theobald}

Culex nigripalpus Theobald, 1901, Mon. Culic., 2:322.

AdUlt female.-Medium sized species. Head: Proboscis dark scaled, usually paler on under side of basal half; palpi short, dark. Broad dorsal region of occiput clothed with narrow pale golden-brown scales and dark erect forked scales; lateral region with a patch of broad pale scales, usually dingy white. Thorax: Integument of scutum brown, covered with fine dark bronzy-brown scales. Pleura with few or no scales; if scales present, rarely more than five or six in any single group. Abdomen: Tergites dark-brown to black scaled dorsally, usually with bronze to metallic blue-green reflection; sharply defined basal white-scaled patches present laterally (occasionally narrow white basal bands dorsally on some segments). Venter pale scaled. Legs: All legs dark scaled with bronze to metallic blue-green reflection, except for whitish 
inner surfaces of femora (hind tibiae frequently streaked with white on inner surfaces and with a few pale scales at apices). Wing: Scales narrow, dark.

ADULT MALE. - Coloration similar to thai of female, but with white basal bands on dorsum of some abdominal segments. TERminalia (Fig. 133). Apical margin of eighth tergite (VIII-T) bearing numerous short stout setae (shorter than those of the lobes of the ninth tergite). Lobes of the ninth tergite (IXTL) prominent, separated by no more than the width of one lobe, each bearing numerous setae. Tenth sternite (X-S) crowned with numerous short spines, the apical ones pointed, the outer ones blunt; basal arm (XS-BA) long, stout, strongly curved, heavily sclerotized. Phallosome (Ph) formed of two large heavily sclerotized plates. Each plate basally with a long stout pointed dorsal arm ( $\mathrm{Ph}$.DA) (not bent at right angles as in C. salinarius) and a slender pointed process nearly as large as the dorsal arm; each plate apically with a curved stout bluntly pointed ventral arm ( $\mathrm{Ph}-\mathrm{VA}$ ), finely denticulate on its middle portion; between the dorsal and ventral arms arise four slender teeth $(\mathrm{Ph}-\mathrm{T})$, the apical one appressed to the ventral arm. Claspette absent. Basistyle (Bs) about two and one-half times as long as basal width, with outer

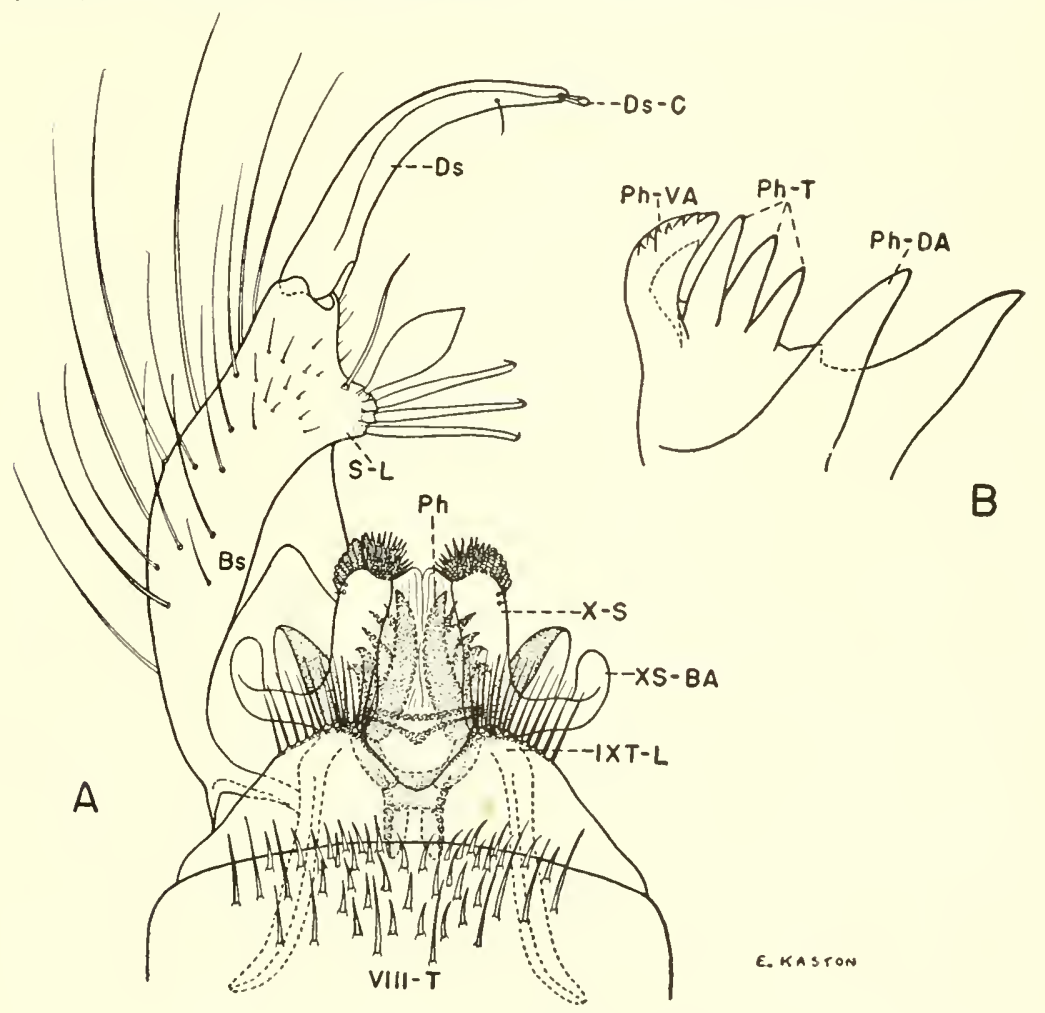

Fig. 133. Culex nigripalpus Theobald. A. Male terminalia. B. Plate of phallosome (flattened). 
margin curved, portion beyond subapical lobe more slender, and apex bluntly rounded; vestiture of numerous setae, longer on outer aspects. Subapical lobe (S-L) prominent, undivided, bearing three long stout pointed rods hooked at tips, a large broad leaf-like filament and a stout seta. Dististyle (Ds) about half as long as basistyle, curved, tapered beyond middle, bluntly pointed at tip; terminal claw (Ds-C) short, blunt.

LARv.A. (Fig. 134).-Head broader than long. Antenna shorter than head, constricted beyond antennal tuft, with portion before constriction spinose, portion beyond constriction darker and with few spines; antennal tuft large, multiple, barbed, inserted at outer third, reaching beyond tip of antenna. Head hairs: Preantennal (A) multiple, barbed, extending near insertion of antennal tuft; lower (B) and upper (C) usually 3-branched, barbed, extending beyond preclypeus; postclypeal (d) single, rather long; sutural (e) and trans-sutural (f) double or multiple; supraorbital single or double. Thorax clothed with fine spicules. Upper lateral abdominal hairs usually 3 to 4-branched on segments I and II, usually double on III to VI. Comb of eighth segment of numerous scales in a patch; individual scale somewhat rounded apically, fringed with

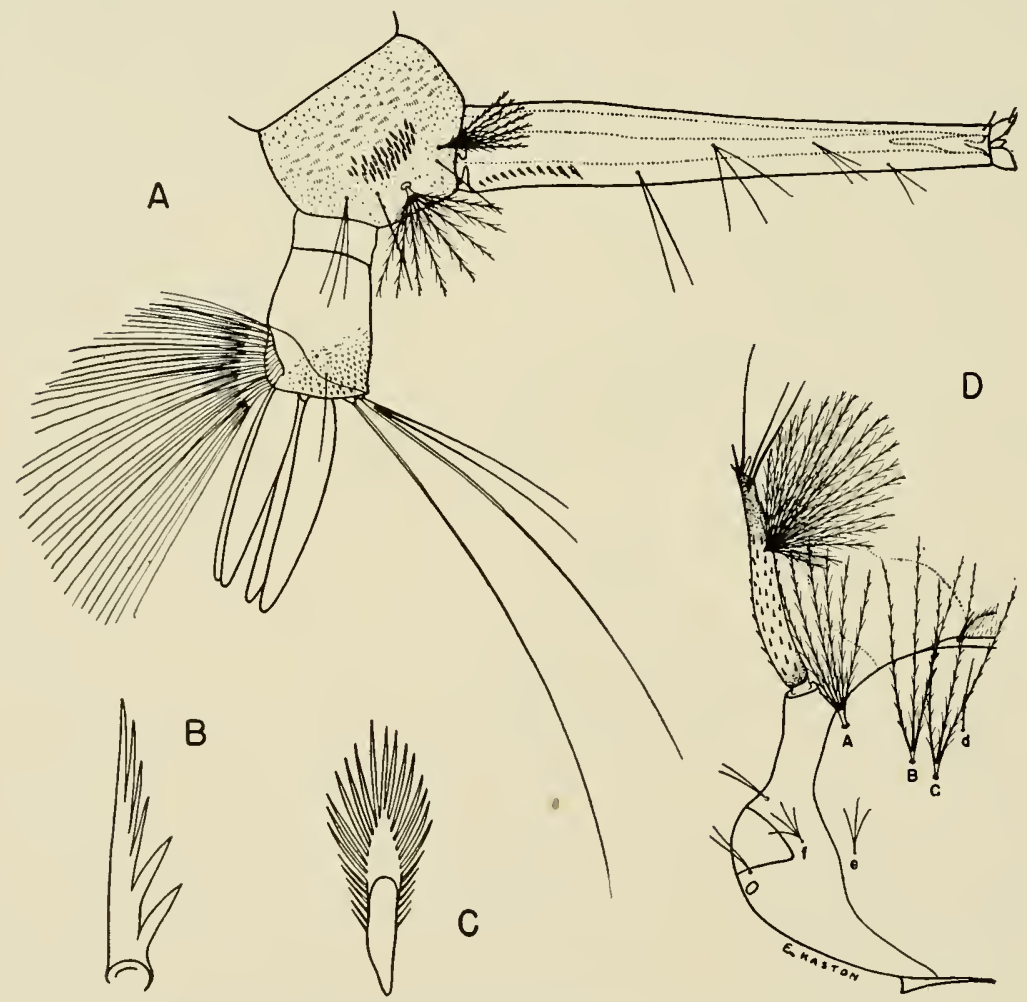

Fig. 134. Larva of Culex nigripalpus Theobald. A, Terminal segments.

B, Pecten tooth. C, Comb scale. D, Head. 
subequal spinules. Siphon slender, six to seven times as long as basal width; pecten of about 10 to 15 rather evenly spaced teeth on basal fourth of siphon; individual tooth with 2 to 5 coarse teeth on one side; paired subventral tufts usually four in number and inserted beyond pecten (proximal tuft as long or longer than basal width of siphon and usually double or single; second and third tufts usually double or triple and inserted somewhat laterally; distal tuft small, 1 to 3 -branched). Anal segment longer than wide, completely ringed by dorsal plate; lateral hair usually single, sometimes double; dorsal brush consisting of a long lower caudal hair and a shorter 2 to 3-branched upper caudal tuft on either side; ventral brush well-developed, confined to the barred area; gills 4 , as long or longer than the segment, each tapering to a blunt point.

Distribution.-Southern United States, southward to Brazil. Southern States: Alabama and Florida (96); Georgia (148); Louisiana (96); Mississippi (125); North Carolina (35); South Carolina (64); Tennessee (125).

BIONOMICS.-The adults are frequently taken in biting collections in Florida and southern Georgia, but are less inclined to bite than $C$. salinarius. The larvae occur in ditches, grassy pools and marshes of a semipermanent or permanent nature. Larvae and adults occur throughout the year in the extreme South (4th Sv. C. Med. Lab. records, 1942-1944, unpublished).

\section{Culex (Culex) pipiens Linnaeus ${ }^{1}$}

Culex pipiens Linnaeus, 1758, Syst. Nat., Ed., 10:602.

ADUlt FEMALE.-Very similar to $C$. quinquefasciatus, but usually with abdominal bands more broadly joined to the lateral basal white patches.

ADULT MALE.-Coloration similar to that of female, but with basal bands of abdomen more broadly joined to the lateral basal patches and not roun.ted on posterior margins. TERMINALIA (Fig. 135). Lobes of winth tergite (IXTL) widely separated, only slightly raised, each bearing numerous setae. Tenth sternite (X-S) crowned with numerous short pointed spines; basal arm (XSBA) variable in length, but usually represented by a short protuberance. Phallosome $(\mathrm{Ph})$ formed of two large sclerotized plates connected at base. Ventral arm (Ph-VA) of each plate large, wing-like (narrower and more heavily sclerotized than in C. quinquefasciatus), curved outwardly, tapered to a point (a much smaller pointed process, similarly curved, present laterally near middle of plate on same plane as ventral arm); dorsal arm (Ph-DA) of each plate long, slender, straight, truncate or bluntly rounded at tip (rarely pointed), directed postero-laterally and obliquely crossing over the wing-like lateral extension of the ventral arm. Claspette absent. Basistyle (Bs) nearly two and onehalf times as long as basal width, with outer margin curved, portion beyond subapical lobe more slender, and apex bluntly rounded; vestiture of numerous setae, longer on outer aspects. Subapical lobe (S-L) prominent, undivided, with armature (in order from anterior margin) as follows: Two long stout rods and one long more slender rod, each pointed and usually slightly hooked

1 Consult Dyar (1928) and Edwards (1932) for synonymy of this species. 
at tip; two stout setae with tips somewhat recurved; a stout rod, about twothirds as long as the first three rods, often with tip minutely hooked; a large broad leaf-like filament; and a stout straight seta. Dististyle (Ds) about half as long as basistyle, curved, gradually tapered beyond middle, bluntly pointed at tip; claw (Ds-C) short, blunt.

LARVA.- The larva appears to be identical with that of Culex quinquefasciatus (Fig. 137).

Distribution.--United States from the Atlantic to the Pacific, except in the extreme South; Canada; northern Europe and Asia; southern portion of South America and East and South Africa. Southern States: Alabama and Georgia (97); Kentucky (140); Mississippi (186); Missouri (1); North Carolina, South Carolina and Tennessee (97); Virginia (49). Other Statcs: California (66); Connecticut (52); Delaware (106); District of Columbia (52); Illinois (42); Indiana (76); Iowa (155, 156); Kansas (79); Maine (90); Maryland (19); Massachusetts (90); Michigan (88); Minnesota (131); Nebraska (178); New Hampshire (90); New Jersey (77); New York (162); Ohio (52); Oregon (44); Pennsylvania (8); Rhode Island (99); Utah (142); Washington (52); Wisconsin (48).

BIONOMics.-Culex pipiens is a domesticated species which commonly in-

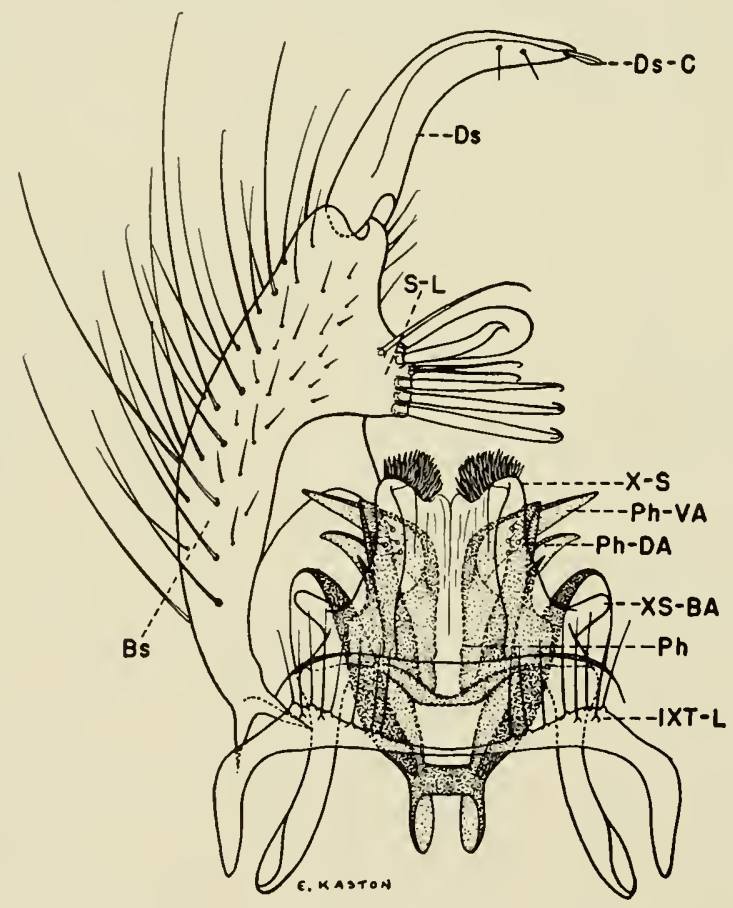

Fig. 135. Male terminalia of Culex pipiens Linnaeus. 
fests houses and bites at night. It is a serious pest throughout most of its range. Larvae occur in rainbarrels, tubs, catch basins, cesspools, ditches, and similar habitats, particularly in urban areas.

MEDICAL IMPORTANCE.-Culex pipiens is a known intermediate host of Wuchereria bancrofti in China, Japan, and Egypt. It is a proven vector of western equine and St. Louis encephalitis viruses in Washington (145) and is known to transmit the organisms causing bird malaria. Heartworm of dogs (Dirofilaria immitis) is also transmitted by this species.

\section{Culex (Culex) quinquefasciatus Say 1}

Culex quinquefasciatus Say, 1823, Jour. Acad. Nat. Sci. Phil., 3:10.

Culex fatigans Wiedmann, 1828, Auss. Zweifl, 1:10.

ADUlt FeMALE.-Medium sized species. Head: Proboscis dark scaled; palpi short, dark. Occiput clothed dorsally with narrow golden scales and numerous erect forked scales (forked scales of central portion usually pale, others dark brown); scales of lateral region of occiput broad, whitish. Thorax: Integument of scutum brown, covered with golden lanceolate scales (coarser than on $C$. restuans, salinarius and nigripalpus), paler on prescutellar space. Abdomen: Tergites dark scaled with bronze to metallic blue-green reflection, except for conspicuous transverse basal bands and basal lateral patches of white scales; each band broadly rounded on posterior margin, constricted laterally, narrowly joining or entirely disconnected from the lateral patches. Venter predominantly whitish scaled, usually speckled with a few brown scales. Legs: Legs dark scaled with bronze to metallic blue-green reflection, except for pale inner surfaces of femora and a few pale scales usually present at tips of tibiae. Wing: Scales narrow, dark.

ADULT MALE.-Coloration similar to that of female, but with basal bands of abdomen broadly joined to the lateral basal patches and not rounded on posterior margins. TERMINALIA (Fig. 136). Lobes of ninth tergite (IXT-L) widely separated, only slightly raised, each bearing numerous setae. Tenth sternite (X-S) crowned with numerous short pointed spines; basal arm (XSBA) variable in length, but usually represented by a short protuberance. Phallosome $(\mathrm{Ph})$ formed of two large moderately sclerotized plates connected at base. Ventral arm (Ph-VA) of each plate large, wing-like (broader and more weakly sclerotized than in C. pipiens), curved outwardly, tapered to a point (a much smaller pointed process, similarly curved, present laterally near middle of plate on same plane as ventral arm); dorsal arm (Ph-DA) of each plate long, slender, straight, pointed or narrowly rounded at tip, directed posteriorly and crossing over the ventral arm nearly at right angle to its wing-like outward extension. Claspette absent. Basistyle (Bs) nearly two and one-half times as long as basal width, with outer margin curved, portion beyond sub. apical lobe more slender, and apex bluntly rounded; vestiture of numerous setae, longer on outer aspects. Subapical lobe (S-L) prominent, undivided, with armature (in order from anterior margin) as follows: two long stout rods

1 Consult Dyar (1928) and Edwards (1932) for additional synonyms of this species. 
and one long more slender rod, each pointed and usually slightly hooked at tip; two stout setae with tips somewhat recurved; a stout rod, about two-thirds as long as the first three rods, often with tip minutely hooked; a large broad leaf-like filament; and a stout straight seta. Dististyle (Ds) about half as long as basistyle, curved, gradually tapered beyond middle, bluntly pointed at tip; claw (Ds-C) short, blunt.

LARVA. (Fig. 137).-Head broader than long. Antenna shorter than head, stout, constricted beyond antennal tuft, with portion before constriction spinose, portion beyond constriction sparsely spined; antennal tuft large, multiple, barbed, inserted at outer third of shaft, reaching beyond tip. Head hairs: Preantennal (A) multiple, barbed, reaching to insertion of antennal tuft; lower (B) and upper (C) usually five or more-branched, extending beyond preclypeus; postclypeal (d) single; sutural (e) usually double; trans-sutural (f) usually triple; supraorbital single or double. Body glabrous. Upper lateral abdominal hairs multiple on segments I and II, double on III to VI. Comb of eighth segment of many scales in a patch; individual scale rounded apically and fringed with subequal spinules. Siphon about four times as long as

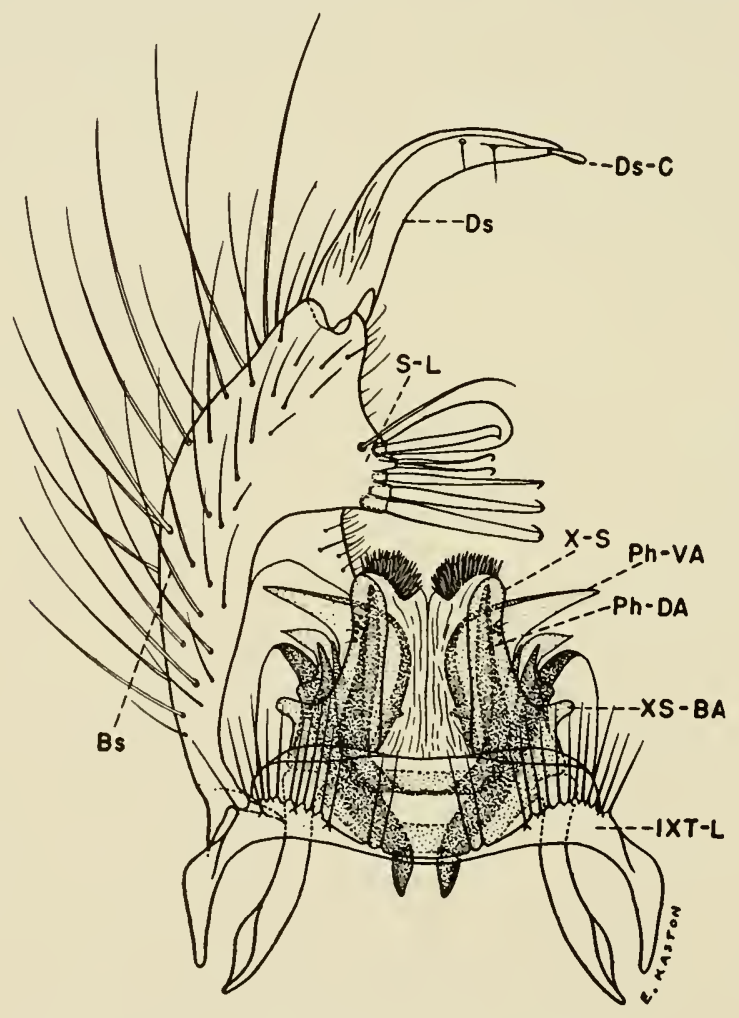

Fig. 136. Male terminalia of Culex quinquefasciatus Say. 
basal width, the basal two-thirds stout, outer third tapered; pecten of about 8 to 12 rather evenly spaced teeth on basal third of siphon; individual tooth with 1 to 5 coarse teeth on one side; four or five pairs of subventral tufts inserted beyond pecten (the subapical tufts inserted laterally; the apical and subapical tufts usually double or triple; proximal tufts multiple, slightly barbed). Anal segment longer than wide, completely ringed by the dorsal plate; lateral hair long, single; dorsal brush consisting of a long lower caudal hair and a shorter 2-branched upper caudal tuft on either side; ventral brush well-developed, confined to the barred area; gills 4 , as long as the segment, each tapering to a blunt point.

DisTRIBUTION.-Occurs throughout the tropical and subtropical regions of the world. Southern States: Alabama (170): Arkansas (30); Florida and Georgia (96); Kentucky (140); Louisiana and Mississippi (96); Missouri

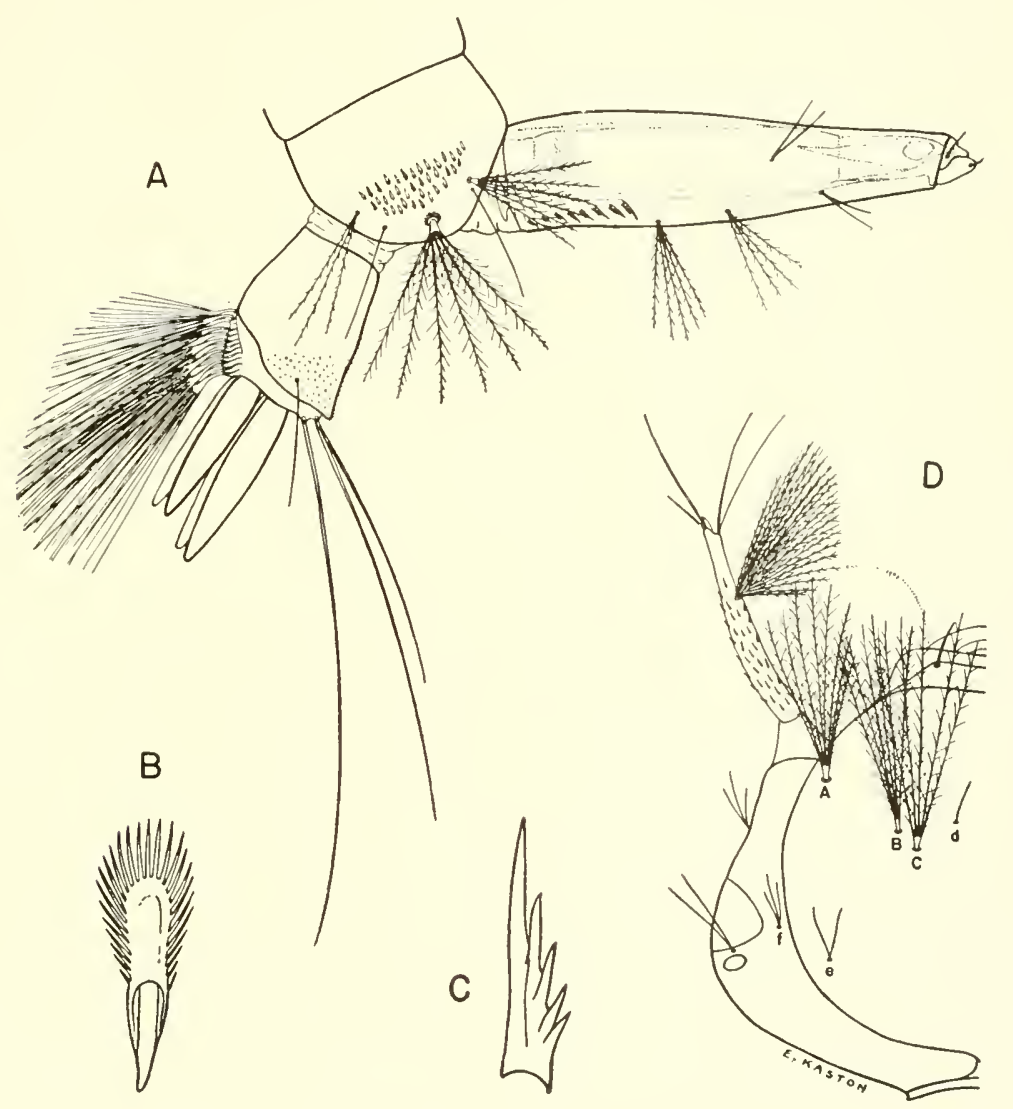

Fig. 137. Larva of Culex quinquefascialus Say. A, Terminal segments. B, Comb scale. C, Pecten tooth. D, Head. 
(1); North Carolina (166); South Carolina (64); Tennessee (96); Virginia (49). Other States: Arizona (52); California (66); District of Columiba and Illinois (52); Iowa (128); Kansas (79); Nebraska (178); New Mexico (9); Ohio (52); Oklahoma (159); Texas (108).

BIONOMICS.-This is a troublesome, domesticated species that commonly infests houses and bites at night. Larvae are found in rainbarrels, tubs, catch basins, cesspools, ditches containing filth, and similar habitats. A heavy population of C. quinquefasciatus is usually associated with insanitary conditions. Larvae and adults occur throughout the year in the extreme South (4th Sv. C. Med. Lab. records, 1942-1944, unpublished).

MEDICAL IMPORTANCE.-Culex quinquefasciatus is an important vector of $W u c h e r e r i a$ bancrofti in tropical and subtropical regions of the world. It is a known vector of the parasites causing bird malaria.

\section{Culex (Culex) Restuans Theobald}

Culex restuans Theobald, 1901, Mon. Culic., 2:142.

adult female.-Medium sized species. Head: Proboscis dark scaled, usually with some pale scales on under side; palpi short, dark. Broad dorsal region of occiput clothed with narrow whitish to golden brown scales, the paler ones bordering the eyes and on the median and posterior portions; numerous dark erect forked scales present. Lateral region of occiput clothed with broad whitish scales. Thorax: Integument of scutum light brown to reddish brown; vestiture of fine golden-brown lanceolate scales, distinctly paler on anterior and lateral margins and on prescutellar space; a pair of small pale-scaled submedian spots usually present near middle of scutum (frequently absent). Pleura with a few patches of broad pale scales. Abdomen: Tergites dark scaled with bronze to metallic blue-green reflection; each tergite except the first with a broad basal band of white to whitish-yellow scales, usually somewhat irregular on posterior margin (rarely as evenly rounded as in C. quinquefasciatus), not appreciably constricted laterally, broadly joining a pair of basal lateral whitish patches. Venter mostly pale scaled. Legs: Legs dark scaled with bronze to metallic bluegreen reflection except for pale inner surfaces of femora, small pale knee spots, pale streaks on inner surfaces of tibiae, and a few white scales at tips of tibiae. Wing: Scales narrow, dark.

ADULT MALE.-Coloration similar to that of female. TERminalia (Fig. 138A). Lobes of ninth tergite (IXT-L) prominent, rounded, each bearing several small setae. Tenth sternite (X-S) crowned with numerous short pointed spines; basal arm (XS-BA) long, stout, usually curved, weakly sclerotized. Phallosome $(\mathrm{Ph})$ formed of two large moderately sclerotized plates. Each plate apically with a long slender ventral arm ( $\mathrm{Ph}$-VA) directed posteriorly. Each plate basally with a short blunt dorsal arm (Ph-DA) curved outwardly to lock with the paramere; a short stout triangular tooth (Ph-T) arising iust posterior to the small dorsal arm. Claspette absent. Basistyle (Bs) about two and one-half times as long as basal width, with outer margin curved, portion 
beyond subapical lobe more slender, and apex bluntly rounded; vestiture of numerous setae, longer on outer aspects. Subapical lobe (S-L) prominent, undivided, bearing three long rods with tips pointed and slightly hooked, a large broad leaf-like filament, and two stout setae, one of which is recurved. Dististyle (Ds) about half as long as basistyle, curved, gradually tapered beyond middle, bluntly pointed at tip; terminal claw (Ds-C) short, blunt.

Larva. (Fig. 139).--Head broader than long. Antenna shorter than head, slightly narrowed beyond antennal tuft, spinose; antennal tuft multiple, barbed, inserted near middle of shaft, barely reaching tip. Head hairs: Preantennal (A) multiple, barbed, reaching beyond insertion of antennal tuft; lower (B) and upper (C) multiple, barbed, extending beyond preclypeus; postclypeal (d) double; sutural (e) single; trans-sutural (f) multiple; supraorbital single or double. Body glabrous. Upper lateral abdominal hairs double on segments I and II, long and single on III to VI. Comb of numerous scales in a patch; individual scale rounded and fringed apically with subequal spines. Siphon four to four and one-half times as long as basal width; pecten of numerous rather

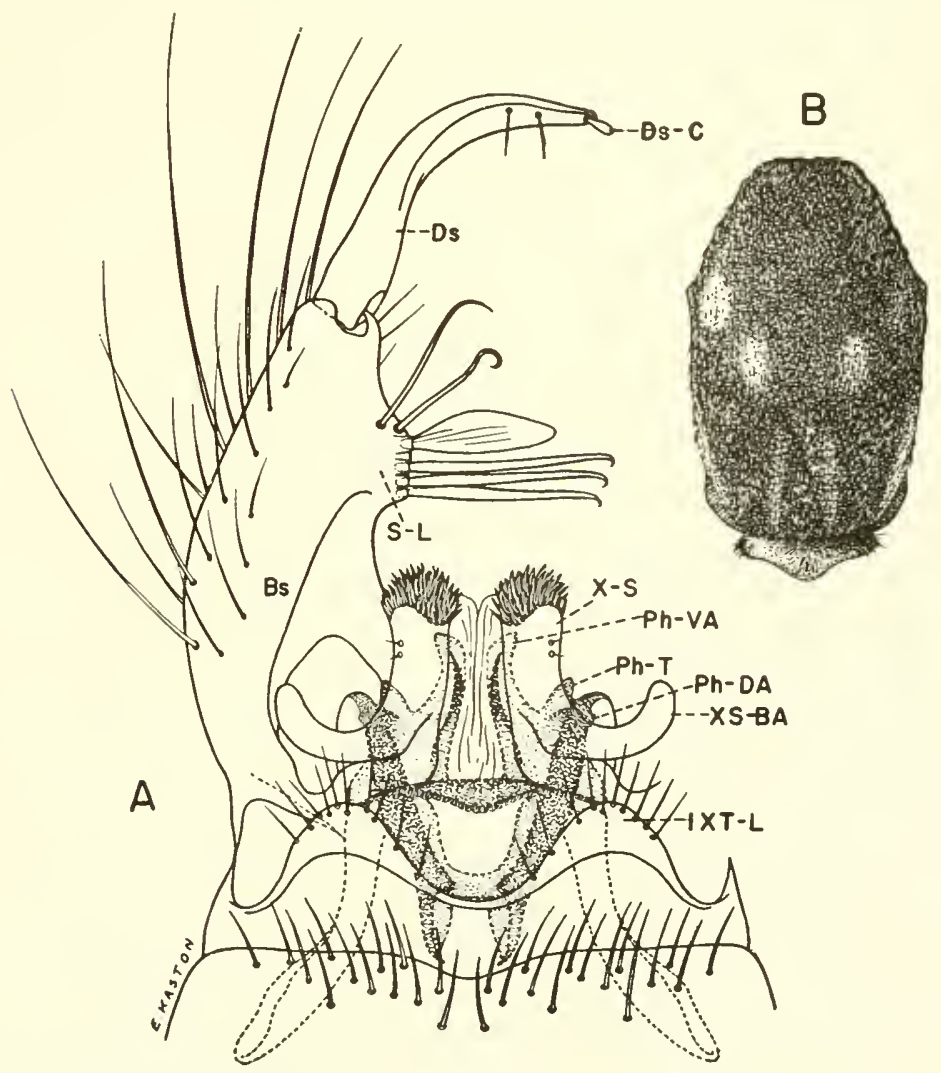

Fig. 138. Culex restuans Theobald. A. Mâle terminalia. B, Scutum and scutellum. 
evenly spaced teeth on basal third of siphon; individual tooth with 1 to 5 coarse teeth on one side; tufts of siphon inserted beyond pecten and represented by three pairs of long hairs irregularly placed and a pair of small subapical tufts of 2 to 3 branches each. Anal segment longer than wide, completely ringed by the dorsal plate; lateral hair single or double; dorsal brush consisting of a single upper and a single lower caudal hair on either side; ventral brush welldeveloped, confined to the barred area; gills 4 , longer than the segment, stout, each tapering to a blunt patch.

Distribution.-Widely distributed in North America from the Gulf of Mexico to southern Canada. Southern States: Alabama (170); Arkansas

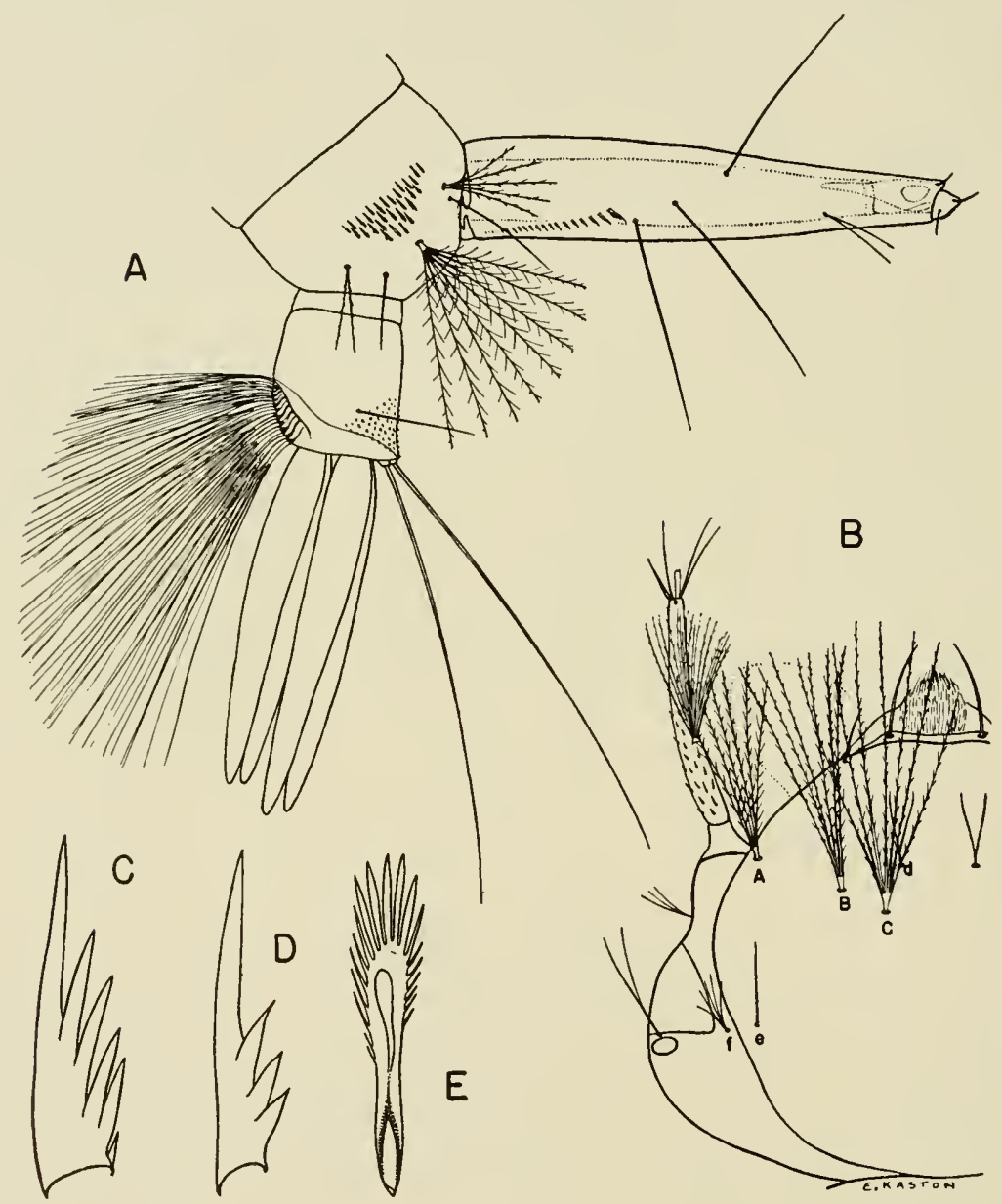

Fig. 139. Larva of Culex restuans Theobald. A, Terminal segments. B, Head. C, and D, Pecten teeth. E, Comb scale. 
(30); Florida (52); Georgia (96); Kentucky (140); Louisiana (96); Mississippi (52) ; Missouri (1); North Carolina (166); South Carolina and Tennessee (96); Virginia (49). Other States: California (66); Connecticut (21); Delaware (106); Illinois (52); Indiana (76); Iowa (155, 156); Kansas (79); Maine, Maryland and Massachusetts (52); Michigan (88); Minnesota (131); Montana (112); Nebraska (178); New Hampshire (52); New Jersey (77); New York (52); North Dakota (127); Ohio (52); Oklahoma (159); Pennsylvania (8); Rhode Island (99); Texas (108); Utah (142); West Virginia (52); Wisconsin (48).

BIONOMICS.-The adults frequently enter houses and are regarded as troublesome biters by some observers, but they do not rival C. quinquefasciatus in this respect. The species reaches its greatesi abundance during the late winter and spring months in the southern states. Larvae and adults occur throughout the year in the southern portion of its range but are rare in southern Florida (4th Sv. C. Med. Lab. records, 1942-1944, unpublished). Larvae are found in a great variety of breeding places, including ditches, pools in streams, rainbarrels and other artificial containers, and woodland pools.

\section{Culex (Culex) salinarius Coquillett}

Culex salinarius Coquillett, 1904. Ent. News, $15: 73$.

Adult Female.-Medium sized species. Head: Proboscis dark scaled; palpi short, dark. Broad dorsal region of occiput clothed with narrow pale golden-brown scales and dark erect forked scales, except for whitish scales narrowly margining eyes; lateral region of occiput with a patch of broad whitish scales. Thorax: Integument of scutum brown, covered with fine golden-brown scales, paler on prescutellar space. Pleura with several small groups of broad pale scales, each group usually comprised of more than six scales. Abdomen: 'Tergites primarily dark brown to black scaled with bronze to metallic bluegreen reflection; narrow dingy-yellow basal bands often present and apices of segments more or less distinctly blended with dingy-yellow scales; seventh and eighth tergites frequently entirely covered with dingy-yellow scales; basal lateral patches rather poorly defined and of similar pale scales. Venter yellowishwhite. Legs: All legs dark scaled with bronze to metallic blue-green reflection, except for whitish inner surfaces of femora. Wing: Scales narrow, dark.

ADULT MALE.-Coloration similar to that of female, but with dorsal abdominal pale bands much broader. TERminalia (Fig. 140). Apical margin of eighth tergite (VIII-T) bearing numerous rather long setae (longer than those of the lobes of the ninth tergite). Lobes of the ninth tergite (IXT-L) widely separated, only slightly raised, each bearing several slender setae. Tenth sternite (X-S) crowned with numerous short pointed spines; basal arm (XSBA) long, stout, strongly curved, heavily sclerotized. Phallosome (Ph) formed of two large heavily sclerotized plates connected at base. Each plate with a stout sharply pointed dorsal arm (Ph-DA) bent medially at right angle, and a stout bluntly pointed ventral arm ( $\mathrm{Ph}$-VA) bearing a small projection on its inner margin near middle; a group of several closely set pointed teeth (Ph-T) arising between the prominent dorsal and ventral arms. Claspette absent. Basi- 
style (Bs) about two and one-half times as long as basal width; outer margin curved, portion beyond subapical lobe more slender, apex bluntly rounded; vestiture of numerous setae, longer on outer aspects. Subapical lobe (S-L) prominent, undivided, bearing three long stout pointed rods usually somewhat hooked at tips, a large broad leaf-like filament, and two stout setae, one of which is recurved. Dististyle (Ds) about half as long as basistyle, curved, tapered beyond middle, bluntly pointed at tip; terminal claw (Ds-C) short, blunt.

LARVA. (Fig. 141).-Head broader than long. Antenna shorter than head, constricted beyond antennal tuft, with portion before constriction spinose, portion beyond constriction darker and with few spines; antennal tuft large, multiple, barbed, inserted at outer third, reaching beyond tip of antenna. Head hairs: Preantennal (A) multiple, barbed, extending to insertion of antennal tuft; lower (B) and upper (C) usually 3-branched, barbed, extending beyond preclypeus; postclypeal (d) rather long, single; sutural (e), and trans-sutural (f) multiple; supraorbital usually double. Thorax glabrous, although occasion-

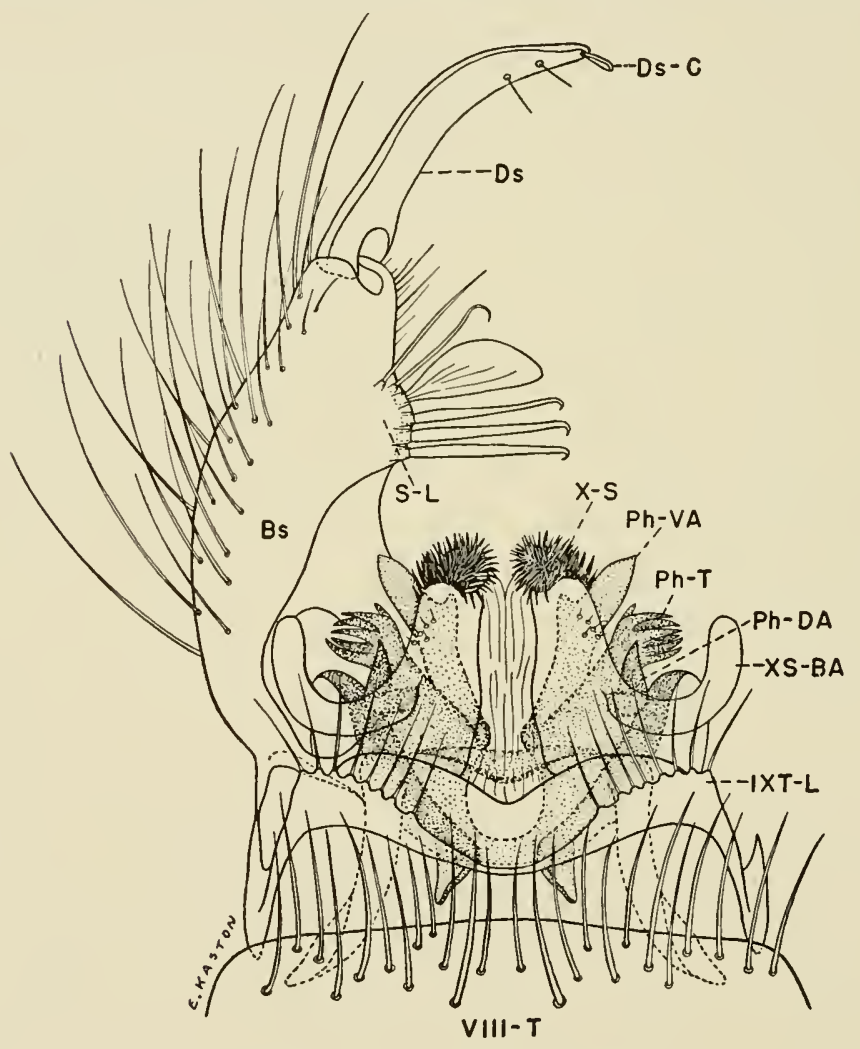

Fig. 140. Male terminalia of Culex salinarius Coquillett. 
ally minute papillated hairs may be present. Upper lateral abdominal hairs 3 branched on segments I and II, usually 2-branched on III to VI. Comb of eighth segment of numerous scales in a patch; individual scale rounded apically, fringed with subequal spinules. Siphon slender, six to seven times as long as basal width; pecten of about 10 to 15 rather evenly spaced teeth on basal fourth of siphon; individual tooth with 2 to 5 coarse teeth on one side; paired subventral tufts usually four in number and inserted beyond pecten (the proximal tuft as long or longer than basal width of siphon, usually 3 or 4-branched,

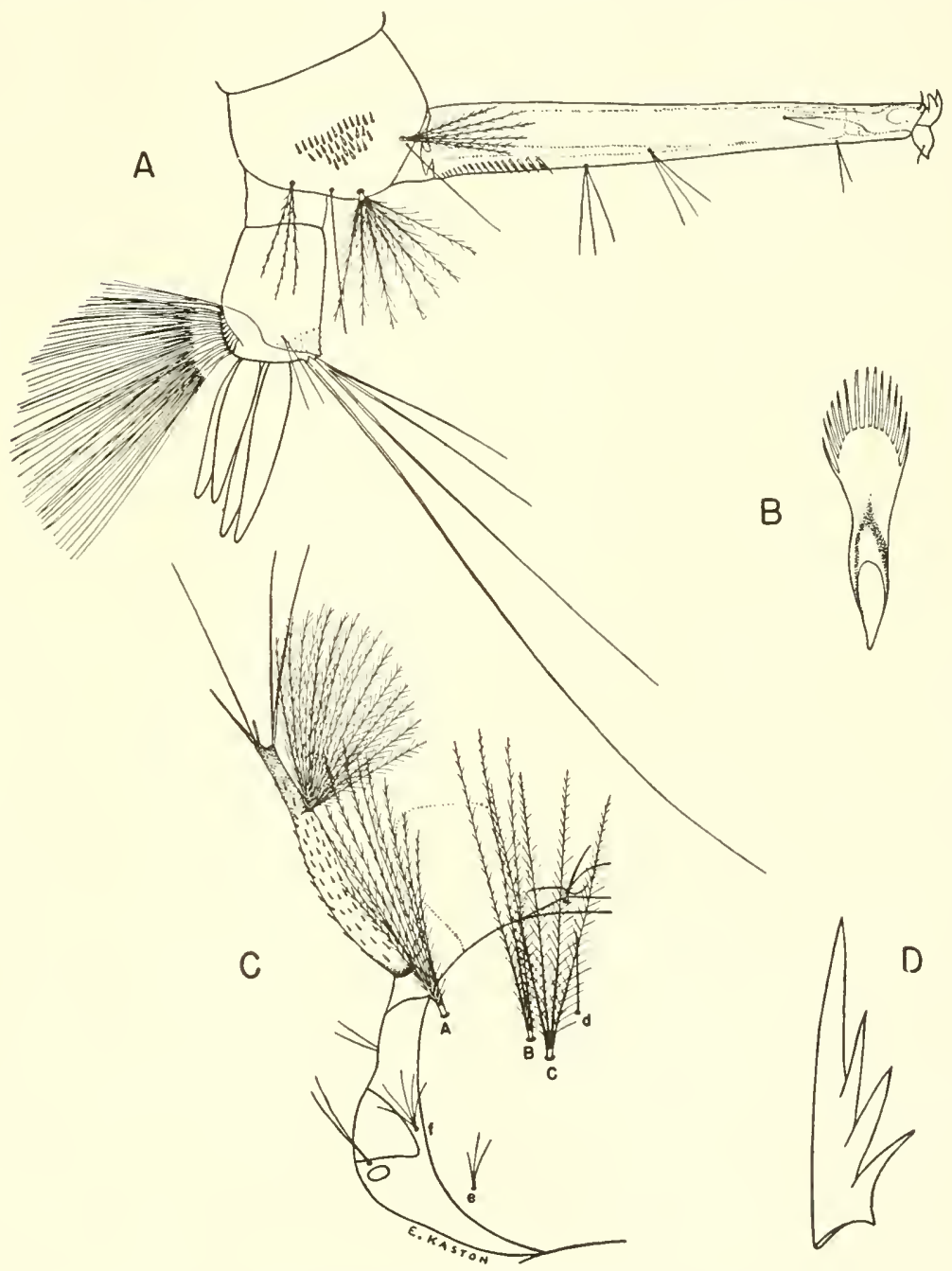

Fig. 1+1. Larva of $C_{1}$ lex salinarius Coquillett. A, Terminal segments.

B, Comb scale. C. Head. D, Pecten tooth. 
occasionally double; second and third tufts usually double or triple and inserted somewhat laterally; distal tuft small, usually double). Anal segment longer than wide, completely ringed by the dorsal plate; lateral hair double, sometimes single; dorsal brush consisting of a long lower caudal hair and a shorter 3 . branched upper caudal tuft on either side; ventral brush well-developed, confined to the barred area; gills 4 , as long as the segment or longer, each tapering to a blunt point.

Distribution.-Eastern United States, north to Massachusetts and west to Utah. Southern States: Alabama (170); Arkansas (30); Florida and Georgia (96); Kentucky (140); Louisiana (96); Mississippi (97); Missouri (1); North Carolina (166); South Carolina (64); Tennessee (97); Virginia (49). Other States: Colorado (128); Connecticut (90); Delaware (106); District of Columbia and Illinois (52); Iowa (155, 156); Kansas (128); Maryland (19); Massachusetts (52); Michigan (88); Minnesota (131); Nebraska (178); New Jersey (77); New Mexico (9); Oklahoma (159); Pennsylvania (52); Rhode Island (99); Texas (108); Utah (142); Wisconsin (48).

BIONOMICS.--The adults are frequently found in outbuildings and other daytime resting places. The females bite readily outdoors and occasionally enter dwellings to bite. Larvae occur in grassy pools, either fresh or brackish water, in ditches, ponds and occasionally in rain barrels. Larvae and adults of Culex salinarius occur throughout the year in the extreme South, but are more common from April to October farther north (4th Sv. C. Med. Lab. records, 1942-1944, unpublished).

\section{Culex (Culex) tarsalis Coquillett}

Culex tarsalis Coquillett, 1896, Can. Ent., 28:43.

adult female.-Medium sized species. Head: Proboscis dark scaled, with a rather broad white band medially; palpus short, dark except for a few white scales at tip and at apex of third segment. Scales of postero-dorsal region of occiput narrow, white, arranged in a triangular patch projecting anteriorly on the mid line; scales dorsally margining eyes narrow, white; scales of submedian areas between the postero-dorsal patch and eye margins narrow, golden brown. Lateral regions of occiput clothed with broad whitish scales. Erect forked scales numerous on dorsal surface of occiput, those on central portion pale. Thorax: Integument of scutum dark brown, clothed dorsally with golden-brown lanceolate scales, narrowly margined anteriorly and laterally with white lanceolate scales. Prescutellar space mostly white scaled, bordered on either side by a narrow submedian white line extending anteriorly to near middle of scutum and terminating in a small white submedian spot (this line may be disrupted near the submedian spot by brown scales). Pleura with a few patches of broad white scales. Abdomen: First tergite mostly pale scaled; remaining tergites with prominent basal bands of white to whitish-yellow scales (these scales may extend toward the posterior margin on the median line, particularly on second segment); the terminal segments often with apical pale scaling as well as basal, the eighth segment frequently entirely pale scaled. Venter pale scaled, with a $\mathrm{V}$-shaped marking of dark scales on each sternite, the base of the $\mathrm{V}$ at the 
median anterior margin. Legs: Hind leg dark scaled with the exception of the following markings: White scales covering inner surface of femur; a conspicuous white streak on outer surface of femur, tibia, and often on first tarsal segment; a patch of white at tip of tibia; wide basal and apical white bands on tarsal segments. Fore and middle legs similarly marked but with tarsal bands narrower on segments 1 to 3, much reduced or entirely lacking on segments 4 and 5. Wing: Scales narrow, dark except for a few white scales on costa and subcosta.

ADULT MALE.-Coloration similat to that of female, but with abdominal white scaling usually more extensive. TERMinALIA (Fig. 142C). Lobes of ninth

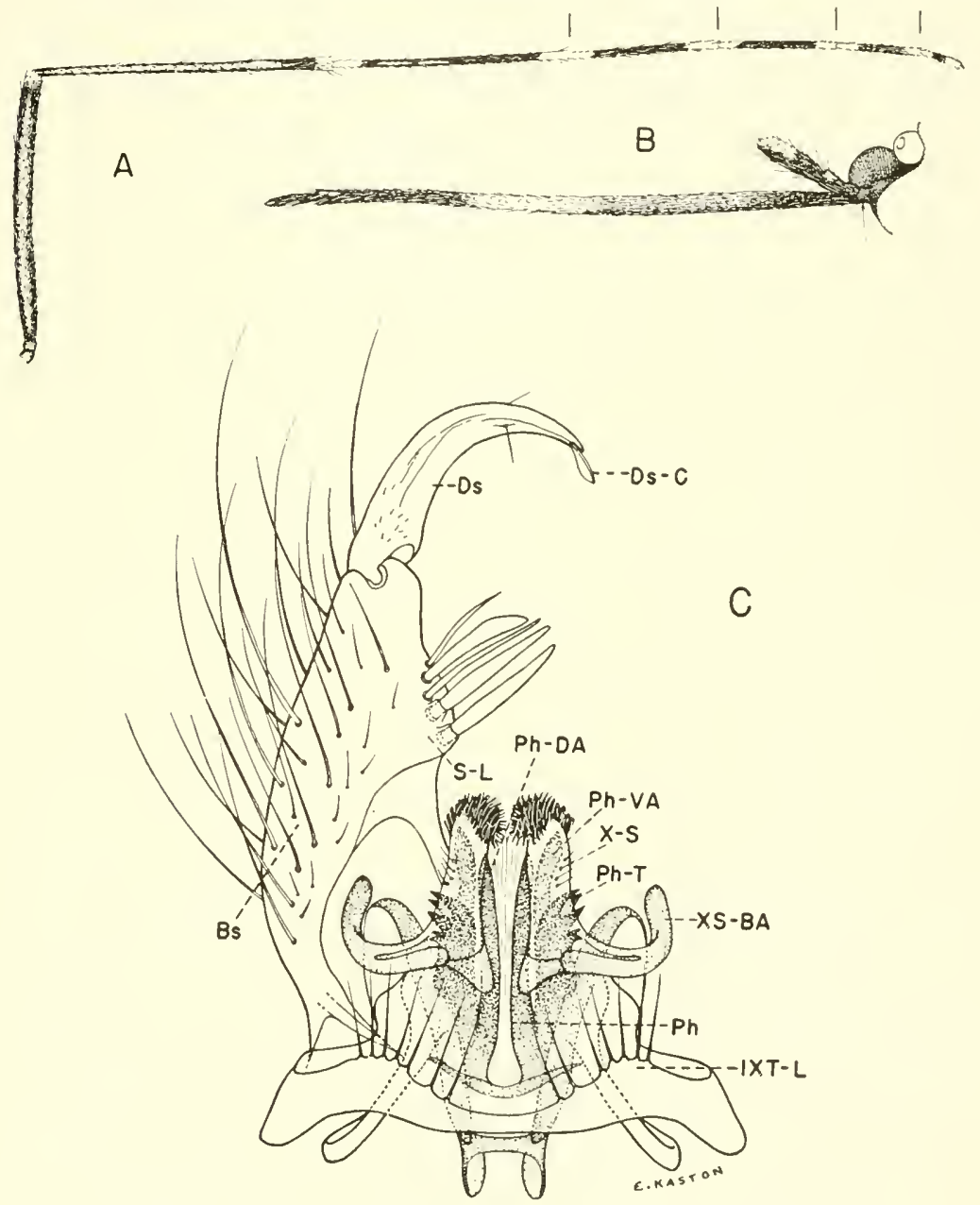

Fig. 142. Culex tarsalis Coquillett. A, Hind leg. B, Palp and proboscis of female. C, Male terminalia. 
tergite (IXT-L) only slightly raised, separated by about the width of one lobe, each bearing several setae. Tenth sternite (X-S) crowned with numerous short spines, the apical ones pointed, the outer ones blunt; basal arm (XS-BA) long, stout, strongly curved, heavily sclerotized. Phallosome (Ph) formed of two large heavily sclerotized plates. Each plate basally with a long pointed dorsal arm (Ph-DA), directed posteriorly and nearly reaching the crown of the tenth

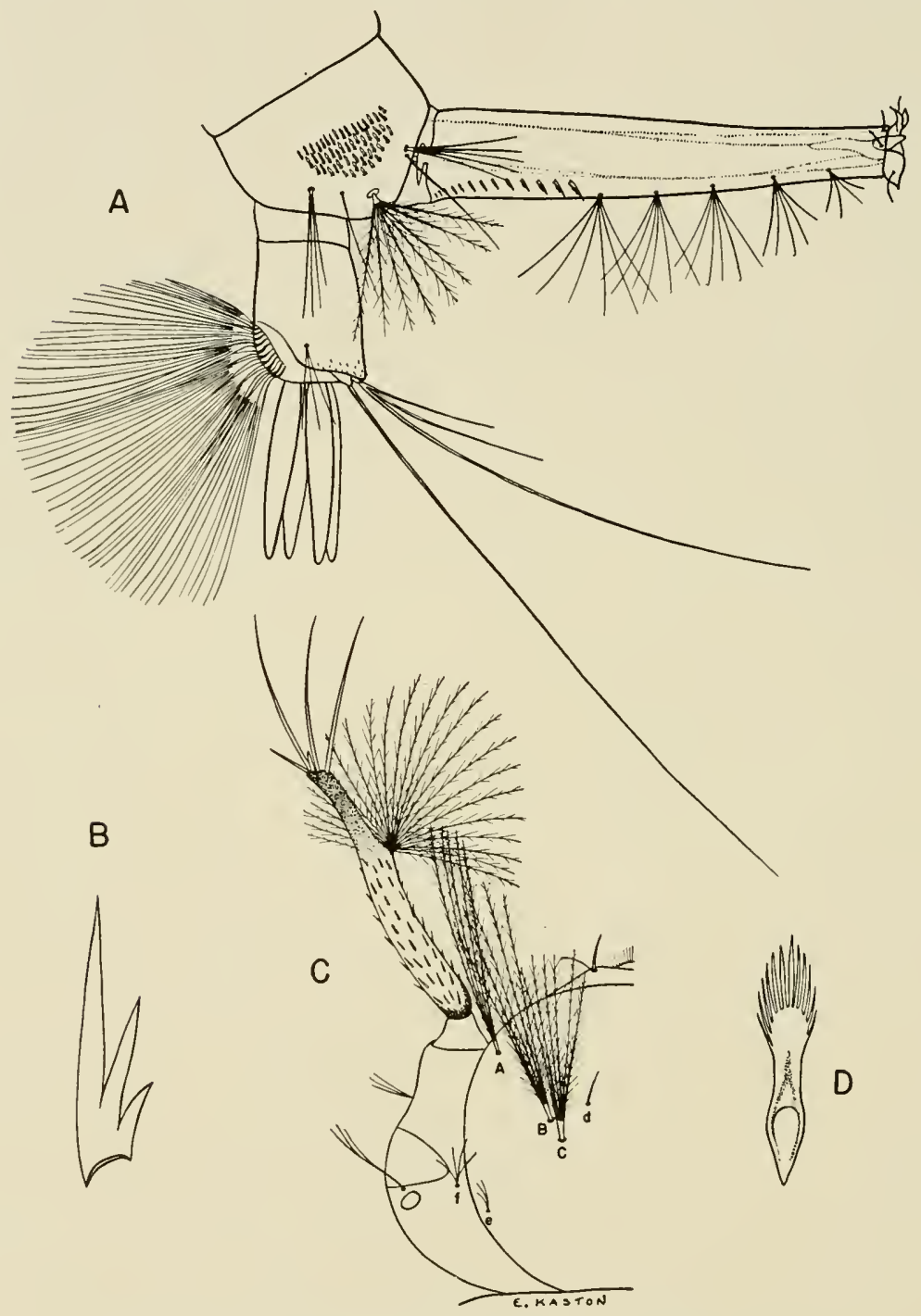

Fig. 143. Larva of Culex tarsalis Coquillett. A, Terminal segments. B. Pecten tooth. C, Head. D, Comb scale. 
sternite; each plate apically with a long bluntly pointed ventral arm (Ph-VA), slightly curved outward and nearly reaching the crown of the tenth sternite; arising laterally near the base of the ventral arm are several stout pointed teeth (Ph-T). Claspette absent. Basistyle (Bs) about two and one-half times as long as basal width, with outer margin curved, portion beyond subapical lobe more slender, and apex bluntly rounded; vestiture of numerous setae, longer on outer aspects. Subapical lobe (S-L) prominent, undivided, armed with two stout rods, two stout setae, and a narrow leaf-like filament. Dististyle (Ds) about half as long as basistyle, curved, gradually tapered, bluntly pointed at tip; claw (Ds-C) short, blunt.

LARva. (Fig. 143).- - Head broader than long. Antenna shorter than head, rather slender, constricted beyond the antennal tuft, with portion before constriction spinose, portion beyond constriction darker and with fewer spines; antennal tuft large, multiple, barbed, inserted at outer third, reaching beyond tip of antenna. Head hairs: Preantennal (A) multiple, barbed, reaching to antennal tuft; lower (B) and upper (C) multiple, barbed, extending beyond preclypeus; postclypeal (d) small, single; sutural (e) and trans-sutural (f) usually multiple; supraorbital usually 2 to 4 -branched beyond base. Thorax glabrous. Upper lateral abdominal hairs usually triple on segments I to VI. Comb of eighth segment of numerous scales in a patch; individual scale rounded apically and fringed with subequal spinules. Siphon about four and one-half to five times as long as basal width; pecten of about 10 to 15 rather evenly spaced teeth on basal third of siphon; individual pecten tooth with 1 to 5 coarse teeth on one side; five pairs of multiple subventral tufts present, all in line, the proximal pair inserted near or slightly before end of pecten. Anal segment longer than wide, completely ringed by the dorsal plate; lateral hair double or triple; dorsal brush bilaterally consisting of a long lower caudal hair and an upper caudal tuft of three hairs, one nearly as long as the lower caudal; ventral brush well-developed, confined to the barred area; gills 4, usually about as long as the segment, each tapering to a blunt point.

Distribution.-Canada; central, southern and western United States; and Mexico. Southern States: Alabama (125); Arkansas (30); Florida and Georgia (125); Kentucky (140); Louisiana (96); Mississippi (125); Missouri (1); South Carolina (125); Tennessee (97); Virginia (54). Other States: Arizona, California, Colorado, Idaho and Illinois (52); Indiana (76); Iowa and Kansas (52); Michigan (88); Minnesota (131); Montana (112); Nebraska (178); Nevada and New Mexico (52); North Dakota (127); Oklahoma (159); Oregon (52); South Dakota (129); Texas (108); Utah (142); Washington (52); Wisconsin (48); Wyoming (129).

BIONOMICS.- The females are fierce biters, attacking at dusk and after dark, and they readily enter houses. The bite is very painful and according to Hearle (78) the swelling and pain last for hours. Larvae occur in either fresh or rather foul water in various types of breeding places, including ground pools, rain water barrels, roadside ditches, and irrigation water.

MEDICAL IMPORTANCE. - This mosquito has been found naturally infected with the viruses of St. Louis and western equine encephalitis, and it has been demonstrated that it is capable of transmitting the infections. 


\section{Culex (Melanoconion) atratus Theobald}

Culex atratus Theobald, 1901, Mon. Culic., 2:55.

adult female.-Small species. Head: Proboscis long, dark, slightly swollen at tip; palpi short, dark. Occiput clothed with broad appressed scales on antero-dorsal and lateral regions (antero-dorsal scales dark; lateral scales pale), and with numerous light-brown lanceolate scales and dark erect forked scales centrally. Thorax: Integument of scutum dark brown, clothed with narrow reddish-brown scales. Abdomen: Tergites dark scaled, with white basal patches laterally; sternite white-banded basally, dark apically. Legs: All legs dark scaled with bronze reflection, except for pale inner surfaces of femora. Wing: Wing scales dark, mostly rather narrow, linear, but mixed with ovate ones on vein 3 and branches of veins 2 and 4 .

ADULT MALE.-Coloration similar to that of female. TERminalia (Fig. 144). Lobes of ninth tergite (IXT-L) large, pyriform, separated by a little less than the width of one lobe, each bearing a number of stout irregularly placed feathered and smooth setae on basal half; apical half bare and tapered. Tenth sternite (X-S) crowned with a comb-like row of blunt spines. Phallosome $(\mathrm{Ph})$ formed of a pair of simple curved divergent blade-like plates,

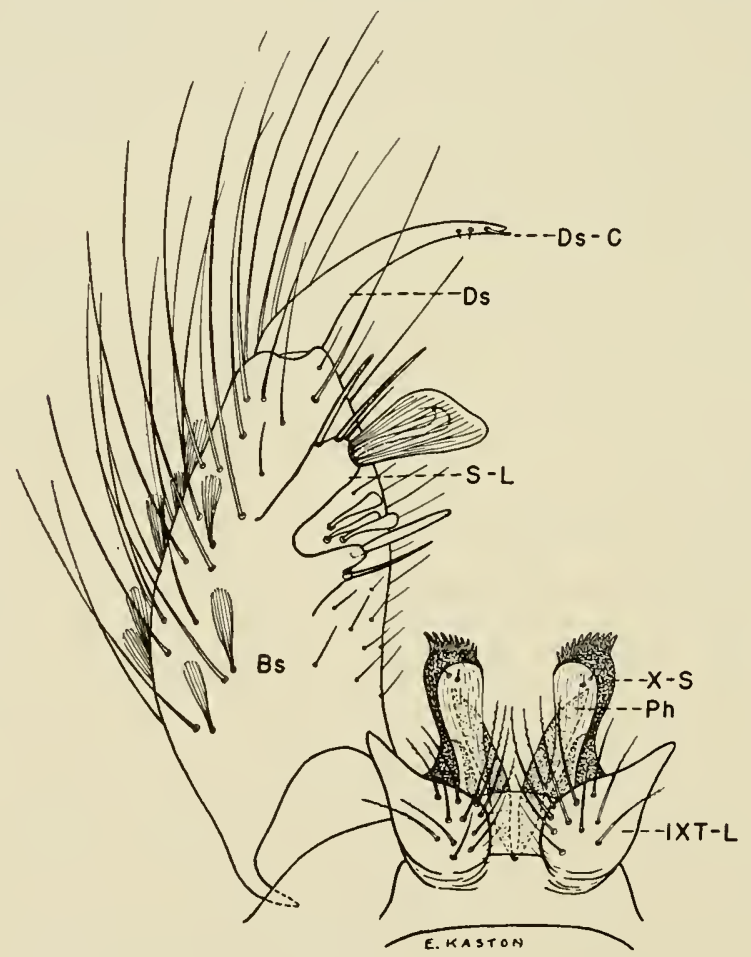

Fig. 144. Male terminalia of Culex atratus Theobald. 
pointed at tips, connected at bases. Claspette absent. Basistyle (Bs) about twice as long as broad, clothed with scales and long setae (setae more numerous and longer beyond middle); three or four short, greatly flattened setae present between trunks of subapical lobe. Subapical lobe (S-L) widely separated into two distinct trunks: Tip of posterior trunk with a large striated deltoid leaflike filament, a flattened recurved spine, two straight spines and one or two smaller setae; anterior trunk with a stout rod at apex and a slender subapical spine arising from a small lateral branch. Dististyle (Ds) about half as long as basistyle, broad at base, tapered and evenly curved to a narrow tip; claw (DsC) a small blunt spine inserted a little before apex.

LARVA. (Fig. 145).- - Head much broader than long. Antenna not quite as long as head, constricted beyond antennal tuft, the portion before constriction

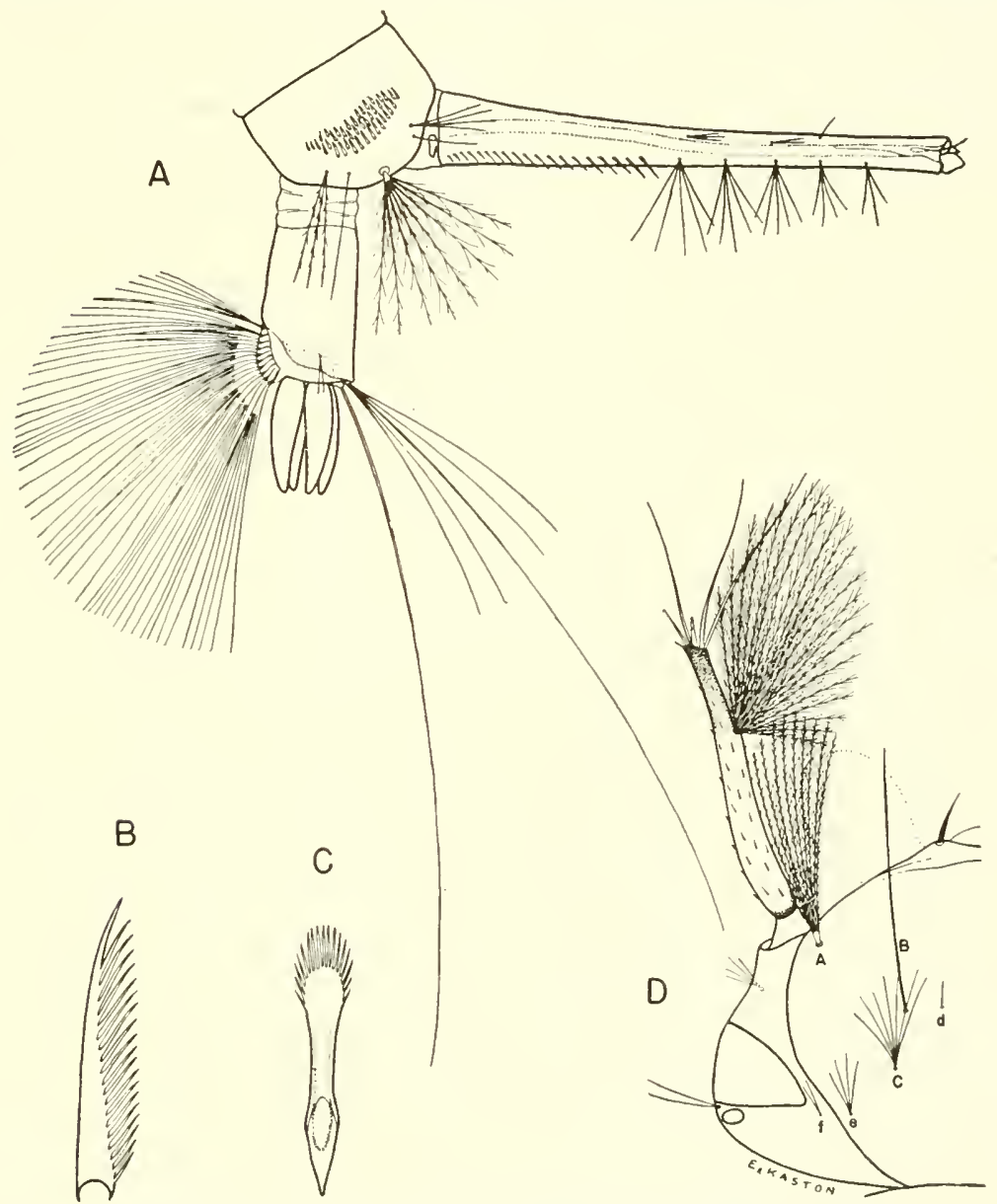

Fig. 145. Larva of Culex atratus Theobald. A, Terminal segments. B, Pecten tooth. C, Comb scale. D, Head. 
spinose and that beyond constriction darker and with fewer spines; antennal tuft large, multiple, barbed, inserted at outer third, reaching considerable beyond tip of antenna. Head hairs: Preantennal (A) multiple, barbed; lower (B) long, single; upper (C) a small 5 to 6-branched tuft; postclypeal (d) small, single; sutural (e) usually multiple; trans-sutural (f) and supraorbital usually double. Body finely spicular. Comb of eighth segment of numerous scales in a patch; individual scale rounded apically and fringed with subequal spinules. Siphon slender, seven to eight times as long as basal width; pecten of numerous teeth reaching beyond basal third of siphon; individual tooth fringed on one side nearly to tip; four or five multiple subventral tufts inserted beyond pecten, the basal tuft longer than width of siphon; three or more minute tufts inserted dorso-laterally on siphon. Anal segment longer than wide, completely ringed by the dorsal plate; lateral hair small, double; dorsal brush consisting of a long lower caudal hair and a shorter 5-branched upper caudal tuft on either side; ventral brush large, posterior to the dorsal plate; gills 4 , shorter than the segment.

Distribution.-Boca Chica Key, Florida (154); Greater Antilles and Virgin Islands.

BIONOMiCs.-Dyar (54) states that Culex atratus breeds in ground pools.

\section{Culex (Melanoconion) erraticus (Dyar and Knab)}

Melanoconion atratus Dyar (not Culex atratus Theobald, 1901), 1905, Jour. N. Y. Ent. Soc., 13:26.

Culex erraticus King and Bradley, 1937. Ann Ent. Soc. Amer., 30:345 (additional synonymy given).

adult Female.-Small species. Head: Proboscis long, dark, slightly swollen at tip; palpi very short, dark. Occiput with large central portion clothed with dark erect forked scales and numerous brown to golden-brown lanceolate scales; antero-dorsal and lateral portions with broad appressed scales, the anterodorsal ones predominantly brown with bronze or metallic blue-green reflection, the lateral ones predominantly dingy-white. Cibarial armature consisting of 7 or 8 teeth (122). Thorax: Integument of scutum dark brown, clothed with lanceolate golden-brown scales (the scales usually paler and coarser than those of C. peccator and C. pilosis). Abdomen: Tergites dark-brown scaled, usually with bronze to metallic blue-green reflection; white basal patches present laterally (occasionally narrow white basal bands present on some segments). Sternites each white-banded basally, the band joining the tergal patch of white scales on either side; dark apically. Legs: All legs dark scaled with bronze to metallic blue-green reflection, except for pale inner surfaces of femora. Wing: Scales all dark. Plume scales narrow; squame scales broader, ligulate to ovate.

ADULT MALE - Coloration similar to that of female. TERMinalia (Fig. 146). Lobes of ninth tergite (IXT-L) large, ovoid, approximate at bases, widely divergent apically, clothed with numerous prominent smooth setae arising from distinct tubercles. Tenth sternite (X-S) crowned with a comb-like row of blunt spines. Phallosome $(\mathrm{Ph})$ of two elongate plates connected basally; each plate with a short stout apical tooth, a strong slender subapical tooth on outer margin, and a stout ventro-lateral tooth at basal third; base of each 
plate giving rise to a long tail-like hook directed ventrally. Claspette absent. Basistyle (Bs) about twice as long as broad, outer margin strongly curved, apex narrow; vestiture of scales and numerous setae. Subapical lobe (S-L) deeply divided into two distinct trunks: Posterior trunk bearing a long flattened pointed strongly recurved spine, a large broadly expanded leaf-like filament, a narrow leaf-like filament, and two blunt slightly flattened rods; anterior trunk cleaft nearly to base into two stout branches, each bearing a long strong rod with tip flattened, curved and pointed (often with a small apical retrorse barb). Dististyle (Ds) about two-thirds as long as basistyle, curved, narrowed a little before middle, becoming broader at apical third (a row of very short, reflexed setae on outer margin near tip), tapered to a pointed slightly reflexed apex; terminal claw (Ds-C) short, curved, blunt.

Larva. (Fig. 147).- Head much broader than long. Antenna about as long as head, constricted beyond the antennal tuft, with portion before constriction spinose, portion beyond constriction darker and more sparsely spined; antennal tuft large, multiple, barbed, inserted at outer third, reaching considerably beyond tip of antenna. Head hairs: Preantennal (A) multiple, barbed, extending to antennal tuft; lower (B) single, barbed, reaching beyond preclypeus; upper (C) short, weak, 4 or more branched; postclypeal (d) small,

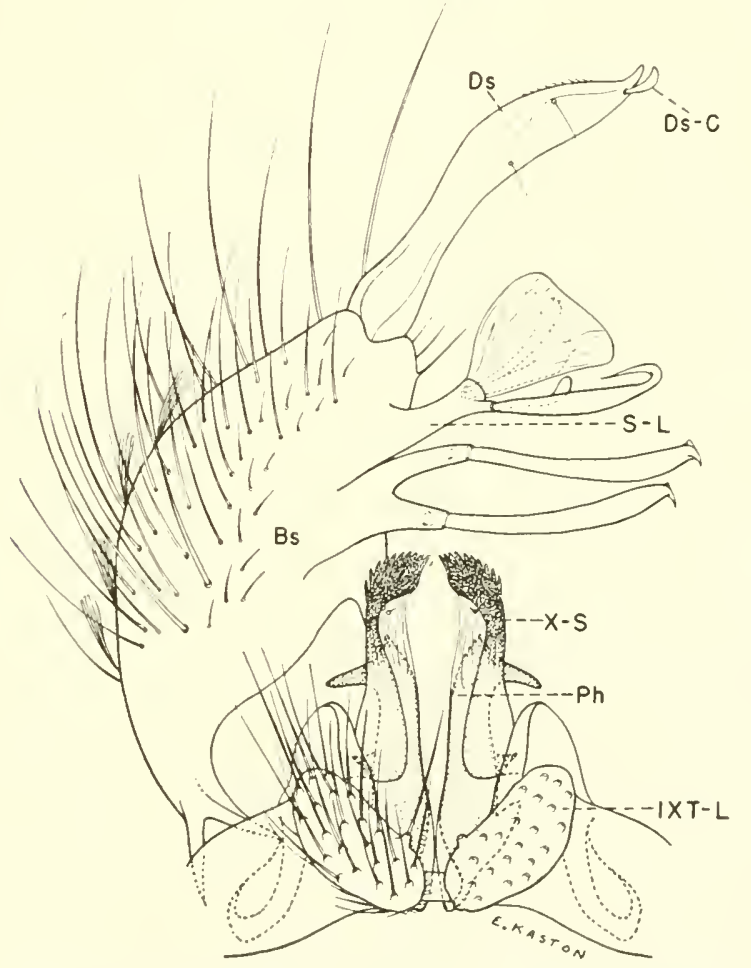

Fig. 146. Male terminalia of Culex erraticus (Dyar and Knab). 
single; sutural (e) and trans-sutural (f) multiple; supraorbital usually double or triple. Body densely clothed with long spicules. Upper lateral abdominal hairs usually double or triple on segments I and II, usually triple on III to VI. Comb of eighth segment of numerous scales in an irregular single or double row; individual scale thorn-shaped, with short lateral spinules. Siphon slender, six to seven times as long as basal width; pecten of numerous teeth on basal third of siphon; individual pecten tooth fringed on one side nearly to tip; about five multiple barbed subventral tufts inserted beyond pecten, with proximal

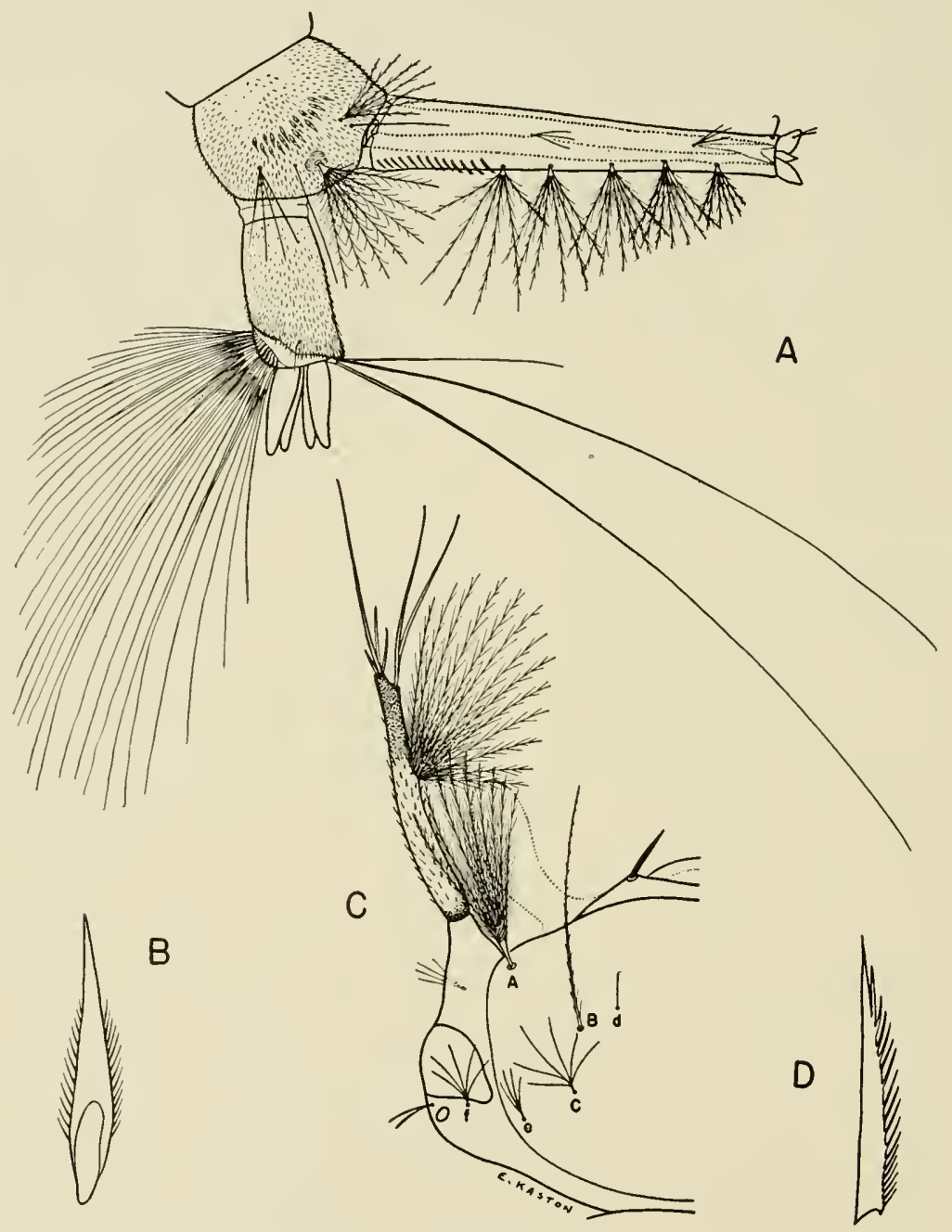

Fig. 147. Larva of Culex erraticus (Dyar and Knab). A, Terminal segments. B, Comb scale. C, Head. D, Pecten tooth. 
tuft much longer than basal width of siphon but no more than one-third its length; two 2 to 3-branched subdorsal tufts present; dorsal preapical spine as long or longer than apical pecten tooth, recurved. Anal segment longer than wide, completely ringed by the dorsal plate; lateral hair single or double; dorsal brush consisting of a long caudal hair and an upper caudal tuft of 2 to 3 hairs on either side (one of the upper caudal hairs nearly as long as the lower caudal); ventral brush well-developed, confined to the barred area; gills 4 , shorter than the segment, each tapering to a blunt point.

Distribution.-Southern United States, north to New York and west to Nebraska and Texas. Southern States: Alabama (170); Arkansas (30): Florida and Georgia (96); Kentucky (140); Louisiana and Mississippi (96); Missouri (1); North Carolina (166); South Carolina and Tennessee (96); Virginia (49). Other States: Delaware (106); Illinois (54); Indiana (76); Iowa (155, 156); Kansas (128); Maryland (19); Nebraska (178); New York (162); Oklahoma (159); Texas (108).

BIONOMICS. - The adults may be found in enormous numbers near favorable breeding places, but are not troublesome biters $(30,82,83)$. Observations made by King et al. (96) in Louisiana indicate that the females may attack man at night outdoors but that they have a preference for the blood of fowl. Larvae occur in grassy areas in ponds, lakes, marshes and streams where they are frequently associated with the larvae of Anopheles, particularly A. quad. rimaculatus. Breeding occurs throughout the year in the extreme South, but is more common from May to October farther north (4th Sv. C. Med. Lab. reports, 1942-1944, unpublished).

\section{Culex (Melanoconion) peccator Dyar and Knab}

Culex peccator Dyar and Knab, 1909, Smiths. Misc. Coll., Quart. Iss., 52:256.

Culex peccator King and Bradley, 1937. Ann. Ent. Soc. Amer., 30:350 (synonymy given).

Adult Female.-Small species. Head: Proboscis long, dark, slightly swollen at tip; palpi very short, dark. Occiput clothed with numerous broad appressed scales antero-dorsally and laterally (the antero-dorsal scales dark trown to black, with bronze or metallic blue-green reflection; the lateral scales whitish), and with a median area of brown lanceolate scales posteriorly; dark erect forked scales numerous. Cibarial armature consisting of 7 or 8 teeth (122). Thorax: Integument of scutum dark brown, clothed with fine brown scales. Abdomen: Tergites dark scaled with bronze to metallic blue-green reflection, and with small white basal patches laterally; sternites primarily pale scaled, becoming darker on apical margins of segments. Legs: All legs dark scaled with bronze to metallic blue-green reflection, except for pale inner surfaces of femora. Wing: Scales all dark. Plume scales narrow; squame scales broader, ligulate to ovate.

ADULT MALE.-Coloration similar to that of female. TERMinalia (Fig. 148). Ninth tergite (IX-T) weak, broader laterally; lobes (IXT-L) well defined, approximate, triangular, cushion-like, bearing numerous long setae arising from distinct tubercles. Tenth sternite (X-S) crowned with a comb-like row of blunt spines. Phallosome (Ph) of two plates connected basally; each 
plate elongate, with a short stout curved tooth at apex and a blunt subapical lateral lobe; base of each plate with a long, tail-like hook directed ventrally. Claspette absent. Basistyle (Bs) very large, nearly spherical, clothed with scales and numerous rather fine setae. (C. peccator may be easily distinguished macroscopically by these greatly swollen basistyles.) Subapical lobe (S-L) deeply divided into two distinct trunks: Posterior trunk with a large greatly expanded leaf-like appendage arising from a stout branch, and three rod-like filaments, each with a lamella apically, arising from smaller branches; anterior trunk bearing an apical rod retrorsely spined at tip, and a lateral rod somewhat flattened and angulate near tip. Dististyle (Ds) about half as long as basistyle, constricted at basal third, quadrately expanded beyond constriction, hirsute on crest; a short, curved spine inserted a little before apex.

LARVA. (Fig. 149).-Head much broader than long. Antenna about as long as head, constricted beyond antennal tuft, with portion before constriction spinose and that beyond constriction darker and more sparsely spined; antennal tuft large, multiple, barbed, inserted at outer third, reaching beyond tip of antenna. Head hairs: Preantennal (A) multiple, barbed, reaching to insertion of antennal tuft; lower (B) single, sparsely barbed, extending beyond preclypeus; upper (C) small, double or triple, occasionally single, less than half as long as lower (B); postclypeal (d) small, single; sutural (e) usually double

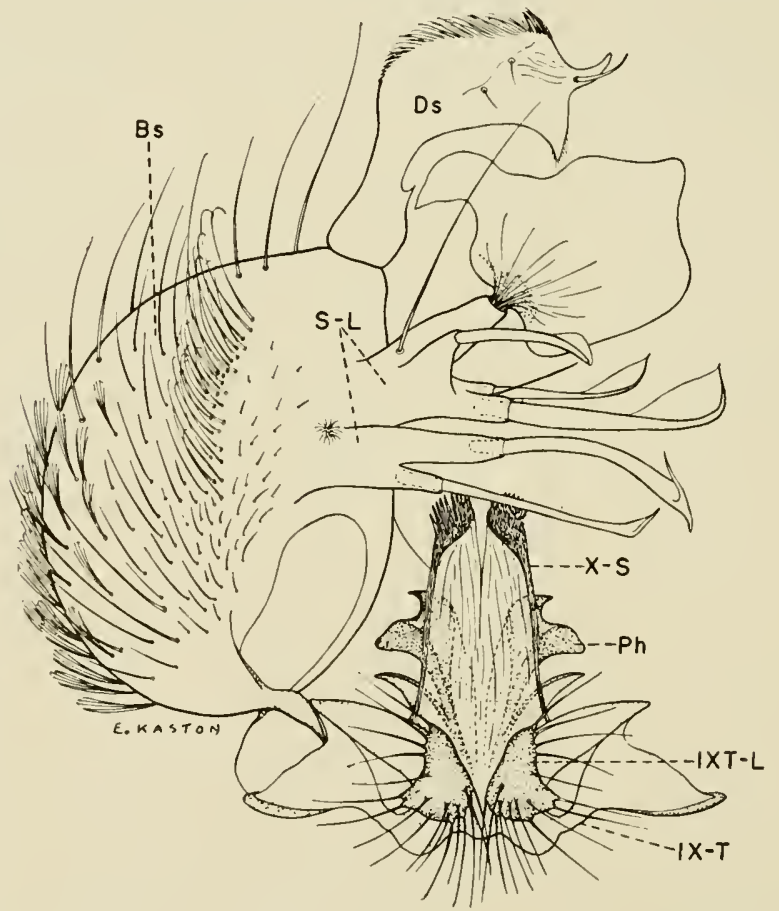

Fig. 148. Male terminalia of Culex peccator Dyar and Knab. 
or triple; trans-sutural (f) usually triple; supraorbital single or double. Body distinctly spicular. Upper lateral abdominal hairs usually double on segments I and II and triple on III to VI. Comb of numerous scales in a patch; individual scale rounded apically and fringed with subequal spinules. Siphon slender, about six times as long as basal width, with a darkly pigmented band at middle; pecten of numerous teeth on the basal third of siphon; individual pecten tooth fringed on one side nearly to tip; about five multiple barbed subventral tufts inserted beyond pecten, the proximal tuft much longer than basal

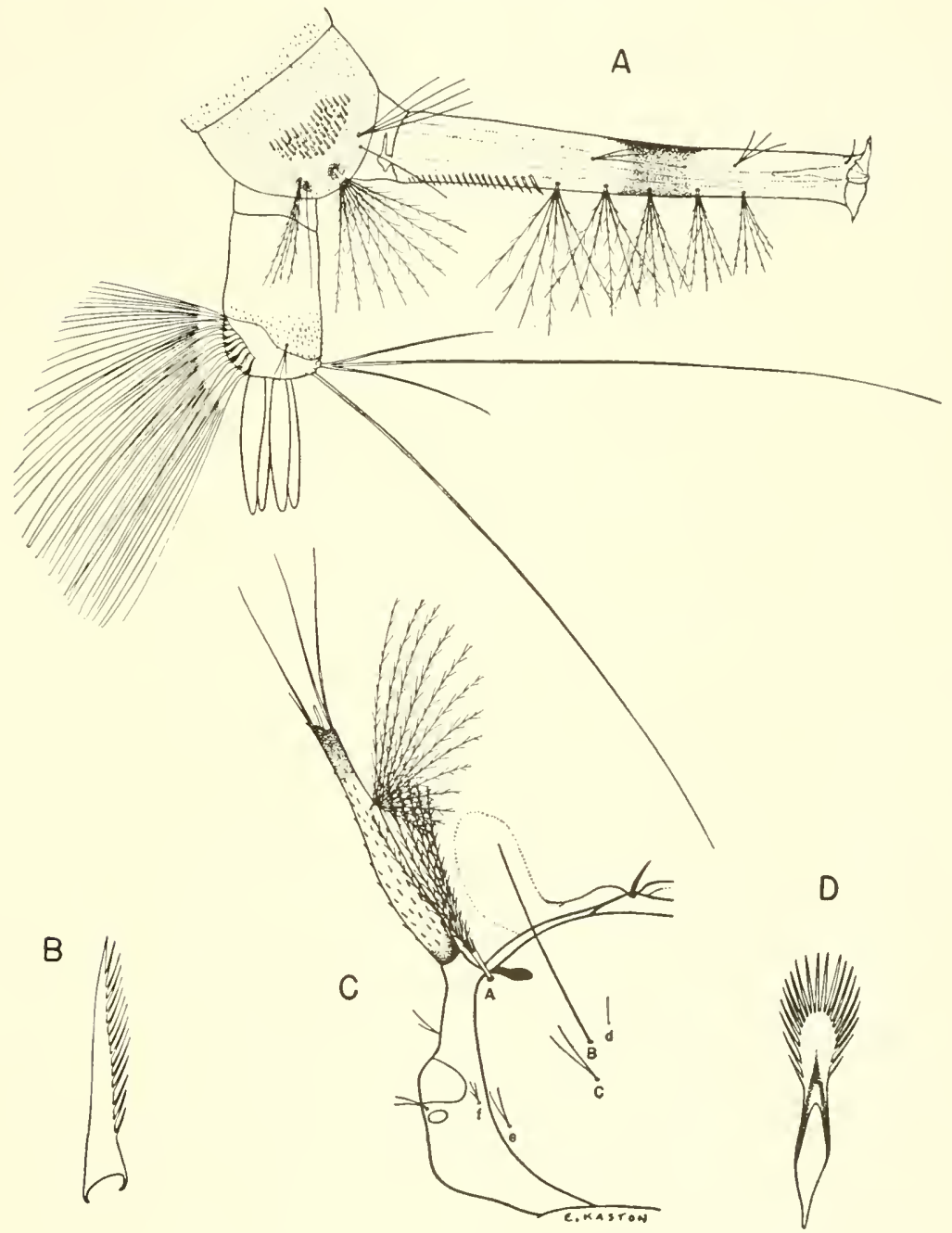

Fig. 149. Larva of Culex peccator Dyar and Knab. A, Terminal segments. B. Pecten tooth. C, Head. D, Comb scale. 
width of siphon; two 2 to 3 -branched subdorsal tufts present; dorsal preapical spine as long or longer than apical pecten tooth, recurved. Anal segment longer than wide, completely ringed by the dorsal plate; lateral hair usually 3-branched; dorsal brush consisting of a long lower caudal hair and an upper caudal tuft of 3 hairs on either side (one of the upper caudal hairs nearly as long as the lower caudal); ventral brush well-developed, confined to the barred area; gills 4 , shorter than the segment, tapered.

Distribution.-Eastern United States from Florida northward to Michigan and westward to Kansas. Southern States: Alabama (96); Arkansas (30); Florida and Georgia (96); Kentucky (140); Louisiana and Mississippi (96); Missouri (1); North Carolina (166); South Carolina and Tennessee (96); Virginia (49). Other States: Delaware (106); Kansas (128); Michigan (88); Oklahoma (159).

BIONOMics.-Adult specimens of $C$. peccator are frequently found in diurnal shelters, particularly in damp situations. They are occasionally taken in New Jersey type light traps. Since the females cannot be separated with certainty from other members of the subgenus Melanoconion in this region, nothing is known of their feeding habits. This is a summer species, but adults have been collected from April to December in the extreme South (4th Sv. C. Med. Lab. records, 1942-1944, unpublished). Larvae occur in stream pools and in marshy areas.

\section{Culex (Melanoconion) pilosus (Dyar and Knab)}

Mochlostyrax pilosus Dyar and Knab, 1906. Jour. N. Y. Ent. Soc., 14:223.

Culex pilosus King and Bradley, 1937, Ann. Ent. Soc. Amer., 30:353 (additional synonymy given).

adult female.-Small species. Head: Proboscis long, dark, slightly swollen at tip; palpi very short, dark. Occiput principally clothed with broad appressed scales, dark brown with bronze to metallic blue-green reflection on antero-dorsal portion, gradually fading to dingy-white laterally; a small median area of pale lanceolate scales present posteriorly; dark erect forked scales numerous. Cibarial armature consisting of 3 teeth (122). Thorax: Integument of scutum dark brown, clothed with fine dark bronzy-brown scales. Abdomen: Tergites dark brown or black scaled dorsally with bronze to metallic blue-green reflection and with basal white-scaled patches laterally (occasionally narrow white basal bands present dorsally on some segments). Sternites each with a white band basally, the band joining the white lateral tergal patches; dark apically. Legs: All legs dark scaled with bronze to metallic blue-green reflection, except for pale inner surfaces of femora. Wing: Scales all dark. Plume scales narrow; squame scales broader, ligulate to ovate.

ADUlt MALE.-Coloration similar to that of female. TERMinalia (Fig. 150). Lobes of ninth tergite (IXT-L) widely separated, slender, finger-like, about four times as long as broad, with tips slightly convergent; vestiture consisting of three or four short setae distributed on each lobe in addition to several small basal setae. Tenth sternite (X-S) crowned with a comb-like row 
of blunt spines. Phallosome ( $\mathrm{Ph})$ of two plates connected basally; each plate elongate, the tip with a short stout apical tooth, a stout subapical tooth on outer margin, and a strong pointed ventral tooth; base of each plate giving rise to a long tail-like hook directed ventrally. Claspette absent. Basistyle (Bs) nearly twice as long as broad, outer margin strongly curved, apex narrow; vestiture of scales and numerous setae. Subapical lobe (S-L) deeply divided into two distinct trunks: Posterior trunk bearing a long flattened pointed strongly recurved spine, three shorter slightly flattened rods, a moderately-broad weakly striated leaf-like filament, and two narrower leaf-like filaments; anterior trunk cleft nearly to base into two stout branches, each bearing a long strong rod with tip flattened, curved, and pointed (often with a small apical retrorse barb). Dististyle (Ds) nearly three-fourths as long as basistyle, curved, narrow medially, outer margin near tip expanded cap-like and bearing a row of very short reflexed setae, tip pointed and slightly reflexed; terminal claw (Ds-C) short, curved, blunt.

LARVA. (Fig. 151).-Head much broader than long. A stalked ovoid gill on ventral side of head at base of antenna. Antenna as long or longer than

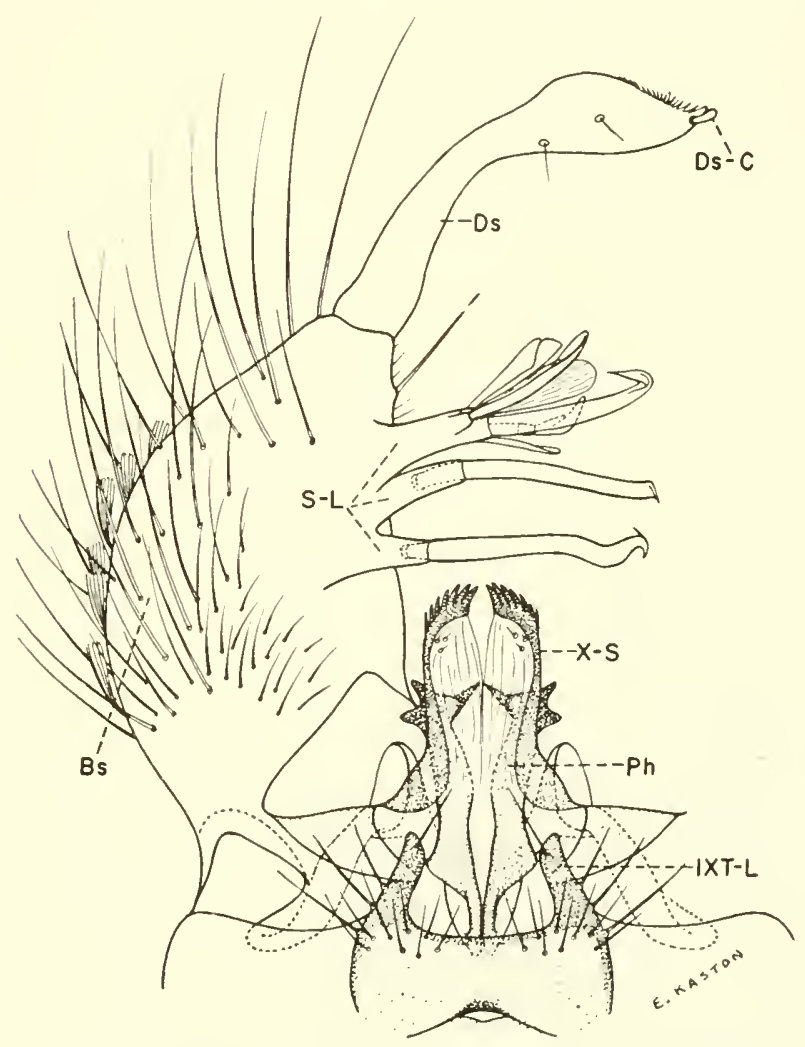

Fig. 150. Male terminalia of Culex pilosus (Dyar and Knab). 
head, constricted beyond antennal tuft, with portion before constriction spinose, portion beyond constriction more sparsely spined; antennal tuft large, multiple, barbed, inserted at outer third, reaching beyond tip of antenna. Head hairs: Preantennal (A) multiple, barbed, reaching near insertion of antennal tuft; lower (B) single, barbed, not reaching beyond preclypeus; upper (C) single, occasionally double, barbed, about half as long as lower (B) and much weaker; postclypeal (d) small, single; sutural (e) and trans-sutural (f) small, multiple; supraorbital usually double or triple. Body spicular. Upper lateral abdominal hairs usually triple on segments I and II and double on III to VI. Comb

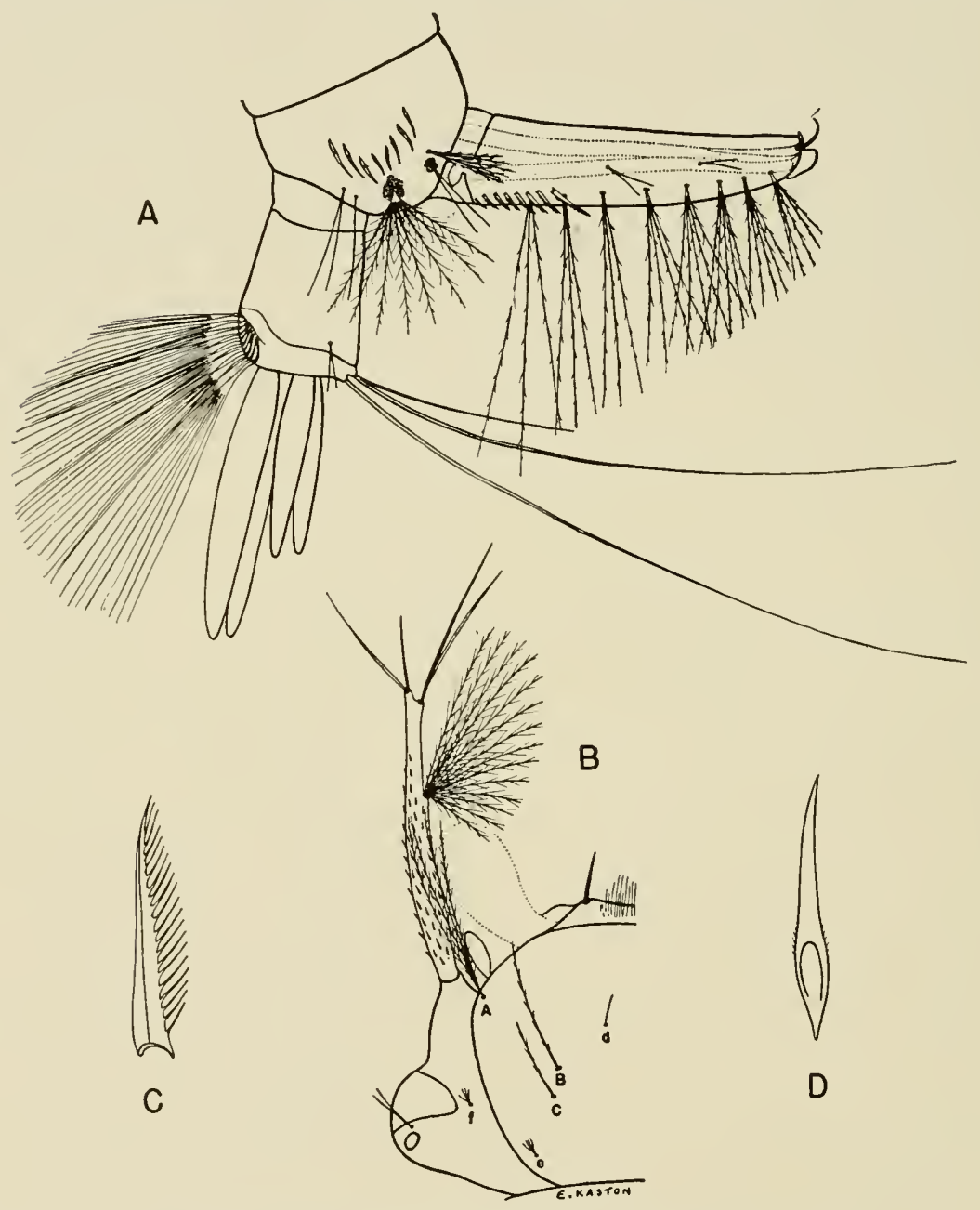

Fig. 151. Larva of Culex pilosus (Dyar and Knab). A, Terminal segments. B, Head. C, Pecten tooth. D, Comb scale. 
of eighth segment of about 8 to 12 scales in a single curved or irregular row; individual scale long, thorn-shaped, nearly smooth, but with a few minute spinules toward base. Siphon three to four times as long as basal width, distinctly upcurved; pecten of about 6 to 9 rather evenly spaced teeth on basal third of siphon; individual pecten tooth fringed on one side to tip; eight pairs of very long multiple barbed subventral tufts present, the basal two inserted within the pecten and nearly as long as siphon; two small 2 to 3 -branched tufts inserted laterally; dorsal preapical spine as long or longer than the apical pecten tooth, recurved. Anal segment a little longer than wide, completely ringed by the dorsal plate; lateral hair usually 2 to 3-branched; dorsal brush bilaterally consisting of a long lower caudal hair and an upper caudal tuft of one long and one short hair; ventral tuft well-developed, confined to the barred area; gills 4 , each tapered to a blunt point, the ventral pair as long or longer than the segment, the dorsal pair shorter.

distribution.--Southern United States, Mexico, Central America, West Indies and northern South America. Southern States: Alabama and Florida (96); Georgia (148); Kentucky (140); Louisiana (96); Mississippi (97); North Carolina (52); South Carolina (64).

BIONOMICS.-Females of this species cannot be separated with certainty from other members of the subgenus Melanoconion, therefore nothing is known of their feeding habits. The larvae occur mostly in pools of a temporary nature, such as ditches, grassy pools and floodwater areas. King et al. (96) state that the eggs are able to withstand drying, an unusual trait in this genus. Larvae have been collected from April to December in the extreme South (4th Sv. C. Med. Lab. records, 1942-1944, unpublished).

\section{Culex (Neoculex) apicalis Adams}

Culex apicalis Adams, 1903. Kans. Univ. Sci. Bull., 2:26.

Adult female.--Rather small species. Head: Proboscis long, dark scaled; palpi short, dark. Broad dorsal region of occiput clothed with whitish to golden lanceolate scales and numerous brown erect forked scales (forked scales of central portion often pale); lateral region of occiput clothed with broad whitish scales. Thorax: Integument of scutum light brown; vestiture of lanceolate scales (somewhat coarser than on C. restuans, salinarius, and nigripalpus), usually light brown, but varying on different specimens from light gray to dark brown; scales of anterior and lateral margins and of prescutellar space paler; frequently a pair of indefinite submedian spots of pale scales present near middle of scutum. Pleura with a few patches of broad white scales. Abdomen: Tergites dark-brown to black scaled with bronze to metallic blue-green reflection; each tergite except the first with a conspicuous apical triangular patch of white scales on either side, usually joined by a narrow dorso-apical band of similar scales. Venter whitish scaled. Legs: Legs dark scaled with bronze to metallic blue. green reflection except for pale inner surfaces of femora and tibiae and a pale streak usually present on one side of segment 1 of tarsi. Wing: Scales narrow, dark. 
ADUlt Male.-Coloration similar to that of female. TERMinalia (Fig. 152A). Lobes of ninth tergite (IXT-L) rounded, about as long as broad, separated by about twice the width of one lobe, each bearing several setae. Tenth sternite (X-S) crowned with short blunt spines, arranged in a somewhat comblike row. Phallosome $(\mathrm{Ph})$ formed of a pair of stout columnar structures (lateral plates) strongly joined like a U-bolt at base and with a narrow transverse bridge at apical fourth; apex of each column blunt, crowned with numerous small denticles. Claspette absent. Basistyle (Bs) about two and one-half times as long as basal width, with apex narrow, bluntly rounded; vestiture of numerous setae, longer on outer aspects. Subapical lobe (S-L) prominent, undivided, bearing two strong rods with tips pointed and recurved; four smaller flattened recurved spines, three of which are serrated on one side; and two stout setae. Dististyle (Ds) about half as long as basistyle, curved, slightly constricted at basal third, a little broader at apical third, then tapered to apex; terminal claw (Ds-C) about one-fifth as long as dististyle, with tip blunt and somewhat flattened.

LARva. (Fig. 153).-Head much broader than long. Antenna about as long as head, constricted beyond antennal tuft, with portion before constriction spinose, portion beyond constriction darker and with coarser and fewer spines;
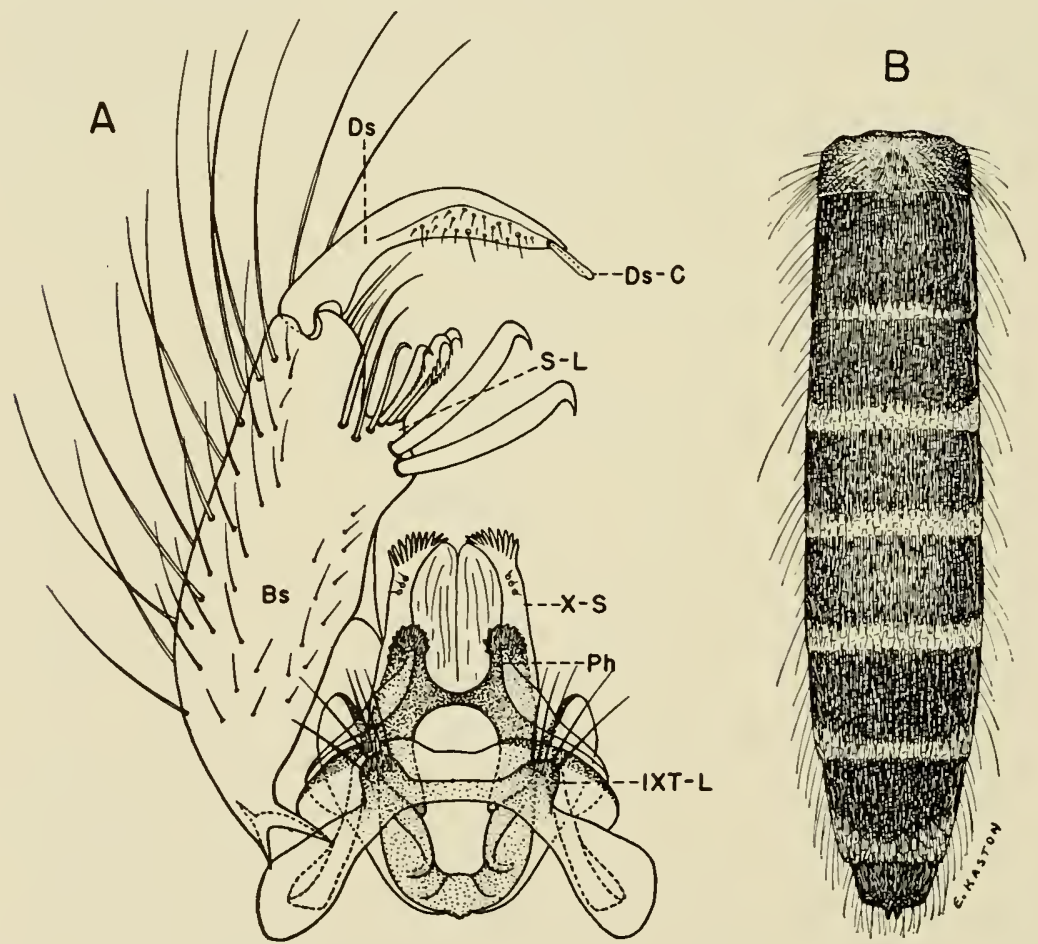

Fig. 152. Culex apicalis Adams. A, Male terminalia. B, Abdomen of female. 
antennal tuft large, multiple, barbed, inserted at outer third of shaft, reaching considerably beyond tip. Head hairs: Preantennal (A) multiple, barbed, extending to insertion of antennal tuft; lower (B) single, barbed, extending beyond insertion of antennal tuft; upper (C) single, barbed, about two-thirds as long as lower $(\mathrm{B})$ (either or both $\mathrm{B}$ and $\mathrm{C}$ occasionally double); postclypeal (d) small, single; sutural (e), trans-sutural (f) and supraorbital double or triple. Body finely spicular. Upper lateral abdominal hairs multiple on segments I and II, double on III to VI. Comb of eighth segment of numerous scales in a patch; individual scale rounded apically, fringed with subequal spinules. Siphon slender, about seven times as long as basal width, slightly expanded at the apex; pecten of 12 to 16 rather evenly spaced teeth on basal third of siphon; individual tooth with 1 to 4 long coarse side teeth; four or five pairs

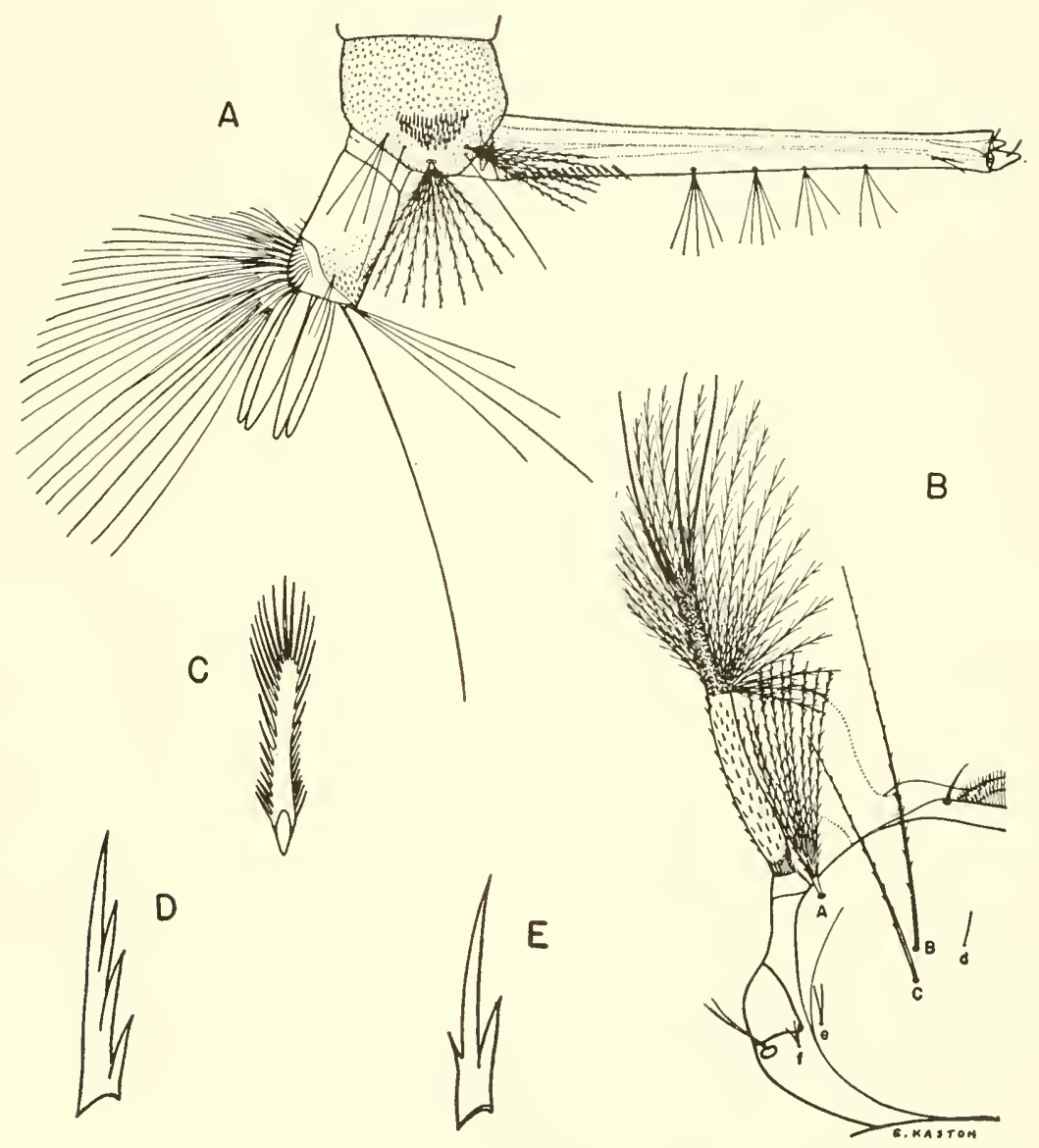

Fig. 153. Larva of Culex apicalis. A, Terminal segments.

B, Head. C. Comb scale. D, and E, Pecten teeth. 
of subventral tufts inserted beyond pecten, the individual hairs little if any longer than diameter of siphon (apical tuft inserted somewhat laterally). Anal segment nearly twice as long as wide, completely ringed by the dorsal plate; lateral hair usually double; dorsal brush consisting of a long lower caudal hair and a shorter 4-branched upper caudal tuft on either side; ventral brush large, with 2 or 3 small tufts preceding the barred area; gills 4 , about as long as the segment, tapered.

Distribution.-Widely distributed in North America, Europe and central Asia. Southern States: Alabama (170); Arkansas (30); Florida (52); Georgia (96); Kentucky (140); Louisiana (96); Mississippi (97); Missouri (1); North Carolina (166); South Carolina (64); Tennessee (170); Virginia (49). Other States: California (66); Colorado (128); Connecticut (21); Delaware (106); District of Columbia and Illinois (52); Indiana (76); Iowa (155, 156); Kansas (79); Maine, Maryland and Massachusetts (52); Michigan (88); Minnesota (131); Montana (112); Nebraska (178); New Hampshire (105); New Jersey (77); New Mexico (9); New York (52); Oklahoma (159); Pennsylvania (52); Rhode Island (99); Texas (108); Wisconsin (48).

BIONOMics.-Culex apicalis is not known to feed on man but has been observed feeding on cold-blooded animals, particularly frogs. The adults are rarely taken inside dwellings but are usually found amongst vegetation and in shelters near their breeding places. The larvae are found in semi-permanent and permanent pools, in streams, and in swamps. Larvae and adults occur throughout the year in the southern portion of its range but are rarely taken in southern Florida (4th Sv. C. Med. Lab. records, 1942-1944, unpublished).

\section{Genus Deinocerites Theobald 1}

Deinocerites Theobald, 1901, Mon. Culic., 2:215.

The genus Deinocerites contains a few species which are confined to the Caribbean area, the Gulf of Mexico and adjacent regions. All the known species breed in saltwater in crab holes and the adults rest in the upper part of the holes. The genus is represented by a single species, $D$. cancer, in the southern United States.

SALIENT CHARACTERS.-Adult: The adults resemble Culex but have the antenna much longer than the proboscis; the first flagellar segment is long in both sexes. Antennae and palpi are long in both males and females. Cerci prominent. Male Terminalia: Ninth tergite with two large elongated lobes. Tenth sternite with a crown of close-set teeth. Phallosome strongly toothed. Basistyle stout. Dististyle short, pubescent, with two terminal claws. Larva: Head with a prominent triangular pouch on either side. Comb of eighth segment consists of many scales in a patch. Siphon of moderate length, with pecten well developed. Dorsal plate sclerotized dorsally and ventrally, indistinct laterally. Two short, bulbous anal gills present.

1 See Edwards (1932) or Dyar (1928) for synonymy. 


\section{Deinocerites CANCER Theobald}

Deinocerites cancer Theobald, 1901, Mon. Culic, 2:215.

Adult FEMALE.-Medium-sized species. Head: Proboscis long, slender, dark scaled; palpi short, dark; antennae much longer than proboscis with the first flagellar segment three times as long as the second and equal in length to the second, third, and fourth segments combined. Occiput clothed with numerous coppery-brown erect forked and lanceolate scales. Thorax: Scutum covered by brown lanceolate scales with a coppery sheen; sternopleura shingled with rather broad, coppery scales. Spiracular and postspiracular bristles absent. Abdomen: Dorsum with dark metallic scales, venter tan; terminal segments somewhat compressed, truncate, with large cerci prominently displayed. Legs: All leg scales dark. Wing: Scales narrow, brown.

ADULT MALE.-Coloration similar to that of female; antennae and palpi similar to those of female, but with first segment of antenna less than twice as long as the second, and not equal in length to the second, third, and fourth segments combined. TERMinalia (Fig. 154). Ninth tergite (IX-T) with dorsal band narrow, curved; lobes (IXT-L) very large, widely separated, heavily sclerotized, flattened and elongate, extending to apical fourth of basistyle, blunt at tip and with numerous small setae on rounded inner surface at

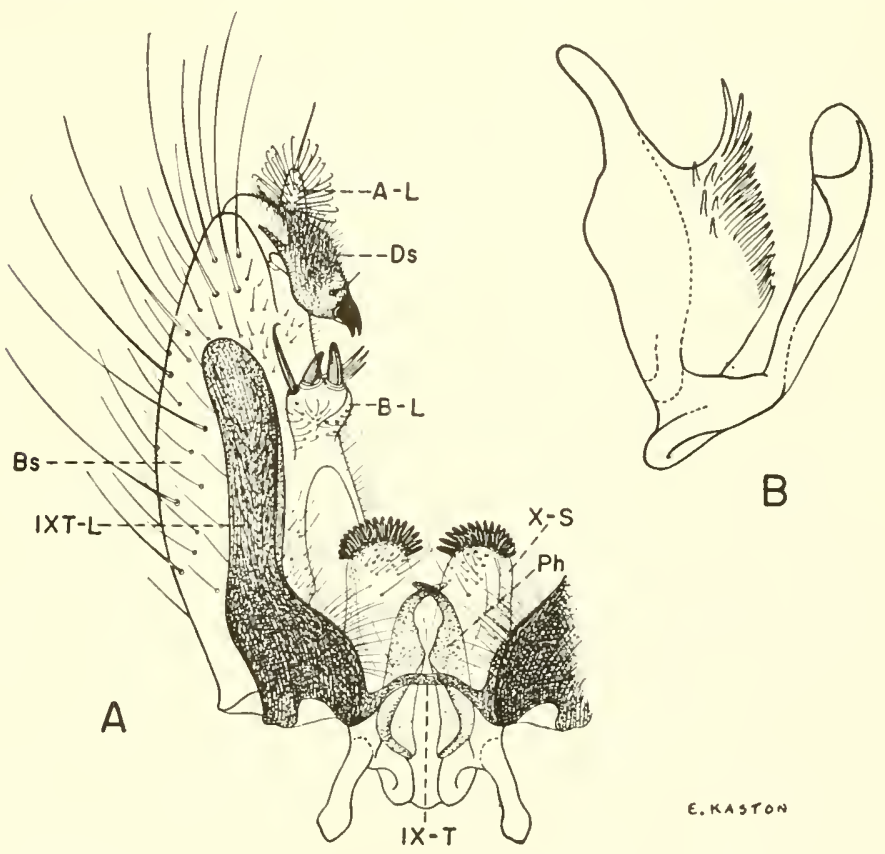

Fig. 154. Deinocerites cancer Theobald. A, Male terminalia. B, Phallosome (side view). 
base. Tenth sternite (X-S) stout, about half as long as lobe of ninth tergite, with a dome-like crown of numerous blunt heavily sclerotized teeth. Phallosome (Ph) large, heavily sclerotized, open dorsally and ventrally, with a pair of strong horn-like curved extensions apically, overlapping near tip; each plate with many long, slender filaments arising rib-like latero-ventrally and projecting ventro-posteriorly. Claspette absent. Basistyle (Bs) about two and one-half times as long as wide, gradually tapering to a rounded apex, clothed with many long and short setae; a fleshy, finger-like, posteriorly directed projection arising from inner margin near apex, probably representing the apical lobe; basal lobe (B-L) rounded, situated near middle of basistyle, bearing two short blunt dark rods and a longer more slender spine, pointed and recurved at tip. Dististyle (Ds) short, dark, densely covered with fine hairs, always held reflexed and not reaching basal lobe, swollen at apical third, obtusely pointed at tip; two stout, downcurved claws, pointed and nearly equal in size, inserted a little before apex.

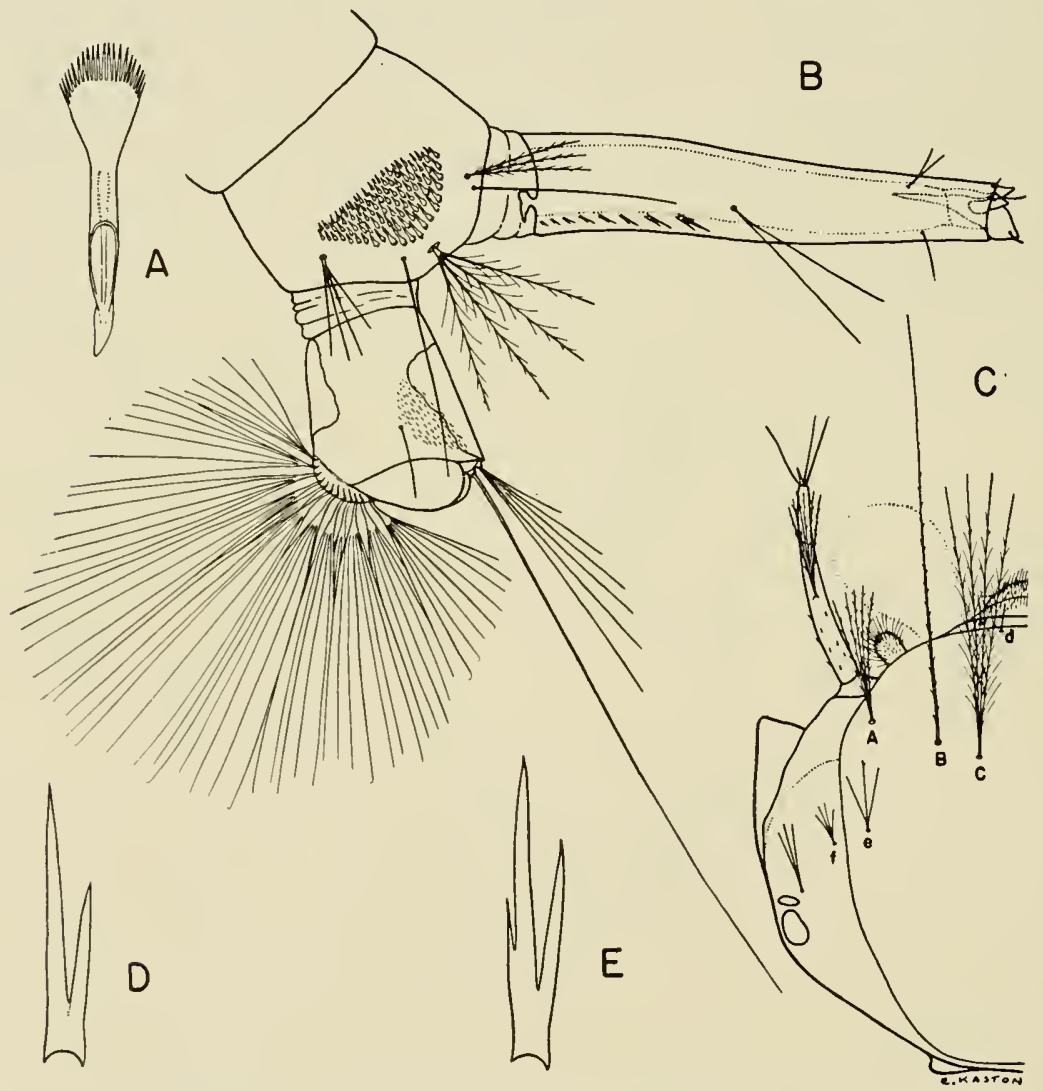

Fig. 155. Larva of Deinocerites cancer Theobald. A, Comb scale.

B, Terminal segments. C, Head. D, and E, Pecten teeth. 
LARVA. (Fig. 155).--Head slightly broader than long, almost circular in outline, with a prominent triangular pouch on either side. Antenna about half as long as the head, sparsely spined basally; antennal tuft multiple, sparsely barbed, inserted near middle, extending near tip of antenna. Eyes small, rounded. Inner preclypeal spine weak. Head hairs: Preantennal (A) multiple, barbed, extending nearly to insertion of antennal tuft; lower (B) very long, single, sparsely barbed; upper (C) shorter, usually 3 to 4-branched, barbed, extending beyond preclypeus; postclypeal (d) small, single, inserted near anterior margin of clypeus; sutural (e) usually triple; trans-sutural (f) multiple; supraorbital usually 2 to 4-branched. Comb of eighth segment of numerous scales in a patch; individual scale abruptly broadened and rounded apically, fringed with subequal spinules. Siphon about four to five times as long as basal width; pecten of 6 to 8 teeth progressively more widely spaced and restricted to basal third of siphon; a long double or triple subventral tuft near middle of siphon and two smaller 1 to 2-branched tufts apically, one of which is inserted subdorsally. Anal segment slightly longer than wide; dorsal plate sclerotized dorsally and ventrally, indistinct laterally; lateral hair single; dorsal brush consisting of a long lower caudal hair and a short multiple upper caudal tuft on either side; ventral brush well developed, confined to the barred area; gills 2, short, bulbous.

distribution.-Southern Florida (52); Antilles, Mexico and Central America.

BIONOMICS.-The adults of this species are frequently found resting in the upper portion of the crab holes in which they breed. Some observers claim that the females occasionally bite humans, but they are seldom encountered except in areas where breeding is heavy. The larvae are found in the holes of land crabs in the coastal salt marshes. T. E. Duffey collected several larvae of this species from a tin can, together with larvae of Aedes triseriatus, during October, 1944, on Jupiter Island near Hobe Sound, Florida. Duffey has also observed numerous specimens in the act of copulation while resting on the sides of crab holes. Larvae have been collected from February to December in Florida (4th Sv. C. Med. Lab. records, 1942-1944, unpublished). 


\section{BIBLIOGRAPHY}

I. Adams, C. F. and WM. M. Gordon. 1943-Notes on The Mosquitoes of Missouri (Diptera, Culicidae). Ent. News 54:232-235.

2. Aitken, T. H. G. 1940-The Genus Psorophora in California (Diptera, Culicidae). Rev. de Entomologia 11:672-682.

3. Aitken, T. H. G. 1945-Studies cn The Anopheline Complex of Western North America. Univ. Calif. Pub. in Ent. 7:273-364.

4. Baisas, F. E. 1938-Notes on Philippine Mosquitoes, Vii. A. Culex (Culex) with banded proboscis and tarsi. B. Anopheles: The pupae of three rare species; the leucosphyrus-subgroup. Monthly Bull. Philippine Hlth. Serv. 18:175-232.

5. Baker, F. C. 1936-A New Species of Orthopodomyia, O. alba, sp. $n$. Proc. Ent. Soc. Wash. 38:1-7.

6. Bang, F. B., G. E. Quinby and T. W. Simpsen. 1940-finopheles walleri Theobald: A Wild-Caught Specimen Harboring Malaria Plasmodia. U. S. Pub. Hlth. Serv. Rpts. 55:119..120.

7. Bang. F. B., G. E. Quinby, and T. W. Simpson. 1943-Studies on Anopheles walheri Theobald Conducted at Reelfoot Lake, Tennessee, 19351941. Amer. Jour. Trop. Med. $23: 247-273$.

8. Banks, H. W. 1937-New and Significant Experiences in Mosquito Control in Delaware County, Pennsylvania. N. J. Mosq. Exterm. Assoc. Prec. $24: 143-147$.

9. Barber, M. A. 1939-Further Observaticns (1938) cn the Anopheles of New Mexico. Amer. Jour. Trop. Med. 19:345-356.

10. Barber. M. A. and J. B. Rice. 1936-Methods of Dissecting and Making Permanent Preparations of the Salivary Glands and Stomachs of Anopheles. Amer. Jour. Hyg. 24:32-40.

11. Barraud, P. J. 1934-Fauna of British India Including Ceylon and Burma. Diptera, Vol. V (Culicidae, Megarhinini and Culicini). London, 403 pp.

12. Bates, M. 1940-The Nomenclature and Taxonomic Status of the Musquitoes of the Anopheles maculipennis Complex. Ann. Ent. Soc. Amer. 33:343-356.

13. Bates, M. 1944-Notes on The Construction and Use of Stable Traps for -Mosquito Studies. Jour. Nat. Mal. Soc. 3:135-145.

14. Bellamy, R. E. 1939-An Anopheline From Inland Georgia Resembling The Brackish-Water Race of Anopheles crucians. Jour. Parasitol. 25:186.

15. Bellamy, R. E. and J. Andrews. 1938 Symposium on Malaria-Part I. The Occurrence of Anopheles walleri Theobald in Georgia. South. Med. Jour. $31: 797$.

16. Bennett, B. L., F. C. Baker and A. W. Sellards. 1939-Susceptibility of The Mosquito Aedes triseriatus to the Virus of Yellow Fever Under Experimental Conditions. Ann. Trop. Med. and Parasitol. 33:101.

17. Beyer, G. E. 1923-Mosquitoes of Louisiana. La. State Bd. Hlth. Quart. Bul. 14:54-84.

18. Beyer, G. E. 1923-A New Species of Anopheles in Louisiana, Anopheles atropos Dyar and Kuab. Amer. Jour. Trop. Med. 3:351-363.

19. Bishopp, F. C.. E. N. Cory and Alan Stone. 1933-Preliminary Results of A Mosquito Survey in The Chesapeake Bay Section. Proc. Ent. Soc. Wash. 35:1-6.

20. Blacklock, D. B. and C. Wilson. 1942-Apparatus for the Collection of Mosquitoes in Ships, with Notes on Methods of Salivary Gland Dissection. Ann. Trop. Med. and Parasitol. 36:53-62.

21. Botsford, R. C. 1936-Progress of Mosquito Control in Connecticut, 1935. N. J. Mosq. Exterm. Assoc. Prcc. 23:151-153.

22. BoYd, MARK F. 1930-An Introduction to Malariology. Harvard Univ. Press, Cambridge, Mass. 437 pp.

23 Boyd, M. F., S. F. Kitchen and J. A. Mulrennan. 1936-On The Relative Susceptibility of The Inland and Coastal Varieties of A. crucians, Wied., to P. falciparum, Welch. Amer. Jour. Trop. Med. 16:159. 
24. Bradey, G. H. 1925-The Larva of Aedes thibaulti Dyar and Knab (Diptera: Culicidae). Insecutor Inscitiae Menstruus 13:89-9!.

25. Bradley, G. H. 1936-On The Identification of Mosquito Larvae of The Genus Anopheles Occurring In The United States (Diptera: Culicidae). South. Med. Jour. $29: 859-861$.

26. Bradley, G. H. and T. E. McNeel. 1935-Mosquito Collections in Florida with the New Jersey Light Trap. Jour. Econ. Ent. 28:780-786.

27. Bradley, G. H., R. F. Fritz and L. E. Perry. 1944-Additional Mosquito Records for the Scutheastern States. Jour. Econ. Ent. 37:109.

28. Brown, Frank R. and J. W. Pearson. 1938-Some Culicidae of the Reelfoot Lake Region. Jour. Tenn. Acad. Sci. 13:126-132.

29. Calrns, D. W. 1936-Mosquitoes of Fort Dupont, Delaware. Military Surgeon $78: 369-386$.

30. Carpenter, S. J. 194l-The Mosquitoes of Arkansas. Ark. State Bd. of Hith., Little Rock. 87 pp., rev. ed.

31. Carpenter, S. J. 1942-Mosquito Studies in Military Establishments in the Seventh Corps Area During 1941. Jour. Econ. Ent. 35:558-561.

32. Carpenter, S. J. 1945-Collection Records of Culex tarsalis (Coq.) in Army Camps in the Southeastern United States During 1942, 1943 and 1944. (ln press.)

33. Carpenter, S. J. 1945-A Simple Method of Packing Mosquito Larvae for Storage or Shipment. (In press.)

34. Carpenter, S. J. and W. W. Middlekauff. 194t-lnland Records of Salt Marsh Mosquitoes. Jour. Econ. Ent. 37:108.

35. Carpenter, S. J., R. W. Chamberlain and J. F. Wanamaker. 1945-New Distribution Records for the Mosquitoes of the Southeastern United States. (In press.)

36. Carr, H. P., J. F. Melendez and E. Ros. 1940-Malaria Reconnaissance of the Province of Oriente in Cuba. Amer. Jour. Trop. Med. 20:81-97.

37. Causey, O. R. 1943-A Method for the Collection, Transportation and Study of Anopheline Eggs and Adults. Amer. Jour. Trop. Med. 23:133-137.

38. Chamberlain, R. W. and T. E. Duffey. 1945-Collection Records of Mansonia titillans (Walker) and Mansonia indubitans Dyar and Shannon in Florida, with Keys to the Species of Mansonia in the United States. (In press.)

39. Christensen, G. R. and F. C. Harmston. 1944-A Preliminary List of the Mosquitoes of Indiana. Jour. Econ, Ent. 37:110-111.

40. Christophers, S. R. 1933-The Fauna of British India. Diptera, Vol. IV (Culicidae, Anophelini). London, $371 \mathrm{pp}$.

41. Christophers, S. R., J. A. Sinton and G. Covell. 1939-How to Do a Malaria Survey. Hith. Bull. No. 14. Calcutta. 208 pp.

42. Clarke, J. Lyell. 1936-Progress of the Mosquito Control Campaign in the Des Plaines Valley Area. N. J. Mosq: Exterm. Assoc. Proc. 23:98-111.

43. Cockerell, T. D. A. 1918-The Mosquitoes of Colorado. Jour. Econ. Ent. $11 ; 195-200$.

44. Cole, F. R. and A. L. Lovett. 1921-An Annotated List of the Diptera (Flies) of Oregon, XV. Calif. Acad. Sci. Proc., ser. 4, 11:197-34t.

45. Crampton, C. C. 1942-External Morphology of the Diptera, Guide to the Insects of Connecticut, part VI, Fasc. 1. Conn. State Geo. and Nat. Hist. Surv. Bul. $64: 10-165$.

46. Day, M. F. 1943--Report on Mosquitoes Collected in St. Louis County During 1942. Trans. St. Louis Acad. Sci. $31: 29-45$.

47. Dethier, V. G. and F. H. Whitley. 194t-Population Studies of Florida Mosquitoes. Jour. Econ. Ent. $37: 480-484$.

48. Dickinson, W. E. 1944-The Mosquitoes of Wisconsin. Milwaukee Pub. Mus. Bul. 8:269-365.

49. Dorer, R. E., W. E. Bickley and H. P. Nicholson. 1944-An Annctated List of the Mosquitoes of Virginia. Mosquito News $4: 48-50$. 
50. Dorsey, C. K. 1944-Mosquito Survey Activities at Camp Peary, Va. Ann. Ent. Soc. Amer. 37:376-387.

51. Dyar, H. G. 1921-The Mosquitoes of Canada. Royal Canad. Inst., Trans. $13: 71-120$.

52. Dyar, H. G. 1922-The Mosquitoes of the United States. Proc. U. S. Nat. Mus. $62: 1-119$.

53. Dyar, H. G. 1924-The Mosquitoes of Colorado. Insecutor Insecitiae Menstruus $12: 39-46$.

54. Dyar, H. G. 1928 -The Mosquitoes of the Americas. Ca:negie Inst. Wash. Pub. 387: 616 pp.

55. Edwards, F. W. 1932-Diptera, Fam. Culicidae, in P. Wystman. Genera insectorum, fas. 194. Bruxelles: V. Verteneuil and L. Desmet. 258 pp.

56. Edwards, F. W. 1941-Mosquitoes of the Ethiopian Region. (Vol. III, Culicine Adults and Pupae). British Museum of Natural History, London. 499 pp.

57. Evans, A. M. 1938-Mosquitoes of the Ethiopian Region. (Vol. II, Anophelini, Adults and Early Stages). British Museum of Natural History, Londen. 417 pp.

58. Eyles, D. E.1944-A Critical Review of the Literature Relating to the Flight and Dispersion Habits of Anopheline Mosquitoes. U. S. Pub. Hlth. Serv. Bul. No. 287. 39 pp.

59. Eyles, D. E. and R. W. Burgess. 1945-Anopheles walleri in South Carolina. Jour. Eccn. Ent. 38:115.

60. Feemster, R. F. and V. A. Getting. 1941-Special Report of the Department of Public Health Relative to Varieties and Prevalence of Mosquitoes in the Commonwealth. Commonwealth of Massachusetts, House Bill 2260. $70 \mathrm{pp}$.

61. Fellton, H. L. 1944-The Breeding of the Salt-Marsh Mosquito in Midwestern States. Jour. Econ. Ent. 37:245-247.

62. Felt, E. P. 1904-Mosquitoes or Culicidae of New York State. N. Y. State Mus. Bul. 79:241-400.

63. Fisk, Frank W. 1939-New Mosquito Records from Key West, Florida. Jour. Econ. Ent. $32: 469$.

64. Fisk, Frank W. and J. H. Le VAN. 1940-Mosquito Collections at Charleston, South Carolina, Using the New Jersey Light Trap. Jour. Econ. Ent. 33: 578-579.

65. Fisk, F. W. and J. H. LE VAN. 1941-Mosquito Collections at Brownsville, Texas. Jour. Econ. Ent. 33:944.

66. Freeborn, S. B.1926-The Mosquitoes of California. Univ. Calif. Pubs., Tech. Bul. Ent. 3:333-460.

67. Freeborn, S. B. and B. Brookman. 1943-Identification Guide to the Mosqnitoes of the Pacific Coast States. U. S. Pub. Hlth. Serv., M. C. W. A., Atlanta, Georgia. 23 pp.

68. Goodwyn, M. H. 1942-Studies on Artificial Resting Places of Anopheles quadrimaculatus Say. Jour. Nat. Mal. Soc. 1:93-99.

69. Griffitts, T. H. D. 1927-Anopheles atropos Dyar and Knab, A Note on its Breeding and Other Habits. U. S. Pub. Hlth. Serv. Rpt's. 42:1903-1905.

70. Gurney, A. B. 1943-A Mosquito Survey of Camp Crowder, Missouri During 1942. Jour. Econ. Ent. 36:927-935.

71. Guyton, F. E. 1935-Pest Mosquito Control in Alabama Under C. W. A. Jour. Econ. Ent. 28:786-790.

72. Hackett, L. W. and A. Missiroli. 1935-The Varieties of Anopheles maculipennis and Their Relation to the Distribution of Malaria in Europe. Riv. di Malariol. $14: 45-109$.

73. Hammon, W. McD. and W. C. Reeves. 1943-Laboratory Transmission of St. Louis Encephalitis Virus by Three Genera of Mosquitoes. Jour. Exp. Med. 78:241-253.

74. Hammon, W. McD. and W. C. Reeves. 1943-Laboratory Transmission of 
Western Equine Encephalomyelitis Virus by Mosquitoes of the Genera Culex and Culiseta. Jour. Exp. Med. 78:425-434.

75. Hammon, W. McD., W. C. Reeves, B. Brookman, and E. M. Izumi. 1942Mosquitoes and Encephalitis in the Yakima Valley, Washington. I. Arthropods Tested and Recovery of Western Equine and St. Louis Viruses from Culex tarsalis Coq. Jour. Inf. Dis. 70:263.

76. HART, J. W. 1944--A Preliminary List of the Mosquitoes of Indiana. Amer. Mid. Nat. $31: 414-416$.

77. Headlee, T. J. 1945-The Mosquitoes of New Jersey and Their Control. Rutgers Univ. Press, 326 pp.

78. Hearle, E. 1926-The Mosquitoes of the Lower Fraser Valley, British Columbia and Their Control. Nat. Res. Council Rpt. No. 17: 94 pp.

79. Hill, N. DeM. 1939-Biological and Taxcnomic Observations on the Mosquitoes of Kansas. Trans. Kan. Acad. Sci. 42:255-265.

80. Hinman, E. H. 1932-A Description of the Larva of Anopheles atropos Dyar and Knab, with Biological Notes on the Species. Proc. Ent. Soc. Wash. $34: 138-142$.

81. Hopkins, G. H. E. 1936-Mosquitoes of the Ethiopian Region. (Vol. I, Larval Bionomics of Mosquitoes and Taxonomy of Culicine Larvae). British Museum of Natural History, London. $498 \mathrm{pp.}$

82. Horsfall, W. R. 1936-Occurrence and Sequence of Mosquitoes in Southeastern Arkansas in 1935. Jour. Econ. Ent. 29:676-679.

83. Horsfall, W. R. 1937-Mosquitoes of Southeastern Arkansas. Jour. Econ. Ent. 30:743-748.

84. Horsfall, W. R. 1939-Habits of Aedes thibaulti Dyar and Knab. (Diptera, Culicidae). Jour. Kans. Ent. Soc. 12:70-71.

85. Horsfall, W. R. 1942-Breeding Habits of a Rice Field Mosquito. Jour. Econ. Ent. $35: 478-482$.

86. Horsfall, W. R. 1942-Biology and Control of Mosquitoes in the Rice Area. Ark. Agr. Exper. Sta. Bul. 427: 46 pp.

87. Howard, L. O., H. G. DYar and F. KNAB. 1912-17-The Mosquitoes of North and Central America and the West Indies. Carnegie Inst. Wash. Pub. 159: 4 vols., 1064 pp.

88. Irwin, H. W. 1941-A Preliminary List of the Culicidae of Michigan. Part I. Culicinae (Diptera). Ent. News 52:101-105.

89. James, M. T. 1942-A Two-Season Light Trap Study of Mosquitoes in Colorado. Jour. Econ. Ent. 35:945.

90. Johnson, C. W. 1925-List of Diptera. Fauna of New England, 15. Boston Soc. Nat. Hist. 7: $326 \mathrm{pp}$.

91. King, W. V. 1937-On the Distribution of Anopheles albimanus and Its Occurrence in the United States. South. Med. Jour. 30:943-946.

92. King, W. V. 1939-Varieties of Anopheles crucians Wied. Amer. Jour. Trop. Med. 19:461-471.

93. King, W. V. and G. H. Bradery. 1937-Notes on Culex erraticus and Related Species in the United States (Diptera, Culicidae). Ann, Ent. Soc. Amer. 30:345-357.

94. King, W. V. and G. H. Bradley. 1941-Distribution of the Nearctic Species of Anopheles. Human Malaria. Amer. Assoc. Adv. Sci. Pub. 15: 71-78.

95. King, W. V. and G. H. Bradley. 1941-General Morphology of Anopheles and Classification of the Nearctic Species. Human Malaria. Amer. Assoc. Adv. Sci. Pub. 15: 63-70.

96. Kinc, W. V., G. H. Bradley and T. E. McNeel. 1944-The Mosquitoes of the Southeastern States. U. S. Dept. Agr., Misc. Pub. 336, rev., 96 pp.

97. King, W. V.. L. Roth, J. Toffaleti, and W. W. Middekauff. 1943New Distribution Records for the Mosquitoes of the Southeastern United States During 1942. Jour. Econ. Ent. 36:573-577.

98. Kitchen, S. F, and G. H. Bradley. 1936-Anopheles walkeri Theobald as a Vector of Plasmodium falciparum (Welch). Amer. Jour. Trop. Med. $16: 579-581$. 
99. Knutson, Herbert. 1943-The Status of the Mosquitoes of the Great Swamp in Rhode Island During 1942. Jour. Econ. Ent. 36:311-319.

100. Комp, W. H. W. 1942-A Technique for Staining, Dissecting and Mounting the Male Terminalia of Mosquitoes. U. S. Pub. Hlth. Serv. Rpts. $57: 1327-1333$.

101. Kumm, H. W. and H. Zuniga. 1942-The Mosquitoes of El Salvador. Amer. Jour. Trop. Med. 22:399-415.

102. Lane, J. and N. L. Cerqueira. 1942-Os Sabetineos Da America (Diptera, Culicidae). Arquivos Zool. Estad. Sao Paulo 7:473-849.

103. Lathrop, Frank H. 1939-Mosquitoes Collected in Maine. Maine Agr. Exp. Sta. Bul. 397:828-829.

104. Leprince, J. A. and A. J. Orenstein. 1916-Mosquito Control in Panama. G. Putnam's Sons, New York and London. 335 pp.

105. Lowry, P. R. 1929-Mosquitoes of New Hampshire, A Preliminary Report. N. H. Agr. Exp. Sta. Bul. 243: 23 pp.

106. MacCreary, D. and L. A. Stearns. 1935-Effect of Drainage Work Accomplished by the CCC Upon the Prevalence of Mosquitoes at Lewes, Delaware, During 1934. N. J. Mosq. Exterm. Assoc. Proc. 22:115-121.

107. Macfie, J. W. S. 1920 The Chaetotaxy of the Pupa of Stegomyia fasciata. Bul. Ent. Res. 10:161-169.

108. McGregor, T. and R. B. Eads. 1943-Mosquitoes of Texas. Jour. Econ. Ent. 36:938-940.

109. McNeEl, T. E. 1931-A Method for Locating the Larvae of the Mosquito Mansonia. Science 74:155.

110. MCNEEL, T. E. 1932-Observations on the Biology of Mansonia perturbans (Walker). Diptera, Culicidae. N. J. Mosq. Exterm. Assoc. Proc. 19:91-96.

111. Magoon, E. H. 1935-A Portable Stable Trap for Capturing Mosquitoes. Bul. Ent. Res. 26:363-372.

112. Mall, G. A. 1934-The Mosquitoes of Montana. Mont. Agr. Exp. Sta. Bul. 288: 72 pp.

113. Marshall, J. F. 1938-The British Mosquitoes. Br. Mus. Nat. Hist., London. $341 \mathrm{pp}$.

114. Martini, E. 1935-Los Mosquitos de Mexico. Dept. Salub. Pub., Bol. Tec. Ser. A: Entom. Med. y Parasitol 1:1-65.

115. Matheson, R. 1934-Notes on Psorophora (Janthinosoma) horridus (Dyar and Knab). Proc. Ent. Soc. Wash. 36:41-43.

116. Matheson, R. 1944-A Handbook of the Mosquitoes of North America. Comstock Pub. Co., 314 pp.

117. Matheson, R., M. F. Boyd and W. K. Stratman-Thomas. 1933-Anopheles walleri Theobald as a Vector of Plasmodium vivax Grassi and Feletti. Amer. Jcur. Hyg. 17:515-516.

118. Mayne, Bruce. 1917-The Malaria Parasite in the Mosquito. The Effects of Low Temperature and Other Factors on Its Development. U. S. Pub. Hith. Serv. Rpts. $32: 1400$.

119. Mayne, Bruce. 1919-The Occurrence of Malaria Parasits in Anopheles crucians in Nature: Percentage of Infection of Anopheles quadrimaculalus and Latest Date Found Infected in Northern Louisiana. U. S. Pub. Hith. Serv. Rpts. 34:1355-1357.

120. Mayne, Bruce and T. H. D. Griffitts. 1931-Anopheles atropos D. and K. A New Potential Carrier of Malaria Organisms. U. S. Pub. Hith. Rpts. 46:3107.

121. Mentzner, R. L. 1938-New Developments in Mosquito Control in Delaware County, Pennsylvania. N. J. Mosq. Exterm. Assoc. Proc. 25:125-130.

122. Michener, C. D. 1944-Differentiation of Females of Certain Species of Culex by Cibarial Armature. Jour. N. Y. Ent. Soc., 52:263-266.

123. Middlekauff, W. W. 1944-A New Species of Aedes From Florida (Diptera: Culicidae). Proc. Ent. Soc. Wash. 46:42-44.

124. Middlekauff, W. W. 1944-A Rapid Method For Making Permanent Mounts of Mosquito Larvae. Science $99 ; 206-207$. 
125. Middlekauff, W. W. and S. J. Carpenter. 1944-New Distribution Records for the Mosquitoes of the Southeastern United States in 1943. Jour. Econ. Ent. 37:88-92.

126. Mulhern, T. D. 1942-New Jersey Mechanical Trap for Mosquito Surveys. N. J. Agr. Exp. Sta. Circ. No. 421: 8 pp.

127. Munro, J. A. and S. Saugstad. 1939-A Preliminary Survey of Mosquitoes in North Dakota. N. D. Agr. Exp. Sta. Bimonthly Bul. 1:7.

128. Olson, T. A. and H. L. Keegan. 1944-New Mosquito Distribution Records From the Seventh Service Command Area. Jour. Econ. Ent. 37:847-848.

129. Olson, T. A. and H. L. KeEgan. 1944-The Mosquito Collecting Program of the Seventh Service Command for 1942-1943. Jour. Econ. Ent. 37: $780-785$.

130. O'Neill, K., L. J. Ogden and D. E. Eyles. 1944-Additional Species of Mosquitoes Found in Texas. Jour. Econ. Ent. 37:555-556.

131. Owen, W. B. 1937-The Mosquitoes of Minnesota, with Special Reference to Their Biologies. Univ. Minn. Agr. Exp. Sta., Tech. Bull. No. 126: 75 pp.

132. Owen, W. B. 19+2-The Biology of Thcobaldia inornala Williston, in Captive Colony. Jour. Econ. Ent. 35:903-907.

133. Perez, Manuel. 1930-An Anopheline Survey of the State of Mississippi. Amer. Jour. Hyg. $11: 696-710$.

134. Portman, Roland IW. 1943-New Mosquito Records for Colorado. Jour. Kans. Ent. Soc. 16:155.

135. Pratt, H. D. 1945-Mansonia indubitans Dyar and Shannon-A New Mosquito Addition to the United States Fauna, In press.

136. Pritchard, A. E. and H. D. Pratt. 1944-1. A Comparison of Light Trap and Animal Bait Trap Anopheline Mosquito Collections in Puerto Rico. I1. A List of the Mosquitoes of Puerto Rico. U. S. Pub. Hith. Rpts. $\mathbf{5 9}: 221-233$.

137. Proctor, William. 1938-Biological Survey of the Mount Desert Region. Wistar Inst. of Anat. and Biology (Insecta), 496 pp.

138. Pryor, J. E. and R. W. Chamberlain, 1944-Differentiating the Larvae of Uranotacnia in the Southeast. Jour. Econ. Ent. 37:543-544.

139. PurI, I. M. 1942-A Practical Entomological Course for Students of Malariology. Hlth. Bul. No. 18. Calculta, 189 pp.

140. Quinby, G. E., R. E. Serfling and J. K. Neel. 1944-Distribution and Prevalence of the Mosquitoes of Kentucky. Jour. Econ. Ent. 37:547-550.

141. Randolph, N. M. and Kellie O'Neil. 1944-The Mosquitoes of Texas. Bul. Texas State Hlth. Dept.. Austin. 100 pp.

142. Rees, D. M. 1943-The Mosquitoes of Utah. Bul. Univ. Utah 33(7): 99 pp.

143. Rees, D. M. 1944-A New Mosquito Record from Utah (Diptera: Culicidae). Pan. Pac. Ent. 20:19.

144. Reeves, IV. C. 1941-The Genus Orthopodomyia Theobald in California. Pan. Pac. Ent. 17:69-72.

145. Reeves, W. C. and W. McD. Hammon. 1944-Feeding Habits of the Proven and Possible Vectors of Western Equine and St. Louis Encephalitis in the Yakima Valley, Wash. Amer. Jour. Trop. Med. 24:131-134.

146. Remington, Charles L. 1945-The Feeding Habits of Uranolacnia lowii Theobald (Diptera: Culicidae). Ent. News 56:32-37, 64-68.

147. Riley, W. A. and W. Chalgren. 1939-The Pest Mosquito Problem in Minneapolis-St. Paul Metropolitan Area. Jour. Econ. Ent. 32:553-557.

148. Root, Francis M. 1924-Notes on the Mosquitoes of Lee County, Georgia. Amer. Jour. Hyg. $4: 449-455$.

149. Ross, E. S. 1943-The Identity of Aedes bimaculetus (Coquillett) and a New Subspecies of Aedes fulvus (Wiedemann) from the United States (Diptera: Culicidae). Proc. Ent. Soc. Wash. 45:143-151.

150. Ross, E. S. and H. R. Roberts. 1943-Mosquito Atlas. Part 1. The Nearctic Anophcles, Important Malaria Vectors of the Americas and Acdes aegypti and Culcx quinquefasciatus. Amer. Ent. Soc., 44 pp. 
151. Roth, L. M. 1943-A Key to the Culex (Diptera: Culicidae) of the Southeastern United States by Male Terminalia, Jour. Kans. Ent. Soc. 16: 117-133.

152. Rотн, L. M. 1944-A Key to the Anopheles of the Southeastern United States, by Male Genitalia (Diptera, Culicidae). Amer. Mid. Nat. $31: 96-110$.

153. Roth, L. M. 1945-The Male and Larva of Psorophora (Janthinosoma) horrida (Dyar and Knab) and a New Species of Psorophora from the United Stales (Diptera: Culicidae). Proc. Ent. Soc. Wash. 47:1-23.

154. Roth, L. M. and F. N. Young. 1944-Culex (Mclanoconion) alralus Theobald in Florida; A New Continental North American Record, with Notes on the Other Melanoconions of the Southeastern United States. Ann. Ent. Soc. Amer. $37: 84-88$.

155. Rowe, J. A. 1942-Preliminary Report on lowa Mosquitoes. Iowa State Coll. Jour. Sci. 16:211-225.

156. Rowe, J. A. 1942-Mosquito Light Trap Catches from Ten Iowa Cities, 1940. lowa State Coll. Jour. Sci. 16:487-518.

157. Rozenoom, L. E. 1939-The Larva of Psorophora (Janthinosoma) horrida (Dyar and Knab) (Diptera: Culicidae). Jour. Parasitol. 25:145-147.

158. Rozeroom, L. E. 1941-Distribution and Ecology of the Anopheles Mosquitoes of the Caribbean Region. Amer. Assoc. Adv. Sci. Pub. 15:98-107.

159. Rozeвoom, L. E. 1942-The Mosquitoes of Oklahoma. Okla. Agr. Exp. Sta., Tech. Bul. T-16: 56 pp.

160. Ruland, LeRoy W. 1940-Report of the Suffolk County Mosquito Extermination Commission. $32 \mathrm{pp}$.

161. Ruland, LeRoy W. 1943-Report of the Suffolk County Mosquito Extermination Commission. $24 \mathrm{pp}$.

162. Ruland, LeRoy W. 1944-Report of the Suffolk County Mosquito Extermination Commission. $16 \mathrm{pp}$.

163. Russell, P. F., L. E. Rozeboom and Alan Stone. 1943-Keys to the Anopheline Mosquitoes of the World. Amer. Ent. Soc., $152 \mathrm{pp}$.

164. Sснмiтt, J. B. 1942-Five Species of Mosquitoes New to New Jersey, Found in Last Five Years. Mosquito News 2:26-29.

165. Schoof, H. F. 1944-Adult Observation Stations to Determine Effectiveness of the Control of Anopheles quadrimaculatus. Jour. Econ. Ent. 37:770-779.

166. Schoof, H. F. and D. F. Ashton. 1944-Notes and New Distribution Records on the Mosquitoes of North Carolina. Jour. Elisha Mitchell Sci. Soc. $60: 1-10$.

167. Schward, H. H. 1939-Biologies of Arkansas Rice Field Mosquitoes. Ark. Agr. Exper. Sta. Bul. 377: 22 pp.

168. Senevet, G. 1930 -Contribution á l'etudé des nymphes de Culicides. Arch. Inst. Pasteur d'Algerie 8:297-382.

169. Shannon, R. C. 1934--The Genus Mansonia in the Amazon Valley. Proc. Ent. Soc. Wash. 36:99-110.

170. Shields, S. E. 1938-Tennessee Valley Mosquito Collections. Jour. Econ. Ent. $31: 426-430$.

171. Shields, S. E. and V. I. Miles. 1937-The Occurrence of Orthopodmyia alba in Alabama (Diptera: Culicidae). Proc. Ent. Soc. Wash. 39:237.

172. Simmons, J. S. 1941-The Transmission of Malaria by the Anopheles Mosquitoes of North America. Amer. Assoc. Adv. Sci. Pub. 15:113-130.

173. Simmons, J. S. and T. H. G. Aitken. 1942-The Anopheles Mosquitoes of the Northern Half of the Western Hemisphere and of the Philippine Islands. Army Med. Bul. 59: 213 pp.

174. Smart, J. 1943-Insects of Medical Importance. Br. Mus. Nat. Hist. London, $269 \mathrm{pp}$.

175. Sмiтн, Gordon E. 1942-The Keg Shelter as a Diurnal Resting Place of Anopheles quadrimaculatus. Jour. Trop. Med. 22:257-269.

176. Stearns, L. A. 1940-The Present Mosquito Control Situation in Delaware. N. J. Mosq. Exterm. Assoc. Proc. 27:106-112. 
177. Stratman-Thomas, W. K. and F. C. B.ker. 1936-Anopheles barberi Coquillett as a Vector of Plasmodium vivax Grassi and Feletti. Amer. Jour. Hyg. $24: 182-183$.

178. Tate, D. H. and D. B. Gates. 1944-The Mosquitoes of Nebraska. Univ. Neb. Coll. Agr. Exp. Sta., Res. Bul. 133: 27 pp.

179. Thibault, J. K. 1910-Notes on the Mosquitoes of Arkansas. Proc. Ent. Soc. Wash. $12: 13-26$.

180. Tulloch, G. S. 1939-A Key to the Mosquitoes of Massachusetts. Psyche $46: 113-136$.

181. Twinn, C .R. 1931-Notes on the Biology of Mosquitoes of Eastern Canada. N. J. Mosq. Exterm. Assoc. Proc. 18:10.

182. VARGas, L. 1940-Clave para Identificar las Larvas de Anopheles Mexicanos. Ciencia $1: 66$.

183. Viosca, Percy. 1924-Report of the Entomologist. Parish of New Orleans and City of New Orleans. Bd. Hith. Ann. Rpt., 1923, $31: 47$.

184. Viosca, Percr. 1925-A Bionomical Study of the Mosquitocs of New Orleans and Southeastern Louisiana. N. J. Mosq. Exterm. Assoc. Proc. 12:34-50.

185. Wanamaker, J. F. 1944-An Improved Method for Mounting Mosquito Larvae. Amer. Jour. Trop. Med. $24: 385-386$.

186. Wanamaker, J. F., R. W. Chamberlain and S. J. Carpenter. 1944--Distribution of Culex pipiens in the Southeastern United States. Jour. Econ. Ent. $37: 106-107$.

187. Wilcox, A. and L. Locan, 1941-The Detection and Differential Diagnosis of Malarial Parasites in the Schizogonous and Sporogonous Cycles. Amer. Assoc. Adv. Sci. Pub. 15:47-62.

188. Wirth, W. W. 1944-Notes on the Occurrence of Anopheles georgianus King in Louisiana. Jour. Econ. Ent. 37:446. 


\section{INDEX}

abdomen: adult, 32, 33; anopheline larvae, 45, 46, 47; culicine larvae, 42-44; larval segmentation, 41; pupä

accessory gland 17

accessory paddle hair acrostichal bristles acus

adult: collection, 6-13; life history, 3, 4; taxonomic characters, 23-37; transporting, mounting and storing, $13-15$

Aedes, 165; apical lobe, 35; basal lobe, 35; bird malaria, 5; claspettes, 36; collecting adults, II; diagram of fourth instar larva, 40; dististyle, 36; eggs, 2, 18; eighth abdominal segment of adult, 33; encephalitides, 6; female abdomen, 33; filariasis, 6; heartworm of dogs, 6; key to adult females, male terminalia and larvae, 166-170; male terminalia, 34; paratergal scales, 29; pleural chaetotaxy, 30; scutellar scales, 29; tenth sternite, 34 ; tenth sternite similar to that of Psorophora, 131; tropical warble fly, 6; yellow fever

Acdes aegupti, 227-230: adult blocd preference, 4; dengue, 5; egg, 48; scale pattern, 29; yellow fever ....... 5

Acdes albopictus: dengue …….......... 5 Aedes at'anticus ........170-173: 214, 217 Aedes atropalpus 220-223

Aedes canadensis 173-176: 194

Aedes cantator 176-179

Aedes cinereus, 234-236. palpi of males similar to those of females .... 25

Aedes dorsalis,

179-182

Aedes dupreei

182-185

Aedes fulvus pallens

185-188

Aedes grossbechi,

Aedes infirmatus

188-191

Aedes mathesoni

191-194

Aedes mitchellae 194

Acdes nigromaculis

Aedes sollicitans, 199-203: light traps

Aedes sticticus

203-206

Acdes stimulans 206-208

Aedes taeniorhynchus, 209-211: light traps Aedes thibau!ti

211-214
Aedes tormentor

214-217

Aedes triseriatus, 224-227: association

with Deinocerites cancer

Aedes trivittatus

217-220

Aedes vexans, 230-233: flight range.. 4

Aedimorphus, subgenus of Aedes ......165 aegypti (see Aedes).

air tube (see siphon).

alba (see Orthopodomyia).

albimanus (see Anopheles).

albopictus (see $A$ edes).

alula

anal cell

anal gills ...........................40, 44, 46, 47

anal hair ........................................40, 42

anal lobe …........................................ 36

anal membrane, .................................... 34

anal segment: adults, 33 ; larvae .......... 43

anal vein .................................................. 31

androphilism .............................. 3

Anopheles, 50: abdomen of larva, 45,

47; adult hibernation, 4; association

with Uranotaenia, 100, Culex erraticus, 265; basal lobe, 35; bird malaria, 5; cibarial armature. 16; claspettes, 36; daytime resting shelters, 7; dististyle, 36; distribution, 50; eggs, 2, 18, 48; filariasis, 6; frontal tuft, 25; head of larva, 43; heartworm of dogs, 6; human malaria, 4 5 ; key to adult females, male terminalia and larvae, 50-52; larval collecting, 19; larval feeding, 2; male terminalia, 36; phallosome, 34; respiratory trumpet of pupa, 37 ; tenth sternite, 34; terminal abdominal segments of pupa, 38; thorax, 44 ; tropical warble fly

Anopheles albimanus ................80-83

Anopheles atropos, 52-55: larval association with $A$. crucians bradleyi, 60 ; light traps 10

Anopheles barberi, 55-58

Anopheles crucians bradlevi .........58-60

Anopheles crucians crucians, 60-64: light traps 10 Anopheles crucians georgianus, ......64-66 Anopheles maculipennis: eggs ........... 18 Anopheles maculipennis freeborni: encephalitides, 6; human malaria ....... 5

Anopheles pseudopunctipennis pseudopunctipennis 66-69 Anopheles punctipennis

69-72 
Anopheles quadrimaculatus, 73-76: association with Uranolaenia sapphirina, 107, Culex erraticus, 265; human malaria, 5; light traps

Anopheles walkeri, habits of adults, 3, 4; larval characters, 44-47; scaling or dorsum and venter, 32; scutellum

Anophelini, tribe of Culicinae, 50; abdominal chaetotaxy of pupa, 39; key, 49; respiratory trumpets, 39; salient characters

antenna: adult, 24, 25; larva, .......41, 42 antennal tuft or hair: Anopheles, 43.

44; culicines, 41,42

antepalmate hair, 45,47

anterior plate 46

anterior pronotal lobe 28

anterior pronotum 27

apical lobe

apicalis (see Culex).

aspirator, 34,35

atlanticus (see Aedes).

atralus (see Culex).

atropalpus (see Aedes).

atropos (see Anopheles).

axillary cell

bahamensis (see Culex).

barberi (see Anopheles).

barred area

basal arm of tenth sternite

basal hairs,

$41,+2,+3,46$

basal lobe 34,35

basal plate 34,35

basistyle, 34,35

bimaculatus (see Aedes fuivus pallens).

bird malaria, 5: transmitted by Culex pipiens, 247, C. quinquefasciatus ...250

Bironella: distribution, 50; palmate hair on mesothorax, 47; palpi, 25; scutellum 50

biting collections 10, 11

bradleyi (see Anopheles).

Bromeliaceae $91,99,114$

canadensis (see Aedes).

cancer (see Deinocerites).

cantator (see Acdes).

capitellum

caudal hairs: lower, $40,43,46,47$; up-

per $\quad 40,43,46,47$

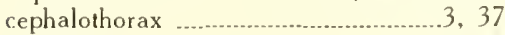

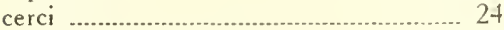

chaetotaxy: of adult, 28, 29; of larva, 39-47; of pupa ........................ 39

Chagasia, genus of Anophelini: distri-

bution, 50; palpi of adults
Chaoborinae, subfamily of Culicidae: key

chloral gum: mounting male terminalia,

15; preparation and use of .........21, 22

chorion ……...................................... 47

cibarial armature .................................. 16

ciliata (see Psorophora).

cinereus (see Acdes).

claspette

claw (see also ungues): on dististyle, $34,35,36$

Climacura, subgenus of Culisela:

107

clypeal hairs: inner, 43, 46; outer, 43 . 46; post ….....................41, $42,43,46$

clypeus: adult, 25; larva .................... 39

collar

collecting methods: adults, 6-13; eggs,

18; larvae .......................... 18-20

comb

40,42

comb scales

40,42

compound eyes

25

confinnis (see Psorophora)

Coquillettidia, subgenus of Mansonia:

127

costal vein

coxae

28,30

cratal tufts

cross-veins

crown of tenth sternite

crucians bradleyi (see Anopheles).

crucians crucians (see Anopheles).

crucians georgianus (see Anopheles).

Culex, 236: accessory paddle hair, 39; adult hibernation, 4; basal lobe, 35; bird malaria, 5; daytime resting shelters, 7; egg, 2, 48; eighth abdominal segment of adult, 33; encephalitides, 6; filariasis, 6; heartworm of dogs, 6 key to adult females, male terminalia and larvae, 237-239; male terminalia, 35 ; phallosome, 34 ; pleural chaetotaxy, 30; preparing male terminalia, 15, prothorax, 27; pulvilli, 31; scutal scales, 29; similarity to Culiseta, 107; to Deinocerites, 274; subapical lobe, 35 ; tenth sternite, 34 ; terminal abdominal segments of pupa, 38: tropical warble fly, 6; ungues, 31: yellow fever

Culex, subgenus of Culex, 237: tenth sternite

Culex apicalis, 271-274: egg raft .... 2

Culex atratus ...........................260-262

Culex bahamensis _....................240-242

Culex erraticus …..................262-265

Culex fatigans (see C. qinquefasciatus).

Culex nigripalpus 242-245: scales on scutum 
Culex peccator, 265-268: scales on scutum 263

Culex pilosus, 268-271: scales on scutum

Culex pipiens, 245-247: bird malaria, 5; encephalitides

Culex quinquefasciatus, 247-250: bird malaria, 5; biting, 253; similarity to C. pipiens 245,246

Culex resiuans, 250-253: scales on scutum ..................... 271

Culex salinarius, 253-256: biting, 245; scales on scutum 271

Culex tarsalis, 256-259: encephalitides

Culex tritaeniorhynchus: encephalitides.. 6

Culicidae, 48: keys to subfamilies ...48, 49

Culicinae, subfamily of Culicidae, 49 : key, 49; key to tribes, 49 ; rotation of male terminalia, 33; wing venation.. 32

culicines ( $=$ tribe Culicini): eggs of, 47, 48; habits of adults, 3, 4; homology of pecten, 47; larval feeding habits, 2; respiratory trumpets of pupae, 39; scales on dorsum and venter, 32; scutellum, 29; taxonomic characters of larvae $41-44$

Culicini, tribe of Culicinae, 88: abdominal chaetotaxy of pupae, 39; key .. 49

Culiseta, 107: egg, 2; bird malaria, 5; encephalitides, 6; key to adult females, male terminalia and larvae. 108; prothorax, 27; tenth sternite .. 34

Culiseta inornata, 108-111: encephalitides

Culiseta melanura

112-114

cyanescens (see Psorophora).

Deinocerites, 274: palpi of males, 25; phallosome

Deinocerites cancer, 275-277: antennae of males, 25 ; scales on sternopleura

dengue 5. 230

Dermatobia hominis, transmitted by mosquitoes

Dirofilaria immitis, transmitted by mosquitoes .... 6,247

discolor (see Psorophora).

discrucians (see Psorophora varipes).

dissection for malaria parasites (see malaria).

dissection of male terminalia (see terminalia).

dististyle $34,35,36$

diverticula: dorsal, 17; ventral ...-.... 17

Dixinae, subfamily of Culicidae, key, 48,49 dorsal anterior submedian hair (see submedian hairs).

dorsal arm of phallosome .35

dorsal arm of tenth sternite ...............34, 35

dorsal brush $40,43,46,47$

dorsal hairs

dorsal lobe of claspette .......................... 36

dorsal plate ....................40, 43, 46, 47

dorsal preapical spine ....................... 40

dorsal submedian prothoracic hairs (see

(submedian prothoracic hairs).

dorsal valve (see median valve).

dorsalis (see Aedes).

dorso-central bristles

28,29

dorsum

dupreci (see Aedes).

egg: characters of, 47-48; collection,

18; life history, 2; raft of Culex

apicalis

egg breaker

egg raft

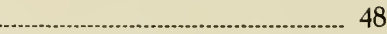

eighth abdominal segment .................... 33

elephantiasis (see filariasis).

empodium

encephalitides

endochorion ................................................ 47

epicranial plate (see occular sclerite).

epicranial suture, 39; anopheline larvae.

equine encephalitis: transmitted by mos-

quitoes $\ldots . .179,223,227,233,247,259$

Eretmapodites: yellow fever

erraticus (see Culex).

euparal

15. 22

exochorion

eye: imaginal, 24,42 ; larval

fatigans (see Culex quinquefasciatus).

femur ........................................... 30

ferox (see Psorophora).

filament of claspette .......................34, 37

filariasis (see also Wuchereria ban-

crofti and $W$. malayi): transmitted

by mosquitoes …............................ 5-6

finger-like process ........................... 43,44

Finlaya, subgenus of Aedes .................165

flagellum ............................................. 25

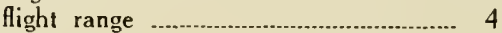

floats ................................................... 47

food: adults, 3; larvae .................... 2

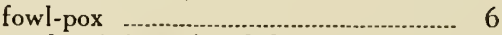

freeborni (see Anopheles).

frill ... 47

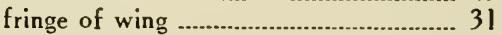

fringe scales ……............................... 32

frons

frontal hairs: inner, 43, 46; middle, 43 . 46; outer .43, 46 
fronto-clypeus, 39; of Anoplicles 43 fulvus pallens (see Aedes).

georgianus (see Anopheles).

Grabhamia, subgenus of Psorophora 130,131

grid (see barred area).

grossbechi (see Aedes).

Haemagogus: tropical warble fly, 6; yellow fever $24,28,32$

halter

head: adult, 24-27; larva $39,41,42,43,44,46$

heartworm of dogs (see Dirofilaria immitis)

hibernation

hind-gut

hirsuteron (see Aedes sticticus).

horrida (see Psorophora).

howardii (see Psorophora).

humeral cross-vein

hypopharynx

identification of specimens

indubitans (see Mansonia).

infirmalus (see Aedes).

inflated siphon

infraorbital hairs

inornata (see Culiseta).

instar

interbasal folds

internal spine

intersegmental membrane

Janthinosoma, subgenus of Psorophora

130,131

killing specimens: adults 14; larvae .... 20

labella

labium: adult, 25; larva

41

labrum

39

labrum-epipharynx …............................ 27

larvae: collecting methods, 18-20; food of, 2: life history, 2, 3; rearing, storing and mounting, 20-23; structures of 39.47

lateral flap 46

lateral hairs: abdomen (culicines) 40. 42; larval thorax (anophelines), 45. 46, 47; larval thorax (culicines), 44; of anal segment ..............40,43,46, 47

lateral teeth of phallosome ................. 35

lateral valves …..................................... 42

leaflets of phallosome ............................ 36

legs ………......................................... 30

leishmaniasis ........................ 6

life history: adult, 3, 4; egg, 2; larva,

2. 3; pupa light traps: New Jersey

Limatus: yellow fever

lobe of ninth tergite

5

(see Psorophora)

longitudinal veins ................................. 31

lower caudal hair (see caudal hairs).

lower head hairs .............................41, 42

lower lateral abdominal hair (see abdominal hair).

lowii (see Uranolaenia).

lutzii (see Psorophora).

maculipentris (see Anopheles).

malaria: bird. 5: dissection for parasites, 16; human 4. 5 male terminalia (see terminalia).

Malpighian tubules

mandibles: adult, 27; larva

Mansonia, 120: egg, 2; eighth abdominal segment of adult, 33; filariasis, 6; larval habits, 3; pleural chaetotaxy, 30; pupal habits, 3; tropical warble $\mathrm{fly}, 6$; yellow fever ….......... 5

Mansonia indubitans, 121-123: dorsal submedian prothoracic hairs

Mansonia perturbans, .............127-130

Mansonia titillans, 124-127: dorsal submedian prothoracic hairs ........... 41

marginal cells ................................ 31

mathesoni (see Aedes).

maxillae: adult. 27: larva

maxillary palps: adult, 24, 25: Anophelini \& Bironella adults, 50; anopheline larvae 43 meatus 37,39 median plate .......................................... 46 median valve ....................................... 42

medical importance of mosquitoes .........4-6

Megarhinini, tribe of Culicinae. 83: absence of comb scales, 42: chaetotaxy of pupal abdomen, 39; key 49

Megarhinus, 83: key to adult males. 84: larval feeding, 2; mouthbrushes. 39 ; pecten, 42 ; scutellum, 29; squama, 31; terminal abdominal segments of pupa, 38; wing venation 32

Megarhinus rutilus ............................ 84

Megarhinus seplentrionalis _...........84-88

Melanoconion, subgenus of Culex: 237 , 268, 271: cibarial armature, 16; tenth sternite 34 melanura (see Culiseta).

mentum

meron $28,29,30$ mesepimeron: 28, 29; generic value of,

mesepimeral bristles ........................... 28

mesonotum ......................................... 29

mesopleural hairs ……..................... 41 
mesosome (see phallosome).

mesothorax: adult, 28; larva 41,44

metameron

28, 30

metanotum

metapleura

..... 28

28,30

metapleural hairs ....................................... 41

metathorax: adult, 30 ; larva .. 41,44

metepisternum …............................... 30

micropylar apparatus ................................ 47

microtrichia

mid-gut

mitchellac (see Aedes).

mitchellii (see Wyeomyia).

mounting technique: adults, 12,14 ; ci-

barial armature, 16; larvae, 21-23;

male terminalia, 15,16 ; media ....21-23

mouth brushes ............................ 29, 42

Myzomyia, subgenus of Anopheles; dis-

tribution

Neoculex, subgenus of Culex, 237:

tenth sternite of

nigripalpus (see Culex).

nigromaculis (see Aedes).

ninth sternite

$33,34,35,36$

ninth tergite

$33,34,35,36$

notched organs (Organs of Nuttall and Shipley) $44,47,50$

Nyssorhynchus, subgenus of Anothe'es: characteristics of male terminalia, 50; distribution 50

occiput

24,25

Ochleratatus, subgenus of Aedes 165

ocular sclerite, 39; of Anopheles

oesophagus

oöcysts

Orthopodomyio. 114: pecten, 42 pleural chaetotaxy, 30; tarsal segments 30,31

Orthopodomyia alba 114-116

Orthopodomyia signifera 116- 119

ovary 17
17

oviduct

paddle hair

37,39

paddles

39,42

palatum

palmate hairs: abdominal, 45, 47;
mesothoracic in Bironella, 46; metamesothoracic in Bironella, 46; meta-
thoracic in Anopheles palps (see maxillary palps).

papilla $41,42,43,44$ parabasal spines 35,36

parameres

paraproct

paratergite peccator (see Culex). pecten penis

perlurbans (see Mansonia).

petiole of wing vein

phallosome

$34,35,36$

pharyngeal pump

pharynx ....................................... 17

pilosus (see Culex).

pinna

pipiens (see Culex).

Pistia

123,127

Plasmodium falciparum, 5: infection in Anopheles albimanus, $83 ; A$. crucians bradley, $60 ; A$. crucians crucians, 64; $A$. pseudopunctipennis pseudopunctipennis, 69; A. punctipennis, 72; $A$. quadrimaculatus, $76 ; A$. walkeri

Plasmodium malariae, 5: infection in Anopheles punctipennis, 72; $A$. quadrimaculatus

Plasmodium vivax, 4: infection in Anopheles albimanus, 83; $A$. atropos, 55; $A$. barberi, 58; $A$. crucians, $64 ; A$. pseudopunctipennis pseudopunctipennis, 69; $A$. punctipennis, 72; $A$. quadrimaculatus, $76 ; A$. walkeri 79 pleurae 28,29

pleural hair groups ................. $41,44,46$

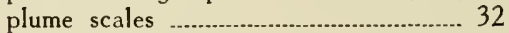

polyvinal alcohol …............................... 22, 23

posterior cell ....................................... 31

posterior pronotum ….............................. 28

postnotum …..........................24, 28, 29

postspiracular area ..........................28, 29

postspiracular bristles .................-_-_-.-... 28

postspiracular hair _............................. 46

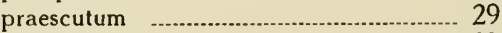

prealar area -........................................... 29

prealar bristles ....-............-.................. 28

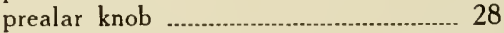

preantennal hairs

preclypeal spines …....................39, 42, 43

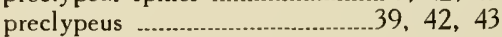

precratal tufts ....................................... 43

prementum .......................................... 41

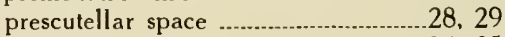

proboscis ............................................ 25

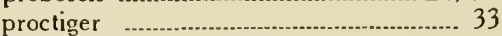

pronotal bristles: anterior, 28; posterior

pronotum 27,28

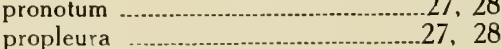

propleural bristles .............................. 28

propleural hairs ................................. 41

prosternum, 27: scaling of probable tax-

onomic value ……......................... 28

prothorax : adult, 27; larva .............41, 44

proventriculus …................................ 17 
pseudopunctipennis pseudopunclipennis (see Anopheles).

Psorophora, 130: collecting adults, 11; dististyle, 36; eggs, 2, 18; eighth abdominal segment of adult, 33; key to adult females, male terminalia and larvae, 131-133; larval feeding. 2; subventral hair tufts, 42 ; tenth sternite, 34; thorax and pleural chaetotaxy, 28, 30; tropical warble Ay, 6; vestiture of legs, 31 ; female abdomen, 33; yellow fever

Psorophora, subgenus of Psorophora

......................................... 13

Psorophora ciliata, 133-136: mouth brushes 39

Psorophora confinnis, 153-156: dististyle, 36; fed upon by $P$. ciliata ...135

Psorophora cyanescens

Psorophora discolor

139-142

sorophora ferox, 142-145, 146: dististyle, 36 ; tropical warble $\mathrm{Aly}$

Psorophora horrida 145-147

Psorophora howardii, 136-139: dististyle, 36; mouth brushes

Psorophora longipalpus

$147-150$

Psorophora lutzii: carrier of tropical warble fly

Psorophora pygmaea

$160-162$

Psorophora signipennis, 162-165: wing fringe

Psorophora varipes, 150-153: dististyle

pulvilli 31

punctipennis (see Anopheles).

pupa: life history. 3 ; taxonomic characters

pygmaea (see Psorophora).

quadrimaculalus (see Anopheles). quinquefasciatus (see Culex).

rearing methods 20

respiratory trumpets 37,39

restuans (see Cule $x$ ).

Rift Valley fever rufilus (see Megarhinus).

sabre: dorsal and ventral 43. 46 salinarius (see Culex).

salivary duct

salivary glands: dissection of sapphirina (see Uranolaenia).

Sarracenia purpurea 96

scabellum

scales: on legs, 31; on wings (fringe, plume, squame)

scape

schizogony scutal angle 28 scutellum, 24, 28, 29: in Anophelini and Chagasia scutum seplentrionalis (see Megarhinus).

shelters, 4 : artificial, 7 ; natural ......... 7 shoulders hairs (see submedian prothoracic hairs).

signifera (see Orthopodomyia).

signipennis (see Psorophora).

siphon, 40, +2: absent in Anophelini

siphonal hair .................................40, 42

siphonal index ...................................... 43

siphonal valves: culicines ….............40,42

smithii (see Wyeomyia).

sollicitans (see Aedes).

spermatheca

spiracles: anopheline larvae, 46, 47;

culicine adults, 27, 28, 30; culicine

larvae 42 spiracular apparatus: Anopheles larvá

spiracular bristles ..........................27, 28

spiracular valves: culicine larvae .... 40,42

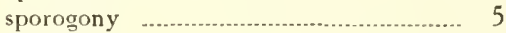

sporozoites ......................................... 16

squama ............................. 31

squame scales ......................................... 32

stable traps …….............................. 10

Stegomyia, subgenus of Aedes _........ 165

Stegomyia fasciata (see $A$ edes aegypti).

stem of claspette ...........................34, 37

sternite _............................................ 32

sternopleuı, 28, 29; of generic value

sternopleural bristles ............................ 28

Stothomyia, subgenus of Anopheles:

distribution

sticticus (see Acdes)

stimulans (see Aedes).

St. Louis encephalitis, mosquito borne.

$6,233,247,259$

storage of specimens: adults. 14: larvae

subapical lobe

sub-basal hairs

$41,42,43,46$

subcostal vein

subdorsal tufts

42

sublateral hairs.

45,47

submarginal cell

31

submedian hairs: of abdomen, 45. 47;

of prothorax (inner, median and outer) 41, 44,46 submentum subsiphonal hair …..........................40, 42 subspiracular area _.... 28, 29 subterminal spines …......................41, 42 subventral hair tuft ........................ 40.42 
supra-alar bristles

..28, 29

supra-orbital hairs $41,42,43,46$

sutural hairs $41,42,43,46$

taeniorhynchus (see Aedes).

tarsalis (see Culex).

tarsus 30

taxonomic characters: adults, 23-37;

larvae, 39-47; pupae

tenth abdominal segment

tenth sternite

$33,34,35,36$

tenth tergite

$33,34,35,36$

tergal plates 45,47

tergite 32

terminal hair of antenna 43,46

terminal spines 41,42

terminalia: female terminalia, 32; preparation for study, 15, 16; taxonomic features

Theobaldia (see Culiseta).

thiboulti (see Aedes).

thorax: adult, 27-32; anopheline larvae, 44, 46; culicine larvae 41

tibia 30

titillans (see Mansonia).

tormentor (see Aedes).

torus

24,25

transporting: adults, 13, 14; larvae .... 20

trans-sutural hairs .................41, 42, 43, 46

Trichoprosopon: carrier of tropical

warble fly

triserialus (see Aedes).

tritaeniorhynchus (see Culex).

trivittatus (see Aedes).

trochanter 30

tularemia 6

ungues 31

upper caudal hair (see caudal hairs).

upper head hairs 41,42

upper lateral abdominal hair (see lateral hairs).
Uranotaenia, 100: egg, 2; palpi of males. 25; phallosome, 34; prothorax, 27; squama, 31; tenth sternite, 34 ; wing venation

Uranotaenia lowii, 101-104: antennae of males, 25; dorsal submedian prothoracic hairs

Uranotaenia sapphirina

104-107

vanduzeei (see $W$ yeomyia).

venter 32

ventral arm of phallosome

ventral brush 35

ventr...40,43, 46, 47

ventral submedian hair .........--....... 44,46

ventro lateral posterior hair .................... 46

vertex .........................................24, 25

vexans (see Aedes).

vitelline membrane

walkeri (see Anopheles).

Western equine encephalitis: mosquito borne 6,111

wing $24,31,32$

Wuchereria bancrofli: mosquito borne $5,247,250$

Wuchereria malayi: mosquito borne .. 6

$W$ yeomyia, 89: antennae of males, 25 ; anterior pronotum, 27; eighth abdominal segment of adult, 33; key to adult females, male terminalia and larvae, 90; palpi of males, 25; pecten, 42; pleural chaetotaxy, 30; postnotal tuft, 30; squama, 31; tropical warble $\mathrm{fl}, 6$; yellow fever 90-93 $W$ yeomyia mitchellii 93.96 $W$ yeomyia smithii, 96-99

Wyeomyia vanduzeei 6-99 yellow fever: transmitted by mosquitoes 5, 227,230 







